1987

\title{
Census of
}

\section{$C$ Agriculture}

$3 \cdot \frac{31 / 4:}{\text { AC8 } / A .8} \cdot 1 /$ PT. 8

GEOGRAPHIC AREA SERIES

Part 8

\section{Delaware}

State and County Data

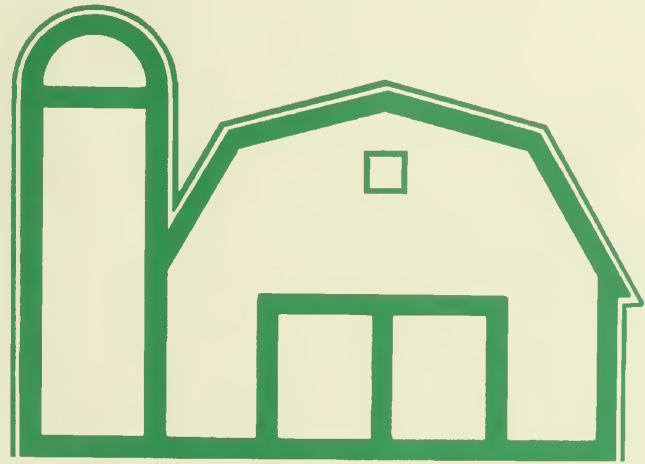

U.S. Department of Commerce

BUREAU OF THE CENSUS 


\section{ACKNOWLEDGMENTS}

This report was prepared in the Agriculture Divisın. Many ther divisions contributed to this preparation: Data Preparation Ferformed the clerical processing; Administrative Services provided the forms design and other administrative services; Publications Services contributed in publication planning and design, editorial review, composition, and printing procurement; Computer Services provided the computer processing facilities; Field provided selected data collection activities; Economic Programming prepared the computer programs; and Economic Surveys assisted in preparation of data collection and processing procedures and computer programs.

Members of the Census Advisory Committee on Agriculture Statistics and representatives of both public and private organizations made significant recommendations which helped establish data content.

Members of various agencies of the U.S. Department of Agriculture provided valuable advice in the planning, publicizing. and processing phases of the census, and in helping farmers and ranchers complete the report forms.

The press, farm magazines, radio and television stations, and farm organizations were most helpful in publicizing the census and encouraging cooperation of farm and ranch operators.

Special tribute is paid to the millions of farm and ranch operators who furnished the information requested. Only through their cooperation was it possible to collect and publish the data in this report.

If you have any questions concerning the statistics in this report, call:

(301) 763-8555 Division Chief

(301) 763-8567 Crops Branch

(301) 763-8569 Livestock Branch

(301) 763-8566 Farm Economics Branch

(301) $763-1113$ General Information

(301) 763-8558 Statistical Methodology 


\section{7 \\ Census of Agriculture}

\section{AC87-A-8 \\ Volume 1 GEOGRAPHIC AREA SERIES}

Part 8

Delaware

State and County Data

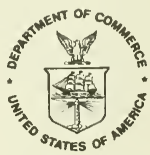

U.S. Department of Commerce C. William Verity, Secretary Donna F. Tuttle, Deputy Secretary Robert Ortner, Under Secretary for Economic Affairs 


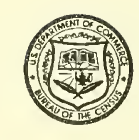

\title{
BUREAU OF THE CENSUS
}

C. L. Kincannon, Deputy Director

\author{
Charles A. Waite, Associate Director for \\ Economic Programs \\ Roger H. Bugenhagen, Assistant Director for \\ Economic and Agriculture Censuses
}

Thomas L. Mesenbourg, Chief, Economic Census Staff

AGRICULTURE DIVISION Charles P. Pautler, Jr., Chief

\section{Library of Congress Cataloging-in-Publication Data}

Census of agriculture (1987). Geographic area series. 1987 census of agriculture. Geographic area series.

Includes indexes.

Supt. of Docs. no.: C 3.31/4:987/v.1

1. Agriculture-Economic aspects-United StatesStatistics. 2. Agriculture-Economic aspects-United States-States-Statistics. 3. Agriculture-Economic aspects-United States-Territories and possessions -Statistics. I. United States. Bureau of the Census. II. Title.

HD1769.C46 $1987 \quad 338.1^{\prime} 0973^{\prime} 021 \quad 88-600103$

For sale by Superintendent of Documents, U.S. Government Printing Office, Washington, DC 20402. 


\section{FIGURES}

1. State Map

2. Profile of State's Agriculture: 1987

3. Percent of Farms and of Value of Products Sold: 1987

4. Farms by Value of Agricultural Products Sold: 1959 to 1987

5. Land Use: 1987

6. Selected Crops Harvested: 1987

7. Value of Livestock and Poultry Sold: 1987

8. Production Expenses: 1987

\section{TABLES}

\section{CHAPTER 1. State Data}

1. Historical Highlights: 1987 and Earlier Census Years

2. Market Value of Agricultural Products Sold: 1987, 1982, and 1978

3. Farm Production Expenses: 1987, 1982, and 1978

4. Net Cash Return From Agricultural Sales: 1987

5. Government Payments and Other Farm-Related Income: 1987 and 1982

6. Commodity Credit Corporation Loans: 1987 and 1982

7. Land Use and Acres Diverted: 1987, 1982, and 1978

8. Land in Farms, Harvested Cropland, and Irrigated Land, by Size of Farm: 1987 and 1982

9. Irrigation: 1987, 1982, and 1978

10. Selected Characteristics of Irrigated and Nonirrigated Farms: 1987 and 1982

11. Value of Land and Buildings: 1987, 1982, and 1978

12. Value of Machinery and Equipment on Place: 1987 and 1982

13. Selected Machinery and Equipment on Place: 1987 and 1982

14. Petroleum Products Expenses: 1987, 1982, and 1978

15. Agricultural Chemicals Used, Including Fertilizer and Lime: 1987, 1982, and 1978

16. Tenure and Characteristics of Operator and Type of Organization for All Farms and Farms Operated by Black and Other Races: 1987, 1982, and 1978

17. Selected Characteristics of Farms Operated by Females, Persons of Spanish Origin, and Specified Racial Groups: 1987 and 1982

18. Selected Characteristics of Farms by Standard Industrial Classification: 1987

19. Selected Characteristics of Abnormal Farms: 1987 and 1982

20. Livestock and Poultry-Inventory and Sales: 1987, 1982, and 1978

21. Poultry-Inventory and Sales: 1987 and 1982

22. Broilers and Started Pullets-Sales: 1987 and 1982

23. Poultry - Inventory and Sales by Size of Flock: 1987

24. Turkeys - Sales by Number Sold Per Farm: 1987

25. Cattle and Calves - Inventory: 1987 and 1982

26. Cattle and Calves-Sales: 1987 and 1982

27. Cattle and Calves - Inventory and Sales by Size of Herd: 1987

28. Cattle and Calves - Inventory and Sales by Size of Cow Herd: 1987

29. Cattle and Calves - Inventory and Sales by Size of Beef Cow Herd: 1987

30. Cattle and Calves - Inventory and Sales by Size of Milk Cow Herd: 1987

31. Cattle and Calves - Sales by Number Sold Per Farm: 1987

32. Hogs and Pigs-Inventory: 1987 and 1982

33. Hogs and Pigs-Sales: 1987 and 1982 
34. Hogs and Pigs - Litters Farrowed: 1987 and 1982

35. Hogs and Pigs-Inventory and Sales by Size of Herd: 1987

36. Hogs and Pigs - Inventory and Sales by Number Sold Per Farm: 1987

37. Hogs and Pigs - Inventory, Sales, and Litters by Total Farrowed: 1987

38. Sheep and Lambs - Inventory and Sales: 1987 and 1982

39. Sheep and Lambs - Inventory and Sales by Size of Flock: 1987

40. Sheep and Lambs - Inventory and Sales by Size of Ewe Flock: 1987

41. Other Livestock and Livestock Products - Inventory and Sales: 1987 and 1982

42. Crops Harvested and Value of Production: 1987 and 1982

43. Specified Crops Harvested-Yield Per Acre Irrigated and Nonirrigated: 1987

44. Specified Crops by Acres Harvested: 1987 and 1982

45. Specified Fruits and Nuts by Acres: 1987 and 1982

46. Nursery and Greenhouse Crops, Mushrooms, and Sod Grown for Sale by Value of Sales: 1987 and

Farms by Concentration of Market Value of Agricultural Products Sold: 1987 .

47. Farms by Concentration of Market Value

49. Summary by Type of Organization: 1987

50. Summary by Age and Principal Occupation of Operator: 1987

51. Summary by Size of Farm: 1987

52. Summary by Value of Agricultural Products Sold: 1987

53. Summary by Standard Industrial Classification of Farm:

1987

\section{CHAPTER 2. County Data}

1. County Summary Highlights: 1987

2. Market Value of Agricultural Products Sold and Farms by Standard Industrial Classification: 1987 and 1982

3. Farm Production Expenses: 1987 and 1982

4. Net Cash Return From Agricultural Sales, Government Payments, Other Farm-Related Income, and

Commodity Credit Corporation Loans: 1987 and 1982

5. Farms, Land in Farms, and Land Use: 1987 and 1982

6. Harvested Cropland by Size of Farm and Acres Harvested: 1987 and 1982

7. Irrigation: 1987 and 1982

8. Machinery and Equipment on Place: 1987 and 1982

9. Agricultural Chemicals Used, Including Fertilizer and Lime: 1987 and 1982

10. Tenure and Characteristics of Operator and Type of Organization: 1987 and 1982

120

11. Cattle and Calves-Inventory and Sales: 1987 and 1982

12. Hogs and Pigs - Inventory, Litters, and Sales: 1987 and 1982

13. Sheep and Horses - Inventory and Sales: 1987 and 1982

14. Poultry - Inventory and Sales: 1987 and 1982

15. Selected Crops: 1987 and 1982

16. Farms With Sales of $\$ 10,000$ or More: 1987 and 1982

17. Milk Goats-Inventory and Sales: 1987 and 1982

18. Angora Goats-Inventory and Sales: 1987 and 1982

19. Mink and Their Pelts - Inventory and Sales: 1987 and 1982

20. Colonies of Bees and Honey-Inventory and Sales: 1987 and 1982

21. Fish Sales: 1987 and 1982

22. Miscellaneous Poultry - Inventory and Sales: 1987 and 1982

23. Miscellaneous Livestock and Animal Specialties - Inventory and Sales: 1987 and 1982

24. Grains - Corn, Sorghum, Wheat, and Other Small Grains: 1987 and 1982

25. Cotton, Tobacco, Soybeans, Dry Beans and Peas, Potatoes, Sugar Crops, and Peanuts: 1987 and 1982.

26. Field Seeds, Grass Seeds, Hay, Forage, and Silage: 1987 and 1982

27. Vegetables, Sweet Corn, and Melons Harvested for Sale: 1987 and 1982

28. Fruits and Nuts: 1987 and 1982

29. Berries Harvested for Sale: 1987 and 1982

30. Nursery and Greenhouse Crops, Mushrooms, and Sod Grown for Sale: 1987 and 1982 


\section{APPENDIXES}

A. General Explanation

B. Places With All Cropland in the Conservation Reserve Program

C. Statistical Methodology

D. Report Form and Information Sheet

Index

Index 1

Publication Program Inside back cover

"Not published for this State. 



\section{INTRODUCTION}

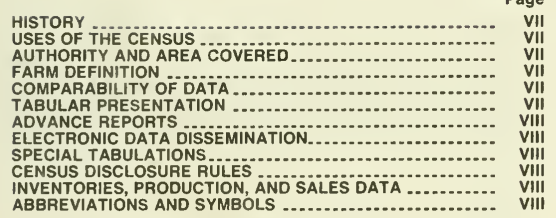

\section{HISTORY}

The 1987 Census of Agriculture is the 23d taken by the U.S. Department of Commerce, Bureau of the Census. The first agriculture census was taken in 1840 as part of the sixth decennial census of population. From 1840 to 1950 , an agriculture census was taken as part of the decennial census. A separate mid-decade census of agriculture was conducted in 1925, 1935, and 1945. From 1954 to 1974, a census of agriculture was taken for the years ending in 4 and 9. In 1976, Congress authorized the census of agriculture to be taken for 1978 and 1982 to adjust the data reference year so that it coincided with the economic censuses covering manufacturing, mining, construction, retail trade, wholesale trade, service industries, and selected transportation activities. This adjustment in timing established the agriculture census on a 5-year cycle collecting data for years ending in 2 and 7.

\section{USES OF THE CENSUS}

The census of agriculture is the leading source of statistics about the Nation's agricultural production and the only source of consistent, comparable data at the county, State, and national levels. Census statistics are used by Congress in developing and changing farm programs and for determining the effects of these programs. Many national and State programs are designed or allocated on the basis of census data, such as funds for extension services, research, and soil conservation projects. Private industry uses census statistics to provide a more effective production and distribution system for the agricultural community.

\section{AUTHORITY AND AREA COVERED}

The census of agriculture is required by law under Title 13, United States Code, sections 142(a) and 191, which directs that a census be taken in 1979,1983, and in every fifth year after 1983 covering the prior year. The 1987 census includes each State, Puerto Rico, Guam, and the Virgin Island of the United States. A census of agriculture will be conducted in American Samoa and the Commonwealth of the Northern Mariana Islands in conjunction with the 1990 Census of Population and Housing.

\section{FARM DEFINITION}

Since 1850 , when minimum criteria defining a farm for census purposes first were established, the farm definition has been changed nine times. The current definition, first used for the 1974 census, is any place from which $\$ 1,000$ or more of agricultural products were produced and sold or normally would have been sold during the census year. The farm definition used for the outlying areas varies according to area. The report for each area includes a discussion of the farm definition.

\section{COMPARABILITY OF DATA}

Data on acreages and inventories for 1987 and 1982 are generally comparable. Dollar figures shown for expenses and agricultural product sales are expressed in current dollars and have not been adjusted for inflation or deflation. In general, data for censuses since 1974 are not fully comparable with data for 1969 and earlier censuses due to changes in the farm definition.

The 1978 U.S., region, and State data shown in the 1978 Census of Agriculture publications included data for farms on the mail list plus estimates from an area sample for farms not on the mail list. For comparability, the 1978 data in the 1987 publications include only farms on the mail list.

\section{TABULAR PRESENTATION}

State data-Tables 1 through 47 in chapter 1 show detailed State-level data usually accompanied by historical data for one or more past censuses. Tables 48 through 53 provide 1987 State data cross-tabulated by various farm classifications.

County data-Chapter 2 presents selected data items by county. Tables 1 through 16 include general data for all counties. The counties are listed in alphabetical order in the column headings. Tables 17 through 36 include only 
counties reporting the data item. Counties not having the item, or with a limited number of farms reporting the item, have data combined and presented as "all other counties."

\section{ADVANCE REPORTS}

Advance reports of 1987 census data have been published separately for each county with 10 farms or more, each State, and the United States. This series provided, at the earliest possible date, final data on major data items together with comparable final data from the 1982 census. Data items are standard across States except information on selected crops harvested, which vary by State according to their relative importance in the State.

\section{ELECTRONIC DATA DISSEMINATION}

The volume 1 data are available on computer tapes and compact disc. The advance report data are available on computer tapes, computer diskettes, and through electronic data services such as the AGRIDATA network, the CENDATA package on Dialog, CompuServe on-line services, and the Census Bureau's State Data Center Bulletin Board. Computer tapes, diskettes, and compact discs are sold by the Customer Services Branch, Data User Services Division, Bureau of the Census, Washington, D.C. 20233 (telephone (301) 763-4100).

\section{SPECIAL TABULATIONS}

Custom designed tabulations can be developed to individual user specifications on a programming cost reimbursable basis. Inquiries about special tabulations should be directed to the Chief, Agriculture Division, Bureau of the Census, Washington, D.C. 20233.

\section{CENSUS DISCLOSURE RULES}

In keeping with the provisions of Title 13, United States Code, no data are published that would disclose the operations of an individual farm. However, the number of farms in a given size category or other classification, such as size of farm, is not considered a release of confidential information and is provided even though other information is withheld.

\section{INVENTORIES, PRODUCTION, AND SALES DATA}

Inventories of livestock, poultry, and machinery and equipment are measured as of December 31 of the census year. Crop and livestock production, sales, and expense data are for the calendar year, except for a few crops (such as citrus) for which the production year overlaps the calendar year.

\section{ABBREVIATIONS AND SYMBOLS}

The following abbreviations and symbols are used throughout the tables:

- $\quad$ Represents zero.

(D) Withheld to avoid disclosing data for individual farms.

(IC) Independent city.

(NA) Not available.

(S) Withheld because estimate did not meet publication standards on the basis of either the response rate (associated relative standard error) or a consistency review.

(X) Not applicable.

(Z) Less than half of the unit shown.

cwt Hundredweight.

sq $\mathrm{ft}$ Square feet. 


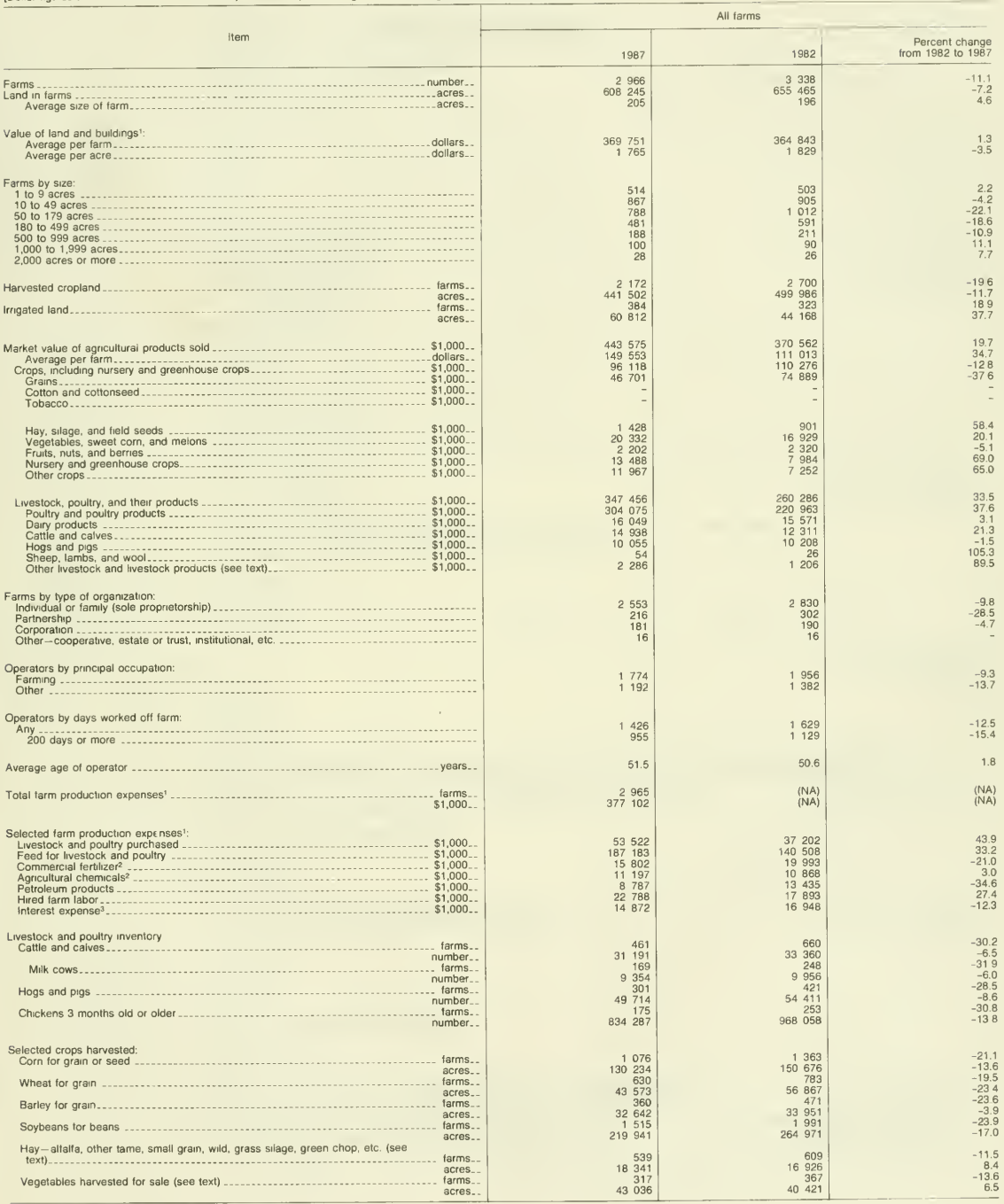

Data are based on a sample of farms.

2Data for 1987 include cost of custom applications

Data for 1982 do not include imputation for item nonresponse. 
Figure 1. State Map

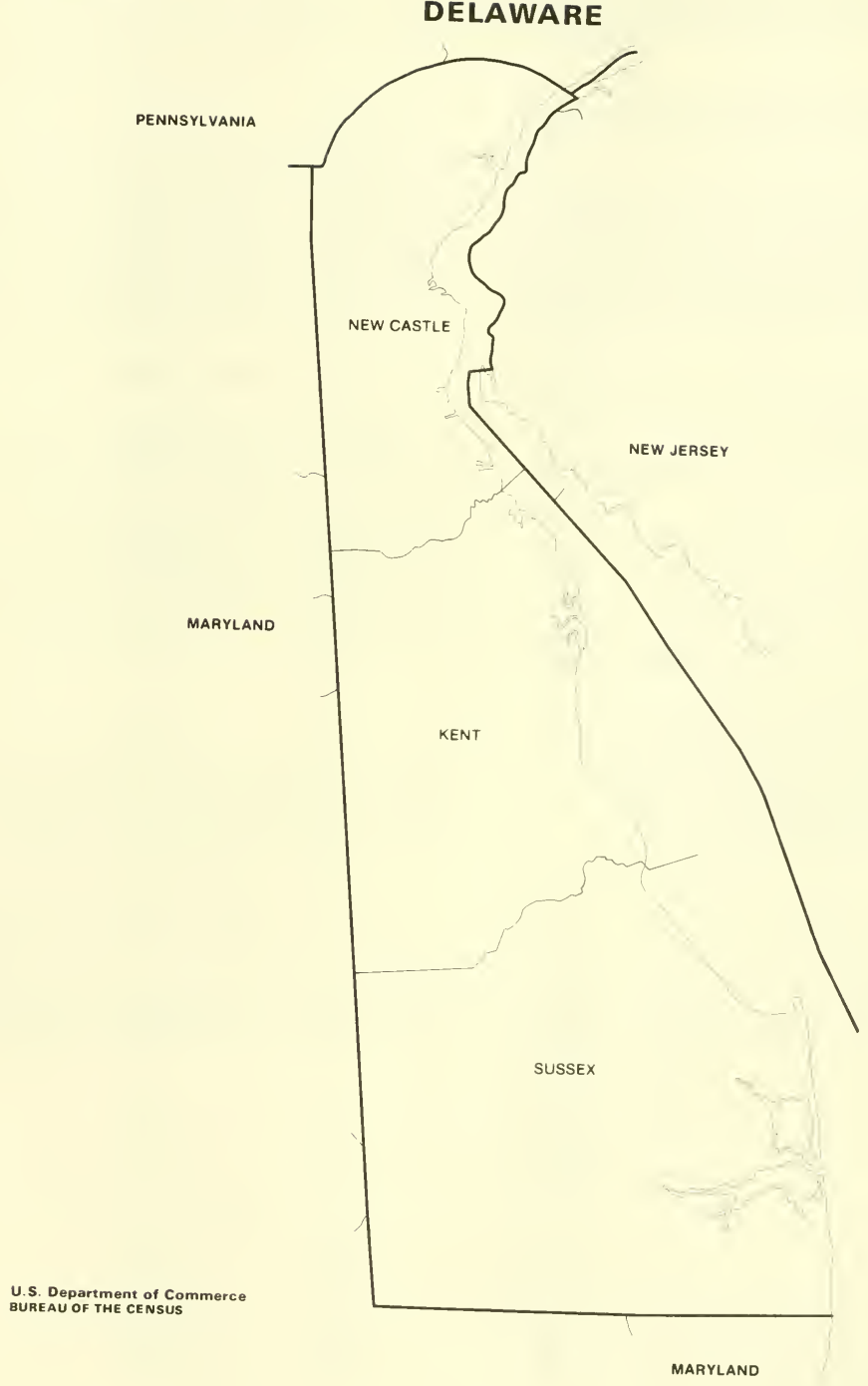


Figure 2. Profile of State's Agriculture: 1987

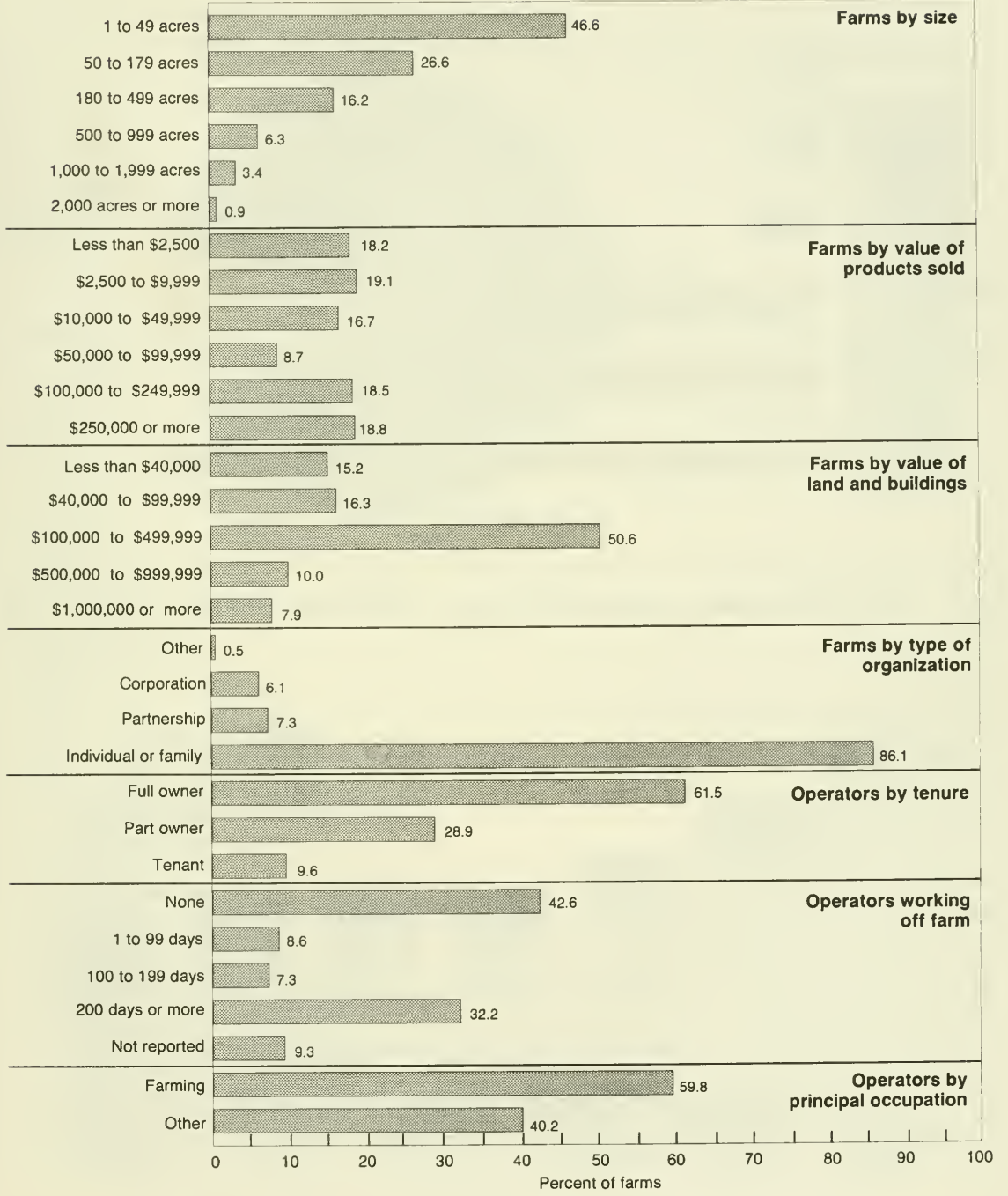


Figure 3. Percent of Farms and of Value of Products Sold: 1987

Value of sales

Less than $\$ 10,000$

0.8

$\$ 10,000$ to $\$ 49,999$

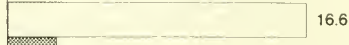

2.7

$\$ 50,000$ to $\$ 99,999$

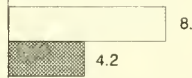

8.7 Number of farms

Value of agricultural products sold

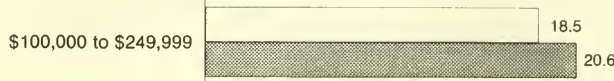

$\$ 250,000$ to $\$ 499,999$

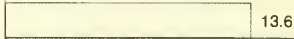

0.6

$\$ 500,000$ or more

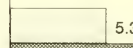

30.2

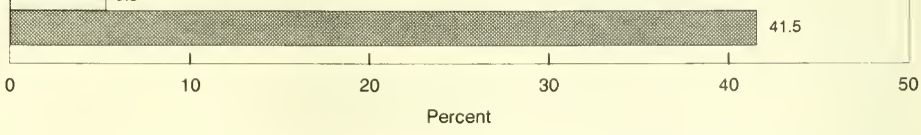

Figure 4. Farms by Value of Agricultural Products Sold: 1959 to 1987

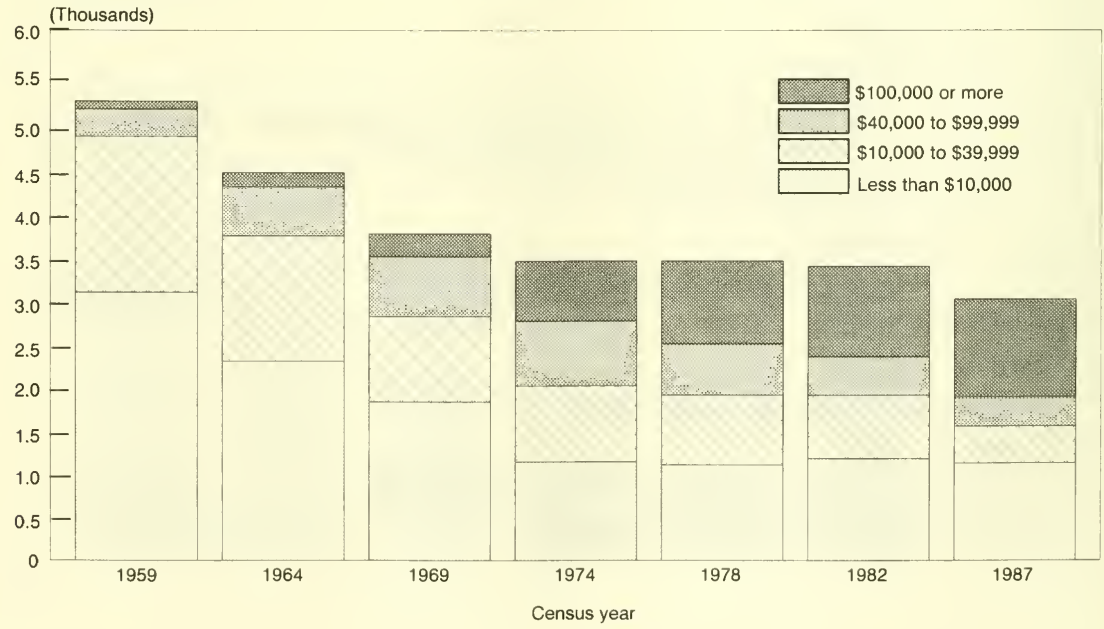


Figure 5. Land Use: 1987

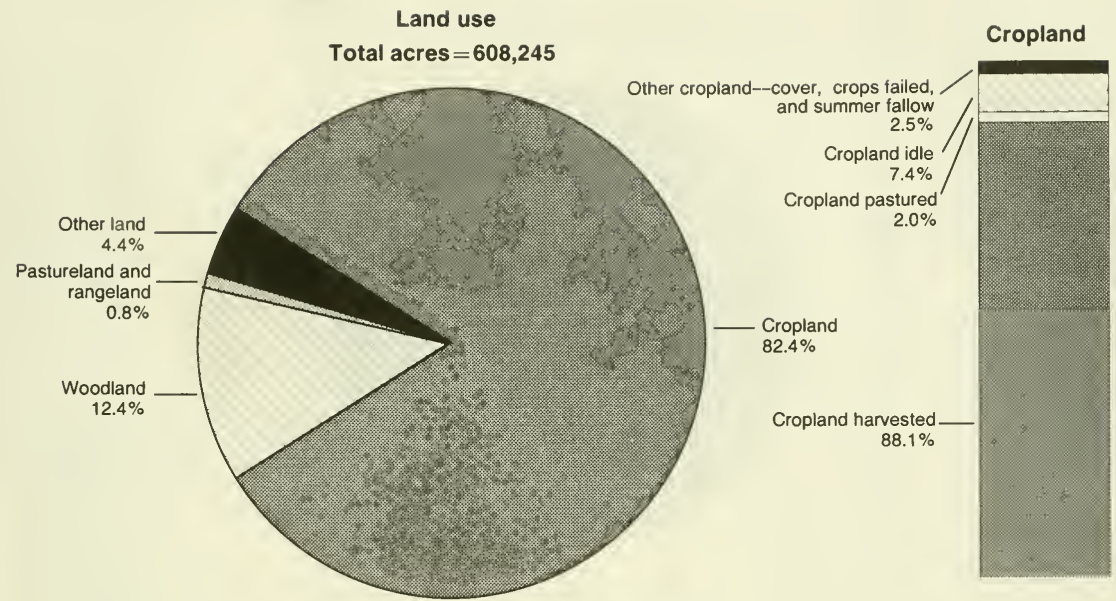

Figure 6. Selected Crops Harvested: 1987

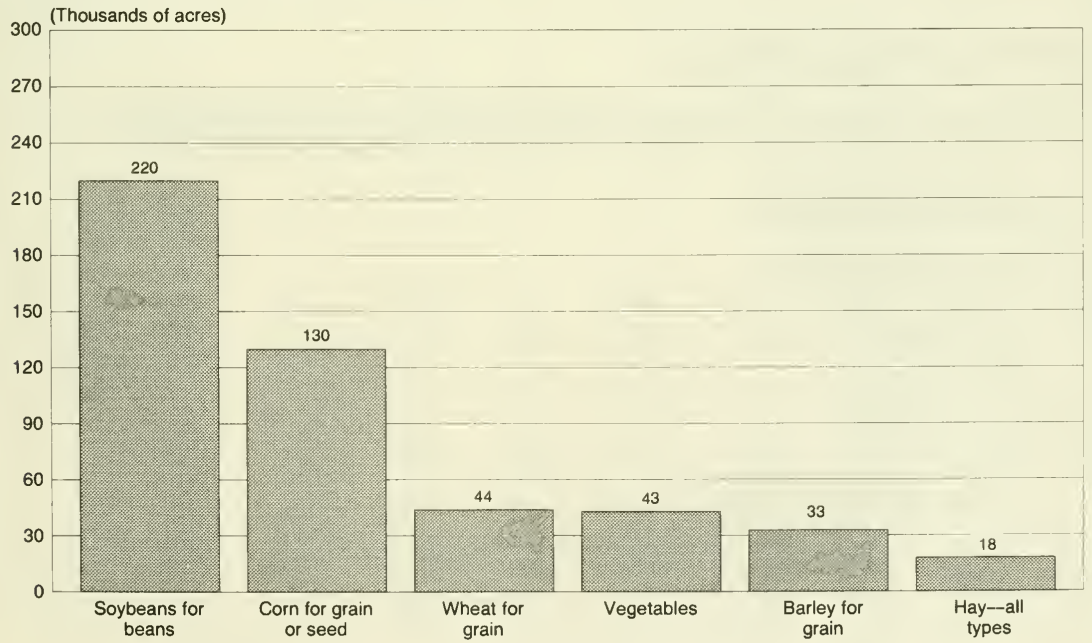


Figure 7. Value of Livestock and Poultry Sold: 1987

\section{State total $=\$ 347,456,000$}

Poultry and poultry products $87.5 \%$

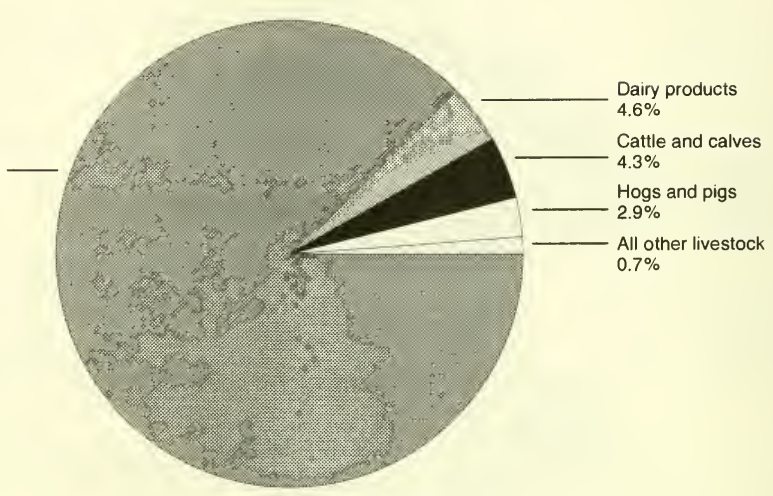

Figure 8. Production Expenses: 1987

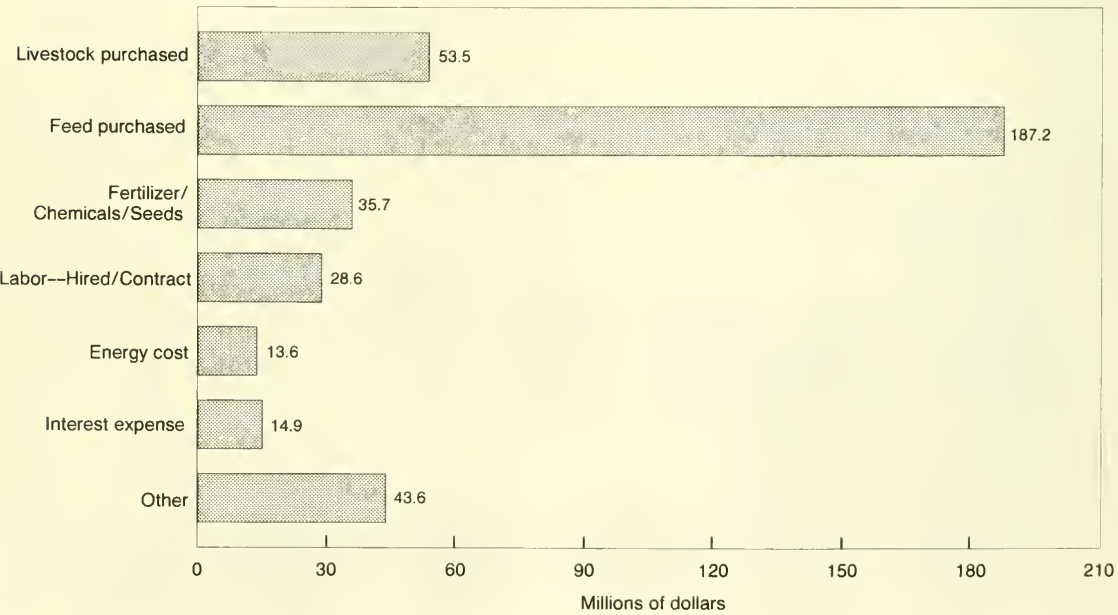


Table 1. Historical Highlights: 1987 and Earlier Census Years

[For meaning of abbreviations and symbols, see introductory text]

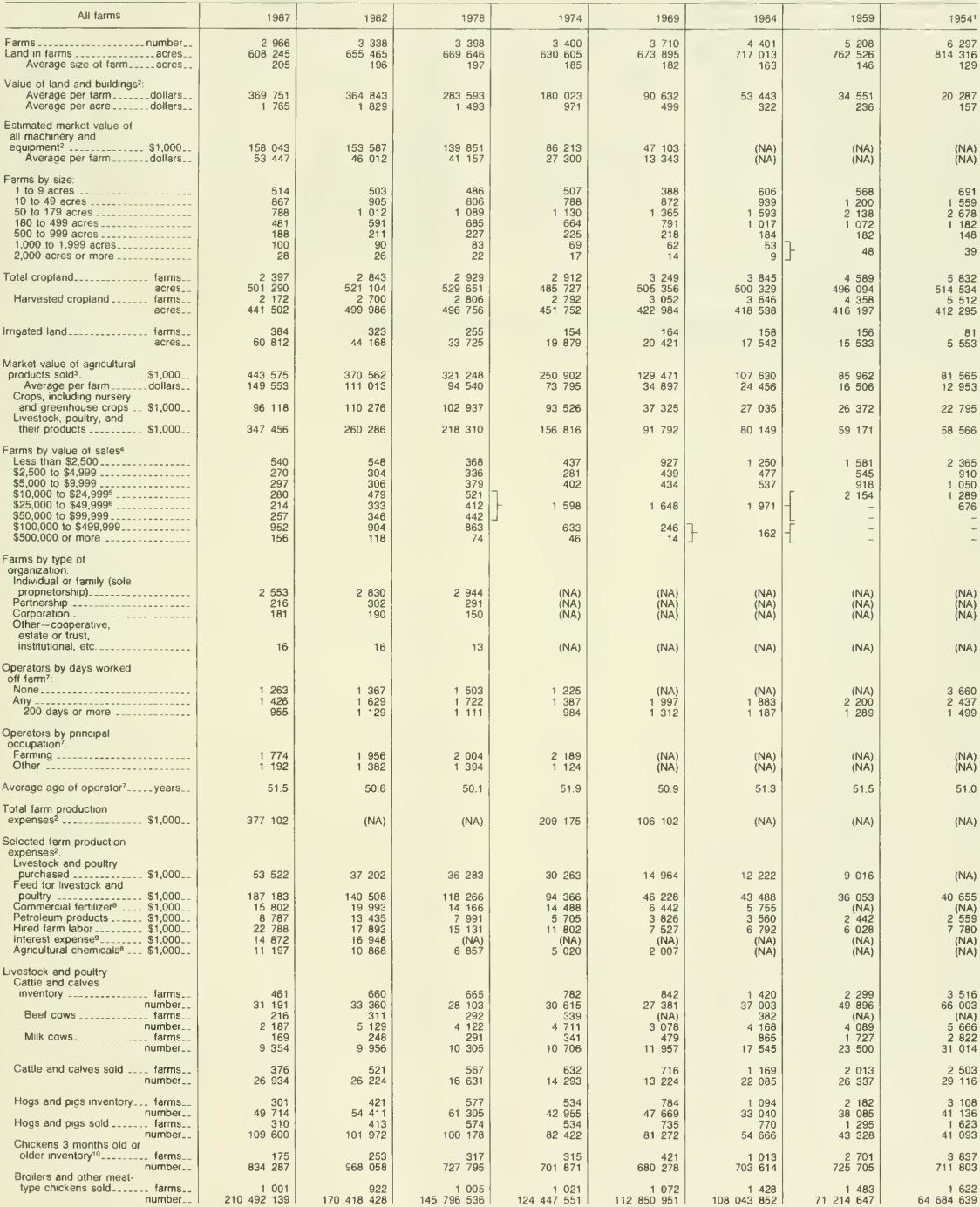


Table 1. Historical Highlights: 1987 and Earlier Census Years-Con.

[For meaning of abbreviations and symbols, see introductory text]

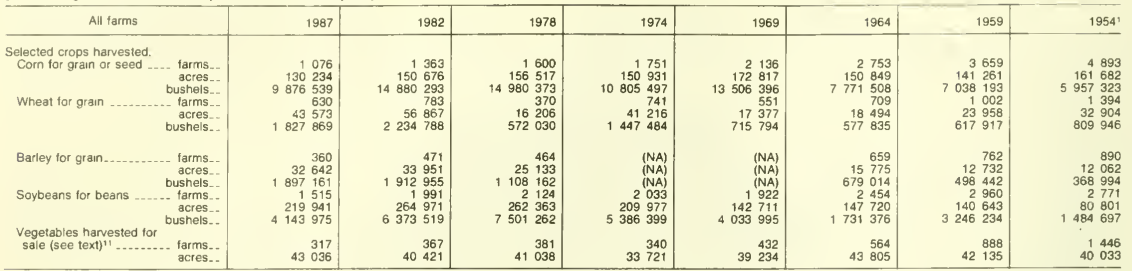

'Data for 1954 exclude Alaska and Hawall

Data are based on a sample of larms

Data for 1974 and prior years include the value of torest
"Data for 1982 and prior years exclude abnormal farms

\$Data for 1959 are for $\$ 10,000$ or more.

BData for 1954 are for $\$ 25,000$ or more

TData for 1974 apply oniy to individual or family operations (sole propnetorship) and partnerships; see text.

Data for 1987 include cost of custom applications; data for agricuitural chemicals exclude the cost of lime for 1987 and 1982

Pata for 1982 do nol include imputation for tiem nonresponse.

10 Data for 1964 and pror years are for chickens 4 months old or older

11Data for 1974 were trom land area used. 
Table 2. Market Value of Agricultural Products Sold: 1987, 1982, and 1978

[For meaning of abbreviations and symbols, see introductory text]

\begin{tabular}{|c|c|c|c|c|}
\hline Item & 1987 & Percent of total in 1987 & 1982 & 1978 \\
\hline 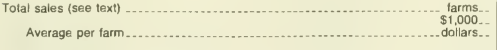 & $\begin{array}{r}2966 \\
443575 \\
149553\end{array}$ & $\begin{array}{r}100.0 \\
100.0 \\
(X)\end{array}$ & \begin{tabular}{rr|}
3 & 338 \\
370 & 562 \\
111 & 013
\end{tabular} & $\begin{array}{rr}3 & 398 \\
321 & 248 \\
94 & 540\end{array}$ \\
\hline 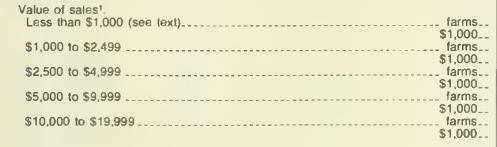 & $\begin{array}{r}280 \\
91 \\
260 \\
429 \\
270 \\
977 \\
297 \\
2059 \\
215 \\
3106\end{array}$ & $\begin{array}{r}9.4 \\
(Z) \\
8.8 \\
.1 \\
9.1 \\
.2 \\
10.0 \\
.5 \\
7.2 \\
.7\end{array}$ & $\begin{array}{r}230 \\
75 \\
318 \\
525 \\
304 \\
1103 \\
306 \\
2190 \\
373 \\
5294\end{array}$ & $\begin{array}{r}101 \\
47 \\
267 \\
450 \\
336 \\
1211 \\
379 \\
2655 \\
394 \\
5575\end{array}$ \\
\hline $\begin{array}{l}\$ 20,000 \text { to } \$ 24,999 \\
\$ 25,000 \text { to } \$ 39,999 \ldots \ldots \ldots \\
\$ 40,000 \text { to } \$ 49.999 \\
\$ 50,000 \text { to } \$ 99,999\end{array}$ & $\begin{array}{r}65 \\
149 \\
141 \\
4 \quad 400 \\
73 \\
3 \quad 232 \\
257 \\
18614\end{array}$ & $\begin{array}{r}2.2 \\
.3 \\
48 \\
10 \\
2.5 \\
.7 \\
87 \\
4.2\end{array}$ & $\begin{array}{r}106 \\
2355 \\
239 \\
7581 \\
94 \\
4 \quad 186 \\
346 \\
25 \quad 502\end{array}$ & $\begin{array}{rr}127 \\
2804 \\
272 \\
8 \quad 637 \\
140 \\
6 \quad 276 \\
442 \\
32 & 505\end{array}$ \\
\hline 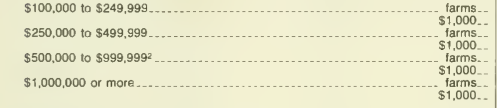 & 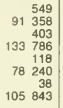 & $\begin{array}{r}18.5 \\
20.6 \\
13.6 \\
30.2 \\
4.0 \\
17.6 \\
1.3 \\
23.9\end{array}$ & $\begin{array}{r}601 \\
98409 \\
303 \\
102 \quad 430 \\
118 \\
120 \quad 911 \\
- \\
-\end{array}$ & 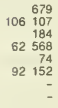 \\
\hline $\begin{array}{l}\text { Sales by commodity or commodity group. } \\
\text { Crops, including nursery and greenhouse crops.................... farms.. }\end{array}$ & $\begin{array}{rr}1 & 992 \\
96 & 118\end{array}$ & $\begin{array}{l}67.2 \\
21.7\end{array}$ & $\begin{array}{r}2501 \\
110276\end{array}$ & $\begin{array}{r}2628 \\
102937\end{array}$ \\
\hline $\begin{array}{l}\text { Corn for grain } \\
\text { Wheat } \\
\text { Soybeans. }\end{array}$ & \begin{tabular}{rr|}
1 & 733 \\
46 & 701 \\
915 \\
18 & 294 \\
626 \\
4 & 438 \\
1 & 497 \\
21 & 654
\end{tabular} & $\begin{array}{r}58.4 \\
10.5 \\
30.8 \\
4.1 \\
21.1 \\
1.0 \\
50.5 \\
4.9\end{array}$ & $\begin{array}{rr}2 & 254 \\
74 & 889 \\
1 & 164 \\
30 & 844 \\
776 \\
6 & 654 \\
1 & 977 \\
34 & 109\end{array}$ & $\begin{array}{r}2380 \\
70 \text { 999 } \\
\text { (NA) } \\
\text { (NA) } \\
\text { (NA) } \\
\text { (NA) } \\
\text { (NA) } \\
\text { (NA) }\end{array}$ \\
\hline 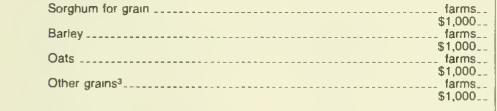 & $\begin{array}{r}18 \\
36 \\
320 \\
2 \quad 190 \\
9 \\
6 \\
40 \\
83\end{array}$ & $\begin{array}{r}.6 \\
(Z) \\
10.8 \\
.5 \\
.3 \\
(Z) \\
1.3 \\
(Z)\end{array}$ & $\begin{array}{r}4 \\
8 \\
\text { (NA) } \\
\text { (NA) } \\
23 \\
23 \\
462 \\
3 \\
251\end{array}$ & $\begin{array}{l}\text { (NA) } \\
\text { (NA) } \\
\text { (NA) } \\
\text { (NA) } \\
\text { (NA) } \\
\text { (NA) } \\
\text { (NA) } \\
\text { (NA) }\end{array}$ \\
\hline $\begin{array}{l}\text { Cotton and cottonseed............. } \\
\text { Tobacco farms... } \\
\text { Hay, silage, and field seeds } \\
\text { Vegetables, sweet corn, and melons }\end{array}$ & $\begin{array}{rr}- & - \\
- & - \\
& - \\
1 & 230 \\
428 \\
316 \\
20 & 332\end{array}$ & $\begin{array}{r}- \\
- \\
- \\
7.8 \\
3 \\
10.7 \\
46\end{array}$ & $\begin{array}{r}- \\
\overline{-} \\
\overline{-} \\
193 \\
901 \\
366 \\
16929\end{array}$ & $\begin{array}{r}- \\
\overline{-} \\
246 \\
812 \\
380 \\
15977\end{array}$ \\
\hline $\begin{array}{l}\begin{array}{l}\text { Fruts, nuts, and berries } \\
\text { Nursery and greenhouse crops.............. }\end{array} \\
\text { Other crops }\end{array}$ & 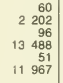 & $\begin{array}{r}2.0 \\
.5 \\
3.2 \\
3.0 \\
1.7 \\
2.7\end{array}$ & $\begin{array}{rr}2 & 66 \\
106 \\
7 & 984 \\
7 & 45 \\
7 & 252\end{array}$ & $\begin{array}{r}56 \\
1502 \\
113 \\
8052 \\
50 \\
5595\end{array}$ \\
\hline $\begin{array}{l}\text { Livestock, poultry, and their products } \ldots \ldots \ldots \\
\text { Poultry and poultry products } \ldots \ldots \ldots \\
\text { Dairy products }\end{array}$ & $\begin{array}{rr}1 & 658 \\
347 & 456 \\
1 & 127 \\
304 & 075 \\
& 139 \\
16 & 049\end{array}$ & $\begin{array}{r}55.9 \\
78.3 \\
38.0 \\
68.6 \\
4.7 \\
3.6\end{array}$ & $\begin{array}{rr}1 & 733 \\
260 & 286 \\
1 & 094 \\
220 & 963 \\
184 & 184 \\
15 & 571\end{array}$ & $\begin{array}{r}1910 \\
218310 \\
1164 \\
191425 \\
211 \\
11437\end{array}$ \\
\hline 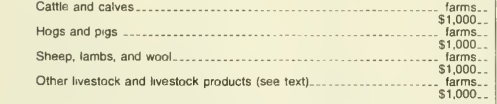 & $\begin{array}{r}376 \\
14 \\
938 \\
310 \\
10 \quad 055 \\
45 \\
54 \\
175 \\
2 \quad 286\end{array}$ & $\begin{array}{r}12.7 \\
3.4 \\
10.5 \\
2.3 \\
1.5 \\
(Z) \\
5.9 \\
.5\end{array}$ & $\begin{array}{r}521 \\
12 \\
311 \\
413 \\
10 \quad 208 \\
32 \\
26 \\
189 \\
1206\end{array}$ & $\begin{array}{r}567 \\
5767 \\
574 \\
8411 \\
27 \\
24 \\
183 \\
1248 \\
\end{array}$ \\
\hline
\end{tabular}

Data for 1982 and 1978 exclude abnormal farms.

2Data for 1982 and 1976 are for $\$ 500,000$ or more.

3Data for 1982 include barley 
Table 3. Farm Production Expenses: 1987, 1982, and 1978

Data are based on a sample of tarms. see text For meanıng of abbrevations and symbols, see introductory text]

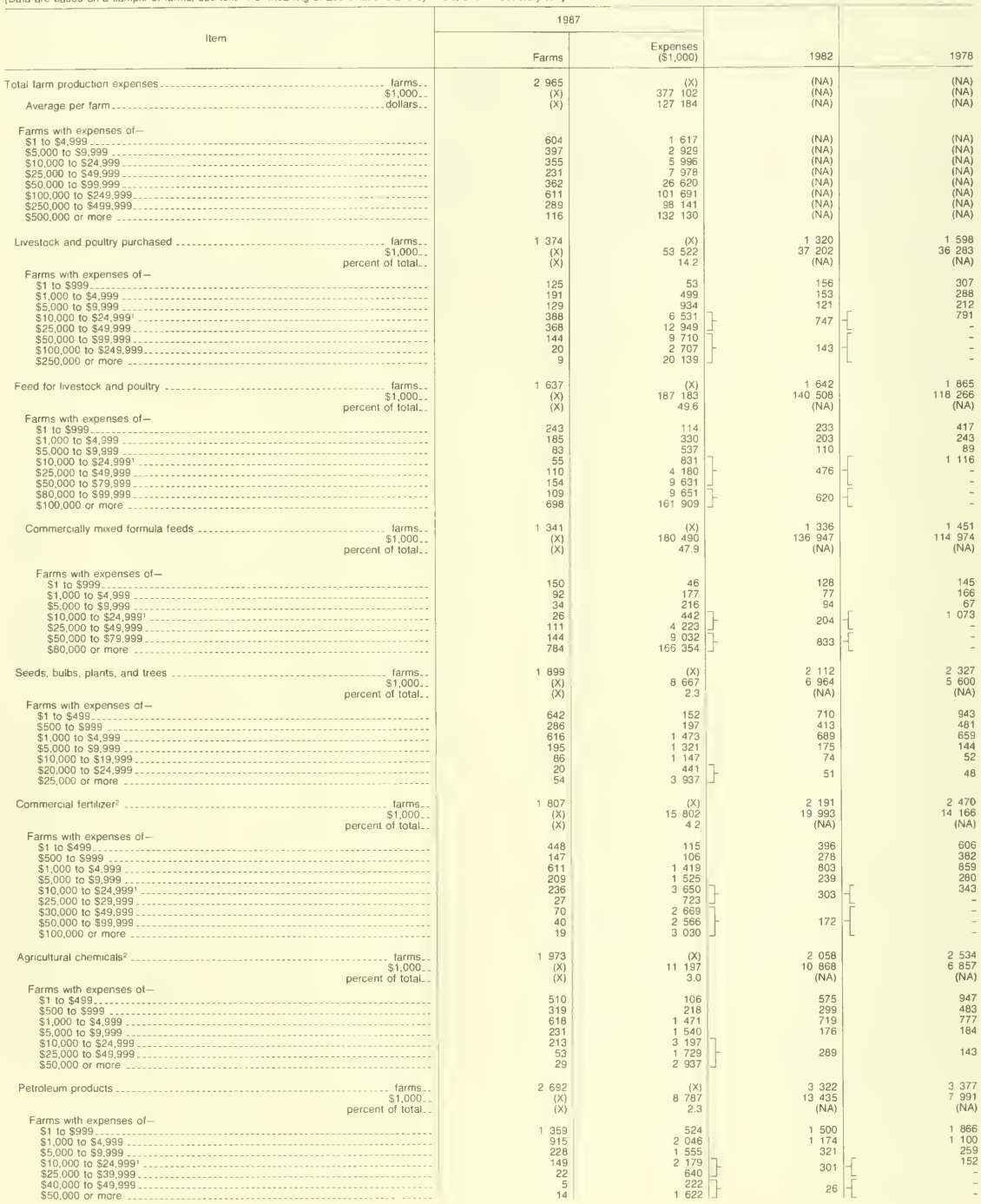

See footnctes at end of tabie 
Table 3. Farm Production Expenses: 1987, 1982, and 1978-Con.

[Data are based on a sample ot farms, see text For meaning of abbreviations and symbols, see introductory texi]

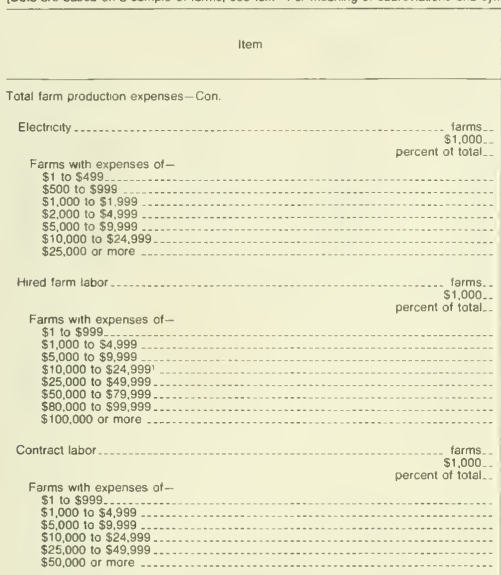

Repaif and maıtenance

Farms with expenses of-

percent of total.

$\$ 1$ to $\$ 999$

$\$ 1,000$ to $\$ 4,999$

$\$ 5,000$ to $\$ 9,999$

$\$ 10,000$ to $\$ 24,999$

$\$ 25,000$ to $\$ 49,999$

Customwork, machine hire, and rental of machunery and equipment $\beta^{3} \ldots \ldots . .$. farms

Farms with expenses of-

percent of iotal

$\$ 1$ to $\$ 999$

$\$ 1,000$ to $\$ 4,999$

$\$ 5.000$ to $\$ 9,999$

$\$ 10.000$ to $\$ 24,999$

$\$ 25,000$ to $\$ 49,999$

$\$ 50,000$ or more

Interest 4

farms

|

1987

Farms $\mid$

Expense
$(\$ 1,000)$

2195

195
$(X)$
$(X)$
722
363
361
581
105
46
17

$(X)$
862
13

1254

2244
4554
$(\mathrm{~N}$
7
7
3
4
5
5

$\begin{array}{rr}2 & 211 \\ 2 & 374 \\ \text { (NA) }\end{array}$

1064

585
339

155

68

1. 593

15131

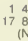

(NA)

594

562
165
272
-

1443

3328

$\begin{array}{ll}3 & 199 \\ 2 & 144\end{array}$

454

11225

$(x)$
5798

1.5

2

549
808
582
5

582
3405

2481

$\left(\begin{array}{l}(x) \\ (x)\end{array}\right.$

(x)

1918

1
3
30
1

1192

$1 \begin{array}{r}192 \\ (x) \\ (x)\end{array}$

672
406
77
29

45

2

\begin{tabular}{r|r}
70 & -1 \\
179 & - \\
296 & 264 \\
(NA) & 1495 \\
68 & (NA) \\
75 & 130 \\
16 & 85 \\
20 & 17 \\
20 & 32
\end{tabular}

$\begin{array}{ll}\text { (NA) } & \text { (NA) } \\ \text { (NA) } & \text { (NA) } \\ \text { (NA) } & \text { (NA) }\end{array}$

$\begin{array}{rl}4 & 32 \\ 2 & 46 \\ 2 & 46 \\ 2 & 23 \\ 2 & 23 \\ 2 & 19 \\ 2 & 36\end{array}$

(NA)

(NA)

(NA)

(NA)

1687
2418
(NA)

$\begin{array}{ll}1 & 27 \\ 2606 \\ \text { (NA) }\end{array}$

$2 \begin{array}{r}(X) \\ 445 \\ 6\end{array}$

1018

583
68

18

percent of total.

Farms with expenses of-

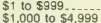

$\$ 1,000$ to $\$ 4,999$

$\$ 5,000$ to $\$ 9,999$

$\$ 10,000$ to $\$ 24,999$

$\$ 25,000$ to $\$ 49,999$

$\$ 100,000$ or more

Interest paid on debt:

Secured by real estate

Not secured by real estate

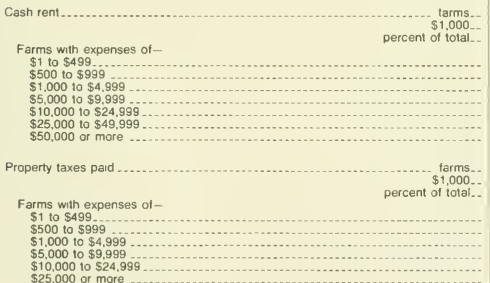

See footnotes at end of table 
Table 3. Farm Production Expenses: 1987, 1982, and 1978-Con.

[Data are based on a sample of farms, see text. For meaning of abbreviations and symbols, see introductory text]

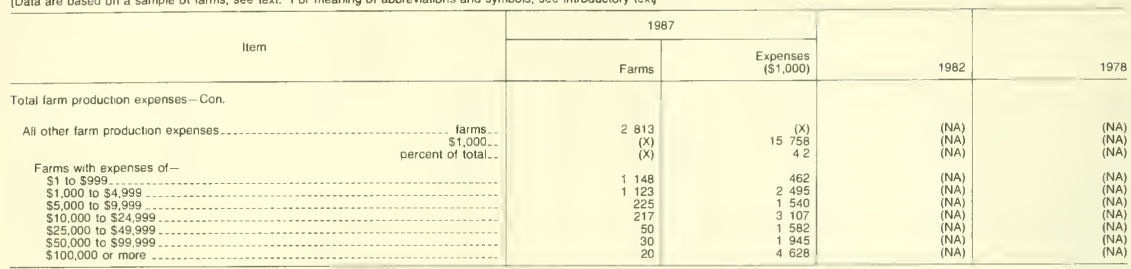

'Data for 1978 are for $\$ 10,000$ or more

2Data for 1987 include cost of custom appications; data for agricultural chemicals exclude the cost of lime for 1987 and 1982

'Data for 1987 exclude cost of custom applications for commercial fertilizer and agricultural chemicals

"Data for 1982 do not include imputation for item nonresponse.

Table 4. Net Cash Return From Agricultural Sales: 1987

[Data are based on a sample of farms, see text. For meaning of abbreviations and symbols, see introductory text]

\begin{tabular}{|c|c|c|c|}
\hline Item & All farms & $\begin{array}{l}\text { Farms with sales } \\
\text { of } \$ 10,000 \text { or more }\end{array}$ & $\begin{array}{l}\text { Farms with sales } \\
\text { of less than } \$ 10,000\end{array}$ \\
\hline 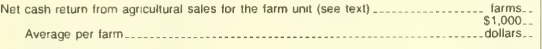 & $\begin{array}{r}2965 \\
65300 \\
22023\end{array}$ & $\begin{array}{r}1866 \\
68350 \\
36629\end{array}$ & $\begin{array}{r}1099 \\
-3050 \\
-2775\end{array}$ \\
\hline $\begin{array}{r}\text { Farms with net gans' } 1 \text {..................... } \\
\text { Average per farm }\end{array}$ & $\begin{array}{rr}1 & 695 \\
76 & 564 \\
45 & 171\end{array}$ & $\begin{array}{rr}1 & 462 \\
76 & 116 \\
52 & 063\end{array}$ & $\begin{array}{r}233 \\
448 \\
1923\end{array}$ \\
\hline $\begin{array}{l}\text { Gain of- } \\
\text { Less than } \$ 1,000 \\
\$ 1,000 \text { to } \$ 4,999 \\
\$ 5,000 \text { to } \$ 9,999 \\
\$ 10,000 \text { to } \$ 24,999 \\
\$ 25000 \text { to } \$ 49,999 \\
\$ 50,000 \text { or more }\end{array}$ & $\begin{array}{l}129 \\
247 \\
195 \\
432 \\
355 \\
337\end{array}$ & $\begin{array}{r}33 \\
125 \\
180 \\
432 \\
355 \\
337\end{array}$ & $\begin{array}{r}96 \\
122 \\
15 \\
- \\
\\
-\end{array}$ \\
\hline 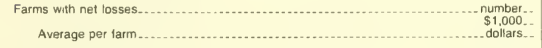 & $\begin{array}{r}1 \\
11270 \\
8870\end{array}$ & $\begin{array}{r}404 \\
7767 \\
19224\end{array}$ & $\begin{array}{r}866 \\
3 \quad 498 \\
4039\end{array}$ \\
\hline $\begin{array}{l}\text { Loss of- } \\
\text { Less than } \$ 1,000 \\
\$ 1,000 \text { to } \$ 4,999 \\
\$ 5,000 \text { to } \$ 9,999 \\
\$ 10,000 \text { to } \$ 24,999 \\
\$ 25,000 \text { to } \$ 49,999\end{array}$ & $\begin{array}{r}233 \\
537 \\
231 \\
181 \\
57 \\
31\end{array}$ & $\begin{array}{r}29 \\
109 \\
78 \\
102 \\
55 \\
31\end{array}$ & $\begin{array}{r}204 \\
428 \\
153 \\
79 \\
2 \\
\end{array}$ \\
\hline
\end{tabular}

iFarms with total production expenses equal to market value of agricultural products sold are included as farms with gains of less than $\$ 1,000$ 
Table 5. Government Payments and Other Farm-Related Income: 1987 and 1982

[For meaning of abbreviations and symbols, see intraductory text]

\begin{tabular}{|c|c|c|c|c|}
\hline \multirow{2}{*}{ Item } & \multicolumn{2}{|c|}{ All farms } & \multicolumn{2}{|c|}{ Farms with sales of $\$ 10,000$ or mare } \\
\hline & Farms & $\begin{array}{r}\text { Value } \\
(\$ 1.000)\end{array}$ & Farms & $\begin{array}{r}\text { Value } \\
(\$ 1,000)\end{array}$ \\
\hline $\begin{array}{l}\text { Government payments } \\
\quad \text { Average per farm }\end{array}$ & $\begin{array}{r}549 \\
(x)\end{array}$ & $\begin{array}{r}7414 \\
13505\end{array}$ & $\begin{array}{r}451 \\
(x)\end{array}$ & $\begin{array}{r}7170 \\
15898\end{array}$ \\
\hline 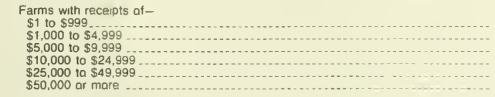 & $\begin{array}{r}58 \\
193 \\
98 \\
122 \\
44 \\
34\end{array}$ & $\begin{array}{r}30 \\
530 \\
671 \\
1810 \\
1 \\
1511 \\
2863\end{array}$ & $\begin{array}{r}25 \\
138 \\
91 \\
119 \\
44 \\
34\end{array}$ & $\begin{array}{r}14 \\
394 \\
623 \\
1765 \\
1511 \\
2863\end{array}$ \\
\hline $\begin{array}{l}\text { Amount recerved in cash } \\
\text { Value of certuficates recerved }\end{array}$ & $\begin{array}{l}478 \\
493\end{array}$ & $\begin{array}{l}3513 \\
3902\end{array}$ & $\begin{array}{l}403 \\
401\end{array}$ & $\begin{array}{l}3421 \\
3749\end{array}$ \\
\hline $\begin{array}{l}\text { Other farm-related incame, grass before taxes and expenses } \\
\text { Average per farm' }\end{array}$ & $\begin{array}{r}757 \\
(\times)\end{array}$ & $\begin{array}{l}3998 \\
5282\end{array}$ & $\begin{array}{r}461 \\
(x)\end{array}$ & $\begin{array}{l}2810 \\
6095\end{array}$ \\
\hline 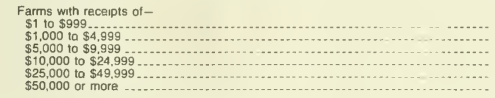 & $\begin{array}{r}269 \\
238 \\
132 \\
100 \\
13 \\
5\end{array}$ & $\begin{array}{r}91 \\
575 \\
873 \\
+424 \\
448 \\
587\end{array}$ & $\begin{array}{r}173 \\
136 \\
73 \\
63 \\
11 \\
5\end{array}$ & $\begin{array}{r}56 \\
318 \\
491 \\
(D) \\
\text { (D) } \\
587\end{array}$ \\
\hline 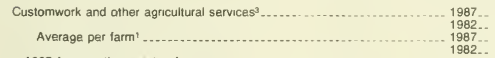 & $\begin{array}{r}272 \\
284 \\
(X) \\
(X)\end{array}$ & 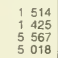 & $\begin{array}{r}178 \\
237 \\
(X) \\
(X)\end{array}$ & $\begin{array}{l}1072 \\
1355 \\
6022 \\
5716\end{array}$ \\
\hline 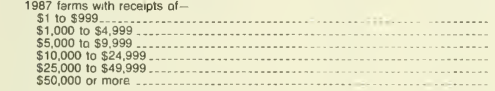 & $\begin{array}{r}52 \\
110 \\
73 \\
29 \\
8\end{array}$ & $\begin{array}{r}26 \\
285 \\
492 \\
427 \\
283\end{array}$ & $\begin{array}{r}37 \\
70 \\
35 \\
29 \\
7 \\
-\end{array}$ & $\begin{array}{r}16 \\
145 \\
(D) \\
427 \\
\text { (D) }\end{array}$ \\
\hline $\begin{array}{l}\text { Rental of farmland } \\
\text { Average per farm }\end{array}$ & $\begin{array}{r}287 \\
(\times)\end{array}$ & $\begin{array}{ll}1 & 151 \\
4 & 009\end{array}$ & $\begin{array}{r}150 \\
(x)\end{array}$ & $\begin{array}{r}663 \\
4417\end{array}$ \\
\hline 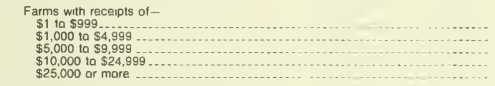 & $\begin{array}{r}112 \\
96 \\
33 \\
42 \\
4\end{array}$ & $\begin{array}{r}43 \\
192 \\
211 \\
551 \\
154\end{array}$ & $\begin{array}{r}63 \\
39 \\
30 \\
15 \\
3\end{array}$ & $\begin{array}{r}22 \\
82 \\
189 \\
\text { (D) } \\
\text { (D) }\end{array}$ \\
\hline $\begin{array}{l}\text { Sales of forest products and Christmas trees } \\
\text { Average per farm' }\end{array}$ & $\begin{array}{r}113 \\
(x)\end{array}$ & $\begin{array}{r}878 \\
7767\end{array}$ & $\begin{array}{r}49 \\
(x)\end{array}$ & 13560 \\
\hline $\begin{array}{l}\text { Farms with receipts of - } \\
\$ 1 \text { to } \$ 999 \\
\$ 1,000 \text { to } \$ 4,999 \\
\$ 5,000 \text { to } \$ 9,999 \\
\$ 100000 \\
\$ 25,000 \text { or more }\end{array}$ & $\begin{array}{r}44 \\
27 \\
29 \\
10 \\
3\end{array}$ & $\begin{array}{r}21 \\
89 \\
192 \\
128 \\
448\end{array}$ & $\begin{array}{r}13 \\
21 \\
2 \\
10 \\
3\end{array}$ & $\begin{array}{r}8 \\
\text { (D) } \\
\text { (D) } \\
128 \\
448\end{array}$ \\
\hline $\begin{array}{l}\text { Other farm-related income sources } \\
\text { Average per farm } 1 \text { 1.... }\end{array}$ & $\begin{array}{r}208 \\
(x)\end{array}$ & $2 \begin{array}{r}456 \\
2191\end{array}$ & $\begin{array}{r}156 \\
(\mathrm{x})\end{array}$ & $\begin{array}{r}411 \\
2633\end{array}$ \\
\hline $\begin{array}{l}\text { Farms with receipts of- } \\
\$ 1 \text { to } \$ 999 \\
\$ 1,000 \text { to } \$ 4,999 \\
\$ 5,000 \text { to } \$ 9,999 \\
\$ 10,000 \text { to } \$ 24,99 . \ldots \\
\$ 25,000 \text { or mare }\end{array}$ & $\begin{array}{r}161 \\
24 \\
9 \\
11 \\
3\end{array}$ & $\begin{array}{r}23 \\
54 \\
67 \\
220 \\
91\end{array}$ & $\begin{array}{r}121 \\
14 \\
7 \\
11 \\
3\end{array}$ & $\begin{array}{r}21 \\
(D) \\
\text { (D) } \\
220 \\
91\end{array}$ \\
\hline
\end{tabular}

'Data are in whole dallars.

${ }^{2}$ Data are based an a sample of farms.

3Data for 1987 are based an a sample af farms; data for 1982 are nonsample and exciude abnarmals fram farms with sales af $\$ 10,000$ or mare. 
Table 6. Commodity Credit Corporation Loans: 1987 and 1982

[For meaning of abbreviations and symbols, see introductory text]

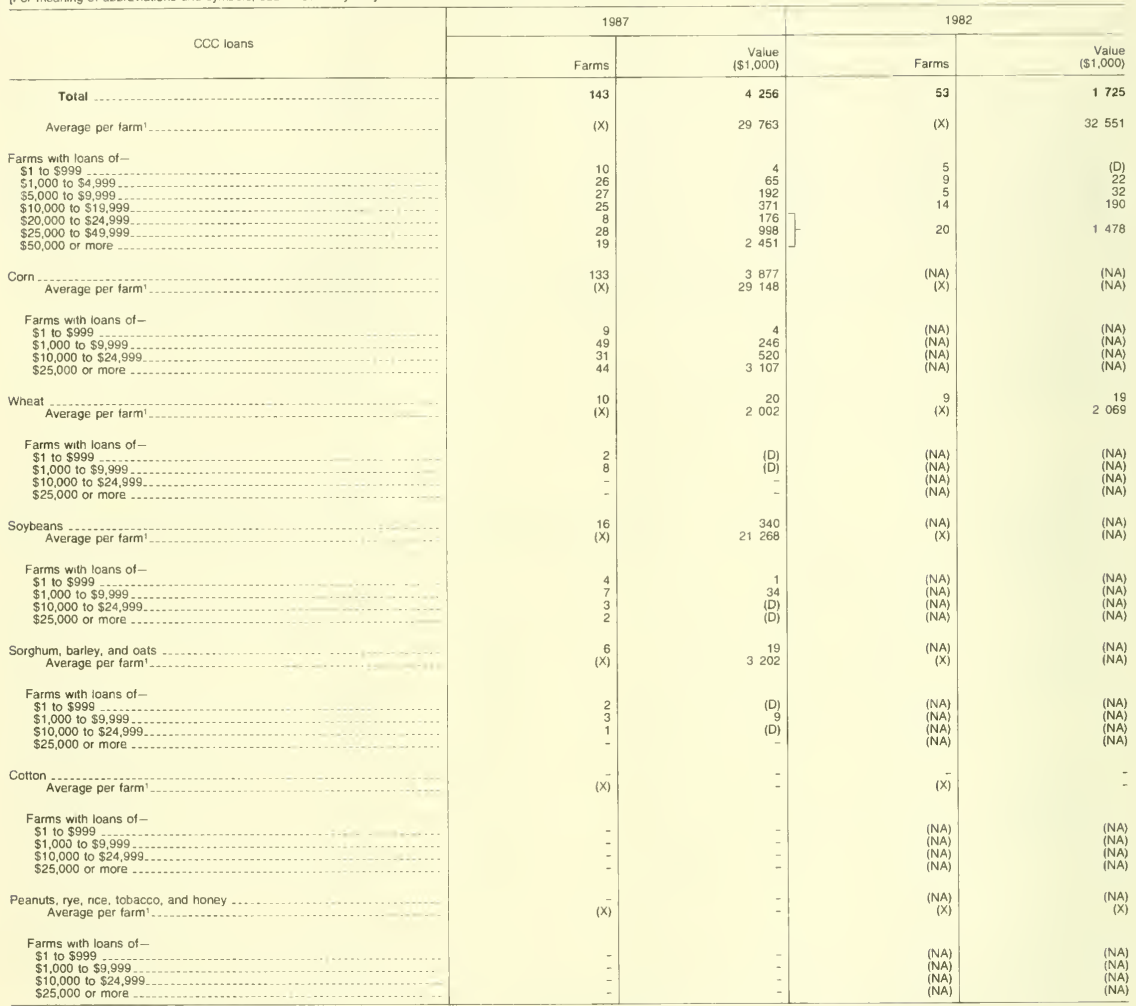

'Data are in whole dollars. 
Table 7. Land Use and Acres Diverted: 1987, 1982, and 1978

[For meaning of abbreviations and symbols, see introductory text]

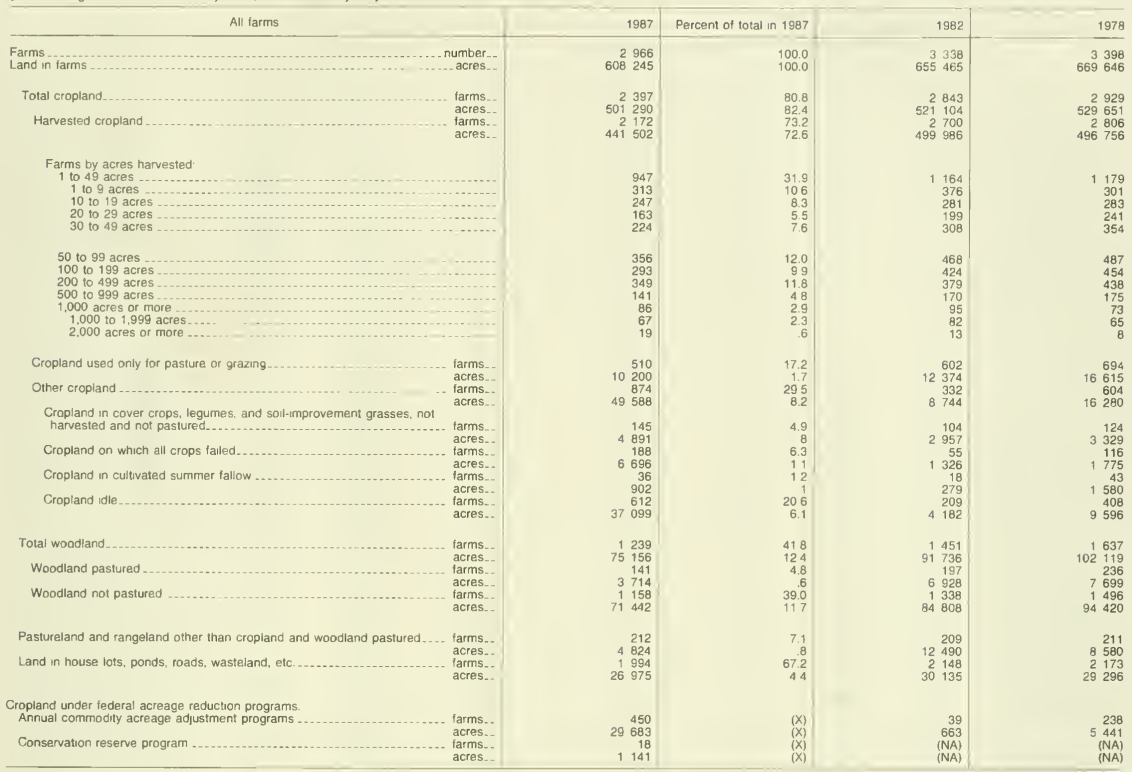


Table 8. Land in Farms, Harvested Cropland, and Irrigated Land, by Size of Farm: 1987 and 1982

[For meaning of abbreviations and symbols, see introductory text]

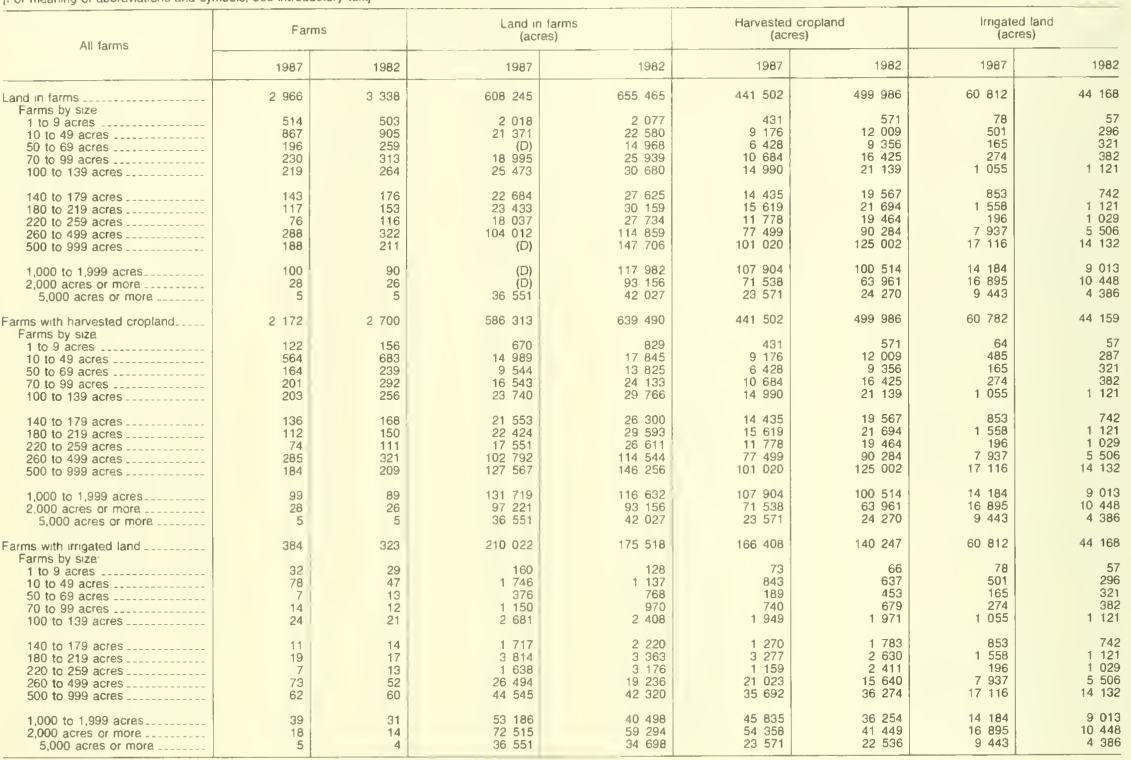

Table 9. Irrigation: 1987,1982 , and 1978

[For meaning of abbreviations and symbols, see introductory text]

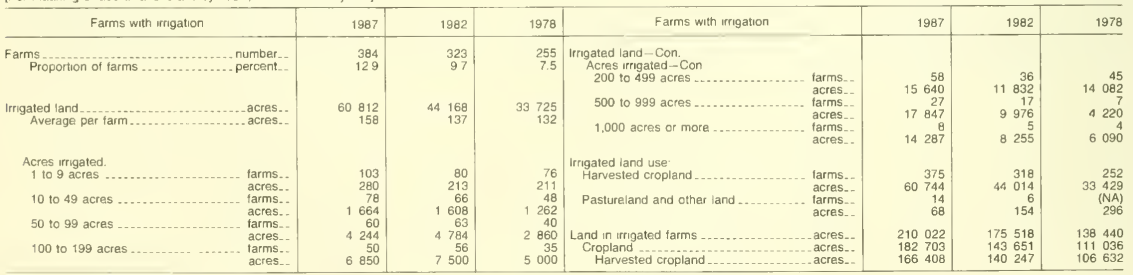


Table 10. Selected Characteristics of Irrigated and Nonirrigated Farms: 1987 and 1982

[For meaning of abbreviations and symbols, see introductory text]

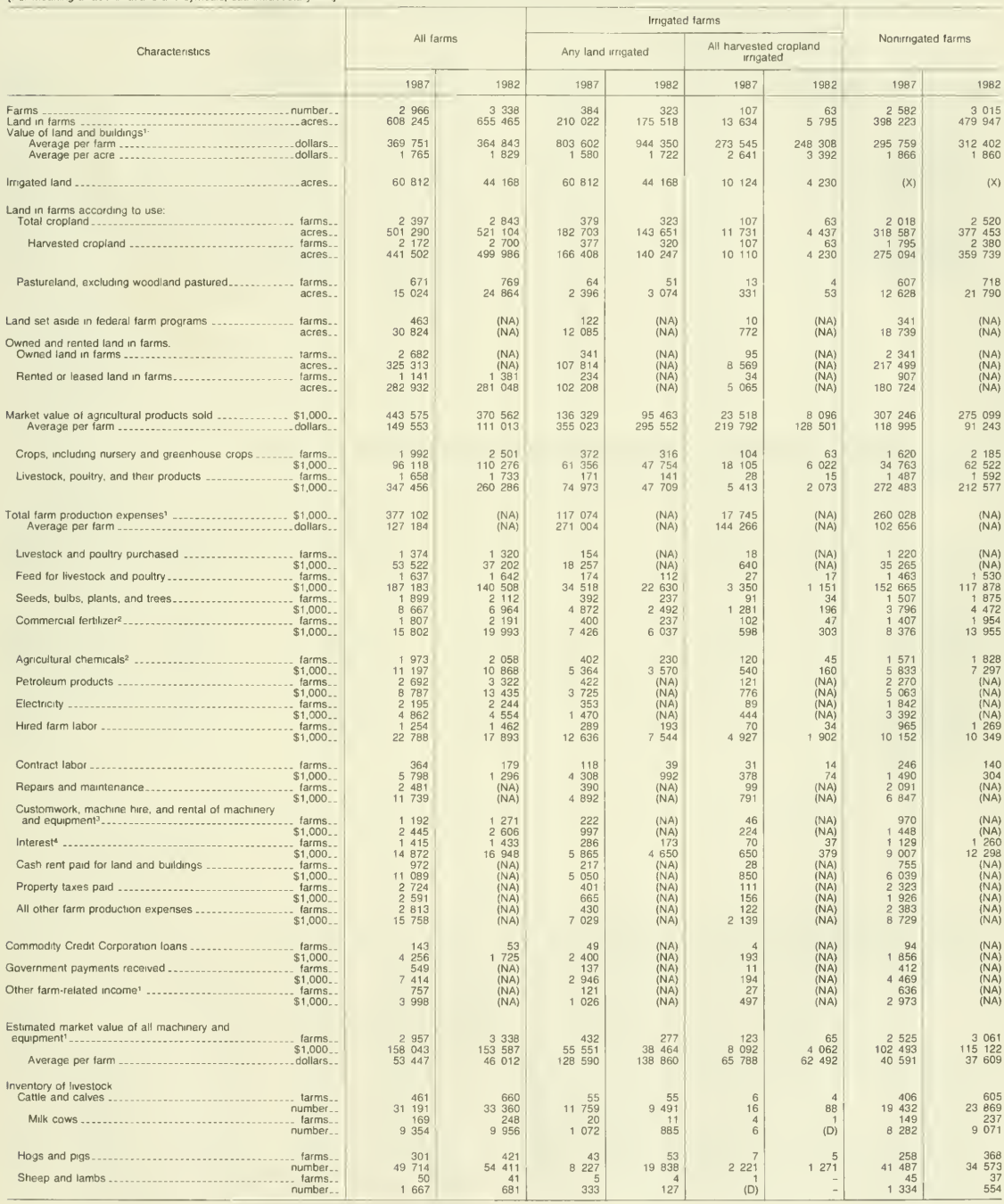

'Data are based on a sample of farms

Data for 1987 include cost of custom applicatons.

Dala for 1987 exclude cost of custom applications for commercial fertilizer and agricultural chemicals.

«Data for 1982 do not include imputation for item nonresponse 
Table 11. Value of Land and Buildings: 1987, 1982, and 1978

[Data are based on a sample of farms, see text For meaning of abbreviations and symbols, see introductory text]

\begin{tabular}{|c|c|c|c|c|}
\hline \multirow{2}{*}{ Value of land and buildings } & \multicolumn{2}{|c|}{1987} & \multirow[b]{2}{*}{1982} & \multirow[b]{2}{*}{1978} \\
\hline & Farms & (\$1,000) & & \\
\hline 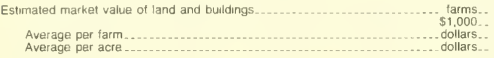 & $\begin{array}{r}2965 \\
(X) \\
(X) \\
(X)\end{array}$ & $\begin{array}{rr}1 & (X) \\
1096 & 313 \\
369 & 751 \\
1 & 765\end{array}$ & $\begin{array}{rr}3 & 338 \\
1 & 217 \\
3647 \\
3643 \\
1 & 829\end{array}$ & $\begin{array}{rr}3 & 399 \\
963 & 934 \\
283 & 593 \\
1 & 493\end{array}$ \\
\hline $\begin{array}{l}\$ 200,000 \text { to } \$ 499,999 \\
\$ 500,000 \text { to } \$ 999,999 \\
\$ 1,000,000 \text { to } \$ 1,999,999 \\
\$ 2,000,000 \text { to } \$ 4,999,999 \\
\$ 5,000,000 \text { or more }\end{array}$ & $\begin{array}{r}778 \\
297 \\
150 \\
71 \\
14\end{array}$ & $\begin{array}{ll}233 & 355 \\
206 & 327 \\
195 & 787 \\
209 & 250 \\
112 & 013\end{array}$ & $\begin{array}{l}789 \\
322 \\
264\end{array}$ & $\begin{array}{l}606 \\
308 \\
187\end{array}$ \\
\hline
\end{tabular}

Table 12. Value of Machinery and Equipment on Place: 1987 and 1982

[Data are based on a sample of farms, see text For meanung of abbreviations and symbols, see introductory text]

\begin{tabular}{|c|c|c|c|c|}
\hline \multirow{2}{*}{ Value of machinery and equipment } & \multicolumn{2}{|c|}{1987} & \multicolumn{2}{|c|}{1982} \\
\hline & Farms & $\begin{array}{r}\text { Value } \\
(\$ 1,000)\end{array}$ & Farms & $\begin{array}{r}\text { Value } \\
(\$ 1.000)\end{array}$ \\
\hline $\begin{array}{l}\text { Estumated market value of all machinery and equipment } \\
\text { Average per tarm }\end{array}$ & $\begin{array}{r}2957 \\
(X)\end{array}$ & $\begin{array}{rr}158 & 043 \\
53 & 447\end{array}$ & $\begin{array}{r}338 \\
(X)\end{array}$ & $\begin{array}{r}153587 \\
46012\end{array}$ \\
\hline $\begin{array}{l}\text { By value group } \\
\$ 1 \text { to } \$ 4,999 \\
\$ 5,000 \text { to } \$ 9,999 \\
\$ 10,000 \text { to } \$ 19,999 \\
\$ 20,000 \text { to } \$ 29,999 \\
\$ 30,000 \text { to } \$ 49,999\end{array}$ & $\begin{array}{l}201 \\
485 \\
494 \\
503 \\
463\end{array}$ & $\begin{array}{rr}628 \\
3 & 183 \\
6 & 500 \\
11 & 450 \\
16 & 660\end{array}$ & $\begin{array}{l}203 \\
757 \\
643 \\
447 \\
472\end{array}$ & $\begin{array}{r}626 \\
4952 \\
9028 \\
10335 \\
16729\end{array}$ \\
\hline $\begin{array}{l}\$ 50,000 \text { to } \$ 69.999 \\
\$ 70,000 \text { to } \$ 99.999 \\
\$ 100,000 \text { to } \$ 199,999 \ldots \ldots \ldots \\
\$ 200,000 \text { to } \$ 499,999 \ldots \ldots\end{array}$ & $\begin{array}{l}203 \\
197 \\
257 \\
129\end{array}$ & $\begin{array}{ll}11 & 155 \\
15 & 422 \\
32 & 226 \\
36 & 828\end{array}$ & $\begin{array}{l}209 \\
228 \\
226 \\
136\end{array}$ & $\begin{array}{ll}11 & 253 \\
18 & 287 \\
30 & 133 \\
35 & 648\end{array}$ \\
\hline $\begin{array}{l}\$ 500,000 \text { to } \$ 999,999 \\
\$ 1,000,000 \text { or more }\end{array}$ & $\begin{array}{r}16 \\
9\end{array}$ & $\begin{array}{r}9500 \\
14490\end{array}$ & 17 & 16596 \\
\hline
\end{tabular}

Data are in whole dollars

Table 13. Selected Machinery and Equipment on Place: 1987 and 1982

[Data are based on a sample of farms, see text. For meaning of abbreviations and symbols, see introductory text]

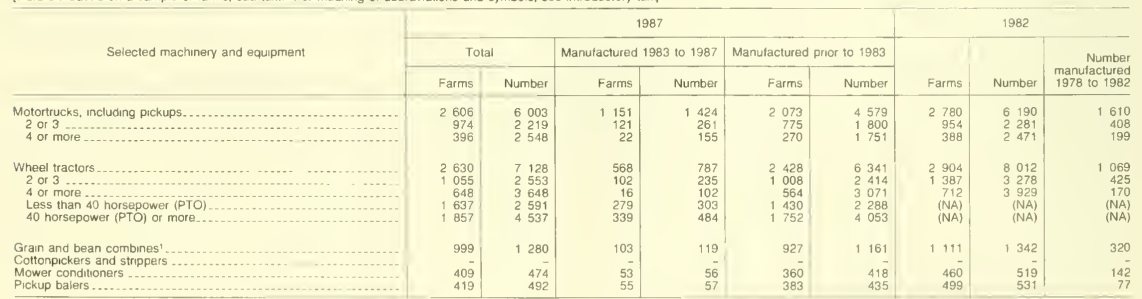

'Data for 1982 include sell-propelled only 
Table 14. Petroleum Products Expenses: 1987, 1982, and 1978

[Data are based on a sample of farms; see text. For meaning of abbreviations and symbols, see introductory text]

\begin{tabular}{|c|c|c|c|c|}
\hline \multirow[b]{2}{*}{ Item } & \multicolumn{2}{|c|}{1987} & \multirow[b]{2}{*}{1982} & \multirow[b]{2}{*}{1978} \\
\hline & Farms & $\begin{array}{r}\text { Expenses } \\
(\$ 1,000)\end{array}$ & & \\
\hline 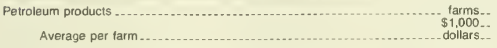 & $\begin{array}{r}2692 \\
(X) \\
(X)\end{array}$ & \begin{tabular}{ll|}
$(X)$ \\
8 & 787 \\
3 & 264
\end{tabular} & $\begin{array}{rr}3 & 322 \\
13 & 435 \\
4 & 044\end{array}$ & $\begin{array}{l}3377 \\
7991 \\
2366\end{array}$ \\
\hline $\begin{array}{c}\text { Gasoline and gasohol } \\
\text { Average per farm }\end{array}$ & $\begin{array}{r}356 \\
(X) \\
(X)\end{array}$ & \begin{tabular}{rr|}
3 & $(x)$ \\
1 & 226 \\
1 & 369
\end{tabular} & \begin{tabular}{ll|l}
3 & 062 \\
5 & 192 \\
1 & 696
\end{tabular} & $\begin{array}{ll}3 & 176 \\
3 & 625 \\
1 & 141\end{array}$ \\
\hline $\begin{array}{l}\text { Farms with expenses of }- \\
\$ 1 \text { to } \$ 499 \\
\$ 500 \text { to } \$ 999 \\
\$ 1,000 \text { to } \$ 1,999 \\
\$ 2,000 \text { to } \$ 4,999 \\
\$ 5,000 \text { to } \$ 9,999 \\
\$ 10,000 \text { to } \$ 24,999 \\
\$ 25,000 \text { or more }\end{array}$ & $\begin{array}{r}1091 \\
449 \\
383 \\
305 \\
94 \\
25 \\
9\end{array}$ & $\begin{array}{l}234 \\
307 \\
499 \\
831 \\
626 \\
316 \\
412\end{array}$ & $\begin{array}{r}1228 \\
482 \\
635 \\
495 \\
161 \\
61\end{array}$ & $\begin{array}{r}1476 \\
770 \\
425 \\
375 \\
96 \\
34\end{array}$ \\
\hline $\begin{array}{r}\text { Diesel fuel } \\
\text { Average per farm }\end{array}$ & $\begin{array}{r}1494 \\
(X) \\
(X)\end{array}$ & $\begin{array}{l}(X) \\
3905 \\
2614\end{array}$ & $\begin{array}{ll}1 & 755 \\
5 & 019 \\
2 & 860\end{array}$ & $\begin{array}{ll}1 & 538 \\
2 & 209 \\
1 & 436\end{array}$ \\
\hline $\begin{array}{l}\text { Farms with expenses of } \\
\$ 1 \text { to } \$ 499 \\
\$ 500 \text { to } \$ 999 \\
\$ 1,000 \text { to } \$ 1,999 \\
\$ 2,000 \text { to } \$ 4,999 \\
\$ 5,000 \text { to } \$ 9,999 \\
\$ 10,000 \text { to } \$ 24,999 \\
\$ 25,000 \text { or more }\end{array}$ & $\begin{array}{r}600 \\
228 \\
207 \\
275 \\
110 \\
59 \\
15\end{array}$ & $\begin{array}{l}133 \\
148 \\
266 \\
860 \\
745 \\
820 \\
935\end{array}$ & $\begin{array}{l}524 \\
358 \\
330 \\
249 \\
181 \\
113\end{array}$ & $\begin{array}{l}691 \\
297 \\
205 \\
270 \\
45 \\
30\end{array}$ \\
\hline 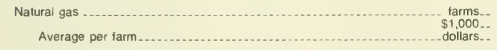 & $\begin{array}{l}93 \\
(X) \\
(X)\end{array}$ & $\begin{array}{r}(x) \\
331 \\
3559\end{array}$ & $\begin{array}{r}27 \\
79 \\
2937\end{array}$ & $\begin{array}{r}33 \\
28 \\
848\end{array}$ \\
\hline $\begin{array}{l}\text { Farms with expenses ot } \\
\$ 1 \text { to } \$ 99 \\
\$ 100 \text { to } \$ 499 \\
\$ 500 \text { to } \$ 999 \\
\$ 1,000 \text { to } \$ 1,999 \\
\$ 2,000 \text { to } \$ 4,999 \\
\$ 5,000 \text { to } \$ 9,999 \\
\$ 10,000 \text { or more }\end{array}$ & $\begin{array}{r}2 \\
30 \\
29 \\
12 \\
10 \\
6 \\
4\end{array}$ & $\begin{array}{r}(\mathrm{D}) \\
(\mathrm{D}) \\
17 \\
16 \\
33 \\
38 \\
220\end{array}$ & $\begin{array}{r}6 \\
12 \\
2 \\
1 \\
5 \\
1\end{array}$ & $\begin{array}{r}1 \\
19 \\
2 \\
9 \\
1 \\
1\end{array}$ \\
\hline 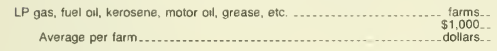 & $\begin{array}{r}1873 \\
(X) \\
(X)\end{array}$ & $\begin{array}{r}(x) \\
325 \\
708\end{array}$ & $\begin{array}{r}(\text { NA) } \\
3 \text { 144 } \\
\text { (NA) }\end{array}$ & $\begin{array}{r}\text { (NA) } \\
2130 \\
(\text { NA) }\end{array}$ \\
\hline $\begin{array}{l}\text { Farms with expenses of } \\
\$ 1 \text { to } \$ 99 \\
\$ 100 \text { to } \$ 499 \\
\$ 500 \text { to } \$ 999 \\
\$ 1,000 \text { to } \$ 1,999 \\
\$ 2,000 \text { to } \$ 4,999 \\
\$ 5,000 \text { to } \$ 9,999 \\
\$ 10,000 \text { or more }\end{array}$ & $\begin{array}{r}643 \\
757 \\
201 \\
120 \\
97 \\
40 \\
15\end{array}$ & $\begin{array}{r}31 \\
177 \\
131 \\
158 \\
270 \\
257 \\
302\end{array}$ & $\begin{array}{l}\text { (NA) } \\
\text { (NA) } \\
\text { (NA) } \\
\text { (NA) } \\
\text { (NA) } \\
\text { (NA) } \\
\text { (NA) }\end{array}$ & $\begin{array}{l}\text { (NA) } \\
\text { (NA) } \\
\text { (NA) } \\
\text { (NA) } \\
\text { (NA) } \\
\text { (NA) } \\
\text { (NA) }\end{array}$ \\
\hline
\end{tabular}

Table 15. Agricultural Chemicals Used, Including Fertilizer and Lime: 1987, 1982, and 1978

[Data are based on a sample of farms; see text. For meaning of abbreviations and symbols, see introductory tex?

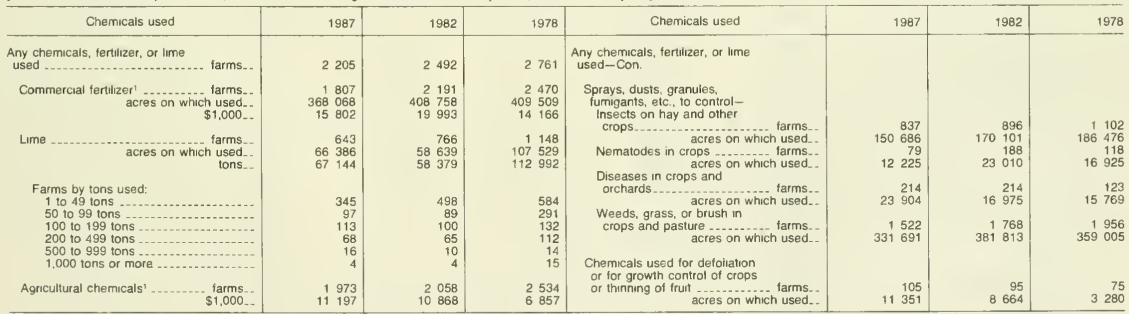

'Data for 1987 include cost of custom apphications; data for agricuitural chemicais exclude the cost of ime for 1987 and 1982. 
Table 16. Tenure and Characteristics of Operator and Type of Organization for All Farms and Farms Operated by Black and Other Races: 1987, 1982, and 1978

[For meaning of abbreviations and symbols, see introductory text]

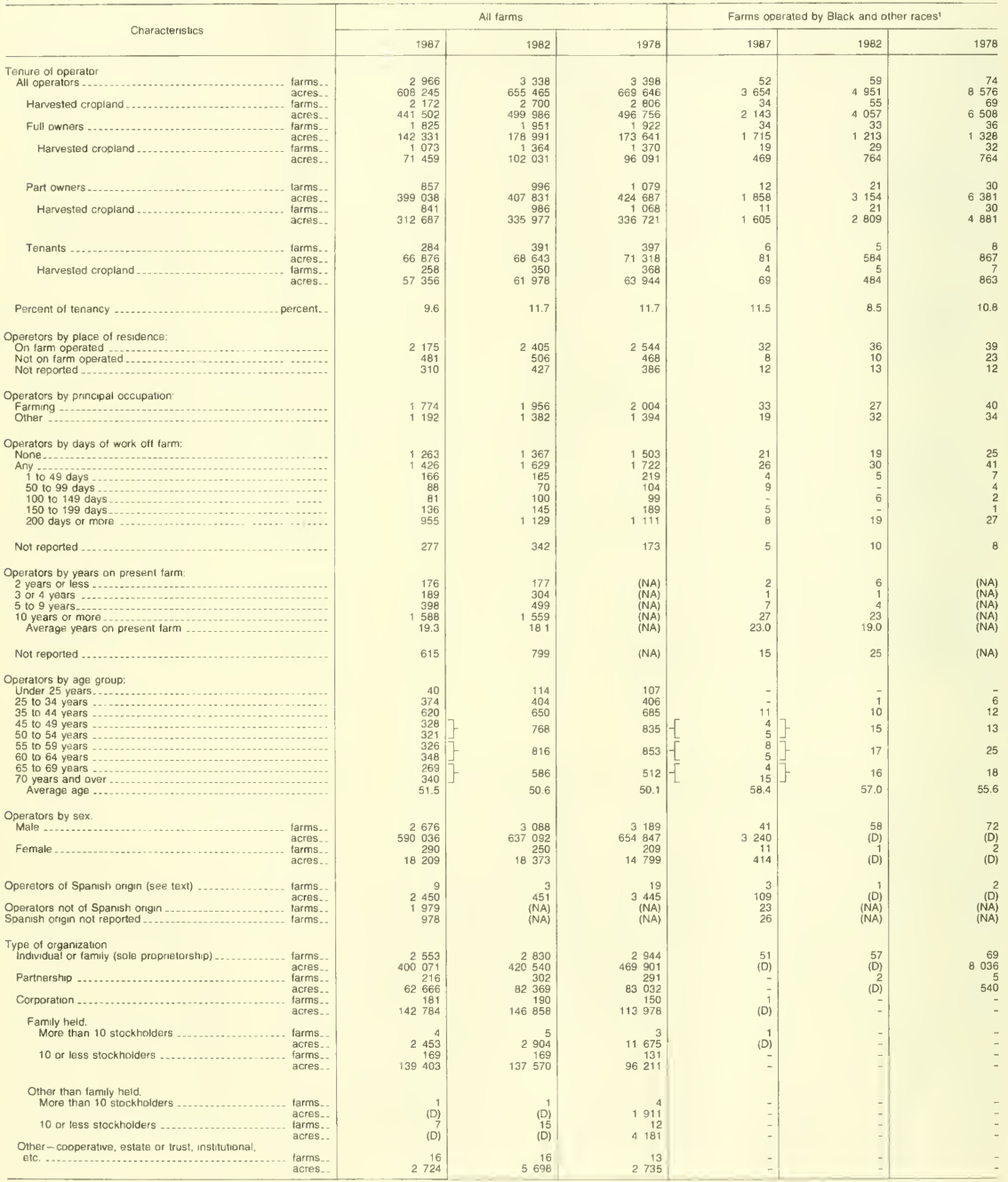

'For classification of social and ethnic groups, see text 


\section{Table 17. Selected Characteristics of Farms Operated by Females, Persons of Spanish Origin, and Specified Racial Groups: 1987 and 1982}

[For meaning of abbreviations and symbols, see introductory text]

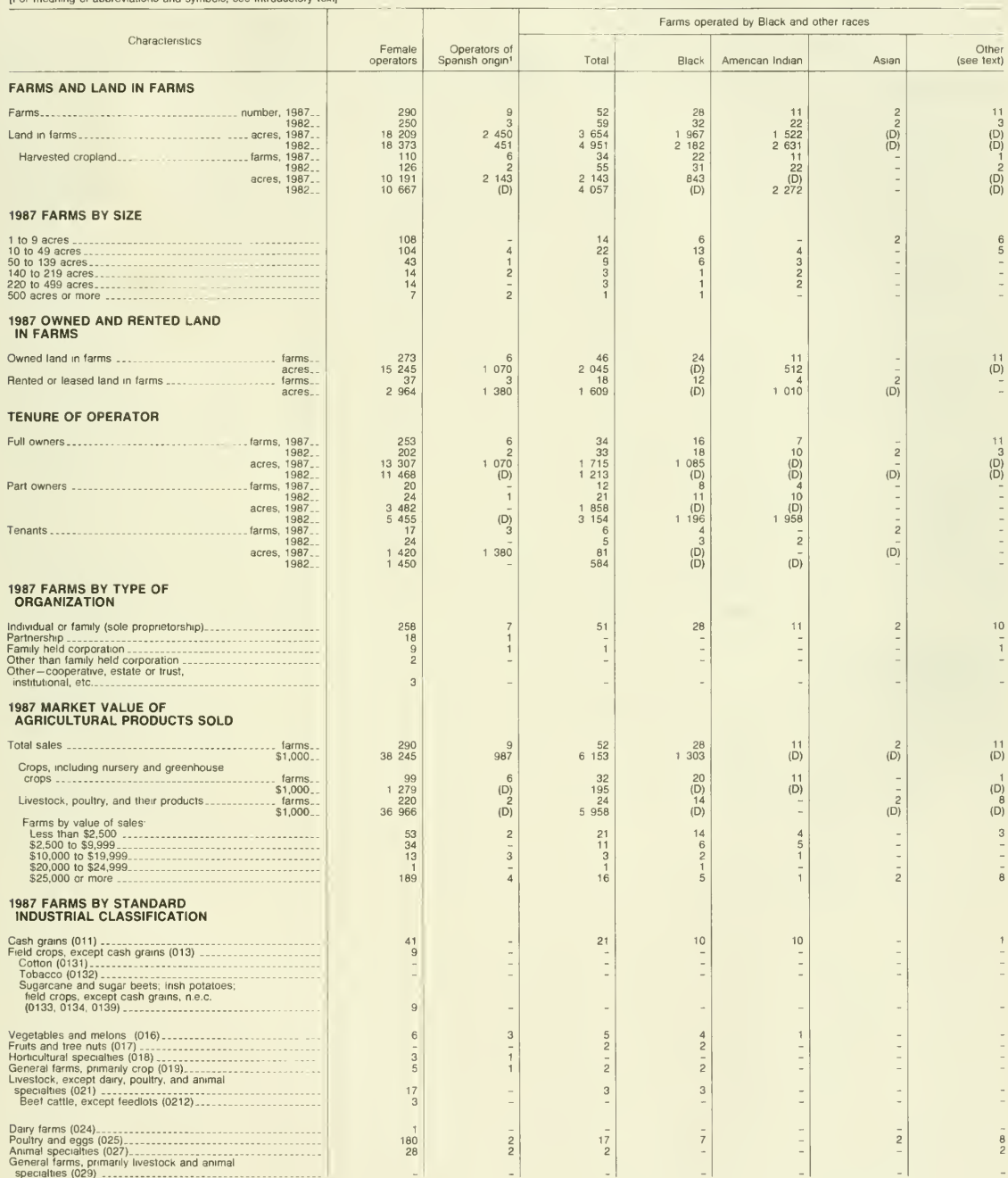

See footnotes at end ot table. 
Table 17. Selected Characteristics of Farms Operated by Females, Persons of Spanish Origin, and Specified Racial Groups: 1987 and 1982-Con.

[For meaning of abbreviations and symbols, see introductory text]

\begin{tabular}{|c|c|c|c|c|c|c|c|}
\hline \multirow[b]{2}{*}{ Characteristics } & \multirow[b]{2}{*}{$\begin{array}{r}\text { Female } \\
\text { operators }\end{array}$} & \multirow[b]{2}{*}{$\begin{array}{l}\text { Cperators of } \\
\text { Spanish onigin' }\end{array}$} & \multicolumn{5}{|c|}{ Farms operated by Black and other races } \\
\hline & & & Total & Black & American Indian & Asıan & $\begin{array}{r}\text { Other } \\
\text { (see text) }\end{array}$ \\
\hline $\begin{array}{l}1987 \text { OPERATOR } \\
\text { CHARACTERISTICS }\end{array}$ & & & & & & & \\
\hline $\begin{array}{l}\text { Operators by place of residence } \\
\text { On farm operated } \\
\text { Not on farm operated. } \\
\text { Not reported }\end{array}$ & $\begin{array}{r}242 \\
22 \\
26\end{array}$ & $\begin{array}{l}8 \\
1 \\
-\end{array}$ & $\begin{array}{r}32 \\
8 \\
12\end{array}$ & $\begin{array}{r}17 \\
6 \\
5\end{array}$ & $\frac{9}{2}$ & $\begin{array}{l}2 \\
-\end{array}$ & $\begin{array}{l}4 \\
2 \\
5\end{array}$ \\
\hline $\begin{array}{l}\text { Operators by principal occupation } \\
\text { Farming } \\
\text { Other }\end{array}$ & $\begin{array}{l}184 \\
106\end{array}$ & $\begin{array}{l}6 \\
3\end{array}$ & $\begin{array}{l}33 \\
19\end{array}$ & $\begin{array}{l}13 \\
15\end{array}$ & $\begin{array}{l}9 \\
2\end{array}$ & $\stackrel{2}{-}$ & $\begin{array}{l}9 \\
2\end{array}$ \\
\hline $\begin{array}{l}\text { Operators by days of work off farm } \\
\text { None } \\
\text { Any } \\
1 \text { to } 99 \text { days } \\
100 \text { to } 199 \text { days } \\
200 \text { days or more }\end{array}$ & $\begin{array}{r}179 \\
133 \\
20 \\
67 \\
46\end{array}$ & $\begin{array}{l}3 \\
6 \\
5 \\
1\end{array}$ & $\begin{array}{r}21 \\
26 \\
13 \\
5 \\
8\end{array}$ & $\begin{array}{r}11 \\
21 \\
4 \\
10 \\
7\end{array}$ & $\begin{array}{l}6 \\
5 \\
3 \\
1 \\
1\end{array}$ & $\begin{array}{l}2 \\
\overline{-} \\
\overline{-}\end{array}$ & $\begin{array}{l}2 \\
8 \\
6 \\
2 \\
-\end{array}$ \\
\hline Not reported $\ldots \ldots \ldots \ldots \ldots$ & 24 & 1 & 5 & 3 & 1 & - & 1 \\
\hline $\begin{array}{l}\text { Operators by years on present farm: } \\
2 \text { years or less } \\
3 \text { or } 4 \text { years } \\
5 \text { to } 9 \text { years } \\
10 \text { years ot more............ } \\
\text { Average years on present farm }\end{array}$ & $\begin{array}{r}31 \\
31 \\
49 \\
123 \\
161\end{array}$ & $\begin{array}{r}\overline{1} \\
1 \\
7 \\
14.9\end{array}$ & \begin{tabular}{r|r}
2 \\
1 \\
7 \\
27 \\
23.0
\end{tabular} & $\begin{array}{r}2 \\
4 \\
13 \\
25.2\end{array}$ & $\begin{array}{r}\overline{1} \\
\overline{9} \\
25.6\end{array}$ & $\overline{2}$ & $\begin{array}{r}- \\
1 \\
17 \\
17.3\end{array}$ \\
\hline Not reported.......... & 56 & - & 15 & 9 & 1 & - & 5 \\
\hline 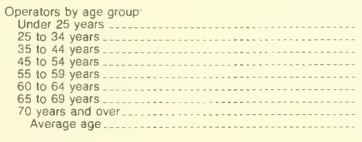 & $\begin{array}{r}2 \\
34 \\
66 \\
61 \\
28 \\
28 \\
22 \\
49 \\
53.1\end{array}$ & $\begin{array}{r}2 \\
4 \\
1 \\
2 \\
- \\
\overline{-} \\
\\
\end{array}$ & $\begin{array}{r}- \\
11 \\
9 \\
8 \\
5 \\
4 \\
15 \\
584\end{array}$ & $\begin{array}{r}- \\
2 \\
5 \\
3 \\
3 \\
4 \\
11 \\
628\end{array}$ & $\begin{array}{r}- \\
1 \\
2 \\
3 \\
1 \\
\overline{4} \\
61.3\end{array}$ & $\begin{array}{l}\overline{-} \\
\overline{2} \\
\overline{-} \\
\overline{-} \\
\text { (D) }\end{array}$ & $\overline{8}$ \\
\hline $\begin{array}{l}\text { Operators by sex } \\
\text { Male } \\
\text { Female }\end{array}$ & $\begin{array}{r}(x) \\
290\end{array}$ & $\begin{array}{l}7 \\
2\end{array}$ & $\begin{array}{l}41 \\
11\end{array}$ & 28 & $\begin{array}{r}10 \\
1\end{array}$ & $\overline{2}$ & $\begin{array}{l}3 \\
8\end{array}$ \\
\hline $\begin{array}{l}\text { Operators of Spanish origin' } \\
1987 \text { COMMODITY CREDIT } \\
\text { CORPORATION LOANS AND } \\
\text { GOVERNMENT PAYMENTS }\end{array}$ & 2 & 9 & 3 & - & - & - & 3 \\
\hline 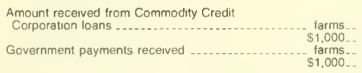 & $\begin{array}{r}3 \\
15 \\
21 \\
117\end{array}$ & (D) & $\overline{-}$ & $\underline{-}$ & $\overline{3}$ & $\begin{array}{l}\overline{-} \\
\overline{-}\end{array}$ & - \\
\hline
\end{tabular}

'See chapter 1 , table 16 for operators not of or not reporting Spanish ongin 
Table 18. Selected Characteristics of Farms by Standard Industrial Classification: 1987

[For meaning of abbreviations and symbols, see introductory text]

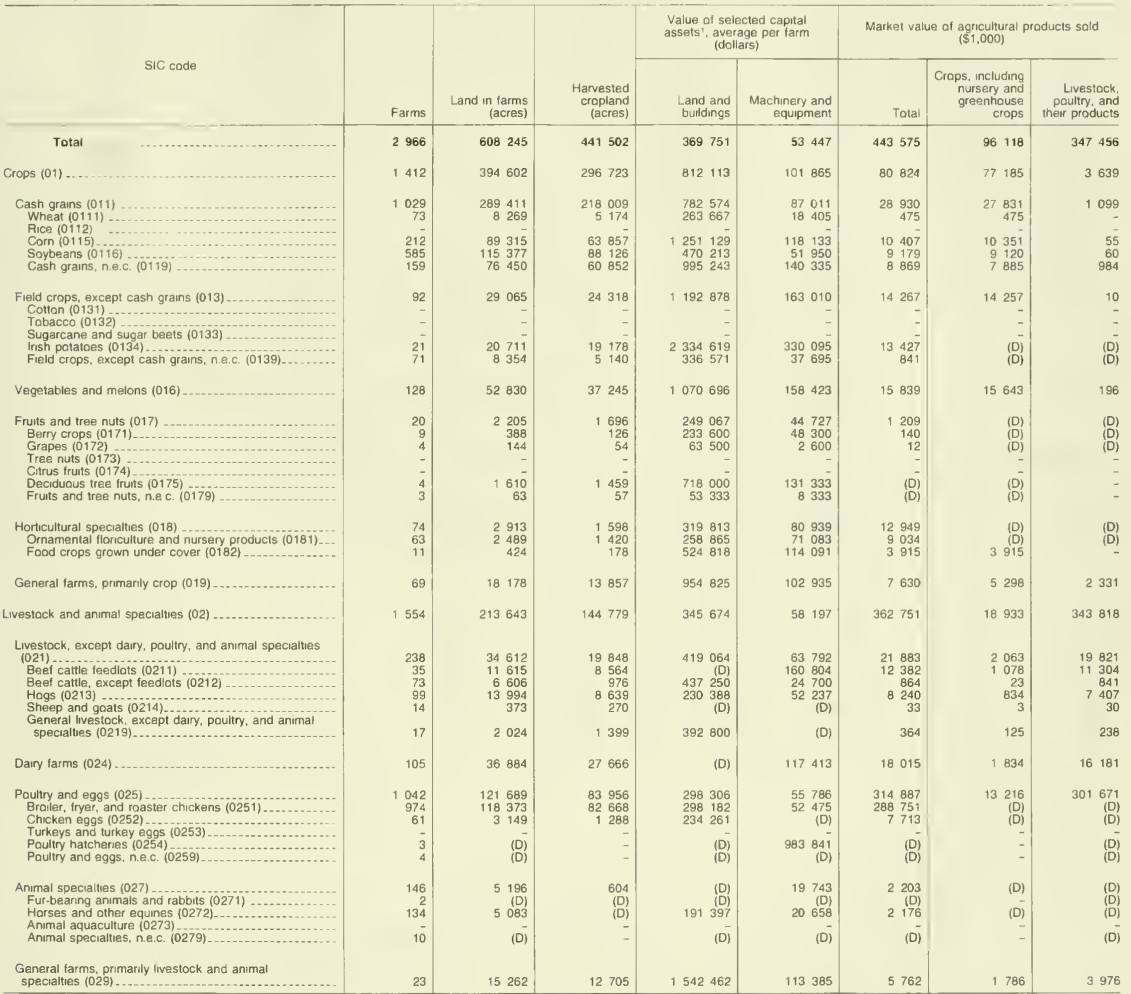

'Data are based on a sample of farms. 
Table 19. Selected Characteristics of Abnormal Farms: 1987 and 1982

[For meaning of abbreviatıons and symbols, see introductory text]

\begin{tabular}{|c|c|c|c|c|c|}
\hline Characteristics & 1987 & 1982 & Characteristics & 1987 & 1982 \\
\hline 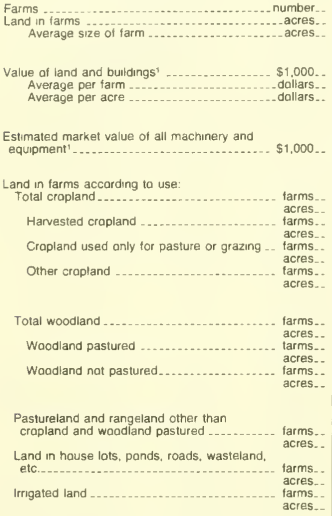 & $\begin{array}{r}\text { (D) } \\
\\
2 \\
\text { (D) } \\
2 \\
\text { (D) } \\
1 \\
\text { (D) } \\
2 \\
\text { (D) }\end{array}$ & $\begin{array}{l}\overline{-} \\
\overline{-} \\
\overline{-} \\
\overline{-} \\
\overline{-}\end{array}$ & 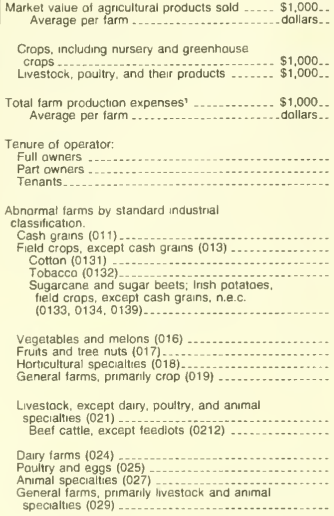 & $\begin{array}{l}\text { (D) } \\
\text { (D) } \\
\text { (D) } \\
\text { (D) } \\
\text { (D) } \\
\text { (D) }\end{array}$ & $\begin{array}{l}\text { - } \\
\text { - } \\
- \\
- \\
- \\
- \\
- \\
-\end{array}$ \\
\hline
\end{tabular}

Data are based on a sample of farms 
Table 20. Livestock and Poultry - Inventory and Sales: 1987, 1982, and 1978

[For meaning of abbreviations and symbols, see introductory text]

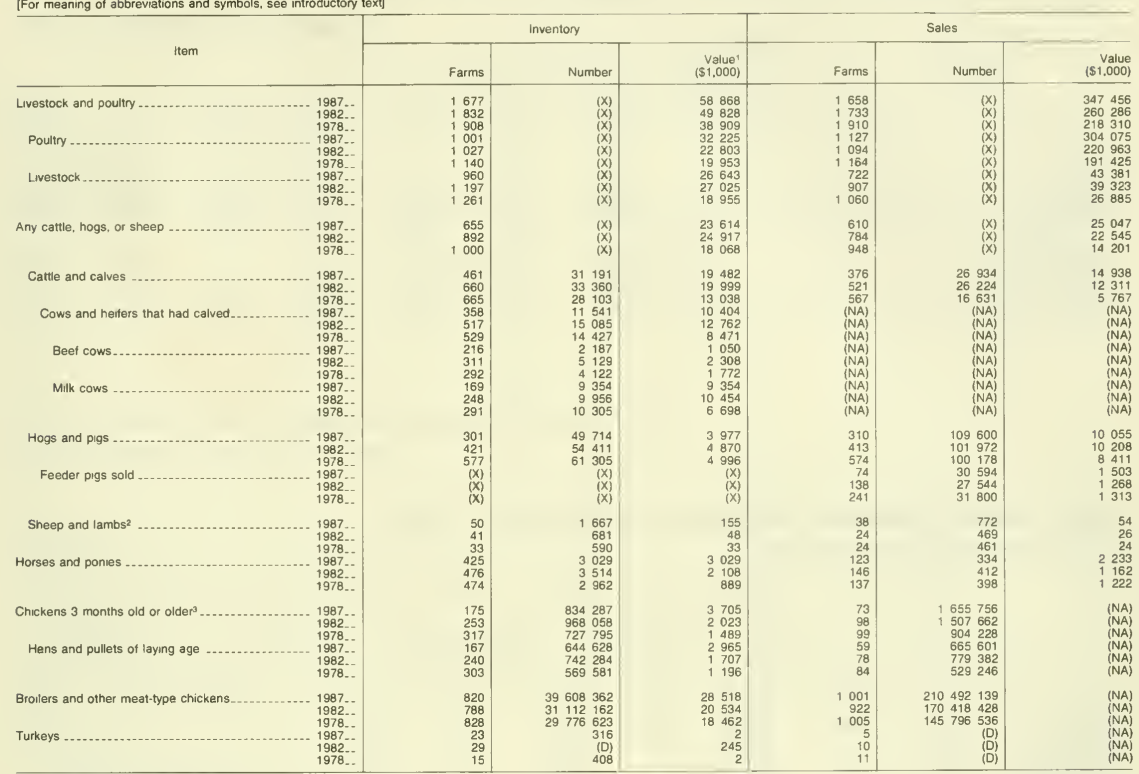

'Data are estimated; see text.

2Value of sales includes sheep, lambs, and wool sold.

3Sales for 1987 include puilets of less than 3 months old.

Table 21. Poultry-Inventory and Sales: 1987 and 1982

[For meaning of abbreviations and symbols, see introductory text]

\begin{tabular}{|c|c|c|c|c|c|c|c|c|c|}
\hline \multirow{2}{*}{ Item } & \multicolumn{2}{|c|}{1987} & \multicolumn{2}{|c|}{1982} & \multirow{2}{*}{ itern } & \multicolumn{2}{|c|}{1987} & \multicolumn{2}{|c|}{1982} \\
\hline & Farms & Number & Farms & Number & & Farms & Number & Farms & Number \\
\hline INVENTORY & & & & & SALES & & & & \\
\hline $\begin{array}{l}\text { Chickens } 3 \text { months old or oider } \\
\text { Farms with- } \\
1 \text { to } 3,199 \\
3,200 \text { to } 9999 . \\
10,000 \text { or more }\end{array}$ & $\begin{array}{r}130 \\
25 \\
20\end{array}$ & \begin{tabular}{rr|}
834 & 287 \\
7 & 328 \\
170 & 255 \\
$656 \quad 704$
\end{tabular} & $\begin{array}{r}253 \\
201 \\
31 \\
21\end{array}$ & $\begin{array}{rr}968 & 058 \\
18 & 703 \\
188 & 845 \\
760 & 510\end{array}$ & $\begin{array}{l}\text { Hens and pullets' } \\
\text { Farms with- } \\
1 \text { to } 3,199 . \\
3,00 \text { to } 9,999 \ldots \ldots \ldots \\
10.000 \text { or more }\end{array}$ & $\begin{array}{l}16 \\
30 \\
27\end{array}$ & $\begin{array}{r}1655756 \\
10418 \\
186514 \\
1458824\end{array}$ & $\begin{array}{l}37 \\
31 \\
30\end{array}$ & $\begin{array}{r}1507662 \\
16719 \\
186313 \\
1304630\end{array}$ \\
\hline $\begin{array}{l}\text { Hens and pullets of laying age } \\
\text { Farms with }- \\
1 \text { to } 99 \\
100 \text { to } 399 \\
400 \text { to } 3.199 \\
3,200 \text { to } 9999 \\
10,000 \text { to } 199999 \\
20,000 \text { to } 49,999 \\
50,000 \text { to } 99,999 \\
100.000 \text { or more }\end{array}$ & \begin{tabular}{r|}
167 \\
109 \\
18 \\
3 \\
22 \\
10 \\
1 \\
3 \\
1
\end{tabular} & 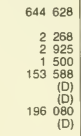 & \begin{tabular}{r|}
240 \\
163 \\
29 \\
8 \\
26 \\
8 \\
3 \\
2 \\
2 \\
1
\end{tabular} & $\begin{array}{r}742284 \\
4893 \\
4220 \\
(D) \\
165924 \\
105600 \\
86000 \\
\text { (D) } \\
\text { (D) }\end{array}$ & $\begin{array}{l}\text { Hens and pullets of laying age ....... } \\
\text { Farms with } \\
1 \text { to } 99 \\
100 \text { to } 399 \\
400 \text { to } 3.199 \\
3,200 \text { to } 9,999 \\
10,000 \text { to } 19999 \\
20,000 \text { to } 49,999 \\
50,000 \text { to } 99,999 \\
100,000 \text { or more }\end{array}$ & $\begin{array}{r}59 \\
9 \\
3 \\
3 \\
30 \\
8 \\
2 \\
3 \\
1\end{array}$ & $\begin{array}{r}665601 \\
\\
252 \\
\text { (D) } \\
6123 \\
187514 \\
103960 \\
\text { (D) } \\
187400 \\
\text { (D) }\end{array}$ & \begin{tabular}{r|r}
78 \\
16 \\
12 \\
12 \\
9 \\
28 \\
6 \\
3 \\
3 \\
3
\end{tabular} & $\begin{array}{r}779382 \\
575 \\
2423 \\
13721 \\
174 \quad 113 \\
76750 \\
74000 \\
\text { (D) } \\
\text { (D) }\end{array}$ \\
\hline $\begin{array}{l}\text { Broilers and other meat-type chickens } \\
\text { Turkeys } \\
\text { For siaughter } \\
\text { Hens kept for breeding ............ }\end{array}$ & $\begin{array}{r}820 \\
23 \\
14 \\
12\end{array}$ & $\begin{array}{r}39608362 \\
316 \\
266 \\
50\end{array}$ & $\begin{array}{r}788 \\
29 \\
22 \\
11\end{array}$ & 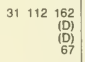 & $\begin{array}{l}\text { Brollers and other meat-type chickens } \\
\text { Turkeys } \\
\text { For slaughter } \\
\text { Hens kept for breeding }\end{array}$ & $\begin{array}{r}1001 \\
5 \\
5 \\
-\end{array}$ & $\begin{array}{rl}210492 & 139 \\
& (\mathrm{D}) \\
& \text { (D) }\end{array}$ & $\begin{array}{r}922 \\
10 \\
9 \\
1\end{array}$ & $\begin{aligned} 170418428 \\
\text { (D) } \\
\text { (D) } \\
\text { (D) }\end{aligned}$ \\
\hline Ducks, geese, and other poultry ........ & 57 & (x) & 53 & $(\mathbf{x})$ & Ducks, geese, and other poultry ........ & 15 & $(x)$ & 39 & $(x)$ \\
\hline
\end{tabular}

'Sales for 1987 include pullets of less than 3 months old. 
Table 22. Broilers and Started Pullets-Sales: 1987 and 1982

[For meaning of abbreviations and symbols, see introductory text]

\begin{tabular}{|c|c|c|c|c|c|c|c|c|}
\hline \multirow{3}{*}{ Number sold } & \multicolumn{4}{|c|}{ Broilers and other meat-type chickens } & \multirow{2}{*}{\multicolumn{2}{|c|}{$\begin{array}{c}\text { Pultets not of laying age } \\
1987\end{array}$}} & \multirow{2}{*}{\multicolumn{2}{|c|}{\begin{tabular}{|c} 
Pullets 3 months old or older not \\
of laying age
\end{tabular}}} \\
\hline & \multicolumn{2}{|c|}{1987} & \multicolumn{2}{|c|}{1982} & & & & \\
\hline & Farms & Number & Farms & Number & Farms & Number & Farms & Number \\
\hline Total... & 1001 & 210492139 & 922 & 170418428 & 17 & 990155 & 21 & 728280 \\
\hline $\begin{array}{l}\text { Farms with- } \\
1 \text { to } 1,999 \\
2,000 \text { to } 15,999 \\
16,000 \text { to } 29,999 \\
30,000 \text { to } 59,999 \\
60,000 \text { to } 99,999\end{array}$ & $\begin{array}{r}6 \\
10 \\
12 \\
82 \\
140\end{array}$ & 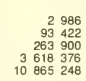 & $\begin{array}{r}19 \\
10 \\
20 \\
76 \\
143\end{array}$ & $\begin{array}{r}(D) \\
(D) \\
457840 \\
3422850 \\
11210657\end{array}$ & $\begin{array}{l}1 \\
6 \\
4 \\
5 \\
-\end{array}$ & $\begin{array}{r}\text { (D) } \\
\text { (D) } \\
78500 \\
191150 \\
\end{array}$ & $\begin{array}{l}- \\
9 \\
5 \\
4 \\
-\end{array}$ & $\begin{array}{r}10- \\
105300 \\
149280\end{array}$ \\
\hline $\begin{array}{r}100,000 \text { to } 199,999 \\
200,000 \text { to } 499,999 \\
200,000 \text { to } 299,999 \\
300,000 \text { to } 499,999 \\
500,000 \text { or more }\end{array}$ & $\begin{array}{r}320 \\
383 \\
264 \\
119 \\
48\end{array}$ & $\begin{array}{rrr}46 & 373 & 405 \\
105 & 230 & 502 \\
60 & 999 & 668 \\
44 & 230 & 834 \\
44 & 044 & 300\end{array}$ & $\begin{array}{r}349 \\
267 \\
\text { (NA) } \\
\text { (NA) } \\
38\end{array}$ & $\begin{array}{rrr}48 & 315 & 461 \\
75 & 699 & 069 \\
& (\text { NA) } \\
& \text { (NA) } \\
31 & 224 & 622\end{array}$ & $\begin{array}{r}\text { (NA) } \\
\text { (NA) } \\
1\end{array}$ & $\begin{array}{l}\text { (NA) } \\
\text { (NA) } \\
\text { (D) }\end{array}$ & $\begin{array}{r}3 \\
- \\
\text { (NA) } \\
\text { (NA) } \\
-\end{array}$ & $\begin{array}{r}376000 \\
\text { (NA) } \\
\text { (NA) }\end{array}$ \\
\hline
\end{tabular}

Table 23. Poultry-Inventory and Sales by Size of Flock: 1987

[For meaning of abbreviations and symbois, see introductory text]

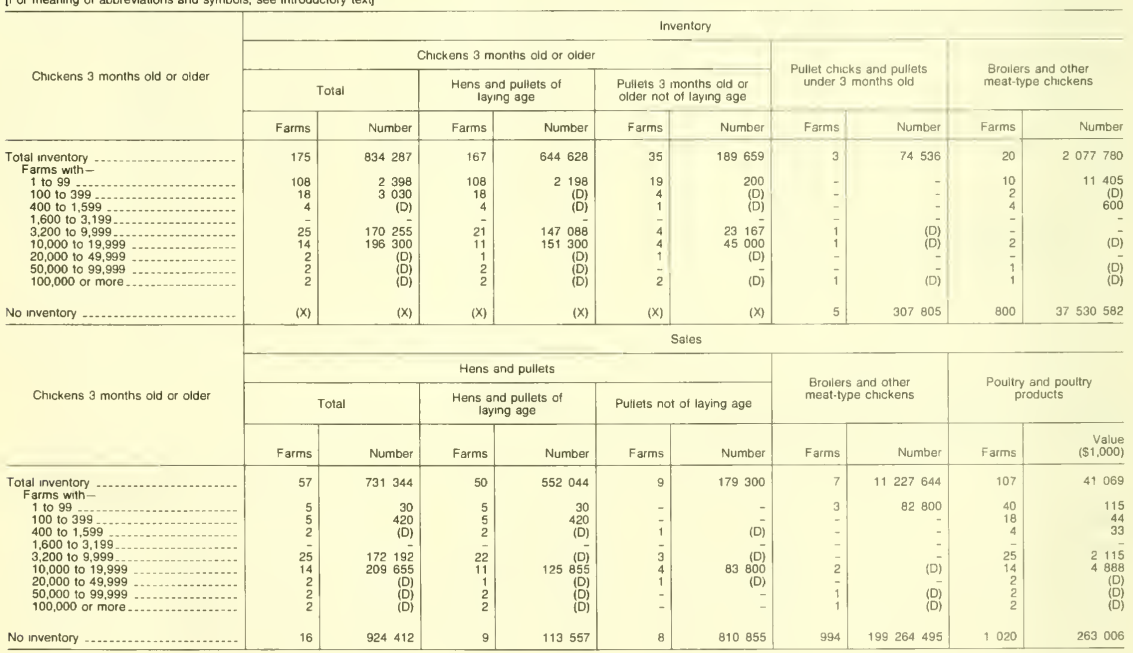


Table 24. Turkeys-Sales by Number Sold Per Farm: 1987

[For meaning of abbreviations and symbols, see introductory text]

\begin{tabular}{|c|c|c|c|c|c|c|}
\hline \multirow{2}{*}{ Turkeys } & \multicolumn{2}{|c|}{ Total } & \multicolumn{2}{|c|}{ Sales for slaughter } & \multicolumn{2}{|c|}{ Sales of hens kept for breeding } \\
\hline & Farms & Number & Farms & Number & Farms & Number \\
\hline $\begin{array}{l}\text { Total sold } \\
\text { Farms with- } \\
1 \text { to } 1.999 \\
2.000 \text { to } 7,999 \\
8,000 \text { to } 15,999 \\
16, \ldots 00 \text { to } 29.999 \\
30,000 \text { to } 59.999 \ldots \ldots \\
60,000 \text { to } 99,999 \ldots \ldots \\
100,000 \text { or more }\end{array}$ & $\begin{array}{l}5 \\
5 \\
- \\
- \\
- \\
- \\
-\end{array}$ & $\begin{array}{r}\text { (D) } \\
\text { (D) } \\
- \\
- \\
- \\
-\end{array}$ & $\begin{array}{l}5 \\
5 \\
- \\
\vdots \\
\vdots \\
-\end{array}$ & $\begin{array}{r}\text { (D) } \\
\text { (D) } \\
\\
- \\
= \\
-\end{array}$ & $\begin{array}{l}- \\
= \\
- \\
z \\
z \\
=\end{array}$ & $\begin{array}{l} \\
\overline{-} \\
\vdots \\
\end{array}$ \\
\hline
\end{tabular}

Table 25. Cattle and Calves-Inventory: 1987 and 1982

[For meaning of abbrevistions and symbols, see introductory text]

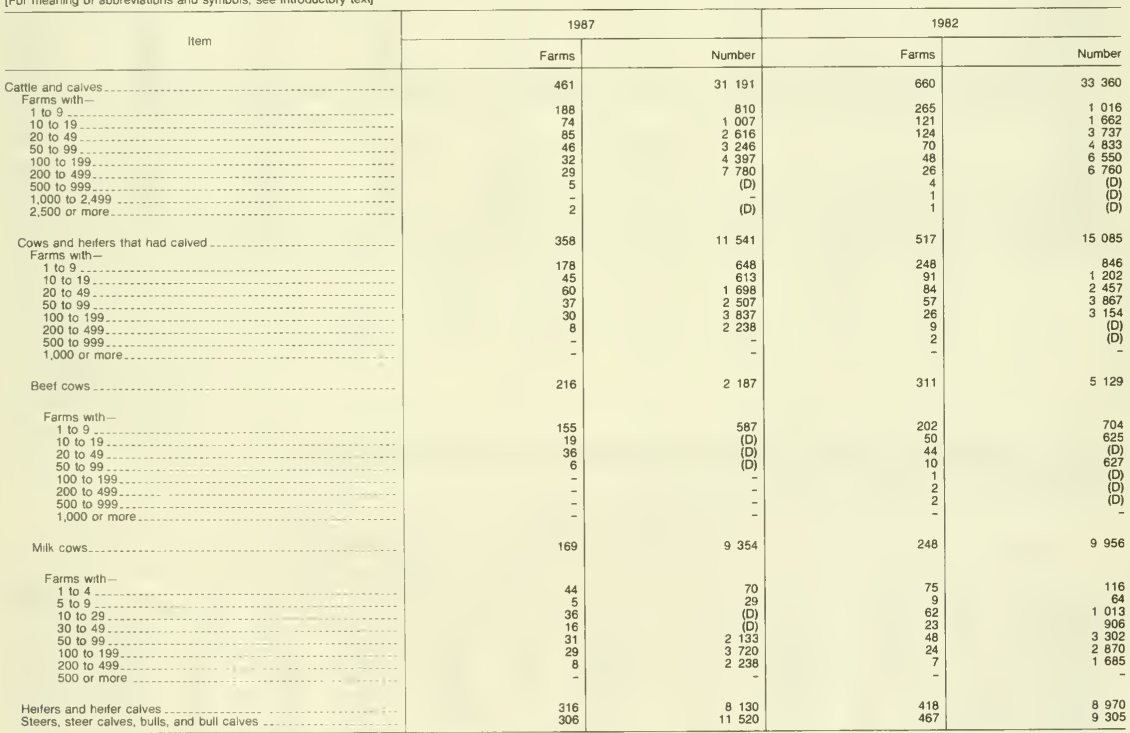


Table 26. Cattle and Calves-Sales: 1987 and 1982

[For meaning of abbreviations and symbois, see introductory text]

\begin{tabular}{|c|c|c|c|c|c|c|}
\hline \multirow[b]{2}{*}{ Item } & \multicolumn{3}{|c|}{1987} & \multicolumn{3}{|c|}{1982} \\
\hline & Farms & Number & $\begin{array}{r}\text { Value } \\
(\$ 1,000)\end{array}$ & Farms & Number & $\begin{array}{r}\text { Value } \\
(\$ 1,000)\end{array}$ \\
\hline 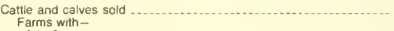 & 376 & 26934 & 14938 & 521 & 26224 & 12311 \\
\hline 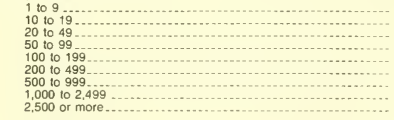 & $\begin{array}{r}178 \\
72 \\
62 \\
39 \\
12 \\
8 \\
2 \\
1 \\
1 \\
2\end{array}$ & 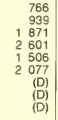 & $\begin{array}{r}314 \\
333 \\
555 \\
821 \\
461 \\
774 \\
\text { (D) } \\
\text { (D) } \\
\text { (D) }\end{array}$ & $\begin{array}{r}248 \\
111 \\
97 \\
43 \\
14 \\
1 \\
6 \\
-1 \\
1\end{array}$ & $\begin{array}{r}954 \\
1562 \\
3021 \\
2980 \\
\text { (D) } \\
\text { (D) } \\
\text { (D) } \\
\text { (D) }\end{array}$ & $\begin{array}{r}337 \\
518 \\
894 \\
914 \\
\text { (D) } \\
\text { (D) } \\
580 \\
\text { (D) }\end{array}$ \\
\hline $\begin{array}{l}\text { Cattle sold } \\
\text { Farms with- }\end{array}$ & 336 & 20377 & 13641 & 417 & 18767 & 11139 \\
\hline 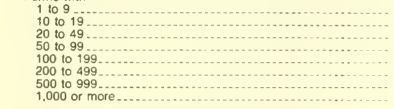 & $\begin{array}{r}209 \\
59 \\
42 \\
17 \\
3 \\
3 \\
1 \\
2\end{array}$ & $\begin{array}{r}791 \\
761 \\
1252 \\
1075 \\
331 \\
798 \\
\text { (D) } \\
\text { (D) }\end{array}$ & $\begin{array}{l}408 \\
363 \\
656 \\
584 \\
240 \\
428 \\
\text { (D) } \\
\text { (D) }\end{array}$ & $\begin{array}{r}262 \\
85 \\
49 \\
10 \\
6 \\
1 \\
3 \\
1\end{array}$ & $\begin{array}{rr} & 975 \\
1 & 137 \\
1 & 393 \\
665 \\
\\
\\
\\
& \text { (D) } \\
& \text { (D) } \\
& \text { (D) } \\
& \text { (D) }\end{array}$ & $\begin{array}{r}476 \\
590 \\
795 \\
403 \\
\text { (D) } \\
\text { (D) } \\
1023 \\
\text { (D) }\end{array}$ \\
\hline Cattle fattened on grain and concentrates sold ................. & 99 & 16835 & 11813 & 129 & 14415 & 8818 \\
\hline 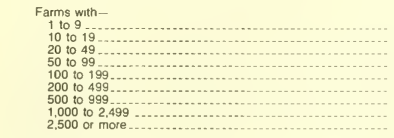 & $\begin{array}{r}72 \\
12 \\
6 \\
4 \\
\overline{2} \\
1 \\
\\
2\end{array}$ & $\begin{array}{r}250 \\
177 \\
227 \\
258 \\
- \\
\text { (D) } \\
\text { (D) } \\
\text { (D) }\end{array}$ & $\begin{array}{r}143 \\
83 \\
140 \\
152 \\
- \\
\text { (D) } \\
\text { (D) } \\
\text { (D) }\end{array}$ & $\begin{array}{r}97 \\
16 \\
7 \\
3 \\
2 \\
1 \\
2 \\
1\end{array}$ & $\begin{array}{l}310 \\
196 \\
236 \\
198 \\
\text { (D) } \\
\text { (D) } \\
\text { (D) } \\
\text { (D) }\end{array}$ & $\begin{array}{l}157 \\
116 \\
151 \\
111 \\
\text { (D) } \\
\text { (D) } \\
\text { (D) } \\
\text { (D) }\end{array}$ \\
\hline $\begin{array}{l}\text { Calves sold } \\
\text { Farms with-.................... }\end{array}$ & 219 & 6557 & 1296 & 351 & 7457 & 1172 \\
\hline 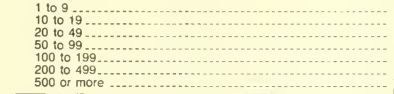 & $\begin{array}{r}119 \\
34 \\
31 \\
25 \\
6 \\
2 \\
2\end{array}$ & $\begin{array}{r}530 \\
459 \\
955 \\
1574 \\
\text { (D) } \\
\text { (D) } \\
\text { (D) }\end{array}$ & $\begin{array}{r}75 \\
65 \\
103 \\
153 \\
53 \\
\text { (D) } \\
\text { (D) }\end{array}$ & $\begin{array}{r}182 \\
66 \\
75 \\
20 \\
5 \\
- \\
3\end{array}$ & $\begin{array}{r}678 \\
885 \\
2310 \\
+252 \\
547 \\
1785\end{array}$ & $\begin{array}{r}90 \\
128 \\
273 \\
127 \\
62 \\
492 \\
49\end{array}$ \\
\hline
\end{tabular}

Table 27. Cattle and Calves - Inventory and Sales by Size of Herd: 1987

[For meaning of abbreviations and symbols, see introductory text]

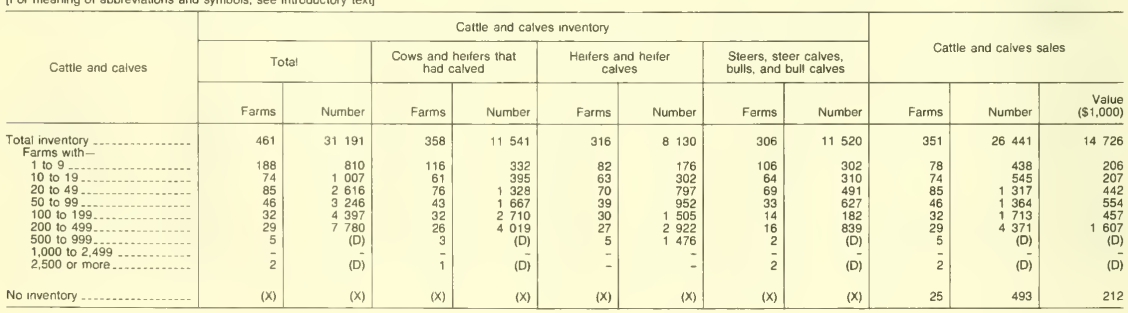


Table 28. Cattle and Calves - Inventory and Sales by Size of Cow Herd: 1987

[For meaning of abbreviations and symbols, see introductory text]

\begin{tabular}{|c|c|c|c|c|c|c|c|c|c|c|c|c|}
\hline \multirow{3}{*}{$\begin{array}{c}\text { Cows and herfers that had } \\
\text { celved }\end{array}$} & \multicolumn{8}{|c|}{ Cattle and calves inventory } & \multirow{2}{*}{\multicolumn{4}{|c|}{ Cattie and calves sales }} \\
\hline & \multicolumn{2}{|c|}{ Total } & \multicolumn{2}{|c|}{$\begin{array}{c}\text { Cows and heifers that } \\
\text { had calved }\end{array}$} & \multicolumn{2}{|c|}{$\begin{array}{l}\text { Heifers and heifer } \\
\text { calves }\end{array}$} & \multicolumn{2}{|c|}{$\begin{array}{l}\text { Steers, steer calves, } \\
\text { bulls, and bull calves }\end{array}$} & & & & \\
\hline & Farms & Number & Farms & Number & Farms & Number & Farms & Number & Farms & Number & \multicolumn{2}{|c|}{$\begin{array}{r}\text { Value } \\
(\$ 1,000)\end{array}$} \\
\hline $\begin{array}{l}\text { Total inventory ... } \\
\text { Farms with- }\end{array}$ & 358 & 24689 & 358 & 11541 & 259 & 7218 & 224 & 5930 & 288 & 15858 & & 142 \\
\hline Farms with- & 113 & 822 & 113 & 248 & 56 & 349 & 59 & 225 & 50 & 416 & & 214 \\
\hline 5 to 9 & 65 & 849 & 65 & 400 & 44 & 177 & 47 & 272 & 58 & 466 & & 180 \\
\hline $\begin{array}{l}10 \text { to } 19 \\
20 \text { to } 29\end{array}$ & 45 & 1096 & 45 & 613 & 42 & 335 & 40 & 148 & 45 & 585 & & $\begin{array}{l}171 \\
168\end{array}$ \\
\hline $\begin{array}{l}20 \text { to } 29 \\
30 \text { to } 49\end{array}$ & $\begin{array}{l}32 \\
28\end{array}$ & 1253 & $\begin{array}{l}32 \\
28\end{array}$ & $\begin{array}{l}717 \\
981\end{array}$ & $\begin{array}{l}23 \\
24\end{array}$ & $\begin{array}{l}300 \\
557\end{array}$ & $\begin{array}{l}24 \\
20\end{array}$ & $\begin{array}{l}236 \\
203\end{array}$ & $\begin{array}{l}32 \\
28\end{array}$ & $\begin{array}{l}526 \\
585\end{array}$ & & $\begin{array}{l}168 \\
240\end{array}$ \\
\hline $\begin{array}{l}30 \text { to } 49 \\
50 \text { to } 99\end{array}$ & $\begin{array}{l}28 \\
37\end{array}$ & $\begin{array}{l}1741 \\
8548\end{array}$ & 37 & 2507 & $\begin{array}{l}24 \\
34\end{array}$ & 1379 & 19 & 4662 & $\begin{array}{l}20 \\
37\end{array}$ & 9358 & 6 & \\
\hline 100 to 199 & 30 & 6866 & 30 & 3837 & 28 & (D) & 14 & (D) & 30 & 2641 & & 731 \\
\hline 200 to 499 & 8 & 3514 & 8 & 2238 & 8 & (D) & 1 & (D) & 8 & 1281 & & 376 \\
\hline 500 to 999 & - & - & $\overline{-}$ & $\overline{-}$ & - & - & $=$ & $\overline{-}$ & - & - & & - \\
\hline 1,000 or more............... & - & & - & & - & & - & & - & & & \\
\hline No inventory .... & 103 & 6502 & $(\mathrm{X})$ & $(\mathrm{X})$ & 57 & 912 & 82 & 5590 & 88 & 11076 & 6 & 796 \\
\hline
\end{tabular}

Table 29. Cattle and Calves - Inventory and Sales by Size of Beef Cow Herd: 1987

[For meaning of abbreviations and symbols, see introductory text]

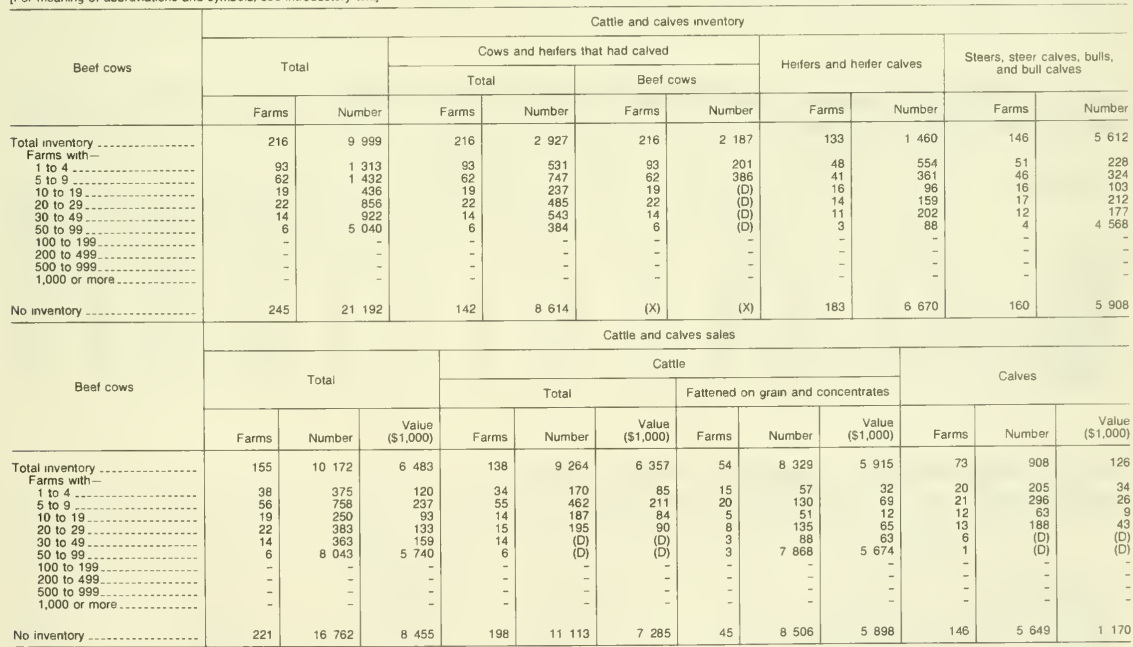


Table 30. Cattle and Calves - Inventory and Sales by Size of Milk Cow Herd: 1987

[For meaning of abbreviations and symbols, see intraductory tex?]

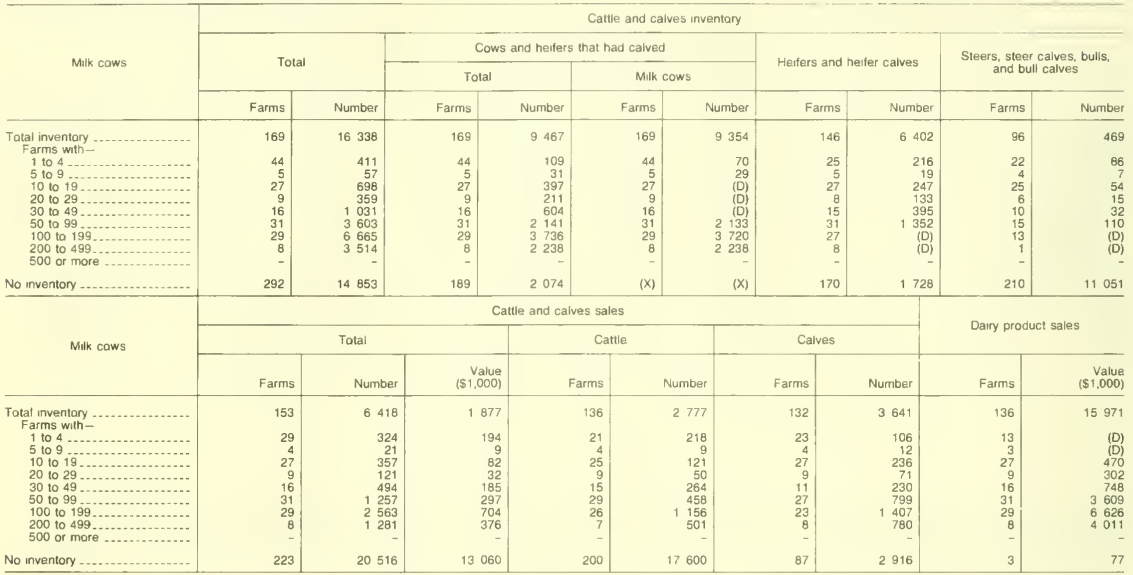

Table 31. Cattle and Calves-Sales by Number Sold Per Farm: 1987

[For meaning of abbreviations and symbols, see introductary text]

\begin{tabular}{|c|c|c|c|c|c|c|c|c|c|c|c|c|}
\hline \multirow{3}{*}{ Cattle and calves } & \multirow{2}{*}{\multicolumn{3}{|c|}{ Cattle and calves }} & \multicolumn{6}{|c|}{ Cattie } & \multirow{2}{*}{\multicolumn{3}{|c|}{ Calves }} \\
\hline & & & & \multicolumn{3}{|c|}{ Total } & \multicolumn{3}{|c|}{ Fattened on grain and concentrates } & & & \\
\hline & Farms & Number & $\begin{array}{r}\text { Value } \\
(\$ 1,000)\end{array}$ & Farms & Number & $\begin{array}{r}\text { Value } \\
(\$ 1,000)\end{array}$ & Farms & Number & $\begin{array}{r}\text { Value } \\
(\$ 1,000)\end{array}$ & Farms & Number & $\begin{array}{r}\text { Value } \\
(\$ 1,000)\end{array}$ \\
\hline $\begin{array}{l}\text { Tatal sold } \\
\text { Farms with- }\end{array}$ & 376 & & 14938 & 336 & 20377 & 13641 & 99 & 16835 & 11813 & 219 & 6557 & 1296 \\
\hline $\begin{array}{l}1 \text { to } 4 \\
5 \text { to } 9 \\
10 \text { to } 19 \\
20 \text { to } 49 \\
50 \text { to } 99 \\
100 \text { to } 199 \\
200 \text { to } 499 \\
500 \text { to } 999 \\
1,000 \text { or mare.... }\end{array}$ & $\begin{array}{r}98 \\
80 \\
72 \\
62 \\
39 \\
12 \\
8 \\
2 \\
3\end{array}$ & $\begin{array}{r}241 \\
525 \\
939 \\
1 \quad 871 \\
2.601 \\
1 \quad 506 \\
2077 \\
\\
\text { (D) } \\
\text { (D) }\end{array}$ & $\begin{array}{l}105 \\
209 \\
333 \\
555 \\
821 \\
461 \\
774 \\
\text { (D) } \\
\text { (D) }\end{array}$ & $\begin{array}{r}84 \\
70 \\
71 \\
55 \\
35 \\
11 \\
7 \\
1 \\
2\end{array}$ & $\begin{array}{r}181 \\
326 \\
599 \\
916 \\
1353 \\
537 \\
\text { (D) } \\
\text { (D) } \\
\text { (D) }\end{array}$ & $\begin{array}{r}96 \\
174 \\
291 \\
429 \\
725 \\
354 \\
\text { (D) } \\
\text { (D) } \\
\text { (D) }\end{array}$ & $\begin{array}{r}37 \\
20 \\
16 \\
10 \\
9 \\
1 \\
3 \\
1 \\
2\end{array}$ & $\begin{array}{r}86 \\
92 \\
127 \\
185 \\
312 \\
\text { (D) } \\
624 \\
\text { (D) } \\
\text { (D) }\end{array}$ & $\begin{array}{r}51 \\
54 \\
77 \\
93 \\
190 \\
\text { (D) } \\
363 \\
\text { (D) } \\
\text { (D) }\end{array}$ & $\begin{array}{r}31 \\
45 \\
45 \\
48 \\
30 \\
12 \\
6 \\
1 \\
1\end{array}$ & $\begin{array}{r}60 \\
199 \\
340 \\
955 \\
1248 \\
969 \\
\text { (D) } \\
\text { (D) } \\
\text { (D) }\end{array}$ & $\begin{array}{r}9 \\
36 \\
42 \\
126 \\
96 \\
107 \\
\text { (D) } \\
\text { (D) } \\
\text { (D) }\end{array}$ \\
\hline
\end{tabular}

Table 32. Hogs and Pigs-Inventory: 1987 and 1982

[For meaning of abbreviations and symbols, see introductory text]

\begin{tabular}{|c|c|c|c|c|}
\hline \multirow{2}{*}{ Item } & \multicolumn{2}{|c|}{1987} & \multicolumn{2}{|c|}{1982} \\
\hline & Farms & Number & Farms & Number \\
\hline $\begin{array}{l}\text { Tatal hogs and pigs .............. } \\
\quad \text { Farms with - }\end{array}$ & 301 & 49714 & 421 & $54 \quad 411$ \\
\hline $\begin{array}{l}1 \\
25 \\
50 \text { to } 4909 \text { to } 199 \\
200 \text { to } 499 \\
500 \text { to } 999 \\
1,000 \text { to } 1,999 \\
2,000 \text { to } 4,999\end{array}$ & $\begin{array}{r}123 \\
56 \\
47 \\
23 \\
23 \\
15 \\
11 \\
3 \\
-\end{array}$ & $\begin{array}{rr}1 & 982 \\
3 & 150 \\
2 & 744 \\
7 & 412 \\
10 & 136 \\
15 & 265 \\
8 & 151 \\
& -\end{array}$ & $\begin{array}{r}200 \\
50 \\
66 \\
42 \\
32 \\
19 \\
9 \\
3 \\
-\end{array}$ & $\begin{array}{rr}1 & 576 \\
1 & 687 \\
4 & 353 \\
5 & 441 \\
9 & 600 \\
12 & 956 \\
11 & 673 \\
7 & 125\end{array}$ \\
\hline $\begin{array}{l}\text { Hogs and pigs used ar to be used far breeding .......................... } \\
\text { Farms with - }\end{array}$ & 203 & 10813 & 287 & 8689 \\
\hline $\begin{array}{l}1 \text { to } 24 \\
50 \text { to } 49 \\
100 \text { to } 199 \ldots \\
200 \text { or more }\end{array}$ & $\begin{array}{r}141 \\
22 \\
16 \\
13 \\
11\end{array}$ & $\begin{array}{ll}1 & 122 \\
& 739 \\
1 & 186 \\
1 & 951 \\
5 & 815\end{array}$ & $\begin{array}{r}231 \\
17 \\
19 \\
13 \\
7\end{array}$ & $\begin{array}{rr}1 & 787 \\
& 552 \\
1 & 284 \\
1 & 655 \\
3 & 411\end{array}$ \\
\hline
\end{tabular}


Table 33. Hogs and Pigs-Sales: 1987 and 1982

[For meaning of abbrevrations and symbols, see introductory text]

\begin{tabular}{|c|c|c|c|c|c|c|}
\hline \multirow[b]{2}{*}{ Hem } & \multicolumn{3}{|c|}{1987} & \multicolumn{3}{|c|}{1982} \\
\hline & Farms & Number & $\begin{array}{r}\text { Value } \\
(\$ 1,000)\end{array}$ & Farms & Number & $\begin{array}{r}\text { Value } \\
(\$ 1.000)\end{array}$ \\
\hline Total hogs and pigs sold & 310 & 109600 & 10055 & 413 & 101972 & 10208 \\
\hline 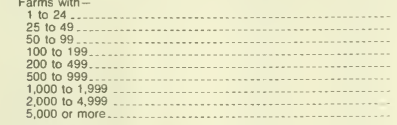 & $\begin{array}{r}77 \\
66 \\
53 \\
41 \\
25 \\
20 \\
15 \\
10 \\
3\end{array}$ & \begin{tabular}{rr|}
2 & 748 \\
3 & 619 \\
5 & 271 \\
7 & 552 \\
13 & 756 \\
19 & 741 \\
29 & 192 \\
27 & 500
\end{tabular} & $\begin{array}{rr}70 \\
207 \\
354 \\
497 \\
729 \\
1249 \\
1709 \\
2863 \\
2377\end{array}$ & \begin{tabular}{r|}
122 \\
79 \\
68 \\
61 \\
39 \\
19 \\
15 \\
8 \\
2
\end{tabular} & $\begin{array}{rr}1 & 303 \\
2 & 819 \\
4 & 637 \\
8 & 005 \\
12 & 471 \\
12 & 861 \\
21 & 728 \\
& \text { (D) } \\
\text { (D) }\end{array}$ & $\begin{array}{r}136 \\
291 \\
415 \\
729 \\
1 \\
139 \\
1404 \\
2183 \\
2 \\
\quad(D) \\
\text { (D) }\end{array}$ \\
\hline $\begin{array}{l}\text { Feeder pigs sold } \\
\text { Farms with }\end{array}$ & 74 & 30594 & 1503 & 138 & 27544 & 1268 \\
\hline $\begin{array}{l}10 \text { to } 49 \\
50 \text { to } 99 \\
100 \text { to } 199 \\
200 \text { to } 499 \\
500 \text { to } 999 \\
1,000 \text { or more }\end{array}$ & $\begin{array}{r}7 \\
30 \\
8 \\
9 \\
5 \\
5 \\
10\end{array}$ & $\begin{array}{rr}665 \\
566 \\
1 \\
107 \\
1233 \\
2995 \\
23 & 984\end{array}$ & $\begin{array}{r}2 \\
25 \\
20 \\
37 \\
50 \\
122 \\
1246\end{array}$ & $\begin{array}{r}14 \\
53 \\
25 \\
21 \\
16 \\
2 \\
7\end{array}$ & $\begin{array}{rr} & 92 \\
1 & 342 \\
1 & 672 \\
2 & 760 \\
2 & (\mathrm{D}) \\
& (\mathrm{D}) \\
15 & 802\end{array}$ & $\begin{array}{r}3 \\
52 \\
70 \\
99 \\
\text { (D) } \\
\text { (D) } \\
789\end{array}$ \\
\hline 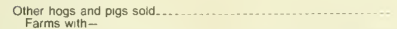 & 285 & 79006 & 8552 & 363 & 74428 & 8940 \\
\hline 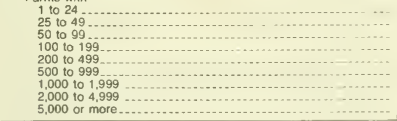 & $\begin{array}{r}92 \\
53 \\
47 \\
34 \\
22 \\
16 \\
12 \\
8 \\
1\end{array}$ & $\begin{array}{rr} & 816 \\
1 & 843 \\
3 & 267 \\
4 & 457 \\
6 & 718 \\
11 & 269 \\
14 & 999 \\
& \text { (D) } \\
& \text { (D) }\end{array}$ & $\begin{array}{r}91 \\
195 \\
362 \\
486 \\
720 \\
1220 \\
1518 \\
\text { (D) } \\
\text { (D) }\end{array}$ & $\begin{array}{l}\text { (NA) } \\
\text { (NA) } \\
\text { (NA) } \\
\text { (NA) } \\
\text { (NA) } \\
\text { (NA) } \\
\text { (NA) } \\
\text { (NA) } \\
\text { (NA) }\end{array}$ & $\begin{array}{l}\text { (NA) } \\
\text { (NA) } \\
\text { (NA) } \\
\text { (NA) } \\
\text { (NA) } \\
\text { (NA) } \\
\text { (NA) } \\
\text { (NA) } \\
\text { (NA) }\end{array}$ & $\begin{array}{l}\text { (NA) } \\
\text { (NA) } \\
\text { (NA) } \\
\text { (NA) } \\
\text { (NA) } \\
\text { (NA) } \\
\text { (NA) } \\
\text { (NA) } \\
\text { (NA) }\end{array}$ \\
\hline
\end{tabular}

Table 34. Hogs and Pigs-Litters Farrowed: 1987 and 1982

[For meaning of abbreviations and symbols, see introductory text]

\begin{tabular}{|c|c|c|c|c|}
\hline \multirow{2}{*}{ Litters } & \multicolumn{2}{|c|}{1987} & \multicolumn{2}{|c|}{1982} \\
\hline & Farms & Number of litters & Farms & Number of litters \\
\hline $\begin{array}{l}\text { Litters farrowed between Dec. } 1 \text { of preceding year and Nov. } 30 \\
\text { Farms with- } \\
1 \text { to } 9 \\
10 \text { to } 19 \\
20 \text { to } 49 \\
50 \text { to } 99 \\
100 \text { to } 199 \\
200 \text { to } 499 \\
500 \text { or more }\end{array}$ & $\begin{array}{r}211 \\
99 \\
31 \\
39 \\
11 \\
11 \\
15 \\
5\end{array}$ & $\begin{array}{r}13197 \\
432 \\
430 \\
1098 \\
858 \\
1499 \\
4366 \\
4514\end{array}$ & $\begin{array}{r}292 \\
146 \\
57 \\
43 \\
21 \\
10 \\
12 \\
3\end{array}$ & $\begin{array}{rr}10 & 887 \\
& 577 \\
& 747 \\
1 & 152 \\
1 & 323 \\
1 & 335 \\
3 & 443 \\
2 & 310\end{array}$ \\
\hline $\begin{array}{l}\text { Dec. } 1 \text { of preceding year and May } 31 \\
\text { June } 1 \text { and Nov } 30\end{array}$ & $\begin{array}{l}179 \\
183\end{array}$ & $\begin{array}{l}6597 \\
6600\end{array}$ & $\begin{array}{l}259 \\
259\end{array}$ & $\begin{array}{l}5875 \\
5012\end{array}$ \\
\hline
\end{tabular}

Table 35. Hogs and Pigs - Inventory and Sales by Size of Herd: 1987

[For meaning of abbreviations and symbols, see introductory text]

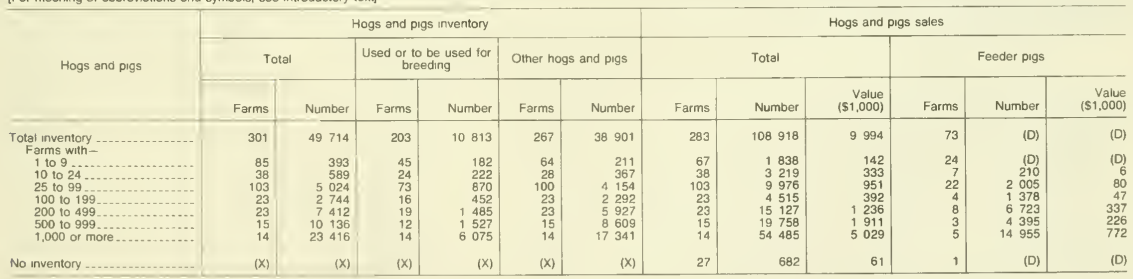


Table 36. Hogs and Pigs - Inventory and Sales by Number Sold Per Farm: 1987

[For meaning of abbreviations and symbols, see introductory text]

\begin{tabular}{|c|c|c|c|c|c|c|c|c|c|c|c|c|}
\hline \multirow{3}{*}{ Hogs and pigs } & \multicolumn{6}{|c|}{ Hogs and pigs inventory } & \multicolumn{6}{|c|}{ Hogs and pags sales } \\
\hline & \multicolumn{2}{|c|}{ Total } & \multicolumn{2}{|c|}{$\begin{array}{c}\text { Used or to be used for } \\
\text { breeding }\end{array}$} & \multicolumn{2}{|c|}{ Other hogs and pigs } & \multicolumn{3}{|c|}{ Total } & \multicolumn{3}{|c|}{ Feeder pigs } \\
\hline & Farms & Number & Farms & Number & Farms & Number & Farms & Number & $\begin{array}{r}\text { Value } \\
(\$ 1.000)\end{array}$ & Farms & Number & $\begin{array}{r}\text { Value } \\
(\$ 1.000)\end{array}$ \\
\hline 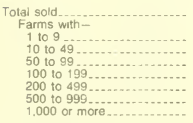 & $\begin{array}{r}283 \\
30 \\
90 \\
52 \\
38 \\
25 \\
20 \\
28\end{array}$ & $\begin{array}{r}49658 \\
365 \\
1898 \\
2413 \\
2762 \\
4186 \\
7005 \\
31029\end{array}$ & $\begin{array}{l}11 \\
61 \\
39 \\
30 \\
18 \\
16 \\
26\end{array}$ & $\begin{array}{r} \\
\quad(D) \\
\text { (D) } \\
342 \\
383 \\
559 \\
718 \\
1487 \\
7 \quad 469\end{array}$ & $\begin{array}{r}249 \\
28 \\
75 \\
48 \\
30 \\
22 \\
18 \\
28\end{array}$ & $\begin{array}{rl} & \text { (D) } \\
1 & \text { (D) } \\
1 & 556 \\
2 & 030 \\
2 & 203 \\
3 & 468 \\
5 & 788 \\
23 & 560\end{array}$ & \begin{tabular}{r|r}
310 \\
42 \\
401 \\
53 \\
41 \\
25 \\
20 \\
28
\end{tabular} & $\begin{array}{rr}109600 \\
\quad 190 \\
2779 \\
3619 \\
5 \quad 271 \\
7552 \\
13756 \\
76433\end{array}$ & $\begin{array}{r}10055 \\
21 \\
256 \\
354 \\
497 \\
729 \\
1249 \\
6949\end{array}$ & \begin{tabular}{r|}
74 \\
29 \\
10 \\
13 \\
4 \\
6 \\
12
\end{tabular} & $\begin{array}{r}30594 \\
\\
525 \\
595 \\
964 \\
931 \\
3180 \\
24 \quad 399\end{array}$ & $\begin{array}{r}1503 \\
19 \\
22 \\
34 \\
33 \\
129 \\
1266\end{array}$ \\
\hline None sold .............................. & 18 & 56 & 2 & (D) & 18 & (D) & $(\mathrm{x})$ & $(\mathrm{x})$ & $(\mathrm{X})$ & (x) & (X) & (X) \\
\hline
\end{tabular}

Table 37. Hogs and Pigs - Inventory, Sales, and Litters by Total Litters Farrowed: 1987

[For meaning of abbreviations and symbols, see introductory text]

\begin{tabular}{|c|c|c|c|c|c|c|c|c|c|c|c|c|}
\hline \multirow{3}{*}{ Litters farrowed } & \multicolumn{6}{|c|}{ Hogs and pigs inventory } & \multicolumn{6}{|c|}{ Hogs and pigs sales } \\
\hline & \multicolumn{2}{|c|}{ Total } & \multicolumn{2}{|c|}{$\begin{array}{l}\text { Used or to be used for } \\
\text { breeding }\end{array}$} & \multicolumn{2}{|c|}{ Other hogs and pigs } & \multicolumn{3}{|c|}{ Total } & \multicolumn{3}{|c|}{ Feeder pigs } \\
\hline & Farms & Number & Farms & Number & Farms & Number & Farms & Number & $\begin{array}{r}\text { Value } \\
(\$ 1,000)\end{array}$ & Farms & Number & $\begin{array}{r}\text { Value } \\
(\$ 1,000)\end{array}$ \\
\hline $\begin{array}{l}\text { Total hitters farrowed } \\
\text { Farms wth- } \\
11 \ldots \\
2 \text { to } 4 \\
5 \text { to } 9 \\
10 \text { to } 19 \\
20 \text { to } 49 \\
50 \text { to } 99 \\
100 \text { to } 199 \\
200 \text { or more }\end{array}$ & \begin{tabular}{r|r}
209 & 1 \\
10 & \\
49 & \\
39 & 30 \\
39 & 11 \\
11 & \\
20 &
\end{tabular} & $\begin{array}{r}44251 \\
186 \\
798 \\
1349 \\
1418 \\
4286 \\
3810 \\
6148 \\
26256\end{array}$ & \begin{tabular}{r|r}
203 & \\
8 & \\
49 & 37 \\
30 & 39 \\
9 & 11 \\
11 & 20
\end{tabular} & $\begin{array}{r}10813 \\
25 \\
205 \\
240 \\
371 \\
+098 \\
701 \\
969 \\
7204\end{array}$ & \begin{tabular}{r|r}
175 & \\
10 & 35 \\
33 & 25 \\
30 & 11 \\
11 & \\
20 &
\end{tabular} & $\begin{array}{rr}33 & 438 \\
& 161 \\
593 \\
1109 \\
1047 \\
3188 \\
3109 \\
5179 \\
19 & 052\end{array}$ & \begin{tabular}{r|r}
209 & \\
9 & \\
49 & 39 \\
31 & \\
39 & 11 \\
11 & \\
20 &
\end{tabular} & \begin{tabular}{rr|}
100 & 204 \\
& 200 \\
1 & 568 \\
1 & 784 \\
4 & 084 \\
8 & 661 \\
6710 \\
11282 \\
$65 \quad 915$
\end{tabular} & $\begin{array}{r}9087 \\
21 \\
145 \\
159 \\
382 \\
819 \\
661 \\
903 \\
5997\end{array}$ & \begin{tabular}{r|}
74 \\
1 \\
21 \\
12 \\
13 \\
10 \\
3 \\
5 \\
9
\end{tabular} & $\begin{array}{rr}30 & 594 \\
& \\
& \text { (D) } \\
& \text { (D) } \\
416 \\
\\
941 \\
1785 \\
1330 \\
4365 \\
21384\end{array}$ & $\begin{array}{r}1503 \\
(D) \\
(D) \\
16 \\
32 \\
66 \\
52 \\
228 \\
1096\end{array}$ \\
\hline \multirow[t]{4}{*}{ No litters farrowed ............... } & 92 & 5463 & - & - & 92 & 5463 & 101 & 9396 & 968 & - & - & - \\
\hline & \multicolumn{12}{|c|}{ Litters farrowed between Dec 1, 1986, and Nov, 30, 1987} \\
\hline & \multicolumn{4}{|c|}{ Total } & \multicolumn{4}{|c|}{ Dec. 1, 1986, and May 31, 1987} & \multicolumn{4}{|c|}{ June 1, 1987, and Nov. 30, 1987} \\
\hline & & Farms & & Litters & \multicolumn{2}{|c|}{ Farms } & \multicolumn{2}{|r|}{ Litters } & \multicolumn{2}{|r|}{ Farms } & \multicolumn{2}{|r|}{ Litters } \\
\hline $\begin{array}{l}\text { Total litters farrowed } \\
\text { Farms with- } \\
1 \text { to } 4 \\
2 \text { to } 4 \\
5 \text { to } 9 \\
10 \text { to } 19 \\
20 \text { to } 49 \\
50 \text { to } 99 \\
100 \text { to } 199 \\
200 \text { or more }\end{array}$ & & \begin{tabular}{r|}
211 \\
11 \\
49 \\
39 \\
31 \\
39 \\
11 \\
11 \\
20
\end{tabular} & & $\begin{array}{r}13197 \\
11 \\
146 \\
146 \\
275 \\
430 \\
1098 \\
858 \\
1499 \\
8880\end{array}$ & & $\begin{array}{l}3 \\
3 \\
2 \\
3 \\
1 \\
1\end{array}$ & & $\begin{array}{r}6597 \\
8 \\
8 \\
76 \\
117 \\
206 \\
539 \\
401 \\
790 \\
4 \quad 460 \\
\end{array}$ & & $\begin{array}{r}183 \\
3 \\
36 \\
38 \\
29 \\
36 \\
11 \\
10 \\
20\end{array}$ & & $\begin{array}{r}6600 \\
3 \\
70 \\
758 \\
224 \\
559 \\
457 \\
709 \\
4420 \\
\end{array}$ \\
\hline
\end{tabular}

Table 38. Sheep and Lambs - Inventory and Sales: 1987 and 1982

[For meaning of abbreviations and symbols, see introductory text]

\begin{tabular}{|c|c|c|c|c|}
\hline \multirow{2}{*}{ Item } & \multicolumn{2}{|c|}{1987} & \multicolumn{2}{|c|}{1982} \\
\hline & Farms & Number & Farms & Number \\
\hline $\begin{array}{l}\text { Sheep and lambs inventory } \\
\text { Farms with- } \\
1 \text { to } 24 \\
25 \text { to } 99 \\
100 \text { to } 299 \\
300 \text { to } 999 \text { to } 2.499 \\
2,000 \text { or more }\end{array}$ & $\begin{array}{r}50 \\
33 \\
12 \\
5 \\
- \\
-\end{array}$ & $\begin{array}{r}1667 \\
228 \\
585 \\
854 \\
- \\
- \\
-\end{array}$ & $\begin{array}{r}41 \\
32 \\
8 \\
1 \\
- \\
- \\
-\end{array}$ & $\begin{array}{r}681 \\
199 \\
\text { (D) } \\
\text { (D) } \\
- \\
-\end{array}$ \\
\hline Ewes 1 year old or older..... & 45 & 1006 & 33 & 358 \\
\hline $\begin{array}{l}\text { Sheep and lambs shorn } \\
\text { Pounds of wool }\end{array}$ & $\begin{array}{l}39 \\
(x)\end{array}$ & $\begin{array}{ll}1 & 099 \\
7 & 081\end{array}$ & $\begin{array}{r}29 \\
(x)\end{array}$ & $\begin{array}{r}474 \\
3033\end{array}$ \\
\hline $\begin{array}{l}\text { Sheep and lambs sold .... lambs, and wool }(\$ 1,000) \\
\text { value of sales from sheep, }\end{array}$ & $\begin{array}{l}38 \\
45\end{array}$ & $\begin{array}{r}772 \\
54\end{array}$ & $\begin{array}{l}24 \\
32\end{array}$ & $\begin{array}{r}469 \\
26\end{array}$ \\
\hline
\end{tabular}


Table 39. Sheep and Lambs - Inventory and Sales by Size of Flock: 1987

[For meaning of abbreviations and symbols, see introductory text]

\begin{tabular}{|c|c|c|c|c|c|c|c|c|c|c|c|}
\hline \multirow{3}{*}{ Sheep and lambs } & \multicolumn{4}{|c|}{ Sheep and lambs inventory } & \multirow{2}{*}{\multicolumn{3}{|c|}{ Sheep and lambs shorn }} & \multicolumn{4}{|c|}{ Sales } \\
\hline & \multicolumn{2}{|c|}{ Total } & \multicolumn{2}{|c|}{ Ewes 1 year old or older } & & & & \multicolumn{2}{|c|}{ Sheep and lambs } & \multicolumn{2}{|c|}{ Sheep, lambs, and wool } \\
\hline & Farms & Number & Farms & Number & Farms & Number & $\begin{array}{r}\text { Pounds of } \\
\text { wool }\end{array}$ & Farms & Number & Farms & $\begin{array}{r}\text { Value } \\
(\$ 1,000)\end{array}$ \\
\hline $\begin{array}{l}\text { Total inventory } \\
\text { Farms with-................. }\end{array}$ & 50 & 1667 & 45 & 1006 & 39 & 1099 & 7081 & 37 & (D) & 44 & (D) \\
\hline $\begin{array}{l}1 \text { to } 24 \\
25 \text { to } 99 \\
100 \text { to } 299 \\
300 \text { to } 999 \\
1,000 \text { to } 2,499 \\
2,500 \text { to } 4.999 \\
5,000 \text { or more }\end{array}$ & $\begin{array}{r}33 \\
12 \\
5 \\
: \\
- \\
- \\
-\end{array}$ & $\begin{array}{r}228 \\
585 \\
854 \\
- \\
- \\
-\end{array}$ & $\begin{array}{r}28 \\
12 \\
5 \\
- \\
= \\
= \\
-\end{array}$ & $\begin{array}{r}164 \\
350 \\
492 \\
- \\
- \\
-\end{array}$ & $\begin{array}{r}22 \\
12 \\
5 \\
- \\
- \\
- \\
-\end{array}$ & $\begin{array}{r}157 \\
402 \\
540 \\
- \\
- \\
-\end{array}$ & $\begin{array}{rr}1 & 076 \\
2 & 998 \\
3 & 007 \\
& \\
& = \\
& = \\
& -\end{array}$ & $\begin{array}{r}20 \\
12 \\
5 \\
- \\
- \\
- \\
-\end{array}$ & $\begin{array}{r}127 \\
319 \\
\text { (D) } \\
- \\
- \\
-\end{array}$ & $\begin{array}{r}27 \\
12 \\
5 \\
- \\
- \\
- \\
-\end{array}$ & $\begin{array}{r}6 \\
19 \\
\text { (D) } \\
- \\
-\end{array}$ \\
\hline No inventory ................ & $(x)$ & $(\mathrm{X})$ & $(\mathrm{X})$ & $(\mathrm{X})$ & - & - & - & 1 & (D) & 1 & (D) \\
\hline
\end{tabular}

Table 40. Sheep and Lambs - Inventory and Sales by Size of Ewe Flock: 1987

[For meaning of abbreviations and symbols, see introductory text]

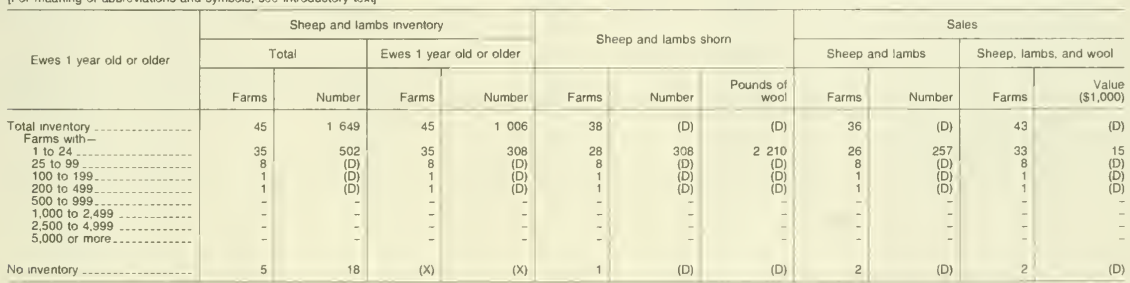


Table 41. Other Livestock and Livestock Products-Inventory and Sales: 1987 and 1982

\begin{tabular}{|c|c|c|c|c|}
\hline \multirow{2}{*}{ Item } & \multicolumn{2}{|c|}{ Inventory } & \multicolumn{2}{|c|}{ Sales } \\
\hline & 1987 & 1982 & 1987 & 1982 \\
\hline 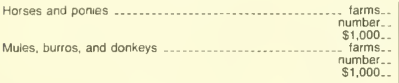 & $\begin{array}{r}425 \\
3029 \\
(X) \\
22 \\
43 \\
(X)\end{array}$ & $\begin{array}{r}476 \\
514 \\
(X) \\
7 \\
14 \\
(X)\end{array}$ & $\begin{array}{r}123 \\
334 \\
2233 \\
2 \\
\text { (D) } \\
\text { (D) }\end{array}$ & $\begin{array}{r}146 \\
412 \\
1 \quad 162 \\
3 \\
7 \\
2\end{array}$ \\
\hline $\begin{array}{l}\text { Colonies of bees } \\
\text { Honey sold } \\
\text { Bees and honey sold } \ldots \ldots \ldots \\
\text { farms }\end{array}$ & $\begin{array}{r}65 \\
(X 43 \\
(X) \\
(X) \\
(X)\end{array}$ & $\begin{array}{r}68 \\
832 \\
(X) \\
(X) \\
(X) \\
(X)\end{array}$ & $\begin{array}{r}5 \\
101 \\
20 \\
17916 \\
23 \\
19\end{array}$ & $\begin{array}{r}3 \\
(D) \\
15 \\
265 \\
\text { (NA) } \\
\text { (D) }\end{array}$ \\
\hline $\begin{array}{l}\text { Angora goats } \\
\text { Mohair sold }\end{array}$ & $\begin{array}{r}62 \\
465 \\
- \\
- \\
(X) \\
(X)\end{array}$ & $\begin{array}{r}62 \\
409 \\
1 \\
(D) \\
(X) \\
(X)\end{array}$ & $\begin{array}{r}29 \\
326 \\
- \\
- \\
-\end{array}$ & $\begin{array}{r}29 \\
153 \\
1 \\
\text { (D) } \\
1 \\
\text { (D) }\end{array}$ \\
\hline 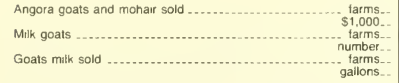 & $\begin{array}{r}(X) \\
(X) \\
22 \\
110 \\
(X) \\
(X)\end{array}$ & $\begin{array}{r}(X) \\
(X) \\
35 \\
208 \\
(X) \\
(X)\end{array}$ & $\begin{array}{r}1 \overline{1} \\
133 \\
2 \\
\text { (D) }\end{array}$ & $\begin{array}{r}\text { (NA) } \\
\text { (D) } \\
16 \\
109 \\
3 \\
\text { (D) }\end{array}$ \\
\hline $\begin{array}{l}\text { Milk goats and goats milk sold } \\
\text { Other goats }\end{array}$ & $\begin{array}{r}(X) \\
(X) \\
47 \\
355 \\
(X)\end{array}$ & $\begin{array}{l}(X) \\
(X) \\
28 \\
(D) \\
(X)\end{array}$ & $\begin{array}{r}13 \\
(\mathrm{D}) \\
19 \\
193 \\
7\end{array}$ & $\begin{array}{l}\text { (NA) } \\
\text { (D) } \\
12 \\
\text { (D) } \\
\text { (D) }\end{array}$ \\
\hline 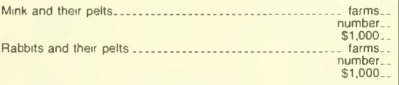 & $\begin{array}{r}- \\
\overline{(X)} \\
19 \\
974 \\
(X)\end{array}$ & $\begin{array}{r}- \\
\bar{x} \\
14 \\
1010 \\
010 \\
(X)\end{array}$ & $\begin{array}{r}- \\
- \\
\overline{8} \\
1925 \\
12\end{array}$ & $\begin{array}{r}- \\
8 \\
\text { (D) } \\
\text { (D) }\end{array}$ \\
\hline $\begin{array}{l}\text { Fish and other aquaculture products sold ............... farms... } \\
\text { Other livestock and irvestock products ................. farms.. } \\
\$ 1,000 \ldots\end{array}$ & $\begin{array}{r}(X) \\
(X) \\
7 \\
(X)\end{array}$ & $\begin{array}{r}(X) \\
(X) \\
(\mathrm{NA}) \\
(X)\end{array}$ & $\begin{array}{l}- \\
\overline{5} \\
9\end{array}$ & (NA) \\
\hline
\end{tabular}


Table 42. Crops Harvested and Value of Production: 1987 and 1982

[For meaning of abbreviations and symbols, see introductory text]

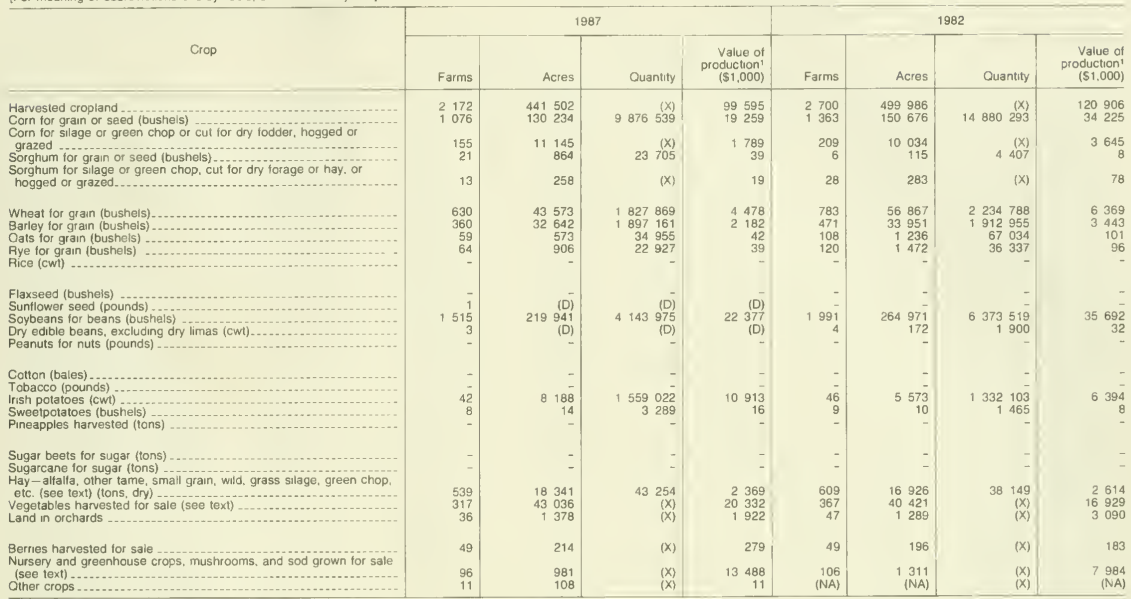

'Data are estumaled; see text

Table 43. Specified Crops Harvested-Yield Per Acre Irrigated and Nonirrigated: 1987

[For meaning of abbrevations and symbols, see introductory text]

\begin{tabular}{|c|c|c|c|c|c|c|c|c|c|c|}
\hline \multirow[b]{2}{*}{ Crop } & \multicolumn{3}{|c|}{ Entre crop irngated } & \multicolumn{4}{|c|}{ Part of crop irrigated } & \multicolumn{3}{|c|}{ None of crop imigated } \\
\hline & Farms & Acres & $\begin{array}{r}\text { Average } \\
\text { yield per } \\
\text { acre }\end{array}$ & Farms & $\begin{array}{r}\text { Acres } \\
\text { itrigated }\end{array}$ & $\begin{array}{r}\text { Acres not } \\
\text { irrigated }\end{array}$ & $\begin{array}{r}\text { Average } \\
\text { yield per } \\
\text { acre }\end{array}$ & Farms & Acres & $\begin{array}{r}\text { Average } \\
\text { yield per } \\
\text { acre }\end{array}$ \\
\hline $\begin{array}{l}\text { Corn for grain or seed (bushels) } \\
\text { Corn for silage or green chop (tons, green) } \\
\text { Sorghum for grain or seed (bushels) } \\
\text { Wheat for grain (bushels) } \\
\text { Oats for grain (bushels) }\end{array}$ & $\begin{array}{r}66 \\
3 \\
1 \\
14 \\
-\end{array}$ & $\begin{array}{r}9665 \\
183 \\
\quad \text { (D) } \\
1 \quad 164 \\
\end{array}$ & $\begin{array}{r}112.1 \\
17.3 \\
(D) \\
54.2 \\
-\end{array}$ & $\begin{array}{r}79 \\
3 \\
19 \\
-\end{array}$ & 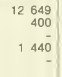 & $\begin{array}{r}12822 \\
500 \\
1059 \\
-\end{array}$ & $\begin{array}{r}96.1 \\
11.1 \\
49.5 \\
-\end{array}$ & $\begin{array}{r}931 \\
148 \\
20 \\
597 \\
59\end{array}$ & $\begin{array}{rr}95 & 098 \\
10 & 025 \\
& (D) \\
39 & 910 \\
573\end{array}$ & $\begin{array}{r}66.7 \\
9.8 \\
(D) \\
41.1 \\
61.0\end{array}$ \\
\hline $\begin{array}{l}\text { Barley for grain (bushels) } \\
\text { Rice (cw) }\end{array}$ & 7 & 863 & 57.2 & 5 & 483 & 597 & 56.9 & 348 & 30699 & 58.2 \\
\hline $\begin{array}{l}\text { Rice (Cw1) } \\
\text { Soybeans for beans (bushels) }\end{array}$ & $2 \overline{7}$ & 3463 & $27 \overline{4}$ & 90 & 8300 & 28.743 & $20 . \overline{1}$ & 1398 & $17943 \overline{5}$ & 18.4 \\
\hline $\begin{array}{l}\text { Peanuts for nuts (pounds) } \\
\text { Dry edible beans, excluding dry limas (cwt) }\end{array}$ & - & & - & $\bar{i}$ & (D) & (D) & (D) & $\overline{2}$ & (D) & (D) \\
\hline $\begin{array}{l}\text { Cotton (bales) } \\
\text { Tobacco (pounds) }\end{array}$ & $\overline{-}$ & - & - & $\overline{-}$ & $\overline{-}-$ & $\overline{-}$ & $\overline{-}$ & $=$ & - & - \\
\hline $\begin{array}{l}\text { Irish potatoes (cwt) } \\
\text { Sugar beets for sugar (tons) }\end{array}$ & 18 & 2001 & 213.0 & 8 & 1645 & 1633 & 191.6 & 16 & 2909 & 173.5 \\
\hline 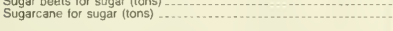 & $\overline{-}$ & & $\overline{-}$ & $\overline{-}$ & - & - & - & $\overline{-}$ & $\therefore$ & - \\
\hline $\begin{array}{l}\text { Altalfa hay (tons, dry) } \\
\text { Small grain hay (tons, dry) }\end{array}$ & 10 & (D) & (D) & 3 & 88 & (D) & (D) & 289 & $\begin{array}{lll}7 & 417 \\
2 & 099\end{array}$ & 3.1 \\
\hline $\begin{array}{l}\text { Tame hay other than altaifa, small grain, and wild hay (see } 10 \times 1 \text { ) } \\
\text { (tons, dry) }\end{array}$ & & (D) & & - & & & & 90 & 2099 & 2.0 \\
\hline 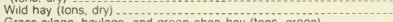 & $\div$ & (D) & (D) & 1 & (D) & (D) & (D) & $\begin{array}{r}272 \\
29\end{array}$ & $\begin{array}{l}4.65 \\
652\end{array}$ & $\begin{array}{l}2.0 \\
1.5\end{array}$ \\
\hline Grass silage, haylage, and green chop hay (tons, green) & 2 & (D) & (D) & 3 & 126 & (D) & (D) & 48 & 2342 & 45 \\
\hline Altalia seed (pounds) & $\overline{-}$ & $12 \cdot 114$ & $(x)$ & 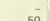 & - & & $-\overline{1}$ & - & & \\
\hline $\begin{array}{l}\text { Vegetables harvested for sale (see text) } \\
\text { Land in orchards }\end{array}$ & $\begin{array}{r}135 \\
5\end{array}$ & $12 \begin{array}{r}114 \\
377\end{array}$ & $\begin{array}{l}(x) \\
(x)\end{array}$ & $\begin{array}{r}50 \\
3\end{array}$ & 11661 & $12 \begin{array}{l}187 \\
760\end{array}$ & $\begin{array}{l}(x) \\
x\end{array}$ & $\begin{array}{r}132 \\
28\end{array}$ & $\begin{array}{r}7074 \\
92\end{array}$ & $\begin{array}{l}(X) \\
(X)\end{array}$ \\
\hline Strawbernes harvested tor sale (pounds) & 23 & 80 & 29497 & 1 & (D) & (D) & (D) & 18 & (D) & (D) \\
\hline
\end{tabular}


Table 44. Specified Crops by Acres Harvested: 1987 and 1982

[For meaning of abbreviations and symbols, see introductory text]

\begin{tabular}{|c|c|c|c|c|c|c|c|c|c|c|}
\hline \multirow{3}{*}{ Crop } & \multicolumn{5}{|c|}{1987} & \multicolumn{5}{|c|}{1982} \\
\hline & \multirow[b]{2}{*}{ Farms } & \multirow[b]{2}{*}{ Acres } & \multirow[b]{2}{*}{ Quanuty } & \multicolumn{2}{|c|}{ Irrigated land } & \multirow[b]{2}{*}{ Farms } & \multirow[b]{2}{*}{ Acres } & \multirow[b]{2}{*}{ Quantity } & \multicolumn{2}{|c|}{ Irrigated land } \\
\hline & & & & Farms & Acres & & & & Farms & Acres \\
\hline $\begin{array}{l}\text { Corn for grain or seed (bushels) } \\
1 \text { to } 14 \text { acres } \\
15 \text { to } 24 \text { acres. } \\
25 \text { to } 49 \text { acres } \\
500 \\
100 \text { to } 99 \text { acres } \\
250 \text { to } 499 \text { acres } \\
500 \text { to } 999 \text { acres } \\
1,000 \text { acres or more } \\
1,000 \text { to } 1999 \text { acres } \\
2,000 \text { to } 2,999 \text { acres } \\
3,000 \text { to } 49999 \text { acres } \\
5,000 \text { acres or more }\end{array}$ & $\begin{array}{r}1076 \\
230 \\
97 \\
195 \\
208 \\
217 \\
81 \\
33 \\
15 \\
13 \\
13 \\
2\end{array}$ & $\begin{array}{rr}130 & 234 \\
1 & 663 \\
1 & 863 \\
6 & 846 \\
14 & 695 \\
33 & 181 \\
27 & 601 \\
22 & 132 \\
22 & 253 \\
(X) \\
(X) \\
(X) \\
(X) \\
(X)\end{array}$ & 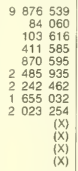 & \begin{tabular}{r|r}
145 & \\
9 & \\
5 & 11 \\
34 & \\
51 & 23 \\
6 & \\
6 & \\
4 & \\
2 & \\
- &
\end{tabular} & 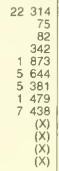 & $\begin{array}{r}1363 \\
308 \\
152 \\
259 \\
242 \\
253 \\
88 \\
49 \\
12 \\
9 \\
3\end{array}$ & $\begin{array}{rr}150 & 676 \\
2 & 354 \\
2 & 881 \\
8 & 744 \\
16 & 907 \\
37 & 157 \\
29 & 040 \\
31 & 302 \\
22 & 291 \\
& (X) \\
& (X)\end{array}$ & 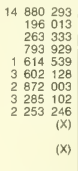 & \begin{tabular}{r|r}
132 & \\
9 & \\
9 & \\
26 & 52 \\
15 & 12 \\
5 & \\
3 & \\
2 &
\end{tabular} & $\begin{array}{r}17771 \\
82 \\
85 \\
242 \\
1782 \\
4718 \\
2487 \\
3226 \\
5149 \\
(X) \\
\\
\\
(X)\end{array}$ \\
\hline $\begin{array}{l}\text { Corn for slage or green chop (tons, green) } \\
1 \text { to } 14 \text { acres. } \\
15 \text { to } 24 \text { acres. } \\
25 \text { to } 49 \text { acres. } \\
50 \\
10009 \text { acres. } \\
250 \text { to } 249 \text { acres } \\
500 \\
1,000 \text { to } 999 \text { acres acres }\end{array}$ & $\begin{array}{r}154 \\
61 \\
11 \\
20 \\
26 \\
26 \\
8 \\
1 \\
1 \\
1\end{array}$ & $\begin{array}{r}11109 \\
489 \\
203 \\
671 \\
1747 \\
3938 \\
\text { (D) } \\
\text { (D) } \\
\text { (D) }\end{array}$ & \begin{tabular}{rl|}
1111 & 491 \\
5 & 372 \\
1 & 621 \\
5.597 \\
20 & 692 \\
35 & 599 \\
(D) \\
(D) \\
(D)
\end{tabular} & \begin{tabular}{l|}
6 \\
1 \\
- \\
- \\
2 \\
2 \\
1 \\
1
\end{tabular} & $\begin{array}{r}583 \\
\text { (D) } \\
- \\
\text { (D) } \\
\text { (D) } \\
\text { (D) }\end{array}$ & \begin{tabular}{r|r}
209 \\
84 \\
25 \\
41 \\
37 \\
19 \\
1 \\
1 \\
1
\end{tabular} & $\begin{array}{r}10034 \\
549 \\
448 \\
1319 \\
2373 \\
2501 \\
\text { (D) } \\
\text { (D) } \\
\text { (D) } \\
\text { (D) }\end{array}$ & $\begin{array}{rl}145 & 796 \\
7 & 586 \\
5 & 785 \\
21 & 254 \\
34 & 871 \\
33 & 530 \\
\text { (D) } \\
\text { (D) } \\
\text { (D) }\end{array}$ & \begin{tabular}{l|l}
6 & \\
3 & \\
- & \\
1 & \\
2 & \\
- & \\
- &
\end{tabular} & $\begin{array}{r}187 \\
26 \\
- \\
\text { (D) } \\
\text { (D) } \\
-\end{array}$ \\
\hline 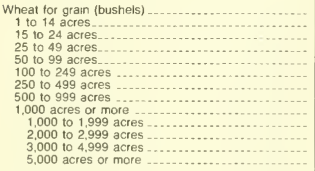 & \begin{tabular}{r|}
630 \\
102 \\
101 \\
152 \\
147 \\
100 \\
23 \\
4 \\
1 \\
1 \\
- \\
- \\
-
\end{tabular} & $\begin{array}{rr}43 & 573 \\
& 896 \\
1 & 975 \\
5 & 303 \\
9 & 791 \\
14 & 652 \\
7 & 781 \\
(D) \\
\\
\text { (D) } \\
\text { (X) } \\
\text { (X) } \\
\text { (X) } \\
\\
(X)\end{array}$ & \begin{tabular}{rr|}
1827 & 869 \\
37 & 259 \\
72 & 155 \\
220 & 290 \\
400 & 545 \\
627 & 090 \\
343 & 538 \\
& $(D)$ \\
& $(D)$ \\
& $(X)$ \\
& $(X)$ \\
& $(X)$ \\
& $(X)$ \\
& $(X)$
\end{tabular} & \begin{tabular}{r|}
33 \\
1 \\
1 \\
4 \\
12 \\
13 \\
2 \\
- \\
- \\
$=$ \\
- \\
- \\
-
\end{tabular} & $\begin{array}{r}2604 \\
\text { (D) } \\
(D) \\
153 \\
641 \\
1363 \\
1 \text { (D) } \\
- \\
- \\
(X) \\
(X) \\
(X) \\
(X) \\
(X)\end{array}$ & \begin{tabular}{r|r}
783 \\
118 \\
107 \\
205 \\
186 \\
125 \\
35 \\
5 \\
2 \\
2 \\
2 \\
-
\end{tabular} & \begin{tabular}{rr|}
56 & 867 \\
1 & 013 \\
2 & 034 \\
6 & 960 \\
12 & 259 \\
18 & 168 \\
11 & 403 \\
& (D) \\
& (D) \\
& (X) \\
& (X)
\end{tabular} & $\begin{array}{r}2234788 \\
34638 \\
69089 \\
266746 \\
483844 \\
729954 \\
473005 \\
\text { (D) } \\
\text { (D) } \\
\text { (X) } \\
\text { (X) }\end{array}$ & $\begin{array}{r}23 \\
3 \\
2 \\
5 \\
10 \\
3 \\
- \\
= \\
-\end{array}$ & $\begin{array}{r}2136 \\
16 \\
\text { (D) } \\
\text { (D) } \\
109 \\
726 \\
\overline{-} \\
(\mathrm{X}) \\
\text { (X) }\end{array}$ \\
\hline 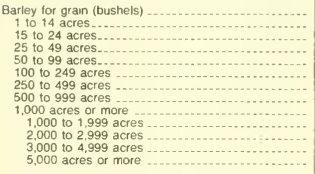 & $\begin{array}{r}360 \\
58 \\
43 \\
90 \\
72 \\
71 \\
19 \\
4 \\
3 \\
3 \\
- \\
- \\
-\end{array}$ & $\begin{array}{r}32642 \\
547 \\
843 \\
3143 \\
4753 \\
10559 \\
6670 \\
2 \quad 383 \\
3744 \\
(X) \\
(X) \\
(x) \\
(x) \\
(x)\end{array}$ & $\begin{array}{rr}1897 & 161 \\
27 & 474 \\
43 & 855 \\
175 & 575 \\
281 & 778 \\
600 & 210 \\
418 & 303 \\
134 & 735 \\
215 & 231 \\
& (X) \\
& (X) \\
& (X) \\
& (X) \\
& (X)\end{array}$ & $\begin{array}{r}12 \\
- \\
- \\
2 \\
2 \\
5 \\
3 \\
- \\
- \\
- \\
- \\
- \\
-\end{array}$ & $\begin{array}{r}346 \\
- \\
- \\
(D) \\
(D) \\
475 \\
651 \\
- \\
- \\
(X) \\
(x) \\
(X) \\
(X) \\
(X)\end{array}$ & \begin{tabular}{r|r}
471 & \\
90 & \\
69 & \\
96 & 98 \\
97 & 16 \\
4 & \\
1 & \\
1 & \\
- &
\end{tabular} & $\begin{array}{rr}33 & 951 \\
713 \\
1309 \\
3284 \\
6590 \\
13534 \\
4900 \\
\text { (D) } \\
\text { (D) } \\
\text { (X) } \\
\\
\quad \text { (X) }\end{array}$ & $\begin{array}{r}1912955 \\
36610 \\
68745 \\
177524 \\
390018 \\
751 \text { 021 } \\
278 \quad 157 \\
\text { (D) } \\
\text { (D) } \\
\text { (X) } \\
\\
\text { (X) }\end{array}$ & $\begin{array}{r}12 \\
1 \\
2 \\
3 \\
2 \\
3 \\
1 \\
- \\
- \\
- \\
-\end{array}$ & $\begin{array}{r}611 \\
\text { (D) } \\
\text { (D) } \\
122 \\
(D) \\
280 \\
(D) \\
\\
(x) \\
(x) \\
(x)\end{array}$ \\
\hline Qats for grain (bushels) ........... & 59 & 573 & 34955 & - & - & 108 & 1236 & 67034 & 1 & (D) \\
\hline $\begin{array}{l}\text { Soybeans for beans (bushels) } \\
1 \text { to } 14 \text { acres } \\
15 \text { to } 24 \text { acres } \\
25 \text { to } 49 \text { acres. } \\
50 \text { to } 99 \text { acres } \\
100 \text { to } 249 \text { acres } \\
250 \text { to } 499 \text { acres } \\
500 \text { to } 999 \text { acres } \\
1,000 \text { acres or more } \\
1,000 \text { to } 1.999 \text { acres } \\
2,000 \text { to } 2.999 \text { acres } \\
3,000 \text { to } 4,999 \text { acres } \\
5,000 \text { acres or more }\end{array}$ & $\begin{array}{r}1515 \\
190 \\
151 \\
262 \\
338 \\
322 \\
158 \\
71 \\
23 \\
22 \\
1 \\
- \\
-\end{array}$ & \begin{tabular}{rr|}
219 & 941 \\
1 & 611 \\
2 & 843 \\
9 & 071 \\
23 & 385 \\
49 & 577 \\
54 & 874 \\
46 & 441 \\
32 & 139 \\
& $(X)$ \\
& $(X)$ \\
& $(X)$ \\
$(X)$ & $(X)$
\end{tabular} & $\begin{array}{rr}4143975 \\
32 & 721 \\
46 & 019 \\
162 & 226 \\
404 & 913 \\
922 & 448 \\
1043 & 622 \\
883 & 654 \\
648 & 372 \\
& (X) \\
& \\
& (X) \\
& (X) \\
& (X)\end{array}$ & \begin{tabular}{r|r}
117 & 3 \\
4 & \\
10 & 17 \\
32 & 32 \\
3 & \\
10 & \\
9 & 1 \\
1 & \\
- &
\end{tabular} & 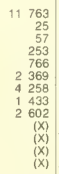 & \begin{tabular}{r|}
1991 \\
273 \\
209 \\
380 \\
403 \\
435 \\
185 \\
86 \\
20 \\
16 \\
4 \\
4
\end{tabular} & \begin{tabular}{rr|}
264 & 971 \\
2 & 359 \\
3 & 956 \\
13 & 334 \\
27 & 985 \\
66 & 470 \\
63 & 353 \\
57 & 140 \\
30 & 368 \\
& $(\times)$ \\
& $(X)$
\end{tabular} & 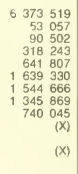 & $\begin{array}{r}84 \\
2 \\
5 \\
5 \\
14 \\
19 \\
23 \\
13 \\
3 \\
2\end{array}$ & $\begin{array}{r}7012 \\
\text { (D) } \\
\text { (D) } \\
147 \\
426 \\
1814 \\
2485 \\
1346 \\
720 \\
\quad 38 \\
\quad(X) \\
\\
(X)\end{array}$ \\
\hline Irish potatoes (cwt) & 42 & 8188 & 1559022 & 26 & 3646 & 46 & 5573 & 1332103 & 21 & 2506 \\
\hline 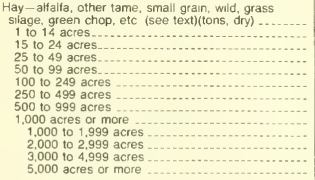 & $\begin{array}{r}539 \\
275 \\
94 \\
71 \\
52 \\
39 \\
6 \\
2 \\
- \\
- \\
- \\
- \\
-\end{array}$ & $\begin{array}{rr}18341 \\
1 & 953 \\
1 & 735 \\
2 & 349 \\
3 & 666 \\
5 & 508 \\
\text { (D) } \\
\text { (D) } \\
\\
\\
(x) \\
(x) \\
(x) \\
(x) \\
(x)\end{array}$ & 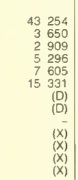 & $\begin{array}{r}20 \\
6 \\
3 \\
2 \\
4 \\
4 \\
1 \\
- \\
- \\
- \\
- \\
- \\
-\end{array}$ & $\begin{array}{r}699 \\
33 \\
48 \\
\text { (D) } \\
(D) \\
(D) \\
(D) \\
- \\
(x) \\
(x) \\
(x) \\
(x) \\
(x)\end{array}$ & \begin{tabular}{r|r}
609 & \\
330 & 100 \\
93 & 52 \\
52 & 27 \\
4 & \\
3 & - \\
- & \\
- &
\end{tabular} & \begin{tabular}{rr|}
16 & 926 \\
2 & 171 \\
1 & 818 \\
3 & 100 \\
3 & 330 \\
3 & 372 \\
1 & 276 \\
1 & 859 \\
& $(x)$ \\
& $(x)$
\end{tabular} & $\begin{array}{rr}38 & 149 \\
4 & 435 \\
4 & 153 \\
6573 \\
9305 \\
8641 \\
2689 \\
2893 \\
2 & 149 \\
& (x) \\
& \\
& (x)\end{array}$ & $\begin{array}{l}6 \\
2 \\
1 \\
3 \\
- \\
= \\
= \\
= \\
=\end{array}$ & $\begin{array}{c}105 \\
\text { (D) } \\
\text { (D) } \\
80 \\
- \\
\vdots \\
\vdots \\
(x) \\
(X)\end{array}$ \\
\hline $\begin{array}{r}\text { Allalfa hay (tons,dry) } \\
1 \text { to } 14 \text { acres.... } \\
15 \text { to } 24 \text { acres. } \\
25 \text { to } 49 \text { acres... } \\
50 \text { to } 99 \text { acres. } \\
100 \text { to } 249 \text { acres } \\
250 \text { to } 499 \text { acres } \\
500 \text { to } 999 \text { acres } \\
1.000 \text { actes or more } \\
1.000 \text { to } 1999 \text { acres } \\
2,000 \text { to } 2,999 \text { acres } \\
3,000 \text { to } 4,999 \text { acres } \\
5,000 \text { acres or more }\end{array}$ & $\begin{array}{r}302 \\
182 \\
46 \\
31 \\
25 \\
16 \\
2 \\
- \\
- \\
- \\
- \\
-\end{array}$ & 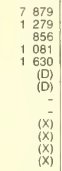 & $\begin{array}{rr}23 & 895 \\
2 & 896 \\
1 & 920 \\
3 & 547 \\
4 & 456 \\
(\mathrm{D}) \\
\\
& (\mathrm{D}) \\
& - \\
& (X) \\
& (X) \\
& (X) \\
(X) \\
(X)\end{array}$ & $\begin{array}{r}13 \\
6 \\
3 \\
- \\
3 \\
1 \\
- \\
- \\
- \\
- \\
\vdots \\
-\end{array}$ & $\begin{array}{r}365 \\
\text { (D) } \\
\text { (D) } \\
\text { (D) } \\
\text { (D) } \\
- \\
- \\
(X) \\
(x) \\
(x) \\
(X) \\
(x)\end{array}$ & $\begin{array}{r}307 \\
199 \\
43 \\
38 \\
20 \\
7 \\
- \\
- \\
- \\
-\end{array}$ & 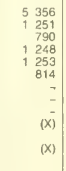 & 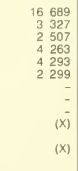 & $\begin{array}{l}3 \\
1 \\
2 \\
- \\
- \\
- \\
- \\
5 \\
- \\
- \\
-\end{array}$ & $\begin{array}{l}38 \\
\text { (D) } \\
\text { (D) } \\
\overline{-} \\
\overline{-} \\
\overline{(x)} \\
(\mathrm{X})\end{array}$ \\
\hline 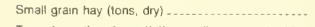 & 95 & 2099 & 4097 & - & - & 67 & 1392 & 2477 & - & - \\
\hline 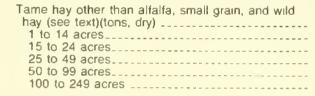 & $\begin{array}{r}275 \\
174 \\
46 \\
37 \\
12 \\
6\end{array}$ & $\begin{array}{r}4811 \\
1161 \\
852 \\
1155 \\
782 \\
861\end{array}$ & $\begin{array}{ll}9 & 774 \\
1 & 888 \\
1 & 748 \\
2 & 019 \\
1 & 677 \\
2 & 442\end{array}$ & $\begin{array}{l}3 \\
1 \\
1 \\
1 \\
- \\
-\end{array}$ & $\begin{array}{r}\text { (D) } \\
\text { (D) } \\
\text { (D) } \\
\text { (D) } \\
-\end{array}$ & $\begin{array}{r}360 \\
226 \\
70 \\
44 \\
13 \\
5\end{array}$ & 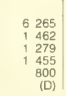 & $\begin{array}{rr}12 & 319 \\
2 & 909 \\
2 & 312 \\
2 & 566 \\
1 & 527 \\
& \text { (D) }\end{array}$ & $\begin{array}{l}4 \\
2 \\
- \\
2 \\
- \\
-1\end{array}$ & $\begin{array}{l}67 \\
\text { (D) } \\
\text { (D) } \\
-\end{array}$ \\
\hline
\end{tabular}


Table 44. Specified Crops by Acres Harvested: 1987 and 1982-Con.

[For meaning of abbrevations and symbois, see introductory text]

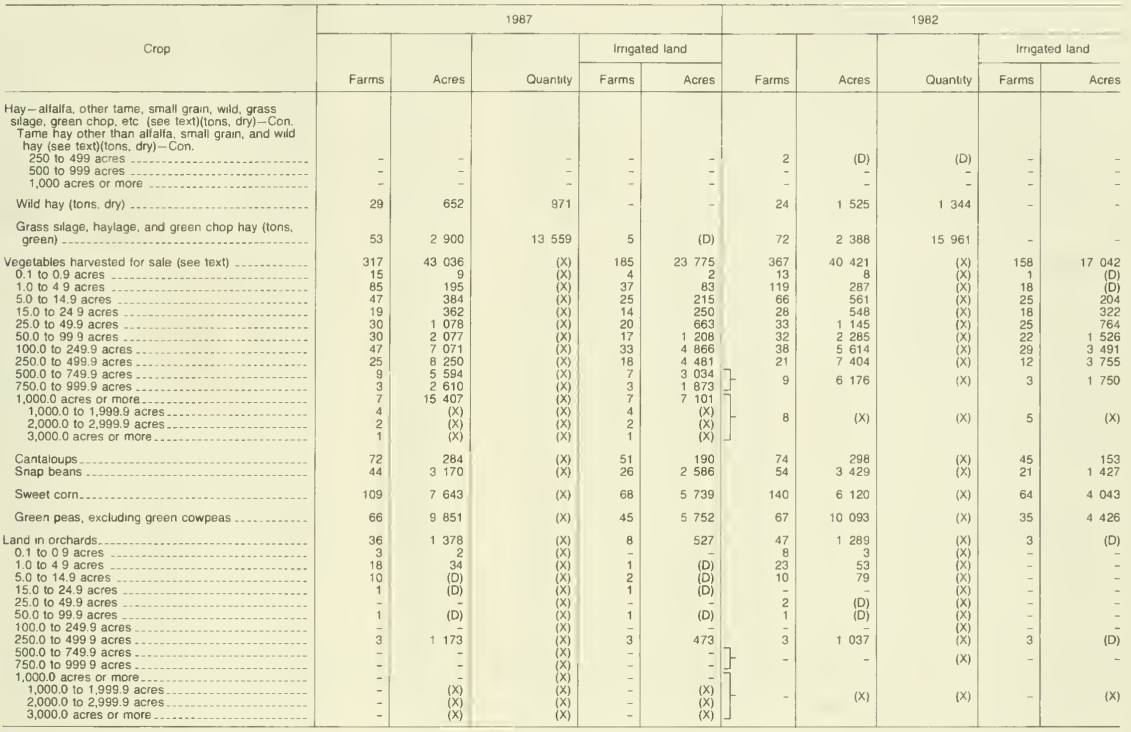

Table 45. Specified Fruits and Nuts by Acres: 1987 and 1982

[Not published for this State]

Table 46. Nursery and Greenhouse Crops, Mushrooms, and Sod Grown for Sale by Value of Sales: 1987 and 1982

[For meaning of abbreviations and symbols, see introductory text]

\begin{tabular}{|c|c|c|c|c|c|c|}
\hline \multirow{2}{*}{ Crop } & \multicolumn{2}{|c|}{ Under glass or other protection } & \multicolumn{2}{|c|}{ In the open } & \multicolumn{2}{|c|}{ Sales } \\
\hline & Farms & Square feet & Farms & Acres & Farms & $\begin{array}{r}\text { Value } \\
(\$ 1,000)\end{array}$ \\
\hline $\begin{array}{l}\text { Nursery and greenhouse crops, mushrooms, and sod } \\
\text { grown for sale (see text) }\end{array}$ & $\begin{array}{l}60 \\
68\end{array}$ & $\begin{array}{lll}1 & 310 & 210 \\
1 & 340 & 520\end{array}$ & $\begin{array}{l}52 \\
61\end{array}$ & $\begin{array}{r}951 \\
1280\end{array}$ & $\begin{array}{r}96 \\
106\end{array}$ & $\begin{array}{rl}13 & 488 \\
7 & 984\end{array}$ \\
\hline 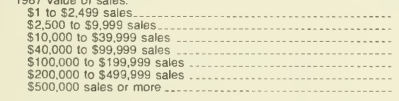 & $\begin{array}{r}4 \\
15 \\
15 \\
10 \\
7 \\
5 \\
4\end{array}$ & $\begin{array}{rr}1 & 632 \\
49 & 229 \\
156 & 240 \\
212 & 241 \\
\text { (D) } \\
\text { (D) } \\
623130\end{array}$ & $\begin{array}{r}10 \\
12 \\
12 \\
7 \\
6 \\
1 \\
4\end{array}$ & \begin{tabular}{r|}
7 \\
37 \\
68 \\
58 \\
$(D)$ \\
$(D)$ \\
600
\end{tabular} & \begin{tabular}{r|r}
13 & 23 \\
24 & 13 \\
9 & \\
6 & \\
8 &
\end{tabular} & $\begin{array}{r}14 \\
127 \\
475 \\
808 \\
1250 \\
1616 \\
9199\end{array}$ \\
\hline $\begin{array}{r}1987 \\
1982 \\
\end{array}$ & $\begin{array}{l}10 \\
17\end{array}$ & $\begin{array}{l}510570 \\
980000\end{array}$ & $\begin{array}{l}(X) \\
(X)\end{array}$ & $\begin{array}{l}(x) \\
(x)\end{array}$ & $\begin{array}{l}10 \\
17\end{array}$ & $\begin{array}{ll}3 & 891 \\
3 & 875\end{array}$ \\
\hline $\begin{array}{l}1987 \\
1982 .\end{array}$ & $\begin{array}{l}12 \\
14\end{array}$ & $\begin{array}{r}114272 \\
71780\end{array}$ & $\begin{array}{l}33 \\
35\end{array}$ & $\begin{array}{r}765 \\
1092\end{array}$ & $\begin{array}{l}35 \\
35\end{array}$ & $\begin{array}{ll}4 & 451 \\
2 & 569\end{array}$ \\
\hline
\end{tabular}


Table 47. Farms by Concentration of Market Value of Agricultural Products Sold: 1987

[For meaning of abbreviations and symbols, see introductory text]

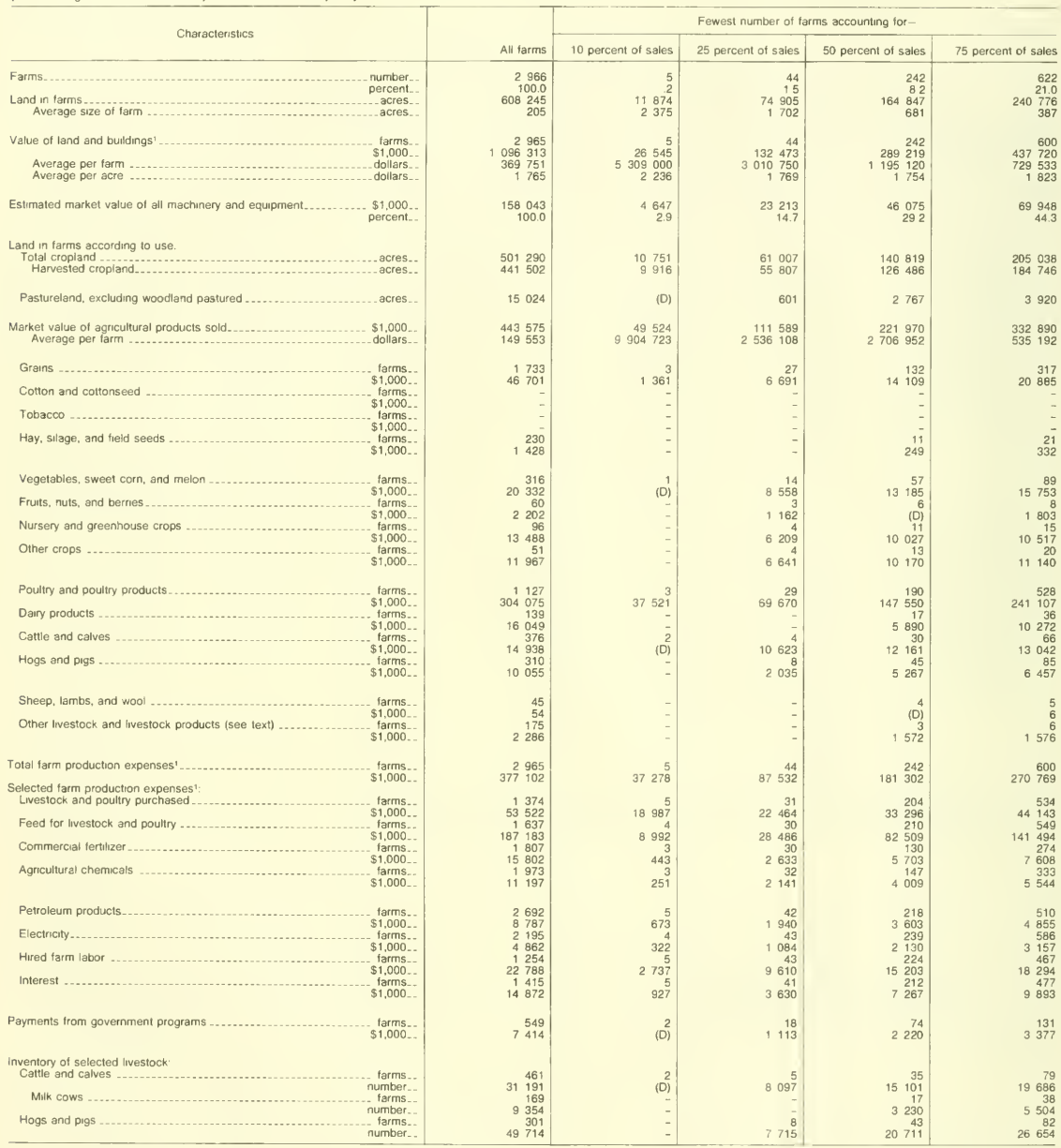

'Data are based on a sample of farms 
This page is intentionally blank to preserve table continuity. 
Table 48. Summary by Tenure of Operator: 1987

[For meanng of abbreviations and symbols, see introductory text]

\begin{tabular}{|c|c|c|c|c|c|c|c|c|}
\hline \multirow{2}{*}{ them } & \multicolumn{4}{|c|}{ All farms } & \multicolumn{4}{|c|}{ Farms with sales of $\$ 10,000$ or more } \\
\hline & Total & Full owners & Part owners & Tenants & Totai & Full owners & Part owners & Tenants \\
\hline \multicolumn{9}{|l|}{ FARMS AND LAND IN FARMS } \\
\hline $\begin{array}{l}\text { Farms . } \\
\text { Land in farms } \\
\text { Average size of farm ................................ }\end{array}$ & $\begin{array}{r}2966 \\
100.0 \\
608245 \\
205\end{array}$ & $\begin{array}{r}1825 \\
615 \\
142 \quad 331 \\
78\end{array}$ & $\begin{array}{r}857 \\
289 \\
399038 \\
466\end{array}$ & $\begin{array}{r}284 \\
96 \\
66 \quad 876 \\
235\end{array}$ & $\begin{array}{r}1859 \\
627 \\
540 \quad 547 \\
291\end{array}$ & $\begin{array}{r}1064 \\
359 \\
98735 \\
93\end{array}$ & 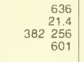 & $\begin{array}{r}159 \\
5.4 \\
59556 \\
375\end{array}$ \\
\hline $\begin{array}{l}\text { MARKET VALUE OF AGRICUL- } \\
\text { TURAL PRODUCTS SOLD }\end{array}$ & & & & & & & & \\
\hline 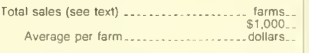 & $\begin{array}{r}2966 \\
443575 \\
149553\end{array}$ & $\begin{array}{r}1825 \\
231606 \\
126908\end{array}$ & $\begin{array}{rr} & 857 \\
178 & 409 \\
208 & 178\end{array}$ & $\begin{array}{rr}33 & 284 \\
118 & 169\end{array}$ & $\begin{array}{rr}1 & 859 \\
440 & 018 \\
236 & 696\end{array}$ & $\begin{array}{r}1064 \\
229413 \\
215614\end{array}$ & $\begin{array}{r}636 \\
177 \quad 495 \\
279080\end{array}$ & $\begin{array}{r}159 \\
33110 \\
208 \quad 240\end{array}$ \\
\hline $\begin{array}{l}\begin{array}{l}\text { Farms by value of sales } \\
\text { Less than } \$ 1,000 \text { (see text) } \\
\$ 1,000 \text { to } \$ 2,499 \\
\$ 2.500 \text { to } \$ 4.999 \\
\$ 5,000 \text { to } \$ 9.999 \\
\$ 10,000 \text { to } \$ 19.999\end{array}\end{array}$ & $\begin{array}{l}280 \\
260 \\
270 \\
297 \\
215\end{array}$ & $\begin{array}{l}213 \\
195 \\
188 \\
165 \\
116\end{array}$ & $\begin{array}{l}45 \\
36 \\
48 \\
92 \\
65\end{array}$ & $\begin{array}{l}22 \\
29 \\
34 \\
40 \\
34\end{array}$ & $\begin{array}{r}- \\
\bar{z} \\
215\end{array}$ & $\begin{array}{r}\overline{-} \\
116\end{array}$ & $\begin{array}{l}\overline{-} \\
\overline{-} \\
6 \bar{x}\end{array}$ & $\begin{array}{l}- \\
4\end{array}$ \\
\hline $\begin{array}{l}\$ 20,000 \text { to } \$ 24,999 \\
\$ 25,000 \text { to } \$ 39,999 \\
\$ 40,000 \text { to } \$ 49,999 \\
\$ 50,000 \text { to } \$ 99,999 \\
\$ 100,000 \text { to } \$ 249,999\end{array}$ & \begin{tabular}{r|}
65 \\
141 \\
73 \\
257 \\
549
\end{tabular} & $\begin{array}{r}27 \\
55 \\
30 \\
159 \\
343\end{array}$ & $\begin{array}{r}30 \\
56 \\
34 \\
77 \\
175\end{array}$ & \begin{tabular}{r|r}
8 & \\
30 & \\
9 & \\
21 & 31
\end{tabular} & $\begin{array}{r}65 \\
141 \\
73 \\
257 \\
549\end{array}$ & $\begin{array}{r}27 \\
55 \\
30 \\
159 \\
343\end{array}$ & $\begin{array}{r}30 \\
56 \\
34 \\
77 \\
175\end{array}$ & $\begin{array}{r}8 \\
30 \\
9 \\
21 \\
31\end{array}$ \\
\hline $\begin{array}{l}\$ 250,000 \text { to } \$ 499,999 \\
\$ 500,000 \text { to } \$ 999,999 . \\
\$ 1,000,000 \text { or more }\end{array}$ & $\begin{array}{r}403 \\
118 \\
38\end{array}$ & $\begin{array}{r}258 \\
62 \\
14\end{array}$ & $\begin{array}{r}130 \\
49 \\
20\end{array}$ & $\begin{array}{r}15 \\
7 \\
4\end{array}$ & $\begin{array}{r}403 \\
118 \\
38\end{array}$ & $\begin{array}{r}258 \\
62 \\
14\end{array}$ & $\begin{array}{r}130 \\
49 \\
20\end{array}$ & $\begin{array}{r}15 \\
7 \\
4\end{array}$ \\
\hline $\begin{array}{c}\text { Grains } \\
\text { Sales of } \$ 50,000 \text { or more } \\
\begin{array}{c}\text { tarms. } \\
\text { tarms. } \\
\$ 1,000\end{array}\end{array}$ & $\begin{array}{rr}1 & 733 \\
46 & 701 \\
242 \\
31412\end{array}$ & $\begin{array}{r}760 \\
6736 \\
1875\end{array}$ & $\begin{array}{r}770 \\
34405 \\
189 \\
25963\end{array}$ & $\begin{array}{r}203 \\
5560 \\
31 \\
3573\end{array}$ & $\begin{array}{r}1070 \\
44580 \\
242 \\
31412\end{array}$ & $\begin{array}{r}353 \\
5605 \\
182 \\
1875\end{array}$ & $\begin{array}{r}599 \\
33715 \\
189 \\
25963\end{array}$ & $\begin{array}{r}118 \\
5259 \\
31 \\
3573\end{array}$ \\
\hline 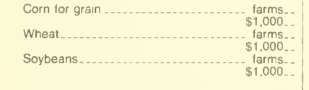 & $\begin{array}{r}915 \\
18294 \\
626 \\
4438 \\
1497 \\
21654\end{array}$ & $\begin{array}{r}307 \\
2260 \\
182 \\
709 \\
614 \\
3464\end{array}$ & $\begin{array}{r}504 \\
13556 \\
367 \\
3202 \\
698 \\
15814\end{array}$ & $\begin{array}{r}104 \\
2478 \\
77 \\
527 \\
185 \\
2376\end{array}$ & $\begin{array}{r}715 \\
17881 \\
481 \\
491 \\
970 \\
20275\end{array}$ & $\begin{array}{r}185 \\
2007 \\
105 \\
586 \\
304 \\
2756\end{array}$ & $\begin{array}{r}447 \\
13434 \\
315 \\
3098 \\
559 \\
15374\end{array}$ & $\begin{array}{r}83 \\
2440 \\
61 \\
507 \\
107 \\
2146\end{array}$ \\
\hline 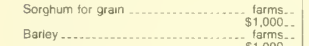 & $\begin{array}{r}18 \\
36 \\
320\end{array}$ & $\begin{array}{r}8 \\
12 \\
76\end{array}$ & $\begin{array}{r}8 \\
\text { (D) } \\
204 \\
62\end{array}$ & $\begin{array}{r}2 \\
\text { (D) } \\
40\end{array}$ & $\begin{array}{r}13 \\
26 \\
272\end{array}$ & $\begin{array}{r}4 \\
2 \\
49\end{array}$ & $\begin{array}{r}7 \\
(D) \\
192\end{array}$ & $\begin{array}{r}2 \\
(D) \\
31\end{array}$ \\
\hline Oats & $\begin{array}{rr}2 & 190 \\
9\end{array}$ & $\begin{array}{r}276 \\
4\end{array}$ & $\begin{array}{r}1767 \\
4\end{array}$ & $\begin{array}{r}147 \\
1\end{array}$ & $2 \begin{array}{r}2127 \\
5\end{array}$ & $\begin{array}{r}246 \\
2\end{array}$ & $\begin{array}{r}1746 \\
2\end{array}$ & $\begin{array}{r}135 \\
1\end{array}$ \\
\hline Other grains ............. & $\begin{array}{r}6 \\
40 \\
83\end{array}$ & $\begin{array}{r}4 \\
9 \\
12\end{array}$ & $\begin{array}{l}(\mathrm{D}) \\
26 \\
56\end{array}$ & $\begin{array}{r}\text { (D) } \\
5 \\
16\end{array}$ & $\begin{array}{r}3 \\
32 \\
76\end{array}$ & $\begin{array}{r}\text { (D) } \\
6 \\
\text { (D) }\end{array}$ & $\begin{array}{l}\text { (D) } \\
22 \\
55\end{array}$ & $\begin{array}{l}\text { (D) } \\
\text { (D) }\end{array}$ \\
\hline Cotton and cottonseed............... farms.. & $=$ & - & - & - & - & - & - & - \\
\hline 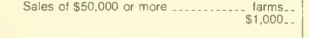 & $\begin{array}{l}- \\
-\end{array}$ & $:$ & $\overline{-}$ & - & - & $:$ & $=$ & $\overline{-}$ \\
\hline 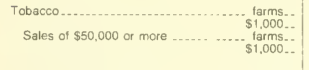 & $\begin{array}{l}- \\
-\end{array}$ & $:$ & $\begin{array}{l}- \\
= \\
=\end{array}$ & $\begin{array}{l}- \\
-\end{array}$ & $\begin{array}{l}- \\
- \\
-\end{array}$ & $\begin{array}{l}\bar{y} \\
\overline{-}\end{array}$ & $\begin{array}{l}= \\
z\end{array}$ & - \\
\hline 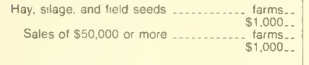 & $\begin{array}{r}230 \\
1428 \\
5 \\
422\end{array}$ & $\begin{array}{r}111 \\
374 \\
-\end{array}$ & $\begin{array}{r}89 \\
883 \\
5 \\
422\end{array}$ & $\begin{array}{r}30 \\
171 \\
- \\
-\end{array}$ & $\begin{array}{r}114 \\
1253 \\
5 \\
422\end{array}$ & $\begin{array}{r}36 \\
258 \\
- \\
-\end{array}$ & $\begin{array}{r}63 \\
851 \\
5 \\
422\end{array}$ & $\begin{array}{r}15 \\
144 \\
- \\
-\end{array}$ \\
\hline $\begin{array}{r}\text { Vegetabies, sweet corn, and melons .... farms.. } \\
\$ 1,000 . . \\
\text { Sales of } \$ 50,000 \text { or more ............. } \\
\$ 1,000 . .\end{array}$ & $\begin{array}{r}316 \\
20332 \\
78 \\
17571\end{array}$ & $\begin{array}{r}119 \\
2352 \\
12 \\
1521\end{array}$ & $\begin{array}{r}159 \\
12620 \\
54 \\
10948\end{array}$ & $\begin{array}{r}38 \\
559 \\
12 \\
5 \quad 102\end{array}$ & 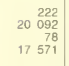 & $\begin{array}{r}57 \\
2218 \\
12 \\
1521\end{array}$ & $\begin{array}{r}139 \\
12555 \\
54 \\
10948\end{array}$ & $\begin{array}{r}26 \\
5318 \\
12 \\
5102\end{array}$ \\
\hline 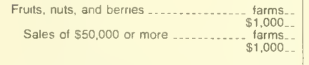 & $\begin{array}{r}60 \\
2202 \\
1.928\end{array}$ & $\begin{array}{r}29 \\
\text { (D) } \\
2 \\
\text { (D) }\end{array}$ & $\begin{array}{r}27 \\
1864 \\
3 \\
\text { (D) }\end{array}$ & $\begin{array}{r}4 \\
(\mathrm{D}) \\
- \\
-\end{array}$ & $\begin{array}{r}38 \\
2163 \\
1928 \\
1\end{array}$ & $\begin{array}{r}15 \\
299 \\
2 \\
(D)\end{array}$ & $\begin{array}{r}21 \\
\text { (D) } \\
3 \\
\text { (D) }\end{array}$ & $\stackrel{2}{(D)}$ \\
\hline $\begin{array}{r}\text { Nursery and greenhouse crops.......... farms.. } \\
\text { Sales of } \$ 50,000 \text { or more ............... tarms.. } \\
\$ 1,000 .\end{array}$ & $\begin{array}{r}96 \\
13488 \\
32 \\
12699\end{array}$ & $\begin{array}{r}65 \\
6275 \\
21 \\
5725\end{array}$ & $\begin{array}{r}21 \\
3944 \\
8 \\
3 \quad 822\end{array}$ & $\begin{array}{r}10 \\
3270 \\
3 \\
3 \quad 152 \\
\end{array}$ & $\begin{array}{r}70 \\
13 \quad 383 \\
32 \\
12699\end{array}$ & $\begin{array}{rr}6 & 43 \\
63 \\
21 \\
5 & 725\end{array}$ & $\begin{array}{r}19 \\
(D) \\
8 \\
3822\end{array}$ & $\begin{array}{r}8 \\
(D) \\
3 \\
3 \quad 152\end{array}$ \\
\hline 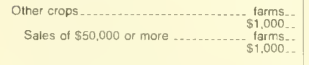 & $\begin{array}{r}51 \\
11967 \\
23 \\
11753\end{array}$ & \begin{tabular}{r|}
14 \\
(D) \\
4 \\
326
\end{tabular} & $\begin{array}{rr} & 30 \\
10 & 894 \\
16 \\
10 & 847\end{array}$ & $\begin{array}{r}7 \\
(D) \\
3 \\
580\end{array}$ & $\begin{array}{rr}11 & 44 \\
11 & 23 \\
11 & 753\end{array}$ & $\begin{array}{r}9 \\
366 \\
4 \\
326\end{array}$ & $\begin{array}{r}28 \\
\text { (D) } \\
16 \\
10847\end{array}$ & $\begin{array}{r}\text { (D) } \\
3 \\
580\end{array}$ \\
\hline $\begin{array}{r}\text { Poultry and poultry products } \ldots . . . . . . . . . \begin{array}{l}\text { farms } \\
\$ 1.000 \\
\text { farms }\end{array} \\
\$ 1.000 .\end{array}$ & 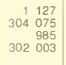 & $\begin{array}{rr}841 \\
203 & 778 \\
734 \\
202 & 112\end{array}$ & $\begin{array}{r}251 \\
90531 \\
225 \\
90 \quad 323\end{array}$ & $\begin{array}{r}35 \\
9767 \\
26 \\
9569\end{array}$ & $\begin{array}{rr}1 & 080 \\
304 & 019 \\
985 \\
302003\end{array}$ & $\begin{array}{r}800 \\
203734 \\
734 \\
202 \quad 112\end{array}$ & $\begin{array}{r}246 \\
\text { (D) } \\
225 \\
90323\end{array}$ & $\begin{array}{r}34 \\
(\mathrm{D}) \\
26 \\
9569\end{array}$ \\
\hline 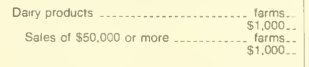 & $\begin{array}{r}139 \\
16 \quad 049 \\
76 \\
14917\end{array}$ & $\begin{array}{r}53 \\
2783 \\
19 \\
2 \quad 352\end{array}$ & $\begin{array}{r}63 \\
(\mathrm{D}) \\
48 \\
10882\end{array}$ & $\begin{array}{r}23 \\
(\mathrm{D}) \\
9 \\
1683\end{array}$ & $\begin{array}{r}126 \\
16 \quad 037 \\
76 \\
14917\end{array}$ & $\begin{array}{r}42 \\
2774 \\
19 \\
2 \quad 352\end{array}$ & $\begin{array}{r}63 \\
101 \\
48 \\
10 \quad 882\end{array}$ & $\begin{array}{r}21 \\
\text { (D) } \\
9 \\
1683\end{array}$ \\
\hline $\begin{array}{l}\text { Cattle and calves........... } \\
\text { Sales ot } \$ 50.000 \text { or more ... }\end{array}$ & $\begin{array}{r}376 \\
14938 \\
15 \\
12681\end{array}$ & $\begin{array}{r}190 \\
1556 \\
5 \\
893\end{array}$ & $\begin{array}{r}145 \\
7362 \\
7 \\
5989\end{array}$ & $\begin{array}{r}\quad 41 \\
6 \quad 020 \\
5 \quad 3 \\
5 \quad 799\end{array}$ & $\begin{array}{r} \\
14585 \\
14 \\
12.58 \\
12681\end{array}$ & $\begin{array}{r}76 \\
1275 \\
5 \\
893\end{array}$ & $\begin{array}{r}127 \\
7321 \\
7 \\
5989\end{array}$ & $\begin{array}{r}31 \\
5989 \\
5 \quad 3 \\
5 \quad 799\end{array}$ \\
\hline
\end{tabular}

See footnotes at end of table 
Table 48. Summary by Tenure of Operator: 1987-Con.

[For meaning of abbrevations and symbols, see introductory text]

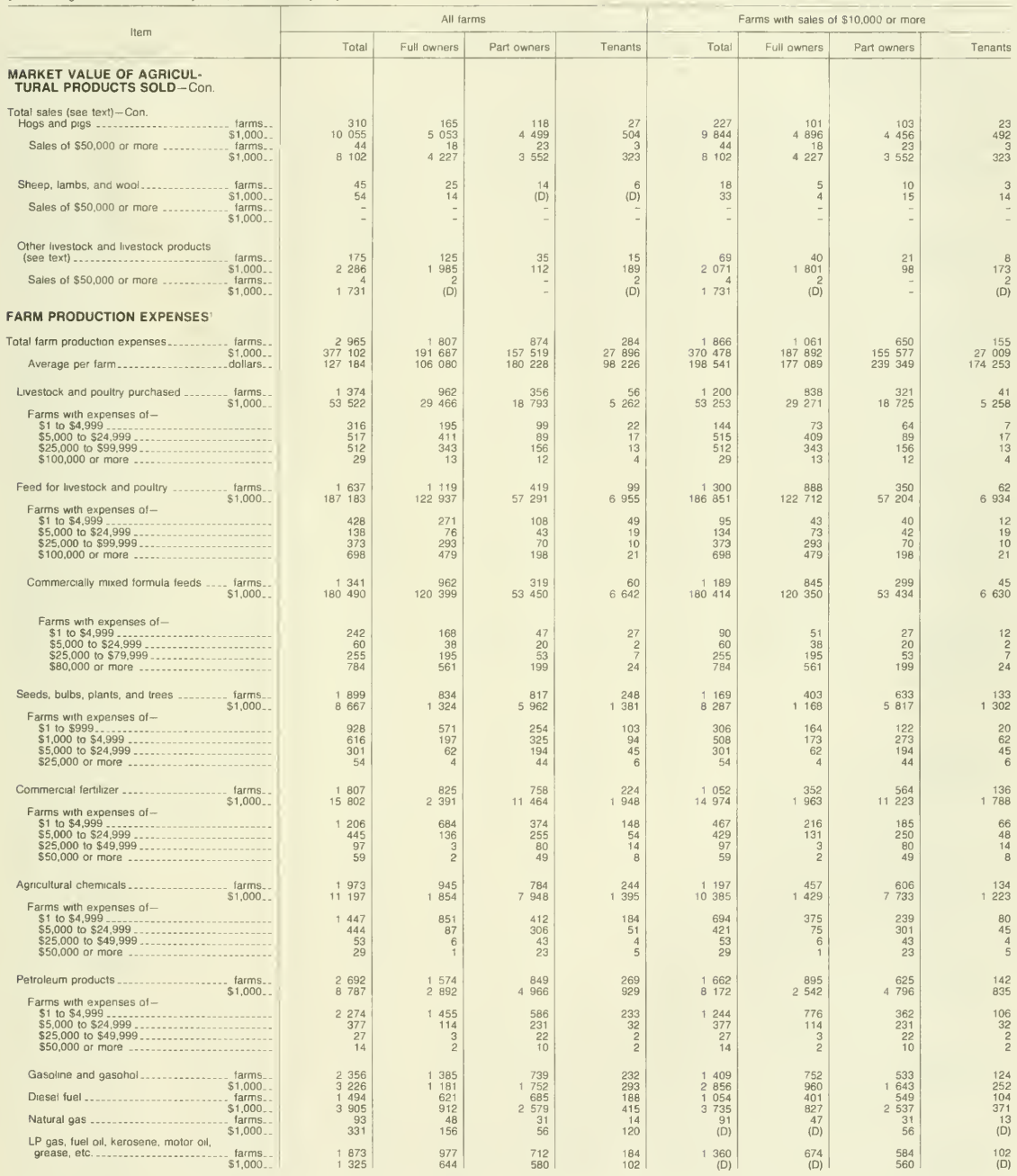


Table 48. Summary by Tenure of Operator: 1987-Con.

[For meanung of abbreviations and symbols, see introductory text]

\begin{tabular}{|c|c|c|c|c|c|c|c|c|}
\hline \multirow{2}{*}{ Item } & \multicolumn{4}{|c|}{ All farms } & \multicolumn{4}{|c|}{ Farms with sales of $\$ 10,000$ or more } \\
\hline & Total & Full owners & Part owners & Tenants & Total & Full owners & Part owners & Tenants \\
\hline \multicolumn{9}{|l|}{$\begin{array}{l}\text { FARM PRODUCTION EXPENSES' } \\
- \text { CON. }\end{array}$} \\
\hline 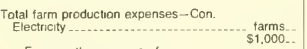 & $\begin{array}{ll}2 & 195 \\
4 & 862\end{array}$ & $\begin{array}{ll}1 & 294 \\
2 & 563\end{array}$ & $\begin{array}{r}714 \\
1861\end{array}$ & $\begin{array}{l}187 \\
439\end{array}$ & $\begin{array}{l}1637 \\
4690\end{array}$ & $\begin{array}{r}928 \\
2449\end{array}$ & $\begin{array}{r}569 \\
1816\end{array}$ & $\begin{array}{l}140 \\
425\end{array}$ \\
\hline 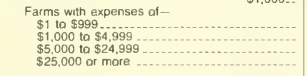 & $\begin{array}{r}1085 \\
942 \\
151 \\
17\end{array}$ & $\begin{array}{r}598 \\
619 \\
72 \\
5\end{array}$ & $\begin{array}{r}361 \\
274 \\
69 \\
10\end{array}$ & $\begin{array}{r}126 \\
49 \\
10 \\
2\end{array}$ & $\begin{array}{r}554 \\
915 \\
151 \\
17\end{array}$ & $\begin{array}{r}257 \\
594 \\
72 \\
5\end{array}$ & $\begin{array}{r}217 \\
273 \\
69 \\
10\end{array}$ & $\begin{array}{r}4 \angle 0 \\
80 \\
48 \\
10 \\
2\end{array}$ \\
\hline $\begin{array}{l}\text { Hired farm labor } \ldots . . . . . .19 a r m s .- \\
\$ 1,000\end{array}$ & 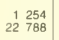 & $\begin{array}{r}689 \\
8036\end{array}$ & $\begin{array}{r}501 \\
11318\end{array}$ & $\begin{array}{r}64 \\
3434\end{array}$ & $\begin{array}{r}1091 \\
22594\end{array}$ & $\begin{array}{r}585 \\
7915\end{array}$ & $\begin{array}{r}451 \\
11246\end{array}$ & $\begin{array}{r}55 \\
3433\end{array}$ \\
\hline $\begin{array}{l}\text { Farms with expenses of - } \\
\$ 1 \text { to } \$ 4.999 \\
\$ 5,000 \text { to } \$ 24,999 \\
\$ 25,000 \text { to } \$ 99,999 \\
\$ 100,000 \text { or more }\end{array}$ & $\begin{array}{r}646 \\
440 \\
133 \\
35\end{array}$ & $\begin{array}{r}407 \\
223 \\
49 \\
10\end{array}$ & $\begin{array}{r}215 \\
192 \\
73 \\
21\end{array}$ & $\begin{array}{r}24 \\
25 \\
11 \\
4\end{array}$ & $\begin{array}{r}488 \\
435 \\
133 \\
35\end{array}$ & $\begin{array}{r}308 \\
218 \\
49 \\
10\end{array}$ & $\begin{array}{r}165 \\
192 \\
73 \\
21\end{array}$ & $\begin{array}{r}15 \\
25 \\
11 \\
4\end{array}$ \\
\hline $\begin{array}{l}\text { Contract labor } \ldots \ldots a n s . . . \\
\$ 1,000 \ldots\end{array}$ & $\begin{array}{r}364 \\
5798\end{array}$ & $\begin{array}{r}226 \\
1418\end{array}$ & $\begin{array}{r}120 \\
4030\end{array}$ & $\begin{array}{r}18 \\
350\end{array}$ & $\begin{array}{r}342 \\
5753\end{array}$ & $\begin{array}{r}213 \\
\text { (D) }\end{array}$ & $\begin{array}{r}112 \\
4002\end{array}$ & $\begin{array}{l}17 \\
\text { (D) }\end{array}$ \\
\hline $\begin{array}{l}\text { Farms with expenses of } \\
\$ 1 \text { to } \$ 999 \\
\$ 1,000 \text { to } \$ 4,999 \\
\$ 5,000 \text { to } \$ 24,999 \\
\$ 25,000 \text { or more }\end{array}$ & $\begin{array}{r}41 \\
161 \\
134 \\
28\end{array}$ & $\begin{array}{r}30 \\
107 \\
80 \\
9\end{array}$ & $\begin{array}{r}9 \\
49 \\
46 \\
16\end{array}$ & \begin{tabular}{r|r}
2 & \\
5 & \\
8 & \\
3 &
\end{tabular} & $\begin{array}{r}31 \\
149 \\
134 \\
28\end{array}$ & $\begin{array}{r}20 \\
104 \\
80 \\
9\end{array}$ & $\begin{array}{r}9 \\
41 \\
46 \\
16\end{array}$ & $\begin{array}{l}2 \\
4 \\
8 \\
3\end{array}$ \\
\hline 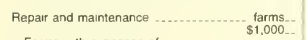 & 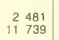 & $\begin{array}{ll}1 & 486 \\
4 & 176\end{array}$ & $\begin{array}{r}783 \\
6 \quad 321\end{array}$ & $\begin{array}{r}212 \\
1242\end{array}$ & $\begin{array}{r}1649 \\
11006\end{array}$ & $\begin{array}{r}933 \\
3694\end{array}$ & $\begin{array}{r}603 \\
6 \quad 144\end{array}$ & $\begin{array}{ll}113 \\
1167\end{array}$ \\
\hline $\begin{array}{l}\begin{array}{l}\text { Farms with expenses of- } \\
\$ 1 \text { to } \$ 4,999 \\
\$ 5,000 \text { to } \$ 24.999 \\
\$ 25.000 \text { to } \$ 49.999 \\
\$ 50,000 \text { or mare }\end{array} \\
\end{array}$ & $\begin{array}{r}1939 \\
455 \\
67 \\
20\end{array}$ & $\begin{array}{r}1272 \\
194 \\
18 \\
2\end{array}$ & $\begin{array}{r}500 \\
226 \\
43 \\
14\end{array}$ & \begin{tabular}{r|r}
167 & -2 \\
35 & -2 \\
6 & - \\
4 &
\end{tabular} & $\begin{array}{r}1111 \\
451 \\
67 \\
20\end{array}$ & $\begin{array}{r}722 \\
191 \\
18 \\
2\end{array}$ & $\begin{array}{r}320 \\
226 \\
43 \\
14\end{array}$ & $\begin{array}{r}69 \\
34 \\
6 \\
4\end{array}$ \\
\hline $\begin{array}{l}\text { Customwork, machine hire, and rental of } \\
\text { machinery and equipment ............. tarms.. }\end{array}$ & $\begin{array}{ll}1 & 192 \\
2 & 445\end{array}$ & $\begin{array}{l}645 \\
848\end{array}$ & $\begin{array}{r}437 \\
1 \quad 289\end{array}$ & $\begin{array}{l}110 \\
308\end{array}$ & $\begin{array}{r}792 \\
2210\end{array}$ & $\begin{array}{l}413 \\
\text { (D) }\end{array}$ & $\begin{array}{r}327 \\
1218\end{array}$ & $\begin{array}{l}52 \\
\text { (D) }\end{array}$ \\
\hline $\begin{array}{l}\text { Farms with expenses of - } \\
\$ 1 \text { to } \$ 999 \\
\$ 1.000 \text { to } \$ 4.999 \\
\$ 5,000 \text { to } \$ 24.999 \\
\$ 25,000 \text { or more }\end{array}$ & $\begin{array}{r}672 \\
406 \\
106 \\
8\end{array}$ & $\begin{array}{r}436 \\
187 \\
21 \\
1\end{array}$ & $\begin{array}{r}180 \\
178 \\
74 \\
5\end{array}$ & \begin{tabular}{r|r}
56 & \\
41 & \\
11 & \\
2 &
\end{tabular} & $\begin{array}{r}355 \\
323 \\
106 \\
8\end{array}$ & $\begin{array}{r}248 \\
143 \\
21 \\
1\end{array}$ & $\begin{array}{r}94 \\
154 \\
74 \\
5\end{array}$ & $\begin{array}{r}13 \\
26 \\
11 \\
2\end{array}$ \\
\hline $\begin{array}{l}\text { Interest expense } \\
\text { farms } \\
\$ 1,000\end{array}$ & $\begin{array}{rr}1 & 415 \\
14 & 872\end{array}$ & $\begin{array}{r}825 \\
6061\end{array}$ & $\begin{array}{r}513 \\
8336\end{array}$ & $\begin{array}{r}77 \\
475\end{array}$ & $\begin{array}{r}1137 \\
14258\end{array}$ & $\begin{array}{r}636 \\
5636\end{array}$ & $\begin{array}{r}443 \\
8185\end{array}$ & $\begin{array}{r}58 \\
437\end{array}$ \\
\hline $\begin{array}{l}\text { Farms with expenses of- } \\
\$ 1 \text { to } \$ 4,999 \\
\$ 5,000 \text { to } \$ 24,999 \\
\$ 25,000 \text { to } \$ 99,999 \\
\$ 100,000 \text { or more }\end{array}$ & $\begin{array}{r}713 \\
580 \\
110 \\
12\end{array}$ & $\begin{array}{r}461 \\
321 \\
41 \\
2\end{array}$ & $\begin{array}{r}201 \\
237 \\
65 \\
10\end{array}$ & $\begin{array}{r}51 \\
22 \\
4 \\
-\end{array}$ & $\begin{array}{r}473 \\
542 \\
110 \\
12\end{array}$ & $\begin{array}{r}295 \\
298 \\
41 \\
2\end{array}$ & $\begin{array}{r}146 \\
222 \\
65 \\
10\end{array}$ & $\begin{array}{r}32 \\
22 \\
4 \\
-\end{array}$ \\
\hline Secured by real estate ................. farms_.. & $\begin{array}{rr}1 & 205 \\
12 & 415\end{array}$ & $\begin{array}{r}752 \\
5254\end{array}$ & $\begin{array}{r}453 \\
7+161\end{array}$ & - & $\begin{array}{r}983 \\
11901\end{array}$ & 4892 & $\begin{array}{r}391 \\
7031\end{array}$ & - \\
\hline $\begin{array}{l}\text { Farms with expenses of- } \\
\$ 1 \text { to } \$ 999 \\
\$ 1.000 \text { to } \$ 4,999 \\
\$ 5,000 \text { to } \$ 24,999 \\
\$ 25,000 \text { or more }\end{array}$ & $\begin{array}{r}139 \\
463 \\
505 \\
98\end{array}$ & $\begin{array}{r}95 \\
329 \\
295 \\
33\end{array}$ & $\begin{array}{r}44 \\
134 \\
210 \\
65\end{array}$ & ${ }^{\prime}$ & $\begin{array}{r}78 \\
334 \\
473 \\
98\end{array}$ & \begin{tabular}{r|r}
53 \\
234 \\
272 \\
33
\end{tabular} & $\begin{array}{r}25 \\
100 \\
201 \\
65\end{array}$ & $\begin{array}{l}- \\
-\end{array}$ \\
\hline Not secured by real estate $\ldots \ldots \ldots . . .1$ farms.. & $\begin{array}{r}500 \\
2457\end{array}$ & $\begin{array}{l}214 \\
806\end{array}$ & $\begin{array}{r}209 \\
1175\end{array}$ & $\begin{array}{r}77 \\
475\end{array}$ & $\begin{array}{r}428 \\
2356\end{array}$ & $\begin{array}{l}180 \\
766\end{array}$ & $\begin{array}{r}190 \\
1153\end{array}$ & $\begin{array}{r}58 \\
437\end{array}$ \\
\hline $\begin{array}{l}\text { Farms with expenses of - } \\
\$ 1 \text { to } \$ 999 \\
\$ 1,000 \text { to } \$ 4,999 \\
\$ 5,000 \text { to } \$ 24,999 \\
\$ 25,000 \text { or more }\end{array}$ & $\begin{array}{r}183 \\
189 \\
113 \\
15\end{array}$ & $\begin{array}{r}90 \\
79 \\
41 \\
4\end{array}$ & $\begin{array}{r}69 \\
83 \\
50 \\
7\end{array}$ & $\begin{array}{r}24 \\
27 \\
22 \\
4\end{array}$ & $\begin{array}{r}158 \\
142 \\
113 \\
15\end{array}$ & $\begin{array}{r}73 \\
62 \\
41 \\
4\end{array}$ & $\begin{array}{r}61 \\
72 \\
50 \\
7\end{array}$ & $\begin{array}{r}24 \\
8 \\
22 \\
4\end{array}$ \\
\hline $\begin{array}{c}\text { Cash rent....................... farms_.. } \\
\$ 1,000 . .\end{array}$ & 11989 & - & $\begin{array}{r}778 \\
8568\end{array}$ & $\begin{array}{r}194 \\
2520\end{array}$ & $\begin{array}{r}707 \\
10709\end{array}$ & $=$ & $\begin{array}{r}585 \\
8321\end{array}$ & $\begin{array}{r}122 \\
2389\end{array}$ \\
\hline $\begin{array}{l}\text { Farms with expenses of - } \\
\$ 1 \text { to } \$ 4,999 \\
\$ 5,000 \text { to } \$ 9,999 \\
\$ 10,000 \text { to } \$ 24,999 \\
\$ 25,000 \text { or more }\end{array}$ & $\begin{array}{l}582 \\
103 \\
184 \\
103\end{array}$ & $\begin{array}{l}- \\
- \\
-\end{array}$ & $\begin{array}{r}470 \\
80 \\
149 \\
79\end{array}$ & $\begin{array}{r}112 \\
23 \\
35 \\
24\end{array}$ & $\begin{array}{r}328 \\
92 \\
184 \\
103\end{array}$ & $\begin{array}{l}- \\
- \\
-\end{array}$ & $\begin{array}{r}287 \\
70 \\
149 \\
79\end{array}$ & $\begin{array}{l}41 \\
22 \\
35 \\
24\end{array}$ \\
\hline Property taxes $\ldots+1,000$. & $\begin{array}{l}2724 \\
2591\end{array}$ & $\begin{array}{l}1791 \\
1 \\
1\end{array} 465$ & $1 \begin{array}{r}872 \\
1056\end{array}$ & $\begin{array}{l}61 \\
71\end{array}$ & $\begin{array}{ll}1 & 745 \\
2 & 101\end{array}$ & $\begin{array}{l}1053 \\
1070\end{array}$ & $\begin{array}{l}649 \\
968\end{array}$ & $\begin{array}{l}43 \\
63\end{array}$ \\
\hline $\begin{array}{l}\text { Farms with expenses of - } \\
\$ 1 \text { to } \$ 4,999 \\
\$ 5,000 \text { to } \$ 9,999 \\
\$ 10,000 \text { to } \$ 24,999 \\
\$ 25,000 \text { ar mare }\end{array}$ & $\begin{array}{r}2666 \\
42 \\
12 \\
4\end{array}$ & $\begin{array}{r}1772 \\
14 \\
4 \\
1\end{array}$ & $\begin{array}{r}835 \\
26 \\
8 \\
3\end{array}$ & $\begin{array}{r}59 \\
2 \\
- \\
-\end{array}$ & $\begin{array}{r}1687 \\
42 \\
12 \\
4\end{array}$ & $\begin{array}{r}1034 \\
14 \\
4 \\
1\end{array}$ & $\begin{array}{r}612 \\
26 \\
8 \\
3\end{array}$ & $\begin{array}{r}41 \\
2 \\
-\end{array}$ \\
\hline All other farm praduction expenses...... farms - $\$ 1,000$. & $\begin{array}{r}2813 \\
15758\end{array}$ & $\begin{array}{ll}1 & 715 \\
6 & 256\end{array}$ & $8 \begin{array}{r}861 \\
815\end{array}$ & 1,237 & 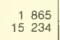 & $\begin{array}{ll}1 & 061 \\
5 & 925\end{array}$ & $\begin{array}{r}649 \\
8179\end{array}$ & $\begin{array}{r}155 \\
1130\end{array}$ \\
\hline $\begin{array}{l}\text { Farms with expenses of } \\
\$ 1 \text { to } \$ 4,999 \\
\$ 5,000 \text { to } \$ 24,999 \\
\$ 25,000 \text { to } \$ 49,999 \\
\$ 50,000 \text { or mare }\end{array}$ & $\begin{array}{r}2271 \\
442 \\
50 \\
50\end{array}$ & $\begin{array}{r}1447 \\
235 \\
20 \\
13\end{array}$ & $\begin{array}{r}628 \\
174 \\
27 \\
32\end{array}$ & $\begin{array}{r}196 \\
33 \\
3 \\
5\end{array}$ & $\begin{array}{r}1324 \\
441 \\
50 \\
50\end{array}$ & $\begin{array}{r}794 \\
234 \\
20 \\
13\end{array}$ & $\begin{array}{r}416 \\
174 \\
27 \\
32\end{array}$ & $\begin{array}{r}114 \\
33 \\
3 \\
5\end{array}$ \\
\hline
\end{tabular}


Table 48. Summary by Tenure of Operator: 1987-Con.

[For meaning of abbrevations and symbols, see intraductary text]

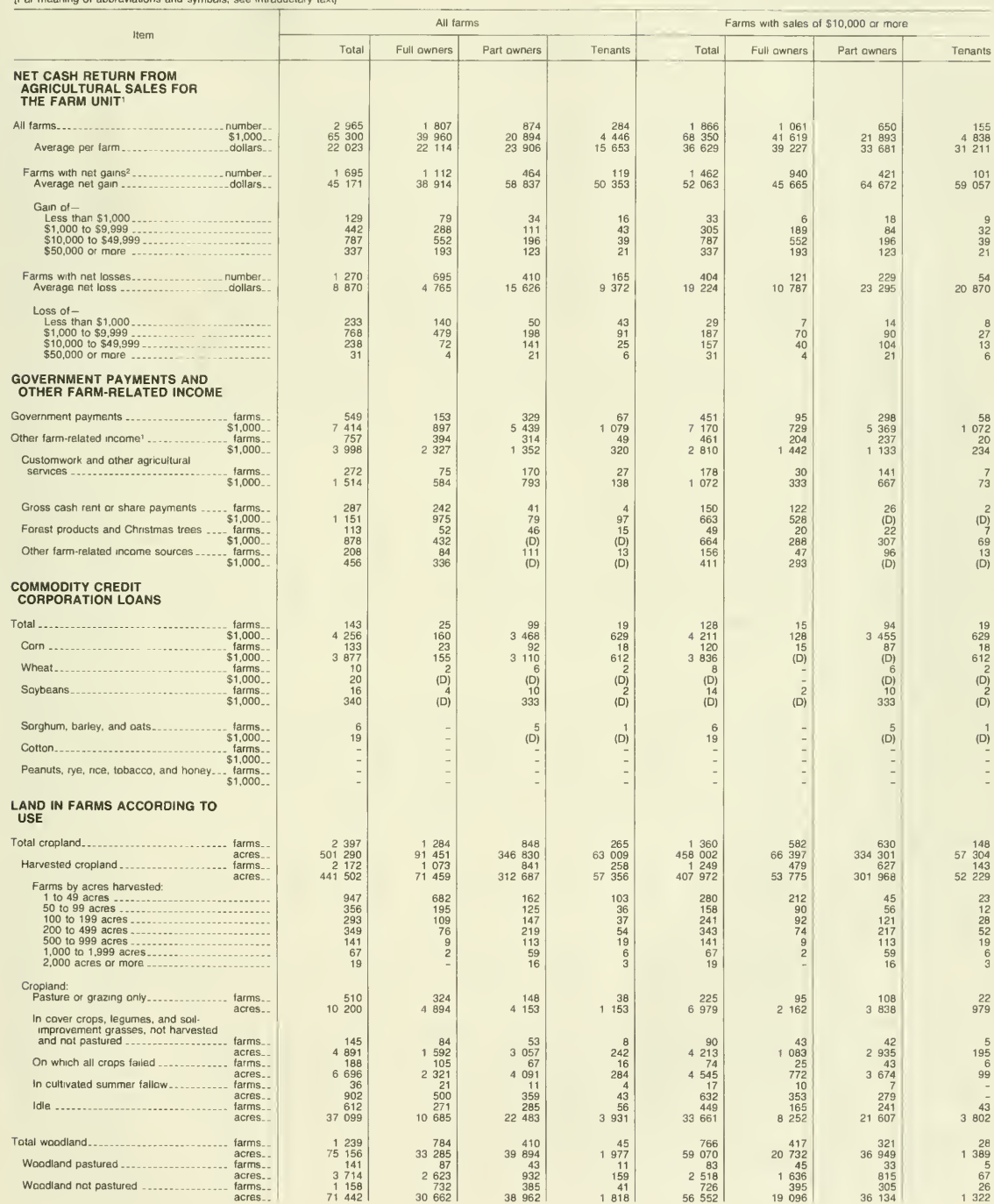


Table 48. Summary by Tenure of Operator: 1987-Con.

[For meaning of abbreviatians and symbols, see introductory text]

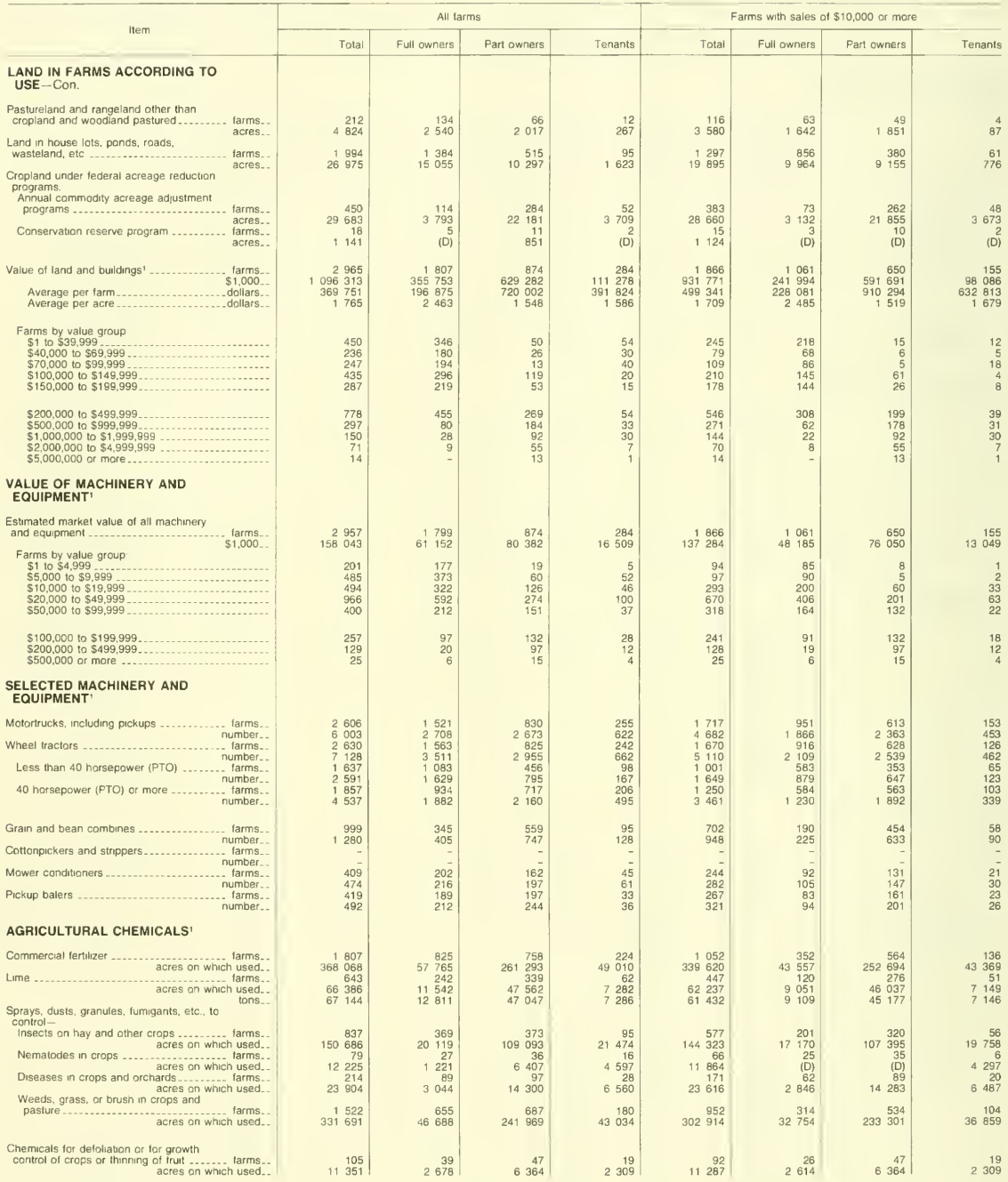


Table 48. Summary by Tenure of Operator: 1987-Con.

[For meaning of abbreviations and symbols, see introductory text]

\begin{tabular}{|c|c|c|c|c|c|c|c|c|}
\hline \multirow{2}{*}{ Item } & \multicolumn{4}{|c|}{ All farms } & \multicolumn{4}{|c|}{ Farms with saies of $\$ 10,000$ or more } \\
\hline & Total & Full owners & Part owners & Tenants & Tolal & Full owners & Part owners & Tenants \\
\hline \multicolumn{9}{|l|}{$\begin{array}{l}\text { TENURE AND RACE OF } \\
\text { OPERATOR }\end{array}$} \\
\hline $\begin{array}{l}\text { All operators } \\
\text { Full owners } \\
\text { Part owners } \\
\text { Tenants ... }\end{array}$ & $\begin{array}{r}2866 \\
1825 \\
857 \\
284\end{array}$ & $\begin{array}{r}1825 \\
1825 \\
-\end{array}$ & $\begin{array}{l}857 \\
85 \overline{7}\end{array}$ & 284 & $\begin{array}{r}1859 \\
1064 \\
636 \\
159\end{array}$ & 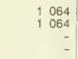 & $\begin{array}{l}636 \\
636\end{array}$ & $\begin{array}{r}159 \\
159\end{array}$ \\
\hline $\begin{array}{l}\text { White } \\
\text { Full owners } \\
\text { Part owners } \\
\text { Tenants .. }\end{array}$ & $\begin{array}{r}2914 \\
1791 \\
845 \\
278\end{array}$ & $\begin{array}{r}1791 \\
1791 \\
- \\
-\end{array}$ & $\begin{array}{l}845 \\
845 \\
-\end{array}$ & 278 & $\begin{array}{r}1839 \\
1052 \\
630 \\
157\end{array}$ & $\begin{array}{r}1 \\
1052 \\
1052 \\
-\end{array}$ & $\begin{array}{r}630 \\
630^{-} \\
-\end{array}$ & $\begin{array}{r}157 \\
157\end{array}$ \\
\hline 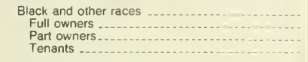 & $\begin{array}{r}52 \\
34 \\
12 \\
6\end{array}$ & $\begin{array}{r}34 \\
34 \\
- \\
-\end{array}$ & $\frac{12}{12}$ & $\begin{array}{l}6 \\
6 \\
6\end{array}$ & $\begin{array}{r}20 \\
12 \\
6 \\
2\end{array}$ & $\begin{array}{r}12 \\
12 \\
-\end{array}$ & $\frac{6}{6}$ & $\frac{2}{2}$ \\
\hline \multicolumn{9}{|l|}{ OWNED AND RENTED LAND } \\
\hline $\begin{array}{r}\text { Land owned } \\
\text { Owned land in farms } \ldots \ldots \ldots\end{array}$ & $\begin{array}{rr}2 & 691 \\
360 & 152 \\
2 & 682 \\
325 & 313\end{array}$ & $\begin{array}{rr}1 & 825 \\
171 & 956 \\
1 & 825 \\
142 & 331\end{array}$ & $\begin{array}{r}857 \\
186836 \\
857 \\
182982\end{array}$ & $\begin{array}{r}9 \\
1360 \\
-\end{array}$ & $\begin{array}{r}1705 \\
293930 \\
1700 \\
273504\end{array}$ & $\begin{array}{rr}1 & 064 \\
116 & 003 \\
1 & 064 \\
98 & 735\end{array}$ & $\begin{array}{rr} & 636 \\
177 & 417 \\
& 636 \\
174 \quad 769\end{array}$ & $\begin{array}{r}5 \\
510 \\
-\end{array}$ \\
\hline $\begin{aligned} \text { Land rented or leased from others ........ farms.. } \\
\text { acres.: } \\
\text { Rented or leased land in farms ......... farms.. } \\
\text { acres.. }\end{aligned}$ & $\begin{array}{rr}1 & 153 \\
285 & 163 \\
1 & 141 \\
282 & 932\end{array}$ & $\begin{array}{r}12 \\
251 \\
-\end{array}$ & $\begin{array}{rr} & 857 \\
216 & 853 \\
& 857 \\
216 & 056\end{array}$ & $\begin{array}{rr}68 & 284 \\
68 & 289 \\
284 \\
66 \quad 876\end{array}$ & $\begin{array}{r}802 \\
269 \\
143 \\
795 \\
267 \\
043\end{array}$ & $\begin{array}{r}7 \\
240 \\
-\end{array}$ & $\begin{array}{rl}636 \\
208 & 164 \\
636 \\
207 & 487\end{array}$ & $\begin{array}{r}159 \\
60739 \\
159 \\
59556\end{array}$ \\
\hline $\begin{array}{r}\text { Land rented or leased to others............ tarms.. } \\
\text { acres.. }\end{array}$ & $37 \begin{array}{r}463 \\
070\end{array}$ & 298976 & 4651 & 2543 & $\begin{array}{rl}277 \\
22 & 526\end{array}$ & $17 \begin{array}{r}233 \\
508\end{array}$ & 3 325 & $\begin{array}{r}8 \\
1693\end{array}$ \\
\hline OPERATOR CHARACTERISTICS & & & & & & & & \\
\hline $\begin{array}{l}\text { Operators by place of residence: } \\
\text { On tarm operated } \\
\text { Not on farm operated } \\
\text { Not reported }\end{array}$ & $\begin{array}{r}2175 \\
481 \\
310\end{array}$ & $\begin{array}{r}1346 \\
229 \\
250\end{array}$ & $\begin{array}{r}689 \\
124 \\
44\end{array}$ & $\begin{array}{r}140 \\
128 \\
16\end{array}$ & $\begin{array}{r}1389 \\
245 \\
225\end{array}$ & $\begin{array}{l}758 \\
117 \\
189\end{array}$ & $\begin{array}{r}536 \\
69 \\
31\end{array}$ & $\begin{array}{r}95 \\
59 \\
5\end{array}$ \\
\hline $\begin{array}{l}\text { Operators by principal occupation: } \\
\text { Farming } \\
\text { Other }\end{array}$ & $\begin{array}{l}1774 \\
1 \\
1\end{array}$ & $\begin{array}{l}999 \\
826\end{array}$ & $\begin{array}{l}608 \\
249\end{array}$ & $\begin{array}{l}167 \\
117\end{array}$ & $\begin{array}{r}1372 \\
487\end{array}$ & $\begin{array}{l}708 \\
356\end{array}$ & $\begin{array}{l}536 \\
100\end{array}$ & $\begin{array}{r}128 \\
31\end{array}$ \\
\hline $\begin{array}{l}\text { Operators by days of work off farm: } \\
\text { None } \\
\text { Any } 10.99 \text { days } \\
100 \text { to } 199 \text { days } \\
200 \text { days or more }\end{array}$ & \begin{tabular}{rr|}
1 & 263 \\
1 & 426 \\
254 \\
217 \\
955
\end{tabular} & $\begin{array}{l}751 \\
867 \\
119 \\
134 \\
614\end{array}$ & $\begin{array}{r}405 \\
392 \\
99 \\
54 \\
239\end{array}$ & $\begin{array}{r}107 \\
167 \\
36 \\
29 \\
102\end{array}$ & $\begin{array}{l}925 \\
732 \\
178 \\
117 \\
437\end{array}$ & $\begin{array}{r}483 \\
437 \\
79 \\
71 \\
287\end{array}$ & $\begin{array}{r}360 \\
226 \\
74 \\
31 \\
121\end{array}$ & $\begin{array}{l}82 \\
69 \\
25 \\
15 \\
29\end{array}$ \\
\hline Not reported ..................... & 277 & 207 & 60 & 10 & 202 & 144 & 50 & 8 \\
\hline 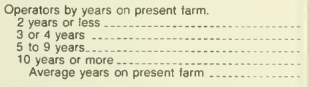 & $\begin{array}{r}176 \\
189 \\
398 \\
1588 \\
193\end{array}$ & $\begin{array}{r}108 \\
127 \\
245 \\
910 \\
18.9\end{array}$ & $\begin{array}{r}30 \\
33 \\
93 \\
568 \\
22.1\end{array}$ & $\begin{array}{r}38 \\
29 \\
60 \\
110 \\
12.8\end{array}$ & $\begin{array}{r}85 \\
119 \\
235 \\
1020 \\
19.5\end{array}$ & $\begin{array}{r}50 \\
84 \\
139 \\
496 \\
177\end{array}$ & $\begin{array}{r}18 \\
21 \\
56 \\
456 \\
23.4\end{array}$ & $\begin{array}{r}17 \\
14 \\
40 \\
68 \\
13.9\end{array}$ \\
\hline Not reported ............... & 615 & 435 & 133 & 47 & 400 & 295 & 85 & 20 \\
\hline 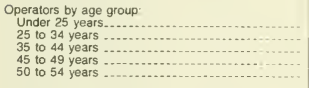 & $\begin{array}{r}40 \\
374 \\
620 \\
328 \\
321\end{array}$ & $\begin{array}{r}12 \\
188 \\
362 \\
197 \\
191\end{array}$ & $\begin{array}{r}12 \\
100 \\
195 \\
96 \\
113\end{array}$ & $\begin{array}{l}16 \\
86 \\
63 \\
35 \\
17\end{array}$ & $\begin{array}{r}22 \\
260 \\
425 \\
211 \\
215\end{array}$ & $\begin{array}{r}10 \\
137 \\
244 \\
119 \\
122\end{array}$ & $\begin{array}{r}4 \\
67 \\
144 \\
76 \\
87\end{array}$ & $\begin{array}{r}8 \\
56 \\
37 \\
16 \\
6\end{array}$ \\
\hline $\begin{array}{l}55 \text { to } 59 \text { years } \\
60 \text { to } 64 \text { years } \\
65 \text { to } 69 \text { years } \\
70 \text { years and over } \\
\text { Average age }\end{array}$ & $\begin{array}{r}326 \\
348 \\
269 \\
340 \\
51.5\end{array}$ & \begin{tabular}{r|}
202 \\
219 \\
185 \\
269 \\
53.3
\end{tabular} & $\begin{array}{r}101 \\
113 \\
68 \\
59 \\
50.3\end{array}$ & $\begin{array}{r}23 \\
16 \\
16 \\
12 \\
43.2\end{array}$ & $\begin{array}{r}197 \\
222 \\
159 \\
148 \\
50.0\end{array}$ & $\begin{array}{r}111 \\
116 \\
101 \\
104 \\
50.8\end{array}$ & $\begin{array}{r}75 \\
93 \\
51 \\
39 \\
50.8\end{array}$ & $\begin{array}{r}11 \\
13 \\
7 \\
5 \\
42.1\end{array}$ \\
\hline $\begin{array}{l}\text { Operators by sex: } \\
\text { Male } \\
\text { Female }\end{array}$ & $\begin{array}{r}2676 \\
290\end{array}$ & $\begin{array}{r}1572 \\
253\end{array}$ & $\begin{array}{r}837 \\
20\end{array}$ & $\begin{array}{r}267 \\
17\end{array}$ & $\begin{array}{r}1656 \\
203\end{array}$ & $\begin{array}{l}883 \\
181\end{array}$ & $\begin{array}{r}625 \\
11\end{array}$ & $\begin{array}{r}148 \\
11\end{array}$ \\
\hline $\begin{array}{l}\text { Operators of Spanish origin (see text) } \\
\text { FARMS BY TYPE OF } \\
\text { ORGANIZATION }\end{array}$ & 9 & 6 & - & 3 & 7 & 4 & - & 3 \\
\hline 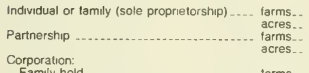 & $\begin{array}{rr}2 & 553 \\
400 & 071 \\
216 \\
62666\end{array}$ & $\begin{array}{r}1625 \\
119669 \\
119 \\
10941\end{array}$ & $\begin{array}{r}701 \\
243828 \\
72 \\
44475\end{array}$ & $\begin{array}{r}227 \\
36574 \\
725 \\
7250\end{array}$ & $\begin{array}{rl}1 & 537 \\
338 & 062 \\
161 \\
58 & 948\end{array}$ & $\begin{array}{r}927 \\
79556 \\
81 \\
8834\end{array}$ & $\begin{array}{rr}493 \\
228 & 243 \\
62 \\
43 \quad 395\end{array}$ & $\begin{array}{rr}117 \\
30 \quad 263 \\
18 \\
6 \quad 719\end{array}$ \\
\hline $\begin{array}{l}\text { Family held } \\
\text { More than } 10 \text { stockholders ............. farms.. } \\
10 \text { or less stockhoiders }\end{array}$ & $\begin{array}{r}173 \\
141856 \\
4 \\
169\end{array}$ & $\begin{array}{r}65 \\
9656 \\
3 \\
62\end{array}$ & 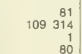 & 22886 & $\begin{array}{r}146 \\
(\mathrm{D}) \\
4 \\
4\end{array}$ & $\begin{array}{r}45 \\
(\mathrm{D}) \\
3 \\
\end{array}$ & $109 \begin{array}{r}78 \\
197 \\
1 \\
77\end{array}$ & $\begin{array}{l}23 \\
\text { (D) }\end{array}$ \\
\hline Other than family held . ............. larms. & 8 & 5 & - & 3 & 5 & 4 & - & 1 \\
\hline $\begin{array}{l}\text { More than } 10 \text { stockholders ........... tarms.: } \\
10 \text { or less stockholders ............ farms.. }\end{array}$ & $\begin{array}{r}928 \\
1 \\
7\end{array}$ & (D) & $\overline{-}$ & $\begin{array}{r}(0) \\
1 \\
2\end{array}$ & (D) & (D) & $\overline{-}$ & (D) \\
\hline $\begin{array}{l}\text { Other-cooperative, estate or trust, } \\
\text { institutional, etc............. }\end{array}$ & $\begin{array}{r}16 \\
2724\end{array}$ & $\begin{array}{l}11 \\
\text { (D) }\end{array}$ & $1421^{3}$ & (D) & $\begin{array}{r}10 \\
2464\end{array}$ & 1043 & $1421^{3}$ & - \\
\hline
\end{tabular}

See footnotes at end of table. 
Table 48. Summary by Tenure of Operator: 1987-Con.

[For meaning of abbreviations and symbols, see introductory text]

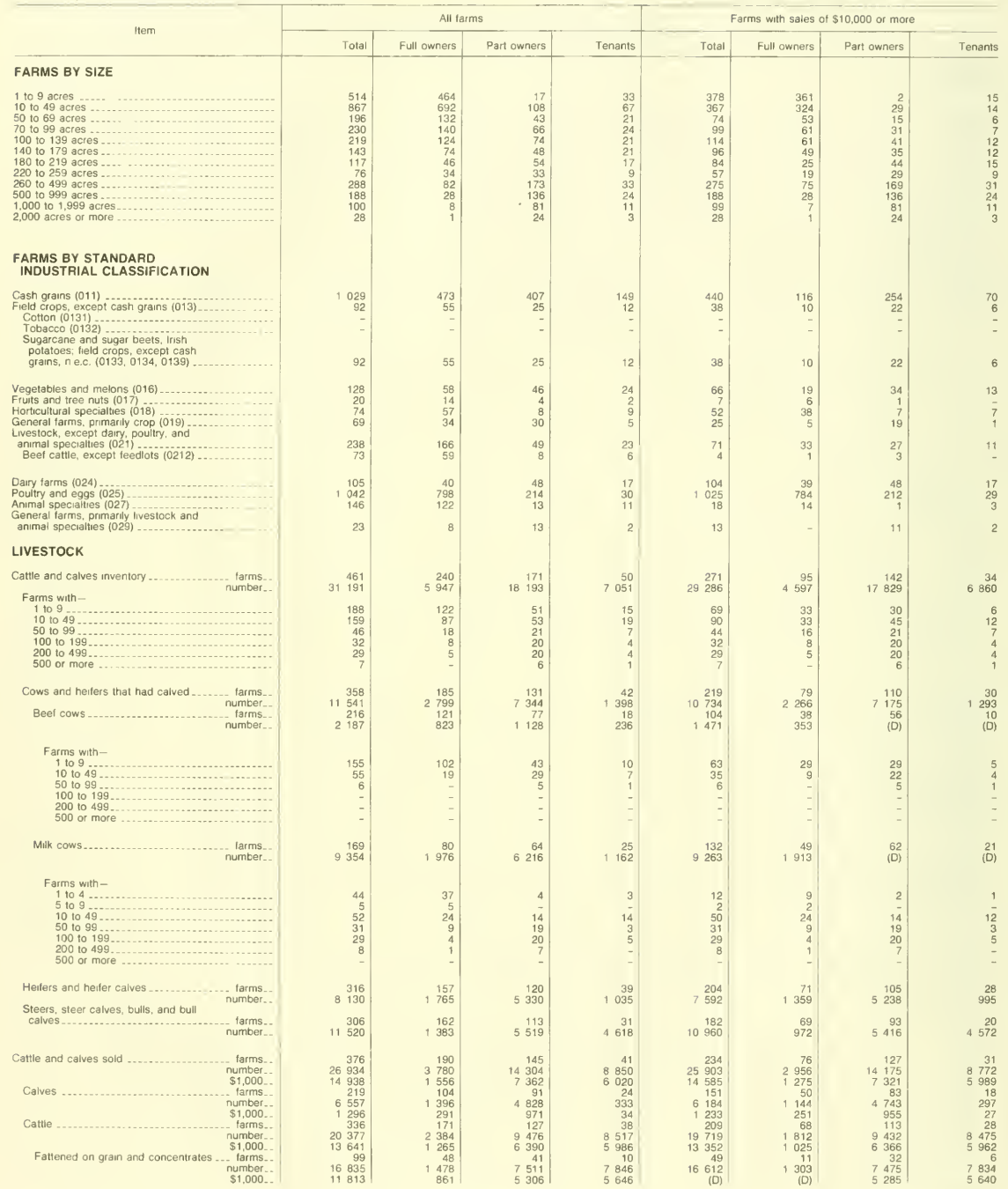

See footnotes at end of table 
Table 48. Summary by Tenure of Operator: 1987-Con.

[For meaning of abbreviations and symbols, see introductory text]

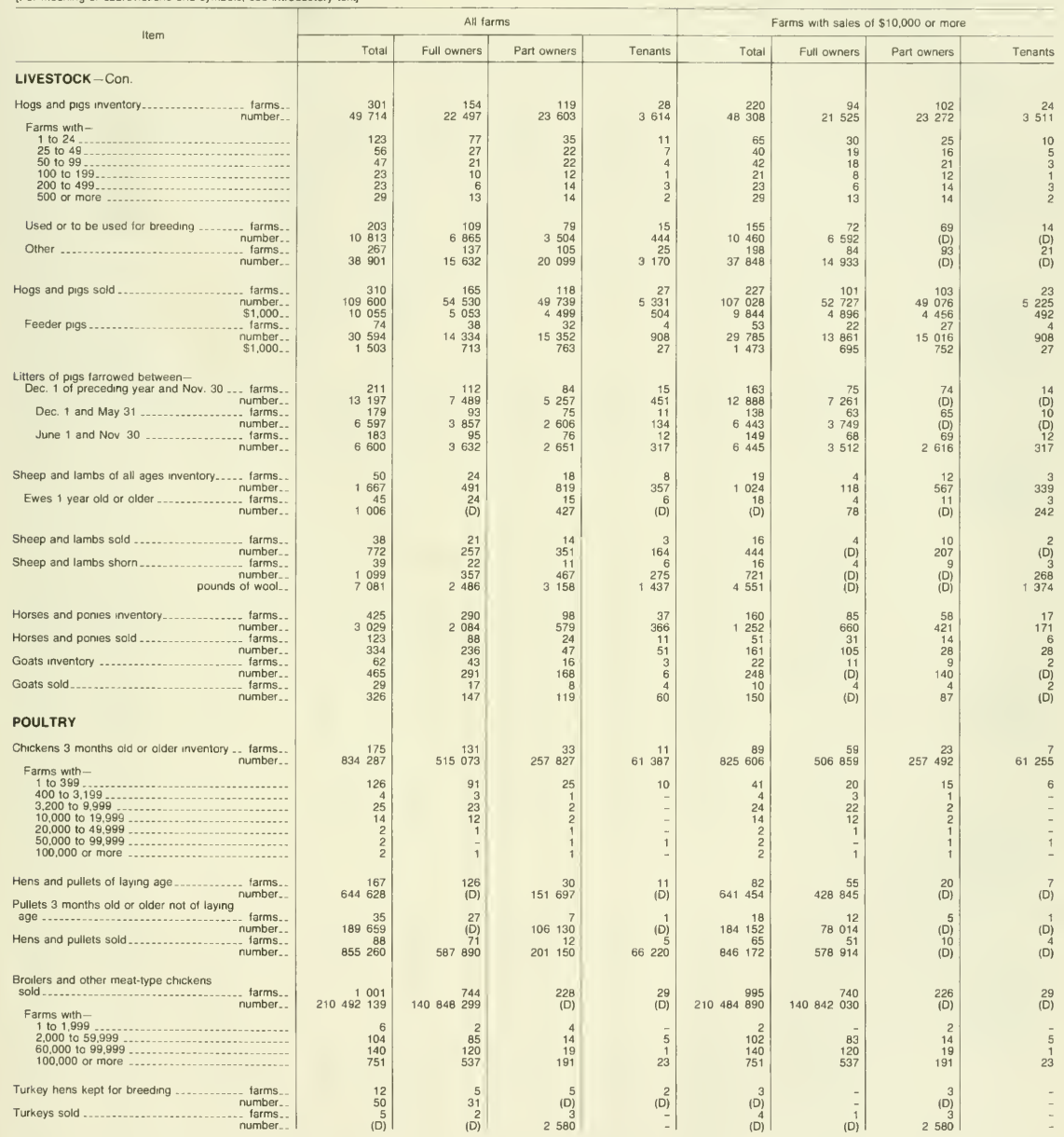


Table 48. Summary by Tenure of Operator: 1987-Con.

[For meaning of abbrevations and symbols, see introductory text]

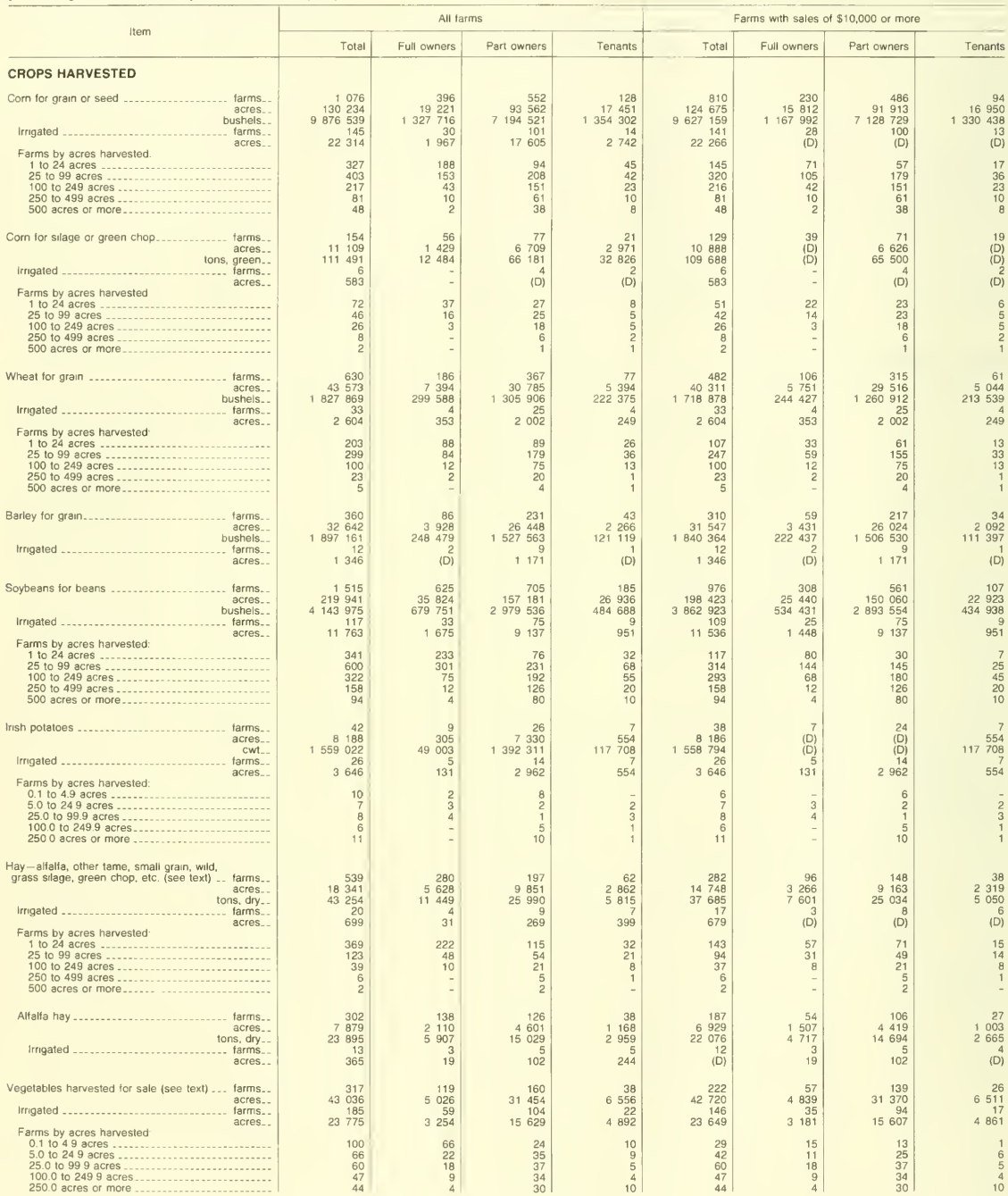


Table 48. Summary by Tenure of Operator: 1987-Con.

[For meaning of abbreviations and symbols, see introductory text]

\begin{tabular}{|c|c|c|c|c|c|c|c|c|}
\hline \multirow{2}{*}{ Item } & \multicolumn{4}{|c|}{ All farms } & \multicolumn{4}{|c|}{ Farms wth sales of $\$ 10,000$ or more } \\
\hline & Total & Full owners & Part owners & Tenants & Total & Full owners & Part owners & Tenants \\
\hline \multicolumn{9}{|l|}{ CROPS HARVESTED-Con. } \\
\hline $\begin{array}{l}\text { Vegetables harvested for sale (see text)- } \\
\text { Con. }\end{array}$ & & & & & & & & \\
\hline 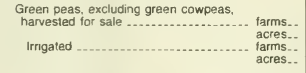 & $\begin{array}{rr}9 & 66 \\
951 \\
5 & 45 \\
5 & 752\end{array}$ & $\begin{array}{r}12 \\
791 \\
7 \\
618\end{array}$ & $\begin{array}{r}75 \\
7 \quad 693 \\
30 \\
4 \quad 089\end{array}$ & $\begin{array}{r}1367 \\
8 \\
1045\end{array}$ & $\begin{array}{r}62 \\
9849 \\
43 \\
\text { (D) }\end{array}$ & $\begin{array}{r}10 \\
(D) \\
7 \\
618\end{array}$ & $\begin{array}{l}43 \\
\text { (D) } \\
28 \\
\text { (D) }\end{array}$ & $\begin{array}{r}9 \\
1367 \\
8 \\
1045\end{array}$ \\
\hline $\begin{array}{r}\text { Sweet corn harvested for sale } \ldots . . . . . . . . \\
\text { farms.. } \\
\text { acres.. } \\
\text { Imigated }\end{array}$ & $\begin{array}{r}109 \\
7643 \\
68 \\
5739\end{array}$ & $\begin{array}{r}36 \\
1537 \\
18 \\
1201\end{array}$ & $\begin{array}{r}62 \\
4788 \\
43 \\
3 \quad 322\end{array}$ & $\begin{array}{rr}1 & 11 \\
317 \\
7 \\
1217\end{array}$ & $\begin{array}{r}81 \\
7535 \\
59 \\
5696\end{array}$ & $\begin{array}{r}17 \\
(D) \\
11 \\
1171\end{array}$ & $\begin{array}{r}55 \\
463 \\
42 \\
\text { (D) }\end{array}$ & $\begin{array}{r}9 \\
\text { (D) } \\
6 \\
\text { (D) }\end{array}$ \\
\hline 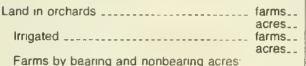 & $\begin{array}{r}36 \\
1378 \\
8 \\
527\end{array}$ & $\begin{array}{r}21 \\
(D) \\
3 \\
43\end{array}$ & $\begin{array}{r}13 \\
1218 \\
5 \\
484\end{array}$ & (D) & $\begin{array}{r}17 \\
1331 \\
8 \\
527\end{array}$ & \begin{tabular}{r|r}
8 \\
119 \\
3 \\
43
\end{tabular} & $\begin{array}{r}129 \\
212 \\
584\end{array}$ & $\overline{-}$ \\
\hline $\begin{array}{l}0.1 \text { to } 49 \text { acres } \\
5.0 \text { to } 249 \text { acres } \\
25.0 \text { to } 99.9 \text { acres } \\
100.0 \text { to } 249.9 \text { acres } \\
250.0 \text { acres or more }\end{array}$ & $\begin{array}{r}21 \\
11 \\
1 \\
-3\end{array}$ & $\begin{array}{r}15 \\
5 \\
1 \\
- \\
-\end{array}$ & $\begin{array}{l}6 \\
4 \\
\overline{-} \\
\overline{3}\end{array}$ & $\begin{array}{l}\dot{2} \\
\dot{-} \\
-\end{array}$ & $\begin{array}{l}6 \\
7 \\
1 \\
\overline{3}\end{array}$ & $\begin{array}{l}4 \\
3 \\
1 \\
- \\
-\end{array}$ & $\begin{array}{l}2 \\
4 \\
- \\
\end{array}$ & $\begin{array}{l}\overline{-} \\
\bar{z}\end{array}$ \\
\hline
\end{tabular}

'Data are based on a sample of farms

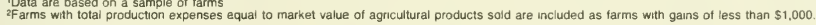


Table 49. Summary by Type of Organization: 1987

[For meaning of abbreviations and symbols, see introductory text]

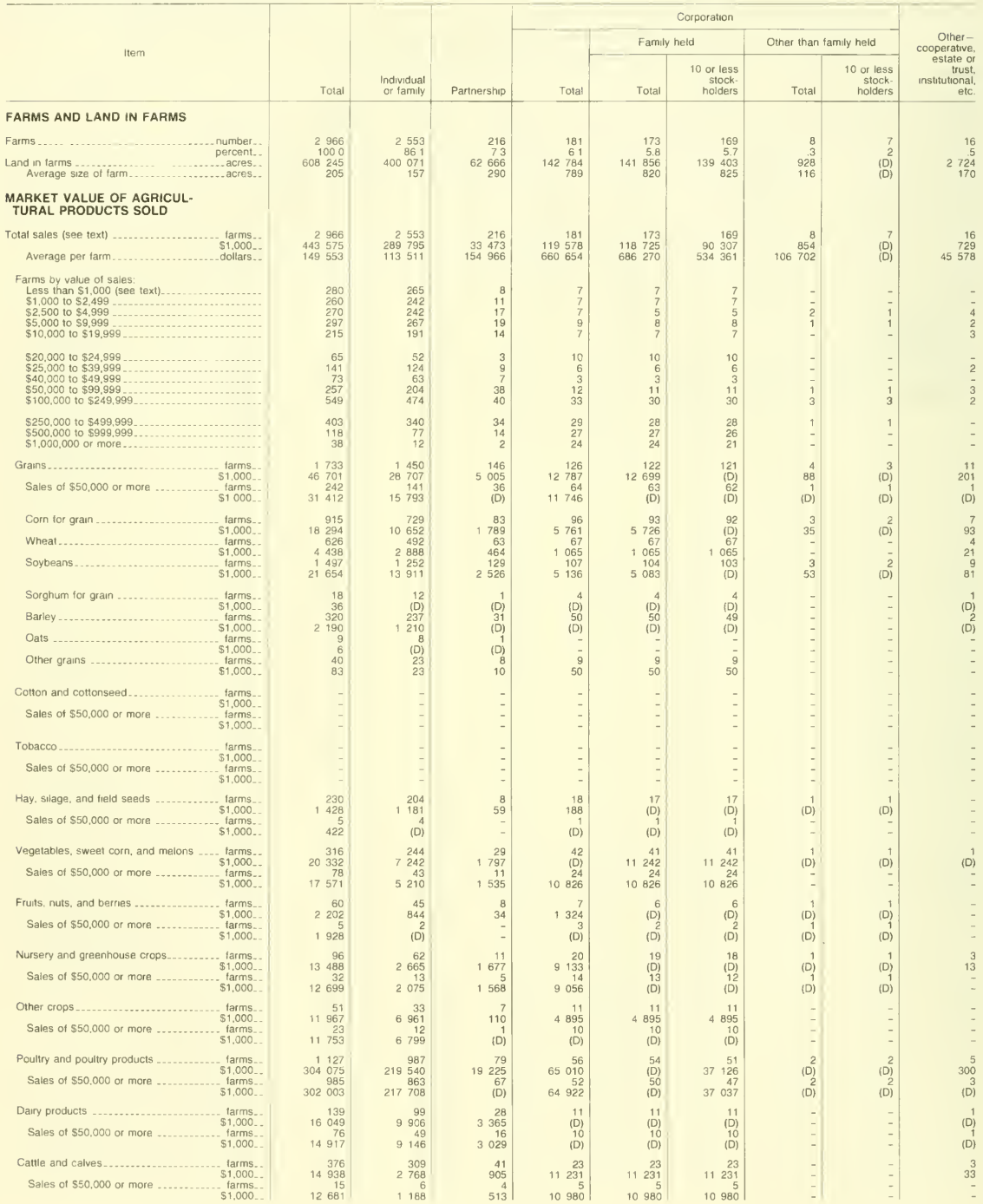


Table 49. Summary by Type of Organization: 1987-Con.

[For meaning of abbreviations and symbols, see introductory text]

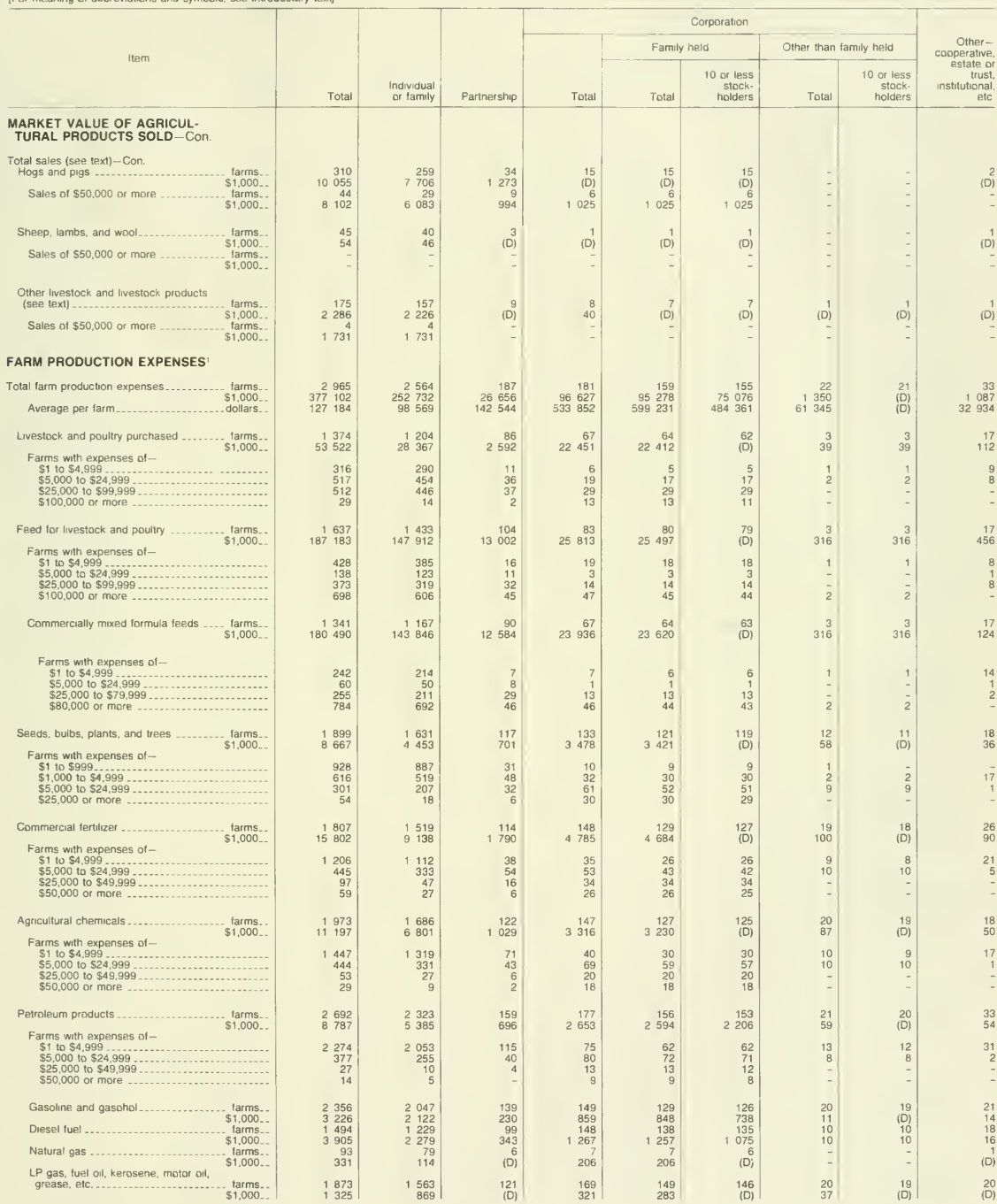


Table 49. Summary by Type of Organization: 1987-Con.

[For meaning of abbreviations and symbols, see introductory text]

\begin{tabular}{|c|c|c|c|c|c|c|c|c|c|}
\hline \multirow{3}{*}{ Item } & \multirow[b]{3}{*}{ Total } & \multirow[b]{3}{*}{$\begin{array}{l}\text { Individual } \\
\text { or family }\end{array}$} & \multirow[b]{3}{*}{ Partnership } & \multicolumn{5}{|c|}{ Corporation } & \multirow{3}{*}{$\begin{array}{l}\text { Other- } \\
\text { cooperative, } \\
\text { estate or } \\
\text { trust. } \\
\text { institutional. } \\
\text { etc }\end{array}$} \\
\hline & & & & & \multicolumn{2}{|c|}{ Family held } & \multicolumn{2}{|c|}{ Other than family held } & \\
\hline & & & & Total & Total & $\begin{array}{r}10 \text { or less } \\
\text { stock- } \\
\text { holders }\end{array}$ & Total & $\begin{array}{c}10 \text { or less } \\
\text { stock- } \\
\text { holders }\end{array}$ & \\
\hline \multicolumn{10}{|l|}{$\begin{array}{l}\text { FARM PRODUCTION EXPENSES } \\
\text { - Con. }\end{array}$} \\
\hline $\begin{array}{l}\text { Total farm production expenses-Con. } \\
\text { Electricity }\end{array}$ & $\begin{array}{l}2195 \\
4862\end{array}$ & $\begin{array}{ll}1 & 882 \\
3 & 118\end{array}$ & $\begin{array}{l}142 \\
419\end{array}$ & $\begin{array}{r}151 \\
1284\end{array}$ & $\begin{array}{r}138 \\
1276\end{array}$ & $\begin{array}{l}135 \\
100\end{array}$ & $\begin{array}{r}13 \\
8\end{array}$ & $\begin{array}{r}12 \\
\text { (D) }\end{array}$ & $\begin{array}{l}20 \\
41\end{array}$ \\
\hline 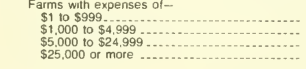 & $\begin{array}{r}1085 \\
942 \\
151 \\
17\end{array}$ & $\begin{array}{r}971 \\
808 \\
101 \\
2\end{array}$ & $\begin{array}{r}53 \\
72 \\
15 \\
2\end{array}$ & $\begin{array}{l}43 \\
61 \\
35 \\
12\end{array}$ & $\begin{array}{l}32 \\
59 \\
35 \\
12\end{array}$ & $\begin{array}{l}32 \\
58 \\
35 \\
10\end{array}$ & $\begin{array}{r}11 \\
2 \\
- \\
-\end{array}$ & $\begin{array}{r}10 \\
2 \\
- \\
-\end{array}$ & $\begin{array}{r}18 \\
1 \\
1\end{array}$ \\
\hline $\begin{array}{l}\text { Hired farm labor } \ldots+\ldots . . .1 \\
\$ \$ 1,000 \ldots\end{array}$ & $\begin{array}{r}1254 \\
22788\end{array}$ & $\begin{array}{l}1006 \\
8768\end{array}$ & $\begin{array}{r}85 \\
1481\end{array}$ & $12 \begin{array}{r}152 \\
458\end{array}$ & $12 \begin{array}{r}141 \\
016\end{array}$ & $11 \begin{array}{r}137 \\
237\end{array}$ & $\begin{array}{r}11 \\
443\end{array}$ & $\begin{array}{r}11 \\
443\end{array}$ & $\begin{array}{l}11 \\
81\end{array}$ \\
\hline $\begin{array}{l}\text { Farms with expenses of - } \\
\$ 1 \text { to } \$ 4,999 \\
\$ 5,000 \text { to } \$ 24,999 \\
\$ 25,000 \text { to } \$ 99,999 \\
\$ 100,000 \text { or more }\end{array}$ & $\begin{array}{r}646 \\
440 \\
133 \\
35\end{array}$ & $\begin{array}{r}579 \\
349 \\
69 \\
9\end{array}$ & \begin{tabular}{r|r}
28 & \\
47 & \\
8 & \\
2 &
\end{tabular} & $\begin{array}{l}31 \\
42 \\
55 \\
24\end{array}$ & $\begin{array}{l}30 \\
41 \\
46 \\
24\end{array}$ & $\begin{array}{l}30 \\
41 \\
45 \\
21\end{array}$ & $\begin{array}{l}1 \\
1 \\
9 \\
-\end{array}$ & $\begin{array}{l}1 \\
1 \\
9 \\
-\end{array}$ & $\begin{array}{l}8 \\
2 \\
1 \\
-\end{array}$ \\
\hline $\begin{array}{l}\text { Contract labor .............................. farms.. } \\
\$ 1,000\end{array}$ & $\begin{array}{r}364 \\
5798\end{array}$ & $\begin{array}{r}289 \\
2236\end{array}$ & $\begin{array}{r}32 \\
\text { (D) }\end{array}$ & $\begin{array}{r}42 \\
3288\end{array}$ & $\begin{array}{l}40 \\
\text { (D) }\end{array}$ & $\begin{array}{l}38 \\
\text { (D) }\end{array}$ & (D) & (D) & (D) \\
\hline $\begin{array}{l}\text { Farms with expenses of- } \\
\$ 1 \text { to } \$ 999 \\
\$ 1,000 \text { to } \$ 4.999 . \\
\$ 5,000 \text { to } \$ 24.999 \\
\$ 25,000 \text { or more }\end{array}$ & $\begin{array}{r}41 \\
161 \\
134 \\
28\end{array}$ & $\begin{array}{r}36 \\
136 \\
102 \\
15\end{array}$ & $\begin{array}{r}2 \\
13 \\
15 \\
2\end{array}$ & $\begin{array}{r}2 \\
12 \\
17 \\
11\end{array}$ & $\begin{array}{r}2 \\
11 \\
16 \\
11\end{array}$ & $\begin{array}{r}2 \\
11 \\
15 \\
10\end{array}$ & $\begin{array}{r}- \\
1 \\
-\end{array}$ & $\begin{array}{r}- \\
1 \\
-\end{array}$ & $\begin{array}{l}1 \\
-\end{array}$ \\
\hline Repar and maintenance $\ldots . . . . . . . .$. farms.. & $\begin{array}{r}2481 \\
11739\end{array}$ & $\begin{array}{ll}2 & 145 \\
7 & 492\end{array}$ & $\begin{array}{r}150 \\
1019\end{array}$ & $\begin{array}{rl}167 \\
3 & 177\end{array}$ & $3 \begin{array}{l}147 \\
142\end{array}$ & $\begin{aligned} 144 \\
3018\end{aligned}$ & $\begin{array}{l}20 \\
36\end{array}$ & $\begin{array}{l}20 \\
36\end{array}$ & $\begin{array}{l}19 \\
51\end{array}$ \\
\hline $\begin{array}{l}\text { Farms with expenses of- } \\
\$ 1 \text { to } \$ 4,999 \\
\$ 5,000 \text { to } \$ 24,999 \\
\$ 25,000 \text { to } \$ 49.999 \\
\$ 50,000 \text { or more }\end{array}$ & $\begin{array}{r}1939 \\
455 \\
67 \\
20\end{array}$ & $\begin{array}{r}1767 \\
340 \\
33 \\
5\end{array}$ & $\begin{array}{r}87 \\
54 \\
8 \\
1\end{array}$ & $\begin{array}{l}69 \\
59 \\
25 \\
14\end{array}$ & $\begin{array}{l}50 \\
58 \\
25 \\
14\end{array}$ & $\begin{array}{l}49 \\
58 \\
24 \\
13\end{array}$ & $\begin{array}{r}19 \\
1 \\
- \\
-\end{array}$ & $\begin{array}{r}19 \\
1 \\
- \\
-\end{array}$ & $\begin{array}{r}16 \\
2 \\
1 \\
-\end{array}$ \\
\hline $\begin{array}{l}\text { Customwork, machine hire, and rental of } \\
\text { machinery and equipment ............. tarms }\end{array}$ & $\begin{array}{ll}1 & 192 \\
2 & 445\end{array}$ & $\begin{array}{l}1058 \\
1677\end{array}$ & $\begin{array}{r}64 \\
169\end{array}$ & $\begin{array}{r}60 \\
590\end{array}$ & $\begin{array}{r}58 \\
\text { (D) }\end{array}$ & $\begin{array}{r}55 \\
508\end{array}$ & (D) & (D) & $\begin{array}{l}10 \\
10\end{array}$ \\
\hline $\begin{array}{l}\text { Farms with expenses of- } \\
\$ 1 \text { to } \$ 999 \\
\$ 1,000 \text { to } \$ 4,999 \\
\$ 5,000 \text { to } \$ 24,999 \\
\$ 25,000 \text { of more }\end{array}$ & $\begin{array}{r}672 \\
406 \\
106 \\
8\end{array}$ & $\begin{array}{r}618 \\
361 \\
77 \\
2\end{array}$ & $\begin{array}{r}36 \\
18 \\
9 \\
1\end{array}$ & $\begin{array}{r}10 \\
25 \\
20 \\
5\end{array}$ & $\begin{array}{r}9 \\
24 \\
20 \\
5\end{array}$ & $\begin{array}{r}8 \\
23 \\
20 \\
4\end{array}$ & $\begin{array}{l}1 \\
1 \\
-\end{array}$ & $\begin{array}{l}1 \\
1 \\
- \\
-\end{array}$ & $\begin{array}{l}8 \\
2 \\
-\end{array}$ \\
\hline 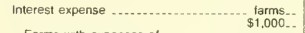 & $\begin{array}{r}1415 \\
14872\end{array}$ & $\begin{array}{ll}1 & 192 \\
9 & 865\end{array}$ & $\begin{array}{l}91 \\
\text { (D) }\end{array}$ & $\begin{array}{r}130 \\
3878\end{array}$ & $\begin{array}{r}127 \\
3862\end{array}$ & $\begin{array}{r}123 \\
3827\end{array}$ & $\begin{array}{r}3 \\
15\end{array}$ & $\begin{array}{r}3 \\
15\end{array}$ & $\left(D^{2}\right)$ \\
\hline $\begin{array}{l}\text { Farms with expenses of- } \\
\$ 1 \text { to } \$ 4,999 \\
\$ 5.000 \text { to } \$ 24,999 \\
\$ 25,000 \text { to } \$ 99,999 \\
\$ 100.000 \text { or more }\end{array}$ & $\begin{array}{r}713 \\
580 \\
110 \\
12\end{array}$ & $\begin{array}{r}638 \\
477 \\
73 \\
4\end{array}$ & $\begin{array}{r}39 \\
42 \\
9 \\
1\end{array}$ & $\begin{array}{r}35 \\
60 \\
28 \\
7\end{array}$ & $\begin{array}{r}34 \\
58 \\
28 \\
7\end{array}$ & $\begin{array}{r}33 \\
55 \\
28 \\
7\end{array}$ & $\begin{array}{l}1 \\
2 \\
- \\
-\end{array}$ & $\begin{array}{l}1 \\
2 \\
-\end{array}$ & $\begin{array}{l}1 \\
1 \\
-\end{array}$ \\
\hline $\begin{array}{c}\text { Secured by real estate ................ farms... } \\
\$ 1,000 . .\end{array}$ & 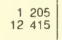 & $\begin{array}{ll}1 & 018 \\
8 & 363\end{array}$ & $\begin{array}{l}81 \\
\text { (D) }\end{array}$ & $\begin{aligned} 105 \\
3074\end{aligned}$ & $\begin{array}{c}103 \\
(D)\end{array}$ & $\begin{array}{l}101 \\
(D)\end{array}$ & (D) & $\left(D^{2}\right.$ & (D) \\
\hline $\begin{array}{l}\text { Farms with expenses of }- \\
\$ 1 \text { to } \$ 999 \\
\$ 1,000 \text { to } \$ 4,999 \\
\$ 5.000 \text { to } \$ 24.999 \\
\$ 25,000 \text { or more }\end{array}$ & $\begin{array}{r}139 \\
463 \\
505 \\
98\end{array}$ & $\begin{array}{r}124 \\
410 \\
423 \\
61\end{array}$ & $\begin{array}{r}8 \\
30 \\
35 \\
8\end{array}$ & $\begin{array}{r}7 \\
22 \\
47 \\
29\end{array}$ & \begin{tabular}{r|r}
6 \\
22 \\
46 \\
29
\end{tabular} & $\begin{array}{r}6 \\
21 \\
45 \\
29\end{array}$ & $\begin{array}{l}1 \\
-1 \\
-\end{array}$ & $\begin{array}{l}1 \\
- \\
- \\
-\end{array}$ & i \\
\hline Not secured by real estate ........... farms.. & $\begin{array}{r}500 \\
2457\end{array}$ & $\begin{array}{r}428 \\
1503\end{array}$ & $\begin{array}{l}24 \\
\text { (D) }\end{array}$ & $\begin{array}{r}46 \\
804\end{array}$ & $\begin{array}{l}44 \\
\text { (D) }\end{array}$ & $\begin{array}{l}42 \\
\text { (D) }\end{array}$ & (D) & (D) & $(\mathrm{D})^{2}$ \\
\hline $\begin{array}{l}\text { Farms with expenses of - } \\
\$ 1 \text { to } \$ 999 \\
\$ 1,000 \text { to } \$ 4,999 \\
\$ 5,000 \text { to } \$ 24,999 \\
\$ 25,000 \text { or more }\end{array}$ & $\begin{array}{r}183 \\
189 \\
113 \\
15\end{array}$ & $\begin{array}{r}165 \\
176 \\
79 \\
8\end{array}$ & $\begin{array}{r}11 \\
3 \\
10\end{array}$ & $\begin{array}{r}7 \\
9 \\
23 \\
7\end{array}$ & \begin{tabular}{r|r}
7 \\
8 \\
22 \\
7
\end{tabular} & $\begin{array}{r}7 \\
8 \\
20 \\
7\end{array}$ & $\begin{array}{l}\overline{1} \\
1 \\
1 \\
-\end{array}$ & $\begin{array}{c}- \\
1 \\
1 \\
-\end{array}$ & $\overline{1}$ \\
\hline $\begin{array}{l}\text { Cash rent___arms.. } \\
\$ \$ 1,000 . .\end{array}$ & 11089 & $\begin{array}{r}803 \\
6611\end{array}$ & $\begin{array}{l}56 \\
\text { (D) }\end{array}$ & $\begin{aligned} 111 \\
3624\end{aligned}$ & 3 $\begin{aligned} 111 \\
624\end{aligned}$ & $\begin{array}{r}110 \\
\text { (D) }\end{array}$ & - & - & (D) \\
\hline $\begin{array}{l}\text { Farms with expenses of- } \\
\qquad 1 \text { to } \$ 4,999 \\
\$ 5,000 \text { to } \$ 9,999 \\
\$ 10,000 \text { to } \$ 24,999 \\
\$ 25,000 \text { or more }\end{array}$ & $\begin{array}{l}582 \\
103 \\
184 \\
103\end{array}$ & $\begin{array}{r}518 \\
80 \\
156 \\
49\end{array}$ & $\begin{array}{r}27 \\
9 \\
11 \\
9\end{array}$ & $\begin{array}{l}35 \\
14 \\
17 \\
45\end{array}$ & $\begin{array}{l}35 \\
14 \\
17 \\
45\end{array}$ & $\begin{array}{l}34 \\
14 \\
17 \\
45\end{array}$ & $\begin{array}{l}- \\
\vdots \\
-\end{array}$ & $\bar{z}$ & $\begin{array}{l}2 \\
\vdots\end{array}$ \\
\hline Property taxes ......... farms... & $\begin{array}{ll}2 & 724 \\
2 & 591\end{array}$ & $\begin{array}{l}2358 \\
1907\end{array}$ & $\begin{array}{l}183 \\
258\end{array}$ & $\begin{array}{l}155 \\
392\end{array}$ & $\begin{array}{r}142 \\
382\end{array}$ & $\begin{array}{l}138 \\
348\end{array}$ & $\begin{array}{l}13 \\
10\end{array}$ & $\begin{array}{l}13 \\
10\end{array}$ & $\begin{array}{l}28 \\
34\end{array}$ \\
\hline $\begin{array}{l}\text { Farms with expenses of - } \\
\$ 1 \text { to } \$ 4,999 \ldots \\
\$ 55,000 \text { to } \$ 9,999 \\
\$ 10,000 \text { to } \$ 24,999 . \ldots\end{array}$ & $\begin{array}{r}2666 \\
42 \\
12 \\
4\end{array}$ & $\begin{array}{r}2325 \\
26 \\
6 \\
1\end{array}$ & $\begin{array}{r}178 \\
4 \\
i\end{array}$ & $\begin{array}{r}136 \\
11 \\
6 \\
2\end{array}$ & $\begin{array}{r}123 \\
11 \\
6 \\
2\end{array}$ & $\begin{array}{r}120 \\
11 \\
6 \\
1\end{array}$ & $\begin{array}{r}13 \\
- \\
-\end{array}$ & $\begin{array}{r}13 \\
- \\
-\end{array}$ & $\begin{array}{r}27 \\
! \\
-\end{array}$ \\
\hline $\begin{array}{c}\text { All other farm production expenses...... farms.. } \\
\text { Farms with expenses of }\end{array}$ & $\begin{array}{r}2813 \\
15758\end{array}$ & $\begin{array}{l}2420 \\
9002\end{array}$ & $\begin{array}{r}184 \\
1259\end{array}$ & $5 \begin{array}{r}181 \\
540\end{array}$ & $\begin{aligned} 159 \\
5271\end{aligned}$ & $4 \begin{array}{r}155 \\
4581\end{array}$ & $\begin{array}{r}22 \\
169\end{array}$ & $\begin{array}{l}21 \\
\text { (D) }\end{array}$ & $\begin{array}{l}28 \\
58\end{array}$ \\
\hline $\begin{array}{l}\$ 1 \text { to } \$ 4,999 \\
\$ 5,000 \text { to } \$ 24,999 \\
\$ 25,000 \text { to } \$ 49,999 \\
\$ 50,000 \text { or more }\end{array}$ & $\begin{array}{r}2271 \\
442 \\
50 \\
50\end{array}$ & $\begin{array}{r}2029 \\
340 \\
33 \\
18\end{array}$ & $\begin{array}{r}129 \\
43 \\
8 \\
4\end{array}$ & $\begin{array}{r}86 \\
58 \\
9 \\
28\end{array}$ & $\begin{array}{r}72 \\
50 \\
9 \\
28\end{array}$ & $\begin{array}{r}72 \\
50 \\
8 \\
25\end{array}$ & $\begin{array}{r}14 \\
8 \\
- \\
-\end{array}$ & $\begin{array}{r}13 \\
8 \\
- \\
-\end{array}$ & $\begin{array}{r}27 \\
! \\
-\end{array}$ \\
\hline
\end{tabular}

See footnotes at end of table 
Table 49. Summary by Type of Organization: 1987-Con.

[For meaning of abbreviatıons and symbols, see introductory text]

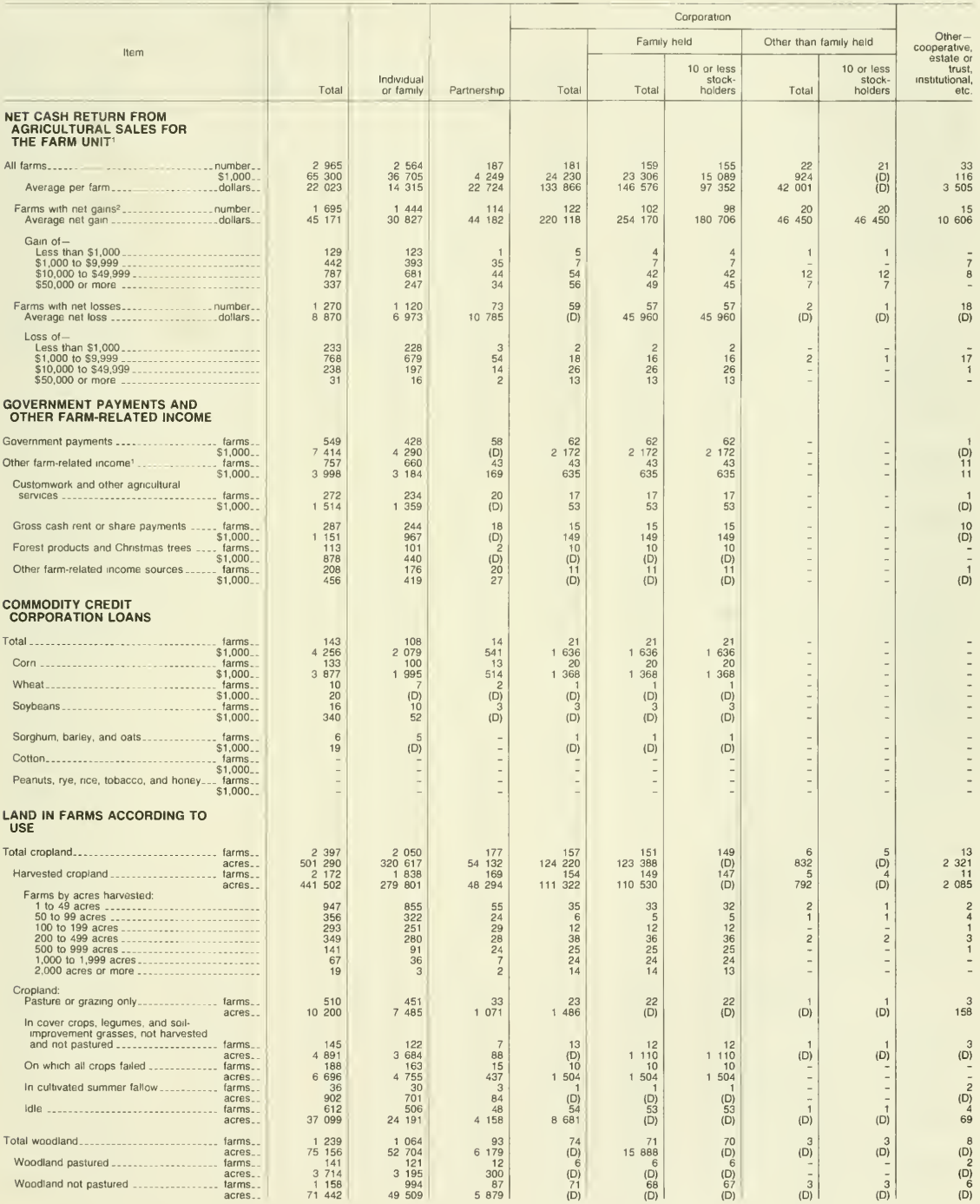


See footnotes at end of table. 
Table 49. Summary by Type of Organization: 1987-Con.

[For meaning of abbreviations and symbals, see intraductory text]

\begin{tabular}{|c|c|c|c|c|c|c|c|c|c|}
\hline \multirow{3}{*}{ Item } & \multirow[b]{3}{*}{ Total } & \multirow[b]{3}{*}{$\begin{array}{l}\text { Individual } \\
\text { or family }\end{array}$} & \multirow[b]{3}{*}{ Parinership } & \multicolumn{5}{|c|}{ Corporation } & \multirow{3}{*}{$\begin{array}{r}\text { Other- } \\
\text { cooperative, } \\
\text { estate or } \\
\text { trust. } \\
\text { institutional, } \\
\text { etc. }\end{array}$} \\
\hline & & & & & \multicolumn{2}{|c|}{ Famuly held } & \multicolumn{2}{|c|}{ Other than family held } & \\
\hline & & & & Total & Total & $\begin{array}{r}10 \text { or less } \\
\text { stock- } \\
\text { holders }\end{array}$ & Total & $\begin{array}{r}10 \text { or less } \\
\text { stock- } \\
\text { holders }\end{array}$ & \\
\hline \multicolumn{10}{|l|}{$\begin{array}{l}\text { TENURE AND RACE OF } \\
\text { OPERATOR }\end{array}$} \\
\hline $\begin{array}{l}\text { All operators } \\
\text { Full owners } \\
\text { Part owners } \\
\text { Tenants }\end{array}$ & $\begin{array}{r}2966 \\
1825 \\
857 \\
284\end{array}$ & $\begin{array}{r}2553 \\
1625 \\
701 \\
227\end{array}$ & $\begin{array}{r}216 \\
119 \\
72 \\
25\end{array}$ & $\begin{array}{r}181 \\
70 \\
81 \\
30\end{array}$ & $\begin{array}{r}173 \\
65 \\
81 \\
27\end{array}$ & \begin{tabular}{r|}
169 \\
62 \\
80 \\
27
\end{tabular} & $\begin{array}{l}8 \\
5 \\
\\
3\end{array}$ & \begin{tabular}{l|l}
7 & \\
5 & - \\
2 &
\end{tabular} & $\begin{array}{r}16 \\
11 \\
3 \\
2\end{array}$ \\
\hline $\begin{array}{l}\text { White } \\
\text { Full owners } \\
\text { Part awners } \\
\text { Tenants }\end{array}$ & $\begin{array}{l}2914 \\
1791 \\
845 \\
278\end{array}$ & $\begin{array}{r}2502 \\
1592 \\
689 \\
221\end{array}$ & $\begin{array}{r}216 \\
119 \\
72 \\
25\end{array}$ & $\begin{array}{r}180 \\
69 \\
81 \\
30\end{array}$ & $\begin{array}{r}172 \\
64 \\
81 \\
27\end{array}$ & \begin{tabular}{r|}
169 \\
62 \\
80 \\
27
\end{tabular} & $\begin{array}{l}8 \\
5 \\
-3\end{array}$ & $\begin{array}{l}7 \\
5 \\
\\
2\end{array}$ & $\begin{array}{r}16 \\
11 \\
3 \\
2\end{array}$ \\
\hline $\begin{array}{l}\text { Black and other races } \\
\text { Full owners } \\
\text { Part awners } \ldots \ldots \\
\text { Tenants } \ldots \ldots\end{array}$ & $\begin{array}{r}52 \\
34 \\
12 \\
6\end{array}$ & $\begin{array}{r}51 \\
33 \\
12 \\
6\end{array}$ & $=$ & $\begin{array}{l}1 \\
1 \\
- \\
-\end{array}$ & $\begin{array}{l}1 \\
1 \\
-\end{array}$ & $\begin{array}{l}- \\
\overline{-} \\
-\end{array}$ & $\begin{array}{l}\vdots \\
\vdots\end{array}$ & 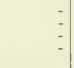 & $\overline{-}$ \\
\hline \multicolumn{10}{|l|}{ OWNED AND RENTED LAND } \\
\hline $\begin{array}{c}\text { Land owned ..................... } \\
\text { Owned land in farms } \\
\begin{array}{l}\text { acres. } \\
\text { farms.- } \\
\text { acres. }\end{array}\end{array}$ & $\begin{array}{rr}2 & 691 \\
360 & 152 \\
2 & 682 \\
325 & 313\end{array}$ & \begin{tabular}{rr|}
2 & 333 \\
251 & 934 \\
2 & 326 \\
222 & 402
\end{tabular} & $\begin{array}{rr}192 \\
31 & 235 \\
191 \\
29 & 117\end{array}$ & $\begin{array}{r}152 \\
74592 \\
7151 \\
71\end{array}$ & $\begin{array}{r}147 \\
73665 \\
146 \\
70828\end{array}$ & $\begin{array}{r}143 \\
71570 \\
142 \\
\text { (D) }\end{array}$ & $\begin{array}{r}5 \\
927 \\
5 \\
882\end{array}$ & $\begin{array}{r}5 \\
927 \\
5 \\
882\end{array}$ & $\begin{array}{rr}2 & 14 \\
2 & 14 \\
2 & 14 \\
2 & 084\end{array}$ \\
\hline $\begin{array}{r}\text { Land rented or leased from others ........ } \begin{array}{l}\text { farms.. } \\
\text { acres. }\end{array} \\
\text { Rented or leased land in farms ......... } \\
\text { tarms.. } \\
\text { acres.. }\end{array}$ & $\begin{array}{rr}1 & 153 \\
285 & 163 \\
1 & 141 \\
282 & 932\end{array}$ & 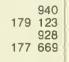 & $\begin{array}{r}97 \\
33 \quad 634 \\
97 \\
33 \quad 549\end{array}$ & $\begin{array}{rr}71 & 111 \\
7161 \\
111 \\
71 & 074\end{array}$ & $\begin{array}{r}108 \\
71715 \\
108 \\
71028\end{array}$ & $\begin{array}{r}107 \\
(D) \\
107 \\
\text { (D) }\end{array}$ & \begin{tabular}{r|}
3 \\
46 \\
3 \\
46
\end{tabular} & $\begin{array}{r}2 \\
\text { (D) } \\
2 \\
(D)\end{array}$ & $\begin{array}{r}5 \\
645 \\
5 \\
640\end{array}$ \\
\hline $\begin{array}{l}\text { Land rented or leased to others............ farms.. } \\
\text { acres.. }\end{array}$ & $37 \begin{array}{r}463 \\
070\end{array}$ & $\begin{array}{r}410 \\
30986\end{array}$ & 2203 & $\begin{array}{r}18 \\
3569\end{array}$ & $\begin{array}{r}17 \\
\text { (D) }\end{array}$ & $\begin{array}{l}17 \\
\text { (D) }\end{array}$ & (D) & (D) & $\begin{array}{r}6 \\
312\end{array}$ \\
\hline \multicolumn{10}{|l|}{ OPERATOR CHARACTERISTICS } \\
\hline $\begin{array}{l}\text { Operators by place of residence } \\
\text { On farm operated } \\
\text { Not an farm aperated } \\
\text { Not reported }\end{array}$ & $\begin{array}{r}2175 \\
481 \\
310\end{array}$ & $\begin{array}{r}1893 \\
391 \\
269\end{array}$ & $\begin{array}{r}154 \\
38 \\
24\end{array}$ & $\begin{array}{r}120 \\
48 \\
13\end{array}$ & $\begin{array}{r}118 \\
43 \\
12\end{array}$ & $\begin{array}{r}117 \\
40 \\
12\end{array}$ & $\begin{array}{l}2 \\
5 \\
1\end{array}$ & $\begin{array}{l}2 \\
5 \\
-\end{array}$ & $\begin{array}{l}8 \\
4 \\
4\end{array}$ \\
\hline $\begin{array}{l}\text { Operators by principal occupation } \\
\text { Farming } \\
\text { Other }\end{array}$ & $\begin{array}{ll}1 & 774 \\
1 & 192\end{array}$ & $\begin{array}{ll}1 & 478 \\
1 & 075\end{array}$ & $\begin{array}{r}158 \\
58\end{array}$ & $\begin{array}{r}128 \\
53\end{array}$ & $\begin{array}{r}125 \\
48\end{array}$ & $\begin{array}{r}122 \\
47\end{array}$ & $\begin{array}{l}3 \\
5\end{array}$ & $\begin{array}{l}3 \\
4\end{array}$ & $\begin{array}{r}10 \\
6\end{array}$ \\
\hline $\begin{array}{l}\text { Operators by days of work oft farm: } \\
\text { Nane. } \\
\text { Any } 10.99 \text { days } \\
100 \text { to t99 days } \\
200 \text { days or more }\end{array}$ & $\begin{array}{rr}1 & 263 \\
1 & 426 \\
254 \\
217 \\
955\end{array}$ & $\begin{array}{r}1054 \\
1270 \\
216 \\
201 \\
853\end{array}$ & $\begin{array}{l}96 \\
95 \\
26 \\
12 \\
57\end{array}$ & $\begin{array}{r}106 \\
58 \\
12 \\
4 \\
42\end{array}$ & $\begin{array}{r}102 \\
54 \\
11 \\
3 \\
40\end{array}$ & $\begin{array}{r}99 \\
53 \\
11 \\
3 \\
39\end{array}$ & $\begin{array}{l}4 \\
4 \\
1 \\
1 \\
2\end{array}$ & $\begin{array}{l}4 \\
3 \\
1 \\
2\end{array}$ & $\begin{array}{l}7 \\
3 \\
\\
\end{array}$ \\
\hline 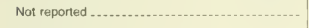 & 277 & 229 & 25 & 17 & 17 & 17 & - & - & 6 \\
\hline $\begin{array}{l}\text { Operators by years on present tarm } \\
2 \text { years or less } \\
3 \text { or } 4 \text { years } \\
5 \text { to } 9 \text { years } \\
10 \text { years or more } \\
\text { Average years on present farm }\end{array}$ & $\begin{array}{r}176 \\
189 \\
398 \\
1588 \\
19.3\end{array}$ & $\begin{array}{r}144 \\
166 \\
336 \\
1371 \\
19.2\end{array}$ & \begin{tabular}{r|}
18 \\
11 \\
33 \\
114 \\
20.0
\end{tabular} & $\begin{array}{r}11 \\
10 \\
26 \\
99 \\
20.5\end{array}$ & $\begin{array}{r}10 \\
8 \\
25 \\
97 \\
20.9\end{array}$ & $\begin{array}{r}10 \\
8 \\
25 \\
93 \\
20.5\end{array}$ & $\begin{array}{r}1 \\
2 \\
1 \\
2 \\
12.3\end{array}$ & $\begin{array}{r}1 \\
2 \\
1 \\
2 \\
12.3\end{array}$ & $\begin{array}{r}3 \\
2 \\
3 \\
4 \\
9.1\end{array}$ \\
\hline Not reported ................................... & 615 & 536 & 40 & 35 & 33 & 33 & 2 & 1 & 4 \\
\hline $\begin{array}{l}\text { Operators by age group } \\
\text { Under } 25 \text { years. } \\
25 \text { to } 34 \text { years } \\
35 \text { to } 44 \text { years } \\
45 \text { to } 49 \text { years } \\
50 \text { to } 54 \text { years } \ldots \ldots\end{array}$ & $\begin{array}{r}40 \\
374 \\
620 \\
328 \\
321\end{array}$ & $\begin{array}{r}38 \\
327 \\
536 \\
279 \\
285\end{array}$ & $\begin{array}{l}1 \\
24 \\
41 \\
20 \\
21\end{array}$ & $\begin{array}{r}1 \\
23 \\
37 \\
28 \\
14\end{array}$ & $\begin{array}{l}1 \\
21 \\
37 \\
26 \\
14\end{array}$ & $\begin{array}{l}1 \\
21 \\
37 \\
26 \\
14\end{array}$ & $\begin{array}{l}\overline{2} \\
\overline{2} \\
\overline{-}\end{array}$ & $\begin{array}{l}\overline{2} \\
\overline{2} \\
-\end{array}$ & $\begin{array}{l}\overline{-} \\
\overline{6} \\
1 \\
1\end{array}$ \\
\hline $\begin{array}{l}55 \text { to } 59 \text { years } \\
60 \text { to } 64 \text { years } \\
65 \text { to } 69 \text { years } \\
70 \text { years and over } \\
\text { Average age }\end{array}$ & $\begin{array}{r}326 \\
348 \\
269 \\
340 \\
51.5\end{array}$ & $\begin{array}{l}274 \\
290 \\
218 \\
306 \\
514\end{array}$ & $\begin{array}{r}31 \\
26 \\
34 \\
18 \\
52.8\end{array}$ & $\begin{array}{r}20 \\
31 \\
13 \\
14 \\
50.8\end{array}$ & \begin{tabular}{r|r}
17 \\
31 \\
13 \\
13 \\
50.9
\end{tabular} & $\begin{array}{r}16 \\
29 \\
12 \\
13 \\
50.7\end{array}$ & $\begin{array}{r}3 \\
\\
1 \\
48.6\end{array}$ & $\begin{array}{r}2 \\
\overline{1} \\
1 \\
47.4\end{array}$ & $\begin{array}{r}1 \\
1 \\
4 \\
2 \\
53.8\end{array}$ \\
\hline $\begin{array}{l}\text { Operators by sex: } \\
\text { Male } \\
\text { Fernale }\end{array}$ & $\begin{array}{r}2676 \\
290\end{array}$ & $2 \underset{258}{295}$ & $\begin{array}{r}198 \\
18\end{array}$ & $\begin{array}{r}170 \\
11\end{array}$ & $\begin{array}{r}164 \\
9\end{array}$ & $\begin{array}{r}160 \\
9\end{array}$ & $\begin{array}{l}6 \\
2\end{array}$ & $\begin{array}{l}5 \\
2\end{array}$ & $\begin{array}{r}13 \\
3\end{array}$ \\
\hline $\begin{array}{l}\text { Operators of Spanish arigin (see text) } \\
\text { FARMS BY TYPE OF } \\
\text { ORGANIZATION }\end{array}$ & 9 & 7 & 1 & 1 & 1 & 1 & - & - & - \\
\hline Individual or family (sole propnetorship) ..... farms.. & $\begin{array}{rl}2 & 553 \\
400 & 071 \\
& 216\end{array}$ & $\begin{array}{rl}2 & 553 \\
400 & 071\end{array}$ & - & - & $=$ & $=$ & - & 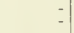 & - \\
\hline $\begin{array}{l}\text { Partnership } \ldots \ldots \ldots \\
\text { acres.. }\end{array}$ & 62666 & & 62666 & $=$ & $\begin{array}{l}- \\
-\end{array}$ & $=$ & $\overline{-}$ & $=$ & $\overline{-}$ \\
\hline $\begin{array}{l}\text { Corporation. } \\
\text { Famity held ..................... farms_- } \\
\text { acres.. }\end{array}$ & $141 \begin{array}{r}173 \\
856\end{array}$ & $\overline{-}$ & $=$ & $\begin{array}{r}173 \\
141856\end{array}$ & $\begin{array}{r}173 \\
141856\end{array}$ & $\begin{array}{r}169 \\
139403\end{array}$ & $=$ & $\overline{-}$ & $=$ \\
\hline $\begin{array}{l}\text { More than } 10 \text { stockhaiders } \ldots \ldots \ldots \ldots \ldots \text { farms } \\
10 \text { or less stockholders } \ldots\end{array}$ & $\begin{array}{r}4 \\
169\end{array}$ & $\overline{-}$ & $=$ & $\begin{array}{r}4 \\
169\end{array}$ & $\begin{array}{r}4 \\
169\end{array}$ & $16 \overline{9}$ & - & - & 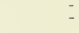 \\
\hline 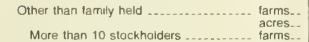 & $\begin{array}{r}8 \\
928 \\
1\end{array}$ & $=$ & 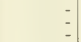 & $\begin{array}{r}8 \\
928 \\
1\end{array}$ & $\begin{array}{l}- \\
-\end{array}$ & $=$ & \begin{tabular}{r|r}
8 \\
928 \\
1
\end{tabular} & (D) & $\overline{-}$ \\
\hline 10 or less stackhalders ................ farms.. & 7 & - & - & 7 & - & - & 7 & 7 & \\
\hline $\begin{array}{l}\text { Other-caaperative, estate ar trust. } \\
\text { institutional, etc .......... tarms.. }\end{array}$ & 16 & - & - & - & - & - & - & - & 16 \\
\hline
\end{tabular}


Table 49. Summary by Type of Organization: 1987-Con.

[For meaning of abbreviations and symbals, see introductory text]

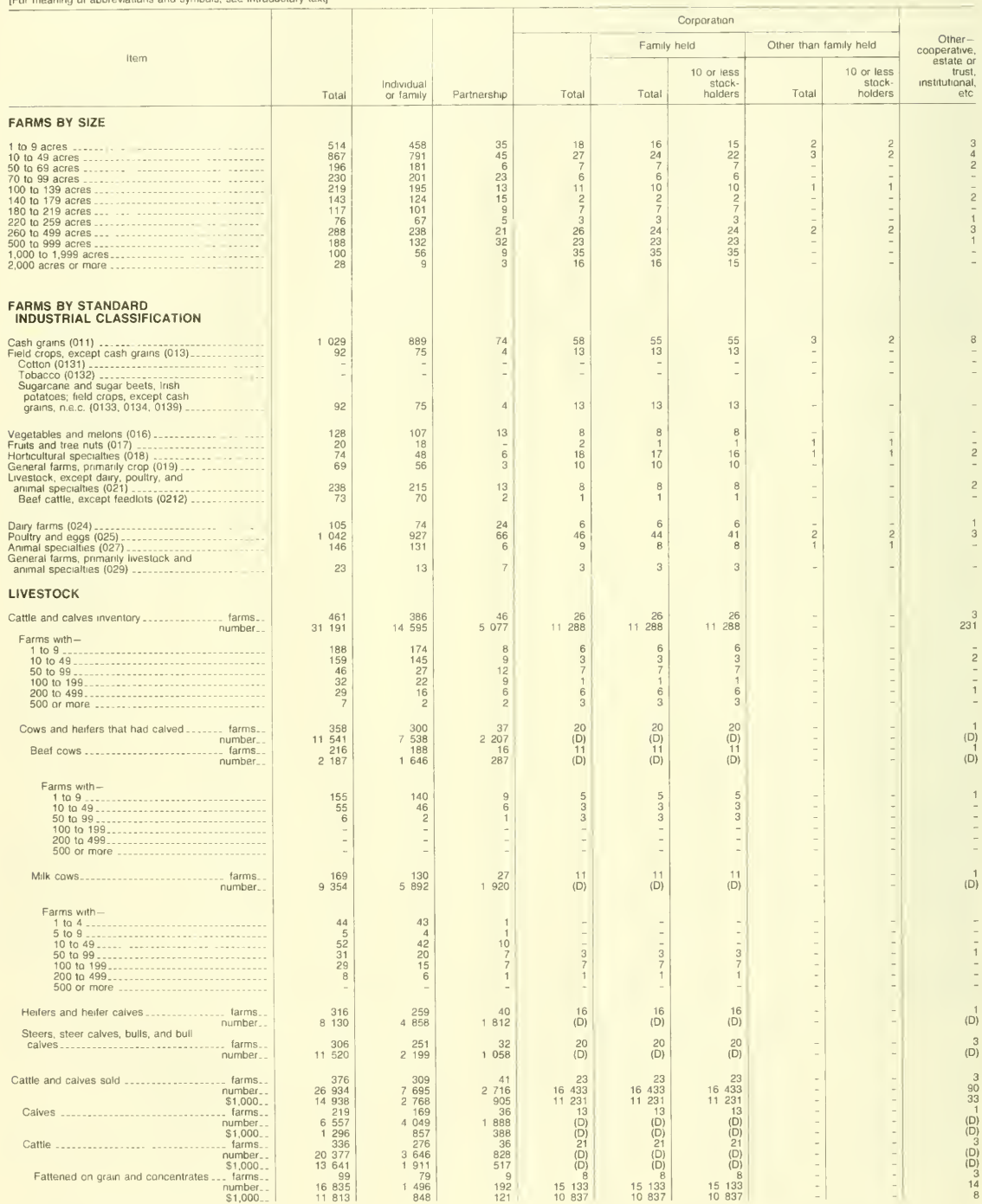


Table 49. Summary by Type of Organization: 1987-Con.

[For meaning of abbreviations and symbols, see introductory text]

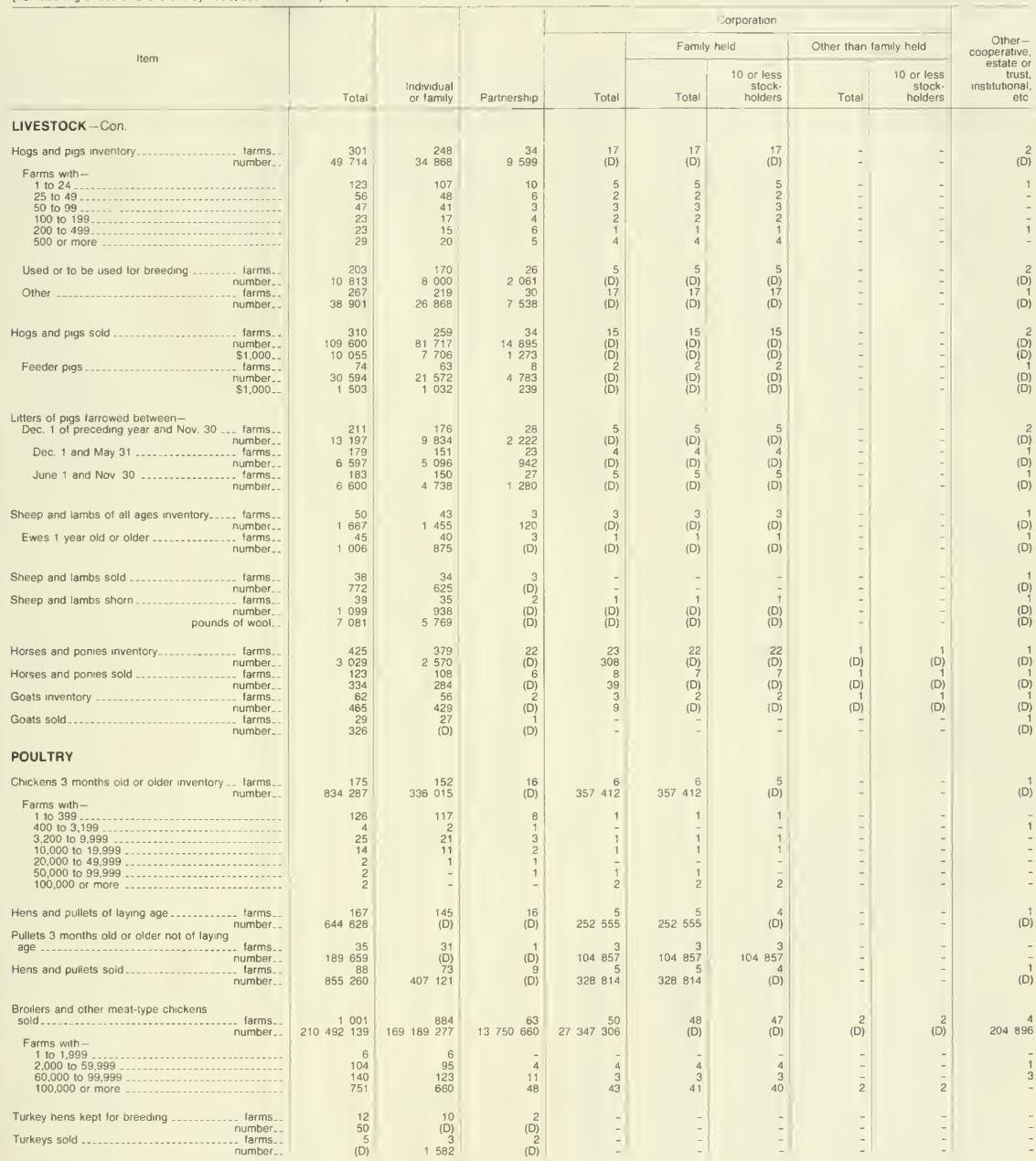

See footnotes at end of table. 
Table 49. Summary by Type of Organization: 1987-Con.

[For meaning of abbreviations and symbols, see introductory text]

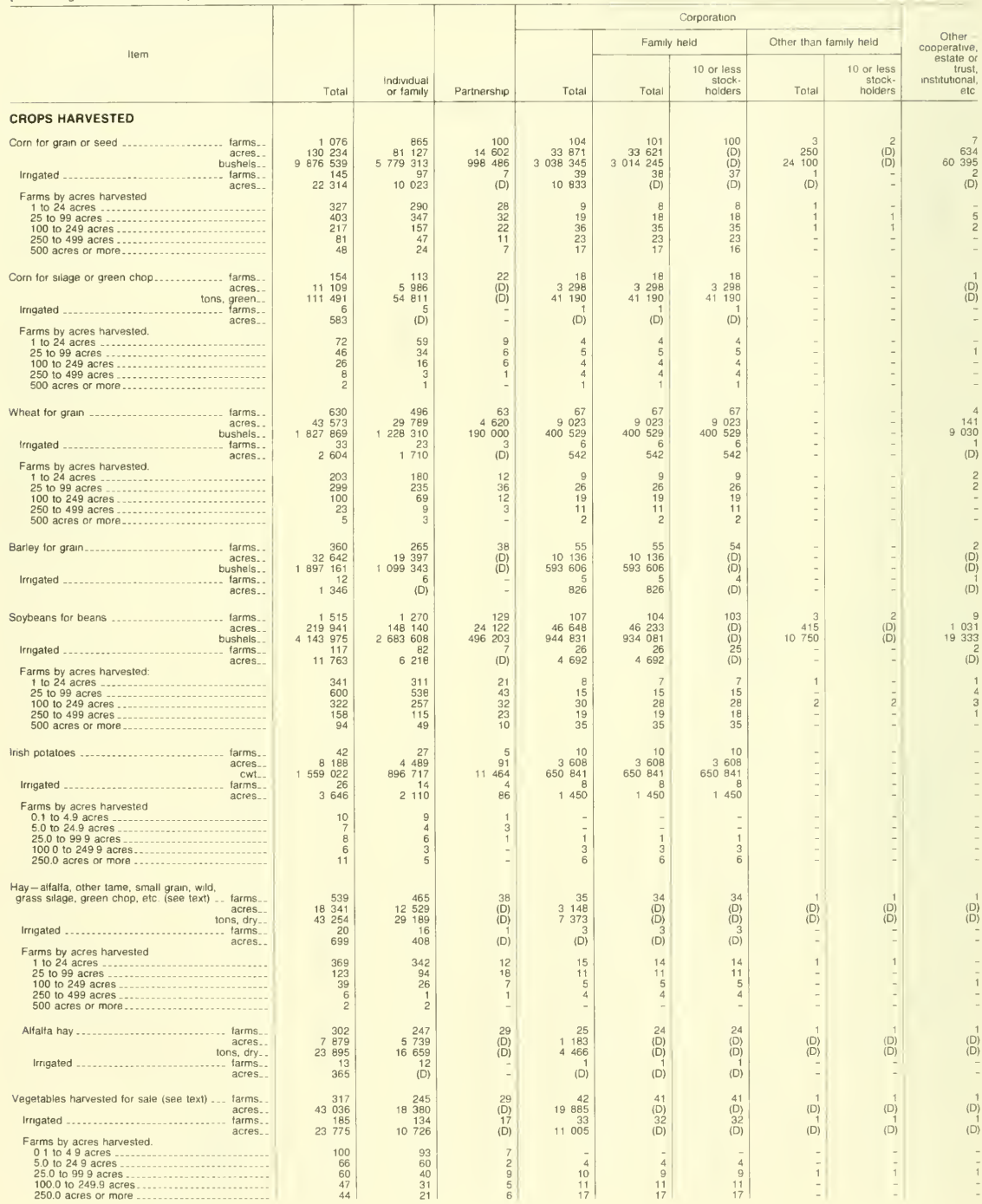

See footnotes at end of table 


\section{Table 49. Summary by Type of Organization: 1987-Con.}

[For meaning of abbreviations and symbols, see introductory text]

\begin{tabular}{|c|c|c|c|c|c|c|c|c|c|}
\hline \multirow{3}{*}{ Item } & \multirow[b]{3}{*}{ Total } & \multirow[b]{3}{*}{$\begin{array}{l}\text { Individual } \\
\text { or family }\end{array}$} & \multirow[b]{3}{*}{ Partnership } & \multicolumn{5}{|c|}{ Corporation } & \multirow{3}{*}{$\begin{array}{l}\text { Other- } \\
\text { cooperative. } \\
\text { estate or } \\
\text { trust. } \\
\text { institutional. } \\
\text { etc. }\end{array}$} \\
\hline & & & & & \multicolumn{2}{|c|}{ Family held } & \multicolumn{2}{|c|}{ Other than family heid } & \\
\hline & & & & Total & Total & $\begin{array}{r}10 \text { or less } \\
\text { stock. } \\
\text { holders }\end{array}$ & Totat & $\begin{array}{l}10 \text { or less } \\
\text { stock. } \\
\text { holders }\end{array}$ & \\
\hline \multicolumn{10}{|l|}{$\begin{array}{l}\text { CROPS HARVESTED - Con. } \\
\text { Vegetables harvested for sale (see lext)- } \\
\text { Con. }\end{array}$} \\
\hline $\begin{array}{c}\begin{array}{c}\text { Green peas, excluding green cowpeas. } \\
\text { harvested for sale }\end{array} \\
\text { trngated }\end{array}$ & $\begin{array}{r}66 \\
9851 \\
45 \\
5752\end{array}$ & $\begin{array}{r}40 \\
3935 \\
25 \\
2353\end{array}$ & $\begin{array}{r}7 \\
1320 \\
5 \\
565\end{array}$ & $\begin{array}{r}19 \\
4996 \\
15 \\
2834\end{array}$ & $\begin{array}{r}19 \\
4596 \\
15 \\
2834\end{array}$ & $\begin{array}{r}19 \\
4596 \\
15 \\
2834\end{array}$ & $\overline{-}$ & $\overline{-}$ & $\begin{array}{l}- \\
-\end{array}$ \\
\hline $\begin{array}{c}\begin{array}{c}\text { Sweet corn harvested tor sale .......... } \\
\text { Imirms... } \\
\text { acres.. } \\
\text { farms.. } \\
\text { acres.. }\end{array}\end{array}$ & $\begin{array}{r}109 \\
7643 \\
68 \\
5 \quad 739\end{array}$ & $\begin{array}{r}80 \\
3752 \\
46 \\
2771\end{array}$ & $\begin{array}{r}11 \\
\text { (D) } \\
5 \\
\text { (D) }\end{array}$ & $\begin{array}{r}17 \\
3514 \\
16 \\
2749\end{array}$ & $\begin{array}{r}16 \\
\text { (D) } \\
15 \\
\text { (D) }\end{array}$ & $\begin{array}{r}16 \\
\text { (D) } \\
15 \\
\text { (D) }\end{array}$ & $\begin{array}{r}1 \\
\text { (D) } \\
1 \\
\text { (D) }\end{array}$ & $\begin{array}{r}1 \\
\text { (D) } \\
1 \\
\text { (D) }\end{array}$ & $\begin{array}{r}1 \\
\text { (D) } \\
1 \\
\text { (D) }\end{array}$ \\
\hline $\begin{array}{c}\text { Land in orchards } \\
\text { urrigated }\end{array}$ & $\begin{array}{r}36 \\
1378 \\
8 \\
527\end{array}$ & $\begin{array}{r}31 \\
\text { (D) } \\
5 \\
64\end{array}$ & $\begin{array}{r}2 \\
\text { (D) } \\
-\end{array}$ & $\begin{array}{r}3 \\
823 \\
3 \\
463\end{array}$ & $\begin{array}{r}2 \\
\text { (D) } \\
2 \\
\text { (D) }\end{array}$ & $\begin{array}{r}{ }^{2} \\
\text { (D) } \\
\text { (D) }\end{array}$ & $\begin{array}{r}1 \\
\text { (D) } \\
1 \\
\text { (D) }\end{array}$ & $\begin{array}{r}1 \\
\text { (D) } \\
1 \\
\text { (D) }\end{array}$ & - \\
\hline $\begin{array}{l}\text { Farms by bearing and nonbeanng acres } \\
0.1 \text { to } 4.9 \text { acres } \\
5.0 \text { to } 249 \text { acres } \\
25.0 \text { to } 99.9 \text { acres } \\
100.0 \text { to } 249.9 \text { acres } \\
250.0 \text { acres or more }\end{array}$ & $\begin{array}{r}21 \\
11 \\
1 \\
5 \\
3\end{array}$ & $\begin{array}{r}21 \\
9 \\
- \\
- \\
1\end{array}$ & $\begin{array}{l}\overrightarrow{2} \\
\overrightarrow{-} \\
-\end{array}$ & 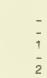 & $\begin{array}{l}- \\
\bar{z} \\
\bar{z}\end{array}$ & $\begin{array}{l}\bar{z} \\
\overline{2}\end{array}$ & $\begin{array}{l}- \\
\overline{1} \\
- \\
-\end{array}$ & $\overline{-}$ & $\begin{array}{l}- \\
-\end{array}$ \\
\hline
\end{tabular}

'Data are based on a sample of farms.

2 Farms with total production expenses equal to market value of agricultural products soid are included as farms with gans of less than $\$ 1,000$. 
Table 50. Summary by Age and Principal Occupation of Operator: 1987 
Table 50. Summary by Age and Principal Occupation of Operator: 1987-Con.

[For meaning of abbreviations and symbols, see introductory text]

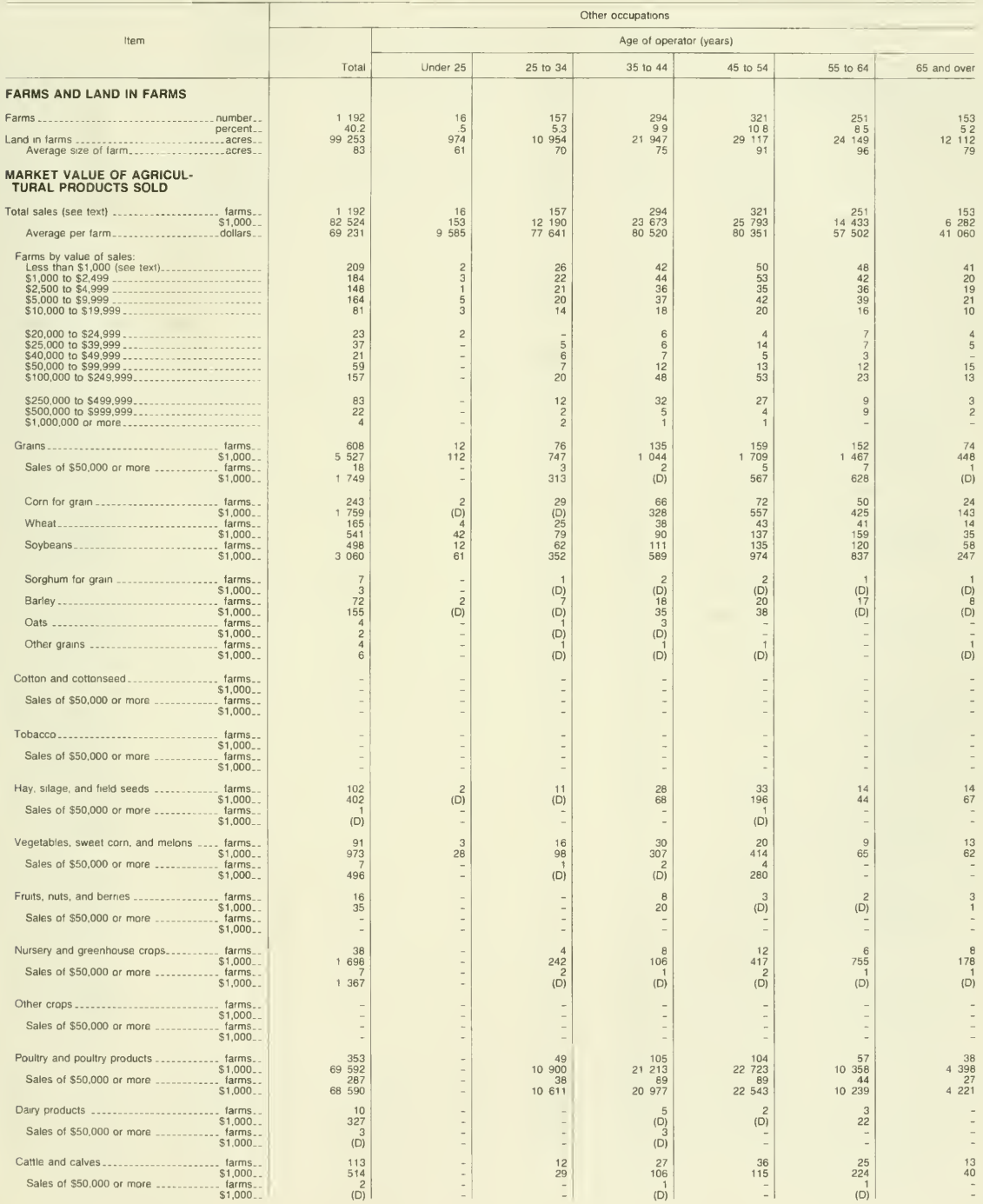


Table 50. Summary by Age and Principal Occupation of Operator: 1987-Con.

[For meaning of abbreviations and symbols, see introductory text]

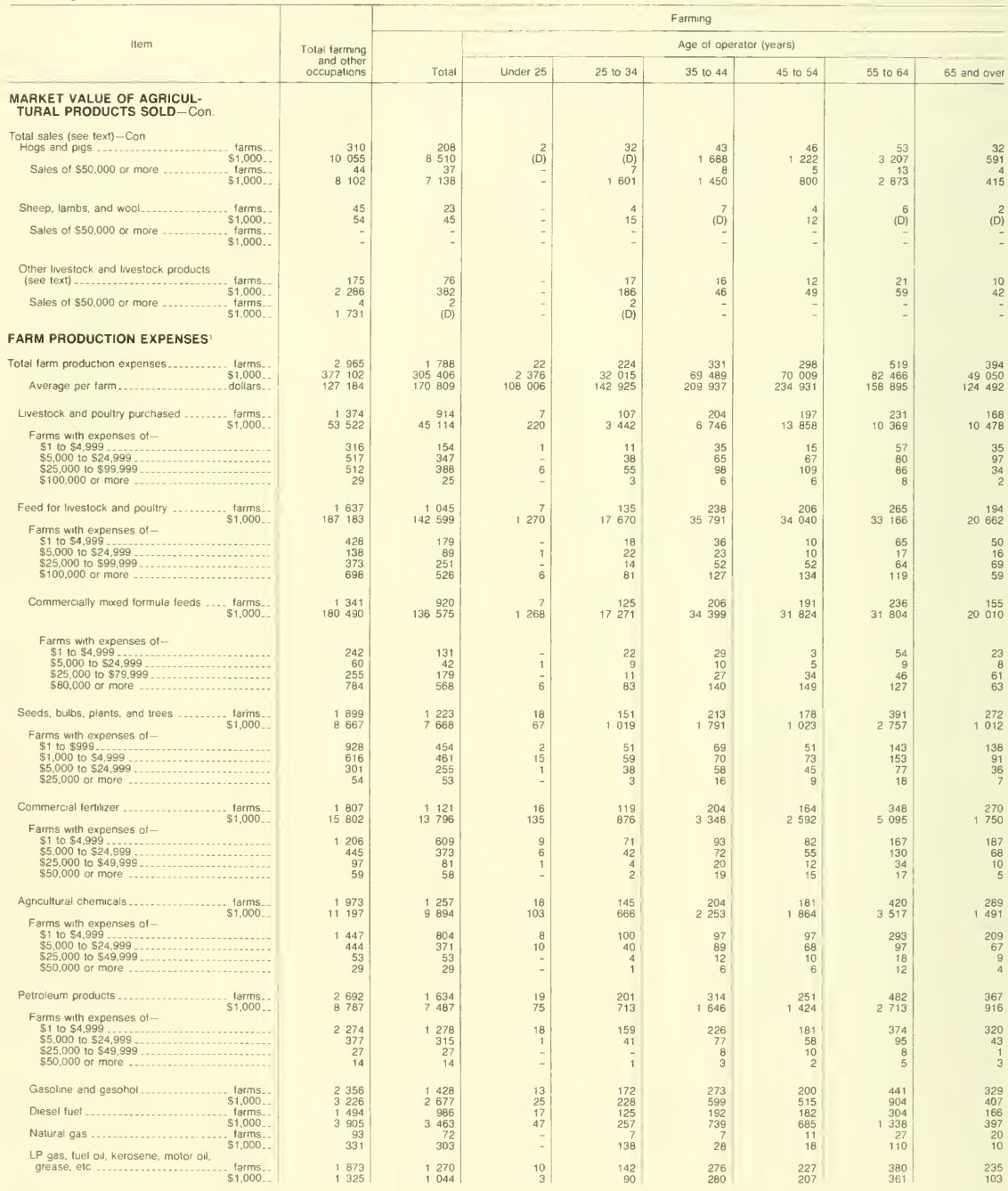


Table 50. Summary by Age and Principal Occupation of Operator: 1987-Con.

[For meaning of abbreviations and symbols, see introductory text]

\begin{tabular}{|c|c|c|c|c|c|c|c|}
\hline \multirow{3}{*}{ Item } & \multicolumn{7}{|c|}{ Other occupations } \\
\hline & \multirow[b]{2}{*}{ Total } & \multicolumn{6}{|c|}{ Age of operator (years) } \\
\hline & & Under 25 & 25 to 34 & 35 to 44 & 45 to 54 & 55 to 64 & 65 and over \\
\hline \multicolumn{8}{|l|}{$\begin{array}{l}\text { MARKET VALUE OF AGRICUL- } \\
\text { TURAL PRODUCTS SOLD-COn. }\end{array}$} \\
\hline 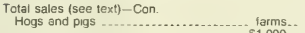 & 102 & & & 27 & & & \\
\hline 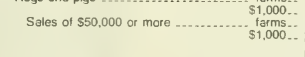 & $\begin{array}{r}1545 \\
7 \\
964\end{array}$ & (D) & $\begin{array}{r}14 \\
105 \\
-\end{array}$ & $\begin{array}{r}431 \\
2 \\
(0)\end{array}$ & $\begin{array}{r}24 \\
136 \\
-\end{array}$ & $\begin{array}{r}24 \\
1 D)_{3} \\
359 \\
559\end{array}$ & $\begin{array}{r}10 \\
218 \\
2 \\
(D)\end{array}$ \\
\hline 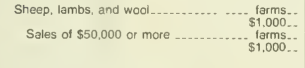 & $\begin{array}{r}22 \\
9 \\
-\end{array}$ & 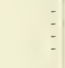 & $\begin{array}{r}2 \\
\text { (D) } \\
-\end{array}$ & $\begin{array}{r}6 \\
(0) \\
-\end{array}$ & $\begin{array}{l}6 \\
4 \\
\vdots \\
-\end{array}$ & $\begin{array}{l}4 \\
2 \\
- \\
-\end{array}$ & $\begin{array}{l}4 \\
1 \\
-\end{array}$ \\
\hline 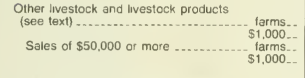 & $\begin{array}{r}99 \\
1904 \\
2 \\
(0)\end{array}$ & 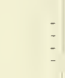 & $\begin{array}{r}10 \\
44 \\
- \\
-\end{array}$ & $\begin{array}{r}29 \\
71 \\
-\end{array}$ & $\begin{array}{r}31 \\
70 \\
-\end{array}$ & $\begin{array}{r}19 \\
848 \\
1 \\
(D)\end{array}$ & $\begin{array}{r}10 \\
871 \\
1 \\
\text { (D) }\end{array}$ \\
\hline \multicolumn{8}{|l|}{ FARM PRODUCTION EXPENSES' } \\
\hline $\begin{array}{l}\text { Total farm production expenses ............. farms.. } \\
\text { Average per farm }\end{array}$ & $\begin{array}{rl}1 & 177 \\
71 & 696 \\
60 & 914\end{array}$ & $\begin{array}{l}- \\
-\end{array}$ & $\begin{array}{r}190 \\
10892 \\
57324\end{array}$ & $\begin{array}{r}306 \\
21772 \\
71 \\
7152\end{array}$ & $\begin{array}{ll}23 & 265 \\
87 & 287 \\
87 & 875\end{array}$ & $\begin{array}{l}264 \\
10647 \\
40330\end{array}$ & $\begin{array}{r}152 \\
5098 \\
33540\end{array}$ \\
\hline $\begin{array}{r}\text { Livestock and poultry purchased ........ farms.. } \\
\$ 1,000 \ldots\end{array}$ & $8 \begin{array}{r}460 \\
8408\end{array}$ & $=$ & $\begin{array}{r}61 \\
1119\end{array}$ & $\begin{array}{r}138 \\
2502\end{array}$ & $\begin{array}{r}150 \\
2981\end{array}$ & $\begin{array}{r}79 \\
1176\end{array}$ & $\begin{array}{r}32 \\
629\end{array}$ \\
\hline $\begin{array}{l}\text { Farms with expenses of } \\
\$ 1 \text { to } \$ 4,999 \\
\$ 5,000 \text { to } \$ 24,999 \\
\$ 25,000 \text { to } \$ 99,999 . \\
\$ 100,000 \text { or more }\end{array}$ & $\begin{array}{r}162 \\
170 \\
124 \\
4\end{array}$ & $\begin{array}{l}\bar{z} \\
\bar{z}\end{array}$ & $\begin{array}{r}18 \\
26 \\
16 \\
1\end{array}$ & $\begin{array}{r}50 \\
52 \\
35 \\
1\end{array}$ & $\begin{array}{r}42 \\
56 \\
51 \\
\end{array}$ & $\begin{array}{r}44 \\
19 \\
15 \\
1\end{array}$ & $\begin{array}{r}8 \\
17 \\
7 \\
-\end{array}$ \\
\hline 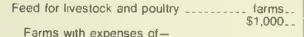 & $\begin{array}{r}592 \\
44585\end{array}$ & $\overline{-}$ & $\begin{array}{r}95 \\
6557\end{array}$ & 13742 & $\begin{array}{r}169 \\
15543\end{array}$ & $\begin{aligned} 137 \\
6004\end{aligned}$ & 2738 \\
\hline $\begin{array}{l}\$ 1 \text { to } \$ 4,999 \\
\$ 5,000 \text { to } \$ 24,999 \ldots \ldots \\
\$ 25,000 \text { to } \$ 99,999 \ldots \ldots\end{array} \$$ & $\begin{array}{r}249 \\
49 \\
122 \\
172\end{array}$ & $\begin{array}{l}\vdots \\
\vdots\end{array}$ & $\begin{array}{l}37 \\
24 \\
15 \\
19\end{array}$ & $\begin{array}{l}41 \\
11 \\
42 \\
56\end{array}$ & $\begin{array}{r}59 \\
4 \\
39 \\
67\end{array}$ & $\begin{array}{r}97 \\
7 \\
13 \\
20\end{array}$ & $\begin{array}{r}15 \\
3 \\
13 \\
10\end{array}$ \\
\hline Commercially mixed formula feeds $\ldots . .$. tarms... & $43 \begin{array}{r}421 \\
915\end{array}$ & - & $\begin{array}{r}61 \\
6438\end{array}$ & $13 \begin{array}{r}120 \\
604\end{array}$ & $15 \begin{array}{r}141 \\
447\end{array}$ & $\begin{array}{r}68 \\
5787\end{array}$ & $\begin{array}{r}31 \\
2639\end{array}$ \\
\hline $\begin{array}{l}\text { Farms with expenses of - } \\
\$ 1 \text { to } \$ 4,999 \\
\$ 5,000 \text { to } \$ 24,999 \\
\$ 25,000 \text { to } \$ 79,999 \\
\$ 80,000 \text { or more }\end{array}$ & $\begin{array}{r}111 \\
18 \\
76 \\
216\end{array}$ & $\begin{array}{l}: \\
:\end{array}$ & $\begin{array}{r}18 \\
9 \\
4 \\
30\end{array}$ & $\begin{array}{r}20 \\
3 \\
24 \\
73\end{array}$ & $\begin{array}{r}34 \\
1 \\
29 \\
77\end{array}$ & $\begin{array}{r}34 \\
2 \\
8 \\
24\end{array}$ & $\begin{array}{r}5 \\
3 \\
11 \\
12\end{array}$ \\
\hline $\begin{array}{c}\text { Seeds, bulbs, plants, and trees .......... farms... } \\
\text { Farms with expenses of }\end{array}$ & $\begin{array}{l}676 \\
999\end{array}$ & $\overline{-}$ & $\begin{array}{l}125 \\
141\end{array}$ & $\begin{array}{l}174 \\
373\end{array}$ & $\begin{array}{l}133 \\
226\end{array}$ & $\begin{array}{l}152 \\
181\end{array}$ & 92 \\
\hline $\begin{array}{l}\$ 1 \text { 10 } \$ 999 \\
\$ 1.000 \text { to } \$ 4.999 \\
\$ 5,000 \text { to } \$ 24,999 \\
\$ 25,000 \text { or more }\end{array}$ & $\begin{array}{r}474 \\
155 \\
46 \\
1\end{array}$ & $\begin{array}{l}\bar{z} \\
\bar{y}\end{array}$ & $\begin{array}{r}92 \\
30 \\
3 \\
-\end{array}$ & $\begin{array}{r}100 \\
59 \\
15 \\
-\end{array}$ & $\begin{array}{r}88 \\
33 \\
11 \\
1\end{array}$ & $\begin{array}{r}122 \\
14 \\
16\end{array}$ & $\begin{array}{r}72 \\
19 \\
1 \\
-\end{array}$ \\
\hline 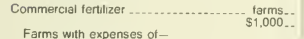 & $\begin{array}{r}686 \\
2006\end{array}$ & 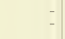 & $\begin{array}{l}123 \\
364\end{array}$ & $\begin{array}{l}177 \\
589\end{array}$ & $\begin{array}{l}128 \\
576\end{array}$ & $\begin{array}{l}163 \\
345\end{array}$ & $\begin{array}{r}95 \\
133\end{array}$ \\
\hline $\begin{array}{l}\$ 1 \text { to } \$ 4,999 \\
\$ 5,000 \text { to } \$ 24,999 \\
\$ 25,000 \text { to } \$ 49,999 \\
\$ 50,000 \text { or more }\end{array}$ & $\begin{array}{r}597 \\
72 \\
16 \\
1\end{array}$ & $\begin{array}{l}\bar{z} \\
\overline{-}\end{array}$ & $\begin{array}{r}103 \\
19 \\
1 \\
-\end{array}$ & $\begin{array}{r}153 \\
18 \\
6 \\
-\end{array}$ & $\begin{array}{r}105 \\
14 \\
9 \\
-\end{array}$ & $\begin{array}{r}144 \\
18 \\
\vdots \\
1\end{array}$ & $\begin{array}{r}92 \\
3 \\
-\end{array}$ \\
\hline $\begin{array}{l}\text { Agricultural chemicals .................. farms.. } \\
\text { Farms with expenses of- }\end{array}$ & $\begin{array}{r}716 \\
1303\end{array}$ & 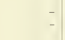 & $\begin{array}{l}130 \\
227\end{array}$ & $\begin{array}{l}183 \\
355\end{array}$ & $\begin{array}{l}137 \\
319\end{array}$ & $\begin{array}{l}161 \\
256\end{array}$ & $\begin{array}{l}105 \\
146\end{array}$ \\
\hline $\begin{array}{l}\text { Farms with expenses of- } \\
\$ 1 \text { to } \$ 4,999 \\
\$ 5,000 \text { to } \$ 24,999 \\
\$ 25,000 \text { to } \$ 49,999 \\
\$ 50,000 \text { or more }\end{array}$ & $\begin{array}{r}643 \\
73 \\
- \\
-\end{array}$ & 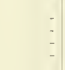 & $\begin{array}{r}114 \\
16 \\
- \\
-\end{array}$ & $\begin{array}{r}165 \\
18 \\
-\end{array}$ & $\begin{array}{r}117 \\
20 \\
-\end{array}$ & $\begin{array}{r}143 \\
18 \\
-\end{array}$ & $\begin{array}{r}104 \\
1 \\
- \\
-\end{array}$ \\
\hline 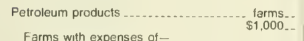 & $\begin{array}{ll}1 & 058 \\
1 & 300\end{array}$ & - & $\begin{array}{l}175 \\
261\end{array}$ & $\begin{array}{l}258 \\
419\end{array}$ & $\begin{array}{l}229 \\
299\end{array}$ & $\begin{array}{l}253 \\
210\end{array}$ & $\begin{array}{l}143 \\
112\end{array}$ \\
\hline $\begin{array}{l}\text { Farms with expenses of- } \\
\$ 1 \text { to } \$ 4,999 \\
\$ 5,000 \text { 10 } \$ 24,999 \\
\$ 25,000 \text { to } \$ \$ 9,999 \\
\$ 50,000 \text { or more }\end{array}$ & $\begin{array}{r}996 \\
62 \\
- \\
-\end{array}$ & 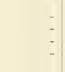 & $\begin{array}{r}161 \\
14 \\
- \\
-\end{array}$ & $\begin{array}{r}235 \\
23 \\
-\end{array}$ & $\begin{array}{r}216 \\
13 \\
- \\
-\end{array}$ & $\begin{array}{r}245 \\
8 \\
-\end{array}$ & $\begin{array}{r}139 \\
4 \\
- \\
-\end{array}$ \\
\hline $\begin{array}{c}\text { Gasoline and gasohol ................... tarms.. } \\
\$ 1,000 \ldots\end{array}$ & $\begin{array}{l}928 \\
549\end{array}$ & $\therefore$ & $\begin{array}{r}157 \\
86\end{array}$ & $\begin{array}{l}216 \\
162\end{array}$ & $\begin{array}{l}206 \\
152\end{array}$ & $\begin{array}{r}227 \\
99\end{array}$ & $\begin{array}{r}122 \\
50\end{array}$ \\
\hline $\begin{array}{c}\text { Diesel fuel ............................... farms } \\
\$ 1.000 .\end{array}$ & $\begin{array}{l}508 \\
442\end{array}$ & $z$ & $\begin{array}{l}83 \\
96\end{array}$ & $\begin{array}{l}161 \\
133\end{array}$ & $\begin{array}{r}81 \\
105\end{array}$ & $\begin{array}{r}124 \\
68\end{array}$ & $\begin{array}{l}59 \\
39\end{array}$ \\
\hline $\begin{array}{l}\text { Natural gas .............. } \\
\$ 1.000 .\end{array}$ & $\begin{array}{l}21 \\
28\end{array}$ & $=$ & $=$ & $\begin{array}{r}13 \\
8\end{array}$ & $\begin{array}{l}4 \\
8\end{array}$ & $\begin{array}{r}3 \\
\text { (D) }\end{array}$ & (D) \\
\hline $\begin{array}{l}\text { grease, etc .................... farms... } \\
\$ 1.000 \ldots\end{array}$ & $\begin{array}{l}603 \\
282\end{array}$ & $\therefore$ & $\begin{array}{r}113 \\
78\end{array}$ & $\begin{array}{l}177 \\
116\end{array}$ & $\begin{array}{l}97 \\
34\end{array}$ & $\begin{array}{l}155 \\
\text { (D) }\end{array}$ & $\begin{array}{l}61 \\
\text { (D) }\end{array}$ \\
\hline
\end{tabular}


Table 50. Summary by Age and Principal Occupation of Operator: 1987-Con.

[For meaning of abbreviations and symbols, see introductory text]

\begin{tabular}{|c|c|c|c|c|c|c|c|c|}
\hline \multirow{3}{*}{ nem } & \multirow{3}{*}{$\begin{array}{l}\text { Total farming } \\
\text { and other } \\
\text { occupations }\end{array}$} & \multicolumn{7}{|c|}{ Farming } \\
\hline & & \multirow[b]{2}{*}{ Total } & \multicolumn{6}{|c|}{ Age of operator (years) } \\
\hline & & & Under 25 & 25 to 34 & 35 to 44 & 45 to 54 & 55 to 64 & 65 and over \\
\hline \multicolumn{9}{|l|}{$\begin{array}{l}\text { FARM PRODUCTION EXPENSES } \\
\text {-CON. }\end{array}$} \\
\hline 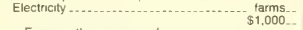 & $\begin{array}{ll}2 & 195 \\
4 & 862\end{array}$ & \begin{tabular}{ll|l}
1 & 431 \\
3 & 917
\end{tabular} & $\begin{array}{l}17 \\
24\end{array}$ & $\begin{array}{l}192 \\
466\end{array}$ & $\begin{array}{l}272 \\
903\end{array}$ & $\begin{array}{l}277 \\
824\end{array}$ & $1 \begin{array}{r}378 \\
1071\end{array}$ & $\begin{array}{l}295 \\
629\end{array}$ \\
\hline $\begin{array}{l}\text { Farms with expenses of } \\
\$ 1 \text { to } \$ 999 \\
\$ 1,000 \text { to } \$ 4,999 \\
\$ 5,000 \text { to } \$ 24,999 \\
\$ 25,000 \text { or more }\end{array}$ & $\begin{array}{r}1085 \\
942 \\
151 \\
17\end{array}$ & $\begin{array}{r}593 \\
695 \\
127 \\
16\end{array}$ & $\begin{array}{l}9 \\
8 \\
- \\
-\end{array}$ & $\begin{array}{r}85 \\
94 \\
12 \\
1\end{array}$ & $\begin{array}{r}84 \\
147 \\
37 \\
4\end{array}$ & $\begin{array}{r}74 \\
174 \\
25 \\
4\end{array}$ & $\begin{array}{r}155 \\
176 \\
43 \\
4\end{array}$ & $\begin{array}{r}186 \\
96 \\
10 \\
3\end{array}$ \\
\hline 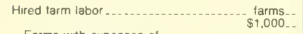 & $\begin{array}{rr}1 & 254 \\
22 & 788\end{array}$ & $20 \begin{array}{r}873 \\
20\end{array}$ & $\begin{array}{r}8 \\
28\end{array}$ & $\begin{array}{r}100 \\
1666\end{array}$ & $\begin{array}{r}189 \\
3991\end{array}$ & $4 \begin{array}{r}169 \\
4046\end{array}$ & 7260 & $\begin{array}{r}147 \\
3200\end{array}$ \\
\hline $\begin{array}{l}\text { Farms with expenses of }- \\
\$ 1 \text { to } \$ 4.999 \\
\$ 5,000 \text { to } \$ 24,999 \\
\$ 25,000 \text { to } \$ 99,999 \\
\$ 100,000 \text { or more }\end{array}$ & $\begin{array}{r}646 \\
440 \\
133 \\
35\end{array}$ & $\begin{array}{r}393 \\
335 \\
112 \\
33\end{array}$ & $\begin{array}{l}7 \\
1 \\
- \\
-\end{array}$ & $\begin{array}{r}46 \\
45 \\
7 \\
2\end{array}$ & $\begin{array}{r}79 \\
69 \\
32 \\
9\end{array}$ & $\begin{array}{r}66 \\
64 \\
33 \\
6\end{array}$ & $\begin{array}{r}109 \\
106 \\
34 \\
11\end{array}$ & $\begin{array}{r}86 \\
50 \\
6 \\
5\end{array}$ \\
\hline Contract labor & $\begin{array}{r}364 \\
5798\end{array}$ & $\begin{array}{r}291 \\
5414\end{array}$ & $\begin{array}{r}4 \\
12\end{array}$ & $\begin{array}{r}32 \\
250\end{array}$ & $\begin{array}{r}69 \\
641\end{array}$ & $\begin{array}{r}59 \\
804\end{array}$ & $\begin{array}{r}86 \\
1092\end{array}$ & $\begin{array}{r}41 \\
2616\end{array}$ \\
\hline $\begin{array}{l}\text { Farms with expenses of - } \\
\$ 1 \text { to } \$ 999 \\
\$ 1,000 \text { to } \$ 4,999 \\
\$ 5,000 \text { to } \$ 24,999 \\
\$ 25,000 \text { or more }\end{array}$ & $\begin{array}{r}41 \\
161 \\
134 \\
28\end{array}$ & $\begin{array}{r}33 \\
124 \\
106 \\
28\end{array}$ & $\begin{array}{l}1 \\
3 \\
- \\
-\end{array}$ & $\begin{array}{r}8 \\
12 \\
9 \\
3\end{array}$ & $\begin{array}{r}2 \\
36 \\
23 \\
8\end{array}$ & $\begin{array}{r}4 \\
30 \\
23 \\
2\end{array}$ & $\begin{array}{r}5 \\
28 \\
42 \\
11\end{array}$ & $\begin{array}{r}13 \\
15 \\
9 \\
4\end{array}$ \\
\hline $\begin{array}{c}\text { Repair and mantenance ............... farms.. } \\
\$ 1,000 \ldots\end{array}$ & $\begin{array}{rr}2 & 481 \\
11 & 739\end{array}$ & $\begin{array}{ll}1 & 546 \\
9 & 853\end{array}$ & $\begin{array}{l}12 \\
35\end{array}$ & $\begin{array}{l}174 \\
910\end{array}$ & $\begin{array}{r}315 \\
2274\end{array}$ & $1 \begin{array}{r}282 \\
958\end{array}$ & $3 \begin{array}{r}460 \\
112\end{array}$ & $\begin{array}{r}303 \\
1564\end{array}$ \\
\hline $\begin{array}{l}\text { Farms with expenses of- } \\
\$ 1 \text { to } \$ 4,999 \\
\$ 5,000 \text { to } \$ 24.999 \\
\$ 25,000 \text { to } \$ 49,999 \\
\$ 50,000 \text { or more }\end{array}$ & $\begin{array}{r}1939 \\
455 \\
67 \\
20\end{array}$ & $\begin{array}{r}1110 \\
354 \\
62 \\
20\end{array}$ & $\begin{array}{r}11 \\
1 \\
- \\
-\end{array}$ & $\begin{array}{r}122 \\
45 \\
6 \\
1\end{array}$ & $\begin{array}{r}204 \\
89 \\
16 \\
6\end{array}$ & $\begin{array}{r}188 \\
78 \\
13 \\
3\end{array}$ & $\begin{array}{r}349 \\
85 \\
21 \\
5\end{array}$ & $\begin{array}{r}236 \\
56 \\
6 \\
5\end{array}$ \\
\hline $\begin{array}{l}\text { Customwork, machine hire, and rental of } \\
\text { machinery and equipment } \\
\text { Farms with expenses of - }\end{array}$ & $\begin{array}{ll}1 & 192 \\
2 & 445\end{array}$ & $\begin{array}{l}771 \\
1975\end{array}$ & $\begin{array}{r}3 \\
10\end{array}$ & $\begin{array}{r}85 \\
289\end{array}$ & $\begin{array}{l}161 \\
368\end{array}$ & $\begin{array}{l}117 \\
272\end{array}$ & $\begin{array}{l}233 \\
614\end{array}$ & $\begin{array}{l}172 \\
422\end{array}$ \\
\hline $\begin{array}{l}\$ 1 \text { to } \$ 999 \\
\$ 1,000 \text { to } \$ 4,999 \\
\$ 5,000 \text { to } \$ 24,999 \\
\$ 25,000 \text { or more }\end{array}$ & $\begin{array}{r}672 \\
406 \\
106 \\
8\end{array}$ & $\begin{array}{r}372 \\
307 \\
84 \\
8\end{array}$ & $\begin{array}{l}1 \\
1 \\
1 \\
-\end{array}$ & $\begin{array}{r}35 \\
35 \\
13 \\
2\end{array}$ & $\begin{array}{r}87 \\
53 \\
19 \\
2\end{array}$ & $\begin{array}{r}51 \\
54 \\
10 \\
2\end{array}$ & $\begin{array}{r}110 \\
92 \\
30 \\
1\end{array}$ & $\begin{array}{r}88 \\
72 \\
11 \\
1\end{array}$ \\
\hline 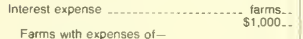 & \begin{tabular}{rr|}
1 & 415 \\
14 & 872
\end{tabular} & 12220 & $\begin{array}{r}9 \\
100\end{array}$ & $1 \begin{array}{r}147 \\
293\end{array}$ & $3 \begin{array}{r}234 \\
054\end{array}$ & $3 \begin{array}{r}201 \\
344\end{array}$ & 3225 & $\begin{array}{r}107 \\
1180\end{array}$ \\
\hline $\begin{array}{l}\$ 1 \text { to } \$ 4,999 \\
\$ 5,000 \text { to } \$ 24,999 \\
\$ 25,000 \text { to } \$ 99,999 \\
\$ 100,000 \text { or more }\end{array}$ & $\begin{array}{r}713 \\
580 \\
110 \\
12\end{array}$ & $\begin{array}{r}389 \\
427 \\
96 \\
11\end{array}$ & $\begin{array}{l}1 \\
8 \\
- \\
-\end{array}$ & $\begin{array}{r}64 \\
76 \\
7 \\
-\end{array}$ & $\begin{array}{r}84 \\
120 \\
28 \\
2\end{array}$ & $\begin{array}{r}64 \\
102 \\
33 \\
2\end{array}$ & $\begin{array}{r}117 \\
79 \\
24 \\
5\end{array}$ & $\begin{array}{r}59 \\
42 \\
4 \\
2\end{array}$ \\
\hline $\begin{array}{l}\text { Secured by real estete ............... farms... } \\
\$ 1,000 \ldots\end{array}$ & $\begin{array}{rr}1 & 205 \\
12 & 415\end{array}$ & $10 \begin{array}{r}805 \\
219\end{array}$ & (D) & $\begin{array}{r}117 \\
\text { (D) }\end{array}$ & 2600 & 2736 & 2797 & $\begin{array}{r}96 \\
937\end{array}$ \\
\hline $\begin{array}{l}\text { Farms with expenses of } \\
\$ 1 \text { to } \$ 999 \\
\$ 1,000 \text { to } \$ 4,999 \\
\$ 5,000 \text { to } \$ 24,999 \\
\$ 25,000 \text { or more }\end{array}$ & $\begin{array}{r}139 \\
463 \\
505 \\
98\end{array}$ & $\begin{array}{r}65 \\
279 \\
376 \\
85\end{array}$ & $\begin{array}{c}- \\
1 \\
7 \\
-\end{array}$ & $\begin{array}{r}8 \\
38 \\
65 \\
6\end{array}$ & $\begin{array}{l}23 \\
58 \\
96 \\
27\end{array}$ & \begin{tabular}{r|}
9 \\
52 \\
100 \\
27
\end{tabular} & $\begin{array}{l}20 \\
82 \\
69 \\
21\end{array}$ & $\begin{array}{r}5 \\
48 \\
39 \\
4\end{array}$ \\
\hline $\begin{array}{c}\text { Not secured by real estate ............ farms.. } \\
\$ 1,000 \ldots\end{array}$ & $2 \begin{array}{r}500 \\
457\end{array}$ & $2 \begin{array}{r}352 \\
2001\end{array}$ & (D) & $\begin{array}{r}77 \\
\text { (D) }\end{array}$ & $\begin{array}{r}92 \\
454\end{array}$ & $\begin{array}{r}64 \\
607\end{array}$ & $\begin{array}{r}83 \\
452\end{array}$ & $\begin{array}{r}34 \\
243\end{array}$ \\
\hline $\begin{array}{l}\text { Farms with expenses of } \\
\$ 1 \text { to } \$ 999 \\
\$ 1,000 \text { to } \$ 4,999 \\
\$ 5,000 \text { to } \$ 24,999 \\
\$ 25,000 \text { or more }\end{array}$ & $\begin{array}{r}183 \\
189 \\
113 \\
15\end{array}$ & $\begin{array}{r}115 \\
133 \\
92 \\
12\end{array}$ & $\begin{array}{l}1 \\
\vdots \\
-\end{array}$ & $\begin{array}{r}35 \\
25 \\
17 \\
-\end{array}$ & $\begin{array}{r}25 \\
34 \\
30 \\
3\end{array}$ & $\begin{array}{r}18 \\
29 \\
12 \\
5\end{array}$ & $\begin{array}{r}24 \\
32 \\
24 \\
3\end{array}$ & $\begin{array}{r}12 \\
13 \\
8 \\
1\end{array}$ \\
\hline Cash rent $\ldots . . .1$ farms & $\begin{array}{rr}972 \\
11 \quad 089\end{array}$ & 9990 & $\begin{array}{r}17 \\
190\end{array}$ & $\begin{array}{r}117 \\
1369\end{array}$ & $\begin{array}{r}144 \\
2644\end{array}$ & $\begin{array}{r}133 \\
1711\end{array}$ & $\begin{array}{r}193 \\
3 \quad 296\end{array}$ & $\begin{array}{r}86 \\
784\end{array}$ \\
\hline $\begin{array}{l}\text { Farms with expenses of } \\
\$ 1 \text { to } \$ 4,999 \\
\$ 5,000 \text { to } \$ 9.999 \\
\$ 10.000 \text { to } \$ 24,999 \\
\$ 25,000 \text { or more }\end{array}$ & $\begin{array}{l}582 \\
103 \\
184 \\
103\end{array}$ & $\begin{array}{r}360 \\
75 \\
157 \\
98\end{array}$ & $\begin{array}{r}2 \\
5 \\
10 \\
-\end{array}$ & $\begin{array}{l}62 \\
14 \\
31 \\
10\end{array}$ & $\begin{array}{l}59 \\
14 \\
42 \\
29\end{array}$ & $\begin{array}{l}81 \\
15 \\
18 \\
19\end{array}$ & $\begin{array}{l}99 \\
22 \\
37 \\
35\end{array}$ & $\begin{array}{r}57 \\
5 \\
19 \\
5\end{array}$ \\
\hline $\begin{array}{l}\text { Property taxes ........... farms.. } \\
\text { Farms with expenses of }\end{array}$ & $\begin{array}{l}2724 \\
2591\end{array}$ & $\begin{array}{ll}1 & 661 \\
1 & 942\end{array}$ & $\begin{array}{l}13 \\
10\end{array}$ & $\begin{array}{l}173 \\
158\end{array}$ & $\begin{array}{l}310 \\
370\end{array}$ & $\begin{array}{l}284 \\
381\end{array}$ & $\begin{array}{l}501 \\
652\end{array}$ & $\begin{array}{l}380 \\
372\end{array}$ \\
\hline $\begin{array}{l}\text { Farms with expenses of } \\
\$ 1 \text { to } \$ 4,999 \\
\$ 5,000 \text { to } \$ 9,999 \\
\$ 10,000 \text { to } \$ 24,999 \ldots \\
\$ 25,000 \text { or more }\end{array}$ & $\begin{array}{r}2666 \\
42 \\
12 \\
4\end{array}$ & $\begin{array}{r}1610 \\
36 \\
11 \\
4\end{array}$ & $\begin{array}{l}13 \\
- \\
- \\
-\end{array}$ & $\begin{array}{r}168 \\
3 \\
2 \\
-\end{array}$ & $\begin{array}{r}299 \\
9 \\
1 \\
1\end{array}$ & $\begin{array}{r}274 \\
6 \\
3 \\
1\end{array}$ & $\begin{array}{r}484 \\
13 \\
3 \\
1\end{array}$ & $\begin{array}{r}372 \\
5 \\
2 \\
1\end{array}$ \\
\hline $\begin{array}{l}\text { All other farm production expenses...... farms.. } \\
\text { Farms with expenses of - }\end{array}$ & $\begin{array}{rr}2 & 813 \\
15 & 758\end{array}$ & $\begin{array}{rr}1 & 759 \\
13 & 382\end{array}$ & $\begin{array}{l}22 \\
98\end{array}$ & $\begin{array}{r}224 \\
1226\end{array}$ & $\begin{array}{r}330 \\
3669\end{array}$ & $\begin{array}{r}295 \\
1868\end{array}$ & $\begin{array}{r}516 \\
4546\end{array}$ & $\begin{array}{r}372 \\
1976\end{array}$ \\
\hline $\begin{array}{l}\$ 1 \text { to } \$ 4,999 \\
\$ 5,000 \text { to } \$ 24,999 \\
\$ 25,000 \text { to } \$ 49,999 \ldots \\
\$ 50,000 \text { or more }\end{array}$ & $\begin{array}{r}2271 \\
442 \\
50 \\
50\end{array}$ & $\begin{array}{r}310 \\
357 \\
44 \\
48\end{array} \mid$ & $\begin{array}{r}20 \\
1 \\
1 \\
-\end{array}$ & $\begin{array}{r}179 \\
38 \\
4 \\
3\end{array}$ & $\begin{array}{r}208 \\
103 \\
4 \\
15\end{array}$ & $\begin{array}{r}196 \\
83 \\
9 \\
7\end{array}$ & $\begin{array}{r}388 \\
90 \\
23 \\
15\end{array}$ & $\begin{array}{r}319 \\
42 \\
3 \\
8\end{array}$ \\
\hline
\end{tabular}


Table 50. Summary by Age and Principal Occupation of Operator: 1987-Con.

[For meaning of abbreviations and symbols, see introductory text]

\begin{tabular}{|c|c|c|c|c|c|c|c|}
\hline \multirow{3}{*}{ Item } & \multicolumn{7}{|c|}{ Other occupations } \\
\hline & \multirow[b]{2}{*}{ Total } & \multicolumn{6}{|c|}{ Age of operator (years) } \\
\hline & & Under 25 & 25 to 34 & 35 to 44 & 45 to 54 & 55 to 64 & 65 and over \\
\hline \multicolumn{8}{|l|}{$\begin{array}{l}\text { FARM PRODUCTION EXPENSES } \\
\text {-CON. }\end{array}$} \\
\hline $\begin{array}{l}\text { Total farm production expenses-Con } \\
\text { Electricity....... tarms. }\end{array}$ & & & & & & & \\
\hline $\begin{array}{c}\text { Electricity } \\
\text { Farms with expenses of }-\end{array} \quad \$ 1,000 .$. & $\begin{array}{l}764 \\
946\end{array}$ & $\overline{-}$ & $\begin{array}{l}115 \\
139\end{array}$ & $\begin{array}{l}222 \\
280\end{array}$ & $\begin{array}{l}166 \\
271\end{array}$ & $\begin{array}{l}167 \\
152\end{array}$ & $\begin{array}{r}94 \\
103\end{array}$ \\
\hline $\begin{array}{l}\text { Farms with expenses of }- \\
\$ 1 \text { to } \$ 999 \\
\$ 1,000 \text { to } \$ 4,999 \\
\$ 5,000 \text { to } \$ 24,999 . \ldots \\
\$ 25,000 \text { or more }\end{array}$ & $\begin{array}{r}492 \\
247 \\
24 \\
1\end{array}$ & $\begin{array}{l}= \\
= \\
-\end{array}$ & $\begin{array}{r}72 \\
37 \\
6 \\
-\end{array}$ & $\begin{array}{r}134 \\
83 \\
5 \\
-\end{array}$ & $\begin{array}{r}85 \\
76 \\
4 \\
1\end{array}$ & $\begin{array}{r}129 \\
34 \\
4 \\
-\end{array}$ & $\begin{array}{r}72 \\
17 \\
5 \\
-\end{array}$ \\
\hline Hired farm labor .................... tarms... & $\begin{array}{r}381 \\
2638\end{array}$ & -1 & $\begin{array}{r}81 \\
518\end{array}$ & $\begin{array}{l}110 \\
569\end{array}$ & $\begin{array}{r}82 \\
403\end{array}$ & $\begin{array}{r}65 \\
748\end{array}$ & $\begin{array}{r}43 \\
400\end{array}$ \\
\hline $\begin{array}{l}\text { Farms with expenses of }- \\
\$ 1 \text { to } \$ 4.999 \\
\$ 5,000 \text { to } \$ 24.999 \\
\$ 25.000 \text { to } \$ 99.999 \\
\$ 100,000 \text { or more }\end{array}$ & $\begin{array}{r}253 \\
105 \\
21 \\
2\end{array}$ & $\begin{array}{l}- \\
- \\
-\end{array}$ & $\begin{array}{r}64 \\
10 \\
7 \\
-\end{array}$ & $\begin{array}{r}73 \\
32 \\
5 \\
-\end{array}$ & $\begin{array}{r}48 \\
31 \\
3 \\
-\end{array}$ & $\begin{array}{r}47 \\
12 \\
5 \\
1\end{array}$ & $\begin{array}{r}21 \\
20 \\
1 \\
1\end{array}$ \\
\hline 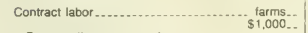 & $\begin{array}{r}73 \\
384\end{array}$ & - & $\begin{array}{r}15 \\
101\end{array}$ & $\begin{array}{r}26 \\
111\end{array}$ & $\begin{array}{r}21 \\
118\end{array}$ & $\begin{array}{r}8 \\
47\end{array}$ & $\begin{array}{l}3 \\
8\end{array}$ \\
\hline $\begin{array}{l}\text { Farms with expenses of }- \\
\$ 1 \text { to } \$ 999 \\
\$ 1,000 \text { to } \$ 4,999 \\
\$ 5,000 \text { to } \$ 24,999 \\
\$ 25,000 \text { or more }\end{array}$ & $\begin{array}{r}8 \\
37 \\
28 \\
-\end{array}$ & $\begin{array}{l}- \\
- \\
-\end{array}$ & $\begin{array}{l}1 \\
8 \\
6 \\
-\end{array}$ & $\begin{array}{r}1 \\
17 \\
8 \\
-\end{array}$ & $\begin{array}{r}5 \\
6 \\
10 \\
-\end{array}$ & $\begin{array}{l}1 \\
3 \\
4 \\
-\end{array}$ & $\overline{3}$ \\
\hline Repar and maintenance ............. farms.. & $\begin{array}{r}935 \\
+886\end{array}$ & - & $\begin{array}{l}137 \\
242\end{array}$ & $\begin{array}{l}263 \\
560\end{array}$ & $\begin{array}{l}236 \\
597\end{array}$ & $\begin{array}{l}216 \\
340\end{array}$ & $\begin{array}{r}83 \\
147\end{array}$ \\
\hline $\begin{array}{l}\text { Farms with expenses of }- \\
\$ 1 \text { to } \$ 4,999 \\
\$ 5,000 \text { to } \$ 24,999 \\
\$ 25,000 \text { to } \$ 49,999 \\
\$ 50,000 \text { or more }\end{array}$ & $\begin{array}{r}829 \\
101 \\
5 \\
-\end{array}$ & $\begin{array}{l}- \\
- \\
- \\
-\end{array}$ & $\begin{array}{r}130 \\
7 \\
- \\
-\end{array}$ & $\begin{array}{r}233 \\
28 \\
2 \\
-\end{array}$ & $\begin{array}{r}195 \\
38 \\
3 \\
-\end{array}$ & $\begin{array}{r}197 \\
19 \\
- \\
-\end{array}$ & $\begin{array}{r}74 \\
9 \\
- \\
-\end{array}$ \\
\hline $\begin{array}{l}\text { Customwork, machine hire, and rental of } \\
\text { machinery and equipment } \\
\text { Farms with expenses of- }\end{array}$ & $\begin{array}{l}421 \\
470\end{array}$ & - & $\begin{array}{l}64 \\
99\end{array}$ & $\begin{array}{l}132 \\
145\end{array}$ & $\begin{array}{l}68 \\
75\end{array}$ & $\begin{array}{l}87 \\
64\end{array}$ & $\begin{array}{l}70 \\
86\end{array}$ \\
\hline $\begin{array}{l}\text { Farms with expenses of }- \\
\$ 1 \text { to } \$ 999 \\
\$ 1.000 \text { to } \$ 4.999 \\
\$ 5.000 \text { to } \$ 24,999 \\
\$ 25.000 \text { or more }\end{array}$ & $\begin{array}{r}300 \\
99 \\
22 \\
-\end{array}$ & $\begin{array}{l}\overline{-} \\
\overline{-}\end{array}$ & $\begin{array}{r}46 \\
9 \\
9 \\
-\end{array}$ & $\begin{array}{r}94 \\
30 \\
8 \\
-\end{array}$ & $\begin{array}{r}38 \\
28 \\
2 \\
-\end{array}$ & $\begin{array}{r}77 \\
9 \\
1 \\
-\end{array}$ & $\begin{array}{r}45 \\
23 \\
2 \\
-\end{array}$ \\
\hline 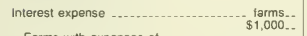 & $\begin{array}{r}492 \\
2652\end{array}$ & $=$ & $\begin{array}{r}83 \\
299\end{array}$ & $\begin{array}{l}169 \\
907\end{array}$ & $\begin{array}{l}117 \\
812\end{array}$ & $\begin{array}{r}93 \\
434\end{array}$ & $\begin{array}{r}30 \\
199\end{array}$ \\
\hline $\begin{array}{l}\text { Farms with expenses of }- \\
\$ 1 \text { to } \$ 4,999 \\
\$ 5,000 \text { to } \$ 24,999 \\
\$ 25,000 \text { to } \$ 99,999 \\
\$ 100,000 \text { or more }\end{array}$ & $\begin{array}{r}324 \\
153 \\
14 \\
1\end{array}$ & $\begin{array}{l}- \\
- \\
- \\
-\end{array}$ & $\begin{array}{r}63 \\
19 \\
1 \\
-\end{array}$ & $\begin{array}{r}113 \\
52 \\
4 \\
-\end{array}$ & $\begin{array}{r}53 \\
59 \\
5 \\
-\end{array}$ & $\begin{array}{r}78 \\
11 \\
3 \\
1\end{array}$ & $\begin{array}{r}17 \\
12 \\
1 \\
-\end{array}$ \\
\hline $\begin{array}{r}\text { Secured by real estate ............. farms.. } \\
\$ 1,000 \ldots\end{array}$ & $2 \begin{array}{r}400 \\
2196\end{array}$ & - & $\begin{array}{r}62 \\
253\end{array}$ & $\begin{array}{l}148 \\
747\end{array}$ & $\begin{array}{r}87 \\
702\end{array}$ & $\begin{array}{r}75 \\
317\end{array}$ & $\begin{array}{r}28 \\
177\end{array}$ \\
\hline $\begin{array}{l}\text { Farms with expenses of- } \\
\$ 1 \text { to } \$ 999 \\
\$ 1,000 \text { to } \$ 4,999 \\
\$ 5,000 \text { to } \$ 24,999 \\
\$ 25,000 \text { or more }\end{array}$ & $\begin{array}{r}74 \\
184 \\
129 \\
13\end{array}$ & $\begin{array}{l}- \\
- \\
-\end{array}$ & $\begin{array}{r}5 \\
38 \\
18 \\
1\end{array}$ & $\begin{array}{r}35 \\
66 \\
44 \\
3\end{array}$ & $\begin{array}{r}2 \\
32 \\
48 \\
5\end{array}$ & $\begin{array}{r}30 \\
34 \\
8 \\
3\end{array}$ & $\begin{array}{r}2 \\
14 \\
11 \\
1\end{array}$ \\
\hline Not secured by real estate ........... farms.. & $\begin{array}{l}148 \\
456\end{array}$ & $=$ & $\begin{array}{l}23 \\
46\end{array}$ & $\begin{array}{r}52 \\
161\end{array}$ & $\begin{array}{r}41 \\
110\end{array}$ & $\begin{array}{r}26 \\
117\end{array}$ & $\begin{array}{r}6 \\
22\end{array}$ \\
\hline $\begin{array}{l}\text { Farms with expenses of }- \\
\$ 1 \text { to } \$ 999 \\
\$ 1,000 \text { to } \$ 4,999 \\
\$ 5,000 \text { to } \$ 24,999 \\
\$ 25,000 \text { or more }\end{array}$ & $\begin{array}{r}68 \\
56 \\
21 \\
3\end{array}$ & $\begin{array}{l}= \\
z \\
=\end{array}$ & $\begin{array}{r}2 \\
21 \\
- \\
-\end{array}$ & $\begin{array}{r}15 \\
27 \\
9 \\
1\end{array}$ & $\begin{array}{r}28 \\
3 \\
10 \\
-\end{array}$ & $\begin{array}{r}18 \\
5 \\
1 \\
2\end{array}$ & $\begin{array}{l}5 \\
1 \\
-\end{array}$ \\
\hline Cash rent ...................... farms . & $\begin{array}{r}282 \\
1094\end{array}$ & $=$ & $\begin{array}{r}79 \\
315\end{array}$ & $\begin{array}{r}85 \\
372\end{array}$ & $\begin{array}{r}54 \\
294\end{array}$ & $\begin{array}{l}35 \\
70\end{array}$ & $\begin{array}{l}29 \\
42\end{array}$ \\
\hline $\begin{array}{l}\text { Farms with expenses of } \\
\$ 1 \text { to } \$ 4,999 \\
\$ 5,000 \text { to } \$ 9,999 \\
\$ 10,000 \text { to } \$ 24,999 \\
\$ 25,000 \text { or more }\end{array}$ & $\begin{array}{r}222 \\
28 \\
27 \\
5\end{array}$ & $\begin{array}{l}= \\
=\end{array}$ & $\begin{array}{r}62 \\
7 \\
8 \\
2\end{array}$ & $\begin{array}{r}62 \\
13 \\
9 \\
1\end{array}$ & $\begin{array}{r}37 \\
7 \\
9 \\
1\end{array}$ & $\begin{array}{r}33 \\
1 \\
1 \\
-\end{array}$ & $\begin{array}{r}28 \\
\overline{1}\end{array}$ \\
\hline Property taxes ................... farms.. & $\begin{array}{r}1063 \\
649\end{array}$ & - & $\begin{array}{r}128 \\
55\end{array}$ & $\begin{array}{l}288 \\
163\end{array}$ & $\begin{array}{l}251 \\
168\end{array}$ & $\begin{array}{l}254 \\
171\end{array}$ & $\begin{array}{r}142 \\
93\end{array}$ \\
\hline $\begin{array}{l}\text { Farms with expenses of } \\
\$ 1 \text { to } \$ 4,999 \\
\$ 5,000 \text { to } \$ 9,999 \\
\$ 10,000 \text { to } \$ 24,999 \\
\$ 25,000 \text { or more }\end{array}$ & $\begin{array}{r}1056 \\
6 \\
1 \\
-\end{array}$ & $\begin{array}{l}- \\
=\end{array}$ & $\begin{array}{r}127 \\
1 \\
- \\
-\end{array}$ & $\begin{array}{r}287 \\
1 \\
- \\
-\end{array}$ & $\begin{array}{r}251 \\
- \\
-\end{array}$ & $\begin{array}{r}250 \\
3 \\
1 \\
-\end{array}$ & $\begin{array}{r}141 \\
1 \\
- \\
-\end{array}$ \\
\hline All other farm production expenses...... farms.. & $\begin{array}{ll}1 & 054 \\
2 & 377\end{array}$ & $\therefore$ & $\begin{array}{l}157 \\
454\end{array}$ & $\begin{array}{l}296 \\
684\end{array}$ & $\begin{array}{l}239 \\
605\end{array}$ & $\begin{array}{l}225 \\
449\end{array}$ & $\begin{array}{l}137 \\
184\end{array}$ \\
\hline $\begin{array}{l}\text { Fatms with expenses of - } \\
\$ 1 \text { to } \$ 4,999 \\
\$ 5,000 \text { to } \$ 24,999 \\
\$ 25,000 \text { to } \$ 49,999 \\
\$ 50,000 \text { or more }\end{array}$ & $\begin{array}{r}961 \\
85 \\
6 \\
2\end{array}$ & $\begin{array}{l}-1 \\
- \\
-\end{array}$ & $\begin{array}{r}141 \\
15 \\
1 \\
-\end{array}$ & $\begin{array}{r}268 \\
28 \\
-\end{array}$ & $\begin{array}{r}212 \\
26 \\
1 \\
-\end{array}$ & $\begin{array}{r}210 \\
10 \\
4 \\
1\end{array}$ & $\begin{array}{r}130 \\
6 \\
\end{array}$ \\
\hline
\end{tabular}


Table 50. Summary by Age and Principal Occupation of Operator: 1987-Con.

[For meaning of abbrevations and symbols. see introductory text]

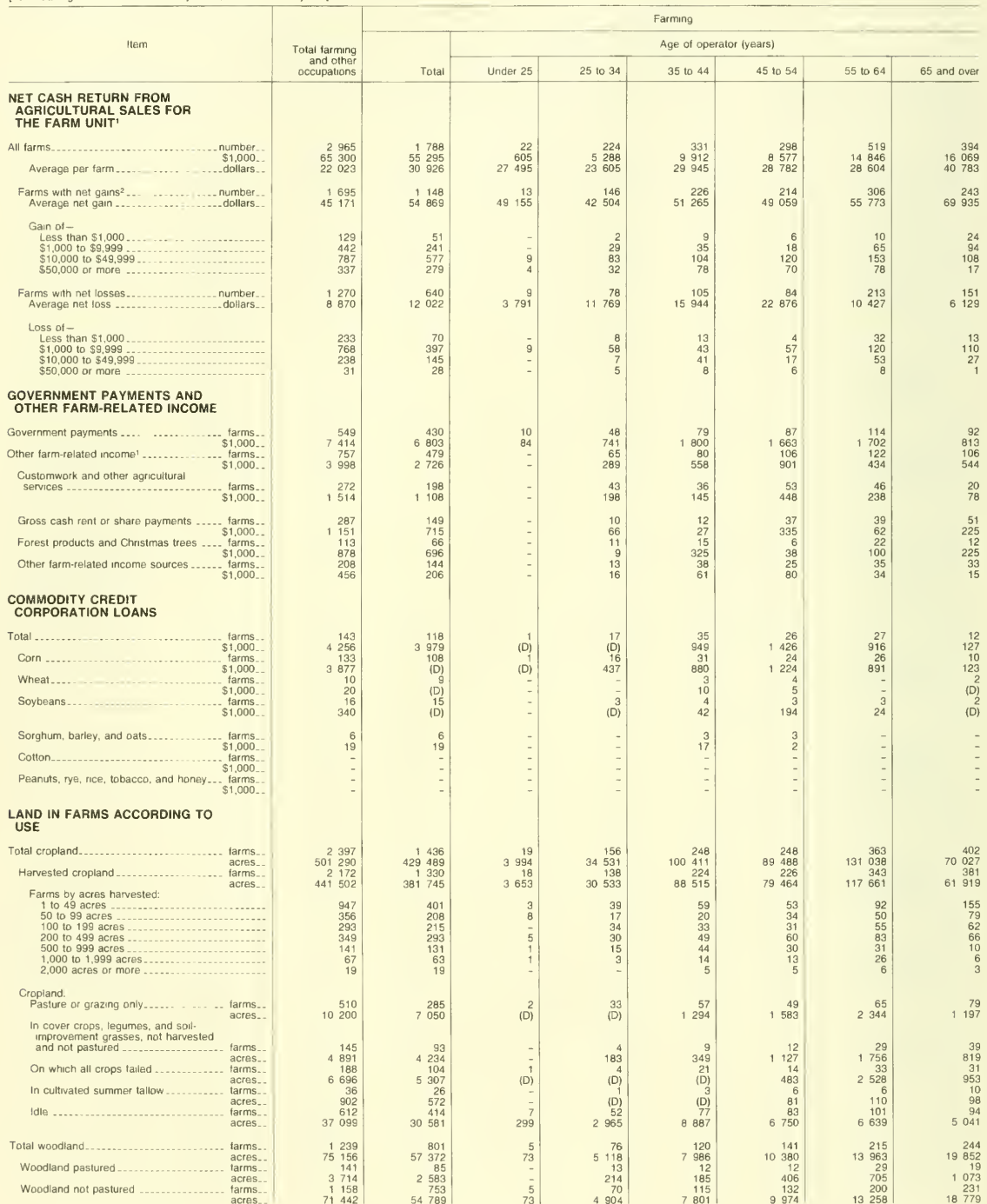

See footnotes at end of table 
Table 50. Summary by Age and Principal Occupation of Operator: 1987-Con.

[For meaning of abbreviations and symbols, see introductory text]

Other occupations

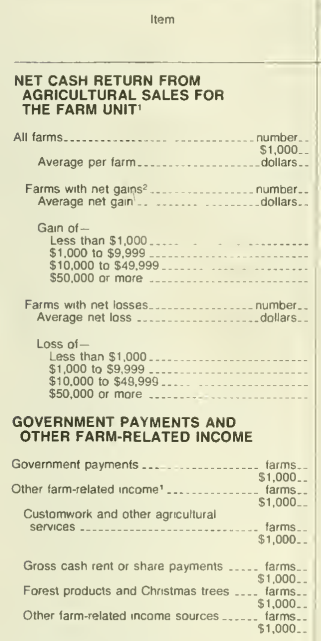

\section{COMMODITY CREDIT}

CORPORATION LOANS

Total

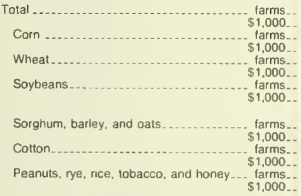

\section{LAND IN FARMS ACCORDING TO}

USE

Total croptand........... farms.

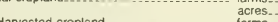

Harvested cropland .

Farms by acres harvested

1 to 49 acres

50 to 99 acres

200 to 499 acres

500 to 999 acres

1.000 to 1.999 acres

2,000 acres or more

Cropland:

Pasture or grazing only............... farms

In cover crops, legumes, and soil-

improvement grasses, not harvested and not pastured

On which all crops falled.

acres.

In cuitivated summer fallow.

$$
\text { Idie }
$$

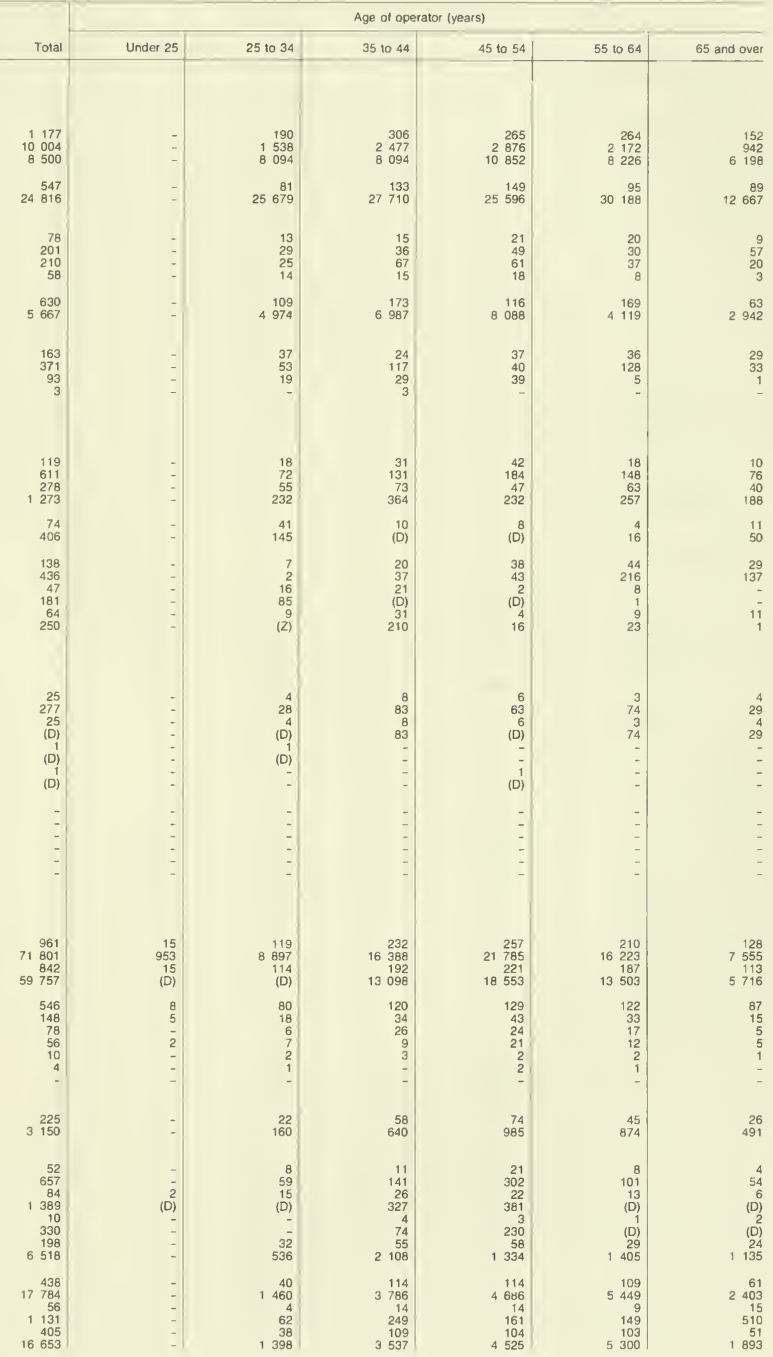


Table 50. Summary by Age and Principal Occupation of Operator: 1987-Con.

[For meaning of abbreviations and symbols, see introductory text]

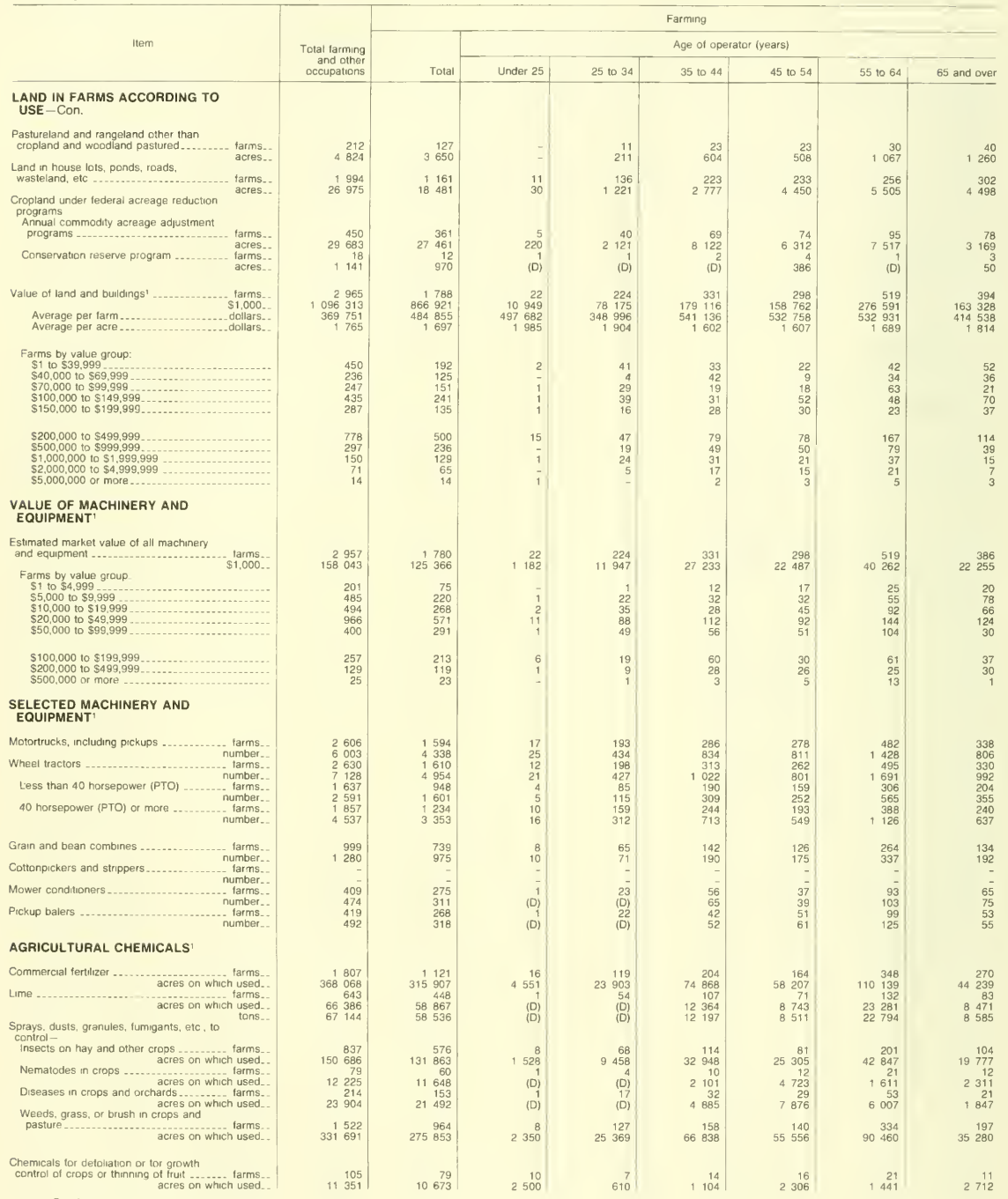


Table 50. Summary by Age and Principal Occupation of Operator: 1987-Con.

[For meaning of abbreviations and symbols. see introductory text]

\begin{tabular}{|c|c|c|c|c|c|c|c|}
\hline \multirow{3}{*}{ Item } & \multicolumn{7}{|c|}{ Other occupations } \\
\hline & \multirow[b]{2}{*}{ Total } & \multicolumn{6}{|c|}{ Age of operalor (years) } \\
\hline & & Under 25 & 25 to 34 & 351044 & 45 to 54 & 55 to 64 & 65 and over \\
\hline \multicolumn{8}{|l|}{$\begin{array}{l}\text { LAND IN FARMS ACCORDING TO } \\
\text { USE-CON. }\end{array}$} \\
\hline $\begin{array}{l}\text { Pastureland and rangeland other than } \\
\text { cropland and woodiand pastured......... farms.. }\end{array}$ & $\begin{array}{r}85 \\
1174\end{array}$ & $=$ & $\begin{array}{r}6 \\
59\end{array}$ & $\begin{array}{r}34 \\
363\end{array}$ & $\begin{array}{r}22 \\
218\end{array}$ & $\begin{array}{r}14 \\
164\end{array}$ & $\begin{array}{r}9 \\
370\end{array}$ \\
\hline $\begin{array}{l}\text { Land in house lots, ponds, roads, } \\
\text { wasteland, etc .......................... farms.. }\end{array}$ & $\begin{array}{r}833 \\
8494\end{array}$ & $\begin{array}{l}11 \\
21\end{array}$ & $\begin{array}{l}108 \\
538\end{array}$ & $\begin{array}{r}195 \\
1410\end{array}$ & $\begin{array}{r}226 \\
2428\end{array}$ & $2 \begin{aligned} 176 \\
2313\end{aligned}$ & $\begin{array}{l}117 \\
1784\end{array}$ \\
\hline 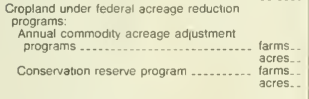 & $\begin{array}{r}89 \\
2222 \\
6 \\
171\end{array}$ & $\bar{z}$ & $\begin{array}{r}14 \\
272 \\
1 \\
\text { (D) }\end{array}$ & $\begin{array}{r}21 \\
498 \\
2 \\
(D)\end{array}$ & $\begin{array}{r}31 \\
764 \\
2 \\
(D)\end{array}$ & $\begin{array}{r}14 \\
381 \\
1 \\
\text { (D) }\end{array}$ & $\begin{array}{r}9{ }^{9} \\
-\end{array}$ \\
\hline 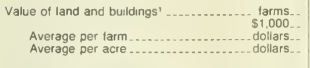 & $\begin{array}{rl}1 & 177 \\
229 & 392 \\
194 & 895 \\
2 & 083\end{array} \mid$ & $\begin{array}{l}\bar{y}- \\
\bar{z}\end{array}$ & $\begin{array}{r}190 \\
33547 \\
176563 \\
2012\end{array}$ & $\begin{array}{r}306 \\
61854 \\
202 \\
202 \\
2151 \\
2151\end{array}$ & $\begin{array}{r}265 \\
53169 \\
200638 \\
2099\end{array}$ & $\begin{array}{r}264 \\
56576 \\
214303 \\
2309\end{array}$ & $\begin{array}{r}152 \\
24246 \\
159 \\
513 \\
1632\end{array}$ \\
\hline 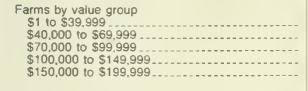 & $\begin{array}{r}258 \\
111 \\
96 \\
194 \\
152\end{array}$ & 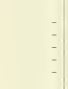 & $\begin{array}{l}39 \\
35 \\
25 \\
31 \\
17\end{array}$ & \begin{tabular}{r|r}
42 & \\
8 & \\
36 & \\
68 & \\
47 &
\end{tabular} & $\begin{array}{l}44 \\
34 \\
17 \\
26 \\
53\end{array}$ & $\begin{array}{r}78 \\
22 \\
5 \\
40 \\
24\end{array}$ & $\begin{array}{l}55 \\
12 \\
13 \\
29 \\
11\end{array}$ \\
\hline $\begin{array}{l}\$ 200,000 \text { to } \$ 499,999 \\
\$ 500,000 \text { to } \$ 999,9999 \\
\$ 1,000,000 \text { to } \$ 1,999,999 \\
\$ 2,000,000 \text { to } \$ 4,999,999 \\
\$ 5,000,000 \text { or more }\end{array}$ & $\begin{array}{r}278 \\
61 \\
21 \\
6 \\
-\end{array}$ & $\begin{array}{l}= \\
=\end{array}$ & $\begin{array}{r}25 \\
9 \\
9 \\
- \\
-\end{array}$ & $\begin{array}{r}74 \\
27 \\
4 \\
- \\
-\end{array}$ & $\begin{array}{r}80 \\
7 \\
2 \\
2 \\
-\end{array}$ & $\begin{array}{r}73 \\
15 \\
4 \\
3 \\
-\end{array}$ & $\begin{array}{r}26 \\
3 \\
2 \\
1 \\
-\end{array}$ \\
\hline \multicolumn{8}{|l|}{$\begin{array}{l}\text { VALUE OF MACHINERY AND } \\
\text { EOUIPMENT }\end{array}$} \\
\hline $\begin{array}{l}\text { Estimated market value of all machinery } \\
\text { and equipment } \\
\text { Farms by vaive group. }\end{array}$ & $\begin{array}{rl}1 & 177 \\
32 & 678\end{array}$ & $\overline{-}$ & 5741 & $\begin{array}{r}306 \\
8589\end{array}$ & 8340 & $\begin{array}{r}264 \\
6513\end{array}$ & $\begin{array}{r}152 \\
3494\end{array}$ \\
\hline 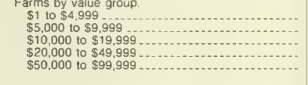 & $\begin{array}{l}126 \\
265 \\
226 \\
395 \\
109\end{array}$ & $\begin{array}{l}z \\
z\end{array}$ & $\begin{array}{l}25 \\
34 \\
44 \\
51 \\
21\end{array}$ & $\begin{array}{r}25 \\
48 \\
63 \\
126 \\
25\end{array}$ & $\begin{array}{l}28 \\
60 \\
49 \\
92 \\
28\end{array}$ & $\begin{array}{l}39 \\
85 \\
29 \\
79 \\
22\end{array}$ & $\begin{array}{r}9 \\
38 \\
41 \\
47 \\
13\end{array}$ \\
\hline $\begin{array}{l}\$ 100,000 \text { to } \$ 199,999 \\
\$ 200,000 \text { to } \$ 49,999 \\
\$ 500,000 \text { or more }\end{array}$ & $\begin{array}{r}44 \\
10 \\
2\end{array}$ & $\overline{-}$ & $\begin{array}{r}13 \\
2 \\
-\end{array}$ & $\frac{18}{1}$ & \begin{tabular}{l|l}
3 & \\
4 & 1 \\
1 &
\end{tabular} & $\begin{array}{l}8 \\
2 \\
-\end{array}$ & $\begin{array}{l}2 \\
2 \\
-\end{array}$ \\
\hline \multicolumn{8}{|l|}{$\begin{array}{l}\text { SELECTED MACHINERY AND } \\
\text { EQUIPMENT }{ }^{1}\end{array}$} \\
\hline Motortrucks, including pickups ............ farms.. & $\begin{array}{l}1 \\
1 \\
1\end{array} 665$ & $=$ & $\begin{array}{l}172 \\
294\end{array}$ & $\begin{array}{l}272 \\
468\end{array}$ & $\begin{array}{l}239 \\
403\end{array}$ & 207 & 122 \\
\hline Wheel tractors ....................... & $\begin{array}{ll}1 & 020 \\
2 & 174\end{array}$ & $=$ & $\begin{array}{l}294 \\
168 \\
313\end{array}$ & $\begin{array}{l}400 \\
260 \\
550\end{array}$ & 213 & 242 & $\begin{array}{l}194 \\
137\end{array}$ \\
\hline Less than 40 horsepower (PTO) ........ farms.. & $\begin{array}{r}21 / 4 \\
689 \\
990\end{array}$ & $=$ & $\begin{array}{r}313 \\
82 \\
120\end{array}$ & $\begin{array}{l}550 \\
162\end{array}$ & $\begin{array}{l}483 \\
166\end{array}$ & $\begin{array}{l}492 \\
178\end{array}$ & $\begin{array}{l}336 \\
101\end{array}$ \\
\hline 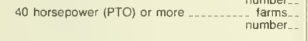 & $\begin{array}{r}990 \\
623 \\
1184\end{array}$ & $\therefore$ & $\begin{array}{l}120 \\
115 \\
193\end{array}$ & $\begin{array}{l}228 \\
169 \\
322\end{array}$ & $\begin{array}{l}237 \\
122 \\
246\end{array}$ & $\begin{array}{l}250 \\
128 \\
242\end{array}$ & $\begin{array}{r}155 \\
89 \\
181\end{array}$ \\
\hline Grain and bean combines .................... tarms... & $\begin{array}{l}260 \\
305\end{array}$ & $\overline{-}$ & $\begin{array}{l}42 \\
50\end{array}$ & $\begin{array}{l}58 \\
66\end{array}$ & $\begin{array}{l}65 \\
73\end{array}$ & $\begin{array}{l}55 \\
69\end{array}$ & 40 \\
\hline $\begin{array}{c}\text { Cottonpickers and strippers } \\
\text { number.............. }\end{array}$ & & $\bar{z}$ & $=$ & $=$ & - & - & - \\
\hline Mower conditioners ................... & $\begin{array}{l}134 \\
163\end{array}$ & - & $\begin{array}{l}30 \\
40\end{array}$ & 30 & 25 & 40 & 9 \\
\hline $\begin{array}{c}\text { Pickup balers ...................... } \\
\text { farms } \\
\text { number. }\end{array}$ & $\begin{array}{l}163 \\
151 \\
174\end{array}$ & 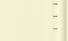 & $\begin{array}{l}40 \\
26 \\
27\end{array}$ & $\begin{array}{l}30 \\
19 \\
26\end{array}$ & $\begin{array}{l}27 \\
35 \\
43\end{array}$ & $\begin{array}{l}57 \\
51 \\
52\end{array}$ & $\begin{array}{l}99 \\
20 \\
26\end{array}$ \\
\hline \multicolumn{8}{|l|}{ AGRICULTURAL CHEMICALS ${ }^{1}$} \\
\hline Commercial fertilizer ................ farms... & $\begin{array}{r}686 \\
52 \quad 161\end{array}$ & - & $9 \begin{array}{r}123 \\
961\end{array}$ & $\begin{array}{r}177 \\
15058\end{array}$ & 12928 & 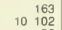 & 4220 \\
\hline $\begin{array}{l}\text { Lime } \\
\text { acres on which used. } \\
\text { tons. }\end{array}$ & $\begin{array}{l}195 \\
7519 \\
8608\end{array}$ & 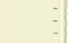 & $\begin{array}{rr}20 \\
1 & 270 \\
1 & 405\end{array}$ & $\begin{array}{r}58 \\
1754 \\
2217\end{array}$ & $\begin{array}{l}144 \\
1854 \\
2187\end{array}$ & $\begin{array}{rl} & 52 \\
2 & 145 \\
2 & 261\end{array}$ & $\begin{array}{r}21 \\
496 \\
538\end{array}$ \\
\hline $\begin{array}{l}\text { Sprays, dusts, granules, fumigants, etc, to } \\
\text { control- } \\
\text { Insects on hay and other crops .......... farms.. }\end{array}$ & & & & & & & \\
\hline $\begin{array}{l}\text { Insects on hay and other crops } \\
\text { acres on which fused. } \\
\text { Nematodes in crops ............ farms. }\end{array}$ & $\begin{array}{r}261 \\
18823 \\
19\end{array}$ & $\therefore$ & $\begin{array}{r}56 \\
4086 \\
12\end{array}$ & $\begin{array}{r}73 \\
6043 \\
2\end{array}$ & $2 \begin{array}{r}50 \\
225 \\
3\end{array}$ & $\begin{array}{r}57 \\
5617 \\
2\end{array}$ & $\begin{array}{r}25 \\
852\end{array}$ \\
\hline $\begin{array}{l}\text { acres on which used.. } \\
\text { Diseases in crops and orchards.......... tarms. }\end{array}$ & $\begin{array}{r}577 \\
61\end{array}$ & $=$ & $\begin{array}{r}360 \\
16\end{array}$ & $(\mathrm{D})$ & $\begin{array}{r}111 \\
7\end{array}$ & (D) & $\overline{5}$ \\
\hline $\begin{array}{l}\text { acres on which used.. } \\
\text { Weeds, grass, or brush in crops and }\end{array}$ & 2412 & - & 1710 & 160 & 147 & 382 & 13 \\
\hline pasture & 55858 & $=$ & $\begin{array}{r}102 \\
7855\end{array}$ & $\begin{array}{ll}18 & \begin{array}{l}45 \\
171\end{array}\end{array}$ & $\begin{array}{r}99 \\
14601\end{array}$ & $\begin{array}{r}140 \\
10514\end{array}$ & $\begin{array}{r}72 \\
4697\end{array}$ \\
\hline $\begin{array}{l}\text { Chemicals for deloliation or for growth } \\
\text { control of crops or thinning of frutt ...... farms. } \\
\text { acres on which used.. }\end{array}$ & $\begin{array}{r}26 \\
678\end{array}$ & 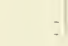 & $420^{7}$ & (D) & $\begin{array}{r}16 \\
221\end{array}$ & $=$ & (D) \\
\hline
\end{tabular}

See footnotes at end of table 
Table 50. Summary by Age and Principal Occupation of Operator: 1987-Con.

[For meaning of abbreviations and symbols, see introductory text]

\begin{tabular}{|c|c|c|c|c|c|c|c|c|}
\hline \multirow{3}{*}{ Item } & \multirow{3}{*}{$\begin{array}{r}\text { Total farming } \\
\text { and other } \\
\text { occupations }\end{array}$} & \multicolumn{7}{|c|}{ Farming } \\
\hline & & \multirow[b]{2}{*}{ Totai } & \multicolumn{6}{|c|}{ Age of operator (years) } \\
\hline & & & Under 25 & 25 to 34 & 351044 & 45 to 54 & 55 to 64 & 65 and over \\
\hline \multicolumn{9}{|l|}{$\begin{array}{l}\text { TENURE AND RACE OF } \\
\text { OPERATOR }\end{array}$} \\
\hline $\begin{array}{l}\text { All operators } \\
\text { Full owners } \\
\text { Part owners } \\
\text { Tenants } \ldots\end{array}$ & $\begin{array}{rl}2 & 966 \\
1 & 825 \\
857 \\
284\end{array}$ & $\begin{array}{r}1774 \\
999 \\
608 \\
167\end{array}$ & $\begin{array}{r}24 \\
9 \\
6 \\
9\end{array}$ & $\begin{array}{r}217 \\
101 \\
65 \\
51\end{array}$ & $\begin{array}{r}326 \\
163 \\
128 \\
35\end{array}$ & $\begin{array}{r}328 \\
175 \\
131 \\
22\end{array}$ & $\begin{array}{r}423 \\
228 \\
165 \\
30\end{array}$ & $\begin{array}{r}456 \\
323 \\
113 \\
20\end{array}$ \\
\hline $\begin{array}{l}\text { White } \\
\text { Full owners } \\
\text { Part owners } \\
\text { Tenants }\end{array}$ & $\begin{array}{r}2914 \\
1791 \\
845 \\
278\end{array}$ & $\begin{array}{r}1741 \\
977 \\
600 \\
164\end{array}$ & $\begin{array}{r}24 \\
9 \\
6 \\
9\end{array}$ & $\begin{array}{r}217 \\
101 \\
65 \\
51\end{array}$ & \begin{tabular}{r|}
319 \\
156 \\
128 \\
35
\end{tabular} & \begin{tabular}{r|}
322 \\
173 \\
129 \\
20
\end{tabular} & \begin{tabular}{r|r}
414 \\
223 \\
162 \\
29
\end{tabular} & $\begin{array}{r}445 \\
315 \\
110 \\
20\end{array}$ \\
\hline $\begin{array}{l}\text { Black and other taces } \\
\text { Full owners } \\
\text { Part owners } \\
\text { Tenants }\end{array}$ & $\begin{array}{r}52 \\
34 \\
12 \\
6\end{array}$ & \begin{tabular}{r|r}
33 & 22 \\
8 & \\
3 &
\end{tabular} & $\begin{array}{l}- \\
\pm \\
- \\
-\end{array}$ & $\begin{array}{l}- \\
- \\
-\end{array}$ & $\begin{array}{l}7 \\
7 \\
- \\
-\end{array}$ & $\begin{array}{l}6 \\
2 \\
2 \\
2\end{array}$ & $\begin{array}{l}9 \\
5 \\
3 \\
1\end{array}$ & $\begin{array}{r}11 \\
8 \\
3 \\
-\end{array}$ \\
\hline \multicolumn{9}{|l|}{ OWNED AND RENTED LAND } \\
\hline 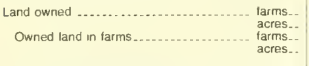 & $\begin{array}{rr}2 & 691 \\
360 & 152 \\
2 & 682 \\
325 & 313\end{array}$ & \begin{tabular}{rr|}
1 & 615 \\
280 & 488 \\
1 & 607 \\
258 & 330
\end{tabular} & $\begin{array}{r}15 \\
835 \\
15 \\
785\end{array}$ & $\begin{array}{rr}167 \\
15 & 018 \\
166 \\
13 & 426\end{array}$ & $\begin{array}{rr}292 \\
46 & 500 \\
291 \\
45 & 117\end{array}$ & $\begin{array}{r}308 \\
53793 \\
306 \\
49882\end{array}$ & $\begin{array}{r}393 \\
83562 \\
393 \\
78 \quad 362\end{array}$ & $\begin{array}{r}440 \\
80780 \\
436 \\
70758\end{array}$ \\
\hline $\begin{array}{r}\text { Land rented or leased from others ........ farms_.. } \\
\text { acres.. } \\
\text { Rented or leased land in farms ........ farms.. } \\
\text { acres.. }\end{array}$ & \begin{tabular}{rr|}
1 & 153 \\
285 & 163 \\
1 & 141 \\
282 & 932
\end{tabular} & $\begin{array}{rr}786 \\
252 & 804 \\
775 \\
250 \quad 662\end{array}$ & $\begin{array}{rr}3 & 15 \\
312 \\
15 \\
312\end{array}$ & $\begin{array}{r}116 \\
27941 \\
116 \\
27655\end{array}$ & $\begin{array}{rl} & 164 \\
67 & 90 \\
163 \\
66 \\
661\end{array}$ & $\begin{array}{r}153 \\
55410 \\
153 \\
54944\end{array}$ & $\begin{array}{r}195 \\
73714 \\
195 \\
73211\end{array}$ & $\begin{array}{rl}143 \\
25 & 237 \\
133 \\
24879\end{array}$ \\
\hline $\begin{array}{l}\text { Land rented or leased to others............ farms_. } \\
\text { acres.. }\end{array}$ & $\begin{array}{r}463 \\
37070\end{array}$ & $24 \stackrel{283}{300}$ & (D) & $\begin{array}{l}24 \\
\text { (D) }\end{array}$ & 1912 & $4 \begin{array}{r}55 \\
377\end{array}$ & 5703 & $10 \begin{array}{l}122 \\
380\end{array}$ \\
\hline \multicolumn{9}{|l|}{ OPERATOR CHARACTERISTICS } \\
\hline $\begin{array}{l}\text { Operators by place of residence: } \\
\text { On farm operated } \\
\text { Not on farm operated } \\
\text { Not reported }\end{array}$ & $\begin{array}{r}2175 \\
481 \\
310\end{array}$ & $\begin{array}{r}1336 \\
231 \\
207\end{array}$ & $\begin{array}{r}13 \\
10 \\
1\end{array}$ & $\begin{array}{r}146 \\
44 \\
27\end{array}$ & $\begin{array}{r}252 \\
36 \\
38\end{array}$ & $\begin{array}{r}258 \\
32 \\
38\end{array}$ & $\begin{array}{r}323 \\
56 \\
44\end{array}$ & $\begin{array}{r}344 \\
53 \\
59\end{array}$ \\
\hline $\begin{array}{l}\text { Operators by principal occupation } \\
\text { Farming } \\
\text { Other }\end{array}$ & $\begin{array}{ll}1 & 774 \\
1 & 192\end{array}$ & 1774 & 24 & 217 & 326 & 328 & 423 & 456 \\
\hline $\begin{array}{l}\text { Operators by days of work oft tarm: } \\
\text { None } \\
\text { Any } 10 . . .19 \text { days } \\
100 \text { to } 199 \text { days } \\
200 \text { days or more }\end{array}$ & $\begin{array}{r}1263 \\
1426 \\
254 \\
217 \\
955\end{array}$ & $\begin{array}{r}1063 \\
434 \\
206 \\
99 \\
129\end{array}$ & $\begin{array}{r}10 \\
12 \\
6 \\
3 \\
3\end{array}$ & $\begin{array}{r}101 \\
98 \\
48 \\
17 \\
33\end{array}$ & $\begin{array}{r}169 \\
113 \\
57 \\
20 \\
36\end{array}$ & $\begin{array}{r}192 \\
94 \\
40 \\
27 \\
27\end{array}$ & \begin{tabular}{r|}
276 \\
82 \\
38 \\
22 \\
22
\end{tabular} & $\begin{array}{r}315 \\
35 \\
17 \\
10 \\
8\end{array}$ \\
\hline Not reported ......................................... & 277 & 277 & 2 & 18 & 44 & 42 & 65 & 106 \\
\hline $\begin{array}{l}\text { Operators by years on present farm: } \\
2 \text { years or less } \\
3 \text { or } 4 \text { years } \\
5 \text { to } 9 \text { years...................... } \\
10 \text { years or more } \\
\text { Average years on present farm }\end{array}$ & $\begin{array}{r}176 \\
189 \\
398 \\
1588 \\
193\end{array}$ & $\begin{array}{r}86 \\
90 \\
194 \\
1023 \\
21.9\end{array}$ & \begin{tabular}{r|r}
10 \\
6 \\
7 \\
$\overline{3}$ \\
3.5
\end{tabular} & $\begin{array}{l}25 \\
35 \\
58 \\
54 \\
7.2\end{array}$ & $\begin{array}{r}18 \\
26 \\
49 \\
164 \\
12.0\end{array}$ & \begin{tabular}{r|}
14 \\
11 \\
35 \\
202 \\
18.6
\end{tabular} & $\begin{array}{r}5 \\
5 \\
34 \\
299 \\
27.4\end{array}$ & $\begin{array}{r}14 \\
7 \\
11 \\
304 \\
35.3\end{array}$ \\
\hline Not reported .......................... & 615 & 381 & 1 & 45 & 69 & 66 & 80 & 120 \\
\hline $\begin{array}{l}\text { Operators by age group } \\
\text { Under } 25 \text { years } \\
25 \text { to } 34 \text { years } \\
35 \text { to } 44 \text { years } \\
45 \text { to } 49 \text { years } \\
50 \text { to } 54 \text { years }\end{array}$ & $\begin{array}{r}40 \\
374 \\
620 \\
328 \\
321\end{array}$ & $\begin{array}{r}24 \\
217 \\
326 \\
153 \\
175\end{array}$ & $\begin{array}{r}24 \\
- \\
- \\
-\end{array}$ & $\begin{array}{r}21 \overline{7} \\
\overline{-} \\
\overline{-}\end{array}$ & $\begin{array}{r}- \\
326 \\
-\end{array}$ & $\begin{array}{r}5 \\
153 \\
175\end{array}$ & $\begin{array}{l}\bar{y} \\
\bar{z} \\
-\end{array}$ & $\begin{array}{l}\bar{z} \\
\overline{-}\end{array}$ \\
\hline 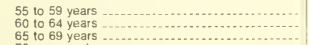 & $\begin{array}{l}326 \\
348 \\
269\end{array}$ & $\begin{array}{l}184 \\
239 \\
208\end{array}$ & $\begin{array}{l}- \\
-\end{array}$ & $\bar{z}$ & $\begin{array}{l}- \\
\overline{-}\end{array}$ & $\overline{-}$ & $\begin{array}{r}184 \\
239 \\
-\end{array}$ & $20 \overline{-}$ \\
\hline 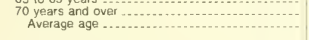 & $\begin{array}{r}209 \\
340 \\
515\end{array}$ & $\begin{array}{l}240 \\
53.0\end{array}$ & $22 . \overline{5}$ & & $39 . \overline{5}$ & $49 . \overline{6}$ & $59 . \overline{7}$ & $\begin{array}{l}248 \\
71.5\end{array}$ \\
\hline $\begin{array}{l}\text { Operators by sex } \\
\text { Male } \\
\text { Female }\end{array}$ & $\begin{array}{r}2676 \\
290\end{array}$ & $\begin{array}{r}1590 \\
184\end{array}$ & $\begin{array}{r}22 \\
2\end{array}$ & $\begin{array}{r}194 \\
23\end{array}$ & $\begin{array}{r}280 \\
46\end{array}$ & $\begin{array}{r}286 \\
42\end{array}$ & $\begin{array}{r}396 \\
27\end{array}$ & $\begin{array}{r}412 \\
44\end{array}$ \\
\hline Operators of Spanısh origin (see text) ................ & 9 & 6 & - & 2 & 2 & - & 2 & - \\
\hline $\begin{array}{l}\text { FARMS BY TYPE OF } \\
\text { ORGANIZATION }\end{array}$ & & & & & & & & \\
\hline Individual or family (sole proprietorship) ..... farms.. & $\begin{array}{rl}2 & 553 \\
400 & 071\end{array}$ & $\begin{array}{rl}1 & 478 \\
317 & 152 \\
158\end{array}$ & $\begin{array}{l}22 \\
(\mathrm{D})\end{array}$ & $\begin{array}{r}186 \\
27944\end{array}$ & $64 \begin{array}{r}268 \\
211 \\
30\end{array}$ & $\begin{array}{r}275 \\
(\mathrm{D}) \\
24\end{array}$ & 91366 & 62085 \\
\hline $\begin{array}{l}\text { Partnership ............................ } \\
\text { farms. } \\
\text { acres . }\end{array}$ & 62666 & $\begin{array}{rr}158 \\
58508\end{array}$ & (D) & $\begin{array}{r}15 \\
\text { (D) }\end{array}$ & 15796 & 7527 & $\begin{array}{r}43 \\
\text { (D) }\end{array}$ & $\begin{array}{r}45 \\
\text { (D) }\end{array}$ \\
\hline $\begin{array}{l}\text { Corporation: } \\
\text { Family held }\end{array}$ & $\begin{array}{rr}141 & 173 \\
856 \\
4\end{array}$ & 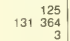 & (D) & $\begin{array}{r}15 \\
(D)\end{array}$ & $31 \begin{array}{rr}25 \\
347\end{array}$ & 28721 & $40 \begin{array}{r}35 \\
064 \\
2\end{array}$ & $21 \begin{array}{r}21 \\
494\end{array}$ \\
\hline $\begin{array}{l}\text { More than } 10 \text { stockholders ............ larms.. } \\
10 \text { or less stockholders ............ larms.. }\end{array}$ & 169 & 122 & i & 15 & 25 & 28 & 33 & 20 \\
\hline $\begin{array}{l}\text { Other than family held ................. farms... } \\
\text { acres.. }\end{array}$ & 928 & $400^{3}$ & $=$ & (D) & - & (D) & (D) & $\overline{-}$ \\
\hline $\begin{array}{l}\text { More than } 10 \text { stockholders ............. farms.. } \\
10 \text { or less stocknolders ............. }\end{array}$ & $\begin{array}{l}1 \\
7\end{array}$ & $\overline{3}$ & - & i & $=$ & i & $i$ & $\overline{-}$ \\
\hline $\begin{array}{l}\text { Other-cooperative, estate or trust, } \\
\text { insitutional, etc ......... farms... } \\
\text { acres.. }\end{array}$ & $\begin{array}{r}16 \\
2724\end{array}$ & $\begin{array}{r}10 \\
1568\end{array}$ & $=1$ & $=1$ & $\begin{array}{r}3 \\
424\end{array}$ & $=1$ & (D) & $\begin{array}{r}6 \\
\text { (D) }\end{array}$ \\
\hline
\end{tabular}


Table 50. Summary by Age and Principal Occupation of Operator: 1987-Con.

[For meaning of abbreviations and symbols, see introductory text]

\begin{tabular}{|c|c|c|c|c|c|c|c|}
\hline \multirow{3}{*}{ ltem } & \multicolumn{7}{|c|}{ Other occupations } \\
\hline & \multirow[b]{2}{*}{ Total } & \multicolumn{6}{|c|}{ Age of operator (years) } \\
\hline & & Under 25 & 25 to 34 & 35 to 44 & 45 to 54 & 55 to 64 & 65 and over \\
\hline \multicolumn{8}{|l|}{$\begin{array}{l}\text { TENURE AND RACE OF } \\
\text { OPERATOR }\end{array}$} \\
\hline $\begin{array}{l}\text { All operators } \\
\text { Full owners } \\
\text { Part owners } \\
\text { Tenants }\end{array}$ & $\begin{array}{r}1192 \\
826 \\
249 \\
117\end{array}$ & $\begin{array}{r}16 \\
3 \\
6 \\
7\end{array}$ & $\begin{array}{r}157 \\
87 \\
35 \\
35\end{array}$ & $\begin{array}{r}294 \\
199 \\
67 \\
28\end{array}$ & $\begin{array}{r}321 \\
213 \\
78 \\
30\end{array}$ & $\begin{array}{r}251 \\
193 \\
49 \\
9\end{array}$ & $\begin{array}{r}153 \\
131 \\
14 \\
8\end{array}$ \\
\hline $\begin{array}{l}\text { White } \\
\text { Full owners } \\
\text { Part owners } \\
\text { Tenants }\end{array}$ & $\begin{array}{r}173 \\
814 \\
245 \\
114\end{array}$ & $\begin{array}{r}16 \\
3 \\
6 \\
7\end{array}$ & $\begin{array}{r}157 \\
87 \\
35 \\
35\end{array}$ & $\begin{array}{r}290 \\
197 \\
65 \\
28\end{array}$ & $\begin{array}{r}318 \\
212 \\
77 \\
29\end{array}$ & $\begin{array}{r}247 \\
190 \\
48 \\
9\end{array}$ & $\begin{array}{r}145 \\
125 \\
14 \\
6\end{array}$ \\
\hline $\begin{array}{l}\text { Black and other races } \\
\text { Full owners } \\
\text { Part owners } \\
\text { Tenants }\end{array}$ & \begin{tabular}{r|}
19 \\
12 \\
4 \\
3
\end{tabular} & $\bar{z}$ & $\begin{array}{l}- \\
- \\
-\end{array}$ & $\begin{array}{l}4 \\
2 \\
2 \\
-\end{array}$ & $\begin{array}{l}3 \\
1 \\
1 \\
1\end{array}$ & $\begin{array}{l}4 \\
3 \\
1 \\
-\end{array}$ & $\begin{array}{l}8 \\
6 \\
2\end{array}$ \\
\hline \multicolumn{8}{|l|}{ OWNED AND RENTED LAND } \\
\hline $\begin{array}{r}\text { Land owned ....................... } \\
\text { Owned land in farms............. } \begin{array}{l}\text { farms.. } \\
\text { acres.. } \\
\text { farms.. } \\
\text { acres.. }\end{array}\end{array}$ & $\begin{array}{rr}1 & 076 \\
79 & 664 \\
1 & 075 \\
66 & 983\end{array}$ & $\begin{array}{r}9 \\
202 \\
9 \\
202\end{array}$ & $\begin{array}{r}122 \\
4188 \\
122 \\
4096\end{array}$ & $\begin{array}{r}266 \\
15 \quad 964 \\
266 \\
14 \\
2225\end{array}$ & $\begin{array}{rr}292 \\
20 & 998 \\
& 291 \\
18 & 128\end{array}$ & $\begin{array}{r}242 \\
24 \\
386 \\
20 \quad 242 \\
20 \\
-278\end{array}$ & $\begin{array}{rr} & 145 \\
13 & 926 \\
& 145 \\
10 & 054\end{array}$ \\
\hline $\begin{array}{l}\text { Land rented or leased from others ......... farms.. } \\
\text { acres.. } \\
\text { Rented or leased land in farms ........ farms. } \\
\text { acres.. }\end{array}$ & \begin{tabular}{ll|} 
& 367 \\
32 & 359 \\
366 \\
32 & 270
\end{tabular} & $\begin{array}{r}13 \\
772 \\
13 \\
772\end{array}$ & $\begin{array}{rr}6 \quad 70 \\
6 \quad 893 \\
6 \quad 858\end{array}$ & $\begin{array}{r}795 \\
7771 \\
95 \\
7722\end{array}$ & $\begin{array}{r}108 \\
10 \quad 989 \\
108 \\
10 \quad 989\end{array}$ & $\begin{array}{r}58 \\
3871 \\
58 \\
3871\end{array}$ & $\begin{array}{rr}23 \\
2063 \\
22 \\
2058\end{array}$ \\
\hline $\begin{array}{l}\text { Land rented or leased to others............ farms.. } \\
\text { acres. }\end{array}$ & $12 \begin{array}{r}180 \\
770\end{array}$ & - & $\begin{array}{r}6 \\
127\end{array}$ & $\begin{array}{r}37 \\
1788\end{array}$ & 2870 & $4 \begin{array}{r}52 \\
108\end{array}$ & 3877 \\
\hline \multicolumn{8}{|l|}{ OPERATOR CHARACTERISTICS } \\
\hline $\begin{array}{l}\text { Operators by place of residence: } \\
\text { On farm operated } \\
\text { Not on farm operated } \\
\text { Not reported }\end{array}$ & $\begin{array}{l}839 \\
250 \\
103\end{array}$ & $\begin{array}{r}6 \\
10 \\
-\end{array}$ & $\begin{array}{r}107 \\
35 \\
15\end{array}$ & $\begin{array}{r}217 \\
56 \\
21\end{array}$ & $\begin{array}{r}227 \\
64 \\
30\end{array}$ & $\begin{array}{r}180 \\
57 \\
14\end{array}$ & $\begin{array}{r}102 \\
28 \\
23\end{array}$ \\
\hline $\begin{array}{l}\text { Operators by principal occupation: } \\
\text { Farming } \\
\text { Other }\end{array}$ & $119 \overline{2}$ & 16 & $15 \overline{7}$ & $29 \overline{4}$ & $32 \overline{1}$ & $25 \overline{1}$ & $15 \overline{3}$ \\
\hline $\begin{array}{l}\text { Operators by days of work off tarm: } \\
\text { None } \\
\text { Any } 1 \text { to } 99 \text { days } \\
100 \text { to } 199 \text { days } \\
200 \text { days or more }\end{array}$ & $\begin{array}{r}200 \\
992 \\
48 \\
118 \\
826\end{array}$ & $\begin{array}{r}- \\
1 \overline{6} \\
1 \\
4 \\
11\end{array}$ & $\begin{array}{r}6 \\
151 \\
7 \\
14 \\
130\end{array}$ & $\begin{array}{r}23 \\
271 \\
5 \\
24 \\
242\end{array}$ & $\begin{array}{r}27 \\
294 \\
14 \\
24 \\
256\end{array}$ & $\begin{array}{r}44 \\
207 \\
14 \\
39 \\
154\end{array}$ & $\begin{array}{r}100 \\
53 \\
7 \\
13 \\
33\end{array}$ \\
\hline Not reported . . . . . . & - & - & - & - & - & - & - \\
\hline $\begin{array}{l}\text { Operators by years on present farm; } \\
2 \text { years or less } \\
3 \text { or } 4 \text { years } \\
5 \text { to } 9 \text { years } \\
10 \text { years or more } \\
\text { Average years on present farm }\end{array}$ & $\begin{array}{r}90 \\
99 \\
204 \\
565 \\
15.5\end{array}$ & $\begin{array}{r}3 \\
3 \\
6 \\
-\overline{3}\end{array}$ & $\begin{array}{l}22 \\
30 \\
41 \\
27 \\
6.3\end{array}$ & $\begin{array}{r}38 \\
42 \\
66 \\
112 \\
9.1\end{array}$ & $\begin{array}{r}9 \\
18 \\
61 \\
162 \\
13.9\end{array}$ & $\begin{array}{r}17 \\
3 \\
25 \\
172 \\
21.6\end{array}$ & $\begin{array}{r}1 \\
3 \\
5 \\
92 \\
348\end{array}$ \\
\hline 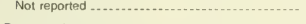 & 234 & 4 & 37 & 36 & 71 & 34 & 52 \\
\hline $\begin{array}{l}\text { Operators by age group: } \\
\text { Under } 25 \text { years } \\
25 \text { to } 34 \text { years } \\
35 \text { to } 44 \text { years } \\
45 \text { to } 49 \text { years } \\
50 \text { to } 54 \text { years }\end{array}$ & $\begin{array}{r}16 \\
157 \\
294 \\
175 \\
146\end{array}$ & $\begin{array}{c}16 \\
- \\
- \\
-\end{array}$ & $\begin{array}{r}15 \overline{7} \\
\overline{-} \\
-\end{array}$ & $\begin{array}{r}- \\
\overline{-} \\
\overline{-} \\
-\end{array}$ & $\begin{array}{r}- \\
- \\
175 \\
146\end{array}$ & $\begin{array}{l}\bar{z} \\
\overline{-}\end{array}$ & $\begin{array}{l}\overline{-} \\
\bar{z}\end{array}$ \\
\hline 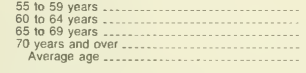 & $\begin{array}{r}142 \\
109 \\
61 \\
92 \\
49.1\end{array}$ & $21 . \overline{4}$ & $30 \overline{2}$ & $\begin{array}{r}- \\
- \\
- \\
- \\
-\end{array}$ & $49, \bar{z}$ & $\begin{array}{r}142 \\
109 \\
-\overline{2}\end{array}$ & $\begin{array}{r}- \\
61 \\
92 \\
72.5\end{array}$ \\
\hline $\begin{array}{l}\text { Operators by sex } \\
\text { Mate } \\
\text { Female }\end{array}$ & $\begin{array}{r}1086 \\
106\end{array}$ & 16 & $\begin{array}{r}146 \\
11\end{array}$ & $\begin{array}{r}274 \\
20\end{array}$ & $\begin{array}{r}302 \\
19\end{array}$ & $\begin{array}{r}222 \\
29\end{array}$ & $\begin{array}{r}126 \\
27\end{array}$ \\
\hline $\begin{array}{l}\text { Operators of Spanish origin (see text) } \ldots . . . \\
\text { FARMS BY TYPE OF } \\
\text { ORGANIZATION }\end{array}$ & 3 & - & - & 2 & 1 & - & - \\
\hline $\begin{array}{l}\text { Individual or family (sole proprietorship) ..... farms_. } \\
\begin{array}{l}\text { acres.. } \\
\text { Partnership ........... } \\
\text { Cormsoration }\end{array}\end{array}$ & $\begin{array}{rr}1 & 075 \\
82 & 919 \\
58 \\
4 \quad 158\end{array}$ & $\begin{array}{r}16 \\
974 \\
- \\
-\end{array}$ & $\begin{array}{r}141 \\
9440 \\
9 \\
\text { (D) }\end{array}$ & $\begin{array}{r}268 \\
19031 \\
11 \\
1411\end{array}$ & $\begin{array}{rr}289 \\
24 & 196 \\
& 17 \\
1 & 194\end{array}$ & $\begin{array}{r}221 \\
19918 \\
14 \\
1071\end{array}$ & $\begin{array}{r}140 \\
960 \\
7 \\
\text { (D) }\end{array}$ \\
\hline Family heid .............. farms.. & $\begin{array}{r}48 \\
10 \quad 492\end{array}$ & $\overline{-}$ & $\begin{array}{rr}1 & 6 \\
1 & 148\end{array}$ & 1. 131 & $3 \begin{array}{r}12 \\
314\end{array}$ & 2365 & 2534 \\
\hline $\begin{array}{l}\text { More than } 10 \text { stockholders ............ tarms.. } \\
10 \text { or less stockholders ............... tarms.. }\end{array}$ & & $\overline{-}$ & $\overline{6}$ & $1 \overline{2}$ & 12 & $\begin{array}{r}1 \\
12\end{array}$ & $\overline{5}$ \\
\hline Other than tamily held ................. farms.. & $\begin{array}{r}5 \\
528\end{array}$ & - & (D) & - & (D) & (D) & (D) \\
\hline $\begin{array}{l}\text { More than } 10 \text { stockholders ............. farms_. } \\
10 \text { or less stockholders .............. farms.. }\end{array}$ & $\begin{array}{l}1 \\
4\end{array}$ & - & $\overline{1}$ & $\overline{-}$ & $\overline{1}$ & $\begin{array}{l}1 \\
1\end{array}$ & $\overline{1}$ \\
\hline $\begin{array}{l}\text { Other-cooperative, estate or trust, } \\
\text { institutional, etc ......... farms.. } \\
\text { acres.. }\end{array}$ & $\begin{array}{rr}6 \\
1 & 156\end{array}$ & $\overline{-}$ & - & $\begin{array}{r}3 \\
374\end{array}$ & (D) & (D) & - \\
\hline
\end{tabular}


Table 50. Summary by Age and Principal Occupation of Operator: 1987-Con.

[For meaning of abbreviations and symbols, see introductory text]

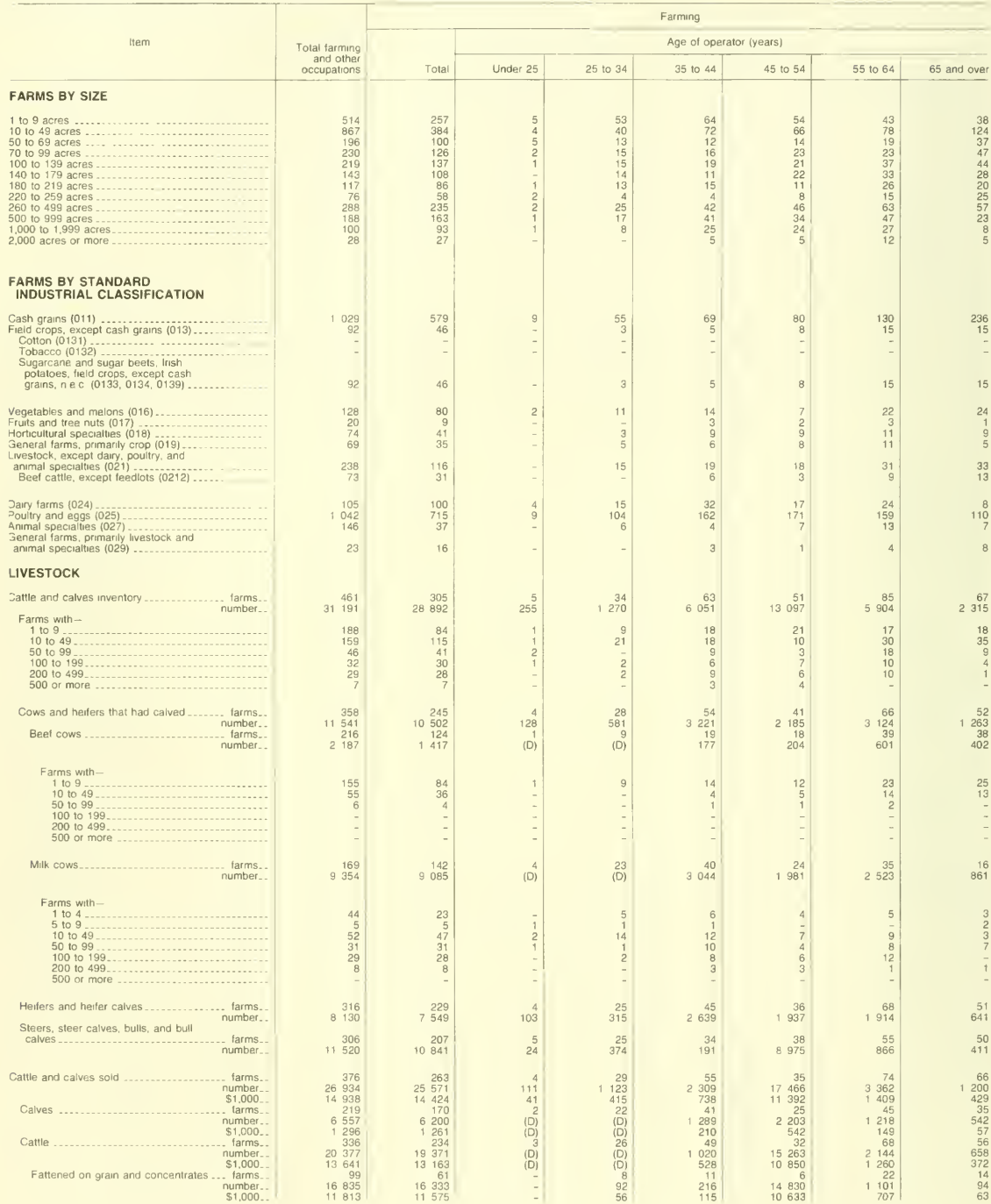


Table 50. Summary by Age and Principal Occupation of Operator: 1987-Con.

[For meaning of abbreviations and symbols, see introductory text]

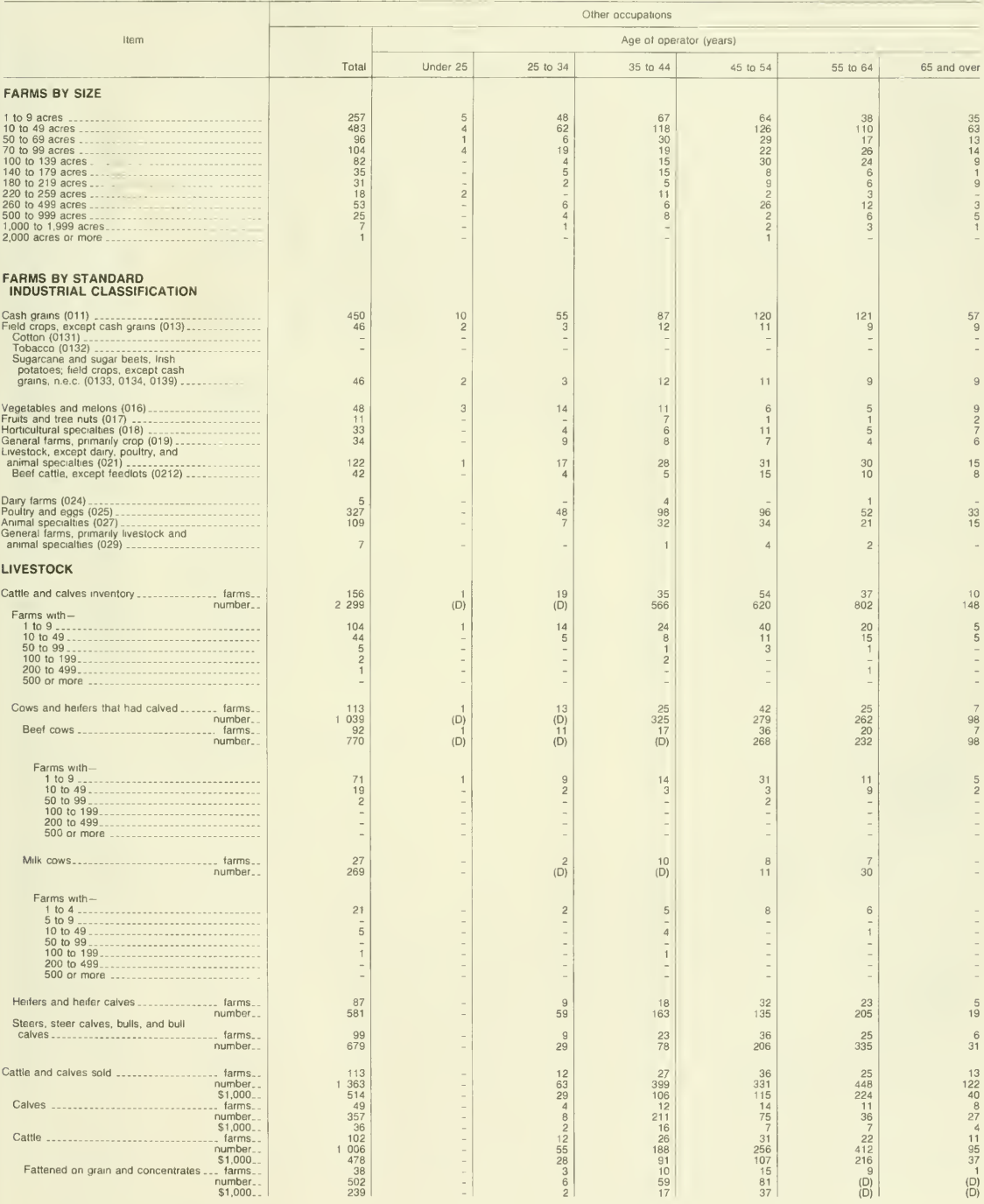


Table 50. Summary by Age and Principal Occupation of Operator: 1987-Con.

[For meaning of abbreviations and symbols, see introductory text]

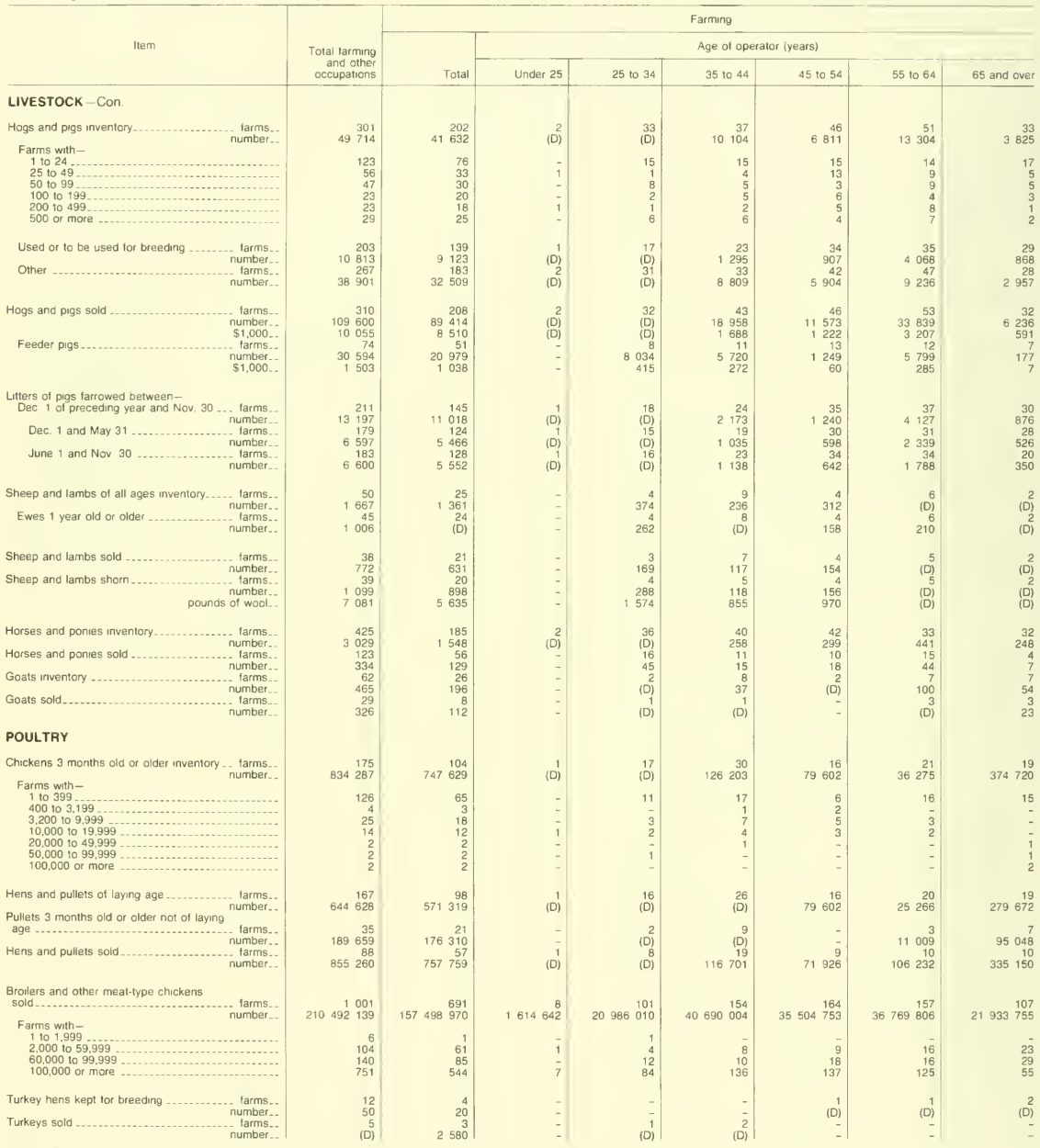


Table 50. Summary by Age and Principal Occupation of Operator: 1987-Con.

[For meaning of abbreviations and symbols, see introductory text]

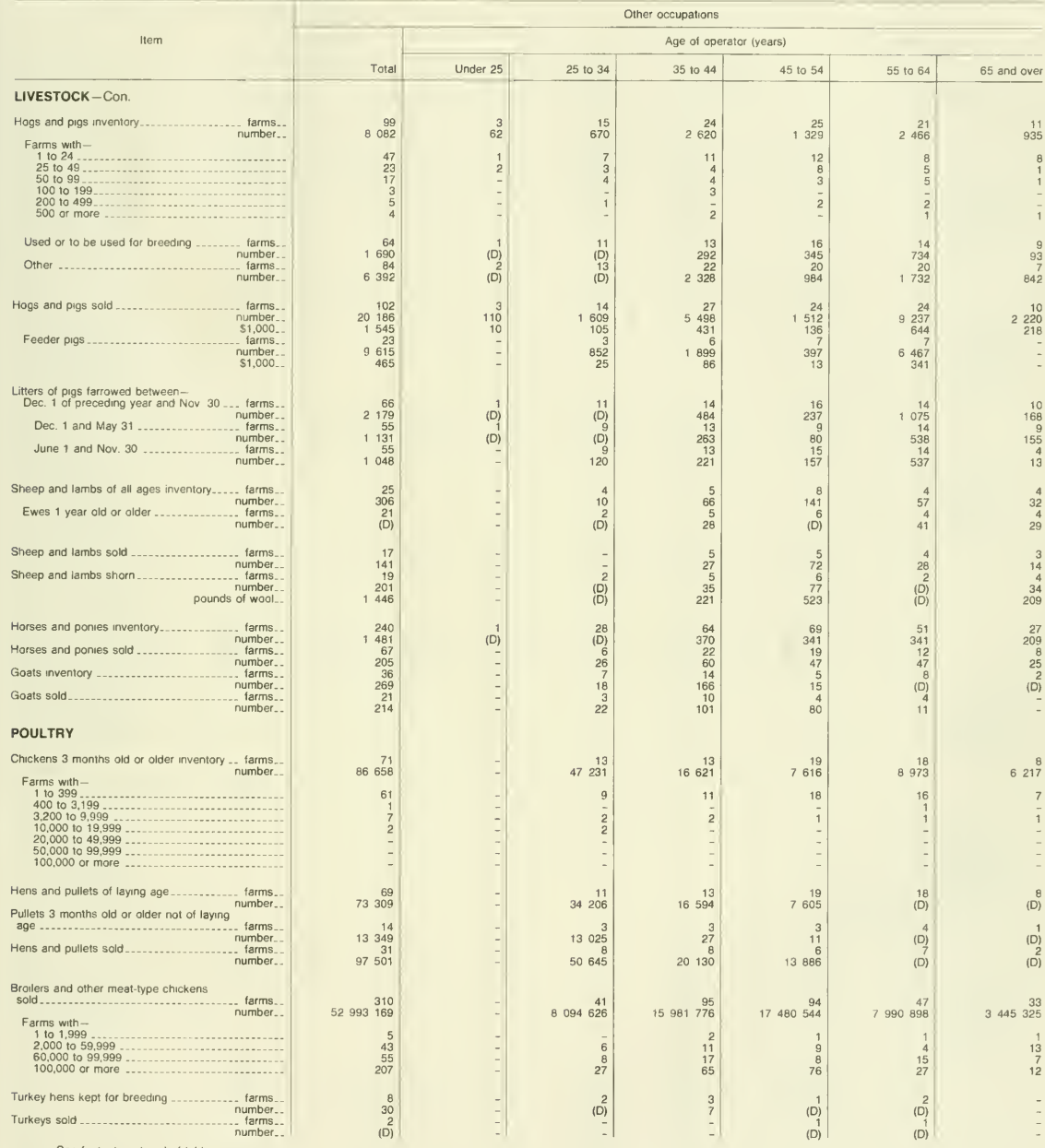


Table 50. Summary by Age and Principal Occupation of Operator: 1987-Con.

[For meaning of abbreviations and symbols, see introductory text]

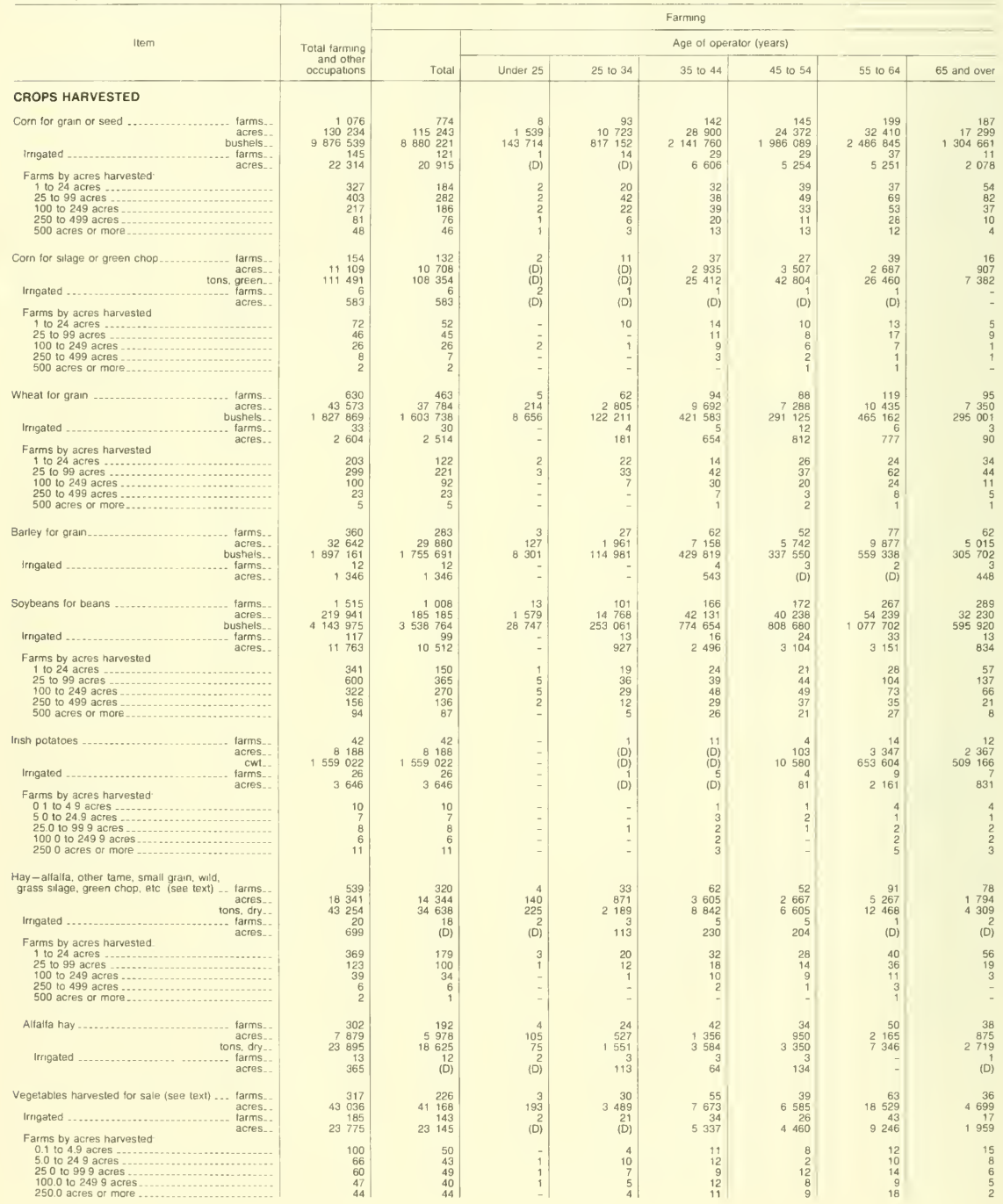

See lootnotes at end of table 
Table 50. Summary by Age and Principal Occupation of Operator: 1987-Con.

[For meaning of abbreviations and symbols, see introductory text]

\begin{tabular}{|c|c|c|c|c|c|c|c|}
\hline \multirow{3}{*}{ Item } & \multicolumn{7}{|c|}{ Other occupations } \\
\hline & \multirow[b]{2}{*}{ Total } & \multicolumn{6}{|c|}{ Age of operator (years) } \\
\hline & & Under 25 & 25 to 34 & 35 to 44 & 45 to 54 & 55 to 64 & 65 and over \\
\hline \multicolumn{8}{|l|}{ CROPS HARVESTED } \\
\hline $\begin{array}{c}\text { Corn for grain or seed } \ldots \ldots \ldots \\
\text { Irrigated }\end{array}$ & $\begin{array}{r}302 \\
14991 \\
996318 \\
24 \\
1399\end{array}$ & $\begin{array}{r}2 \\
\text { (D) } \\
\text { (D) } \\
-\end{array}$ & $\begin{array}{r}39 \\
\text { (D) } \\
\text { (D) } \\
1 \\
\text { (D) }\end{array}$ & $\begin{array}{r}3290 \\
196 \quad 051 \\
6 \\
(0)\end{array}$ & $\begin{array}{r}87 \\
4095 \\
288 \quad 855 \\
10 \\
427\end{array}$ & $\begin{array}{r}63 \\
3200 \\
237 \quad 319 \\
7 \\
435\end{array}$ & $\begin{array}{r}31 \\
1683 \\
105852 \\
-\end{array}$ \\
\hline 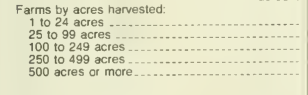 & $\begin{array}{r}143 \\
121 \\
31 \\
5 \\
2\end{array}$ & $\begin{array}{l}\bar{z} \\
- \\
-\end{array}$ & $\begin{array}{r}20 \\
12 \\
5 \\
2\end{array}$ & $\begin{array}{r}34 \\
39 \\
7 \\
- \\
-\end{array}$ & $\begin{array}{r}41 \\
36 \\
9 \\
1 \\
-\end{array}$ & $\begin{array}{r}32 \\
23 \\
6 \\
2 \\
-\end{array}$ & $\begin{array}{r}16 \\
9 \\
4 \\
2 \\
-\end{array}$ \\
\hline 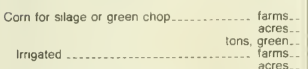 & $\begin{array}{r}22 \\
401 \\
137 \\
\end{array}$ & $\begin{array}{l}- \\
-\end{array}$ & $\begin{array}{r}4 \\
20 \\
107\end{array}$ & $\begin{array}{r}5 \\
1360 \\
362\end{array}$ & $\begin{array}{r}6 \\
43 \\
612 \\
\end{array}$ & $\begin{array}{r}6 \\
\text { (D) } \\
\text { (D) }\end{array}$ & $\begin{array}{l}1 \\
(D) \\
(D)\end{array}$ \\
\hline $\begin{array}{l}\text { Irrigated } \\
\text { Farms by acres harvested: }\end{array}$ & $=$ & : & - & - & $=$ & - & $\overline{-}$ \\
\hline 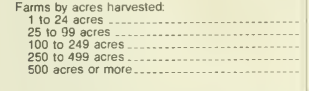 & $\begin{array}{r}20 \\
1 \\
-1 \\
-\end{array}$ & $\begin{array}{l}\vdots \\
\vdots \\
-\end{array}$ & $\begin{array}{l}4 \\
= \\
:\end{array}$ & $\begin{array}{l}4 \\
- \\
1 \\
-\end{array}$ & $\begin{array}{l}6 \\
: \\
- \\
-\end{array}$ & $\begin{array}{l}5 \\
1 \\
- \\
- \\
-\end{array}$ & $\begin{array}{l}1 \\
- \\
-\end{array}$ \\
\hline $\begin{array}{r}\text { Wheat for grain ................. farms.. } \\
\text { acres.. } \\
\text { bushels.. }\end{array}$ & $\begin{array}{rr}5 & 167 \\
224 & 131\end{array}$ & $\begin{array}{r}4 \\
308 \\
18820\end{array}$ & $\begin{array}{r}25 \\
833 \\
33787\end{array}$ & $\begin{array}{rr}38 \\
1 & 045 \\
37 & 964\end{array}$ & $\begin{array}{rr}43 \\
1538 \\
54 & 873 \\
\end{array}$ & $\begin{array}{rr}1 & 41 \\
64 & 634 \\
64 & 600\end{array}$ & $\begin{array}{r}16 \\
531 \\
14087\end{array}$ \\
\hline 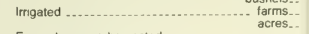 & $\begin{array}{r}3 \\
90\end{array}$ & - & - & (0) & (D) & $=$ & $=$ \\
\hline 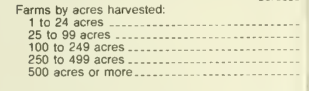 & $\begin{array}{r}81 \\
78 \\
8 \\
- \\
-\end{array}$ & $\begin{array}{l}4 \\
\vdots\end{array}$ & $\begin{array}{r}14 \\
10 \\
1 \\
- \\
-\end{array}$ & $\begin{array}{r}19 \\
19 \\
- \\
-\end{array}$ & $\begin{array}{r}21 \\
20 \\
2 \\
- \\
-\end{array}$ & $\begin{array}{r}19 \\
19 \\
3 \\
- \\
-\end{array}$ & $\begin{array}{l}8 \\
6 \\
2 \\
- \\
-\end{array}$ \\
\hline $\begin{array}{r}\text { Barley for gran................... farms.. } \\
\text { acres. } \\
\text { bushels.. } \\
\text { farms }\end{array}$ & $\begin{array}{r}77 \\
2762 \\
141470\end{array}$ & $\begin{array}{r}2 \\
\text { (D) } \\
\text { (D) }\end{array}$ & $\begin{array}{l}7 \\
(0) \\
(0)\end{array}$ & $\begin{array}{r}20 \\
603 \\
34708\end{array}$ & $\begin{array}{r}20 \\
733 \\
33858\end{array}$ & $\begin{array}{r}19 \\
939 \\
43864\end{array}$ & $\begin{array}{r}317 \\
18 \quad 574\end{array}$ \\
\hline 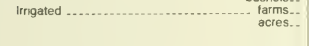 & - & - & $=$ & & $=$ & - & - \\
\hline $\begin{array}{r}\text { Soybeans for beans ................. } \\
\text { acres.. } \\
\text { bushels.. }\end{array}$ & $\begin{array}{rr}507 \\
34 \quad 756 \\
605 \quad 211\end{array}$ & $\begin{array}{r}12 \\
773 \\
13654\end{array}$ & $\begin{array}{r}62 \\
4588 \\
67543\end{array}$ & $\begin{array}{r}113 \\
7686 \\
115031\end{array}$ & $\begin{array}{rr}137 \\
11 & 017 \\
198 & 489\end{array}$ & $\begin{array}{rr}8 & 122 \\
160 & 733\end{array}$ & $\begin{array}{r}61 \\
2565 \\
49761\end{array}$ \\
\hline $\begin{array}{l}\text { Irrigated } \\
\text {...................... tarms. } \\
\text { acres }\end{array}$ & $\begin{array}{r}18 \\
1251\end{array}$ & - & (D) & $\begin{array}{r}5 \\
217\end{array}$ & $\begin{array}{r}7 \\
794\end{array}$ & $\begin{array}{r}3 \\
165\end{array}$ & (D) \\
\hline 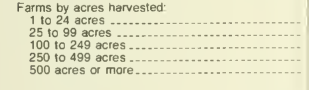 & $\begin{array}{r}191 \\
235 \\
52 \\
22 \\
7\end{array}$ & $\begin{array}{l}4 \\
6 \\
2 \\
- \\
-\end{array}$ & $\begin{array}{r}24 \\
26 \\
6 \\
6 \\
-\end{array}$ & $\begin{array}{r}37 \\
60 \\
9 \\
6 \\
1\end{array}$ & $\begin{array}{r}42 \\
68 \\
19 \\
5 \\
3\end{array}$ & $\begin{array}{r}52 \\
49 \\
14 \\
5 \\
2\end{array}$ & $\begin{array}{r}32 \\
26 \\
2 \\
i\end{array}$ \\
\hline Irish potatoes .......................... tarms_. & - & $\therefore$ & - & - & $\overline{-}$ & - & - \\
\hline Irrigated $\ldots$ & 5 & $=$ & - & $=$ & - & $=$ & - \\
\hline $\begin{array}{l}\text { Farms by acres harvested: } \\
0.1 \text { to } 49 \text { acres } \\
50 \text { to } 249 \text { acres } \\
25.0 \text { to } 999 \text { acres } \\
100.0 \text { to } 2499 \text { acres. } \\
250.0 \text { acres or more }\end{array}$ & $\begin{array}{l}\overline{-} \\
- \\
\overline{-} \\
\overline{-} \\
-\end{array}$ & $\begin{array}{l}z \\
z \\
z \\
z\end{array}$ & $\begin{array}{l}- \\
- \\
\overline{-} \\
-\end{array}$ & $\begin{array}{l}- \\
\vdots \\
\vdots \\
z\end{array}$ & $\begin{array}{l}\overline{-} \\
= \\
\overline{-} \\
=\end{array}$ & $\begin{array}{l}\overline{-} \\
= \\
= \\
= \\
-\end{array}$ & $\begin{array}{l}- \\
\vdots \\
-\end{array}$ \\
\hline $\begin{array}{l}\text { Hay - alfalfa, other tame, small grain, wild, } \\
\text { grass silage, green chop, etc. (see lext) .. tarms.. }\end{array}$ & 219 & $2^{2}$ & 32 & $\begin{array}{r}54 \\
711\end{array}$ & $\begin{array}{r}69 \\
644\end{array}$ & $\begin{array}{r}41 \\
515\end{array}$ & 21 \\
\hline $\begin{array}{c}\text { acres_- } \\
\text { tons, dry.- }\end{array}$ & $\begin{array}{rl}3 & 997 \\
8616\end{array}$ & $\begin{array}{l}\text { (D) } \\
\text { (D) }\end{array}$ & (D) & $\begin{array}{r}711 \\
1010 \\
\end{array}$ & 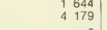 & $\begin{array}{r}515 \\
1083\end{array}$ & $\begin{array}{l}657 \\
1540\end{array}$ \\
\hline $\begin{array}{l}\text { Irrigated } \\
\text {................. farms.- } \\
\text { acres. }\end{array}$ & (D) & - & - & (D) & - & $\overline{-}$ & $=$ \\
\hline $\begin{array}{l}\text { Farms by acres harvested: } \\
1 \text { to } 24 \text { acres } \\
25 \text { to } 99 \text { acres } \\
100 \text { to } 249 \text { acres } \\
250 \text { to } 499 \text { acres } \\
500 \text { acres or more }\end{array}$ & $\begin{array}{r}190 \\
23 \\
5 \\
- \\
1\end{array}$ & $\begin{array}{l}2 \\
\overline{-} \\
-\end{array}$ & $\begin{array}{r}28 \\
4 \\
- \\
-\end{array}$ & $\begin{array}{r}47 \\
7 \\
- \\
-\end{array}$ & $\begin{array}{r}59 \\
6 \\
3 \\
1\end{array}$ & $\begin{array}{r}37 \\
4 \\
- \\
-\end{array}$ & $\begin{array}{r}17 \\
2 \\
2 \\
:\end{array}$ \\
\hline 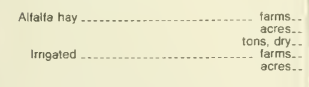 & $\begin{array}{rr}110 \\
1 & 901 \\
5 & 270 \\
1 \\
\\
\text { (D) }\end{array}$ & $\begin{array}{c}2 \\
\text { (D) } \\
\text { (D) } \\
-\end{array}$ & $\begin{array}{l}16 \\
\text { (D) } \\
\text { (D) } \\
-\end{array}$ & $\begin{array}{r}27 \\
282 \\
523 \\
1 \\
10)\end{array}$ & $\begin{array}{r}94 \\
241 \\
953 \\
- \\
-\end{array}$ & $\begin{array}{r}25 \\
214 \\
594 \\
- \\
-\end{array}$ & $\begin{array}{r}6 \\
241 \\
710 \\
-\end{array}$ \\
\hline $\begin{array}{l}\text { Vegetables harvested for sale (see lext) ... tarms.. } \\
\text { acres_. }\end{array}$ & $\begin{array}{r}91 \\
1867\end{array}$ & $\begin{array}{r}3 \\
24\end{array}$ & $\begin{array}{r}16 \\
289\end{array}$ & $\begin{array}{r}30 \\
542\end{array}$ & $\begin{array}{r}20 \\
844\end{array}$ & $\begin{array}{r}9 \\
57\end{array}$ & $\begin{array}{r}13 \\
111\end{array}$ \\
\hline 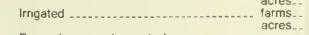 & $\begin{array}{r}401 \\
430\end{array}$ & $\begin{array}{r}24 \\
3 \\
24\end{array}$ & $\begin{array}{r}209 \\
30 \\
30\end{array}$ & $\begin{array}{r}17 \\
157\end{array}$ & $\begin{array}{r}844 \\
10 \\
369\end{array}$ & (D) & (D) \\
\hline $\begin{array}{l}\text { Farms by acres harvested } \\
0.1 \text { to } 4.9 \text { acres } \\
5.0 \text { to } 24.9 \text { acres } \\
25.0 \text { to } 99.9 \text { acres } \\
100.0 \text { to } 249.9 \text { acres. } \\
250.0 \text { acres or more }\end{array}$ & $\begin{array}{r}50 \\
23 \\
11 \\
7 \\
-\end{array}$ & $\begin{array}{l}2 \\
1 \\
\vdots \\
-\end{array}$ & $\begin{array}{l}9 \\
6 \\
\overline{1} \\
-\end{array}$ & $\begin{array}{r}18 \\
6 \\
4 \\
2 \\
-\end{array}$ & $\begin{array}{l}7 \\
4 \\
5 \\
4 \\
-\end{array}$ & $\begin{array}{l}6 \\
2 \\
1 \\
- \\
-\end{array}$ & $\begin{array}{l}8 \\
4 \\
1 \\
\\
-\end{array}$ \\
\hline
\end{tabular}

See footnotes at end of table. 
Table 50. Summary by Age and Principal Occupation of Operator: 1987-Con.

[For meaning of abbreviations and symbols, see introductory text]

\begin{tabular}{|c|c|c|c|c|c|c|c|c|}
\hline \multirow{3}{*}{ Htem } & \multirow{3}{*}{$\begin{array}{r}\text { Total farming } \\
\text { and other } \\
\text { occupations }\end{array}$} & \multicolumn{7}{|c|}{ Farming } \\
\hline & & \multirow[b]{2}{*}{ Total } & \multicolumn{6}{|c|}{ Age of operator (years) } \\
\hline & & & Under 25 & 251034 & 351044 & 45 to 54 & 55 to 64 & 65 and over \\
\hline \multicolumn{9}{|l|}{ CROPS HARVESTED - CON. } \\
\hline \multicolumn{9}{|l|}{$\begin{array}{l}\text { Vegetables harvested for sale (see text)- } \\
\text { Con }\end{array}$} \\
\hline $\begin{array}{l}\begin{array}{l}\text { Green peas, excluding green cowpeas, } \\
\text { harvested for sale }\end{array} \\
\text { Imngated }\end{array}$ & $\begin{array}{r}66 \\
9851 \\
45 \\
5752\end{array}$ & $\begin{array}{r}59 \\
9603 \\
43 \\
(\mathrm{D})\end{array}$ & (D) & $\begin{array}{r}8 \\
\text { (D) } \\
5 \\
\text { (D) }\end{array}$ & $\begin{array}{r}18 \\
1849 \\
15 \\
1560\end{array}$ & $\begin{array}{r}6 \\
998 \\
4 \\
650\end{array}$ & $\begin{array}{r}20 \\
4925 \\
15 \\
2458\end{array}$ & $\begin{array}{r}6 \\
955 \\
4 \\
599\end{array}$ \\
\hline 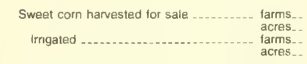 & $\begin{array}{r}109 \\
7643 \\
68 \\
5 \quad 739\end{array}$ & $\begin{array}{r}75 \\
7205 \\
59 \\
5594\end{array}$ & $\begin{array}{r}2 \\
\text { (D) } \\
2 \\
\text { (D) }\end{array}$ & $\begin{array}{r}12 \\
\text { (D) } \\
9 \\
(D)\end{array}$ & $\begin{array}{r}18 \\
1408 \\
13 \\
1185\end{array}$ & $\begin{array}{r}18 \\
1731 \\
15 \\
1418\end{array}$ & $\begin{array}{r}25 \\
3321 \\
16 \\
2327\end{array}$ & $\begin{array}{r}10 \\
254 \\
4 \\
209\end{array}$ \\
\hline $\begin{array}{l}\text { Land in orchards ............ farms.. } \\
\text { Irrigated } \\
\text { acres. } \\
\text { acres. }\end{array}$ & $\begin{array}{r}36 \\
+378 \\
8 \\
527\end{array}$ & $\begin{array}{r}20 \\
1340 \\
8 \\
527\end{array}$ & $\overline{-}$ & 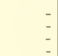 & $\begin{array}{r}3 \\
24 \\
3 \\
24\end{array}$ & $\begin{array}{r}6 \\
849 \\
3 \\
\text { (D) }\end{array}$ & $\begin{array}{r}5 \\
431 \\
2 \\
\text { (D) }\end{array}$ & $\begin{array}{r}6 \\
36 \\
-\end{array}$ \\
\hline $\begin{array}{l}\text { Farms by bearing and nonbearing acres. } \\
0.1 \text { to } 4.9 \text { acres } \\
50 \text { to } 24.9 \text { acres } \\
250 \text { to } 999 \text { acres } \\
100.0 \text { to } 249.9 \text { acres } \\
250.0 \text { acres or more }\end{array}$ & $\begin{array}{r}21 \\
11 \\
1 \\
- \\
3\end{array}$ & $\begin{array}{l}921 \\
9 \\
1 \\
3\end{array}$ & $\begin{array}{l}- \\
\overline{-} \\
-\end{array}$ & $\begin{array}{l}- \\
\overline{-} \\
-\end{array}$ & $\begin{array}{l}1 \\
2 \\
- \\
- \\
-\end{array}$ & $\begin{array}{l}2 \\
2 \\
\overline{2}\end{array}$ & \begin{tabular}{l|}
3 \\
$\overline{1}$ \\
\\
1
\end{tabular} & $\begin{array}{l}3 \\
3 \\
-\end{array}$ \\
\hline
\end{tabular}

See footnotes at end of table. 


\section{Table 50. Summary by Age and Principal Occupation of Operator: 1987-Con.}

[For meaning of abbreviations and symbols, see introductory text]

\begin{tabular}{|c|c|c|c|c|c|c|c|}
\hline \multirow{3}{*}{ Item } & \multicolumn{7}{|c|}{ Other occupations } \\
\hline & \multirow[b]{2}{*}{ Total } & \multicolumn{6}{|c|}{ Age of operator (years) } \\
\hline & & Under 25 & 25 to 34 & 35 to 44 & 45 to 54 & 55 to 64 & 65 and over \\
\hline \multicolumn{8}{|l|}{ CROPS HARVESTED-CON. } \\
\hline $\begin{array}{l}\text { Vegetables harvested for sale (see text)- } \\
\text { Con. }\end{array}$ & & & & & & & \\
\hline $\begin{array}{c}\begin{array}{c}\text { Green peas, excluding green cowpeas, } \\
\text { harvested for sale }\end{array} \\
\text { Imgated }\end{array}$ & $\begin{array}{r}7 \\
248 \\
2 \\
\text { (D) }\end{array}$ & $\begin{array}{l}- \\
\overline{-}\end{array}$ & $\begin{array}{r}2 \\
(D) \\
-\end{array}$ & $\begin{array}{r}3 \\
2 \\
2 \\
\text { (D) }\end{array}$ & (D) & $\begin{array}{l}- \\
\overline{-} \\
\overline{-}\end{array}$ & (D) \\
\hline $\begin{array}{r}\text { Sweet corn harvested for sale ........... } \\
\text { Irrigated } \\
\text { acres... } \\
\text { farms.. } \\
\text { acres.. }\end{array}$ & $\begin{array}{r}24 \\
437 \\
9 \\
146\end{array}$ & $\begin{array}{l}1 \\
\text { (D) } \\
1 \\
\text { (D) }\end{array}$ & $\begin{array}{r}6 \\
(D) \\
3 \\
23\end{array}$ & $\begin{array}{r}7 \\
83 \\
2 \\
\text { (D) }\end{array}$ & $\begin{array}{r}5 \\
287 \\
3 \\
87\end{array}$ & $\begin{array}{r}1 \\
\text { (D) } \\
- \\
-\end{array}$ & $\begin{array}{r}4 \\
21 \\
- \\
-\end{array}$ \\
\hline $\begin{array}{l}\text { Farms by bearing and nonbearing acres: } \\
0.1 \text { to } 49 \text { acres } \\
5.0 \text { to } 24.9 \text { acres } \\
25.0 \text { to } 99.9 \text { acres } \\
100.0 \text { to } 2499 \text { acres } \\
250.0 \text { acres or more }\end{array}$ & $\begin{array}{r}- \\
12 \\
4 \\
- \\
- \\
-\end{array}$ & $\begin{array}{l}- \\
- \\
- \\
-\end{array}$ & $\begin{array}{l}- \\
- \\
- \\
- \\
- \\
-\end{array}$ & $\begin{array}{l}- \\
4 \\
2 \\
- \\
- \\
-\end{array}$ & $\begin{array}{l}- \\
1 \\
- \\
- \\
-\end{array}$ & $\begin{array}{l}- \\
+ \\
- \\
- \\
- \\
-\end{array}$ & $\begin{array}{l}- \\
6 \\
2 \\
- \\
-\end{array}$ \\
\hline
\end{tabular}

'Data are based on a sample of tarms

${ }^{2} \mathrm{Farms}$ with total praduction expenses equal to market value of agricultural products sold are included as farms with gains of less than $\$ 1,000$. 
Table 51. Summary by Size of Farm: 1987

[For meaning of abbrevations and symbols, see introductory text]

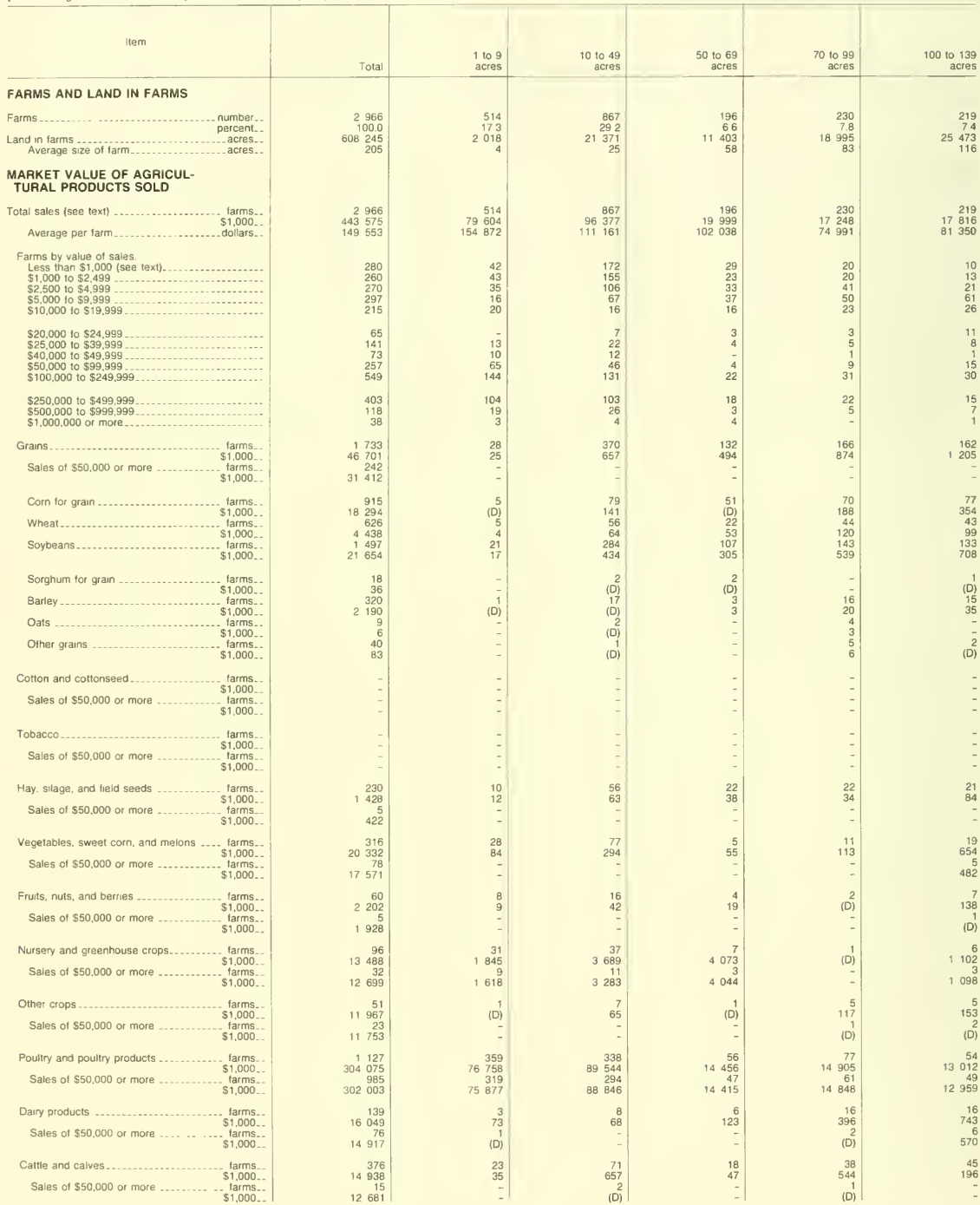

See footnotes at end of table 
Table 51. Summary by Size of Farm: 1987-Con.

[For meaning of abbreviations and symbols, see introductory text]

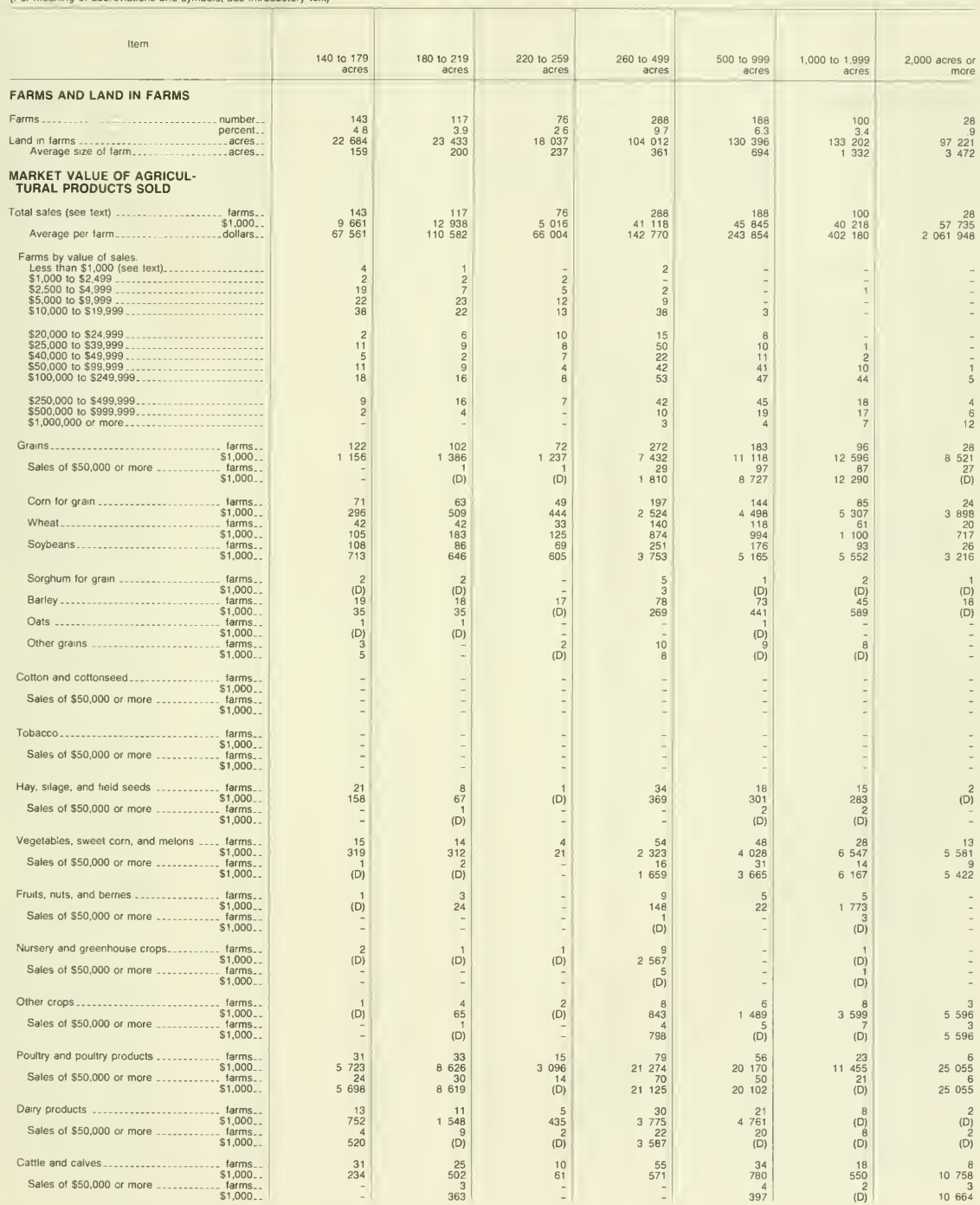


Table 51. Summary by Size of Farm: 1987-Con.

[For meaning of abbreviations and symbols. see introductory text]

\begin{tabular}{|c|c|c|c|c|c|c|}
\hline Item & Total & $\begin{array}{l}1 \text { to } 9 \\
\text { acres }\end{array}$ & $\begin{array}{r}10 \text { to } 49 \\
\text { acres } \\
\end{array}$ & $\begin{array}{r}50 \text { to } 69 \\
\text { acres }\end{array}$ & $\begin{array}{r}70 \text { to } 99 \\
\text { acres }\end{array}$ & $\begin{array}{r}100 \text { to } 139 \\
\text { acres }\end{array}$ \\
\hline \multicolumn{7}{|l|}{$\begin{array}{l}\text { MARKET VALUE OF AGRICUL- } \\
\text { TURAL PRODUCTS SOLD - Con. }\end{array}$} \\
\hline 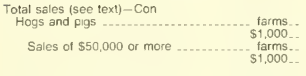 & $\begin{array}{r}310 \\
10 \quad 055 \\
44 \\
8102\end{array}$ & $\begin{array}{r}37 \\
649 \\
5 \\
439\end{array}$ & $\begin{array}{r}81 \\
1043 \\
3 \\
634\end{array}$ & $\begin{array}{r}24 \\
680 \\
1 \\
(D)\end{array}$ & $\begin{array}{r}26 \\
174 \\
1 \\
(D)\end{array}$ & $\begin{array}{r}28 \\
348 \\
2 \\
(D)\end{array}$ \\
\hline 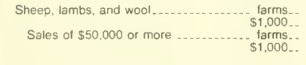 & $\begin{array}{r}45 \\
54 \\
- \\
-\end{array}$ & $\begin{array}{r}7 \\
(D) \\
-\end{array}$ & $\begin{array}{l}8 \\
3 \\
- \\
-\end{array}$ & $\begin{array}{r}2 \\
\left(D^{2}\right) \\
-\end{array}$ & $\begin{array}{r}6 \\
12 \\
- \\
-\end{array}$ & $\stackrel{3}{11}$ \\
\hline 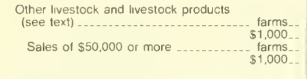 & $\begin{array}{r}175 \\
2286 \\
1731\end{array}$ & $\begin{array}{r}37 \\
110 \\
-\end{array}$ & $\begin{array}{r}63 \\
252 \\
- \\
-\end{array}$ & $\begin{array}{r}10 \\
9 \\
- \\
-\end{array}$ & $\begin{array}{r}22 \\
57 \\
- \\
-\end{array}$ & $\begin{array}{r}19 \\
173 \\
2 \\
(D)\end{array}$ \\
\hline \multicolumn{7}{|l|}{ FARM PRODUCTION EXPENSES } \\
\hline $\begin{array}{c}\text { Total farm production expenses ............ farms... } \\
\text { s1,000.. } \\
\text { Average per farm ................... doilars.. }\end{array}$ & $\begin{array}{rr}2 & 965 \\
377 & 102 \\
127 & 184\end{array}$ & $\begin{array}{r}523 \\
64713 \\
123734\end{array}$ & $\begin{array}{r}785 \\
79841 \\
101709\end{array}$ & $\begin{array}{rr}16 & 179 \\
93 & 737\end{array}$ & $\begin{array}{r}298 \\
15760 \\
52886\end{array}$ & $\begin{array}{l}187 \\
14726 \\
78751\end{array}$ \\
\hline Livestock and poultry purchased _........ tarms... & $\begin{array}{rl}1 & 374 \\
53 & 522\end{array}$ & $\begin{array}{r}370 \\
8558\end{array}$ & $\begin{array}{r}406 \\
15823\end{array}$ & $\begin{array}{r}67 \\
1855\end{array}$ & $\begin{array}{r}98 \\
2143\end{array}$ & $\begin{array}{r}76 \\
1655\end{array}$ \\
\hline $\begin{array}{l}\text { Farms with expenses of - } \\
\$ 1 \text { to } \$ 4,999 \\
\$ 5.000 \text { to } \$ 24.999 \\
\$ 25,000 \text { to } \$ 99.999 . \ldots \\
\$ 100.000 \text { or more }\end{array}$ & $\begin{array}{r}316 \\
517 \\
512 \\
29\end{array}$ & $\begin{array}{r}48 \\
190 \\
128 \\
4\end{array}$ & $\begin{array}{r}102 \\
145 \\
154 \\
5\end{array}$ & \begin{tabular}{r|}
15 \\
28 \\
22 \\
2
\end{tabular} & $\begin{array}{r}38 \\
21 \\
38 \\
1\end{array}$ & $\begin{array}{r}27 \\
24 \\
24 \\
1\end{array}$ \\
\hline Feed lor hivestock and poultry .......... farms.. & $\begin{array}{rr}1 & 637 \\
187 & 183\end{array}$ & $\begin{array}{r}398 \\
47037\end{array}$ & $49 \begin{array}{r}500 \\
399\end{array}$ & $9 \begin{array}{r}85 \\
961\end{array}$ & $9 \begin{array}{r}124 \\
9988\end{array}$ & 9203 \\
\hline 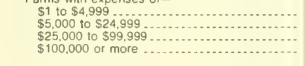 & $\begin{array}{l}428 \\
138 \\
373 \\
698\end{array}$ & $\begin{array}{r}49 \\
32 \\
123 \\
194\end{array}$ & $\begin{array}{r}164 \\
18 \\
118 \\
200\end{array}$ & \begin{tabular}{r|}
37 \\
2 \\
17 \\
29
\end{tabular} & $\begin{array}{l}52 \\
12 \\
16 \\
44\end{array}$ & $\begin{array}{l}20 \\
18 \\
13 \\
40\end{array}$ \\
\hline $\begin{array}{c}\text { Commercialily mixed formula feeds ..... farms... } \\
\$ 1,000 \ldots . .\end{array}$ & $\begin{array}{rr}1 & 341 \\
180 & 490\end{array}$ & $\begin{array}{r}367 \\
46429\end{array}$ & $48 \begin{array}{r}391 \\
905\end{array}$ & $9 \begin{array}{r}59 \\
242\end{array}$ & $9 \begin{array}{l}101 \\
9709\end{array}$ & $\begin{array}{r}69 \\
8923\end{array}$ \\
\hline 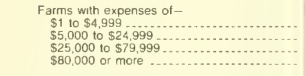 & $\begin{array}{r}242 \\
60 \\
255 \\
784\end{array}$ & $\begin{array}{r}42 \\
15 \\
74 \\
236\end{array}$ & $\begin{array}{r}71 \\
3 \\
89 \\
228\end{array}$ & $\begin{array}{r}11 \\
2 \\
10 \\
36\end{array}$ & $\begin{array}{r}34 \\
8 \\
8 \\
51\end{array}$ & $\begin{array}{r}15 \\
1 \\
10 \\
43\end{array}$ \\
\hline $\begin{array}{l}\text { Seeds, bulbs, plants, and trees } \ldots . . . . . . . \\
\$ 1,000 \ldots\end{array}$ & \begin{tabular}{ll|l}
1 & 899 \\
8 & 667
\end{tabular} & $\begin{array}{l}95 \\
49\end{array}$ & $\begin{array}{l}368 \\
362\end{array}$ & $\begin{array}{l}129 \\
615\end{array}$ & $\begin{array}{l}214 \\
175\end{array}$ & $\begin{array}{l}157 \\
234\end{array}$ \\
\hline $\begin{array}{l}\text { Farms with expenses of - } \\
\$ 1 \text { to } \$ 999 \\
\$ 1,000 \text { to } \$ 4,999 \\
\$ 5,000 \text { to } \$ 24,999 \\
\$ 25,000 \text { or more }\end{array}$ & $\begin{array}{r}928 \\
616 \\
301 \\
54\end{array}$ & $\begin{array}{r}88 \\
4 \\
3 \\
-\end{array}$ & \begin{tabular}{r|r}
339 \\
17 \\
10 \\
2
\end{tabular} & $\begin{array}{r}100 \\
27 \\
\overline{2}\end{array}$ & $\begin{array}{r}162 \\
50 \\
2 \\
-\end{array}$ & $\begin{array}{r}81 \\
70 \\
5 \\
1\end{array}$ \\
\hline $\begin{array}{l}\text { Commercal fertilizer ................. farms... } \\
\$ 1,000 .\end{array}$ & $\begin{array}{r}1807 \\
15802\end{array}$ & $\begin{array}{r}120 \\
46\end{array}$ & $\begin{array}{l}338 \\
167\end{array}$ & $\begin{array}{l}112 \\
211\end{array}$ & $\begin{array}{l}204 \\
350\end{array}$ & $\begin{array}{l}131 \\
378\end{array}$ \\
\hline $\begin{array}{l}\text { Farms with expenses of } \\
\$ 1 \text { to } \$ 4,999 \\
\$ 5,00010 \$ 24,999 \\
\$ 25,000 \text { to } \$ 49,999 \\
\$ 50,000 \text { or more }\end{array}$ & $\begin{array}{r}1206 \\
445 \\
97 \\
59\end{array}$ & $\begin{array}{r}119 \\
1 \\
-\end{array}$ & $\begin{array}{r}337 \\
1 \\
-\end{array}$ & $\begin{array}{r}111 \\
\overline{1}\end{array}$ & $\begin{array}{r}199 \\
5 \\
- \\
-\end{array}$ & $\begin{array}{r}108 \\
23 \\
- \\
-\end{array}$ \\
\hline $\begin{array}{l}\text { Agricultural chemicals } \\
.\end{array}$ & $\begin{array}{rr}1 & 973 \\
11 & 197\end{array}$ & $\begin{array}{r}137 \\
68\end{array}$ & $\begin{array}{l}425 \\
233\end{array}$ & $\begin{array}{l}144 \\
161\end{array}$ & $\begin{array}{l}208 \\
247\end{array}$ & $\begin{array}{l}128 \\
268\end{array}$ \\
\hline $\begin{array}{l}\text { Farms with expenses of - } \\
\$ 1 \text { to } \$ 4,999 \\
\$ 5,000 \text { to } \$ 24,999 \\
\$ 25,000 \text { to } \$ 9,999 \\
\$ 50,000 \text { or more }\end{array}$ & $\begin{array}{r}1447 \\
444 \\
53 \\
29\end{array}$ & $\begin{array}{r}136 \\
1 \\
- \\
-\end{array}$ & $\begin{array}{r}420 \\
5 \\
-\end{array}$ & $\begin{array}{r}142 \\
1 \\
1 \\
-\end{array}$ & $\begin{array}{r}206 \\
2 \\
-\end{array}$ & $\begin{array}{r}114 \\
14 \\
- \\
-\end{array}$ \\
\hline $\begin{aligned} & \text { Petroleum products } \text { tarms.................... } \\
& \$ 11,000 . .\end{aligned}$ & $\begin{array}{ll}2 & 692 \\
8 & 787\end{array}$ & $\begin{array}{l}422 \\
652\end{array}$ & $\begin{array}{r}690 \\
1160\end{array}$ & $\begin{array}{l}167 \\
373\end{array}$ & $\begin{array}{l}276 \\
328\end{array}$ & $\begin{array}{l}170 \\
265\end{array}$ \\
\hline 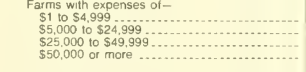 & $\begin{array}{r}274 \\
377 \\
27 \\
14\end{array}$ & $\begin{array}{r}388 \\
33 \\
1 \\
-\end{array}$ & $\begin{array}{r}662 \\
24 \\
2 \\
2\end{array}$ & $\begin{array}{r}159 \\
6 \\
1 \\
1\end{array}$ & $\begin{array}{r}270 \\
6 \\
- \\
-\end{array}$ & $\begin{array}{r}163 \\
7 \\
:\end{array}$ \\
\hline Gasoline and gasohol................. farms... & 2 356 & 354 & 596 & 149 & 261 & 154 \\
\hline Diesel fuel ......................... tarms. & $\begin{array}{l}3226 \\
1 \\
1\end{array} 494$ & $\begin{array}{l}269 \\
103\end{array}$ & $\begin{array}{l}456 \\
204\end{array}$ & $\begin{array}{r}115 \\
83\end{array}$ & $\begin{array}{l}187 \\
180\end{array}$ & $\begin{array}{l}126 \\
114\end{array}$ \\
\hline 51,000 & 3905 & 116 & $\begin{array}{r}365 \\
27\end{array}$ & 84 & 88 & 74 \\
\hline Natural gas ............................. farms.. & $\begin{array}{r}93 \\
331\end{array}$ & $\begin{array}{l}12 \\
26\end{array}$ & $\begin{array}{r}27 \\
117\end{array}$ & (D) & (D) & $\begin{array}{l}7 \\
4\end{array}$ \\
\hline $\begin{array}{l}\text { LP gas, fuel oil, kerosene, motor oil, } \\
\text { grease, etc ...................... tarms. } \\
\$ 1,000 .\end{array}$ & $\begin{array}{l}1873 \\
1 \\
1 \\
325\end{array}$ & $\begin{array}{l}260 \\
240\end{array}$ & $\begin{array}{l}316 \\
223\end{array} \mid$ & $\begin{array}{l}89 \\
\text { (D) }\end{array}$ & $\begin{array}{l}202 \\
\text { (D) }\end{array}$ & $\begin{array}{r}136 \\
61\end{array}$ \\
\hline
\end{tabular}


Table 51. Summary by Size of Farm: 1987-Con.

[For meaning of abbreviations and symbols, see introductory text]

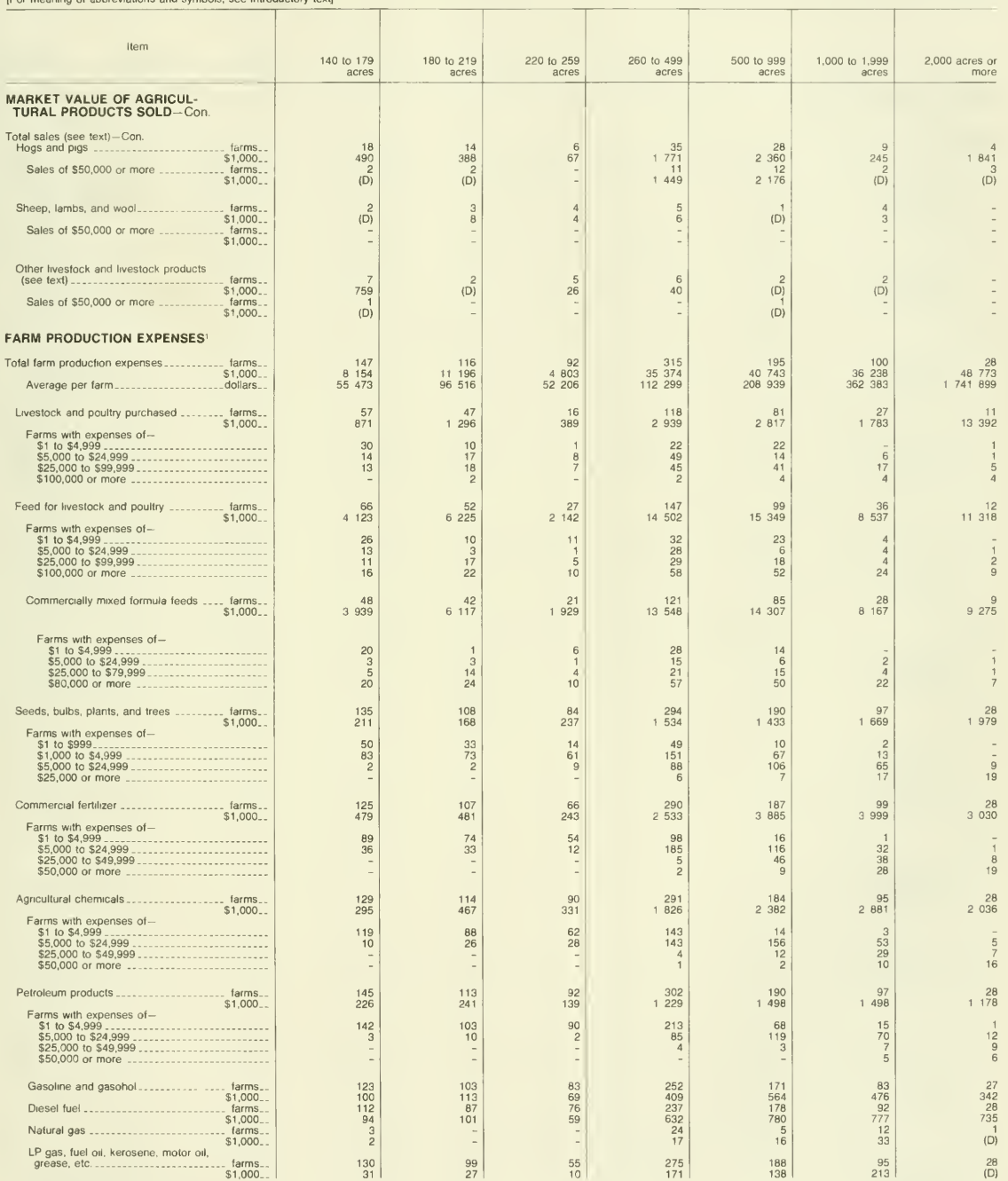

See footnotes at end of table. 
Table 51. Summary by Size of Farm: 1987-Con.

[For meaning of abbreviations and symbols, see introductory text]

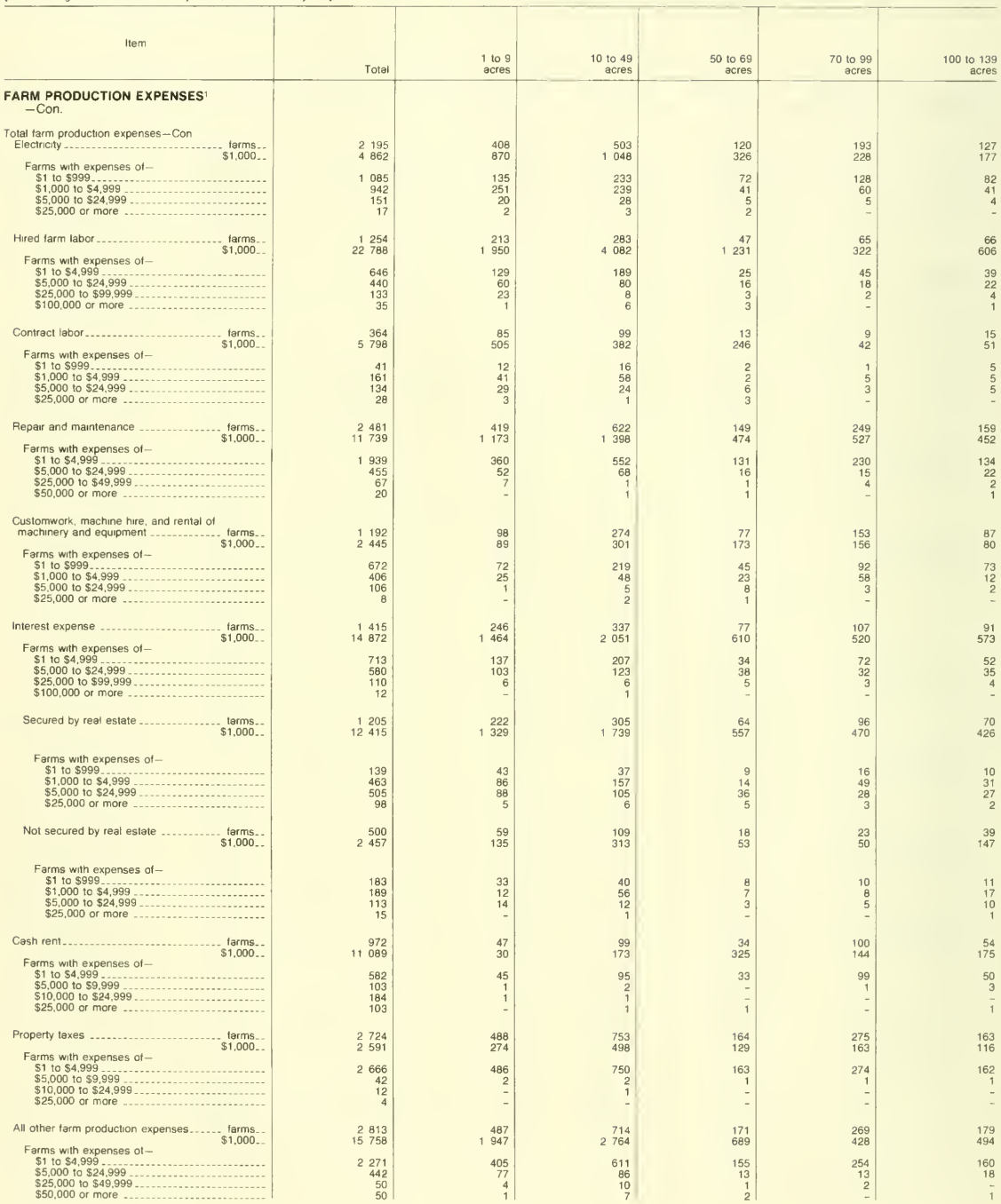


Table 51. Summary by Size of Farm: 1987-Con.

[For meaning of abbreviations and symbols, see introductory text]

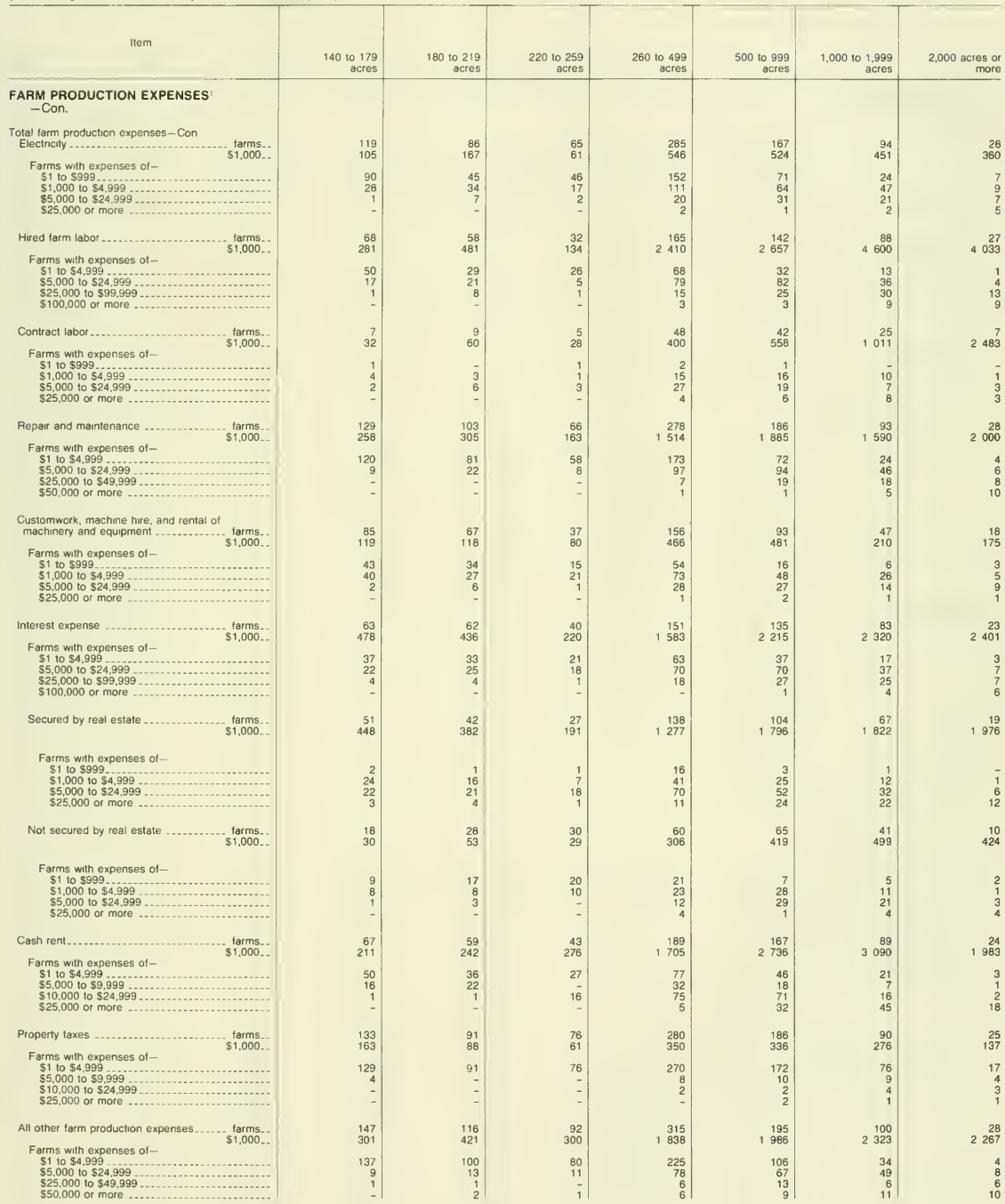


Table 51. Summary by Size of Farm: 1987-Con.

[For meaning of abbreviations and symbols, see introductory text]

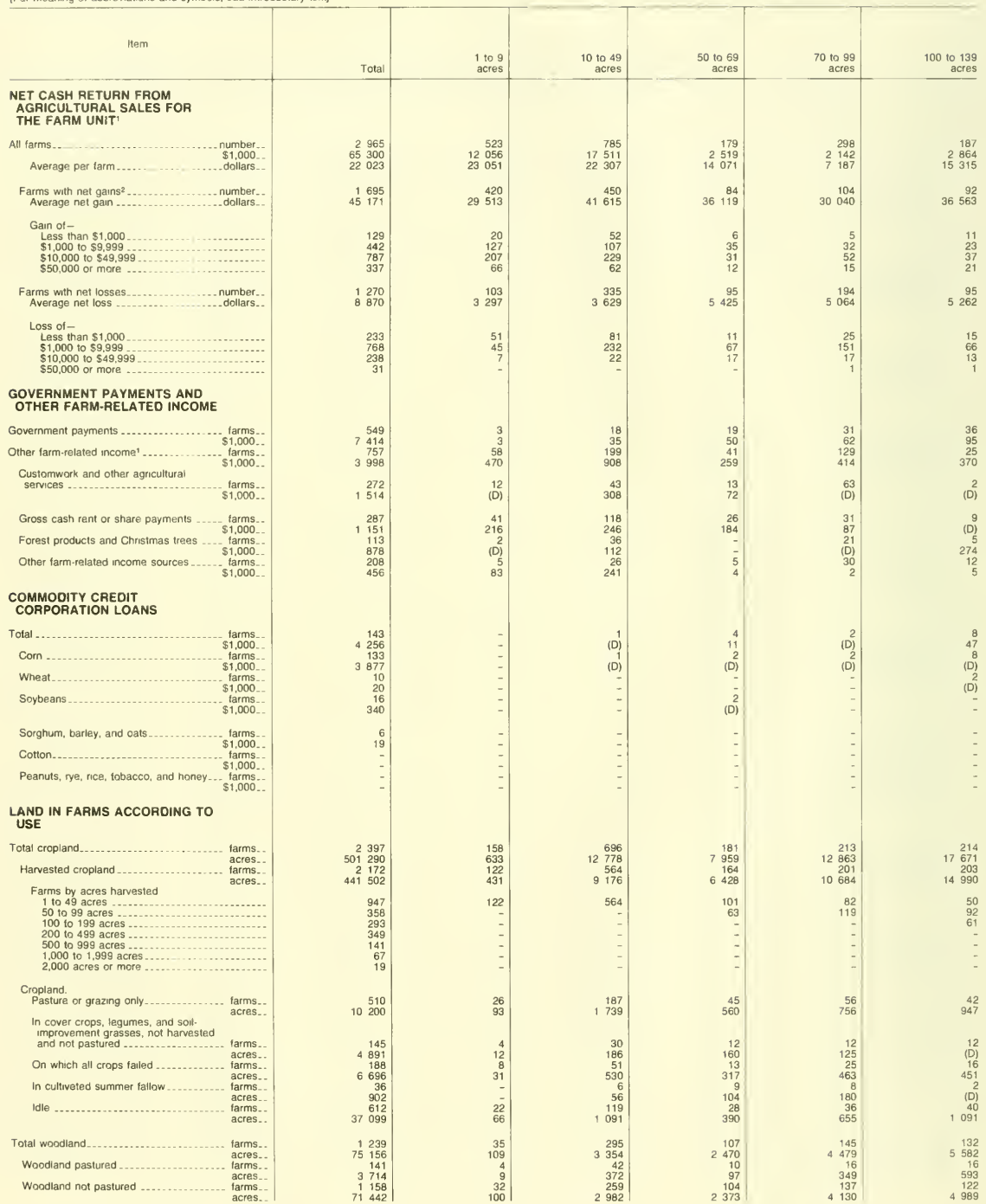

See footnotes at end of table. 
Table 51. Summary by Size of Farm: 1987-Con.

[For meaning of abbrevations and symbols, see introductory text]

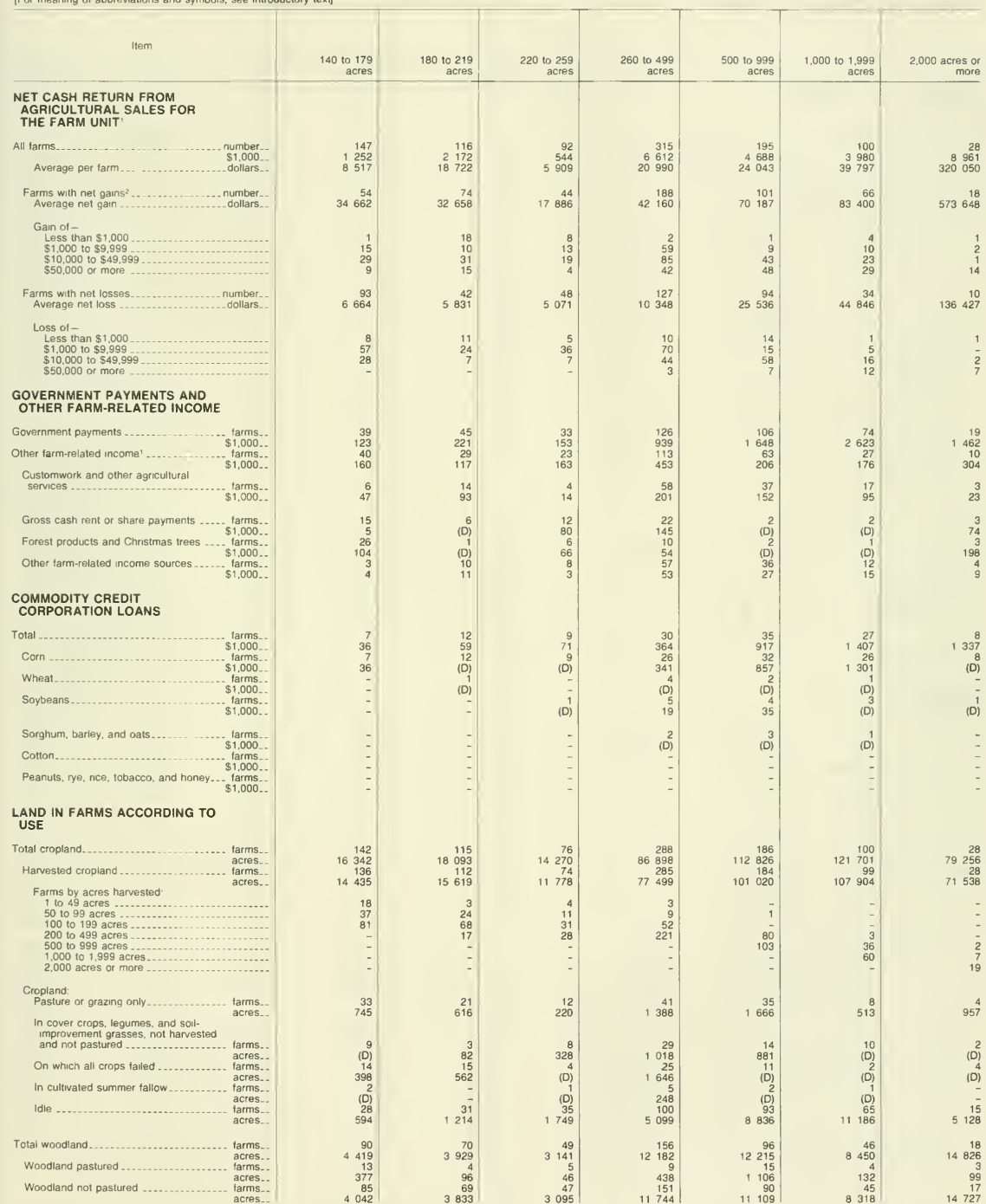


Table 51. Summary by Size of Farm: 1987-Con.

[For meaning of abbreviations and symbols, see introductory text]

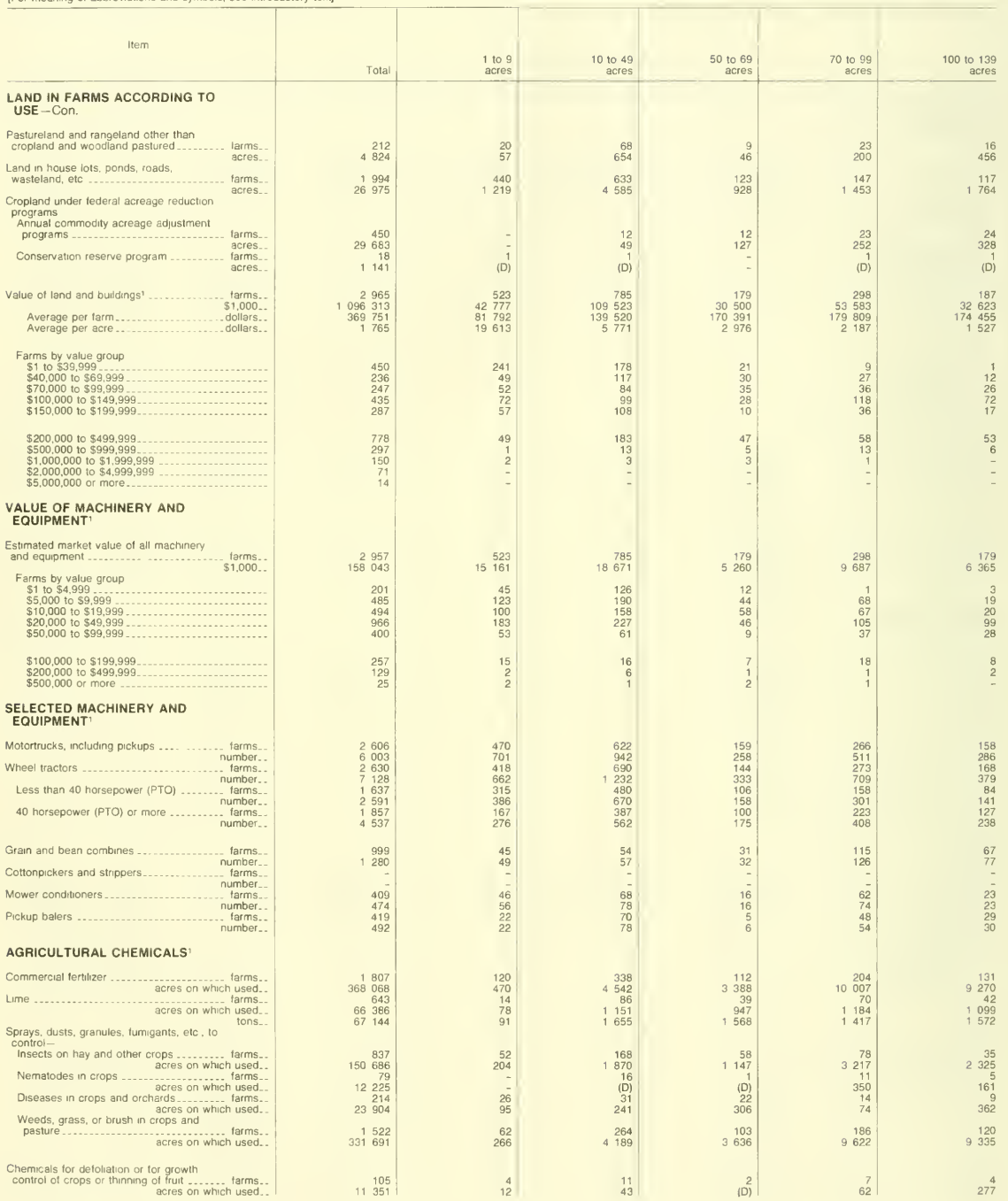


Table 51. Summary by Size of Farm: 1987-Con.

[For meaning of abbreviations and symbols, see introductory text]

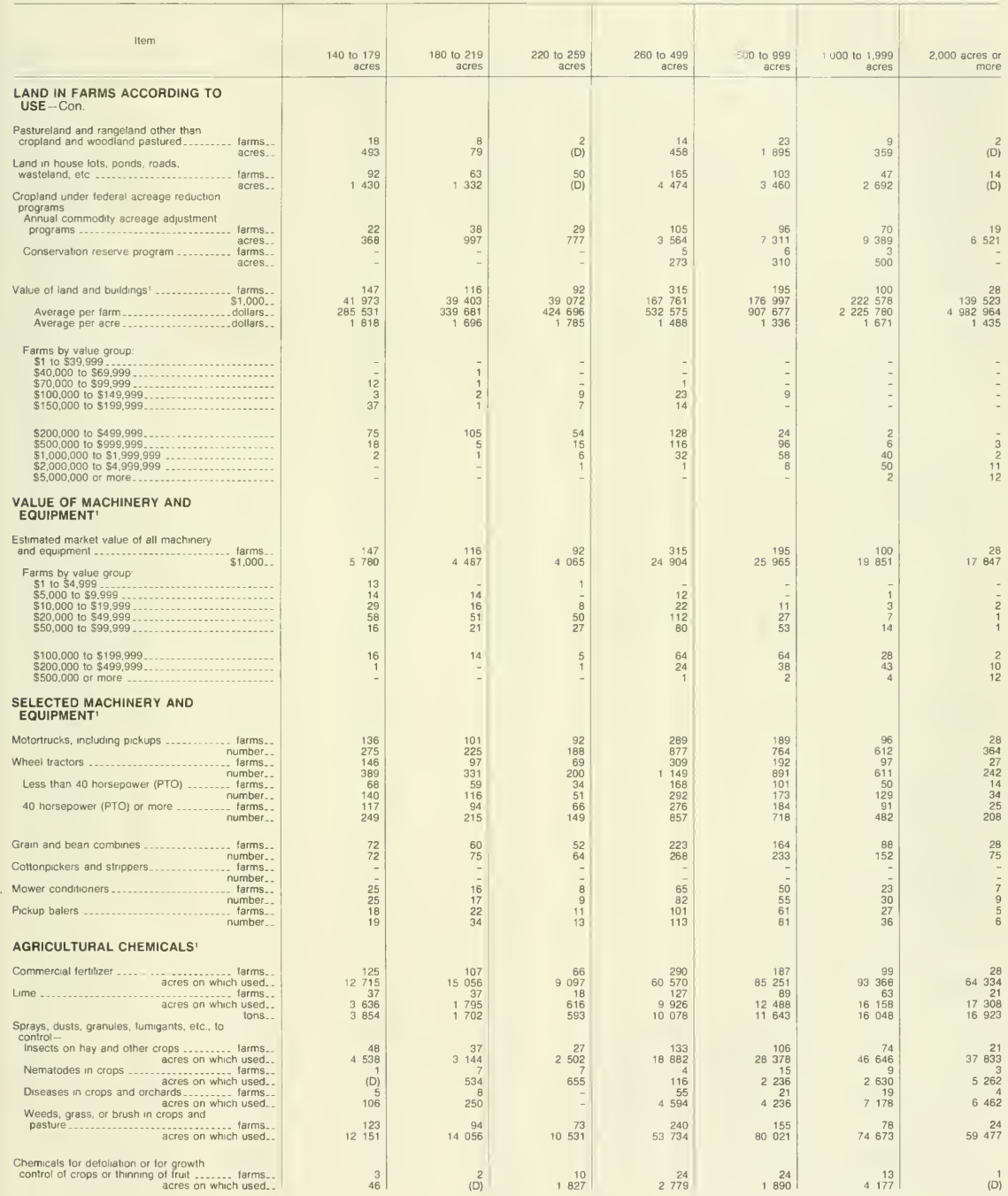


Table 51. Summary by Size of Farm: 1987-Con.

[For meaning of abbreviations and symbols, see introductory text]

\begin{tabular}{|c|c|c|c|c|c|c|}
\hline Item & Total & $\begin{array}{l}1 \text { to } 9 \\
\text { acres }\end{array}$ & $\begin{array}{r}10 \text { to } 49 \\
\text { acres }\end{array}$ & $\begin{array}{r}50 \text { to } 69 \\
\text { acres }\end{array}$ & $\begin{array}{r}70 \text { to } 99 \\
\text { acres }\end{array}$ & $\begin{array}{r}1,0 \text { to } 139 \\
\text { acres }\end{array}$ \\
\hline \multicolumn{7}{|l|}{$\begin{array}{l}\text { TENURE AND RACE OF } \\
\text { OPERATOR }\end{array}$} \\
\hline $\begin{array}{l}\text { All operators } \\
\text { Full owners } \\
\text { Part owners } \\
\text { Tenants }\end{array}$ & $\begin{array}{r}2966 \\
1825 \\
857 \\
284\end{array}$ & $\begin{array}{r}514 \\
464 \\
17 \\
33\end{array}$ & $\begin{array}{r}867 \\
692 \\
108 \\
67\end{array}$ & $\begin{array}{r}196 \\
132 \\
43 \\
21\end{array}$ & $\begin{array}{r}230 \\
140 \\
66 \\
24\end{array}$ & $\begin{array}{r}219 \\
124 \\
74 \\
21\end{array}$ \\
\hline $\begin{array}{l}\text { White } \\
\text { Full owners } \\
\text { Part owners } \ldots \ldots \ldots \\
\text { Tenants }\end{array}$ & \begin{tabular}{ll|}
2 & 914 \\
1 & 791 \\
845 \\
278
\end{tabular} & $\begin{array}{r}500 \\
456 \\
15 \\
29\end{array}$ & $\begin{array}{r}845 \\
673 \\
107 \\
65\end{array}$ & $\begin{array}{r}193 \\
130 \\
42 \\
21\end{array}$ & $\begin{array}{r}228 \\
139 \\
65 \\
24\end{array}$ & $\begin{array}{r}215 \\
122 \\
72 \\
21\end{array}$ \\
\hline $\begin{array}{l}\text { Black and other taces } \\
\text { Full owners } \\
\text { Part owners } \\
\text { Tenants }\end{array}$ & $\begin{array}{r}52 \\
34 \\
12 \\
6\end{array}$ & $\begin{array}{r}14 \\
8 \\
2 \\
4\end{array}$ & \begin{tabular}{r|}
22 \\
19 \\
1 \\
2
\end{tabular} & $\begin{array}{l}3 \\
2 \\
1 \\
-\end{array}$ & \begin{tabular}{l|l}
2 & \\
1 \\
1 \\
-
\end{tabular} & $\begin{array}{l}4 \\
2 \\
2 \\
-\end{array}$ \\
\hline \multicolumn{7}{|l|}{ OWNED AND RENTED LAND } \\
\hline 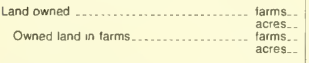 & \begin{tabular}{rr|}
2 & 691 \\
360 & 152 \\
2 & 682 \\
325 & 313
\end{tabular} & $\begin{array}{r}481 \\
5285 \\
481 \\
1805\end{array}$ & $\begin{array}{rr}802 \\
25 & 403 \\
& 800 \\
17 & 929\end{array}$ & $\begin{array}{r}176 \\
11 \\
324 \\
175 \\
8786\end{array}$ & $\begin{array}{r}208 \\
17946 \\
13 \quad 206 \\
758\end{array}$ & $\begin{array}{rr}199 \\
23 & 338 \\
& 198 \\
18 & 908\end{array}$ \\
\hline 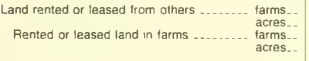 & $\begin{array}{rr}1 & 153 \\
285 & 163 \\
1 & 141 \\
282 & 932\end{array}$ & $\begin{array}{r}51 \\
291 \\
50 \\
213\end{array}$ & $\begin{array}{r}181 \\
3991 \\
175 \\
3442\end{array}$ & $\begin{array}{rr}2 & 64 \\
& 64 \\
2 & 617\end{array}$ & $\begin{array}{r}92 \\
5 \\
345 \\
90 \\
5 \quad 237\end{array}$ & $\begin{array}{r}96 \\
6 \quad 682 \\
95 \\
6 \quad 565\end{array}$ \\
\hline $\begin{array}{l}\text { Land rented or leased to others............ tarms.. } \\
\text { acres.. }\end{array}$ & $37 \begin{array}{r}463 \\
070\end{array}$ & $\begin{array}{r}86 \\
3558\end{array}$ & 8023 & 2758 & 4296 & $\begin{array}{r}32 \\
447\end{array}$ \\
\hline \multicolumn{7}{|l|}{ OPERATOR CHARACTERISTICS } \\
\hline $\begin{array}{l}\text { Operators by place of residence } \\
\text { On farm operated } \\
\text { Not an farm operated } \\
\text { Not reported }\end{array}$ & $\begin{array}{r}175 \\
481 \\
310\end{array}$ & $\begin{array}{r}328 \\
65 \\
121\end{array}$ & $\begin{array}{r}635 \\
153 \\
79\end{array}$ & \begin{tabular}{r|}
143 \\
41 \\
12
\end{tabular} & $\begin{array}{r}178 \\
35 \\
17\end{array}$ & $\begin{array}{r}164 \\
34 \\
21\end{array}$ \\
\hline $\begin{array}{l}\text { Operators by principal occupation } \\
\text { Farming } \\
\text { Other }\end{array}$ & $\begin{array}{ll}1 & 774 \\
1 & 192\end{array}$ & $\begin{array}{l}257 \\
257\end{array}$ & $\begin{array}{l}384 \\
483\end{array}$ & $\begin{array}{r}100 \\
96\end{array}$ & $\begin{array}{l}126 \\
104\end{array}$ & $\begin{array}{r}137 \\
82\end{array}$ \\
\hline $\begin{array}{l}\text { Operators by days of work off farm: } \\
\text { Nane. } \\
\text { Any } 10.99 \text { days } \\
100 \text { to } 199 \text { days } \\
200 \text { days or more }\end{array}$ & $\begin{array}{r}1263 \\
1426 \\
254 \\
217 \\
955\end{array}$ & $\begin{array}{r}186 \\
279 \\
39 \\
43 \\
197\end{array}$ & $\begin{array}{r}312 \\
475 \\
63 \\
58 \\
354\end{array}$ & $\begin{array}{r}70 \\
116 \\
24 \\
17 \\
75\end{array}$ & $\begin{array}{r}95 \\
124 \\
21 \\
24 \\
79\end{array}$ & $\begin{array}{r}91 \\
105 \\
22 \\
13 \\
70\end{array}$ \\
\hline Not reported ........................... & 277 & 49 & 80 & 10 & 11 & 23 \\
\hline $\begin{array}{l}\text { Operators by years on present farm: } \\
2 \text { years or less } \\
3 \text { or } 4 \text { years } \\
5 \text { to } 9 \text { years. } \\
10 \text { years or more } \\
\text { Average years on present farm }\end{array}$ & $\begin{array}{r}176 \\
189 \\
398 \\
1588 \\
19.3\end{array}$ & $\begin{array}{r}47 \\
54 \\
68 \\
178 \\
12.6\end{array}$ & $\begin{array}{r}66 \\
62 \\
159 \\
402 \\
16.7\end{array}$ & $\begin{array}{r}14 \\
12 \\
27 \\
109 \\
20.4\end{array}$ & $\begin{array}{r}7 \\
16 \\
34 \\
133 \\
206\end{array}$ & $\begin{array}{r}11 \\
6 \\
24 \\
138 \\
216\end{array}$ \\
\hline Not reported & 615 & 167 & 178 & 34 & 40 & 40 \\
\hline $\begin{array}{l}\text { Operators by age group } \\
\text { Under } 25 \text { years. } \\
25 \text { to } 34 \text { years } \\
35 \text { to } 44 \text { years } \\
45 \text { to } 49 \text { years } \\
50 \text { to } 54 \text { years }\end{array}$ & $\begin{array}{r}40 \\
374 \\
620 \\
328 \\
321\end{array}$ & $\begin{array}{r}10 \\
101 \\
131 \\
64 \\
54\end{array}$ & $\begin{array}{r}8 \\
102 \\
190 \\
98 \\
94\end{array}$ & $\begin{array}{r}6 \\
19 \\
42 \\
23 \\
20\end{array}$ & $\begin{array}{r}6 \\
34 \\
35 \\
30 \\
15\end{array}$ & $\begin{array}{r}1 \\
19 \\
34 \\
27 \\
24\end{array}$ \\
\hline $\begin{array}{l}55 \text { to } 59 \text { years } \\
60 \text { to } 64 \text { years } \\
65 \text { to } 69 \text { years } \\
70 \text { years and over } \\
\text { Average age }\end{array}$ & $\begin{array}{r}326 \\
348 \\
269 \\
340 \\
515\end{array}$ & $\begin{array}{r}40 \\
41 \\
33 \\
40 \\
47.1\end{array}$ & $\begin{array}{r}98 \\
90 \\
76 \\
111 \\
518\end{array}$ & $\begin{array}{r}19 \\
17 \\
17 \\
33 \\
528\end{array}$ & $\begin{array}{r}22 \\
27 \\
29 \\
32 \\
52.4\end{array}$ & $\begin{array}{r}26 \\
35 \\
22 \\
31 \\
54.1\end{array}$ \\
\hline $\begin{array}{l}\text { Operators by sex } \\
\text { Male } \\
\text { Female }\end{array}$ & $\begin{array}{r}2676 \\
290\end{array}$ & $\begin{array}{l}406 \\
108\end{array}$ & $\begin{array}{l}763 \\
104\end{array}$ & $\begin{array}{r}176 \\
20\end{array}$ & $\begin{array}{r}220 \\
10\end{array}$ & $\begin{array}{r}206 \\
13\end{array}$ \\
\hline $\begin{array}{l}\text { Operators of Spanish origin (see text) } \\
\text { FARMS BY TYPE OF } \\
\text { ORGANIZATION }\end{array}$ & 9 & - & 4 & 1 & - & - \\
\hline $\begin{array}{l}\text { Individual or tamily (sole proprietorship) ..... farms.. } \\
\text { acres... } \\
\text { Partnership .... } \\
\text { Corporation: }\end{array}$ & \begin{tabular}{rr|}
2 & 553 \\
400 & 071 \\
& 216 \\
62 & 666
\end{tabular} & $\begin{array}{r}458 \\
1833 \\
35 \\
124\end{array}$ & $\begin{array}{rr}791 \\
19 & 425 \\
& 45 \\
1 & 146\end{array}$ & 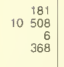 & $\begin{array}{r}201 \\
16 \quad 629 \\
1 \quad 23 \\
1884\end{array}$ & $\begin{array}{r}195 \\
22 \quad 850 \\
13 \\
1 \quad 388\end{array}$ \\
\hline 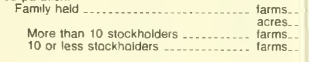 & $\begin{array}{r}173 \\
141856 \\
4 \\
169\end{array}$ & $\begin{array}{r}16 \\
46 \\
1 \\
15\end{array}$ & $\begin{array}{r}24 \\
595 \\
2 \\
22\end{array}$ & $\begin{array}{r}7 \\
\text { (D) } \\
\overline{7}\end{array}$ & $\begin{array}{r}6 \\
482 \\
\overline{6}\end{array}$ & $\begin{array}{r}10 \\
\text { (D) } \\
10\end{array}$ \\
\hline Other than family held ................ farms.. & $\begin{array}{r}8 \\
928\end{array}$ & (D) & $\begin{array}{r}3 \\
65\end{array}$ & $=$ & - & (0) \\
\hline $\begin{array}{l}\text { More than } 10 \text { stockhoiders ............ farms.. } \\
10 \text { or less stockholders }\end{array}$ & $\begin{array}{l}1 \\
7\end{array}$ & $\overline{2}$ & $\begin{array}{l}1 \\
2\end{array}$ & $=$ & 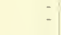 & 1 \\
\hline $\begin{array}{l}\text { Other-cooperative, estate or trust. } \\
\text { institutional, etc............... farms.. } \\
\text { acres... }\end{array}$ & $\begin{array}{r}16 \\
2724\end{array}$ & $\begin{array}{r}3 \\
\text { (D) }\end{array}$ & $\begin{array}{r}4 \\
140\end{array}$ & (D) & - & - \\
\hline
\end{tabular}

See footnotes at end of table 
Table 51. Summary by Size of Farm: 1987-Con.

[For meaning of abbreviations and symbols, see intraductory text]

\begin{tabular}{|c|c|c|c|c|c|c|c|}
\hline Item & $\begin{array}{r}140 \text { to } 179 \\
\text { acres }\end{array}$ & $\begin{array}{r}180 \text { to } 219 \\
\text { acres }\end{array}$ & $\begin{array}{r}220 \text { to } 259 \\
\text { acres }\end{array}$ & $\begin{array}{r}260 \text { to } 499 \\
\text { acres }\end{array}$ & $\begin{array}{r}500 \text { to } 999 \\
\text { acres }\end{array}$ & $\begin{array}{r}1,000 \text { to } 1,999 \\
\text { acres }\end{array}$ & $\begin{array}{r}2.000 \text { acres or } \\
\text { more }\end{array}$ \\
\hline \multicolumn{8}{|l|}{$\begin{array}{l}\text { TENURE AND RACE OF } \\
\text { OPERATOR }\end{array}$} \\
\hline $\begin{array}{l}\text { All operators } \\
\text { Full owners } \\
\text { Part owners } \\
\text { Tenants }\end{array}$ & $\begin{array}{r}143 \\
74 \\
48 \\
21\end{array}$ & $\begin{array}{r}117 \\
46 \\
54 \\
17\end{array}$ & $\begin{array}{r}76 \\
34 \\
33 \\
9\end{array}$ & $\begin{array}{r}288 \\
82 \\
173 \\
33\end{array}$ & $\begin{array}{r}188 \\
28 \\
136 \\
24\end{array}$ & $\begin{array}{r}100 \\
8 \\
81 \\
11\end{array}$ & $\begin{array}{r}28 \\
1 \\
24 \\
3\end{array}$ \\
\hline $\begin{array}{l}\text { White } \\
\text { Full cwners . } \\
\text { Part awners } \\
\text { Tenants }\end{array}$ & $\begin{array}{r}141 \\
73 \\
47 \\
21\end{array}$ & $\begin{array}{r}116 \\
46 \\
53 \\
17\end{array}$ & $\begin{array}{r}76 \\
34 \\
33 \\
9\end{array}$ & $\begin{array}{r}285 \\
82 \\
170 \\
33\end{array}$ & $\begin{array}{r}187 \\
27 \\
136 \\
24\end{array}$ & $\begin{array}{r}100 \\
8 \\
81 \\
11\end{array}$ & $\begin{array}{r}28 \\
1 \\
24 \\
3\end{array}$ \\
\hline $\begin{array}{l}\text { Black and other races } \\
\text { Full owners } \\
\text { Part awners } \ldots \ldots \ldots \\
\text { Tenants }\end{array}$ & $\begin{array}{l}2 \\
1 \\
1 \\
-\end{array}$ & \begin{tabular}{l|l}
1 \\
$\overline{1}$ \\
-
\end{tabular} & $\begin{array}{l}- \\
- \\
-\end{array}$ & $\begin{array}{l}3 \\
\overrightarrow{3} \\
-\end{array}$ & $\begin{array}{l}1 \\
1 \\
- \\
-\end{array}$ & $\begin{array}{l}= \\
\overline{-} \\
\overline{-}\end{array}$ & $\begin{array}{l}- \\
\overline{-}\end{array}$ \\
\hline \multicolumn{8}{|l|}{ OWNED AND RENTED LAND } \\
\hline $\begin{array}{r}\text { Land owned } \ldots w n e d \text { land in farms ................... } \\
\text { farms. } \\
\text { acres.. }\end{array}$ & $\begin{array}{rr}123 \\
17 & 472 \\
122 \\
15 & 581\end{array}$ & $\begin{array}{rr}101 \\
16 \quad 936 \\
100 \\
14 \quad 496\end{array}$ & $\begin{array}{rr}67 \\
13 & 455 \\
& 67 \\
12 & 456\end{array}$ & $\begin{array}{ll}62 & 255 \\
931 \\
255 \\
60 & 116\end{array}$ & $\begin{array}{ll} & 164 \\
63 & 573 \\
& 164 \\
60 & 441\end{array}$ & $\begin{array}{r}89 \\
53642 \\
89 \\
52681\end{array}$ & $\begin{array}{rr}26 \\
48 \quad 847 \\
25 \\
48 \quad 356\end{array}$ \\
\hline $\begin{array}{r}\text { Land rented or leased from others ......... farms.. } \\
\text { acres.. } \\
\text { Rented or leased land in tarms .......... farms.. } \\
\text { acres.. }\end{array}$ & $\begin{array}{rr}69 \\
7 & 103 \\
7 & 69 \\
7 & 103\end{array}$ & $\begin{array}{r}71 \\
8937 \\
8 \\
8937\end{array}$ & $\begin{array}{r}42 \\
5586 \\
42 \\
5581\end{array}$ & 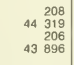 & 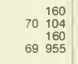 & $\begin{array}{r}92 \\
80 \\
521 \\
92 \\
80521\end{array}$ & $\begin{array}{r}27 \\
49 \quad 447 \\
27 \\
48 \quad 865\end{array}$ \\
\hline $\begin{array}{l}\text { Land rented or leased to others............ farms.. } \\
\text { acres.. }\end{array}$ & 1891 & 2440 & 1004 & 3238 & $\begin{array}{rr}10 \\
281\end{array}$ & $961^{4}$ & $\begin{array}{r}3 \\
1073\end{array}$ \\
\hline \multicolumn{8}{|l|}{ OPERATOR CHARACTERISTICS } \\
\hline $\begin{array}{l}\text { Operators by place of residence. } \\
\text { On farm operated } \\
\text { Not on farm operated } \\
\text { Not reported }\end{array}$ & $\begin{array}{r}116 \\
19 \\
8\end{array}$ & $\begin{array}{r}83 \\
31 \\
3\end{array}$ & $\begin{array}{r}63 \\
9 \\
4\end{array}$ & $\begin{array}{r}225 \\
46 \\
17\end{array}$ & $\begin{array}{r}141 \\
30 \\
17\end{array}$ & $\begin{array}{l}78 \\
12 \\
10\end{array}$ & $\begin{array}{r}21 \\
6 \\
1\end{array}$ \\
\hline $\begin{array}{l}\text { Operators by principal occupatian } \\
\text { Farming } \\
\text { Other }\end{array}$ & $\begin{array}{r}108 \\
35\end{array}$ & $\begin{array}{l}86 \\
31\end{array}$ & $\begin{array}{l}58 \\
18\end{array}$ & $\begin{array}{r}235 \\
53\end{array}$ & $\begin{array}{r}163 \\
25\end{array}$ & $\begin{array}{r}93 \\
7\end{array}$ & 27 \\
\hline $\begin{array}{l}\text { Operators by days of work off farm } \\
\text { None } \\
\text { Any } \\
1 \text { to } 99 \text { days } \\
100 \text { to } 199 \text { days } \\
200 \text { days or more }\end{array}$ & $\begin{array}{r}67 \\
60 \\
12 \\
5 \\
43\end{array}$ & $\begin{array}{r}55 \\
48 \\
9 \\
17 \\
22\end{array}$ & \begin{tabular}{r|r}
35 \\
23 \\
2 \\
8 \\
13
\end{tabular} & $\begin{array}{r}155 \\
109 \\
21 \\
23 \\
65\end{array}$ & $\begin{array}{r}113 \\
58 \\
28 \\
4 \\
26\end{array}$ & $\begin{array}{r}58 \\
27 \\
13 \\
4 \\
10\end{array}$ & $\begin{array}{r}26 \\
2 \\
\\
1 \\
1\end{array}$ \\
\hline Not reported ......................... & 16 & 14 & 18 & 24 & 17 & 15 & - \\
\hline 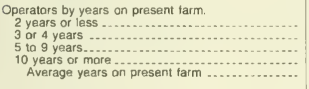 & $\begin{array}{r}6 \\
5 \\
20 \\
90 \\
21.5\end{array}$ & $\begin{array}{r}3 \\
8 \\
17 \\
72 \\
20.8\end{array}$ & $\begin{array}{r}5 \\
4 \\
6 \\
47 \\
24.4\end{array}$ & $\begin{array}{r}7 \\
13 \\
19 \\
202 \\
24.5\end{array}$ & \begin{tabular}{r|r}
6 \\
5 \\
18 \\
130 \\
23.3
\end{tabular} & $\begin{array}{r}3 \\
4 \\
6 \\
64 \\
23.7\end{array}$ & $\begin{array}{r}1 \\
2 \overline{3} \\
31.4\end{array}$ \\
\hline Nat reported ............................. & 22 & 17 & 14 & 47 & 29 & 23 & 4 \\
\hline $\begin{array}{l}\text { Operators by age group: } \\
\text { Under } 25 \text { years } \\
25 \text { to } 34 \text { years } \\
35 \text { to } 44 \text { years } \\
45 \text { to } 49 \text { years } \\
50 \text { to } 54 \text { years }\end{array}$ & $\begin{array}{r}19 \\
26 \\
5 \\
25\end{array}$ & $\begin{array}{r}1 \\
15 \\
20 \\
7 \\
13\end{array}$ & $\begin{array}{r}4 \\
4 \\
15 \\
6 \\
4\end{array}$ & $\begin{array}{l}2 \\
31 \\
48 \\
29 \\
43\end{array}$ & $\begin{array}{l}1 \\
21 \\
49 \\
18 \\
18\end{array}$ & $\begin{array}{r}1 \\
9 \\
25 \\
17 \\
9\end{array}$ & $\begin{array}{l}- \\
\overline{5} \\
4 \\
2\end{array}$ \\
\hline 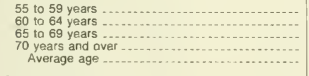 & $\begin{array}{r}17 \\
22 \\
12 \\
17 \\
53.0\end{array}$ & $\begin{array}{r}20 \\
12 \\
10 \\
19 \\
53.8\end{array}$ & $\begin{array}{r}6 \\
12 \\
11 \\
14 \\
54.6\end{array}$ & $\begin{array}{r}32 \\
43 \\
34 \\
26 \\
52.9\end{array}$ & \begin{tabular}{r|}
24 \\
29 \\
14 \\
14 \\
50.8
\end{tabular} & $\begin{array}{r}15 \\
15 \\
7 \\
2 \\
49.4\end{array}$ & $\begin{array}{r}7 \\
5 \\
4 \\
1 \\
54.5\end{array}$ \\
\hline $\begin{array}{l}\text { Operators by sex } \\
\text { Male } \\
\text { Female }\end{array}$ & $\begin{array}{r}135 \\
8\end{array}$ & $\begin{array}{r}111 \\
6\end{array}$ & $\begin{array}{r}71 \\
5\end{array}$ & $\begin{array}{r}279 \\
9\end{array}$ & $\begin{array}{r}182 \\
6\end{array}$ & $\begin{array}{r}99 \\
1\end{array}$ & 28 \\
\hline $\begin{array}{l}\text { Operators of Spanish origin (see text) } \\
\text { FARMS BY TYPE OF } \\
\text { ORGANIZATION }\end{array}$ & - & 2 & - & - & 1 & 1 & $\rightarrow$ \\
\hline Individual or family (sole proprietorship) ..... farms.. & $19 \begin{array}{r}124 \\
660\end{array}$ & $20 \begin{array}{rr}101 \\
20 & 135\end{array}$ & 15925 & 85259 & $89 \begin{array}{r}132 \\
054\end{array}$ & $\begin{array}{r}56 \\
75 \quad 177\end{array}$ & 23616 \\
\hline $\begin{array}{l}\text { Partnership . ............... tarms.. } \\
\text { acres.. }\end{array}$ & $\begin{array}{r}15 \\
2404\end{array}$ & $\begin{array}{r}9 \\
1856\end{array}$ & $\begin{array}{rr}1 & 5 \\
182\end{array}$ & $\begin{array}{r}21 \\
7984\end{array}$ & 23540 & 11985 & 8.805 \\
\hline $\begin{array}{l}\text { Corporation: } \\
\text { Family held }\end{array}$ & (D) & 1442 & (D) & 8872 & $\begin{array}{r}23 \\
\text { (D) }\end{array}$ & $46 \quad 040$ & 64800 \\
\hline $\begin{array}{l}\text { More than } 10 \text { stockhoiders ............ farms.- } \\
10 \text { or less stockholders }\end{array}$ & $\overline{2}$ & & $\overline{3}$ & 24 & $2 \overline{3}$ & $3 \overline{5}$ & $\begin{array}{r}1 \\
15\end{array}$ \\
\hline Other than family held ................ farms.. & - & - & $\overline{-}$ & ${ }^{2}$ & $\overline{-}$ & $z$ & - \\
\hline $\begin{array}{l}\text { More than } 10 \text { stockholders ............ farms.. } \\
10 \text { or less stockholders } \ldots . . . . . . . . . . . \text { farms .. }\end{array}$ & $=$ & - & $=$ & $\overline{2}$ & $\begin{array}{l}- \\
-\end{array}$ & $\overline{-}$ & - \\
\hline $\begin{array}{l}\text { Other-cooperative, estate ar trust, } \\
\text { institutional, etc ........ farms.. }\end{array}$ & (D) & - & (D) & $\begin{array}{r}3 \\
\text { (D) }\end{array}$ & (D) & -1 & - \\
\hline
\end{tabular}


Table 51. Summary by Size of Farm: 1987-Con.

[For meaning of abbreviations and symbols, see introductory text]

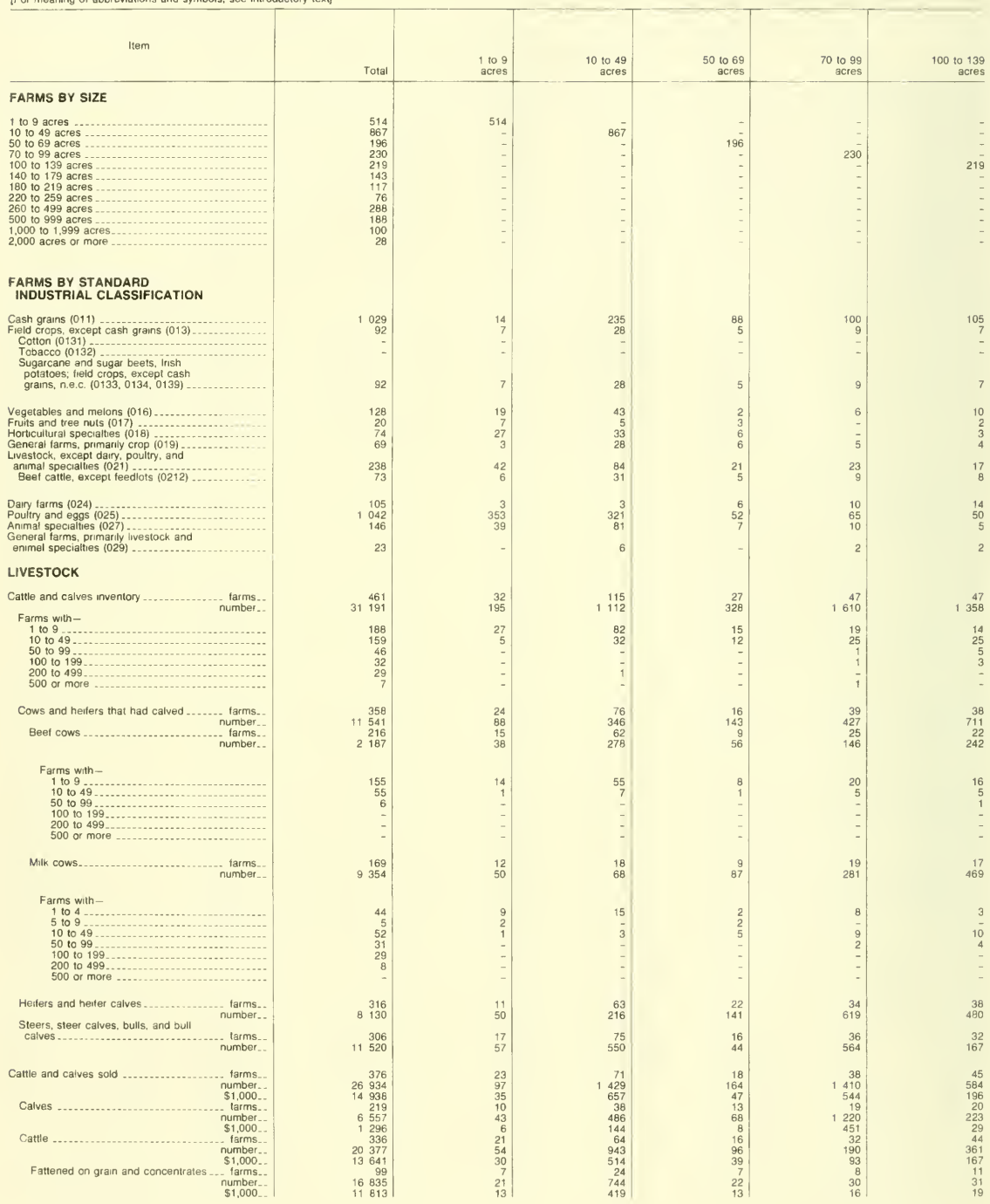


Table 51. Summary by Size of Farm: 1987-Con.

[Far meanung of abbreviatıons and symbols, see introductory text]

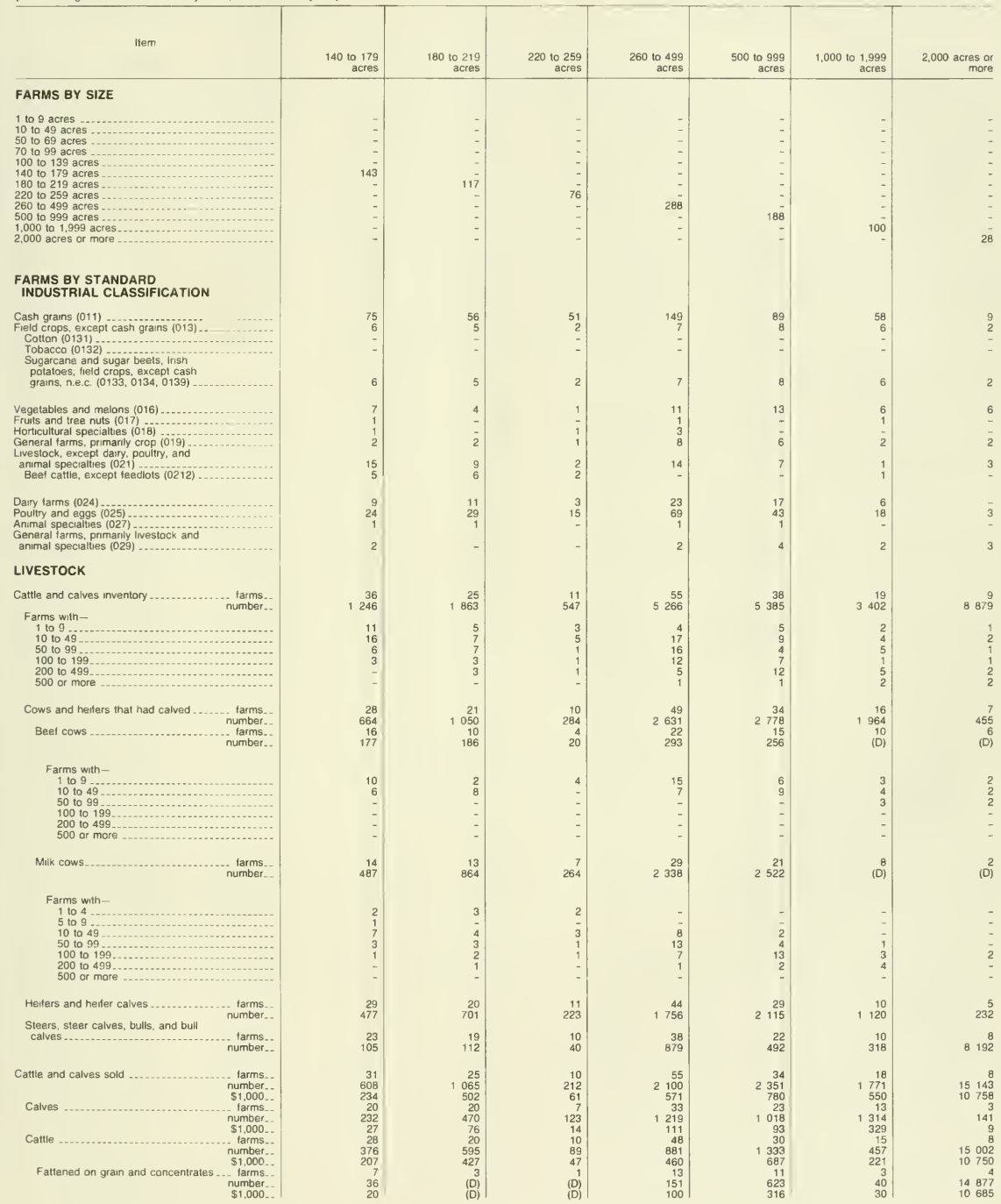


Table 51. Summary by Size of Farm: 1987-Con.

[For meaning of abbreviations and symbols, see introductory text]

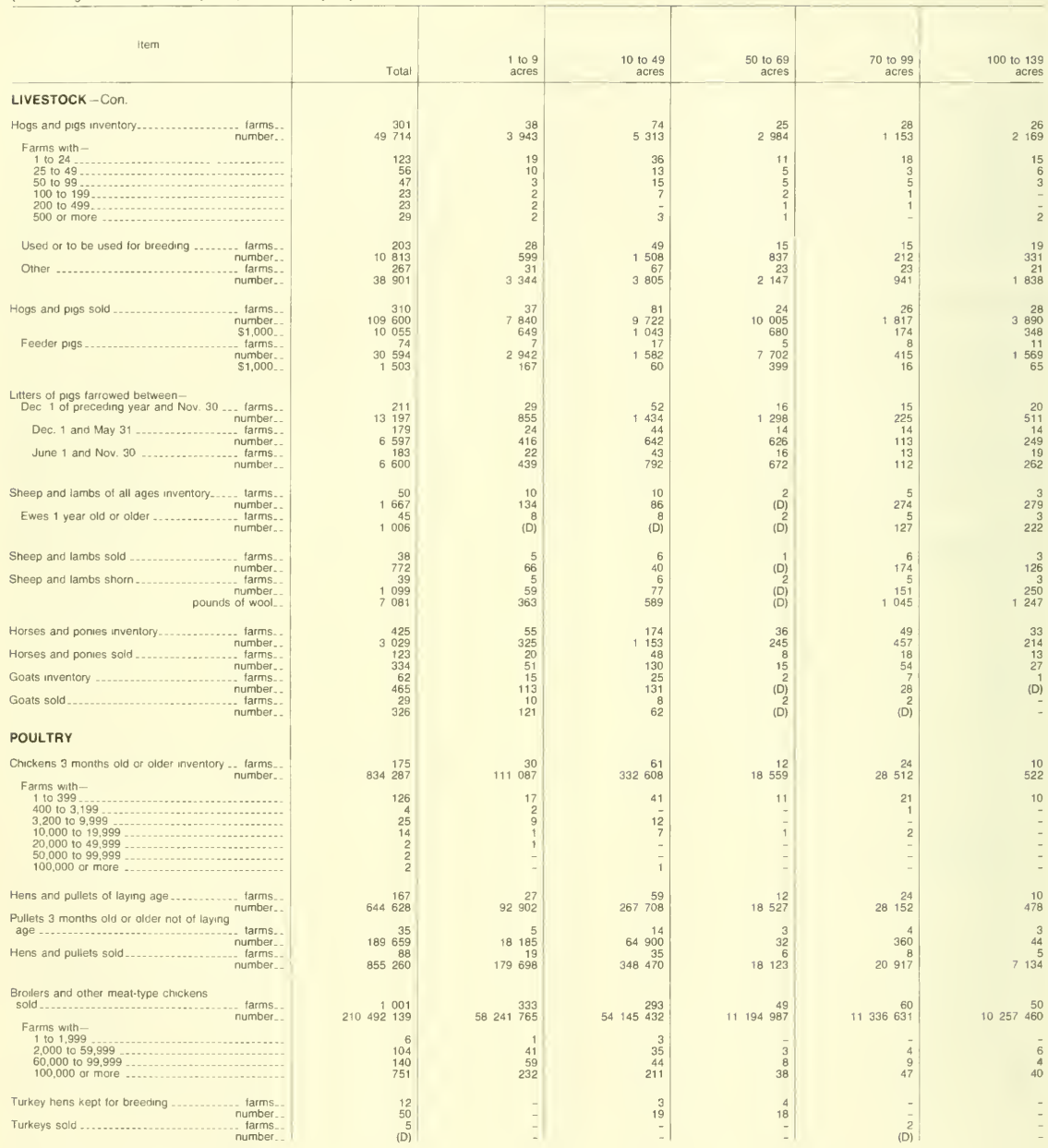


Table 51. Summary by Size of Farm: 1987-Con.

[For meaning of abbreviations and symbols, see introductory text]

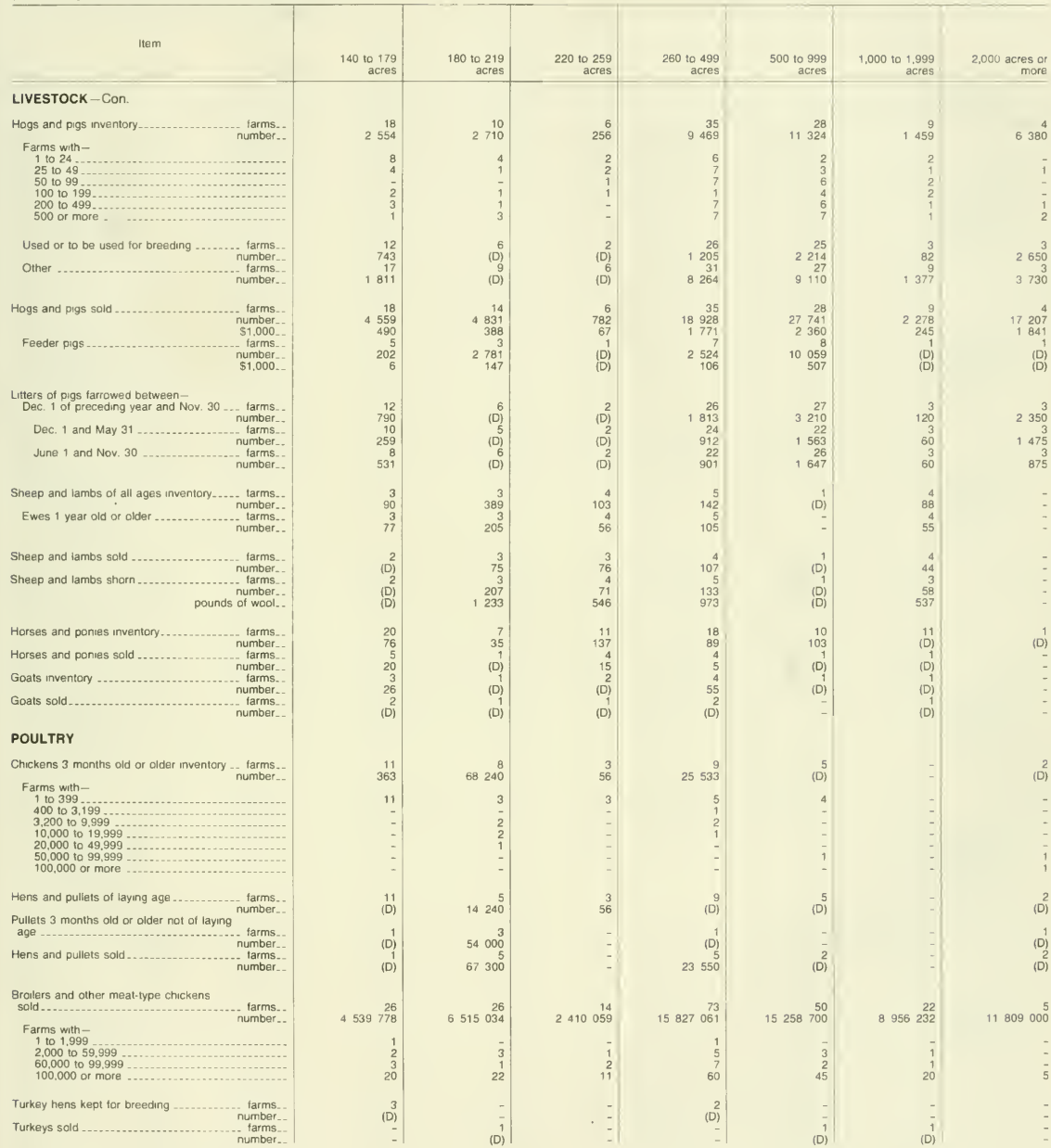

See footnotes at end of table 
Table 51. Summary by Size of Farm: 1987-Con.

[For meaning of abbreviations and symbols, see introductory text]

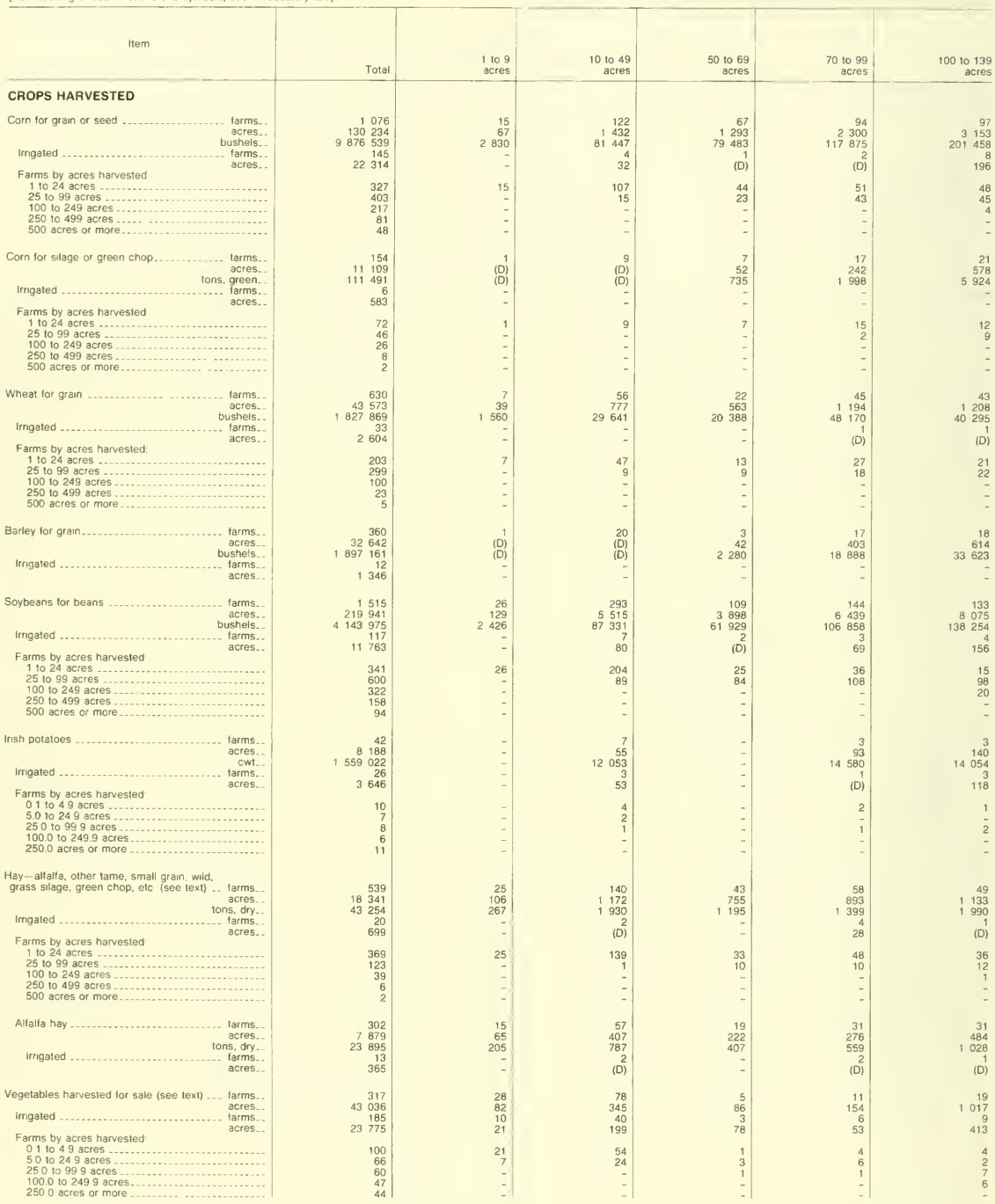


Table 51. Summary by Size of Farm: 1987-Con.

[For meaning of abbrewations and symbols, see introductory text]

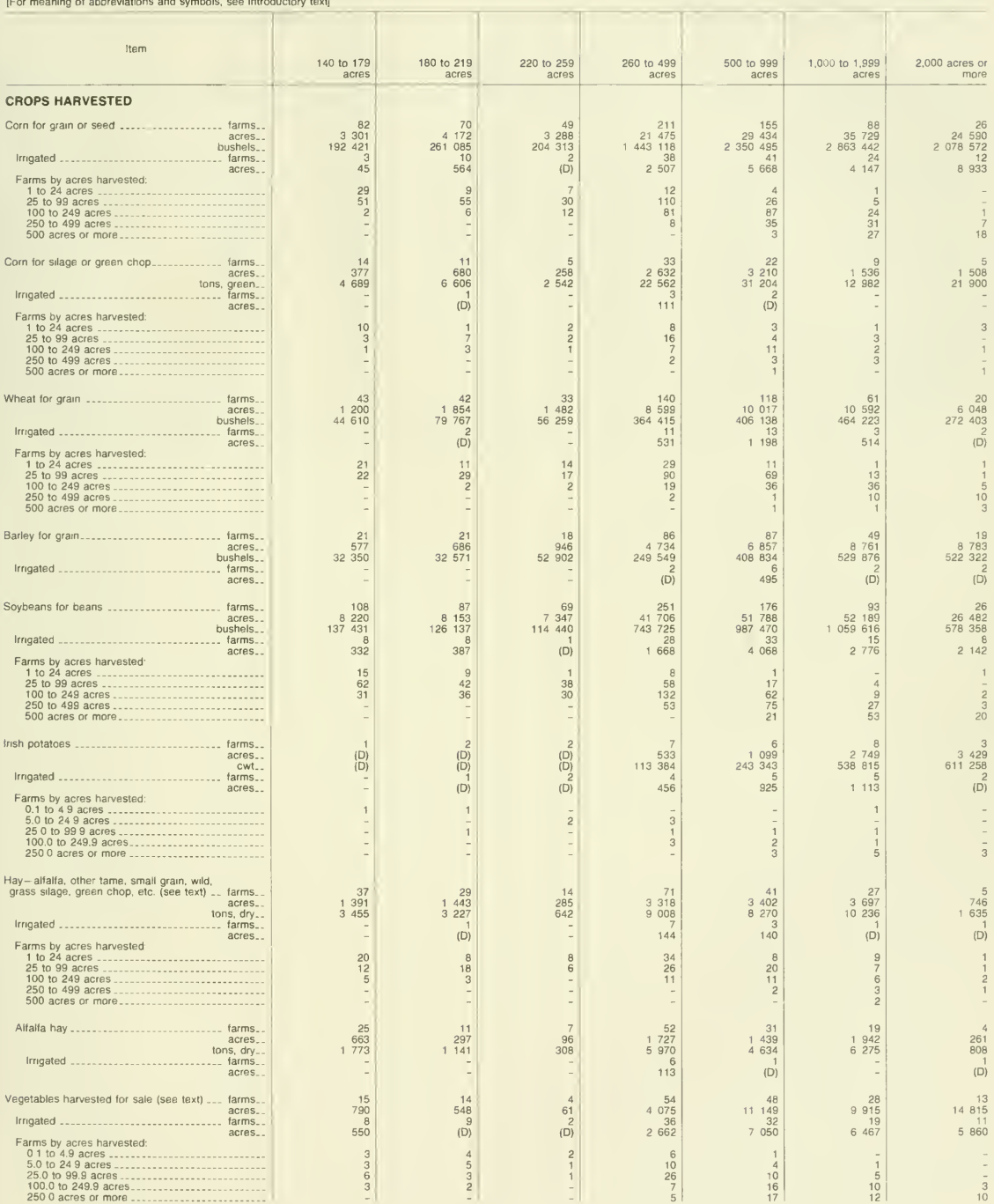


Table 51. Summary by Size of Farm: 1987-Con.

[For meaning of abbreviations and symbols, see introductory text]

\begin{tabular}{|c|c|c|c|c|c|c|}
\hline Item & Total & $\begin{array}{l}1 \text { to } \theta \\
\text { acres }\end{array}$ & $\begin{array}{r}10 \text { to } 49 \\
\text { acres }\end{array}$ & $\begin{array}{r}50 \text { to } 69 \\
\text { acres }\end{array}$ & $\begin{array}{r}70 \text { to } 99 \\
\text { acres }\end{array}$ & $\begin{array}{r}100 \text { to } 139 \\
\text { acres }\end{array}$ \\
\hline \multicolumn{7}{|l|}{$\begin{array}{l}\text { CROPS HARVESTED-Con. } \\
\text { Vegetables harvested for sale (see text)- } \\
\text { Con. }\end{array}$} \\
\hline $\begin{array}{c}\begin{array}{c}\text { Green peas, excluding green cowpeas, } \\
\text { harvested for sale }\end{array} \\
\text { trngated }\end{array}$ & $\begin{array}{r}66 \\
9851 \\
45 \\
5752\end{array}$ & $\begin{array}{r}2 \\
\text { (D) } \\
2 \\
\text { (D) }\end{array}$ & (D) & $\begin{array}{r}1 \\
\text { (D) } \\
1 \\
\text { (D) }\end{array}$ & $\begin{array}{r}1 \\
\text { (D) } \\
-\end{array}$ & $\begin{array}{r}1 \\
\text { (D) } \\
1 \\
\text { (D) }\end{array}$ \\
\hline $\begin{array}{r}\text { Sweet corn harvested for sale } \ldots . . . . . . . \\
\text { frrigated } \\
\text { acres... } \\
\text { farms... } \\
\text { acres.. }\end{array}$ & $\begin{array}{r}109 \\
7643 \\
68 \\
5 \quad 739\end{array}$ & $\begin{array}{r}8 \\
21 \\
1 \\
\text { (D) }\end{array}$ & $\begin{array}{r}24 \\
\text { (D) } \\
10 \\
61\end{array}$ & $\begin{array}{r}1 \\
\text { (D) } \\
1 \\
\text { (D) }\end{array}$ & $\begin{array}{l}\overline{-} \\
\overline{-}\end{array}$ & $\begin{array}{r}8 \\
279 \\
3 \\
105\end{array}$ \\
\hline $\begin{array}{l}\begin{array}{l}\text { Land in orchards .... } \\
\text { Ifrigated }\end{array} \\
\begin{array}{l}\text { Farms by bearing and nonbearing acres. } \\
0.1 \text { to } 4.9 \text { acres } \\
50 \text { to } 249 \text { acres } \\
25.0 \text { to } 999 \text { acres } \\
1000 \text { to } 249.9 \text { acres } \\
250.0 \text { acres } \\
\text { farms.. }\end{array} \\
\end{array}$ & $\begin{array}{r}36 \\
1378 \\
8 \\
527 \\
21 \\
11 \\
1 \\
\\
3\end{array}$ & $\begin{array}{r}6 \\
20 \\
- \\
- \\
4 \\
2 \\
- \\
- \\
-\end{array}$ & $\begin{array}{r}10 \\
17 \\
- \\
- \\
10 \\
- \\
- \\
-\end{array}$ & $\begin{array}{l}6 \\
\text { (D) } \\
- \\
- \\
3 \\
3 \\
- \\
- \\
-\end{array}$ & $\begin{array}{r}\text { (D) } \\
- \\
- \\
1 \\
- \\
- \\
-\end{array}$ & $\begin{array}{r}5 \\
25 \\
3 \\
24 \\
3 \\
2 \\
\\
\end{array}$ \\
\hline
\end{tabular}

See footnotes at end of table. 
Table 51. Summary by Size of Farm: 1987-Con.

[For meaning of abbreviations and symbols, see introductory text]

\begin{tabular}{|c|c|c|c|c|c|c|c|}
\hline Item & $\begin{array}{r}140 \text { to } 179 \\
\text { acres }\end{array}$ & $\begin{array}{r}180 \text { to } 219 \\
\text { acres }\end{array}$ & $\begin{array}{r}220 \text { to } 259 \\
\text { acres }\end{array}$ & $\begin{array}{r}260 \text { to } 499 \\
\text { acres }\end{array}$ & $\begin{array}{r}500 \text { to } 999 \\
\text { acres }\end{array}$ & $\begin{array}{r}1,000 \text { to } 1,999 \\
\text { acres }\end{array}$ & $\begin{array}{r}2.000 \text { acres or } \\
\text { more }\end{array}$ \\
\hline \multicolumn{8}{|l|}{$\begin{array}{l}\text { CROPS HARVESTED - Con. } \\
\text { Vegetables harvested for sale (see text)- } \\
\text { Con }\end{array}$} \\
\hline $\begin{array}{c}\begin{array}{c}\text { Green peas, excluding green cowpeas, } \\
\text { harvested for sale }\end{array} \\
\text { Irngated }\end{array}$ & $\begin{array}{r}5 \\
201 \\
2 \\
(D)\end{array}$ & $\begin{array}{r}3 \\
136 \\
2 \\
(D)\end{array}$ & $\overline{-}$ & $\begin{array}{r}6 \\
517 \\
5 \\
382\end{array}$ & $\begin{array}{rr}21 \\
2678 \\
14 \\
1716\end{array}$ & $\begin{array}{rr}2 & 15 \\
105 \\
12 \\
1 & 485\end{array}$ & $\begin{array}{rr}4 & 97 \\
4 & 6 \\
1 & 990\end{array}$ \\
\hline 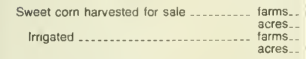 & $\begin{array}{r}6 \\
400 \\
5 \\
345\end{array}$ & $\begin{array}{r}3 \\
65 \\
2 \\
(D)\end{array}$ & $\begin{array}{r}1 \\
\text { (D) } \\
-\end{array}$ & $\begin{array}{r}18 \\
1061 \\
14 \\
696\end{array}$ & $\begin{array}{r}17 \\
1033 \\
13 \\
903\end{array}$ & $\begin{array}{r}18 \\
3087 \\
15 \\
2639\end{array}$ & $\begin{array}{r}5 \\
1577 \\
4 \\
917\end{array}$ \\
\hline $\begin{array}{l}\text { Land in orchards } \\
\text { Irrigated ....... } \\
\text { Farms by bearing and nonbearing acres } \\
0.1 \text { to } 4.9 \text { acres } \\
5.0 \text { to } 249 \text { acres } \\
25.0 \text { to } 999 \text { acres } \\
100.0 \text { to } 249.9 \text { acres. } \\
250.0 \text { acres or more }\end{array}$ & $\begin{array}{l}\overline{-} \\
\overline{-} \\
\overline{-} \\
\overline{-}\end{array}$ & $\begin{array}{l}- \\
\overline{-}\end{array}$ & $\begin{array}{l}\overline{-} \\
\overline{-} \\
\overline{-} \\
\overline{-} \\
\overline{-}\end{array}$ & $\begin{array}{r}1 \\
\text { (D) } \\
1 \\
\text { (D) }\end{array}$ & $\begin{array}{r}3 \\
28 \\
- \\
- \\
- \\
- \\
-\end{array}$ & $\begin{array}{r}183 \\
4 \\
\text { (D) } \\
- \\
1 \\
- \\
-\end{array}$ & $\begin{array}{l}- \\
- \\
- \\
-\end{array}$ \\
\hline
\end{tabular}

'Data are based on a sample of farms.

2 Farms with total production expenses equal to market value of agricuitural products sold are included as farms with gains of less than $\$ 1,000$. 
Table 52. Summary by Value of Agricultural Products Sold: 1987

[For meaning of abbreviations and symbols, see introductory text]

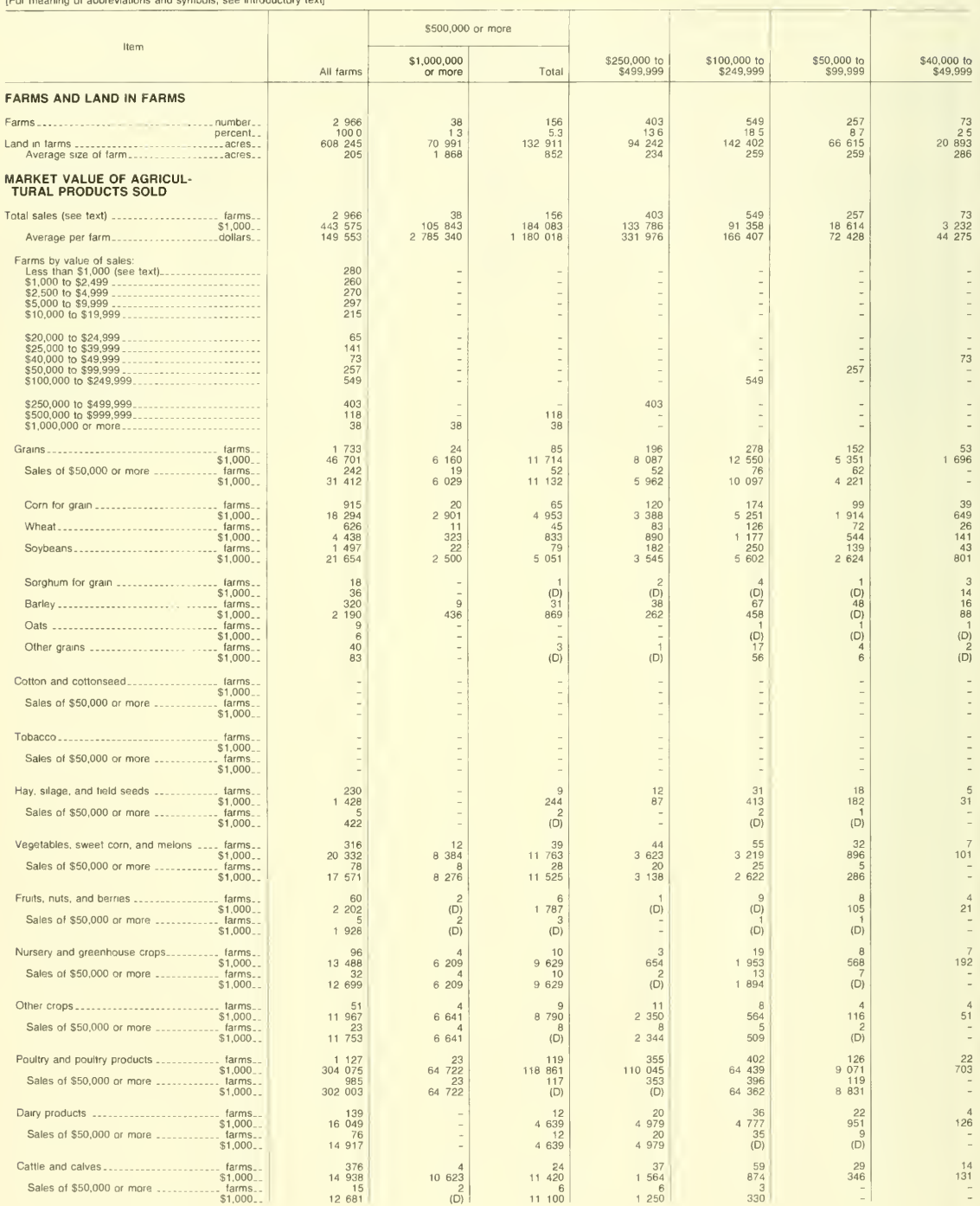

See footnotes at end of table 
Table 52. Summary by Value of Agricultural Products Sold: 1987-Con.

[For meaning of abbreviations and symbols, see introductory text]

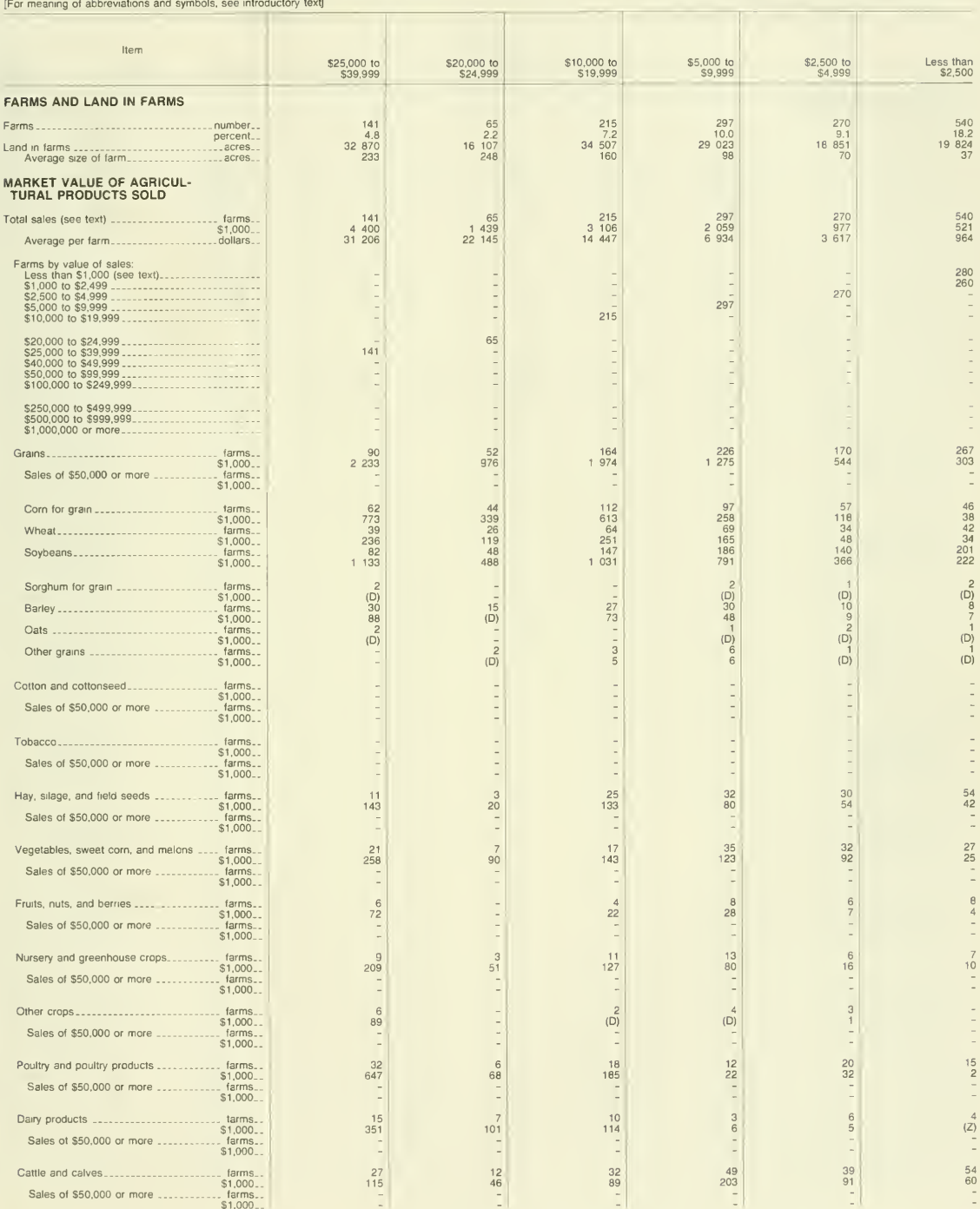

See footnotes at end of table 
Table 52. Summary by Value of Agricultural Products Sold: 1987-Con.

[For meaning of abbreviations and symbols, see introductory text]

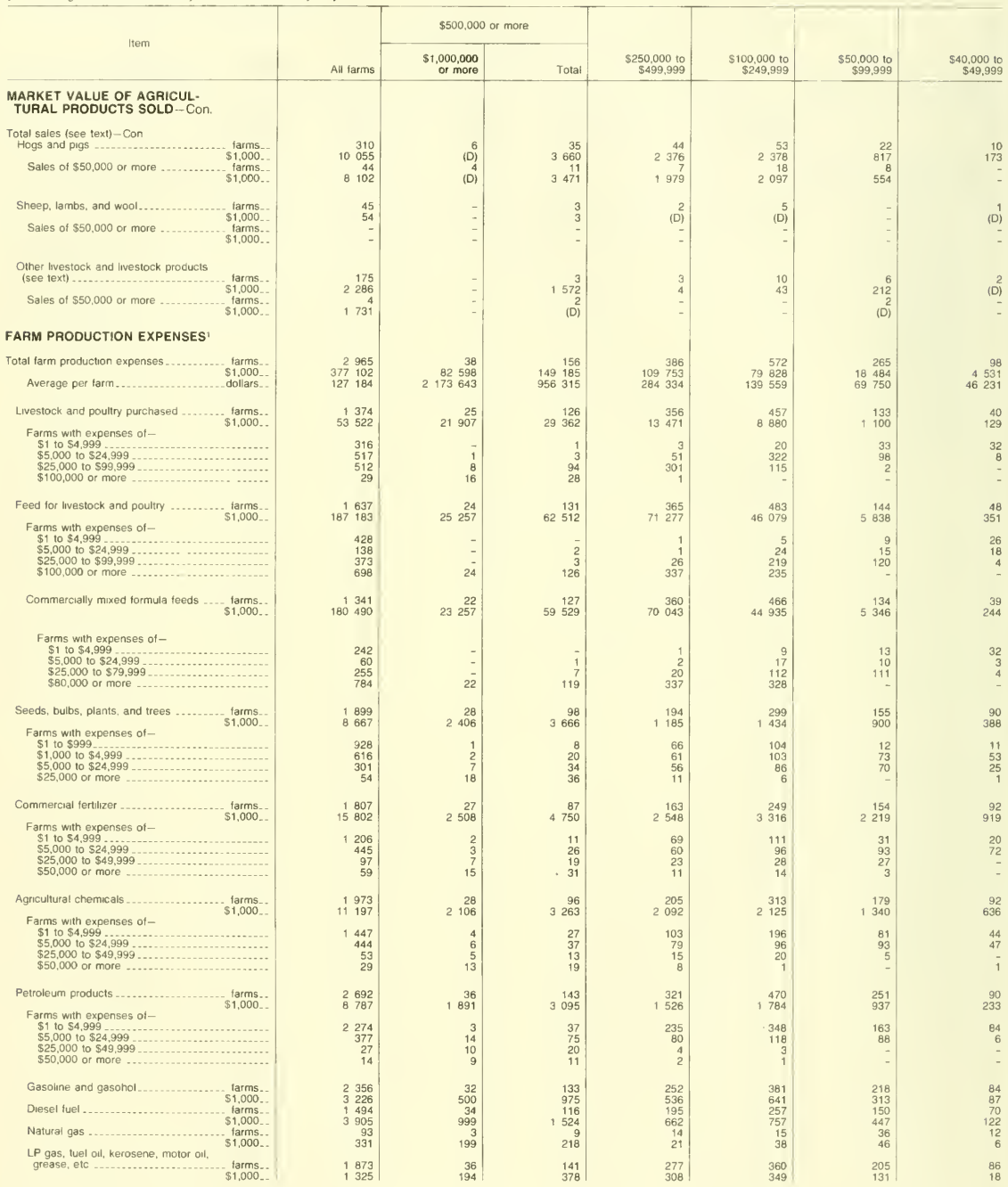


Table 52. Summary by Value of Agricultural Products Sold: 1987-Con.

[For meaning of abbreviations and symbals, see intraductory text]

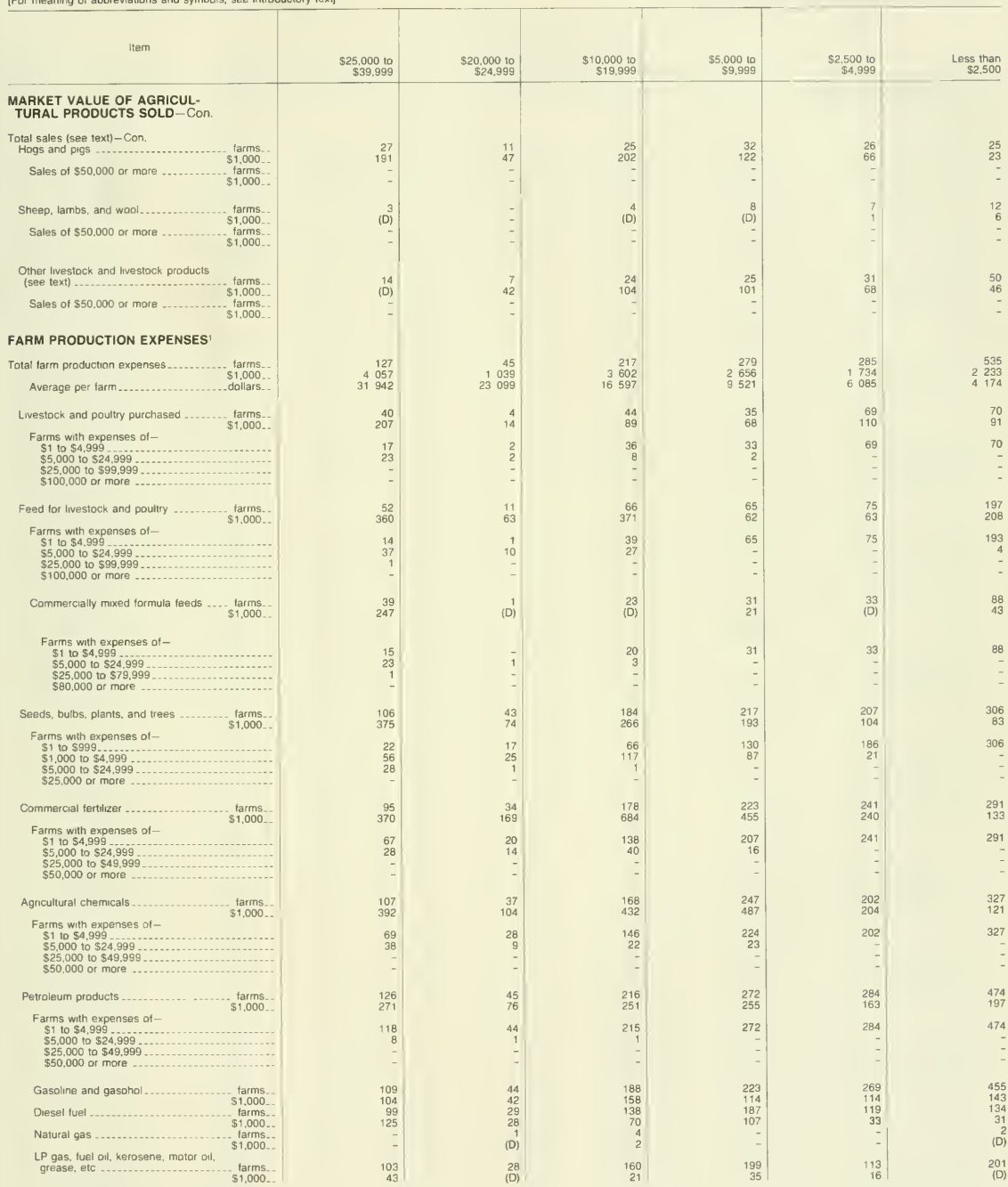

See footnotes at end of table 
Table 52. Summary by Value of Agricultural Products Sold: 1987-Con.

[For meaning of abbreviations and symbols, see introductory text]

\begin{tabular}{|c|c|c|c|c|c|c|c|}
\hline \multirow{2}{*}{ hem } & \multirow[b]{2}{*}{ All tarms } & \multicolumn{2}{|c|}{$\$ 500,000$ or more } & \multirow[b]{2}{*}{$\begin{array}{r}\$ 250,000 \text { to } \\
\$ 499,999\end{array}$} & \multirow[b]{2}{*}{$\begin{array}{r}\$ 100,000 \text { to } \\
\$ 249.999\end{array}$} & \multirow[b]{2}{*}{$\begin{array}{r}\$ 50,000 \text { to } \\
\$ 99,999\end{array}$} & \multirow[b]{2}{*}{$\begin{array}{r}\$ 40,000 \text { to } \\
\$ 49.999\end{array}$} \\
\hline & & $\begin{array}{r}\$ 1,000,000 \\
\text { or more }\end{array}$ & Total & & & & \\
\hline \multicolumn{8}{|l|}{$\begin{array}{l}\text { FARM PRODUCTION EXPENSES }{ }^{1} \\
\text {-CON. }\end{array}$} \\
\hline $\begin{array}{l}\text { Total farm production expenses-Con } \\
\text { Electricity ........ }\end{array}$ & $\begin{array}{ll}2 & 195 \\
4 & 862\end{array}$ & $\begin{array}{r}37 \\
1039\end{array}$ & $\begin{array}{r}154 \\
1816\end{array}$ & $\begin{array}{r}375 \\
1 \quad 192\end{array}$ & $\begin{array}{r}523 \\
1182\end{array}$ & $\begin{array}{l}199 \\
241\end{array}$ & $\begin{array}{l}74 \\
54\end{array}$ \\
\hline $\begin{array}{l}\text { Farms with expenses of } \\
\$ 1 \text { to } \$ 999 \\
\$ 1,000 \text { to } \$ 4,999 \\
\$ 5,000 \text { to } \$ 24,999 \ldots \ldots \\
\$ 25,000 \text { or more }\end{array}$ & $\begin{array}{r}1085 \\
942 \\
151 \\
17\end{array}$ & $\begin{array}{r}2 \\
-52 \\
13\end{array}$ & $\begin{array}{r}5 \\
51 \\
82 \\
16\end{array}$ & $\begin{array}{r}13 \\
326 \\
36 \\
-\end{array}$ & $\begin{array}{r}83 \\
418 \\
21 \\
1\end{array}$ & $\begin{array}{r}129 \\
58 \\
12 \\
-\end{array}$ & $\begin{array}{r}56 \\
18 \\
- \\
-\end{array}$ \\
\hline $\begin{array}{l}\text { Hired farm labor ................... farms... } \\
\$ 1,000 . .\end{array}$ & $\begin{array}{rr}1 & 254 \\
22 & 788\end{array}$ & $9 \begin{array}{r}37 \\
943\end{array}$ & $\begin{array}{r}151 \\
13993\end{array}$ & $3 \begin{array}{r}281 \\
3906\end{array}$ & $\begin{array}{r}302 \\
2957\end{array}$ & $\begin{array}{r}158 \\
1020\end{array}$ & $\begin{array}{r}66 \\
229\end{array}$ \\
\hline $\begin{array}{l}\text { Farms with expenses of- } \\
\$ 1 \text { to } \$ 4,999 \\
\$ 5,000 \text { to } \$ 24.999 \\
\$ 25,000 \text { to } \$ 99.999 . \ldots \\
\$ 100,000 \text { or more }\end{array}$ & $\begin{array}{r}646 \\
440 \\
133 \\
35\end{array}$ & $\begin{array}{r}2 \\
5 \\
11 \\
19\end{array}$ & $\begin{array}{l}16 \\
55 \\
47 \\
33\end{array}$ & $\begin{array}{r}77 \\
159 \\
45 \\
-\end{array}$ & $\begin{array}{r}170 \\
94 \\
36 \\
2\end{array}$ & $\begin{array}{r}86 \\
69 \\
3 \\
-\end{array}$ & $\begin{array}{r}54 \\
10 \\
2 \\
-\end{array}$ \\
\hline Contract labor ...................... farms... & $\begin{array}{r}364 \\
5798\end{array}$ & $3 \begin{array}{r}14 \\
3162\end{array}$ & 4020 & $\begin{array}{r}135 \\
1171\end{array}$ & $\begin{array}{r}84 \\
433\end{array}$ & $\begin{array}{r}37 \\
114\end{array}$ & (D) \\
\hline $\begin{array}{l}\text { Farms with expenses of } \\
\$ 1 \text { to } \$ 999 \\
\$ 1,000 \text { to } \$ 4,999 \\
\$ 5,000 \text { to } \$ 24,999 \\
\$ 25,000 \text { or more }\end{array}$ & $\begin{array}{r}41 \\
161 \\
134 \\
28\end{array}$ & $\begin{array}{l}- \\
1 \\
6 \\
7\end{array}$ & $\begin{array}{l}14 \\
47 \\
14\end{array}$ & $\begin{array}{r}5 \\
69 \\
50 \\
11\end{array}$ & $\begin{array}{r}16 \\
37 \\
28 \\
3\end{array}$ & $\begin{array}{r}4 \\
25 \\
8 \\
-\end{array}$ & $\begin{array}{l}1 \\
1 \\
- \\
-\end{array}$ \\
\hline Repar and mantenance ............. tarms... & $\begin{array}{rr}2 & 481 \\
11 & 739\end{array}$ & 2534 & $\begin{array}{r}145 \\
4229\end{array}$ & $2 \begin{array}{r}366 \\
259\end{array}$ & $2 \begin{array}{r}498 \\
2616\end{array}$ & $\begin{array}{l}249 \\
959\end{array}$ & $\begin{array}{r}94 \\
315\end{array}$ \\
\hline $\begin{array}{l}\text { Farms with expenses of } \\
\$ 1 \text { to } \$ 4,999 \\
\$ 5,000 \text { to } \$ 24,999 \\
\$ 25,000 \text { to } \$ 49,999 \\
\$ 50.000 \text { or more }\end{array}$ & $\begin{array}{r}1939 \\
455 \\
67 \\
20\end{array}$ & $\begin{array}{r}4 \\
9 \\
10 \\
14\end{array}$ & $\begin{array}{l}39 \\
54 \\
32 \\
20\end{array}$ & $\begin{array}{r}214 \\
134 \\
18 \\
-\end{array}$ & $\begin{array}{r}339 \\
142 \\
17 \\
-\end{array}$ & $\begin{array}{r}169 \\
80 \\
- \\
-\end{array}$ & $\begin{array}{r}89 \\
5 \\
- \\
-\end{array}$ \\
\hline $\begin{array}{l}\text { Farms with expenses of- } \\
\$ 1 \text { to } \$ 999 \\
\$ 1,000 \text { to } \$ 4,999 \\
\$ 5,000 \text { to } \$ 24,999 \\
\$ 25,000 \text { or more }\end{array}$ & $\begin{array}{r}672 \\
406 \\
106 \\
8\end{array}$ & $\begin{array}{l}2 \\
4 \\
8 \\
4\end{array}$ & $\begin{array}{r}12 \\
32 \\
23 \\
5\end{array}$ & $\begin{array}{r}48 \\
74 \\
25 \\
2\end{array}$ & $\begin{array}{r}136 \\
74 \\
31 \\
1\end{array}$ & $\begin{array}{l}43 \\
47 \\
10 \\
-\end{array}$ & $\begin{array}{r}25 \\
24 \\
10 \\
-\end{array}$ \\
\hline Interest expense ............... & $\begin{array}{r}1415 \\
14872\end{array}$ & $3 \begin{array}{r}35 \\
363\end{array}$ & $\begin{array}{r}139 \\
6203\end{array}$ & $\begin{array}{r}291 \\
299\end{array}$ & $2 \begin{array}{r}369 \\
2851\end{array}$ & $\begin{array}{r}153 \\
1081\end{array}$ & $\begin{array}{r}29 \\
161\end{array}$ \\
\hline $\begin{array}{l}\text { Farms with expenses of } \\
\$ 1 \text { to } \$ 4,999 \\
\$ 5,000 \text { to } \$ 24.999 \\
\$ 25,000 \text { to } \$ 99,999 \\
\$ 100.000 \text { or more }\end{array}$ & $\begin{array}{r}713 \\
580 \\
110 \\
12\end{array}$ & $\begin{array}{r}3 \\
7 \\
17 \\
8\end{array}$ & $\begin{array}{l}20 \\
49 \\
58 \\
12\end{array}$ & $\begin{array}{r}83 \\
179 \\
29 \\
-\end{array}$ & $\begin{array}{r}158 \\
194 \\
17 \\
-\end{array}$ & $\begin{array}{r}87 \\
62 \\
4 \\
-\end{array}$ & $\begin{array}{r}19 \\
9 \\
1 \\
-\end{array}$ \\
\hline Secured by real estate ............. farms... & $\begin{array}{rr}1 & 205 \\
12 & 415\end{array}$ & 2729 & $5 \begin{array}{r}129 \\
5265\end{array}$ & 2838 & $\begin{array}{r}326 \\
2281\end{array}$ & $\begin{array}{l}130 \\
831\end{array}$ & $\begin{array}{r}27 \\
118\end{array}$ \\
\hline $\begin{array}{l}\text { Farms with expenses of } \\
\$ 1 \text { to } \$ 999 \\
\$ 1.000 \text { to } \$ 4.999 \\
\$ 5.000 \text { to } \$ 24,999 \\
\$ 25,000 \text { or more }\end{array}$ & $\begin{array}{r}139 \\
463 \\
505 \\
98\end{array}$ & $\begin{array}{r}- \\
1 \\
5 \\
23\end{array}$ & \begin{tabular}{r|}
2 \\
16 \\
48 \\
63
\end{tabular} & $\begin{array}{r}5 \\
81 \\
156 \\
23\end{array}$ & $\begin{array}{r}36 \\
117 \\
164 \\
9\end{array}$ & $\begin{array}{r}19 \\
58 \\
51 \\
2\end{array}$ & $\begin{array}{r}6 \\
13 \\
8 \\
-\end{array}$ \\
\hline $\begin{array}{c}\text { Not secured by real estate ............ tarms... } \\
\$ 1,000 \ldots\end{array}$ & $\begin{array}{r}500 \\
2457\end{array}$ & $\begin{array}{r}12 \\
637\end{array}$ & $\begin{array}{r}35 \\
938\end{array}$ & $\begin{array}{r}97 \\
461\end{array}$ & $\begin{array}{l}126 \\
570\end{array}$ & $\begin{array}{r}61 \\
250\end{array}$ & $\begin{array}{l}11 \\
43\end{array}$ \\
\hline $\begin{array}{l}\text { Farms with expenses of- } \\
\$ 1 \text { to } \$ 999 \\
\$ 1,000 \text { to } \$ 4,999 \\
\$ 5,000 \text { to } \$ 24,999 \\
\$ 25,000 \text { or more }\end{array}$ & $\begin{array}{r}183 \\
189 \\
113 \\
15\end{array}$ & $\begin{array}{l}1 \\
1 \\
4 \\
6\end{array}$ & $\begin{array}{r}6 \\
2 \\
17 \\
10\end{array}$ & $\begin{array}{r}25 \\
35 \\
36 \\
1\end{array}$ & $\begin{array}{r}39 \\
41 \\
43 \\
3\end{array}$ & $\begin{array}{r}6 \\
41 \\
14 \\
-\end{array}$ & $\begin{array}{l}4 \\
6 \\
1\end{array}$ \\
\hline 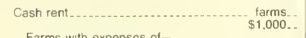 & 11089 & $2 \begin{array}{r}23 \\
230\end{array}$ & $\begin{array}{r}78 \\
4006\end{array}$ & $\begin{array}{r}129 \\
1838\end{array}$ & $\begin{array}{rr}176 \\
2152\end{array}$ & $\begin{array}{r}89 \\
1206\end{array}$ & $\begin{array}{r}64 \\
585\end{array}$ \\
\hline $\begin{array}{l}\text { Farms with expenses of } \\
\$ 1 \text { to } \$ 4,999 \\
\$ 5,000 \text { to } \$ 9.999 \\
\$ 10,000 \text { to } \$ 24.999 \ldots \ldots \\
\$ 25,000 \text { or more }\end{array}$ & $\begin{array}{l}582 \\
103 \\
184 \\
103\end{array}$ & $\begin{array}{r}2 \\
3 \\
4 \\
14\end{array}$ & \begin{tabular}{r|r}
18 & 6 \\
14 \\
40
\end{tabular} & $\begin{array}{l}54 \\
20 \\
32 \\
23\end{array}$ & $\begin{array}{r}101 \\
16 \\
32 \\
27\end{array}$ & $\begin{array}{l}17 \\
23 \\
37 \\
12\end{array}$ & $\begin{array}{r}29 \\
7 \\
27 \\
1\end{array}$ \\
\hline Property taxes ....................... tarms & $\begin{array}{ll}2 & 724 \\
2 & 591\end{array}$ & $\begin{array}{r}36 \\
207\end{array}$ & $\begin{array}{l}151 \\
506\end{array}$ & $\begin{array}{l}378 \\
450\end{array}$ & $\begin{array}{l}549 \\
536\end{array}$ & $\begin{array}{l}258 \\
241\end{array}$ & $\begin{array}{r}87 \\
106\end{array}$ \\
\hline $\begin{array}{l}\text { Farms with expenses of }- \\
\$ 1 \text { to } \$ 4,999 \\
\$ 5,000 \text { to } \$ 9.999 \\
\$ 10,000 \text { to } \$ 24,999 \ldots \ldots \ldots \\
\$ 25,000 \text { or more }\end{array}$ & $\begin{array}{r}2666 \\
42 \\
12 \\
4\end{array}$ & $\begin{array}{r}24 \\
6 \\
5 \\
1\end{array}$ & $\begin{array}{r}121 \\
21 \\
8 \\
1\end{array}$ & $\begin{array}{r}368 \\
7 \\
1 \\
2\end{array}$ & $\begin{array}{r}537 \\
10 \\
1 \\
1\end{array}$ & $\begin{array}{r}256 \\
1 \\
1 \\
-\end{array}$ & $\begin{array}{r}85 \\
1 \\
1 \\
-\end{array}$ \\
\hline All other farm production expenses ....... tarms.. & $\begin{array}{r}2813 \\
15758\end{array}$ & 4204 & $\begin{array}{r}7156 \\
161\end{array}$ & $\begin{array}{r}386 \\
3070\end{array}$ & $2 \begin{array}{r}572 \\
2979\end{array}$ & 1085 & $\begin{array}{r}98 \\
270\end{array}$ \\
\hline $\begin{array}{l}\text { Farms with expenses of - } \\
\$ 1 \text { to } \$ 4,999 \\
\$ 5,000 \text { to } \$ 24,999 \\
\$ 25,000 \text { to } \$ 49,999 \\
\$ 50,000 \text { or more }\end{array}$ & $\begin{array}{r}2271 \\
442 \\
50 \\
50\end{array}$ & $\begin{array}{r}1 \\
13 \\
6 \\
18\end{array}$ & $\begin{array}{l}36 \\
67 \\
16 \\
37\end{array}$ & $\begin{array}{r}231 \\
128 \\
17 \\
10\end{array}$ & $\begin{array}{r}386 \\
175 \\
8 \\
3\end{array}$ & $\begin{array}{r}209 \\
48 \\
8 \\
-\end{array}$ & $\begin{array}{r}87 \\
10 \\
1 \\
-\end{array}$ \\
\hline
\end{tabular}


Table 52. Summary by Value of Agricultural Products Sold: 1987-Con.

[For meaning of abbreviations and symbols, see introductory text]

\begin{tabular}{|c|c|c|c|c|c|c|}
\hline Item & $\begin{array}{r}\$ 25,000 \text { to } \\
\$ 39,999\end{array}$ & $\begin{array}{r}\$ 20,000 \text { to } \\
\$ 24,999\end{array}$ & $\begin{array}{r}\$ 10,000 \text { to } \\
\$ 19.999\end{array}$ & $\begin{array}{r}\$ 5,000 \text { 10 } \\
\$ 9,999\end{array}$ & $\begin{array}{r}\$ 2,500 \text { to } \\
\$ 4,999\end{array}$ & $\begin{array}{l}\text { Less than } \\
\$ 2,500\end{array}$ \\
\hline \multicolumn{7}{|l|}{$\begin{array}{l}\text { FARM PRODUCTION EXPENSES } 1 \\
\text {-CON. }\end{array}$} \\
\hline Total farm production expenses - Con. & & & & & & \\
\hline $\begin{array}{c}\text { Electricity } \\
\text { Farms with expenses of }\end{array} \quad \$ 1,000$. & $\begin{array}{r}100 \\
90\end{array}$ & $\begin{array}{l}39 \\
19\end{array}$ & $\begin{array}{r}173 \\
96\end{array}$ & $\begin{array}{r}177 \\
60\end{array}$ & $\begin{array}{r}151 \\
55\end{array}$ & $\begin{array}{r}230 \\
58\end{array}$ \\
\hline $\begin{array}{l}\$ 1 \text { to } \$ 999 \\
\$ 1,000 \text { to } \$ 4,999 \\
\$ 25,000 \text { to } \$ 24,999 \\
\$ 25,000 \text { or more }\end{array}$ & $\begin{array}{r}77 \\
23 \\
- \\
-\end{array}$ & $\begin{array}{r}36 \\
3 \\
- \\
-\end{array}$ & $\begin{array}{r}155 \\
18 \\
- \\
-\end{array}$ & $\begin{array}{r}169 \\
8 \\
- \\
-\end{array}$ & $\begin{array}{r}138 \\
13 \\
- \\
-\end{array}$ & $\begin{array}{r}224 \\
6 \\
- \\
-\end{array}$ \\
\hline Hired farm labor ........................ tarms. & $\begin{array}{r}48 \\
225\end{array}$ & $\begin{array}{l}18 \\
88\end{array}$ & $\begin{array}{r}67 \\
177\end{array}$ & $\begin{array}{l}66 \\
76\end{array}$ & $\begin{array}{l}34 \\
38\end{array}$ & $\begin{array}{l}63 \\
80\end{array}$ \\
\hline $\begin{array}{l}\text { Farms with expenses ot }- \\
\$ 1 \text { to } \$ 4,999 \\
\$ 5,000 \text { to } \$ 24,999 \\
\$ 25,000 \text { to } \$ 99,999 . \ldots . \\
\$ 100,000 \text { or more }\end{array}$ & $\begin{array}{r}28 \\
20 \\
- \\
-\end{array}$ & $\begin{array}{l}9 \\
9 \\
- \\
-\end{array}$ & $\begin{array}{r}48 \\
19 \\
- \\
-\end{array}$ & $\begin{array}{r}61 \\
5 \\
- \\
-\end{array}$ & $\begin{array}{l}34 \\
- \\
-\end{array}$ & $\begin{array}{l}63 \\
- \\
-\end{array}$ \\
\hline 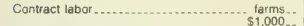 & $\begin{array}{r}7 \\
10\end{array}$ & (D) & 1 & $\begin{array}{l}10 \\
29\end{array}$ & (D) & 11 \\
\hline $\begin{array}{l}\text { Farms with expenses of }- \\
\$ 1 \text { to } \$ 999 \\
\$ 1,000 \text { to } \$ 4,999 \\
\$ 5,000 \text { to } \$ 24,999 \\
\$ 25,000 \text { or more }\end{array}$ & $\begin{array}{l}4 \\
2 \\
1 \\
-\end{array}$ & $\begin{array}{r}1 \\
- \\
- \\
-\end{array}$ & (U) & $\begin{array}{l}29 \\
2 \\
8 \\
-\end{array}$ & $\overline{1}$ & $\begin{array}{l}14 \\
8 \\
3 \\
- \\
-\end{array}$ \\
\hline Repar and maintenance ............... farms.. & $\begin{array}{r}94 \\
250\end{array}$ & $\begin{array}{l}45 \\
77\end{array}$ & $\begin{array}{l}158 \\
301\end{array}$ & $\begin{array}{l}216 \\
232\end{array}$ & $\begin{array}{l}224 \\
182\end{array}$ & $\begin{array}{l}392 \\
320\end{array}$ \\
\hline $\begin{array}{l}\text { Farms with expenses of } \\
\$ 1 \text { to } \$ 4,999 \\
\$ 5,000 \text { to } \$ 24,999 \\
\$ 25,000 \text { to } \$ 49,999 \\
\$ 50,000 \text { or more }\end{array}$ & $\begin{array}{r}74 \\
20 \\
- \\
-\end{array}$ & $\begin{array}{r}45 \\
- \\
-\end{array}$ & $\begin{array}{r}142 \\
16 \\
- \\
-\end{array}$ & $\begin{array}{r}215 \\
1 \\
-\end{array}$ & $\begin{array}{r}222 \\
2 \\
-\end{array}$ & $\begin{array}{r}391 \\
1 \\
-\end{array}$ \\
\hline Customwork, machine hire, and rental of & & & & & & \\
\hline $\begin{array}{l}\text { machinery and equipment .............. farms.. } \\
\text { Farms with expenses of }-\end{array}$ & $\begin{array}{l}36 \\
88\end{array}$ & $\begin{array}{r}14 \\
(D)\end{array}$ & $\begin{array}{l}120 \\
185\end{array}$ & $\begin{array}{r}107 \\
80\end{array}$ & $\begin{array}{r}113 \\
81\end{array}$ & $\begin{array}{r}180 \\
73\end{array}$ \\
\hline $\begin{array}{l}\$ 1 \text { to } \$ 999 \\
\$ 1,000 \text { to } \$ 4,999 \\
\$ 5,000 \text { to } \$ 24,999 \\
\$ 25,000 \text { or more }\end{array}$ & $\begin{array}{r}14 \\
15 \\
7 \\
-\end{array}$ & $\begin{array}{r}13 \\
1 \\
- \\
-\end{array}$ & $\begin{array}{r}64 \\
56 \\
- \\
-\end{array}$ & $\begin{array}{r}70 \\
37 \\
- \\
-\end{array}$ & $\begin{array}{r}92 \\
21 \\
-\end{array}$ & $\begin{array}{r}155 \\
25 \\
- \\
-\end{array}$ \\
\hline 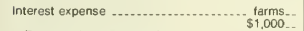 & $\begin{array}{r}61 \\
379\end{array}$ & $\begin{array}{l}26 \\
92\end{array}$ & $\begin{array}{r}69 \\
193\end{array}$ & $\begin{array}{l}101 \\
178\end{array}$ & $\begin{array}{l}51 \\
95\end{array}$ & $\begin{array}{l}126 \\
342\end{array}$ \\
\hline $\begin{array}{l}\text { Farms with expenses of } \\
\$ 1 \text { to } \$ 4,999 \\
\$ 5,000 \text { to } \$ 24,999 \\
\$ 25,000 \text { to } \$ 99,999 \ldots \ldots \ldots \\
\$ 100,000 \text { or more }\end{array}$ & $\begin{array}{r}34 \\
27 \\
- \\
-\end{array}$ & $\begin{array}{r}18 \\
8 \\
- \\
-\end{array}$ & $\begin{array}{r}54 \\
14 \\
1 \\
-\end{array}$ & $\begin{array}{r}95 \\
6 \\
- \\
\end{array}$ & $\begin{array}{r}50 \\
1 \\
- \\
-\end{array}$ & $\begin{array}{r}95 \\
31 \\
-\end{array}$ \\
\hline Secured by real estate .............. farms.. & $\begin{array}{r}58 \\
351\end{array}$ & $\begin{array}{l}14 \\
68\end{array}$ & $\begin{array}{r}34 \\
150\end{array}$ & $\begin{array}{r}67 \\
126\end{array}$ & $\begin{array}{l}34 \\
69\end{array}$ & $\begin{array}{l}121 \\
319\end{array}$ \\
\hline $\begin{array}{l}\text { Farms with expenses of - } \\
\$ 1 \text { to } \$ 999 \\
\$ 1.000 \text { to } \$ 4,999 \\
\$ 5.000 \text { to } \$ 24,999 \\
\$ 25.000 \text { or more }\end{array}$ & $\begin{array}{r}2 \\
29 \\
27 \\
-\end{array}$ & $\begin{array}{l}\overline{8} \\
6 \\
-\end{array}$ & $\begin{array}{r}8 \\
12 \\
13 \\
1\end{array}$ & $\begin{array}{r}19 \\
42 \\
6 \\
-\end{array}$ & $\begin{array}{r}7 \\
26 \\
1 \\
-\end{array}$ & $\begin{array}{l}35 \\
61 \\
25 \\
-\end{array}$ \\
\hline Not secured by real estate ........... farms.. & $\begin{array}{l}25 \\
27\end{array}$ & $\begin{array}{l}20 \\
24\end{array}$ & $\begin{array}{l}53 \\
43\end{array}$ & $\begin{array}{l}40 \\
52\end{array}$ & $\begin{array}{l}18 \\
25\end{array}$ & $\begin{array}{l}14 \\
23\end{array}$ \\
\hline $\begin{array}{l}\text { Farms with expenses of } \\
\$ 1 \text { to } \$ 999 \\
\$ 1,000 \text { to } \$ 4,999 \\
\$ 5,000 \text { to } \$ 24,999 \\
\$ 25,000 \text { or more }\end{array}$ & $\begin{array}{r}19 \\
6 \\
- \\
-\end{array}$ & $\begin{array}{r}17 \\
1 \\
2 \\
-\end{array}$ & $\begin{array}{r}42 \\
10 \\
1 \\
-\end{array}$ & $\begin{array}{r}16 \\
24 \\
- \\
-\end{array}$ & $\begin{array}{r}8 \\
10 \\
- \\
-\end{array}$ & $\begin{array}{r}1 \\
13 \\
- \\
-\end{array}$ \\
\hline Cash rent ......................... farms... & $\begin{array}{r}67 \\
655\end{array}$ & $\begin{array}{l}17 \\
90\end{array}$ & $\begin{array}{r}87 \\
177\end{array}$ & $\begin{array}{r}97 \\
195\end{array}$ & $\begin{array}{r}77 \\
121\end{array}$ & $\begin{array}{l}91 \\
64\end{array}$ \\
\hline $\begin{array}{l}\text { Farms with expenses of } \\
\$ 1 \text { to } \$ 4,999 \\
\$ 5,000 \text { to } \$ 9,999 \\
\$ 10000 \text { to } \$ 24,999 \\
\$ 25,000 \text { or more }\end{array}$ & $\begin{array}{r}22 \\
6 \\
39 \\
-\end{array}$ & $\begin{array}{r}2 \\
14 \\
1 \\
-\end{array}$ & $\frac{85}{2}$ & $\begin{array}{r}96 \\
1 \\
- \\
-\end{array}$ & $\begin{array}{r}67 \\
10 \\
-\end{array}$ & $\begin{array}{l}91 \\
- \\
-\end{array}$ \\
\hline Property taxes .......... & $\begin{array}{r}102 \\
83\end{array}$ & $\begin{array}{l}36 \\
24\end{array}$ & $\begin{array}{l}184 \\
156\end{array}$ & $\begin{array}{l}241 \\
116\end{array}$ & $\begin{array}{l}258 \\
124\end{array}$ & $\begin{array}{l}480 \\
251\end{array}$ \\
\hline $\begin{array}{l}\text { Farms with expenses of }- \\
\$ 1 \text { 10 } \$ 4,999, \ldots 9 . \\
\$ 5,000 \text { to } \$ 9,999 \\
\$ 10,000 \text { to } \$ 24,999 \\
\$ 25,000 \text { or more }\end{array}$ & $\begin{array}{r}101 \\
1 \\
- \\
-\end{array}$ & $\begin{array}{l}36 \\
- \\
-\end{array}$ & $\begin{array}{r}183 \\
1 \\
- \\
-\end{array}$ & $\begin{array}{r}241 \\
- \\
- \\
-\end{array}$ & $\begin{array}{r}258 \\
- \\
-\end{array}$ & 480 \\
\hline $\begin{array}{c}\text { All other farm production expenses...... farms.- } \\
\text { Farms with expenses of - }\end{array}$ & $\begin{array}{l}126 \\
302\end{array}$ & $\begin{array}{r}45 \\
144\end{array}$ & $\begin{array}{l}217 \\
223\end{array}$ & $\begin{array}{l}262 \\
173\end{array}$ & $\begin{array}{l}248 \\
154\end{array}$ & $\begin{array}{l}438 \\
198\end{array}$ \\
\hline $\begin{array}{l}\$ 1 \text { to } \$ 4,999 \\
\$ 5,000 \text { to } \$ 24,999 \\
\$ 25,000 \text { to } \$ 49,999 . \ldots . \\
\$ 50,000 \text { or more }\end{array}$ & $\begin{array}{r}121 \\
5 \\
- \\
-\end{array}$ & $\begin{array}{r}38 \\
7 \\
- \\
-\end{array}$ & $\begin{array}{r}216 \\
1 \\
-\end{array}$ & $\begin{array}{r}261 \\
1 \\
- \\
-\end{array}$ & $\begin{array}{r}248 \\
- \\
-\end{array}$ & ${ }^{438}$ \\
\hline
\end{tabular}


Table 52. Summary by Value of Agricultural Products Sold: 1987-Con.

[For meaning of abbreviations and symbols, see introductory text]

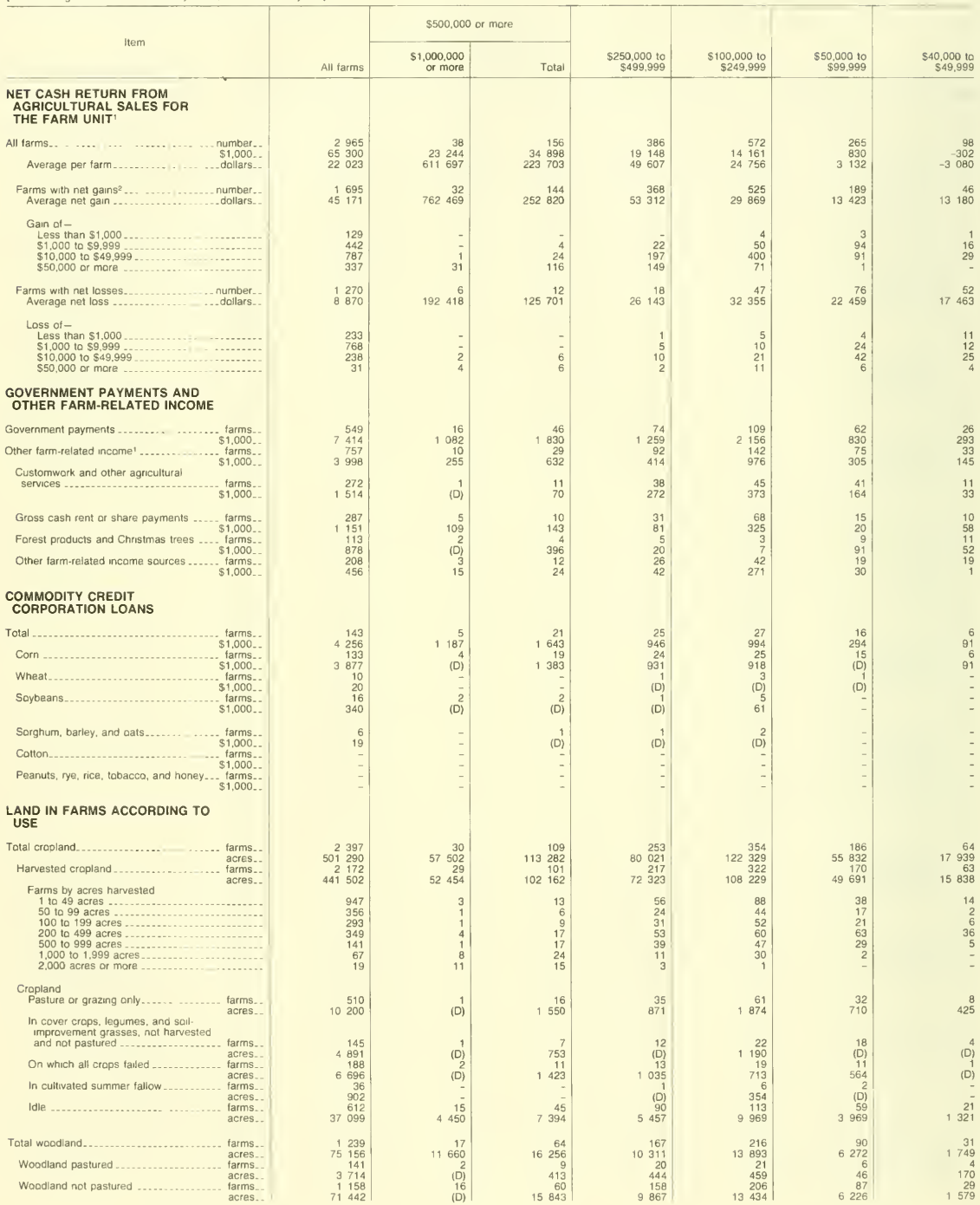

See foatnotes at end of table 
Table 52. Summary by Value of Agricultural Products Sold: 1987-Con.

[For meaning of abbreviations and symbols, see introductory text]

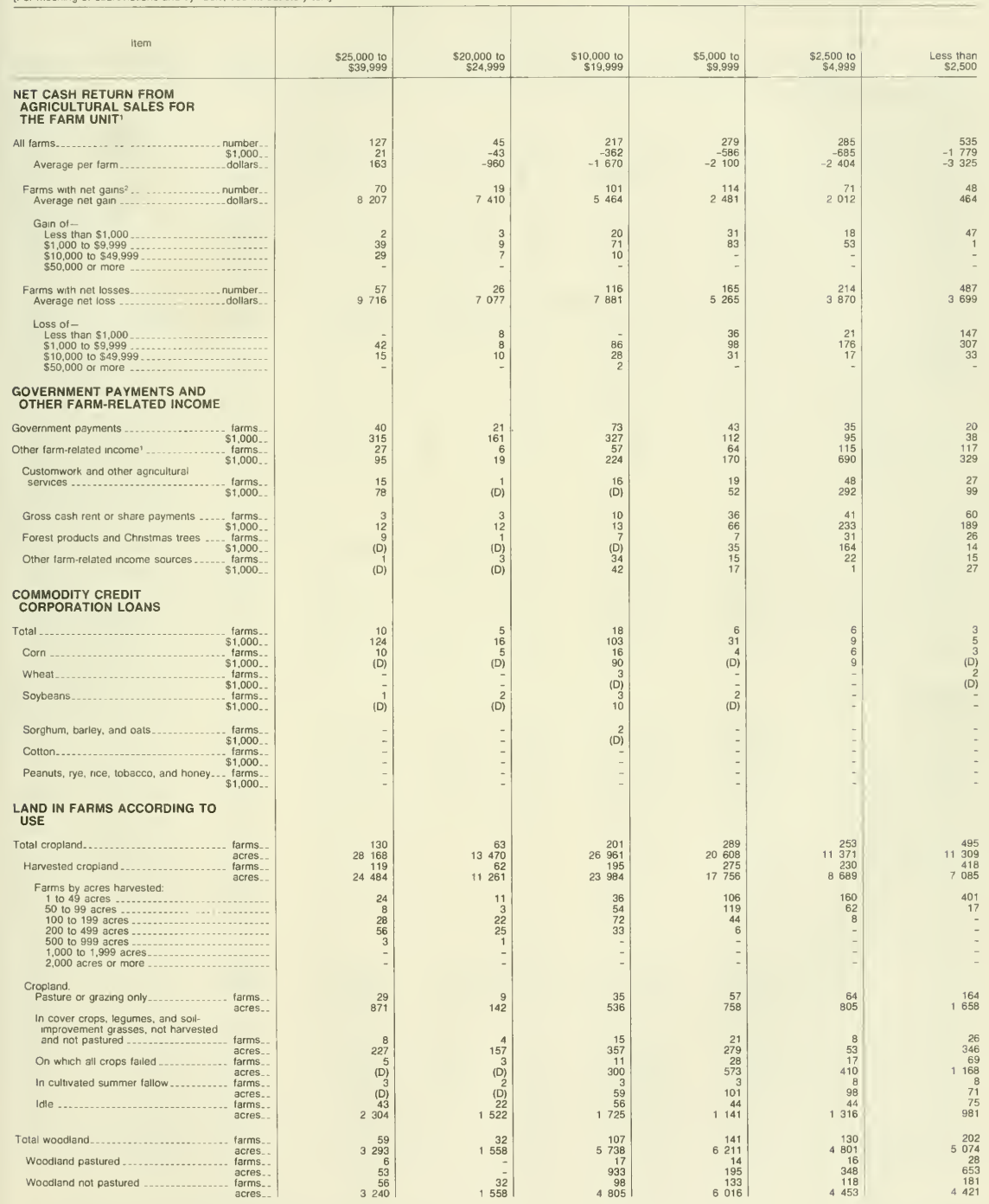

See footnotes at end of table. 
Table 52. Summary by Value of Agricultural Products Sold: 1987-Con.

[For meaning of abbreviations and symbols, see introductory text]

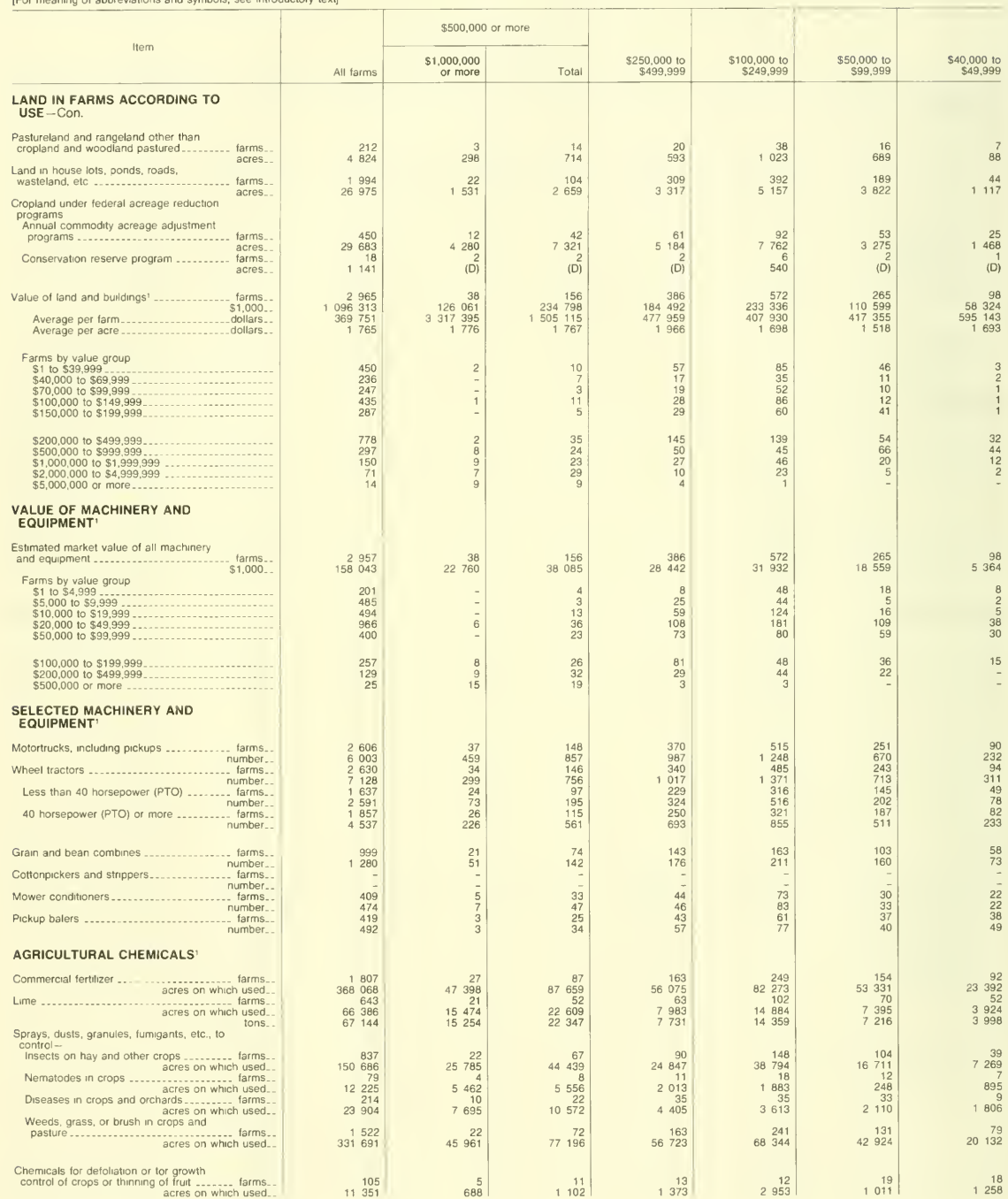


Table 52. Summary by Value of Agricultural Products Sold: 1987-Con.

[For meaning of abbreviations and symbols, see introductory text]

\begin{tabular}{|c|c|c|c|c|c|c|c|}
\hline \multirow{2}{*}{ Item } & \multirow[b]{2}{*}{ All farms } & \multicolumn{2}{|c|}{$\$ 500,000$ or more } & \multirow[b]{2}{*}{$\begin{array}{r}\$ 250,000 \text { to } \\
\$ 499.999\end{array}$} & \multirow[b]{2}{*}{$\begin{array}{r}\$ 100,000 \text { to } \\
\$ 249,999\end{array}$} & \multirow[b]{2}{*}{$\begin{array}{r}\$ 50,000 \text { to } \\
\$ 99,999\end{array}$} & \multirow[b]{2}{*}{$\begin{array}{r}\$ 40,000 \text { to } \\
\$ 49,999\end{array}$} \\
\hline & & $\begin{array}{r}\$ 1,000,000 \\
\text { or more }\end{array}$ & Total & & & & \\
\hline \multicolumn{8}{|l|}{$\begin{array}{l}\text { TENURE AND RACE OF } \\
\text { OPERATOR }\end{array}$} \\
\hline $\begin{array}{l}\text { All operators } \\
\text { Full owners } \\
\text { Part owners } \\
\text { Tenants }\end{array}$ & $\begin{array}{r}2966 \\
1825 \\
857 \\
284\end{array}$ & $\begin{array}{r}38 \\
14 \\
20 \\
4\end{array}$ & $\begin{array}{r}156 \\
76 \\
69 \\
11\end{array}$ & $\begin{array}{r}403 \\
258 \\
130 \\
15\end{array}$ & $\begin{array}{r}549 \\
343 \\
175 \\
31\end{array}$ & $\begin{array}{r}257 \\
159 \\
77 \\
21\end{array}$ & $\begin{array}{r}73 \\
30 \\
34 \\
9\end{array}$ \\
\hline $\begin{array}{l}\text { White } \\
\text { Full owners } \\
\text { Part owners } \\
\text { Tenants } \ldots\end{array}$ & $\begin{array}{r}2914 \\
1791 \\
845 \\
278\end{array}$ & $\begin{array}{r}37 \\
13 \\
20 \\
4\end{array}$ & $\begin{array}{r}149 \\
69 \\
69 \\
11\end{array}$ & $\begin{array}{r}401 \\
257 \\
129 \\
15\end{array}$ & $\begin{array}{r}546 \\
342 \\
173 \\
31\end{array}$ & $\begin{array}{r}257 \\
159 \\
77 \\
21\end{array}$ & $\begin{array}{r}71 \\
29 \\
33 \\
9\end{array}$ \\
\hline $\begin{array}{l}\text { Black and other races } \\
\text { Full owners } \\
\text { Part owners } \\
\text { Tenants }\end{array}$ & $\begin{array}{r}52 \\
34 \\
12 \\
6\end{array}$ & $\begin{array}{l}1 \\
1 \\
- \\
-\end{array}$ & $\begin{array}{l}7 \\
7 \\
- \\
-\end{array}$ & $\begin{array}{l}2 \\
1 \\
1 \\
-\end{array}$ & $\begin{array}{l}3 \\
1 \\
2 \\
-\end{array}$ & $\begin{array}{l}- \\
\overline{-}\end{array}$ & $\begin{array}{l}2 \\
1 \\
1 \\
-\end{array}$ \\
\hline \multicolumn{8}{|l|}{ OWNED AND RENTED LAND } \\
\hline 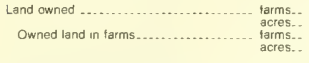 & $\begin{array}{rr}2 & 691 \\
360 & 152 \\
2 & 682 \\
325 & 313\end{array}$ & $\begin{array}{r}35 \\
41 \quad 284 \\
34 \\
40318\end{array}$ & $\begin{array}{r}146 \\
73330 \\
70419 \\
704\end{array}$ & $\begin{array}{r}389 \\
45977 \\
388 \\
41686\end{array}$ & $\begin{array}{r}518 \\
72631 \\
518 \\
66624\end{array}$ & $\begin{array}{r}236 \\
36998 \\
236 \\
35458\end{array}$ & $\begin{array}{r}64 \\
11 \quad 513 \\
64 \\
10 \quad 837\end{array}$ \\
\hline $\begin{aligned} \text { Land rented or leased from others ........ farms_. } \\
\text { acres.. } \\
\text { Rented or leased land in farms ......... facrs.- } \\
\text { acres.. }\end{aligned}$ & $\begin{array}{rr}1 & 153 \\
285 & 163 \\
1 & 141 \\
282 & 932\end{array}$ & $\begin{array}{rr} & 24 \\
31 & 325 \\
24 \\
30673\end{array}$ & \begin{tabular}{rr|} 
& 80 \\
63 & 144 \\
80 \\
$62 \quad 492$
\end{tabular} & $\begin{array}{rr}146 \\
53051 \\
145 \\
52556\end{array}$ & $\begin{array}{r}209 \\
76085 \\
206 \\
75778\end{array}$ & $\begin{array}{r}98 \\
31 \quad 510 \\
98 \\
31 \quad 157\end{array}$ & $\begin{array}{r}43 \\
10 \quad 056 \\
43 \\
10056\end{array}$ \\
\hline $\begin{array}{l}\text { Land rented or leased to others............. farms.. } \\
\text { acres.. }\end{array}$ & $\begin{array}{r}463 \\
37070\end{array}$ & $\begin{array}{r}5 \\
1618\end{array}$ & $\begin{array}{r}20 \\
3563\end{array}$ & $\begin{array}{r}64 \\
4786\end{array}$ & 6314 & $\begin{array}{r}31 \\
1893\end{array}$ & $\begin{array}{r}7 \\
676\end{array}$ \\
\hline \multicolumn{8}{|l|}{ OPERATOR CHARACTERISTICS } \\
\hline $\begin{array}{l}\text { Operators by place of residence } \\
\text { On tarm operated } \\
\text { Not on farm operated } \\
\text { Not reported }\end{array}$ & $\begin{array}{r}2175 \\
481 \\
310\end{array}$ & $\begin{array}{r}23 \\
11 \\
4\end{array}$ & $\begin{array}{r}112 \\
25 \\
19\end{array}$ & $\begin{array}{r}305 \\
32 \\
66\end{array}$ & $\begin{array}{r}410 \\
60 \\
79\end{array}$ & $\begin{array}{r}198 \\
33 \\
26\end{array}$ & $\begin{array}{r}54 \\
12 \\
7\end{array}$ \\
\hline 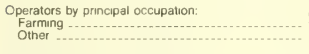 & $\begin{array}{ll}1 & 774 \\
1 & 192\end{array}$ & $\begin{array}{r}34 \\
4\end{array}$ & $\begin{array}{r}130 \\
26\end{array}$ & $\begin{array}{r}320 \\
83\end{array}$ & $\begin{array}{l}392 \\
157\end{array}$ & $\begin{array}{r}198 \\
59\end{array}$ & $\begin{array}{l}52 \\
21\end{array}$ \\
\hline $\begin{array}{l}\text { Operators by days of work off farm. } \\
\text { None. } \\
\text { Any } 1099 \text { days } \\
100 \text { to } 199 \text { days } \\
200 \text { days or more }\end{array}$ & $\begin{array}{r}1263 \\
1426 \\
254 \\
217 \\
955\end{array}$ & $\begin{array}{r}26 \\
8 \\
-2 \\
6\end{array}$ & $\begin{array}{r}101 \\
44 \\
16 \\
9 \\
19\end{array}$ & $\begin{array}{r}217 \\
151 \\
48 \\
20 \\
83\end{array}$ & $\begin{array}{r}264 \\
218 \\
38 \\
33 \\
147\end{array}$ & $\begin{array}{r}137 \\
82 \\
18 \\
17 \\
47\end{array}$ & $\begin{array}{r}33 \\
29 \\
7 \\
2 \\
20\end{array}$ \\
\hline Not reported & 277 & 4 & 11 & 35 & 67 & 38 & 11 \\
\hline $\begin{array}{l}\text { Operators by years on present farm } \\
2 \text { years or less } \\
3 \text { or } 4 \text { years } \\
5 \text { to } 9 \text { years. } \\
10 \text { years or more } \\
\text { Average years on present farm }\end{array}$ & $\begin{array}{r}176 \\
189 \\
398 \\
1588 \\
193\end{array}$ & \begin{tabular}{r|r}
1 \\
1 \\
2 \\
23 \\
23.3
\end{tabular} & \begin{tabular}{r|r}
7 \\
8 \\
11 \\
96 \\
20.5
\end{tabular} & $\begin{array}{r}23 \\
22 \\
47 \\
211 \\
17.4\end{array}$ & $\begin{array}{r}26 \\
39 \\
71 \\
290 \\
194\end{array}$ & $\begin{array}{r}10 \\
18 \\
34 \\
132 \\
21.6\end{array}$ & $\begin{array}{r}3 \\
5 \\
12 \\
41 \\
195\end{array}$ \\
\hline 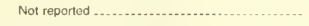 & 615 & 11 & 34 & 100 & 123 & 63 & 12 \\
\hline $\begin{array}{l}\text { Operators by sge group. } \\
\text { Under } 25 \text { years. } \\
25 \text { to } 34 \text { years } \\
35 \text { to } 44 \text { years } \\
45 \text { to } 49 \text { years } \\
50 \text { to } 54 \text { years } \ldots\end{array}$ & $\begin{array}{l}40 \\
374 \\
620 \\
328 \\
321\end{array}$ & $\begin{array}{l}- \\
4 \\
8 \\
2 \\
4\end{array}$ & $\begin{array}{l}14 \\
14 \\
18 \\
17\end{array}$ & $\begin{array}{r}6 \\
69 \\
111 \\
55 \\
57\end{array}$ & $\begin{array}{r}4 \\
63 \\
139 \\
70 \\
67\end{array}$ & $\begin{array}{r}4 \\
31 \\
45 \\
20 \\
25\end{array}$ & $\begin{array}{r}7 \\
11 \\
14 \\
7 \\
7\end{array}$ \\
\hline $\begin{array}{l}55 \text { to } 59 \text { years } \\
60 \text { to } 64 \text { years } \\
65 \text { to } 69 \text { years } \\
70 \text { years and over } \\
\text { Average age }\end{array}$ & $\begin{array}{r}326 \\
348 \\
269 \\
340 \\
51.5\end{array}$ & $\begin{array}{r}9 \\
7 \\
3 \\
1 \\
51.6\end{array}$ & $\begin{array}{r}29 \\
19 \\
8 \\
2 \\
486\end{array}$ & $\begin{array}{r}40 \\
42 \\
14 \\
9 \\
463\end{array}$ & $\begin{array}{r}55 \\
63 \\
48 \\
40 \\
50.0\end{array}$ & $\begin{array}{r}31 \\
36 \\
33 \\
32 \\
53.1\end{array}$ & $\begin{array}{r}7 \\
16 \\
7 \\
4 \\
51.3\end{array}$ \\
\hline $\begin{array}{l}\text { Operators by sex: } \\
\text { Male } \\
\text { Female }\end{array}$ & $\begin{array}{r}2676 \\
290\end{array}$ & 38 & $\begin{array}{r}145 \\
11\end{array}$ & $\begin{array}{r}350 \\
53\end{array}$ & $\begin{array}{r}487 \\
62\end{array}$ & $\begin{array}{r}209 \\
48\end{array}$ & $\begin{array}{r}72 \\
1\end{array}$ \\
\hline $\begin{array}{l}\text { Operators of Spanish origin (see text) } \\
\text { FARMS BY TYPE OF } \\
\text { ORGANIZATION }\end{array}$ & 9 & - & - & 2 & 1 & - & - \\
\hline $\begin{array}{l}\text { Individual or family (sole propnetorship) _... farms.. } \\
\text { acres.. } \\
\text { Partnership ............ larms.. }\end{array}$ & $\begin{array}{r}2553 \\
400 \quad 071 \\
216 \\
62666\end{array}$ & $\begin{array}{r}12 \\
(\mathrm{D}) \\
2\end{array}$ & $\begin{array}{r}89 \\
40 \quad 538 \\
16 \\
12 \quad 252\end{array}$ & 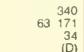 & $\begin{array}{r}474 \\
98 \quad 784 \\
40 \\
14 \quad 235\end{array}$ & $\begin{array}{r}204 \\
45984 \\
38 \\
\text { (D) }\end{array}$ & $\begin{array}{r}63 \\
18063 \\
1637\end{array}$ \\
\hline $\begin{array}{l}\text { Corporation } \\
\text { Family held }\end{array}$ & 173 & 24 & 51 & 28 & $\begin{array}{r}14.230 \\
30\end{array}$ & $\begin{array}{r}11 \\
7003\end{array}$ & 3 \\
\hline $\begin{array}{l}\text { More than } 10 \text { stockhoiders ........... farms. } \\
10 \text { or less stockholders }\end{array}$ & $\begin{array}{r}141 \quad 856 \\
4 \\
169\end{array}$ & $\begin{array}{r}53 \quad 352 \\
3 \\
21\end{array}$ & $\begin{array}{r}80 \quad 121 \\
4 \\
47\end{array}$ & $\begin{array}{r}18204 \\
28\end{array}$ & $\begin{array}{r}27819 \\
30\end{array}$ & $\begin{array}{r}7003 \\
11\end{array}$ & $\frac{1}{3}$ \\
\hline Other than family held ................ tarms. & $\begin{array}{r}8 \\
928\end{array}$ & - & $\overline{-}$ & (D) & (D) & (D) & $\overline{-}$ \\
\hline $\begin{array}{l}\text { More than } 10 \text { stockholders ............ farms.. } \\
10 \text { or less stockholders }\end{array}$ & $\frac{1}{7}$ & - & - & $\overline{1}$ & $\overline{3}$ & $\overline{1}$ & $=$ \\
\hline $\begin{array}{l}\text { Other-cooperative, estate or trust, } \\
\text { institutional, etc }\end{array}$ & $\begin{array}{r}16 \\
2724\end{array}$ & - & $=$ & - & (D) & $\frac{3}{7}$ & - \\
\hline
\end{tabular}


Table 52. Summary by Value of Agricultural Products Sold: 1987-Con.

[For meaning of abbreviations and symbols, see introductory text]

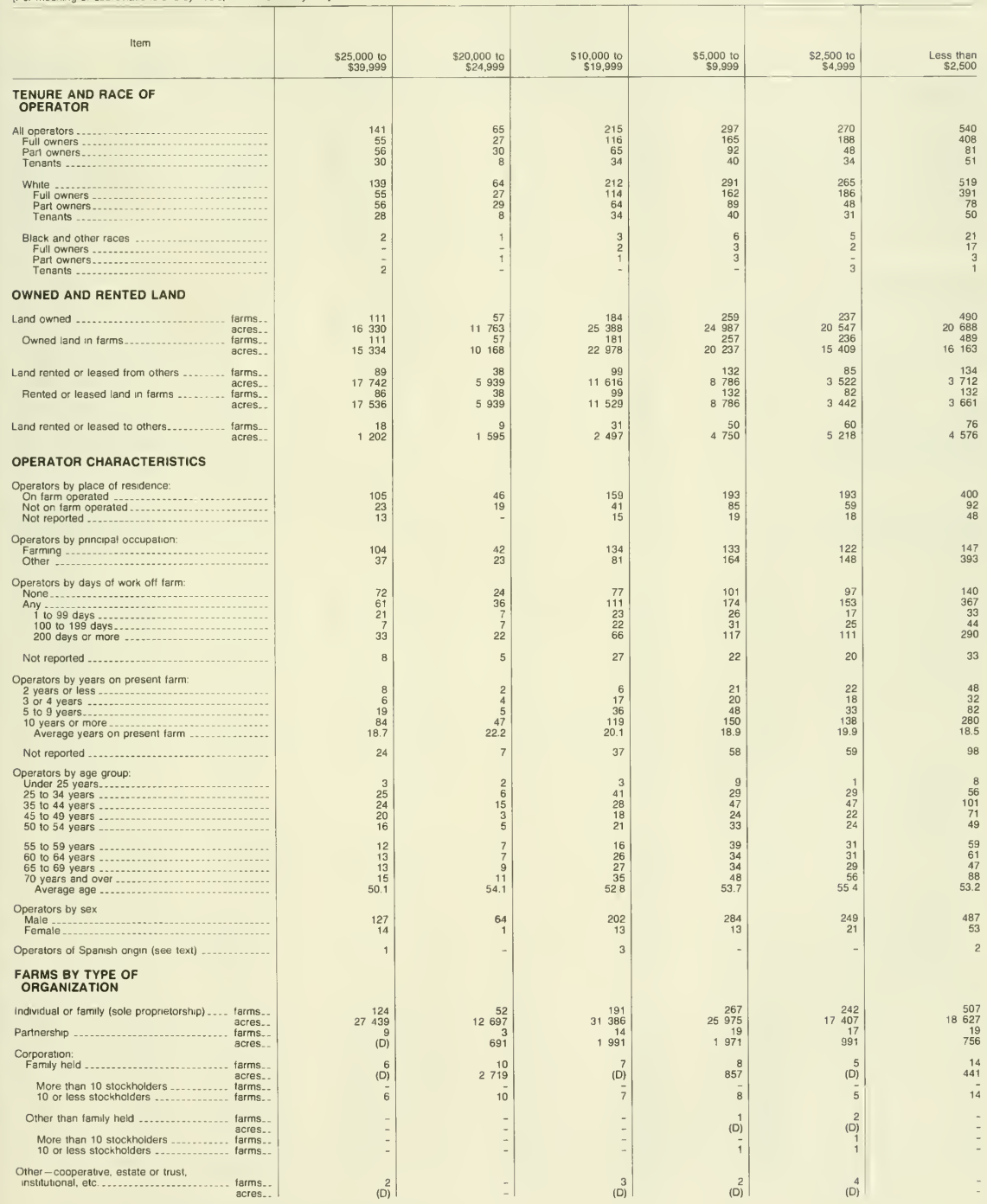

See footnotes at end of table. 
Table 52. Summary by Value of Agricultural Products Sold: 1987-Con.

[For meanung of abbreviations and symbols, see introductory text]

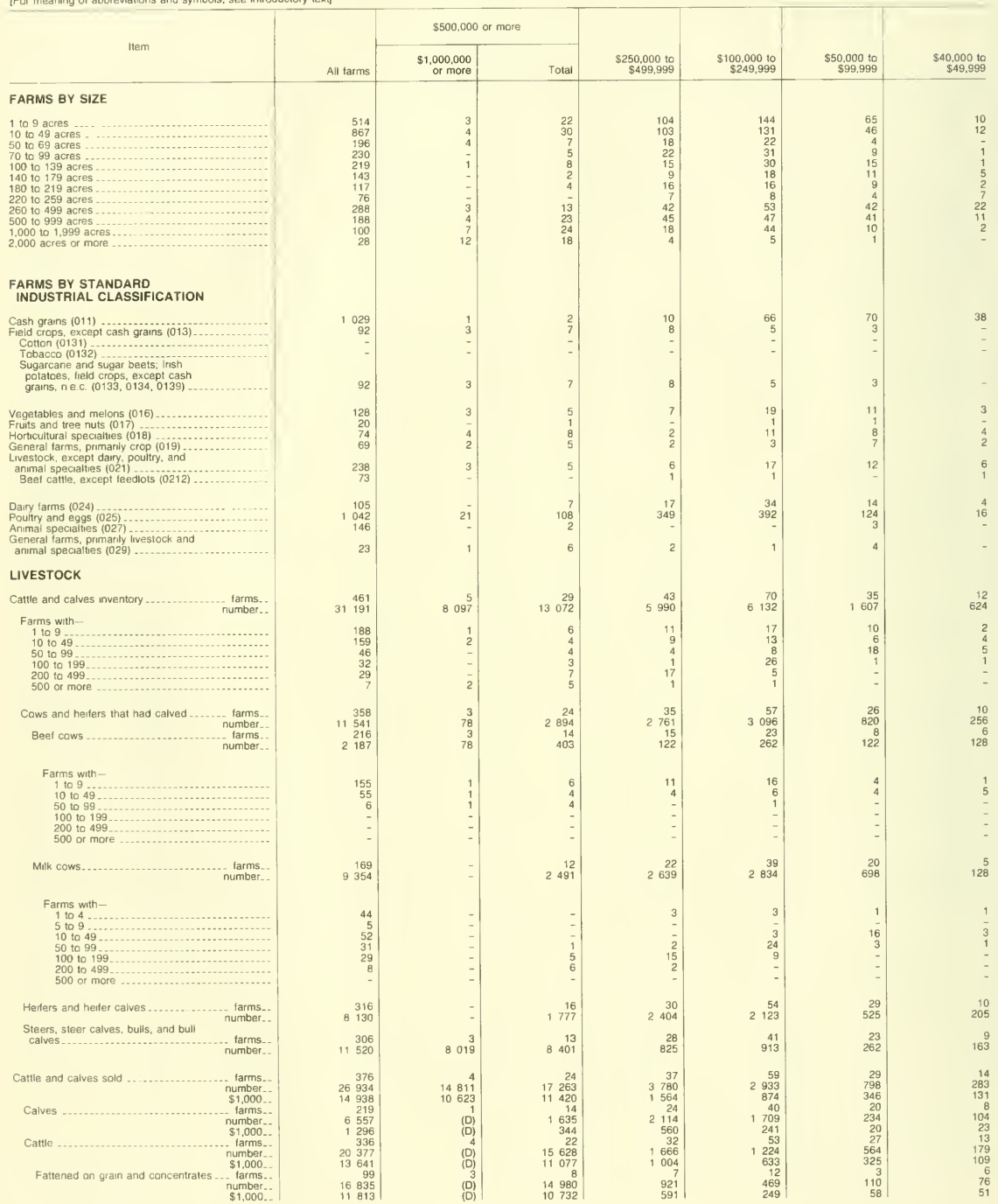

Sea footnotes at end of table 
Table 52. Summary by Value of Agricultural Products Sold: 1987-Con.

[For meaning of abbreviations and symbols, see introductory text]

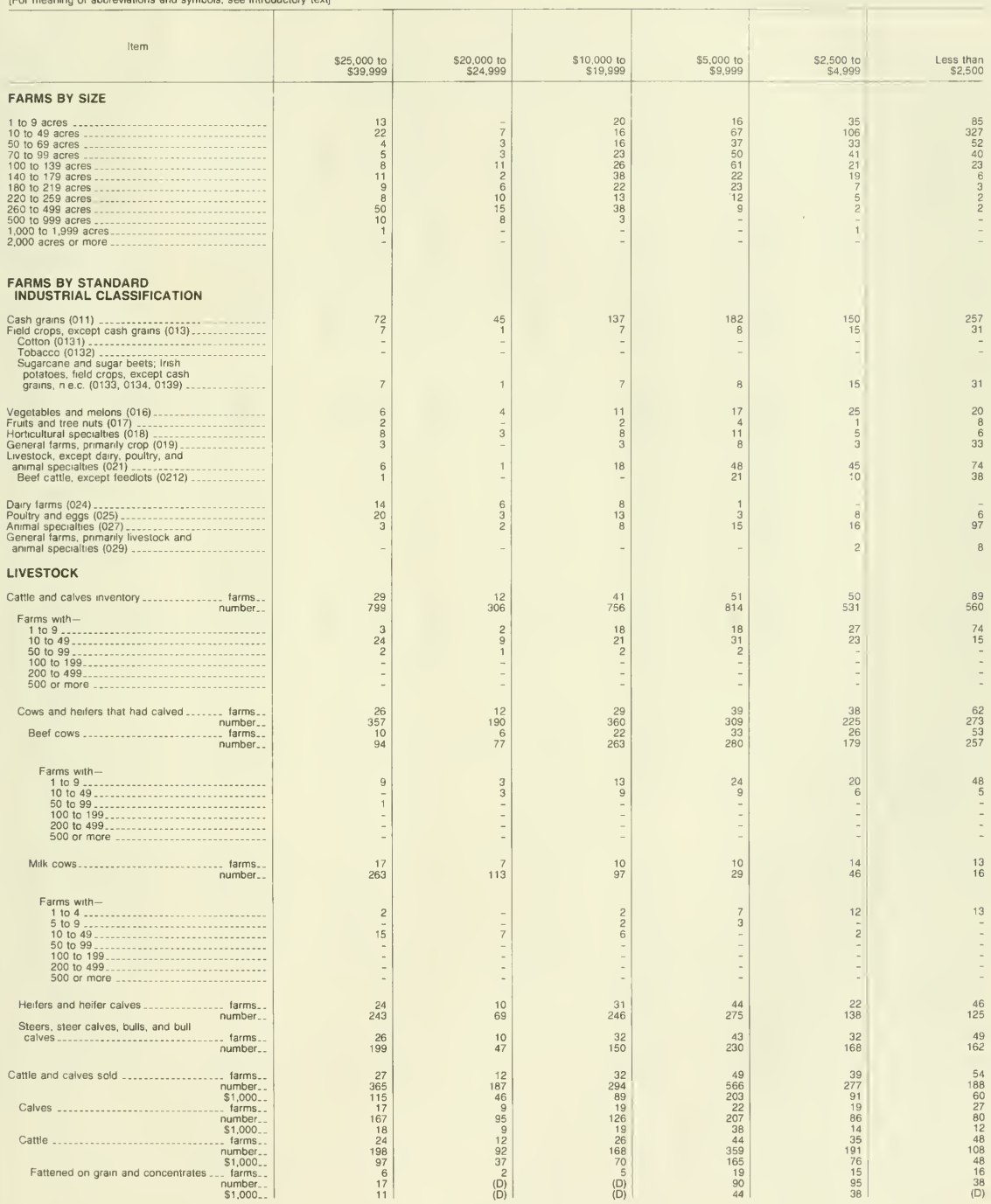


Table 52. Summary by Value of Agricultural Products Sold: 1987-Con.

[For meaning of abbreviations and symbols, see introductory text]

\begin{tabular}{|c|c|c|c|c|c|c|c|}
\hline \multirow{2}{*}{ Hem } & \multirow[b]{2}{*}{ All farms } & \multicolumn{2}{|c|}{$\$ 500,000$ or more } & \multirow[b]{2}{*}{$\begin{array}{r}\$ 250,000 \text { to } \\
\$ 499,999 \\
\end{array}$} & \multirow[b]{2}{*}{$\begin{array}{r}\$ 100,000 \text { to } \\
\$ 249,399 \\
\end{array}$} & \multirow[b]{2}{*}{$\begin{array}{r}\$ 50,000 \text { to } \\
\$ 99,999\end{array}$} & \multirow[b]{2}{*}{$\begin{array}{r}\$ 40,000 \text { to } \\
\$ 49,999 \\
\end{array}$} \\
\hline & & $\begin{array}{r}\$ 1,000,000 \\
\text { or more }\end{array}$ & Total & & & & \\
\hline \multicolumn{8}{|l|}{ LIVESTOCK - Con. } \\
\hline Hogs and pigs inventory .................... tarms.. & $49 \begin{array}{r}301 \\
714\end{array}$ & $7 \begin{array}{r}6 \\
7265\end{array}$ & 14541 & 10330 & $14 \begin{array}{r}52 \\
754\end{array}$ & $\begin{array}{rl}21 & 21 \\
5 & 057\end{array}$ & $\begin{array}{r}10 \\
1114\end{array}$ \\
\hline 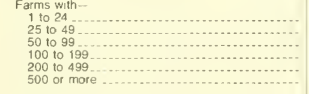 & $\begin{array}{r}123 \\
56 \\
47 \\
23 \\
23 \\
29\end{array}$ & $\begin{array}{l}- \\
\overline{2} \\
\overline{1} \\
3\end{array}$ & $\begin{array}{l}8 \\
4 \\
5 \\
4 \\
5 \\
7\end{array}$ & $\begin{array}{r}10 \\
10 \\
6 \\
10 \\
2 \\
6\end{array}$ & $\begin{array}{r}17 \\
6 \\
10 \\
\overline{7} \\
12\end{array}$ & $\begin{array}{l}4 \\
1 \\
4 \\
3 \\
5 \\
4\end{array}$ & $\begin{array}{l}2 \\
3 \\
1 \\
1 \\
3 \\
-\end{array}$ \\
\hline Used or to be used for breeding .......... farms.. & $10 \begin{array}{r}203 \\
813\end{array}$ & 2716 & 3941 & $2 \begin{array}{r}34 \\
2115\end{array}$ & 2770 & $1 \begin{array}{r}19 \\
1012\end{array}$ & $\begin{array}{r}6 \\
141\end{array}$ \\
\hline Other $\ldots \ldots \ldots$ tarms.. & 38901 & $\begin{array}{r}6 \\
4549\end{array}$ & 10600 & 8215 & $\begin{array}{r}46 \\
11984\end{array}$ & 4045 & $\begin{array}{r}10 \\
973\end{array}$ \\
\hline 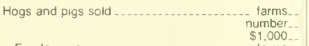 & $\begin{array}{r}310 \\
109600 \\
10 \quad 055\end{array}$ & $\begin{array}{r}6 \\
18394 \\
1946\end{array}$ & $\begin{array}{r}35 \\
37745 \\
3660\end{array}$ & $\begin{array}{r}44 \\
26106 \\
2376\end{array}$ & $\begin{array}{r}53 \\
26333 \\
2378\end{array}$ & $\begin{aligned} 22 \\
9 \\
764 \\
817\end{aligned}$ & $\begin{array}{r}10 \\
2474 \\
173\end{array}$ \\
\hline Feeder pigs ............................. & $\begin{array}{rl} & 74 \\
30 & 594 \\
1503\end{array}$ & (D) & $\begin{array}{r}7 \quad 6 \\
7433 \\
380\end{array}$ & $\begin{array}{r}14 \\
9850 \\
482\end{array}$ & $\begin{array}{r}10 \\
6841 \\
339\end{array}$ & $\begin{array}{r}35 \\
427 \\
184\end{array}$ & $\begin{array}{r}4 \\
1558 \\
63\end{array}$ \\
\hline \multicolumn{8}{|l|}{$\begin{array}{l}\text { Litters of pigs farrowed between- } \\
\text { Dec } 1 \text { of preceding year and Nov } 30 \text {.... farms.. }\end{array}$} \\
\hline & $\begin{array}{rl}13 & 197 \\
179\end{array}$ & 2411 & $\begin{array}{r}4301 \\
17\end{array}$ & $\begin{aligned} 3331 \\
31\end{aligned}$ & $\begin{array}{r}3326 \\
36\end{array}$ & $\begin{array}{r}1201 \\
18\end{array}$ & $\begin{array}{r}218 \\
7\end{array}$ \\
\hline $\begin{array}{c}\text { June } 1 \text { and Nov } 30 \ldots \ldots \\
\text { number.-............... } \\
\text { number... }\end{array}$ & $\begin{array}{l}6597 \\
183 \\
6600\end{array}$ & $\begin{array}{r}1490 \\
4 \\
921\end{array}$ & $\begin{array}{rr}2424 \\
20 \\
1877\end{array}$ & $\begin{array}{r}1615 \\
35 \\
1716\end{array}$ & $\begin{array}{r}1299 \\
37 \\
2027\end{array}$ & $\begin{array}{r}750 \\
17 \\
451\end{array}$ & $\begin{array}{r}117 \\
6 \\
101\end{array}$ \\
\hline $\begin{array}{r}\text { Sheep and lambs of all ages inventory...... farms.. } \\
\text { number.. }\end{array}$ & $\begin{array}{r}50 \\
1667\end{array}$ & - & $\begin{array}{r}3 \\
85\end{array}$ & 93 & 203 & 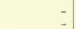 & (D) \\
\hline $\begin{array}{c}\text { Ewes } t \text { year old or older ................. farms... } \\
\text { number.. }\end{array}$ & 1006 & $\overline{-}$ & 52 & (D) & $\begin{array}{r}203 \\
51 \\
151\end{array}$ & - & (D) \\
\hline Sheep and lambs sold .......................... farms. & $\begin{array}{r}38 \\
77 ?\end{array}$ & - & 3 & 2 & 5 & - & 1 \\
\hline $\begin{array}{c}\text { Sheep and lambs shorn } \\
\text { number.- } \\
\text { farms................. } \\
\text { number. }\end{array}$ & $\begin{array}{r}772 \\
39 \\
1099 \\
7081\end{array}$ & $\begin{array}{l}z \\
z\end{array}$ & $\begin{array}{r}42 \\
3 \\
58 \\
537\end{array}$ & $\begin{array}{l}(\mathrm{D}) \\
2 \\
(\mathrm{D}) \\
\text { (D) }\end{array}$ & $\begin{array}{r}100 \\
3 \\
105 \\
795\end{array}$ & $\begin{array}{l}\overline{-} \\
\overline{-}\end{array}$ & $\begin{array}{l}\text { (D) } \\
\text { (D) } \\
\text { (D) }\end{array}$ \\
\hline Horses and ponies inventory ................. farms. & $\begin{array}{r}425 \\
3.029\end{array}$ & 1 & 99 & 17 & 41 & 21 & 3 \\
\hline Horses and ponies sold $\ldots \ldots \ldots$ farms & $\begin{array}{r}3029 \\
123\end{array}$ & (D) & $\begin{aligned} 17 \\
2\end{aligned}$ & 2 & $\begin{array}{r}274 \\
8\end{array}$ & $\begin{array}{r}98 \\
5\end{array}$ & 56 \\
\hline Goats inventory & $\begin{array}{r}334 \\
62\end{array}$ & - & (D) & (D) & 20 & $\begin{array}{r}12 \\
3\end{array}$ & (D) \\
\hline 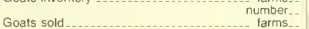 & $\begin{array}{r}02 \\
465 \\
29\end{array}$ & $\bar{z}$ & (D) & (D) & 60 & 27 & (D) \\
\hline Goats sold & $\begin{array}{r}29 \\
326\end{array}$ & - & (D) & - & $\left(D^{2}\right)$ & (D) & (D) \\
\hline \multicolumn{8}{|l|}{ POULTRY } \\
\hline $\begin{array}{l}\text { Chickens } 3 \text { months old or older inventory ... farms.. } \\
\text { number.. }\end{array}$ & $\begin{array}{rr}834 & 175 \\
887\end{array}$ & 359404 & $462 \quad 404$ & 91440 & $\begin{array}{r}24 \\
187256\end{array}$ & 22059 & $23+24$ \\
\hline 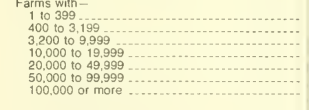 & $\begin{array}{r}126 \\
4 \\
25 \\
14 \\
2 \\
2 \\
2 \\
2\end{array}$ & $\begin{array}{l}- \\
\overline{1} \\
\overline{1} \\
1 \\
2\end{array}$ & $\begin{array}{l}- \\
\overline{2} \\
2 \\
1 \\
2 \\
2\end{array}$ & $\begin{array}{l}2 \\
- \\
6 \\
- \\
- \\
-\end{array}$ & \begin{tabular}{r|r}
4 \\
1 \\
13 \\
5 \\
1 \\
\\
-
\end{tabular} & $\begin{array}{l}3 \\
\overline{3} \\
- \\
- \\
- \\
-\end{array}$ & $\begin{array}{l}5 \\
\frac{5}{2} \\
1 \\
- \\
-\end{array}$ \\
\hline Hens and puliets of laying age ............ farms.. & $\begin{array}{r}167 \\
644 \quad 628\end{array}$ & (D) & $\begin{array}{r}6 \\
357547\end{array}$ & $\begin{array}{r}8 \\
\text { (D) }\end{array}$ & 137242 & $\begin{array}{r}5 \\
(D)\end{array}$ & (D) \\
\hline $\begin{array}{l}\text { Pullets } 3 \text { months old of older not of laying } \\
\text { age }\end{array}$ & 35 & 2 & & 1 & & 2 & 2 \\
\hline 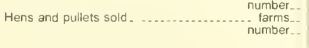 & $\begin{array}{r}189659 \\
88 \\
855 \quad 260\end{array}$ & \begin{tabular}{rr|} 
& (D) \\
331 & 414
\end{tabular} & $\begin{array}{r}104 \\
857 \\
7 \\
417 \quad 754\end{array}$ & $\begin{array}{r}(\mathrm{D}) \\
73030\end{array}$ & $\begin{array}{r}50 \quad 012 \\
24 \\
241 \quad 856\end{array}$ & $\begin{array}{r}(D) \\
5 \\
28003\end{array}$ & $\begin{array}{r}(D) \\
5 \\
20411\end{array}$ \\
\hline \multicolumn{8}{|l|}{$\begin{array}{l}\text { Brolers and other meat-type chickens } \\
\text { sold .................................... tarms. }\end{array}$} \\
\hline Farms with- & 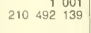 & 26585344 & 67781899 & $85756 \quad \begin{array}{l}348 \\
853\end{array}$ & 49071654 & $\begin{array}{l}6982899 \\
121\end{array}$ & 443526 \\
\hline 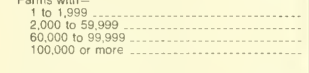 & $\begin{array}{r}6 \\
104 \\
140 \\
751\end{array}$ & $\begin{array}{r}- \\
\overline{-} \\
19\end{array}$ & 111 & \begin{tabular}{r|r}
- \\
1 \\
1 \\
346
\end{tabular} & $\begin{array}{r}\overline{5} \\
79 \\
293\end{array}$ & $\begin{array}{r}6 \overrightarrow{6} \\
60 \\
1\end{array}$ & $\overline{13}$ \\
\hline Turkey hens kept for breeding ................. farms... & $\begin{array}{l}12 \\
50\end{array}$ & $\overline{-}$ & $\overline{-}$ & $=$ & $\overline{-}$ & :- & (D) \\
\hline farms.. & $\begin{array}{r}5 \\
\text { (D) }\end{array}$ & $z$ & (D) & $z$ & (D) & 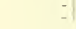 & - \\
\hline
\end{tabular}


Table 52. Summary by Value of Agricultural Products Sold: 1987-Con.

[For meaning of abbreviations and symbols, see introductory text]

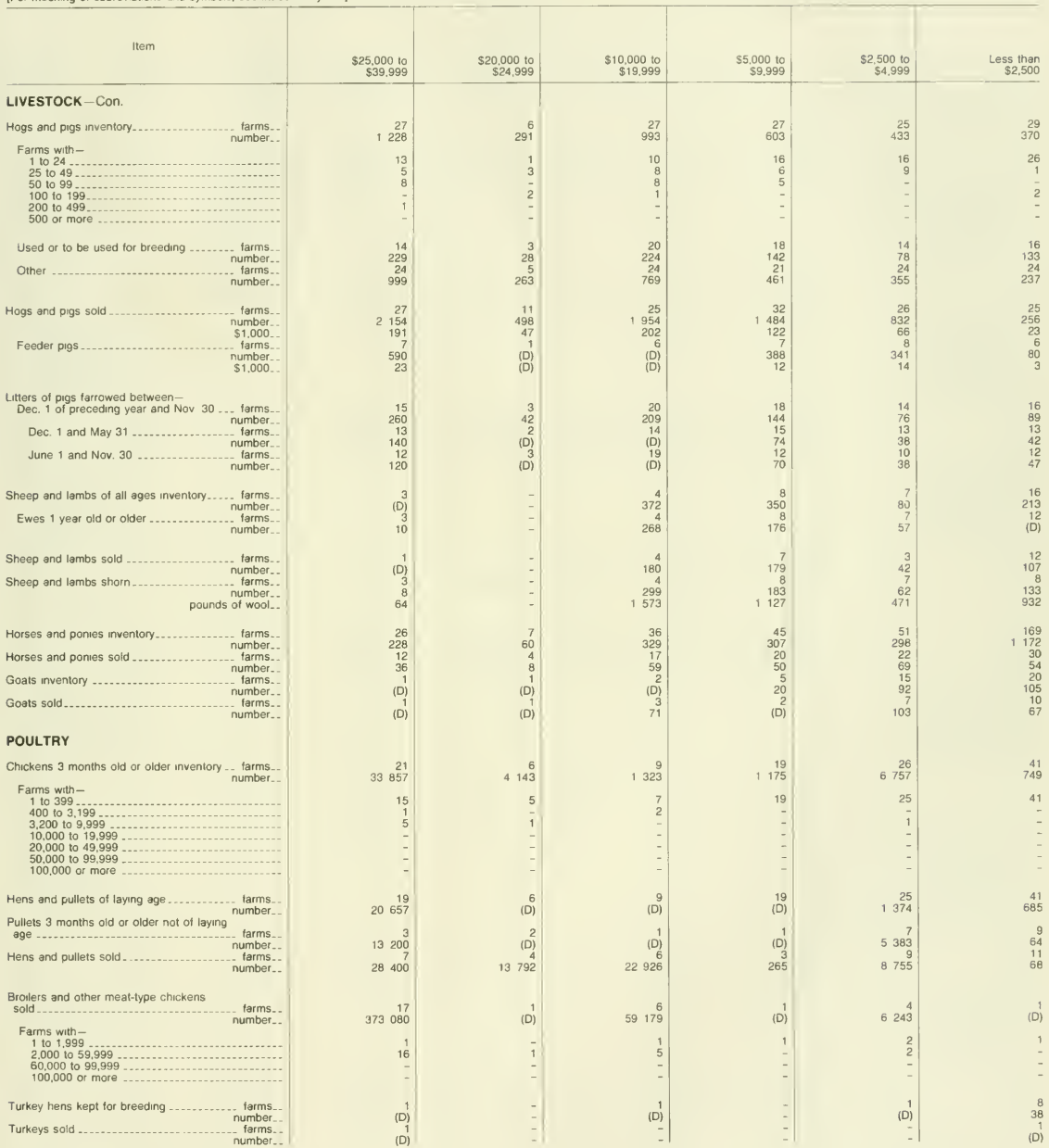

See footnotes at end of table 
Table 52. Summary by Value of Agricultural Products Sold: 1987-Con.

[For meaning of abbreviations and symbols, see introductory text]

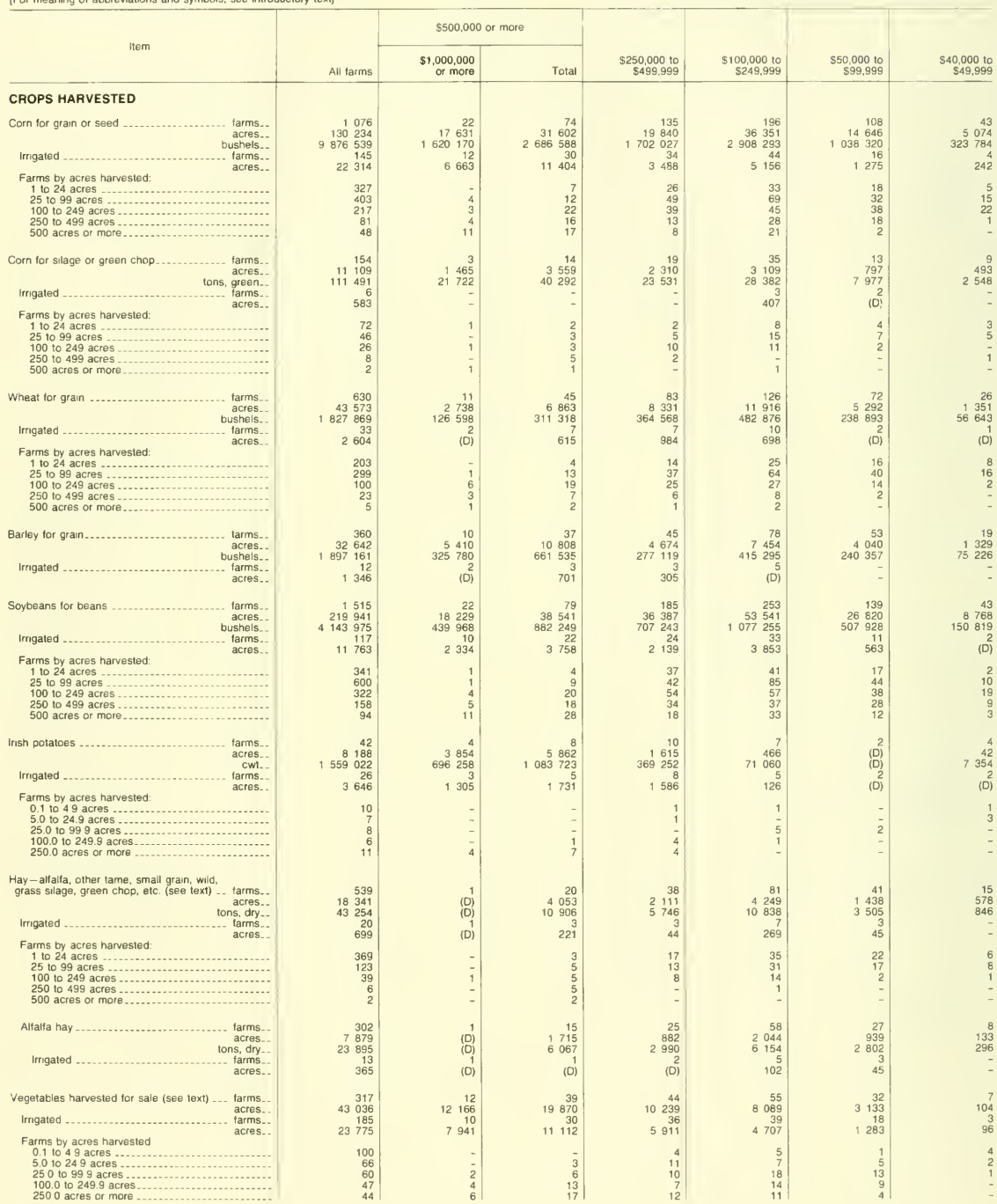


Table 52. Summary by Value of Agricultural Products Sold: 1987-Con.

[For meaning of abbreviations and symbols, see introductory text]

\begin{tabular}{|c|c|c|c|c|c|c|}
\hline Item & $\begin{array}{r}\$ 25,000 \text { to } \\
\$ 39.999\end{array}$ & $\begin{array}{r}\$ 20,000 \text { to } \\
\$ 24.999\end{array}$ & $\begin{array}{r}\$ 10,000 \text { to } \\
\$ 19,999\end{array}$ & $\begin{array}{r}\$ 5,000 \text { to } \\
\$ 9.999\end{array}$ & $\begin{array}{r}\$ 2,500 \text { 10 } \\
\$ 4.999\end{array}$ & $\begin{array}{l}\text { Less than } \\
\$ 2.500\end{array}$ \\
\hline \multicolumn{7}{|l|}{ CROPS HARVESTED } \\
\hline $\begin{array}{c}\text { Corn for grain or seed .................. farms.. } \\
\text { acres.. } \\
\text { bushels. } \\
\text { Irrigated } \\
\text { acres... }\end{array}$ & $\begin{array}{r}77 \\
620 \\
420497 \\
9 \\
576\end{array}$ & $\begin{array}{r}51 \\
3 \quad 814 \\
206 \quad 011 \\
4 \\
125\end{array}$ & $\begin{array}{r}126 \\
649 \\
341 \quad 639 \\
- \\
-\end{array}$ & $\begin{array}{rr}2112 \\
2967 \\
151 & 362 \\
2 \\
\text { (D) }\end{array}$ & $\begin{array}{r}81 \\
72866 \\
72256 \\
1 \\
\text { (D) }\end{array}$ & $\begin{array}{r}73 \\
726 \\
25762 \\
1 \\
\text { (D) }\end{array}$ \\
\hline $\begin{array}{l}\text { Farms by acres harvested. } \\
1 \text { to } 24 \text { acres } \\
25 \text { to } 99 \text { acres } \\
100 \text { to } 249 \text { acres } \\
250 \text { to } 499 \text { acres } \\
500 \text { acres or more.............. }\end{array}$ & $\begin{array}{r}16 \\
32 \\
27 \\
2\end{array}$ & $\begin{array}{r}14 \\
20 \\
15 \\
2 \\
-\end{array}$ & $\begin{array}{r}26 \\
91 \\
8 \\
1 \\
-\end{array}$ & $\begin{array}{r}62 \\
49 \\
1 \\
- \\
-\end{array}$ & $\begin{array}{r}52 \\
29 \\
- \\
-\end{array}$ & $\begin{array}{r}68 \\
5 \\
- \\
-\end{array}$ \\
\hline Corn for silage or green chop ............ farms.. & $\begin{array}{r}17 \\
315 \\
773\end{array}$ & $\begin{array}{r}8 \\
139 \\
1\end{array}$ & $\begin{array}{r}14 \\
166 \\
1067\end{array}$ & $\begin{array}{r}13 \\
163 \\
163\end{array}$ & $\begin{array}{r}7 \\
44\end{array}$ & $\begin{array}{r}5 \\
14\end{array}$ \\
\hline $\begin{array}{l}\text { tons, green } \\
\text { Irngated }\end{array}$ & $\begin{array}{r}3773 \\
1 \\
\text { (D) }\end{array}$ & $\begin{array}{r}1218 \\
-\end{array}$ & $\begin{array}{r}1967 \\
-\end{array}$ & $\begin{array}{r}1106 \\
-\end{array}$ & $\begin{array}{r}585 \\
- \\
-\end{array}$ & 112 \\
\hline $\begin{array}{l}\text { Farms by acres harvested. } \\
1 \text { to } 24 \text { acres } \\
25 \text { to } 99 \text { acres } \\
100 \text { to } 249 \text { acres } \\
250 \text { to } 499 \text { acres } \\
500 \text { acres or more }\end{array}$ & $\begin{array}{r}14 \\
3 \\
- \\
-\end{array}$ & $\begin{array}{l}6 \\
2 \\
- \\
= \\
-\end{array}$ & \begin{tabular}{r|r}
12 & \\
2 & - \\
- &
\end{tabular} & $\begin{array}{l}9 \\
4 \\
- \\
- \\
-\end{array}$ & $\begin{array}{l}7 \\
- \\
- \\
-\end{array}$ & $\begin{array}{l}5 \\
- \\
-\end{array}$ \\
\hline $\begin{array}{r}\text { Wheat for grain ................ tarms.. } \\
\text { acres.. } \\
\text { bushels.. }\end{array}$ & $\begin{array}{r}39 \\
2509 \\
103666\end{array}$ & $\begin{array}{rr}26 \\
1373 \\
51 & 140\end{array}$ & $\begin{array}{r}65 \\
2676 \\
109774\end{array}$ & $\begin{array}{r}69 \\
1971 \\
69 \\
117\end{array}$ & $\begin{array}{r}35 \\
778 \\
22067\end{array}$ & $\begin{array}{r}44 \\
513 \\
17807\end{array}$ \\
\hline 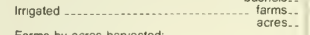 & $\begin{array}{r}5 \\
172\end{array}$ & (D) & $=$ & $=$ & $=$ & - \\
\hline $\begin{array}{l}\text { Farms by acres harvested: } \\
\begin{array}{l}1 \text { to } 24 \text { acres } \\
25 \text { to } 99 \text { acres } \\
100 \\
250 \\
250 \\
500 \text { to } 499 \text { acres } \\
500 \text { acres or more }\end{array}\end{array}$ & $\begin{array}{r}5 \\
26 \\
8 \\
- \\
-\end{array}$ & $\begin{array}{r}6 \\
17 \\
3 \\
- \\
-\end{array}$ & $\begin{array}{r}29 \\
34 \\
2 \\
- \\
-\end{array}$ & $\begin{array}{r}33 \\
36 \\
- \\
- \\
-\end{array}$ & $\begin{array}{r}21 \\
14 \\
- \\
-\end{array}$ & $\begin{array}{r}42 \\
2 \\
- \\
-\end{array}$ \\
\hline 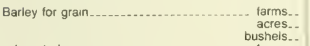 & $\begin{array}{rr}1 & 32 \\
78 & 859\end{array}$ & $\begin{array}{r}17 \\
504 \\
27 \\
921\end{array}$ & $\begin{array}{r}129 \\
64 \\
642\end{array}$ & $\begin{array}{r}32 \\
862 \\
43513\end{array}$ & $\begin{array}{r}10 \\
155 \\
7962\end{array}$ & $\begin{array}{r}8 \\
78 \\
5322\end{array}$ \\
\hline $\begin{array}{l}\text { Irrigated } \ldots+\ldots \ldots+\ldots \\
\text { tarms.- } \\
\text { acres.- }\end{array}$ & (D) & - & - & - & - & - \\
\hline $\begin{array}{c}\text { Soybeans for beans _................. } \begin{array}{c}\text { farms } \\
\text { acres.- } \\
\text { bushels }\end{array}\end{array}$ & $\begin{array}{rr}82 \\
14479 \\
219 & 515\end{array}$ & 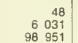 & $\begin{array}{r}147 \\
13856 \\
218963\end{array}$ & $\begin{array}{r}186 \\
11628 \\
156 \quad 949\end{array}$ & $\begin{array}{rr}1441 \\
5443 \\
74 & 831\end{array}$ & $\begin{array}{r}212 \\
4447 \\
49272\end{array}$ \\
\hline 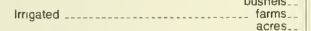 & $\begin{array}{r}219515 \\
13 \\
\text { (D) }\end{array}$ & $\begin{array}{r}98 \\
4 \\
239\end{array}$ & $\begin{array}{rl}218 & 963 \\
- & -\end{array}$ & 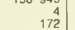 & $=$ & $\begin{array}{r}49272 \\
4 \\
55\end{array}$ \\
\hline $\begin{array}{l}\text { Farms by acres harvested. } \\
1 \text { to } 24 \text { acres } \\
25 \text { to } 99 \text { acres } \\
100 \\
250 \text { to } 249 \text { acres } \\
599 \\
500 \text { acres or more }\end{array}$ & $\begin{array}{r}\text { (U) } \\
6 \\
15 \\
40 \\
21\end{array}$ & $\begin{array}{r}22 \\
21 \\
5 \\
-\end{array}$ & $\begin{array}{r}10 \\
87 \\
44 \\
6\end{array}$ & $\begin{array}{r}32 \\
129 \\
25 \\
- \\
-\end{array}$ & $\begin{array}{r}47 \\
90 \\
4 \\
- \\
-\end{array}$ & $\begin{array}{r}145 \\
67 \\
- \\
-\end{array}$ \\
\hline 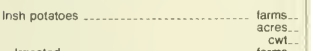 & $\begin{array}{r}5 \\
73 \\
12885\end{array}$ & $\because$ & $\begin{array}{r}2 \\
\text { (D) } \\
\text { (D) }\end{array}$ & $\begin{array}{r}2 \\
\text { (D) } \\
\text { (D) }\end{array}$ & (D) & $\overline{-}$ \\
\hline 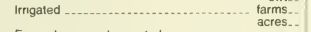 & $\begin{array}{r}4 \\
69\end{array}$ & $=$ & - & $=$ & $=$ & $=$ \\
\hline $\begin{array}{l}\text { Farms by acres harvested } \\
0.1 \text { to } 4.9 \text { acres } \\
5.0 \text { to } 24.9 \text { acres } \\
25.0 \text { to } 99.9 \text { acres } \\
100.0 \text { to } 249.9 \text { acres.... } \\
250.0 \text { acres or more }\end{array}$ & $\begin{array}{l}1 \\
3 \\
1 \\
- \\
-\end{array}$ & $\begin{array}{l}- \\
- \\
- \\
-\end{array}$ & $\begin{array}{l}2 \\
- \\
= \\
-\end{array}$ & $\begin{array}{l}2 \\
- \\
- \\
-\end{array}$ & $\begin{array}{l}2 \\
- \\
- \\
-\end{array}$ & $\begin{array}{l}- \\
\overline{-}\end{array}$ \\
\hline $\begin{array}{r}\begin{array}{r}\text { Hay-alfalfa. other tame, small grain. wild, } \\
\text { grass silage, green chop, etc. (see text) }\end{array} \\
\text { to farms.. } \\
\text { acres. } \\
\text { tons, dry.- }\end{array}$ & $\begin{array}{r}30 \\
862 \\
2601 \\
-\end{array}$ & $\begin{array}{r}12 \\
212 \\
512\end{array}$ & $\begin{array}{rr}1 & 45 \\
2 & 731 \\
2 & 1\end{array}$ & $\begin{array}{rr} & 67 \\
1 & 463 \\
2 & 173 \\
1\end{array}$ & $\begin{array}{r}52 \\
779 \\
1377 \\
\end{array}$ & $\begin{array}{rr}138 \\
1 & 351 \\
2 & 019\end{array}$ \\
\hline $\begin{array}{l}\text { Ifrigated } \ldots . . .1 . \\
\text { Farms by acres harvested }\end{array}$ & - & - & (D) & (D) & (D) & (D) \\
\hline $\begin{array}{l}\text { Farms by acres harvested } \\
1 \text { to } 24 \text { acres } \\
25 \text { to } 99 \text { acres } \\
100 \text { to } 249 \text { acres } \\
250 \text { to } 499 \text { acres } \\
500 \text { acres or more }\end{array}$ & $\begin{array}{r}22 \\
5 \\
3 \\
- \\
-\end{array}$ & $\begin{array}{r}10 \\
2 \\
- \\
- \\
-\end{array}$ & \begin{tabular}{r|r}
28 \\
13 \\
4 \\
- \\
-
\end{tabular} & $\begin{array}{r}48 \\
17 \\
2 \\
- \\
-\end{array}$ & $\begin{array}{r}45 \\
7 \\
- \\
- \\
-\end{array}$ & $\begin{array}{r}133 \\
5 \\
- \\
-\end{array}$ \\
\hline $\begin{array}{c}\text { Alfalfa hay ............ } \\
\text { Irrigated } \ldots \\
\text { acres.. } \\
\text { tons, dry.. } \\
\text { acres.. } \\
\text { acres. }\end{array}$ & $\begin{array}{r}22 \\
572 \\
2136\end{array}$ & \begin{tabular}{r|r}
7 \\
63 \\
175 \\
- \\
-
\end{tabular} & $\begin{array}{r}25 \\
581 \\
1456 \\
1 \\
\text { (D) }\end{array}$ & $\begin{array}{r}35 \\
345 \\
691 \\
- \\
-\end{array}$ & $\begin{array}{r}30 \\
252 \\
607 \\
- \\
-\end{array}$ & $\begin{array}{r}50 \\
353 \\
521 \\
1 \\
(D)\end{array}$ \\
\hline 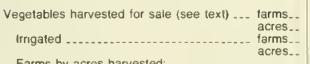 & $\begin{array}{r}21 \\
719 \\
9 \\
415\end{array}$ & $\begin{array}{r}7 \\
332 \\
2 \\
\text { (D) }\end{array}$ & $\begin{array}{r}17 \\
233 \\
9 \\
\text { (D) }\end{array}$ & $\begin{array}{r}35 \\
134 \\
18 \\
59\end{array}$ & $\begin{array}{r}32 \\
112 \\
10 \\
47\end{array}$ & $\begin{array}{l}28 \\
70 \\
11 \\
20\end{array}$ \\
\hline $\begin{array}{l}\text { Farms by acres harvested } \\
0.1 \text { to } 4.9 \text { acres } \\
5.00 \text { to } 24.9 \text { acres } \\
25.0 \text { to } 99.9 \text { acres } \\
100.0 \text { to } 249.9 \text { acres. } \\
250.0 \text { acres or more }\end{array}$ & $\begin{array}{l}6 \\
6 \\
7 \\
2 \\
-\end{array}$ & \begin{tabular}{l|}
- \\
3 \\
2 \\
2 \\
-
\end{tabular} & $\begin{array}{l}9 \\
5 \\
3 \\
- \\
-\end{array}$ & $\begin{array}{r}23 \\
12 \\
- \\
- \\
-\end{array}$ & $\begin{array}{r}26 \\
6 \\
- \\
- \\
-\end{array}$ & $\begin{array}{r}22 \\
6 \\
- \\
-\end{array}$ \\
\hline
\end{tabular}


Table 52. Summary by Value of Agricultural Products Sold: 1987-Con.

[For meaning of abbreviations and symbols, see introductory text]

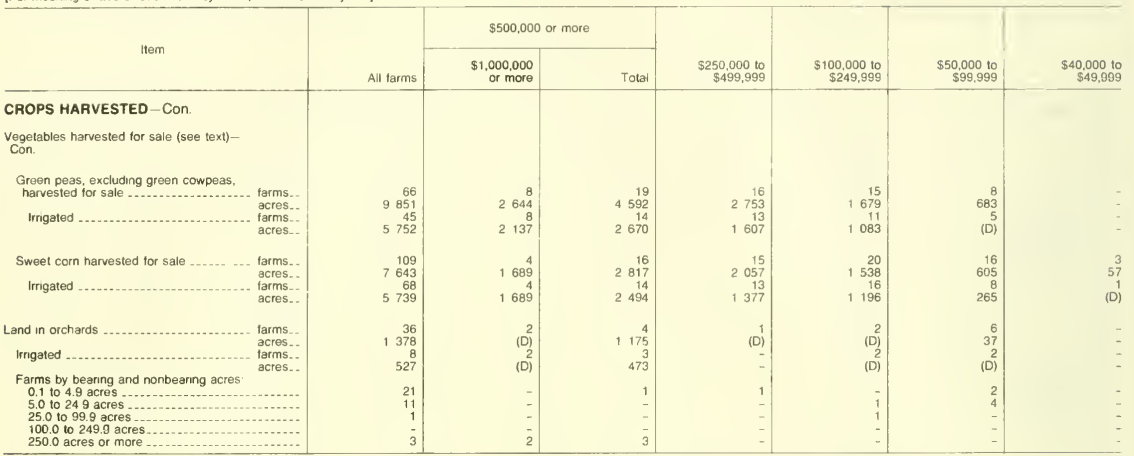

See footnotes at end of table 
Table 52. Summary by Value of Agricultural Products Sold: 1987-Con.

[For meaning of abbreviations and symbols, see introductory text]

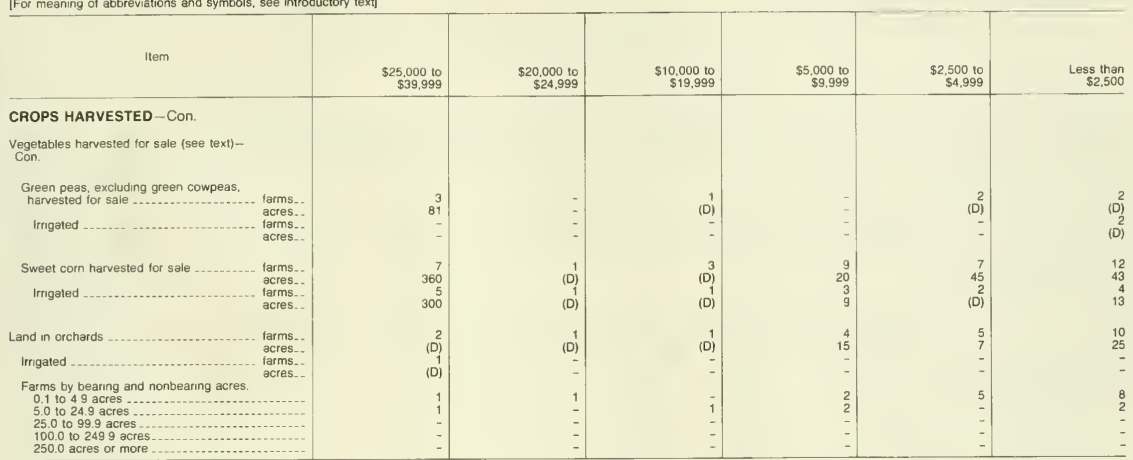

'Data are based on a sample of farms

2Farms with total production expenses equal to market value of agricultural products sold are included as farms with gains of less than $\$ 1,000$. 


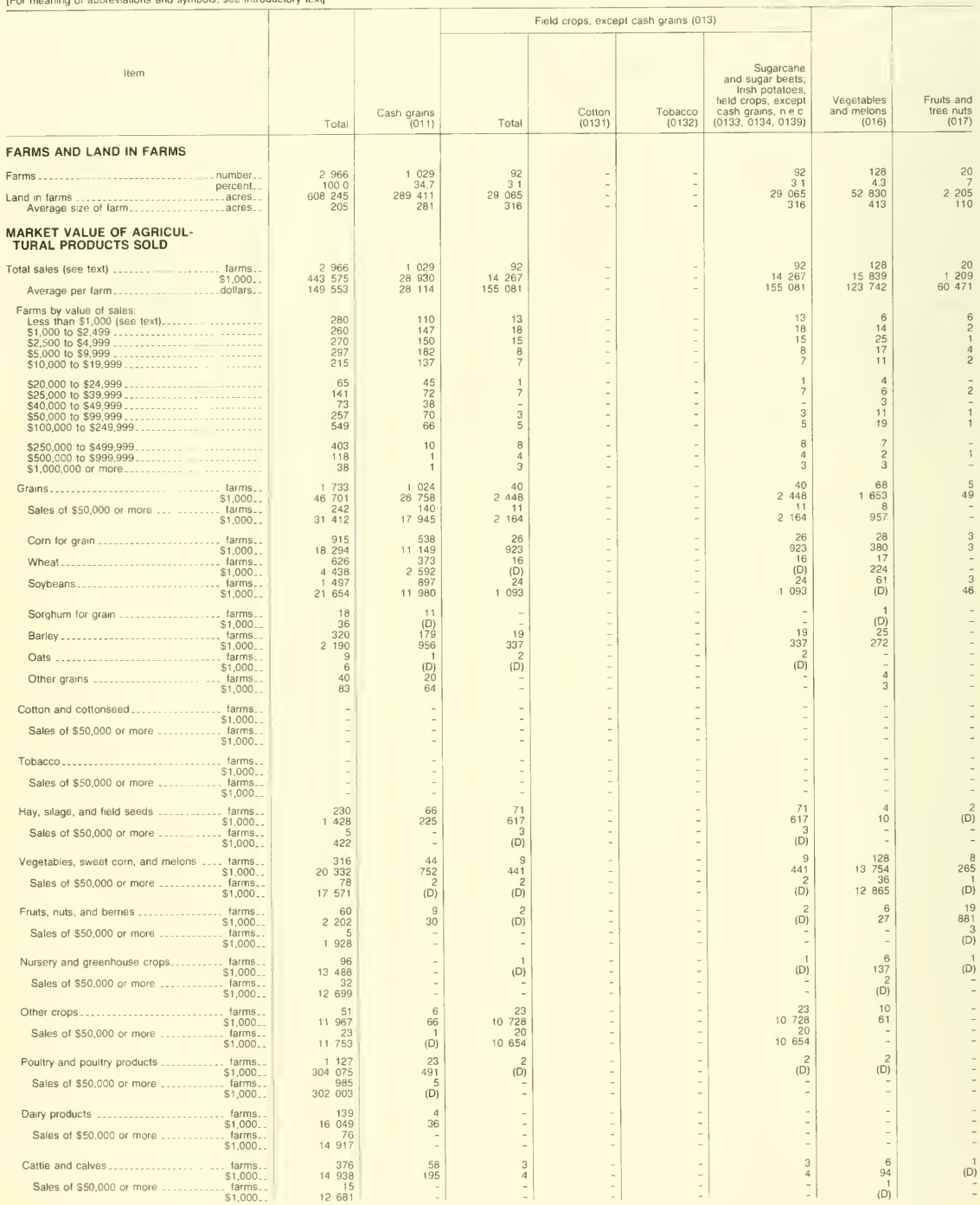

See footnotes at end of table 
Table 53. Summary by Standard Industrial Classification of Farm: 1987-Con.

[For meaning of abbreviations and symbols, see introductory text]

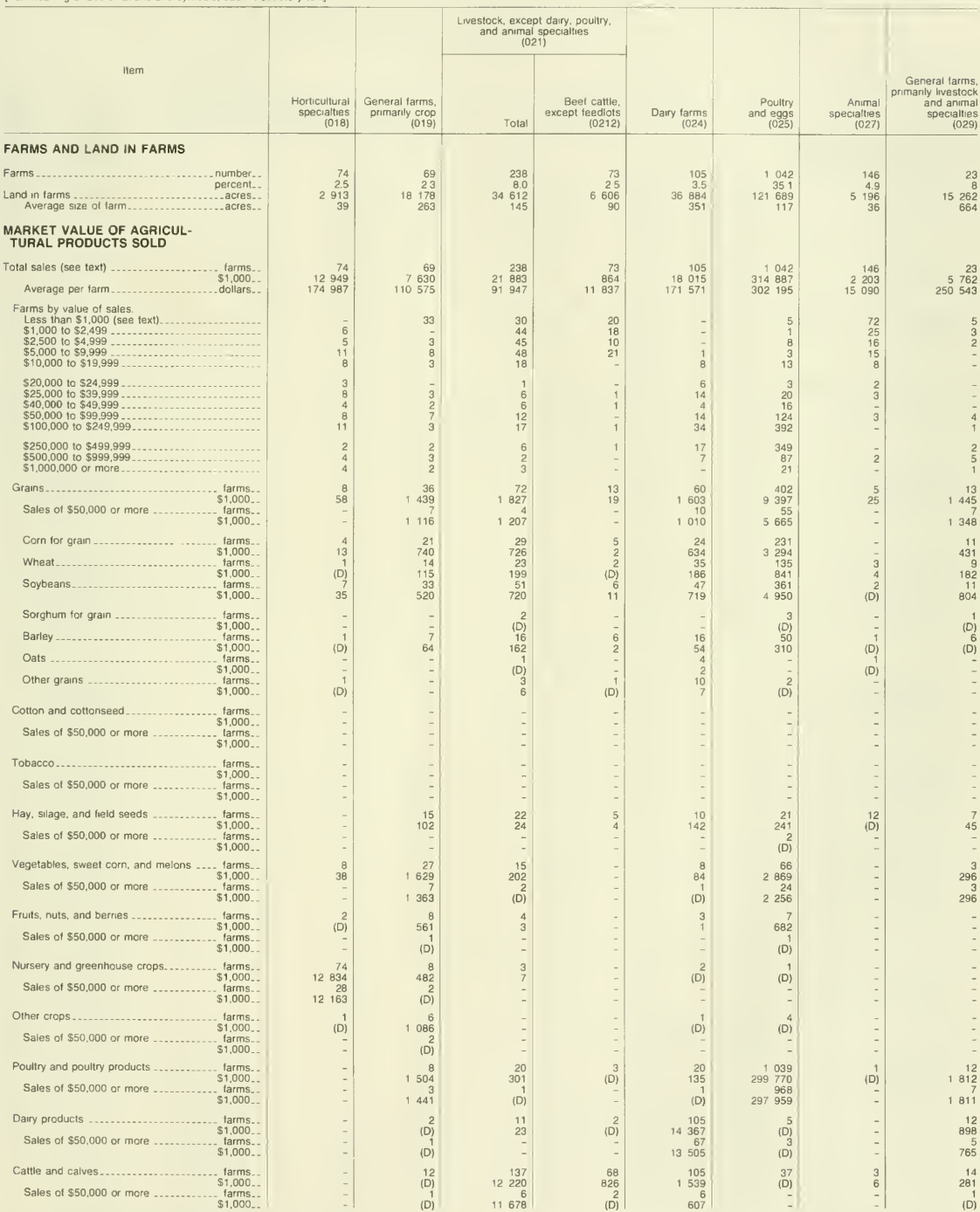

See footnotes at end of table 
Table 53. Summary by Standard Industrial Classification of Farm: 1987-Con.

[For meaning of abbreviations and symbols, see introductory text]

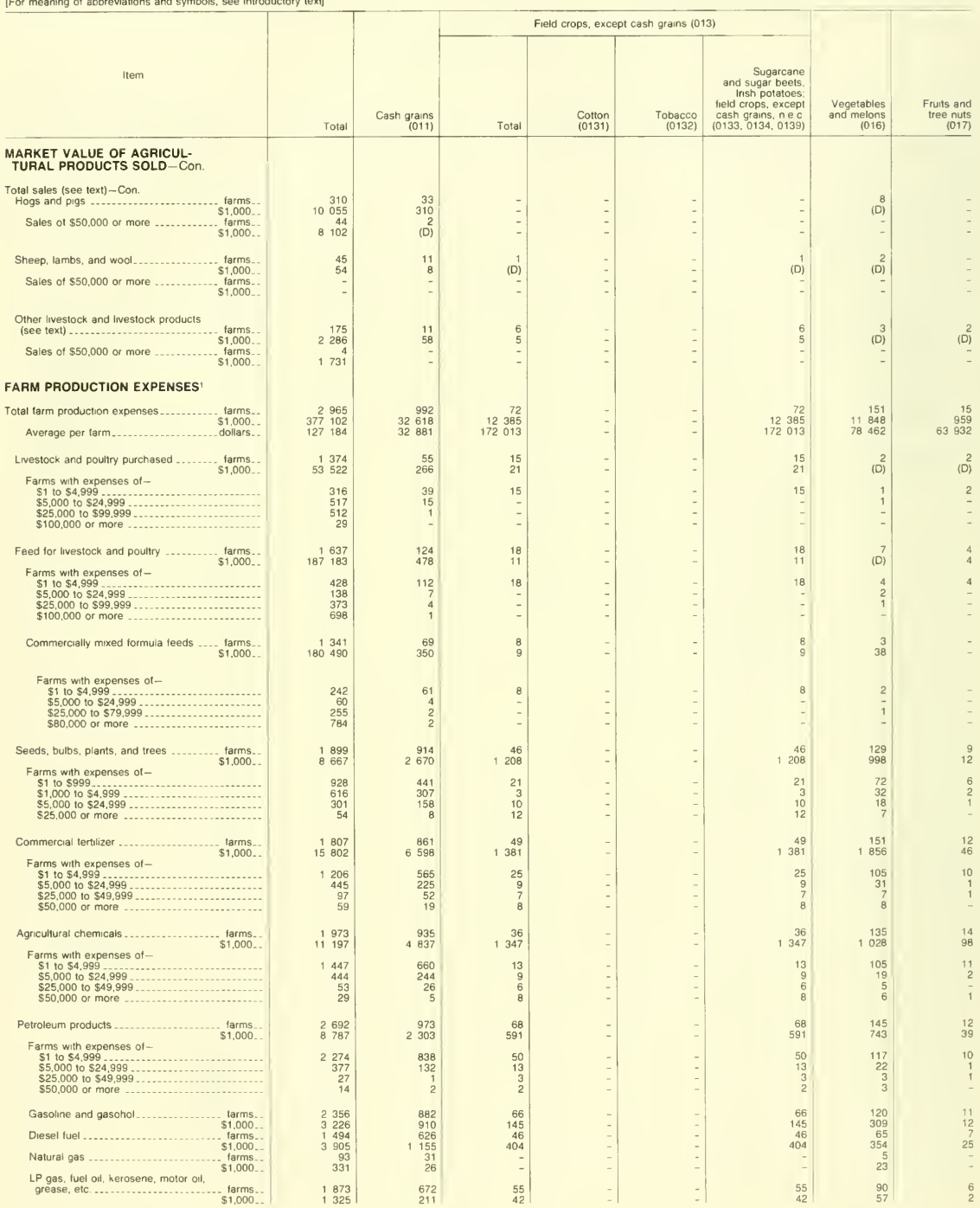

See footnotes at end of table 
Table 53. Summary by Standard Industrial Classification of Farm: 1987-Con.

[For meaning of abbreviatıons and symbols, see introductory text]

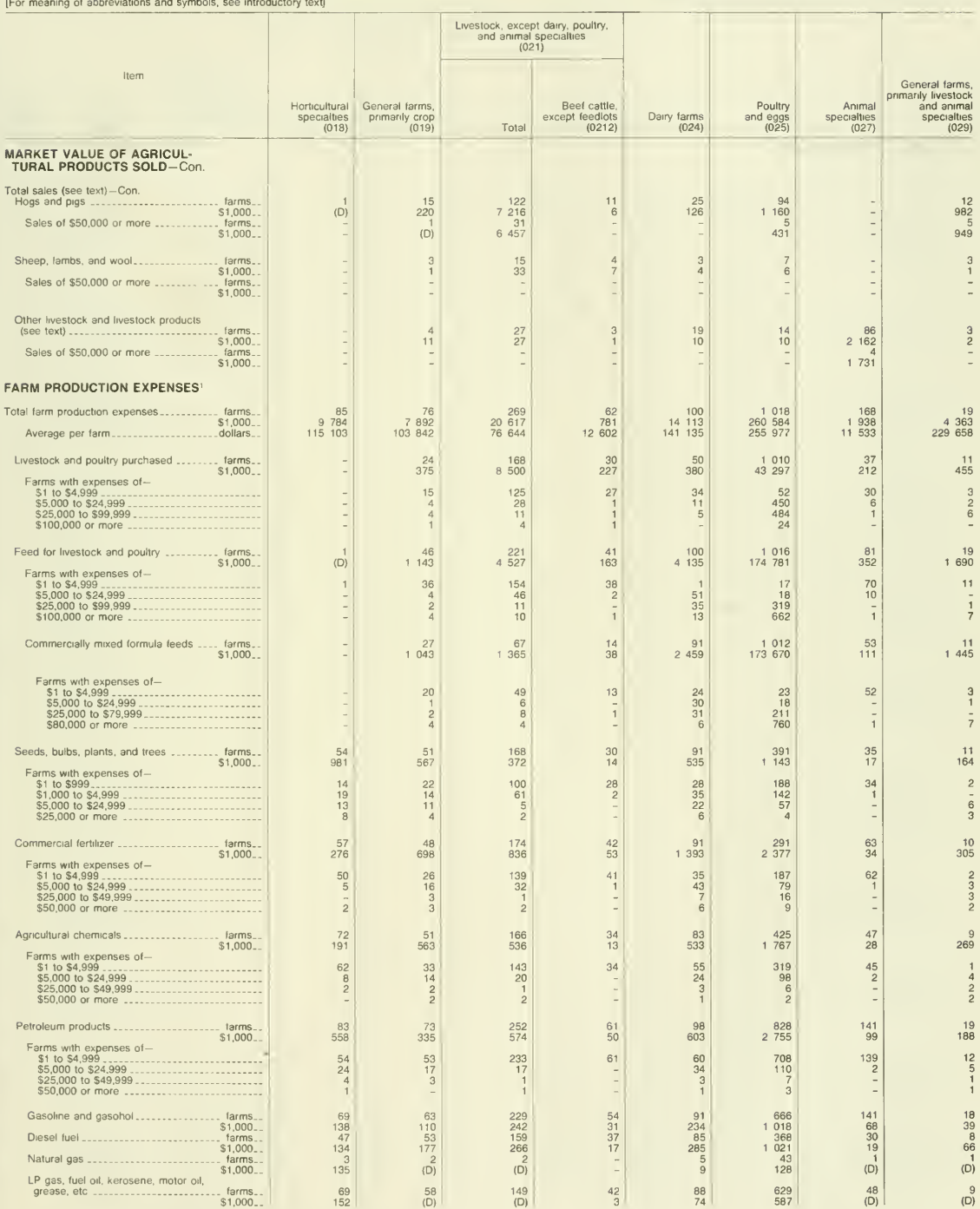

See tootnotes at end of table 
Table 53. Summary by Standard Industrial Classification of Farm: 1987-Con.

[For meaning of abbreviations and symbols, see introductory text]

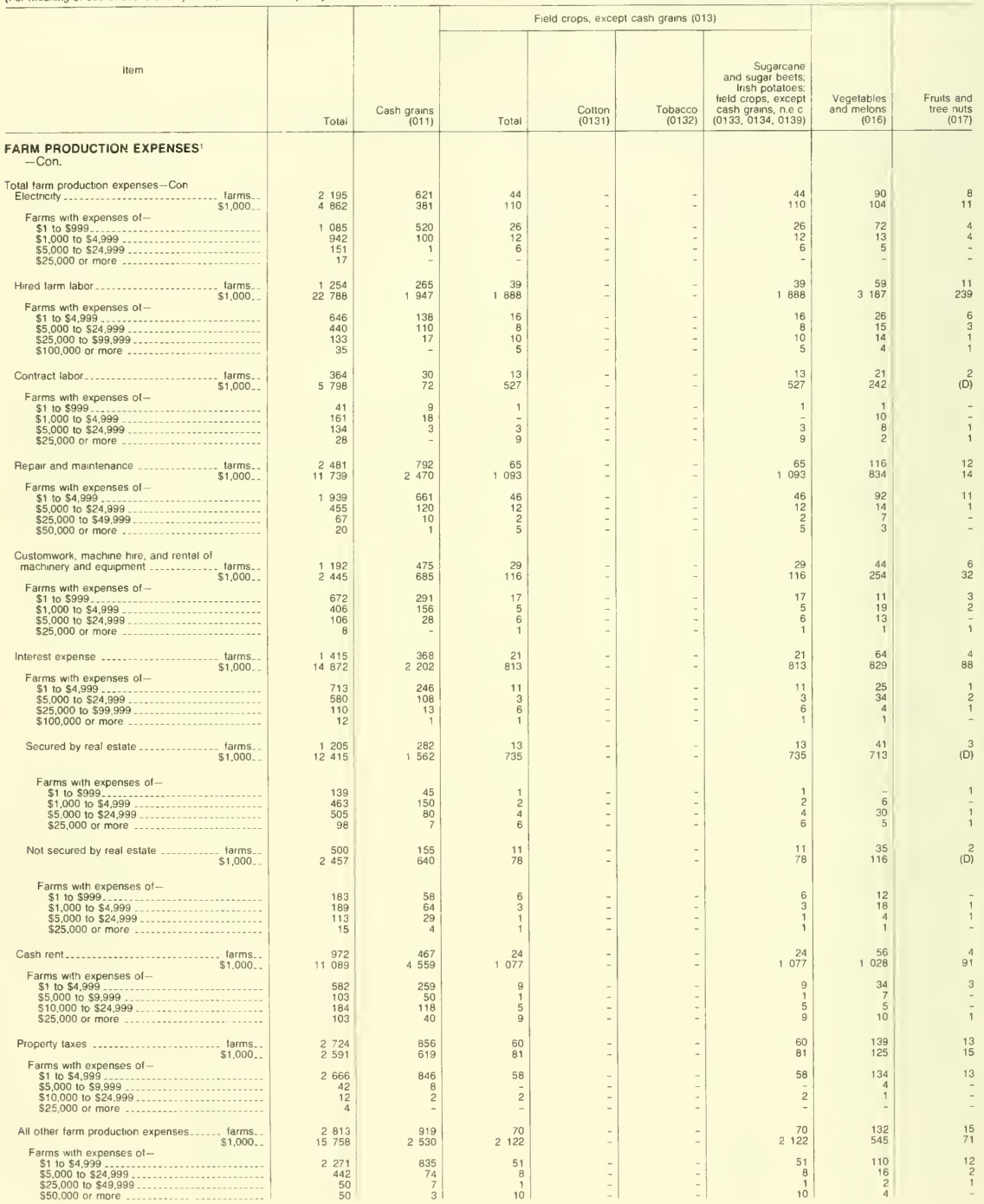


Table 53. Summary by Standard Industrial Classification of Farm: 1987-Con.

[For meaning of abbreviations and symbols, see introductory text]

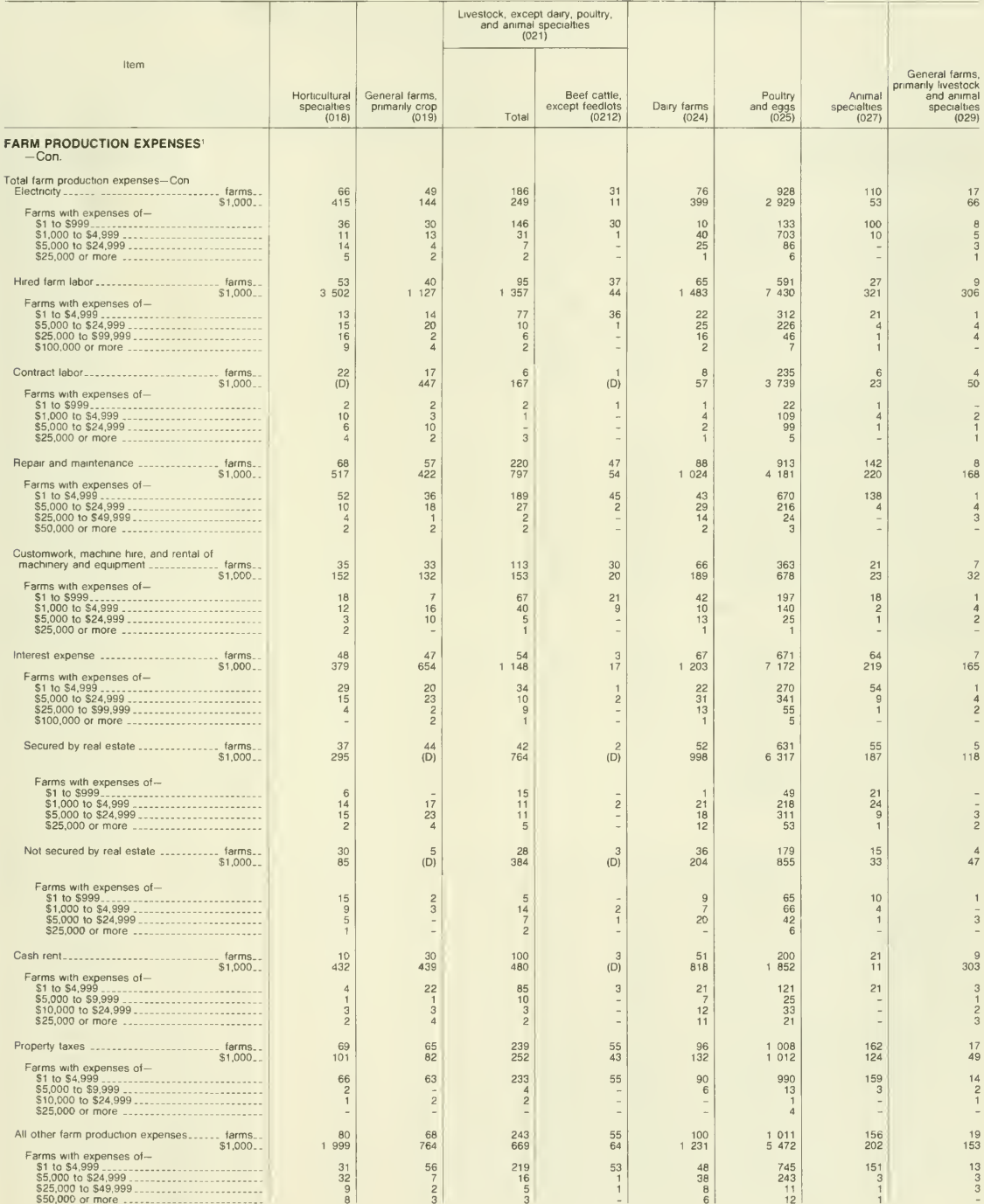

See footnotes at end of tabie 
Table 53. Summary by Standard Industrial Classification of Farm: 1987-Con.

[For meaning of abbreviations and symbols, see introductory text]

\begin{tabular}{|c|c|c|c|c|c|c|c|c|}
\hline \multirow[b]{2}{*}{ Item } & \multirow[b]{2}{*}{ Total } & \multirow[b]{2}{*}{$\begin{array}{r}\text { Cash grains } \\
(011)\end{array}$} & \multicolumn{4}{|c|}{ Field crops, except cash grains (013) } & \multirow[b]{2}{*}{$\begin{array}{r}\text { Vegetables } \\
\text { and melons } \\
(016)\end{array}$} & \multirow[b]{2}{*}{$\begin{array}{l}\text { Fruits and } \\
\text { tree nuts } \\
(017)\end{array}$} \\
\hline & & & Total & $\begin{array}{l}\text { Cotton } \\
(0131)\end{array}$ & $\begin{array}{r}\text { Tobacco } \\
(0132)\end{array}$ & $\begin{array}{r}\text { Sugarcane } \\
\text { and sugar beets: } \\
\text { Irish potatoes: } \\
\text { held crops, excepi } \\
\text { cash grains, ne.c. } \\
(0133,0134,0139)\end{array}$ & & \\
\hline \multicolumn{9}{|l|}{$\begin{array}{l}\text { NET CASH RETURN FROM } \\
\text { AGRICULTURAL SALES FOR } \\
\text { THE FARM UNIT }\end{array}$} \\
\hline 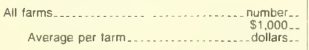 & $\begin{array}{r}2965 \\
65300 \\
22023\end{array}$ & $\begin{array}{r}992 \\
-2311 \\
-2329\end{array}$ & 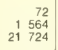 & $\overline{-}$ & $\begin{array}{l}- \\
-\end{array}$ & $\begin{array}{rr}1 & 72 \\
21 & 564 \\
21\end{array}$ & $\begin{array}{rr}151 \\
3643 \\
24 & 127\end{array}$ & $\begin{array}{r}15 \\
204 \\
13600\end{array}$ \\
\hline 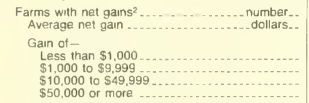 & $\begin{array}{r}1695 \\
45 \quad 171 \\
129 \\
442 \\
787 \\
337\end{array}$ & $12 \begin{array}{r}291 \\
974\end{array}$ & $54 \begin{array}{r}32 \\
585\end{array}$ & $\bar{z}$ & $\begin{array}{l}- \\
- \\
-\end{array}$ & $54 \begin{array}{r}32 \\
585\end{array}$ & 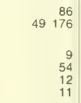 & 46821 \\
\hline 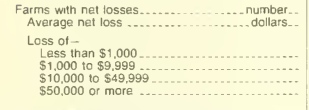 & $\begin{array}{rr}1 & 270 \\
8 & 870 \\
& 233 \\
768 \\
238 \\
31\end{array}$ & $\begin{array}{r}701 \\
8682 \\
143 \\
390 \\
148 \\
20\end{array}$ & $\begin{array}{r}40 \\
564 \\
3 \\
34 \\
3 \\
-\end{array}$ & $\vdots$ & $\begin{array}{l}- \\
-\end{array}$ & $\begin{array}{r}40 \\
564 \\
3 \\
34 \\
34 \\
3 \\
-\end{array}$ & $\begin{array}{r}65 \\
9016 \\
\\
17 \\
42 \\
4 \\
2\end{array}$ & $\begin{array}{r}3010 \\
\\
6 \\
3 \\
1 \\
-\end{array}$ \\
\hline $\begin{array}{l}\text { GOVERNMENT PAYMENTS AND } \\
\text { OTHER FARM-RELATED INCOME }\end{array}$ & & & & & & & & \\
\hline $\begin{array}{l}\text { Government payments ................. farms. } \\
\$ 1.000 .\end{array}$ & $\begin{array}{r}549 \\
7414\end{array}$ & $\begin{array}{r}332 \\
4646\end{array}$ & $\begin{array}{r}18 \\
289\end{array}$ & - & -1 & $\begin{array}{r}18 \\
289\end{array}$ & $\begin{array}{r}19 \\
273\end{array}$ & 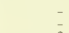 \\
\hline $\begin{array}{l}\text { Other farm-related income' ................ farms... } \\
\$ 1,000 .\end{array}$ & $\begin{array}{r}757 \\
3998\end{array}$ & $\begin{array}{r}277 \\
1234\end{array}$ & $\begin{array}{r}29 \\
83\end{array}$ & $=$ & 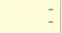 & $\begin{array}{r}29 \\
83\end{array}$ & $\begin{array}{r}40 \\
316\end{array}$ & (D) \\
\hline $\begin{array}{l}\text { Customwork and other agricultural } \\
\text { services }\end{array}$ & $\begin{array}{r}272 \\
1514 \\
\end{array}$ & $\begin{array}{l}121 \\
600\end{array}$ & $\begin{array}{l}14 \\
42\end{array}$ & $\overline{-}$ & 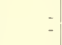 & $\begin{array}{l}14 \\
42\end{array}$ & $\begin{array}{l}25 \\
84\end{array}$ & - \\
\hline 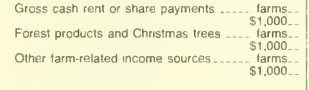 & $\begin{array}{r}287 \\
1151 \\
113 \\
878 \\
208 \\
456\end{array}$ & $\begin{array}{r}83 \\
303 \\
33 \\
285 \\
86 \\
46\end{array}$ & \begin{tabular}{r|r}
2 \\
$10)^{10}$ \\
$10)$ \\
18 \\
14
\end{tabular} & $\begin{array}{l}\bar{z} \\
\vdots \\
\vdots\end{array}$ & $\begin{array}{l}z \\
\bar{z} \\
z \\
z\end{array}$ & $\begin{array}{r}2 \\
(D) \\
10 \\
(D) \\
8 \\
14\end{array}$ & $\begin{array}{r}6 \\
2 \\
14 \\
221 \\
8 \\
9\end{array}$ & $\begin{array}{r}3 \\
6 \\
1 \\
\text { (D) } \\
-\end{array}$ \\
\hline $\begin{array}{l}\text { COMMODITY CREDIT } \\
\text { CORPORATION LOANS }\end{array}$ & & & & & & & & \\
\hline 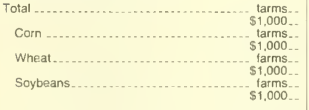 & $\begin{array}{r}143 \\
4256 \\
133 \\
3877 \\
10 \\
20 \\
16 \\
340\end{array}$ & $\begin{array}{r}88 \\
2142 \\
84 \\
2068 \\
7 \\
(D) \\
9 \\
53\end{array}$ & $\begin{array}{r}4 \\
245 \\
4 \\
245 \\
- \\
- \\
-\end{array}$ & $\begin{array}{l}- \\
\vdots \\
\vdots \\
\vdots\end{array}$ & $\begin{array}{l}= \\
\overline{-} \\
= \\
= \\
= \\
-\end{array}$ & $\begin{array}{r}24 \\
24 \\
4 \\
245 \\
: \\
:\end{array}$ & $\begin{array}{r}7 \\
95 \\
5 \\
80 \\
2 \\
2 \\
\text { (D) } \\
2 \\
\text { (D) }\end{array}$ & $\begin{array}{l}\bar{z} \\
\bar{z} \\
\bar{z}\end{array}$ \\
\hline 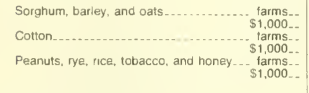 & $\begin{array}{r}6 \\
19 \\
- \\
- \\
-\end{array}$ & $\begin{array}{r}4 \\
(D) \\
: \\
-\end{array}$ & $\begin{array}{l}- \\
\overline{-} \\
-\end{array}$ & $\begin{array}{l}- \\
\vdots \\
\vdots\end{array}$ & $\begin{array}{l}z \\
z \\
z\end{array}$ & $\begin{array}{l}z \\
: \\
\vdots \\
:\end{array}$ & $\bar{\vdots}$ & $\begin{array}{l}\bar{z} \\
\bar{z} \\
\bar{z}\end{array}$ \\
\hline $\begin{array}{l}\text { LAND IN FARMS ACCORDING TO } \\
\text { USE }\end{array}$ & & & & & & & & \\
\hline 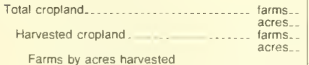 & $\begin{array}{rl}2 & 397 \\
501 & 290 \\
2 & 172 \\
441502 & \end{array}$ & $\begin{array}{rr}1 & 029 \\
246 & 935 \\
1 & 029 \\
218 & 009\end{array}$ & $\begin{array}{r}92 \\
26 \quad 244 \\
92 \\
24318\end{array}$ & $\begin{array}{l}= \\
- \\
-\end{array}$ & $\begin{array}{l}- \\
\bar{z} \\
=\end{array}$ & $\begin{array}{rr}26 & 92 \\
244 \\
92 & 92 \\
218\end{array}$ & $\begin{array}{r}128 \\
40999 \\
128 \\
37 \quad 245\end{array}$ & $\begin{array}{r}20 \\
1828 \\
1696 \\
1696\end{array}$ \\
\hline 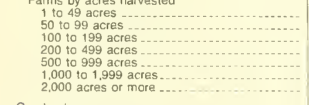 & $\begin{array}{r}947 \\
356 \\
293 \\
349 \\
141 \\
67 \\
19\end{array}$ & $\begin{array}{r}359 \\
218 \\
151 \\
189 \\
71 \\
37 \\
4\end{array}$ & $\begin{array}{r}47 \\
9 \\
14 \\
8 \\
7 \\
5 \\
2\end{array}$ & $\begin{array}{l}- \\
- \\
= \\
= \\
= \\
-\end{array}$ & $\begin{array}{l}= \\
\vdots \\
z \\
z\end{array}$ & $\begin{array}{r}47 \\
9 \\
14 \\
8 \\
8 \\
7 \\
5 \\
2\end{array}$ & $\begin{array}{r}69 \\
6 \\
20 \\
14 \\
9 \\
5 \\
5\end{array}$ & $\begin{array}{r}16 \\
2 \\
7 \\
5 \\
1 \\
-\end{array}$ \\
\hline $\begin{array}{l}\text { Cropland. } \\
\text { Pasture or grazing only ................ farms.. } \\
\text { acres.. }\end{array}$ & $10 \begin{array}{r}510 \\
1000\end{array}$ & $\begin{array}{r}95 \\
1453\end{array}$ & $\begin{array}{r}23 \\
441\end{array}$ & - & $\overline{-}$ & $\begin{array}{r}23 \\
441\end{array}$ & 420 & $\begin{array}{r}4 \\
41\end{array}$ \\
\hline 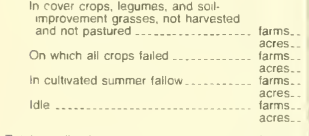 & $\begin{array}{r}145 \\
4891 \\
188 \\
6696 \\
36 \\
902 \\
612 \\
37099\end{array}$ & $\begin{array}{r}70 \\
2867 \\
66 \\
2040 \\
19 \\
609 \\
288 \\
21957\end{array}$ & $\begin{array}{r}5 \\
165 \\
7 \\
230 \\
- \\
- \\
18 \\
1090\end{array}$ & $\begin{array}{l}z \\
z \\
z \\
z \\
z\end{array}$ & $\begin{array}{l}\bar{z} \\
\vdots \\
\vdots \\
\vdots\end{array}$ & $\begin{array}{r}5 \\
165 \\
7 \\
230 \\
- \\
- \\
18 \\
1090\end{array}$ & $\begin{array}{r}9 \\
(D) \\
18 \\
1289 \\
1 \\
(D) \\
30 \\
1659\end{array}$ & $\begin{array}{r}- \\
3 \\
9 \\
\\
5 \\
82\end{array}$ \\
\hline 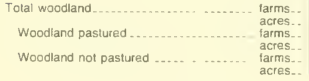 & $\begin{array}{rl}1 & 239 \\
75 & 156 \\
& 141 \\
3 & 714 \\
1 & 158 \\
71 & 442\end{array}$ & $\begin{array}{r}467 \\
29509 \\
34 \\
1370 \\
443 \\
28139\end{array}$ & $\begin{array}{r}38 \\
1 \quad 644 \\
7 \\
78 \\
32 \\
1566\end{array}$ & $\begin{array}{l}- \\
\vdots \\
\vdots\end{array}$ & $\begin{array}{l}\vdots \\
\vdots\end{array}$ & $\begin{array}{r}38 \\
1 \quad 644 \\
7 \\
78 \\
32 \\
1566\end{array}$ & $\begin{array}{r}50 \\
9675 \\
2 \\
\text { (D) } \\
49 \\
\text { (D) }\end{array}$ & $\begin{array}{r}8 \\
285 \\
- \\
8 \\
285\end{array}$ \\
\hline
\end{tabular}


Table 53. Summary by Standard Industrial Classification of Farm: 1987-Con. 
Table 53. Summary by Standard Industrial Classification of Farm: 1987-Con.

[For meaning of abbreviations and symbols. see introductory text]

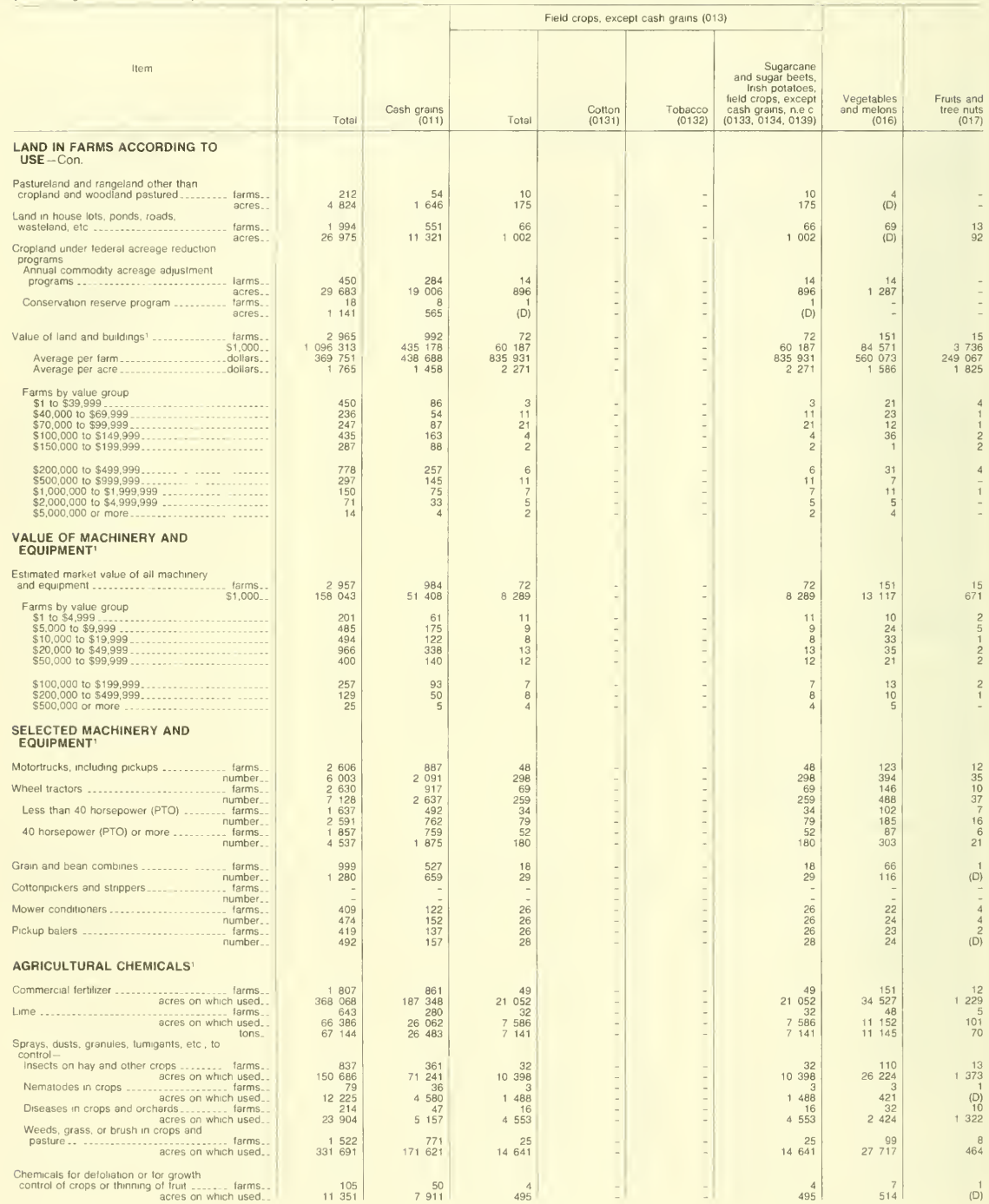

See footnotes at end of tabie 
Table 53. Summary by Standard Industrial Classification of Farm: 1987--Con.

[For meaning of abbreviations and symbols, see introductory text]

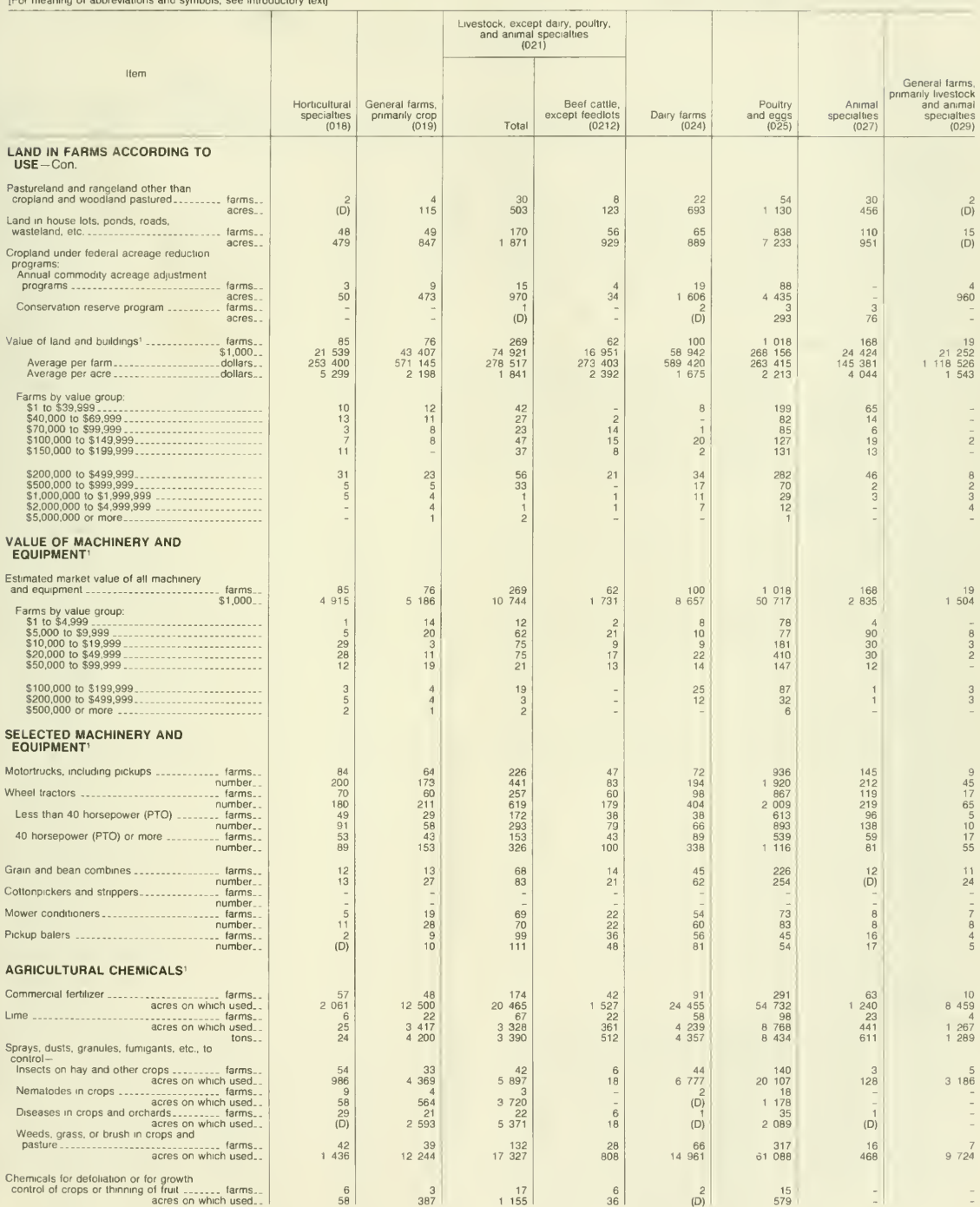

See footnotes at end of table 
Table 53. Summary by Standard Industrial Classification of Farm: 1987-Con.

[For meaning of abbrevations and symbois, see introductory text]

\begin{tabular}{|c|c|c|c|c|c|c|c|c|}
\hline \multirow[b]{2}{*}{ Item } & \multirow[b]{2}{*}{ Total } & \multirow[b]{2}{*}{$\begin{array}{r}\text { Cash grains } \\
(011)\end{array}$} & \multicolumn{4}{|c|}{ Field crops, except cash grains (013) } & \multirow[b]{2}{*}{$\begin{array}{r}\text { Vegetables } \\
\text { and melons } \\
(016)\end{array}$} & \multirow[b]{2}{*}{$\begin{array}{l}\text { Fruits and } \\
\text { tree nuts } \\
\quad(017)\end{array}$} \\
\hline & & & Total & $\begin{array}{l}\text { Colton } \\
\text { (0131) }\end{array}$ & $\begin{array}{r}\text { Tobacco } \\
(0132)\end{array}$ & $\begin{array}{r}\text { Sugarcane } \\
\text { and sugar beets. } \\
\text { Irish potatoes. } \\
\text { field crops, except } \\
\text { cash grains, n e.c. } \\
(0133,0134,0139)\end{array}$ & & \\
\hline \multicolumn{9}{|l|}{$\begin{array}{l}\text { TENURE AND RACE OF } \\
\text { OPERATOR }\end{array}$} \\
\hline $\begin{array}{l}\text { All operators } \\
\quad \text { Full owners } \\
\text { Part owners } \\
\text { Tenants }\end{array}$ & $\begin{array}{r}2966 \\
1825 \\
857 \\
284\end{array}$ & $\begin{array}{r}1029 \\
473 \\
407 \\
149\end{array}$ & $\begin{array}{l}92 \\
55 \\
25 \\
12\end{array}$ & $\begin{array}{l}\bar{z} \\
\bar{z}\end{array}$ & $\bar{z}$ & $\begin{array}{l}92 \\
55 \\
25 \\
12\end{array}$ & $\begin{array}{r}128 \\
58 \\
46 \\
24\end{array}$ & $\begin{array}{r}20 \\
14 \\
4 \\
2\end{array}$ \\
\hline $\begin{array}{l}\text { White } \\
\text { Full owners } \\
\text { Part owners } \\
\text { Tenants }\end{array}$ & $\begin{array}{r}2914 \\
1791 \\
845 \\
278\end{array}$ & $\begin{array}{r}1008 \\
460 \\
401 \\
147\end{array}$ & $\begin{array}{l}92 \\
55 \\
25 \\
12\end{array}$ & $\begin{array}{l}- \\
=\end{array}$ & $\begin{array}{l}\overline{-} \\
\overline{-}\end{array}$ & $\begin{array}{l}92 \\
55 \\
25 \\
12\end{array}$ & $\begin{array}{r}123 \\
56 \\
45 \\
22\end{array}$ & $\begin{array}{r}18 \\
14 \\
2 \\
2\end{array}$ \\
\hline $\begin{array}{l}\text { Black and other races } \\
\text { Full owners } \\
\text { Part owners } \\
\text { Tenants }\end{array}$ & $\begin{array}{r}52 \\
34 \\
12 \\
6\end{array}$ & $\begin{array}{r}21 \\
13 \\
6 \\
2\end{array}$ & $\begin{array}{l}- \\
- \\
-\end{array}$ & $\begin{array}{l}- \\
\overline{-} \\
-\end{array}$ & :- & $\begin{array}{l}- \\
- \\
-\end{array}$ & $\begin{array}{l}5 \\
2 \\
1 \\
2\end{array}$ & $\overline{2}$ \\
\hline \multicolumn{9}{|l|}{ OWNED AND RENTED LAND } \\
\hline $\begin{array}{r}\text { Land owned .......................... } \begin{array}{l}\text { larms.. } \\
\text { acres.. }\end{array} \\
\text { Owned land in farms................ } \\
\text { larms.. } \\
\text { acres.. }\end{array}$ & $\begin{array}{rr}2 & 691 \\
360 & 152 \\
2 & 682 \\
325 & 313\end{array}$ & 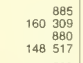 & $\begin{array}{rr}80 \\
14 \quad 663 \\
80 \\
13 \quad 259\end{array}$ & $\begin{array}{l}\bar{z} \\
\bar{z}\end{array}$ & : & $\begin{array}{rr}14 & 863 \\
14 & 80 \\
13 & 259\end{array}$ & $\begin{array}{rr}104 \\
26 \quad 978 \\
104 \\
26 \quad 576\end{array}$ & $\begin{array}{r}18 \\
1848 \\
18 \\
1 \quad 448\end{array}$ \\
\hline 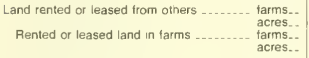 & $\begin{array}{rr}1 & 153 \\
285 & 163 \\
1 & 141 \\
282 & 932\end{array}$ & $\begin{array}{r}560 \\
141 \quad 742 \\
556 \\
140 \quad 894\end{array}$ & $\begin{array}{r}38 \\
15811 \\
37 \\
15806\end{array}$ & $\overline{-}$ & $\begin{array}{l}\bar{z} \\
\overline{-}\end{array}$ & $\begin{array}{r}38 \\
15 \quad 811 \\
37 \\
15806\end{array}$ & $\begin{array}{r}72 \\
(D) \\
70 \\
26 \quad 254\end{array}$ & $\begin{array}{r}7 \\
812 \\
6 \\
757\end{array}$ \\
\hline $\begin{array}{r}\text { Land rented or leased to others............ tarms.. } \\
\text { acres.. }\end{array}$ & $37 \begin{array}{r}463 \\
070\end{array}$ & 12640 & $\begin{array}{r}18 \\
1409\end{array}$ & - & 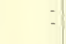 & $\begin{array}{r}18 \\
1409\end{array}$ & (D) & $\begin{array}{r}5 \\
455\end{array}$ \\
\hline \multicolumn{9}{|l|}{ OPERATOR CHARACTERISTICS } \\
\hline $\begin{array}{l}\text { Operators by place of residence } \\
\text { On farm operated } \\
\text { Not on farm operated } \\
\text { Not reported }\end{array}$ & $\begin{array}{r}175 \\
481 \\
310\end{array}$ & $\begin{array}{r}685 \\
265 \\
79\end{array}$ & $\begin{array}{r}69 \\
20 \\
3\end{array}$ & $\ddot{-}$ & $\begin{array}{l}- \\
-\end{array}$ & $\begin{array}{r}69 \\
20 \\
3\end{array}$ & $\begin{array}{l}89 \\
27 \\
12\end{array}$ & $\begin{array}{r}18 \\
2 \\
-\end{array}$ \\
\hline $\begin{array}{l}\text { Operators by principal occupation } \\
\text { Farming } \\
\text { Other }\end{array}$ & $\begin{array}{ll}1 & 774 \\
1 & 192\end{array}$ & $\begin{array}{l}579 \\
450\end{array}$ & $\begin{array}{l}46 \\
46\end{array}$ & - & $\begin{array}{l}- \\
-\end{array}$ & $\begin{array}{l}46 \\
46\end{array}$ & $\begin{array}{l}80 \\
48\end{array}$ & $\begin{array}{r}9 \\
11\end{array}$ \\
\hline $\begin{array}{l}\text { Operators by days of work oft farm } \\
\text { None } \\
\text { Any } 1 \text { to } 99 \text { days } \\
100 \text { to } 199 \text { days } 10 . \\
200 \text { days or more } \ldots \ldots\end{array}$ & $\begin{array}{r}1263 \\
1426 \\
254 \\
217 \\
955\end{array}$ & $\begin{array}{r}393 \\
533 \\
80 \\
86 \\
367\end{array}$ & $\begin{array}{r}42 \\
46 \\
7 \\
3 \\
36\end{array}$ & $\begin{array}{l}5 \\
z\end{array}$ & $\begin{array}{l}- \\
z \\
z \\
-\end{array}$ & $\begin{array}{r}42 \\
46 \\
7 \\
3 \\
36\end{array}$ & $\begin{array}{l}57 \\
58 \\
13 \\
12 \\
33\end{array}$ & $\begin{array}{r}9 \\
11 \\
1 \\
3 \\
7\end{array}$ \\
\hline 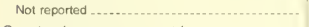 & 277 & 103 & 4 & - & - & 4 & 13 & - \\
\hline $\begin{array}{l}\text { Operators by years on present farm } \\
2 \text { years or less } \\
3 \text { or } 4 \text { years } \\
5 \text { to } 9 \text { years } \\
10 \text { years or more } \\
\text { Average years on present farm }\end{array}$ & $\begin{array}{r}176 \\
189 \\
398 \\
1588 \\
193\end{array}$ & $\begin{array}{r}53 \\
55 \\
110 \\
610 \\
224\end{array}$ & $\begin{array}{r}8 \\
7 \\
12 \\
55 \\
208\end{array}$ & $\begin{array}{l}\overline{-} \\
\bar{z}\end{array}$ & $\begin{array}{l}\overline{-} \\
\bar{y} \\
\overline{-}\end{array}$ & $\begin{array}{r}8 \\
7 \\
12 \\
55 \\
20.8\end{array}$ & $\begin{array}{r}11 \\
6 \\
19 \\
59 \\
18.6\end{array}$ & $\begin{array}{r}2 \\
2 \\
5 \\
8 \\
11.6\end{array}$ \\
\hline 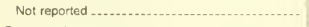 & 615 & 201 & 10 & - & - & 10 & 33 & 3 \\
\hline $\begin{array}{l}\text { Operators by age group } \\
\text { Under } 25 \text { years } \\
25 \text { to } 34 \text { years } \\
35 \text { to } 44 \text { years } \\
45 \text { to } 49 \text { years } \\
50 \text { to } 54 \text { years }\end{array}$ & $\begin{array}{r}40 \\
374 \\
620 \\
328 \\
321\end{array}$ & $\begin{array}{r}19 \\
110 \\
156 \\
107 \\
93\end{array}$ & $\begin{array}{r}2 \\
6 \\
17 \\
14 \\
5\end{array}$ & $\begin{array}{l}\bar{z} \\
\bar{z}\end{array}$ & 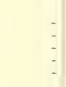 & $\begin{array}{r}2 \\
6 \\
17 \\
14 \\
5\end{array}$ & $\begin{array}{r}5 \\
25 \\
25 \\
8 \\
5\end{array}$ & $\begin{array}{r}- \\
10 \\
1 \\
2\end{array}$ \\
\hline 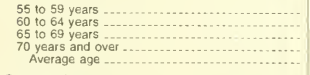 & $\begin{array}{l}326 \\
348 \\
269 \\
340 \\
515\end{array}$ & $\begin{array}{r}108 \\
143 \\
128 \\
165 \\
543\end{array}$ & $\begin{array}{r}12 \\
12 \\
10 \\
14 \\
548\end{array}$ & $\begin{array}{l}- \\
\overline{-} \\
-\end{array}$ & $\begin{array}{l}- \\
\overline{-} \\
\overline{-}\end{array}$ & $\begin{array}{r}12 \\
12 \\
10 \\
14 \\
548\end{array}$ & $\begin{array}{r}14 \\
13 \\
11 \\
22 \\
50.2\end{array}$ & $\begin{array}{r}2 \\
2 \\
2 \\
1 \\
50.7\end{array}$ \\
\hline $\begin{array}{l}\text { Operators by sex } \\
\text { Male } \\
\text { Female }\end{array}$ & $\begin{array}{r}2676 \\
290\end{array}$ & $\begin{array}{r}988 \\
41\end{array}$ & $\begin{array}{r}83 \\
9\end{array}$ & 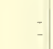 & $\overline{-}$ & $\begin{array}{r}83 \\
9\end{array}$ & $\begin{array}{r}122 \\
6\end{array}$ & $\stackrel{20}{-}$ \\
\hline $\begin{array}{l}\text { Operators of Spanish onigin (see text) } \ldots . . . \\
\text { FARMS BY TYPE OF } \\
\text { ORGANIZATION }\end{array}$ & 9 & - & - & - & - & - & 3 & - \\
\hline $\begin{array}{l}\text { individual or family (sole proprietorship) ..... tarms.. } \\
\text { acres.. }\end{array}$ & $\begin{array}{r}2553 \\
400071\end{array}$ & $\begin{array}{r}889 \\
206 \quad 855\end{array}$ & 19251 & 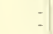 & $\overline{-}$ & 19251 & $\begin{array}{r}107 \\
22 \quad 476\end{array}$ & $\begin{array}{r}18 \\
\text { (D) }\end{array}$ \\
\hline 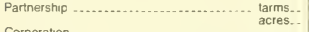 & 62666 & & $\begin{array}{r}4 \\
798\end{array}$ & 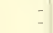 & 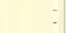 & $\begin{array}{r}4 \\
798\end{array}$ & & - \\
\hline $\begin{array}{l}\begin{array}{l}\text { Corporation } \\
\text { Family held } \ldots \ldots \ldots \ldots\end{array} \\
\text { acres. }\end{array}$ & $141 \quad 856$ & $55 \quad 357$ & $\begin{array}{r}13 \\
9016\end{array}$ & $\overline{-}$ & - & $\begin{array}{rr}13 \\
9 & 016\end{array}$ & 25431 & (D) \\
\hline $\begin{array}{l}\text { More than } 10 \text { stockholders .......... tarms.. } \\
10 \text { or less stockhoiders ............. tarms.. }\end{array}$ & $\begin{array}{r}4 \\
169\end{array}$ & $5 \overline{5}$ & 13 & - & $\overline{-}$ & $1 \overline{3}$ & 8 & 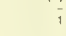 \\
\hline $\begin{array}{l}\text { Other than family held .................. farms.. } \\
\text { acres.. }\end{array}$ & $\begin{array}{r}8 \\
928\end{array}$ & $\begin{array}{r}3 \\
520\end{array}$ & - & $\overline{-}$ & 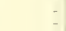 & - & $\overline{-}$ & (D) \\
\hline 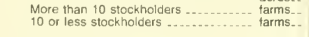 & $\begin{array}{l}1 \\
7\end{array}$ & $\begin{array}{l}1 \\
2\end{array}$ & - & $\overline{-}$ & - & $\overline{-}$ & - & $\frac{1}{1}$ \\
\hline $\begin{array}{l}\text { Other-cooperative, estate or trust, } \\
\text { institutional, etc. .......... farms.. }\end{array}$ & $\begin{array}{r}16 \\
2724\end{array}$ & 2097 & - & 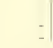 & $\underline{-}$ & - & 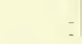 & $\overline{-}$ \\
\hline
\end{tabular}


Table 53. Summary by Standard Industrial Classification of Farm: 1987-Con.

[For meaning of abbreviations and symbols, see introductory text]

\begin{tabular}{|c|c|c|c|c|c|c|c|c|}
\hline \multirow{2}{*}{ Item } & \multirow[b]{2}{*}{$\begin{array}{r}\text { Horticultural } \\
\text { specialties } \\
(018)\end{array}$} & \multirow[b]{2}{*}{$\begin{array}{r}\text { General farms. } \\
\text { primarily crop } \\
(019)\end{array}$} & \multicolumn{2}{|c|}{$\begin{array}{l}\text { Livestock, except darry, poultry, } \\
\text { and animal specialties } \\
\qquad(021)\end{array}$} & \multirow[b]{2}{*}{$\begin{array}{r}\text { Dary farms } \\
(024)\end{array}$} & \multirow[b]{2}{*}{$\begin{array}{r}\text { Poultry } \\
\text { and eggs } \\
(025)\end{array}$} & \multirow[b]{2}{*}{$\begin{array}{r}\text { Animal } \\
\text { specialties } \\
(027)\end{array}$} & \multirow[b]{2}{*}{$\begin{array}{r}\text { General farms, } \\
\text { primarily livestock } \\
\text { and animal } \\
\text { specialties } \\
(029)\end{array}$} \\
\hline & & & Total & $\begin{array}{r}\text { Beef cattie, } \\
\text { except feediots } \\
\text { (0212) }\end{array}$ & & & & \\
\hline \multicolumn{9}{|l|}{$\begin{array}{l}\text { TENURE AND RACE OF } \\
\text { OPERATOR }\end{array}$} \\
\hline $\begin{array}{l}\text { All operators } \\
\text { Full owners } \\
\text { Part owners } \\
\text { Tenants }\end{array}$ & $\begin{array}{r}74 \\
57 \\
8 \\
9\end{array}$ & $\begin{array}{r}69 \\
34 \\
30 \\
5\end{array}$ & $\begin{array}{r}238 \\
166 \\
49 \\
23\end{array}$ & $\begin{array}{r}73 \\
59 \\
8 \\
6\end{array}$ & $\begin{array}{r}105 \\
40 \\
48 \\
17\end{array}$ & $\begin{array}{r}1042 \\
798 \\
214 \\
30\end{array}$ & $\begin{array}{r}146 \\
122 \\
13 \\
11\end{array}$ & $\begin{array}{r}23 \\
8 \\
13 \\
2\end{array}$ \\
\hline $\begin{array}{l}\text { White } \\
\text { Full owners } \\
\text { Part owners } \\
\text { Tenants }\end{array}$ & $\begin{array}{r}74 \\
57 \\
8 \\
9\end{array}$ & $\begin{array}{r}67 \\
32 \\
30 \\
5\end{array}$ & $\begin{array}{r}235 \\
163 \\
49 \\
23\end{array}$ & $\begin{array}{r}73 \\
59 \\
8 \\
6\end{array}$ & $\begin{array}{r}105 \\
40 \\
48 \\
17\end{array}$ & $\begin{array}{r}1025 \\
786 \\
211 \\
28\end{array}$ & $\begin{array}{r}144 \\
120 \\
13 \\
11\end{array}$ & $\begin{array}{r}23 \\
8 \\
13 \\
2\end{array}$ \\
\hline $\begin{array}{l}\text { Black and other races } \\
\text { Full owners } \\
\text { Part owners } \\
\text { Tenants } \\
\text { OWNED AND RENTED LAND }\end{array}$ & $\begin{array}{l}- \\
\overline{-} \\
-\end{array}$ & $\begin{array}{l}2 \\
2 \\
- \\
-\end{array}$ & $\begin{array}{l}3 \\
3 \\
- \\
-\end{array}$ & $\begin{array}{l}- \\
- \\
-\end{array}$ & $=$ & $\begin{array}{r}17 \\
12 \\
3 \\
2\end{array}$ & $\begin{array}{l}2 \\
2 \\
- \\
-\end{array}$ & $\begin{array}{l}\text { - } \\
\overline{-}\end{array}$ \\
\hline $\begin{aligned} & \text { Land owned } \text { Owned land in farms..................... } \\
& \text { farms.. } \\
& \text { acres.. }\end{aligned}$ & $\begin{array}{rr}65 \\
2 \quad 820 \\
65 \\
2 \quad 174\end{array}$ & $\begin{array}{rr}65 \\
11 & 374 \\
64 \\
10 \quad 860\end{array}$ & 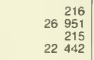 & $\begin{array}{r}67 \\
7 \quad 420 \\
67 \\
5 \quad 331\end{array}$ & $\begin{array}{r}89 \\
20 \quad 113 \\
88 \\
19 \quad 365\end{array}$ & $\begin{array}{rr}1 & 013 \\
83 & 946 \\
1 & 012 \\
71 & 696\end{array}$ & $\begin{array}{r}135 \\
6416 \\
135 \\
4642\end{array}$ & $\begin{array}{r}21 \\
4734 \\
21 \\
4 \quad 334\end{array}$ \\
\hline $\begin{array}{l}\text { Land rented or leased from others ......... farms.. } \\
\qquad \begin{array}{l}\text { acres.. } \\
\text { Rented or leased land in farms .......... } \\
\text { farms... }\end{array}\end{array}$ & $\begin{array}{r}17 \\
959 \\
17 \\
739\end{array}$ & $\begin{array}{r}35 \\
753 \\
75 \\
7318\end{array}$ & $\begin{array}{rr}72 \\
12 & 602 \\
72 \\
12 & 170\end{array}$ & $\begin{array}{rr}14 \\
1275 \\
14 \\
1275\end{array}$ & 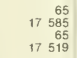 & $\begin{array}{r}248 \\
50 \quad 545 \\
244 \\
49993\end{array}$ & $\begin{array}{r}24 \\
568 \\
24 \\
554\end{array}$ & $\begin{array}{r}15 \\
(\mathrm{D}) \\
15 \\
10928\end{array}$ \\
\hline $\begin{array}{l}\text { Land rented or leased to others ............ farms.. } \\
\text { acres. }\end{array}$ & $\begin{array}{r}10 \\
866\end{array}$ & $\begin{array}{r}7 \\
549\end{array}$ & 4941 & 2089 & $\begin{array}{r}11 \\
814\end{array}$ & 12802 & 1788 & (D) \\
\hline \multicolumn{9}{|l|}{ OPERATOR CHARACTERISTICS } \\
\hline $\begin{array}{l}\text { Operators by place of residence: } \\
\text { On farm operated } \\
\text { Not on farm operated } \\
\text { Not reported } \\
\text { Operators by principal occupation: }\end{array}$ & $\begin{array}{r}53 \\
18 \\
3\end{array}$ & $\begin{array}{r}58 \\
5 \\
6\end{array}$ & $\begin{array}{r}198 \\
23 \\
17\end{array}$ & $\begin{array}{r}64 \\
5 \\
4\end{array}$ & $\begin{array}{r}96 \\
3 \\
6\end{array}$ & $\begin{array}{r}774 \\
96 \\
172\end{array}$ & $\begin{array}{r}115 \\
21 \\
10\end{array}$ & $\begin{array}{r}20 \\
1 \\
2\end{array}$ \\
\hline $\begin{array}{l}\text { Farming } \\
\text { Other ....... }\end{array}$ & $\begin{array}{l}41 \\
33\end{array}$ & $\begin{array}{l}35 \\
34\end{array}$ & $\begin{array}{l}116 \\
122\end{array}$ & $\begin{array}{l}31 \\
42\end{array}$ & $\begin{array}{r}100 \\
5\end{array}$ & $\begin{array}{l}715 \\
327\end{array}$ & $\begin{array}{r}37 \\
109\end{array}$ & $\begin{array}{r}16 \\
7\end{array}$ \\
\hline $\begin{array}{l}\text { Operators by days of work off farm: } \\
\text { None } \\
\text { Any } \\
1 \text { to } 99 \text { days } \\
100 \text { to } 199 \text { days } \\
200 \text { days or more }\end{array}$ & $\begin{array}{r}38 \\
32 \\
6 \\
4 \\
22\end{array}$ & $\begin{array}{r}31 \\
34 \\
7 \\
1 \\
26\end{array}$ & $\begin{array}{r}83 \\
141 \\
22 \\
25 \\
94\end{array}$ & $\begin{array}{r}27 \\
40 \\
2 \\
8 \\
30\end{array}$ & $\begin{array}{r}60 \\
36 \\
25 \\
3 \\
8\end{array}$ & \begin{tabular}{r|r}
495 \\
431 \\
82 \\
69 \\
280
\end{tabular} & $\begin{array}{l}42 \\
96 \\
11 \\
11 \\
74\end{array}$ & $\begin{array}{r}13 \\
8 \\
- \\
\overline{8}\end{array}$ \\
\hline Not reported ....................................... & 4 & 4 & 14 & 6 & 9 & 116 & 8 & 2 \\
\hline $\begin{array}{l}\text { Operators by years on present farm: } \\
2 \text { years or less } \\
3 \text { or } 4 \text { years } \\
5 \text { to } 9 \text { years... } \\
10 \text { years or more } \\
\text { Average years on present farm }\end{array}$ & $\begin{array}{r}4 \\
6 \\
13 \\
44 \\
16.4\end{array}$ & $\begin{array}{r}5 \\
2 \\
10 \\
44 \\
18.3\end{array}$ & $\begin{array}{r}17 \\
16 \\
43 \\
124 \\
176\end{array}$ & $\begin{array}{r}2 \\
2 \\
15 \\
45 \\
20.7\end{array}$ & $\begin{array}{r}4 \\
7 \\
14 \\
68 \\
19.7\end{array}$ & $\begin{array}{r}58 \\
81 \\
139 \\
490 \\
17.5\end{array}$ & $\begin{array}{r}13 \\
7 \\
32 \\
71 \\
14.1\end{array}$ & $\begin{array}{r}1 \\
1 \\
15 \\
244\end{array}$ \\
\hline Not reported & 7 & 8 & 38 & 9 & 12 & 274 & 23 & 6 \\
\hline $\begin{array}{l}\text { Operators by age group. } \\
\text { Under } 25 \text { years } \\
25 \text { to } 34 \text { years } \ldots \ldots \ldots \\
35 \text { to } 44 \text { years } \ldots \ldots \\
45 \text { to } 49 \text { years } \\
50 \text { to } 54 \text { years }\end{array}$ & $\begin{array}{r}7 \\
75 \\
15 \\
14 \\
6\end{array}$ & $\begin{array}{r}-14 \\
14 \\
5 \\
10\end{array}$ & $\begin{array}{r}1 \\
32 \\
47 \\
25 \\
24\end{array}$ & $\begin{array}{r}4 \\
11 \\
13 \\
5\end{array}$ & $\begin{array}{r}4 \\
15 \\
36 \\
6 \\
11\end{array}$ & $\begin{array}{r}9 \\
152 \\
260 \\
124 \\
143\end{array}$ & $\begin{array}{r}13 \\
36 \\
22 \\
19\end{array}$ & $\begin{array}{l}- \\
4 \\
2 \\
3\end{array}$ \\
\hline $\begin{array}{l}55 \text { to } 59 \text { years } \\
60 \text { to } 64 \text { years } \\
65 \text { to } 69 \text { years } \ldots \text { years and over } \\
70 . \\
\text { Average age }\end{array}$ & $\begin{array}{r}8 \\
8 \\
6 \\
10 \\
51.9\end{array}$ & $\begin{array}{r}5 \\
10 \\
8 \\
3 \\
489\end{array}$ & $\begin{array}{r}36 \\
25 \\
13 \\
35 \\
51.9\end{array}$ & $\begin{array}{r}15 \\
4 \\
3 \\
18 \\
55.9\end{array}$ & $\begin{array}{r}11 \\
14 \\
6 \\
2 \\
27.0\end{array}$ & $\begin{array}{r}105 \\
106 \\
73 \\
70 \\
489\end{array}$ & $\begin{array}{r}20 \\
14 \\
9 \\
13 \\
508\end{array}$ & $\begin{array}{r}5 \\
1 \\
3 \\
5 \\
57.6\end{array}$ \\
\hline $\begin{array}{l}\text { Operators by sex } \\
\text { Male } \\
\text { Femaie }\end{array}$ & $\begin{array}{r}71 \\
3\end{array}$ & $\begin{array}{r}64 \\
5\end{array}$ & $\begin{array}{r}221 \\
17\end{array}$ & $\begin{array}{r}70 \\
3\end{array}$ & $\begin{array}{r}104 \\
1\end{array}$ & $\begin{array}{l}862 \\
180\end{array}$ & $\begin{array}{r}118 \\
28\end{array}$ & 23 \\
\hline $\begin{array}{l}\text { Operators of Spanish origin (see text) } \\
\text { FARMS BY TYPE OF } \\
\text { ORGANIZATION }\end{array}$ & 1 & 1 & - & - & - & 2 & 2 & - \\
\hline $\begin{array}{l}\text { Individual or family (sole proprietorship) .... farms.. } \\
\text { Partnership }\end{array}$ & $\begin{array}{r}48 \\
1514 \\
6 \\
65\end{array}$ & $\begin{array}{r}56 \\
7 \quad 171 \\
3 \\
2399\end{array}$ & $\begin{array}{r}215 \\
\text { (D) } \\
13 \\
116\end{array}$ & $\begin{array}{r}6 \quad 70 \\
6 \quad 267 \\
2\end{array}$ & $\begin{array}{rr} & 74 \\
21 & 430 \\
& 24 \\
10 & 849\end{array}$ & $\begin{array}{r}927 \\
89585 \\
66 \\
0 \quad 072\end{array}$ & $\begin{array}{r}131 \\
4789 \\
6 \\
212\end{array}$ & $\begin{array}{r}13 \\
3 \quad 706 \\
7 \\
7\end{array}$ \\
\hline $\begin{array}{l}\text { Corporation } \\
\text { Family held }\end{array}$ & 17 & 10 & 8 & (D) & 6 & 10072 & 212 & \\
\hline $\begin{array}{l}\text { More than to stockholders ............. } \\
10 \text { or less stockholders .............. farms. }\end{array}$ & $\begin{array}{r}1212 \\
1 \\
16\end{array}$ & $8 \overline{-} 608$ & 11212 & (D) & (D) & $\begin{array}{r}21994 \\
3 \\
41\end{array}$ & (D) & $4 \frac{506}{3}$ \\
\hline 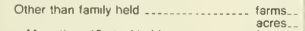 & (D) & - & $\overline{-}$ & - & - & (D) & (D) & - \\
\hline $\begin{array}{l}\text { More than } 10 \text { stockholders ............ farms.. } \\
10 \text { or less stockholders }\end{array}$ & $i$ & $=$ & $\overline{-}$ & - & $\overline{-}$ & $\frac{-}{2}$ & $\frac{1}{1}$ & $\overline{-}$ \\
\hline $\begin{array}{l}\text { Other-cooperative, estate or trust. } \\
\text { institutional, etc............ farms.. }\end{array}$ & (D) & - & $2^{2}$ & - & (D) & (D) & $\overline{-}$ & - \\
\hline
\end{tabular}

See tootnotes at end of table. 
Table 53. Summary by Standard Industrial Classification of Farm: 1987-Con.

[For meaning of abbreviations and symbols, see intraductory text]

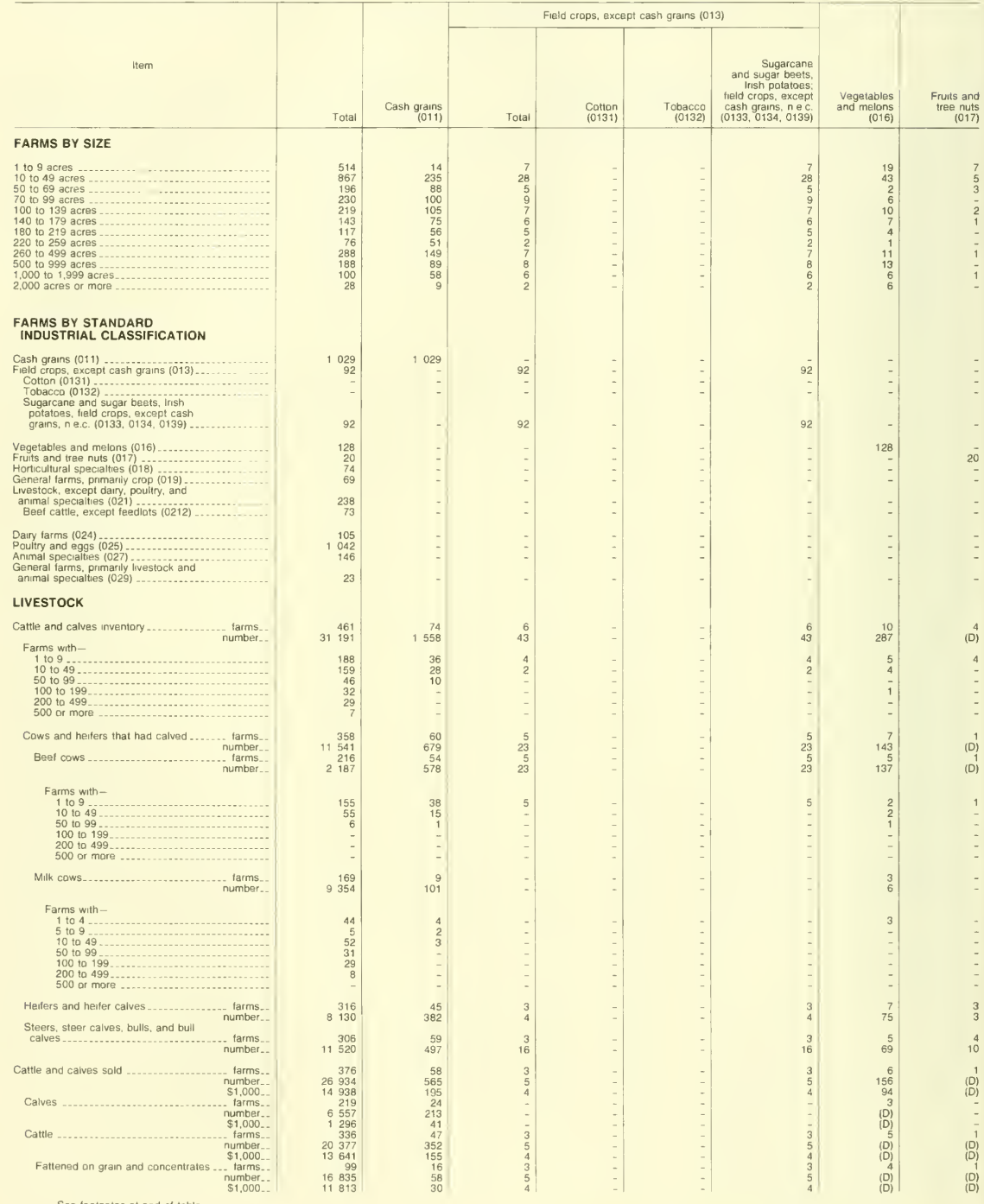

See footnates at end of table 
Table 53. Summary by Standard Industrial Classification of Farm: 1987-Con.

[For meaning of abbreviations and symbols, see introductory text]

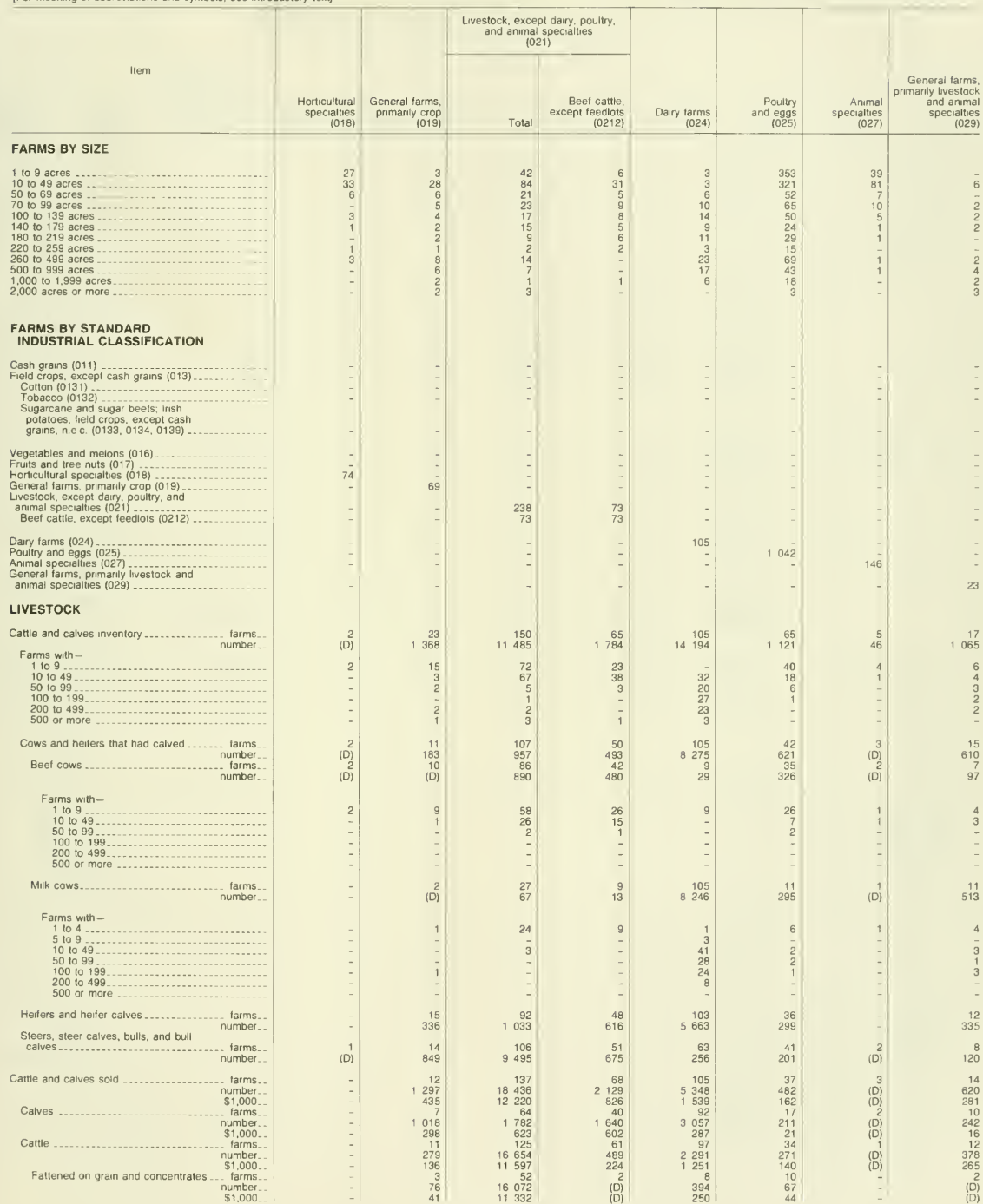

See footnotes at end of table 
Table 53. Summary by Standard Industrial Classification of Farm: 1987-Con.

[For meaning af abbrevations and symbals, see introductory text]

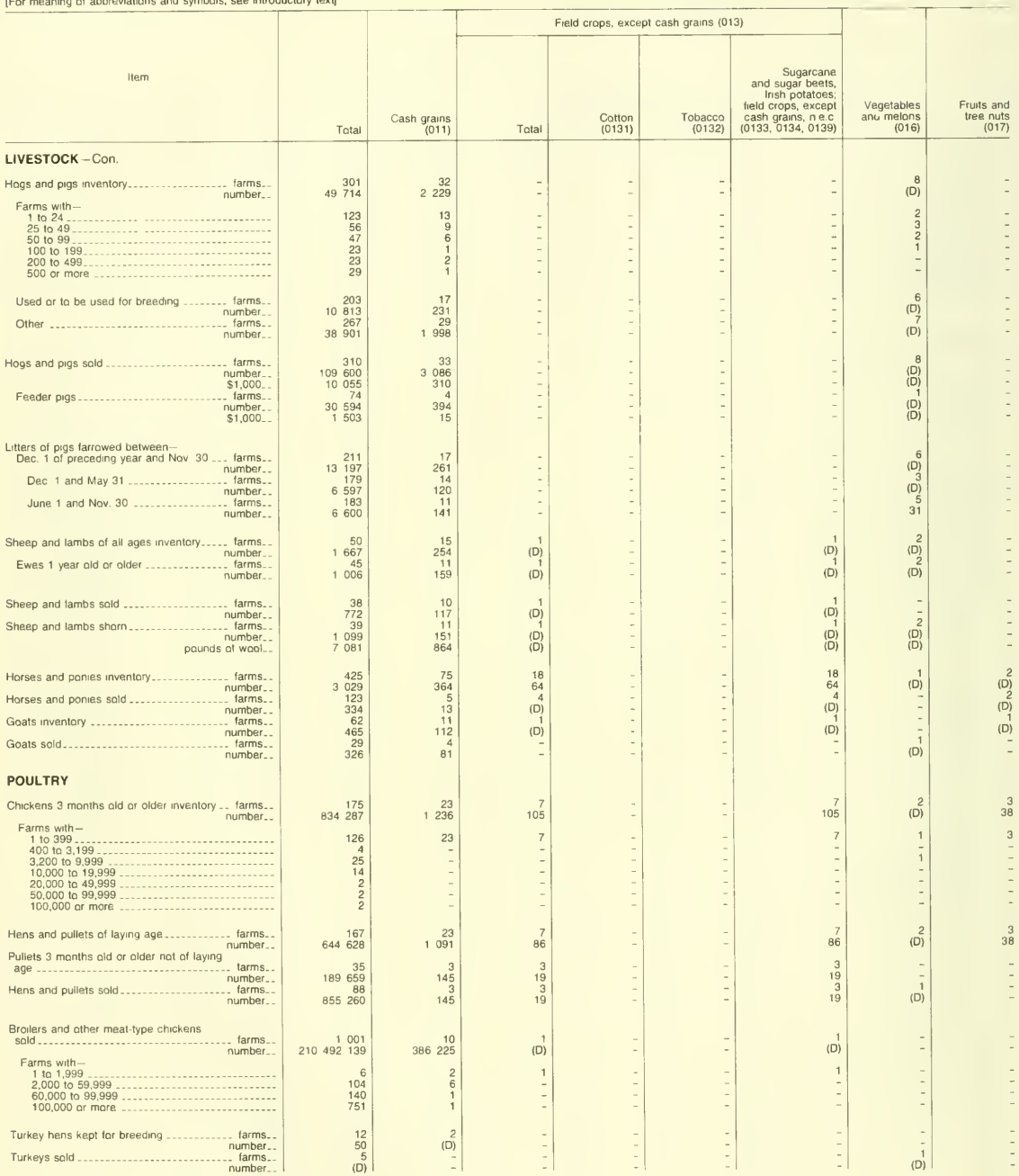


Table 53. Summary by Standard Industrial Classification of Farm: 1987-Con.

[For meaning of abbreviations and symbols, see introductory text]

\begin{tabular}{|c|c|c|c|c|c|c|c|c|}
\hline \multirow{2}{*}{ Item } & \multirow[b]{2}{*}{$\begin{array}{r}\text { Horticuitural } \\
\text { specialties } \\
(018)\end{array}$} & \multirow[b]{2}{*}{$\begin{array}{r}\text { General farms, } \\
\text { primarily crop } \\
\text { (019) } \\
\end{array}$} & \multicolumn{2}{|c|}{$\begin{array}{l}\text { Livestock, except dary, poultry, } \\
\text { and animal specialties } \\
\qquad(021)\end{array}$} & \multirow[b]{2}{*}{$\begin{array}{r}\text { Dairy farms } \\
(024)\end{array}$} & \multirow[b]{2}{*}{$\begin{array}{r}\text { Poultry } \\
\text { and eggs } \\
(025) \\
\end{array}$} & \multirow[b]{2}{*}{$\begin{array}{r}\text { Animal } \\
\text { specialties } \\
\text { (027) }\end{array}$} & \multirow{2}{*}{$\begin{array}{r}\text { General farms, } \\
\text { primarily livestock } \\
\text { and animal } \\
\text { specialties } \\
(029) \\
\end{array}$} \\
\hline & & & Total & $\begin{array}{r}\text { Beef cattle, } \\
\text { except feedlots } \\
(0212)\end{array}$ & & & & \\
\hline \multicolumn{9}{|l|}{ LIVESTOCK - Con. } \\
\hline Hogs and pigs inventory...................... farms... & (D) & 1202 & $32 \begin{array}{l}121 \\
760\end{array}$ & $\begin{array}{l}11 \\
59\end{array}$ & $\begin{array}{r}25 \\
795\end{array}$ & $\begin{array}{r}90 \\
642\end{array}$ & - & $\begin{array}{r}10 \\
5555\end{array}$ \\
\hline 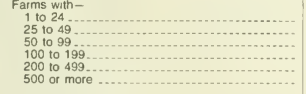 & $\begin{array}{l}1 \\
- \\
: \\
- \\
-\end{array}$ & $\begin{array}{l}7 \\
1 \\
3 \\
1 \\
1 \\
1\end{array}$ & $\begin{array}{r}47 \\
22 \\
13 \\
6 \\
11 \\
22\end{array}$ & $\begin{array}{l}11 \\
- \\
\vdots \\
-\end{array}$ & \begin{tabular}{r|r}
17 & \\
2 & \\
4 & \\
1 & 1 \\
1 &
\end{tabular} & $\begin{array}{r}33 \\
18 \\
18 \\
13 \\
6 \\
2\end{array}$ & $\begin{array}{l}- \\
z \\
- \\
-\end{array}$ & $\begin{array}{l}3 \\
1 \\
1 \\
5 \\
2 \\
3\end{array}$ \\
\hline Used or to be used for breeding .......... farms... & (D) & $\begin{array}{r}10 \\
230\end{array}$ & 8211 & $\begin{array}{r}4 \\
14\end{array}$ & $\begin{array}{r}9 \\
69\end{array}$ & $\begin{array}{r}63 \\
1348\end{array}$ & - & $\begin{array}{r}7 \\
668\end{array}$ \\
\hline Other ................. farms.. & (D) & $\begin{array}{r}8 \\
972\end{array}$ & $\begin{array}{rl}110 \\
24 & 549\end{array}$ & $\begin{array}{r}9 \\
45\end{array}$ & $\begin{array}{r}23 \\
726\end{array}$ & $\begin{array}{r}81 \\
5494\end{array}$ & $=$ & $\begin{array}{r}8 \\
4887\end{array}$ \\
\hline $\begin{array}{c}\text { Hogs and pigs sold ..................... farms.. } \\
\text { number. } \\
\$ 1,000\end{array}$ & (D) & $\begin{array}{rr}2 & 15 \\
271 \\
220\end{array}$ & $\begin{array}{r}122 \\
78988 \\
7216\end{array}$ & $\begin{array}{r}11 \\
114 \\
6\end{array}$ & $\begin{array}{r}25 \\
1381 \\
126\end{array}$ & $\begin{array}{r}94 \\
13310 \\
1160\end{array}$ & $=$ & $10 \begin{array}{r}12 \\
182 \\
982\end{array}$ \\
\hline 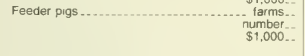 & $\begin{array}{r}- \\
- \\
-\end{array}$ & $\begin{array}{r}4 \\
400 \\
16\end{array}$ & $\begin{array}{r}7216 \\
35 \\
23 \\
1253 \\
1218\end{array}$ & $\begin{array}{r}6 \\
4 \\
80 \\
3\end{array}$ & $\begin{array}{r}126 \\
7 \\
(D) \\
(D)\end{array}$ & $\begin{array}{r}1 \\
160 \\
3337 \\
138\end{array}$ & $\begin{array}{l}- \\
- \\
-\end{array}$ & $\begin{array}{r}982 \\
3 \\
2381 \\
104\end{array}$ \\
\hline \multicolumn{9}{|l|}{$\begin{array}{l}\text { Litters of pigs larrowed between- } \\
\text { Dec. } 1 \text { of preceding year and Nov. } 30 \text {.... farms.. }\end{array}$} \\
\hline 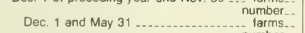 & (D) & $\begin{array}{r}263 \\
10\end{array}$ & 9849 & $\begin{array}{r}10 \\
2\end{array}$ & $\begin{array}{r}88 \\
9\end{array}$ & $\begin{array}{r}1553 \\
57\end{array}$ & - & 1124 \\
\hline June 1 and Nov $30 \quad$ number.. & (D) & $\begin{array}{r}129 \\
10\end{array}$ & 4999 & (D) & $\begin{array}{r}9 \\
45\end{array}$ & 704 & - & 572 \\
\hline number... & - & $\begin{array}{r}10 \\
134\end{array}$ & $\begin{array}{r}81 \\
4850\end{array}$ & $\left(D^{2}\right)$ & $\begin{array}{r}8 \\
43\end{array}$ & $\begin{array}{r}62 \\
849\end{array}$ & - & $\begin{array}{r}6 \\
552\end{array}$ \\
\hline Sheep and lambs of all ages inventory...... farms.. & - & $\begin{array}{r}3 \\
38\end{array}$ & $\begin{array}{r}15 \\
947\end{array}$ & $\begin{array}{r}4 \\
278\end{array}$ & 92 & $\begin{array}{r}8 \\
259\end{array}$ & - & $40^{3}$ \\
\hline $\begin{array}{c}\text { Ewes } 1 \text { year old or older ................. farms.. } \\
\text { number }\end{array}$ & $=$ & \begin{tabular}{r|r}
3 \\
22
\end{tabular} & $\begin{array}{r}15 \\
532\end{array}$ & $\begin{array}{r}4 \\
137\end{array}$ & $\begin{array}{r}3 \\
64\end{array}$ & (D) & $\overline{-}$ & $\begin{array}{r}3 \\
39\end{array}$ \\
\hline Sheep and lambs sold ........................ & - & 1 & 13 & 3 & 3 & 7 & - & 3 \\
\hline 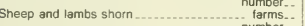 & $=$ & (D) & $\begin{array}{r}408 \\
11\end{array}$ & $\begin{array}{r}61 \\
2\end{array}$ & $\begin{aligned} 82 \\
2\end{aligned}$ & $\begin{array}{r}112 \\
6\end{array}$ & $\overline{-}$ & (D) \\
\hline pounds of wool... & - & $\begin{array}{r}23 \\
217\end{array}$ & $\begin{array}{r}653 \\
3844\end{array}$ & $\begin{array}{l}\text { (D) } \\
\text { (D) }\end{array}$ & (D) & $\begin{array}{r}124 \\
1064\end{array}$ & - & $\begin{array}{r}44 \\
320\end{array}$ \\
\hline $\begin{array}{l}\text { Horses and ponies inventory ................... farms_. } \\
\text { number }\end{array}$ & 4 & 24 & 71 & $\begin{array}{r}25 \\
16\end{array}$ & 37 & 55 & 129 & 9 \\
\hline Horses and ponies sold ................... & 8 & $\begin{array}{r}198 \\
3\end{array}$ & $\begin{array}{r}320 \\
12\end{array}$ & $\begin{array}{r}116 \\
2\end{array}$ & $\begin{array}{r}282 \\
16\end{array}$ & $\begin{array}{r}214 \\
7\end{array}$ & $\begin{array}{r}1511 \\
74\end{array}$ & ${ }^{46}-$ \\
\hline Goats inventory ............. & - & 16 & $\begin{array}{l}31 \\
25\end{array}$ & (D) & 22 & 12 & 230 & $\overline{3}$ \\
\hline Goals inventory ..........- & - & $\begin{array}{l}4 \\
6\end{array}$ & 203 & 19 & (D) & $\begin{array}{l}13 \\
93\end{array}$ & (D) & 34 \\
\hline Goats sold .............. & - & (D) & 170 & (D) & (D) & $\begin{array}{r}3 \\
44\end{array}$ & (D) & $=$ \\
\hline \multicolumn{9}{|l|}{ POULTRY } \\
\hline $\begin{array}{l}\text { Chickens } 3 \text { months old or older inventory ... farms.. } \\
\text { number. }\end{array}$ & - & 112265 & $\begin{array}{r}41 \\
824\end{array}$ & $\begin{array}{r}13 \\
211\end{array}$ & 20000 & $\begin{array}{lr}693 & 54 \\
223\end{array}$ & $\begin{array}{r}8 \\
(D)\end{array}$ & $\begin{array}{r}6 \\
437\end{array}$ \\
\hline $\begin{array}{r}1 \text { to } 399 \\
400 \text { to } 3.199\end{array}$ & $:$ & 4 & 41. & 13 & $\begin{array}{r}23 \\
2\end{array}$ & $\begin{array}{r}10 \\
2\end{array}$ & 8 & 6 \\
\hline 10,000 to 99,99999 & $z$ & $z$ & : & $:$ & $i$ & $\begin{array}{l}24 \\
13\end{array}$ & $=$ & - \\
\hline $\begin{array}{l}50,000 \text { to } 99,999 \\
100.000 \text { or more }\end{array}$ & $\overline{-}$ & $\bar{i}$ & $\overline{-}$ & $=$ & $\overline{-}$ & & 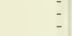 & 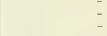 \\
\hline Hens and pullets of laying age ............. farms.. & - & (D) & $\begin{array}{r}41 \\
744\end{array}$ & $\begin{array}{r}13 \\
\text { (D) }\end{array}$ & 1725 & $574 \begin{array}{r}47 \\
308\end{array}$ & $\begin{array}{r}8 \\
141\end{array}$ & $\begin{array}{r}6 \\
(D)\end{array}$ \\
\hline $\begin{array}{l}\text { Pullets } 3 \text { months old or oider not of laying farms. } \\
\text { age }\end{array}$ & -1 & 1 & 7 & 2 & & & 2 & \\
\hline $\begin{array}{c}\text { number.- } \\
\text { Hens and pullets soid } \\
\text { number. } \\
\text { number }\end{array}$ & $\begin{array}{l}- \\
- \\
-\end{array}$ & $\begin{array}{l}\text { (D) } \\
\text { (D) }\end{array}$ & $\begin{array}{r}80 \\
8 \\
90\end{array}$ & $\begin{aligned} \text { (D) } \\
2 \\
\text { (D) }\end{aligned}$ & $\begin{array}{l}18280 \\
11 \\
19086\end{array}$ & $\begin{array}{r}118915 \\
57 \\
715700\end{array}$ & $\begin{array}{l}\text { (D) } \\
\text { (D) }\end{array}$ & $\begin{array}{l}\text { (D) } \\
\text { (D) }\end{array}$ \\
\hline $\begin{array}{l}\text { Broilers and other meat-type chickens } \\
\text { sold }\end{array}$ & & & & & & & & \\
\hline Farms with- $\quad$ number.- & - & 382400 & (D) & $:-$ & (D) & $\begin{array}{lll}208 & 073 & 974 \\
\end{array}$ & 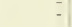 & 1395500 \\
\hline $\begin{array}{l}1 \text { to } 1,999 \\
2,000 \text { to } 59,999 \\
60.000 \text { to } 99,999 \\
100,000 \text { or more }\end{array}$ & $\begin{array}{l}: \\
z\end{array}$ & $\begin{array}{l}\overline{1} \\
\frac{-}{2}\end{array}$ & $\begin{array}{l}3 \\
1 \\
i\end{array}$ & $\begin{array}{l}: \\
-\end{array}$ & $\begin{array}{l}- \\
1 \\
-\end{array}$ & $\begin{array}{r}9 \overline{5} \\
139 \\
740\end{array}$ & $\begin{array}{l}\bar{y} \\
\overline{-}\end{array}$ & $\overline{5}$ \\
\hline $\begin{array}{l}\text { Turkey hens kept for breeding ................ farms.. } \\
\text { number.. }\end{array}$ & 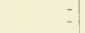 & (D) & $\begin{array}{l}4 \\
8 \\
0\end{array}$ & (D) & (D) & (D) & - & (D) \\
\hline $\begin{array}{c}\text { farms } \\
\text { number.. }\end{array}$ & -1 & (D) 1 & $\left(D^{2}\right)$ & (D) & (D) & $=$ & - & - \\
\hline
\end{tabular}


Table 53. Summary by Standard Industrial Classification of Farm: 1987-Con.

[For meaning of abbreviations and symbols, see introductory text]

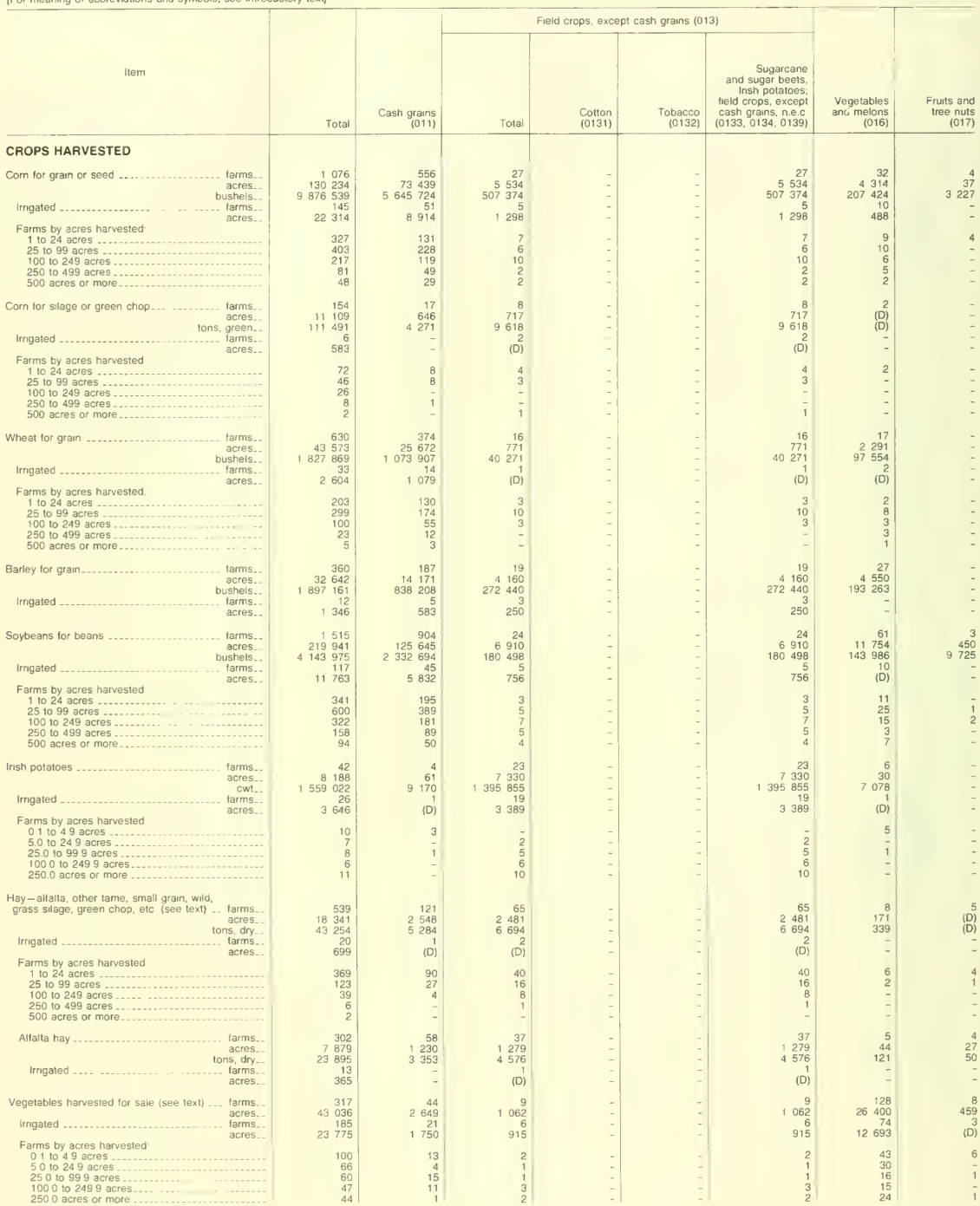


Table 53. Summary by Standard Industrial Classification of Farm: 1987-Con.

[For mearung of abbravations and symbols, see introductory text]

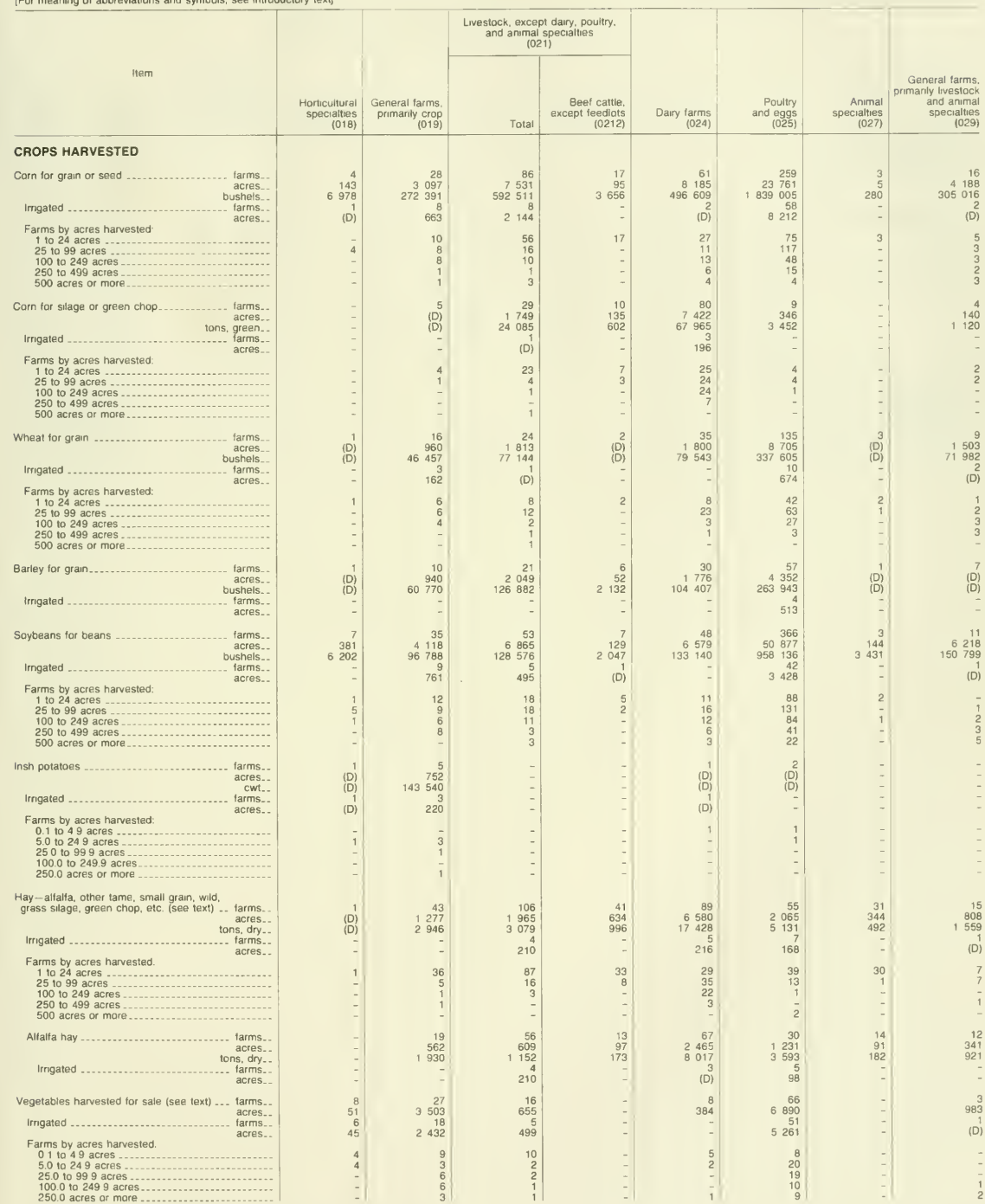

See footnotes at end of table 
Table 53. Summary by Standard Industrial Classification of Farm: 1987-Con.

[For meaning of abbreviations and symbols, see introductory text]

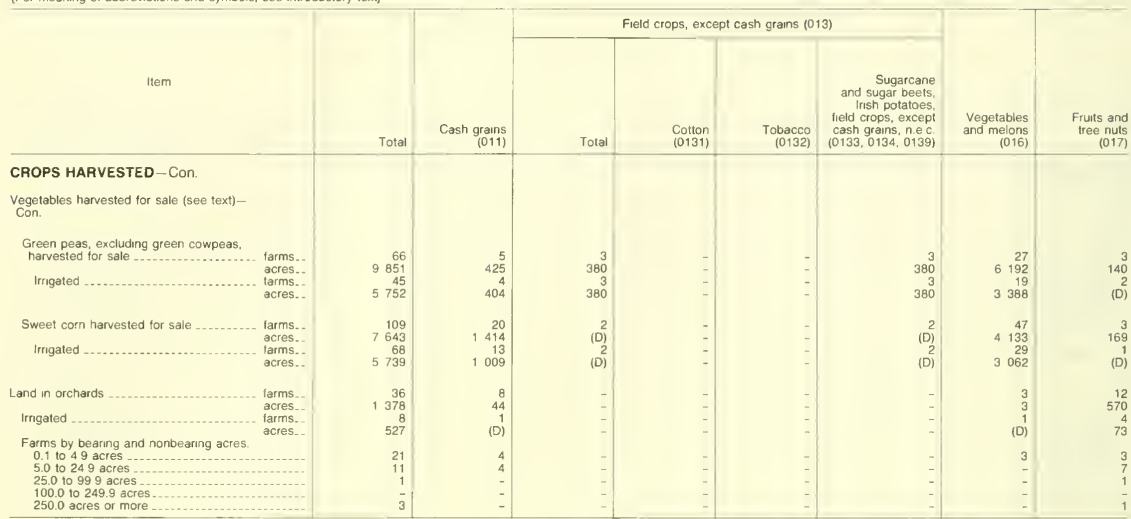

See footnotes at end of table 
Table 53. Summary by Standard Industrial Classification of Farm: 1987-Con.

[For meaning of abbreviations and symbols, see introductory text]

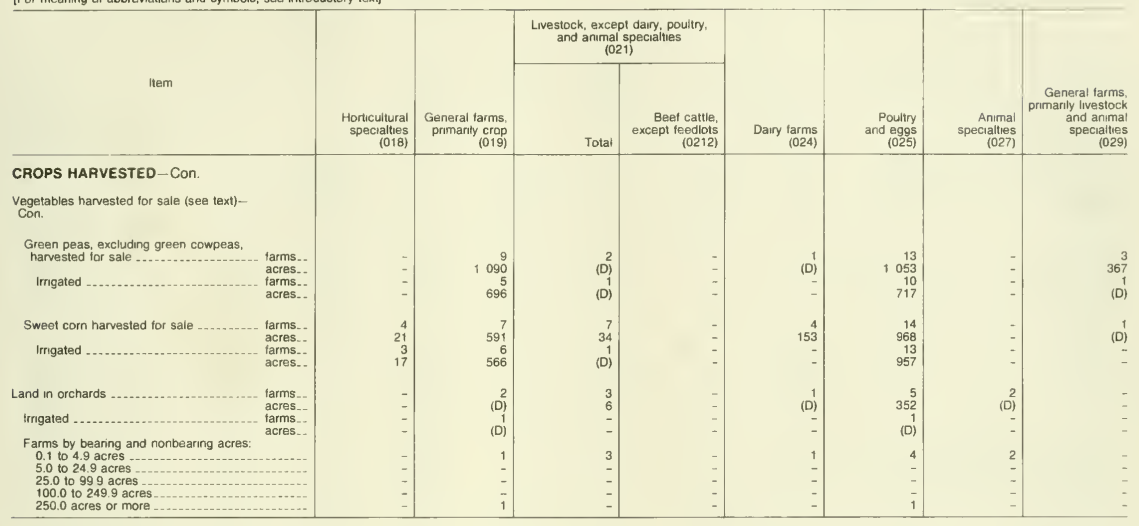

'Data are based on a sample of farms.

${ }^{2} \mathrm{Farms}$ with total production expenses equal to market value of agncultural products sold are included as farms with gains of less than $\$ 1,000$.

1987 CENSUS OF AGRICULTURE -STATE DATA

DELAWARE

139 
Table 1. County Summary Highlights: 1987

[For meaning of abbreviations and symbols, see introductory texi]

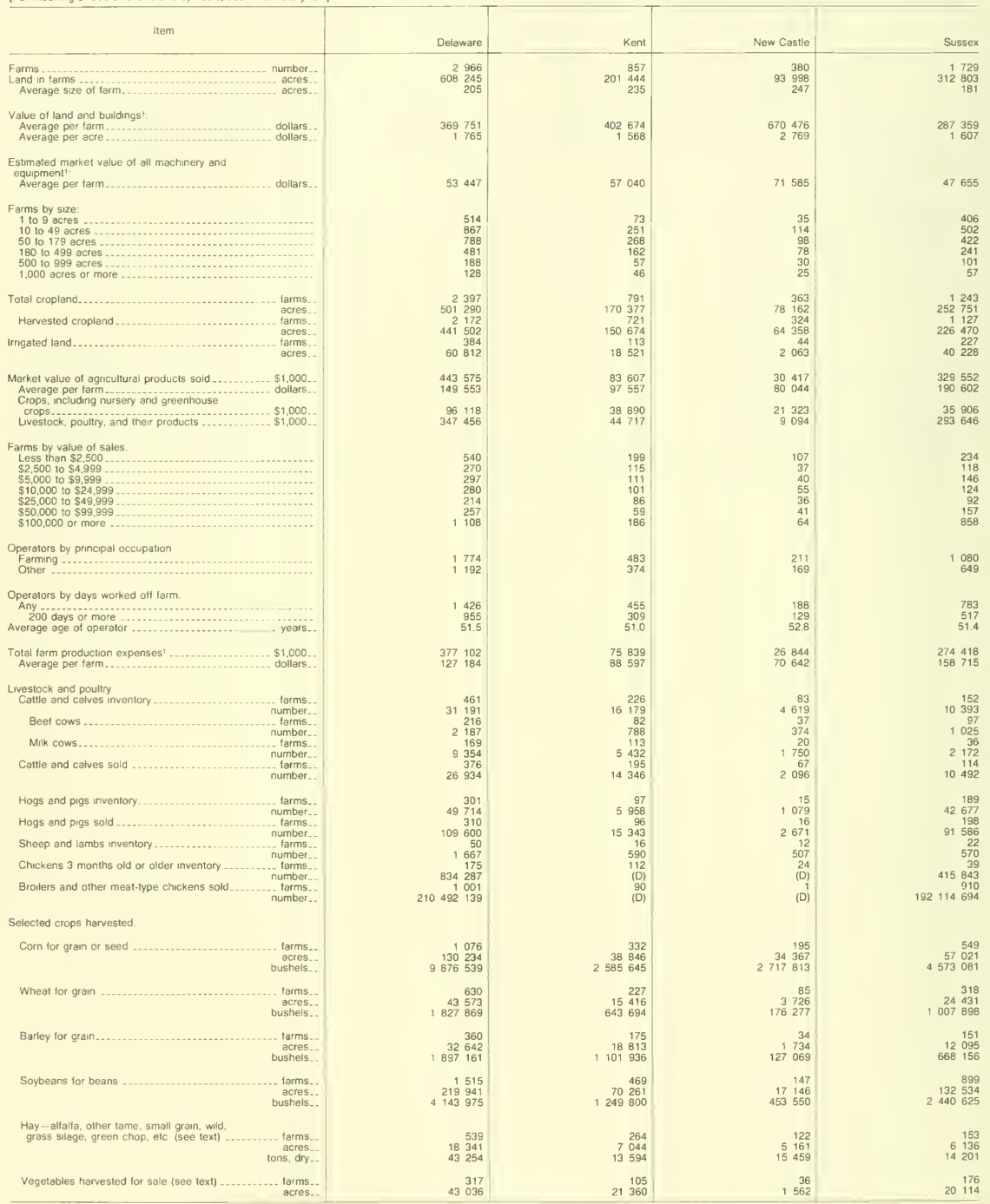

- Data are based on a sample of farms 


\section{Table 2. Market Value of Agricultural Products Sold and Farms by Standard Industrial Classification: 1987 and 1982}

[For meaning of abbreviations and symbols, see introductory text]

\begin{tabular}{|c|c|c|c|c|}
\hline Item & Delaware & Kent & New Castle & Sussex \\
\hline \multicolumn{5}{|l|}{$\begin{array}{l}\text { MARKET VALUE OF AGRICUL- } \\
\text { TURAL PRODUCTS SOLD }\end{array}$} \\
\hline 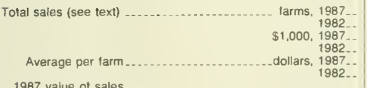 & $\begin{array}{rr}2 & 966 \\
3 & 338 \\
443 & 575 \\
370 & 562 \\
149 & 553 \\
111 & 013\end{array}$ & \begin{tabular}{rr|}
1 & 857 \\
83 & 609 \\
73 & 447 \\
97 & 557 \\
72 & 792
\end{tabular} & $\begin{array}{ll} & 380 \\
30 & 417 \\
27 & 151 \\
80 & 044 \\
55 & 638\end{array}$ & $\begin{array}{rr}1 & 729 \\
1 & 841 \\
329 & 552 \\
269 & 964 \\
190 & 602 \\
146 & 640\end{array}$ \\
\hline $\begin{array}{c}\begin{array}{c}1987 \text { value of sales. } \\
\text { Less than } \$ 1,000 \\
\$ 1,000 \text { to } \$ 2,499\end{array} \\
\$ 2.500 \text { to } \$ 4,999 \\
\$ 5,000 \text { to } \$ 9,999\end{array}$ & $\begin{array}{r}280 \\
91 \\
260 \\
429 \\
270 \\
977 \\
297 \\
2059\end{array}$ & $\begin{array}{r}109 \\
33 \\
90 \\
153 \\
115 \\
401 \\
111 \\
754\end{array}$ & $\begin{array}{r}61 \\
14 \\
46 \\
66 \\
37 \\
135 \\
40 \\
268\end{array}$ & $\begin{array}{r}110 \\
45 \\
124 \\
209 \\
118 \\
440 \\
146 \\
1037\end{array}$ \\
\hline 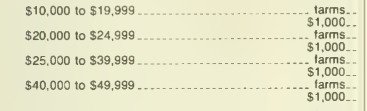 & $\begin{array}{r}215 \\
3 \quad 106 \\
65 \\
439 \\
141 \\
4 \quad 400 \\
73 \\
3232\end{array}$ & $\begin{array}{r}78 \\
1103 \\
23 \\
525 \\
53 \\
1 \quad 629 \\
143 \\
1462\end{array}$ & $\begin{array}{r}42 \\
629 \\
13 \\
288 \\
25 \\
792 \\
11 \\
487\end{array}$ & $\begin{array}{r}95 \\
1374 \\
29 \\
627 \\
63 \\
1979 \\
929 \\
1282\end{array}$ \\
\hline $\begin{array}{l}\$ 50,000 \text { to } \$ 99,999 \ldots \ldots \ldots \\
\$ 100,000 \text { to } \$ 249,999 \ldots \ldots \ldots \\
\$ 250,000 \text { to } \$ 499,999 \ldots \ldots \ldots \\
\$ 500,000 \text { or more }\end{array}$ & 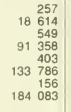 & $\begin{array}{rr}59 \\
455 \\
88 \\
13508 \\
69 \\
23 \\
609 \\
29 \\
36 & 274\end{array}$ & $\begin{array}{r}241 \\
2986 \\
63 \\
623 \\
9 \\
3019 \\
12 \\
14 \quad 808\end{array}$ & $\begin{array}{r}157 \\
11473 \\
418 \\
70 \quad 927 \\
325 \\
107 \quad 158 \\
115 \\
133001\end{array}$ \\
\hline 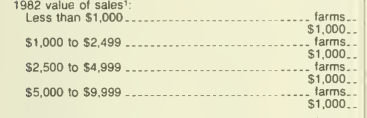 & $\begin{array}{r}230 \\
75 \\
318 \\
525 \\
304 \\
1103 \\
306 \\
2190\end{array}$ & $\begin{array}{r}89 \\
29 \\
96 \\
153 \\
105 \\
382 \\
121 \\
865\end{array}$ & $\begin{array}{r}66 \\
15 \\
65 \\
104 \\
49 \\
180 \\
51 \\
366\end{array}$ & $\begin{array}{r}75 \\
31 \\
157 \\
268 \\
150 \\
542 \\
134 \\
959\end{array}$ \\
\hline 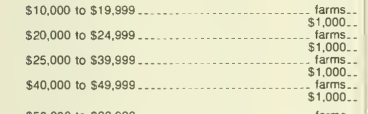 & $\begin{array}{rr}573 \\
5294 \\
106 \\
2355 \\
239 \\
7581 \\
94 \\
4 \quad 186\end{array}$ & $\begin{array}{r}145 \\
2060 \\
45 \\
1022 \\
107 \\
3342 \\
30 \\
1344\end{array}$ & $\begin{array}{r}61 \\
882 \\
12 \\
267 \\
37 \\
1159 \\
10 \\
440\end{array}$ & $\begin{array}{r}167 \\
2 \quad 352 \\
49 \\
1066 \\
95 \\
3079 \\
5 \\
54 \\
2402\end{array}$ \\
\hline $\begin{array}{l}\$ 50,000 \text { to } \$ 99,999 \ldots \ldots . . . \\
\$ 100,000 \text { to } \$ 249,999 \ldots \ldots \\
\$ 250,000 \text { to } \$ 499,999 \ldots \ldots \\
\$ 500,000 \text { or more }\end{array}$ & $\begin{array}{r}346 \\
25502 \\
601 \\
98 \quad 409 \\
303 \\
102 \quad 430 \\
118 \\
120911\end{array}$ & $\begin{array}{rr}7 & 96 \\
138 \\
93 \\
14 & 964 \\
60 \\
20 & 577 \\
22 & 22 \\
21 & 571\end{array}$ & $\begin{array}{rr}56 \\
4094 \\
59 \\
9817 \\
18 \\
6 \quad 279 \\
4 \\
3 \quad 549\end{array}$ & $\begin{array}{r}194 \\
14 \\
270 \\
449 \\
73 \quad 628 \\
225 \\
75 \quad 574 \\
92 \\
95 \quad 791\end{array}$ \\
\hline 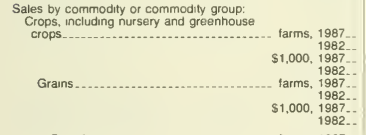 & $\begin{array}{rr}1 & 992 \\
2 & 501 \\
96 & 118 \\
110 & 276 \\
1 & 733 \\
2 & 254 \\
46 & 701 \\
74 & 889\end{array}$ & $\begin{array}{r}633 \\
807 \\
38 \quad 890 \\
41335 \\
539 \\
723 \\
14551 \\
23940\end{array}$ & \begin{tabular}{rr|}
288 \\
389 \\
21 & 323 \\
20 & 385 \\
210 \\
304 \\
8 & 071 \\
13 & 144
\end{tabular} & $\begin{array}{rr}1 & 071 \\
1 & 305 \\
35 & 906 \\
48 & 556 \\
& 984 \\
1 & 227 \\
24 & 080 \\
37 & 805\end{array}$ \\
\hline $\begin{array}{rr}\text { Corn for grain .................... farms, } 1987 \ldots & 1982 . \\
& \$ 1,000,1987 \ldots \\
& 1982 \ldots \\
\text { Wheat } & \text { tarms, } 1987 \ldots \\
& 1982 . \\
& \$ 1,000,1987 \ldots \\
& 1982 \ldots\end{array}$ & $\begin{array}{rr}1 & 915 \\
18 & 294 \\
30 & 844 \\
626 \\
776 \\
74 \\
4 & 438 \\
6 & 654\end{array}$ & $\begin{array}{r}245 \\
393 \\
4921 \\
10918 \\
226 \\
308 \\
1546 \\
2319\end{array}$ & $\begin{array}{r}172 \\
234 \\
5201 \\
8295 \\
85 \\
100 \\
428 \\
672\end{array}$ & $\begin{array}{rr} & 498 \\
537 \\
8 & 171 \\
11 & 632 \\
315 \\
368 \\
2 & 464 \\
3 & 663\end{array}$ \\
\hline 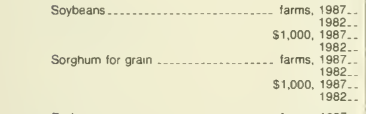 & \begin{tabular}{rr|}
1 & 497 \\
1 & 977 \\
21 & 654 \\
34 & 109 \\
18 \\
\\
\\
\\
\\
\\
36 \\
8
\end{tabular} & $\begin{array}{r}462 \\
612 \\
6756 \\
9147 \\
\overline{2} \\
\text { (D) }\end{array}$ & $\begin{array}{r}147 \\
207 \\
3224 \\
3936 \\
- \\
- \\
-\end{array}$ & $\begin{array}{rr} & 888 \\
1 & 158 \\
12 & 574 \\
21 & 026 \\
& 18 \\
& 2 \\
& 36 \\
& \text { (D) }\end{array}$ \\
\hline 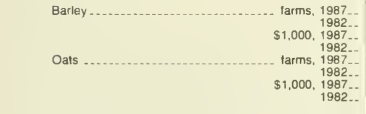 & $\begin{array}{r}320 \\
\text { (NA) } \\
2190 \\
\text { (NA) } \\
9 \\
23 \\
6 \\
23\end{array}$ & $\begin{array}{r}160 \\
\text { (NA) } \\
1307 \\
\text { (NA) } \\
6 \\
14 \\
4 \\
\text { (D) }\end{array}$ & $\begin{array}{r}29 \\
\text { (NA) } \\
111 \\
\text { (NA) } \\
1 \\
6 \\
\text { (D) } \\
6\end{array}$ & $\begin{array}{r}131 \\
\text { (NA) } \\
772 \\
\text { (NA) } \\
2 \\
3 \\
\text { (D) } \\
\text { (D) }\end{array}$ \\
\hline 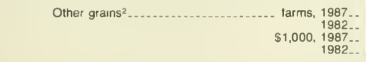 & $\begin{array}{r}40 \\
462 \\
83 \\
3251\end{array}$ & $\begin{array}{r}14 \\
200 \\
17 \\
1542\end{array}$ & $\begin{array}{r}3 \\
45 \\
(D) \\
236\end{array}$ & $\begin{array}{r}23 \\
217 \\
(D) \\
1473\end{array}$ \\
\hline
\end{tabular}

See footnotes at end of table 
Table 2. Market Value of Agricultural Products Sold and Farms by Standard Industrial Classification: 1987 and 1982-Con.

[For meaning of abbreviations and symbols, see intraductory text]

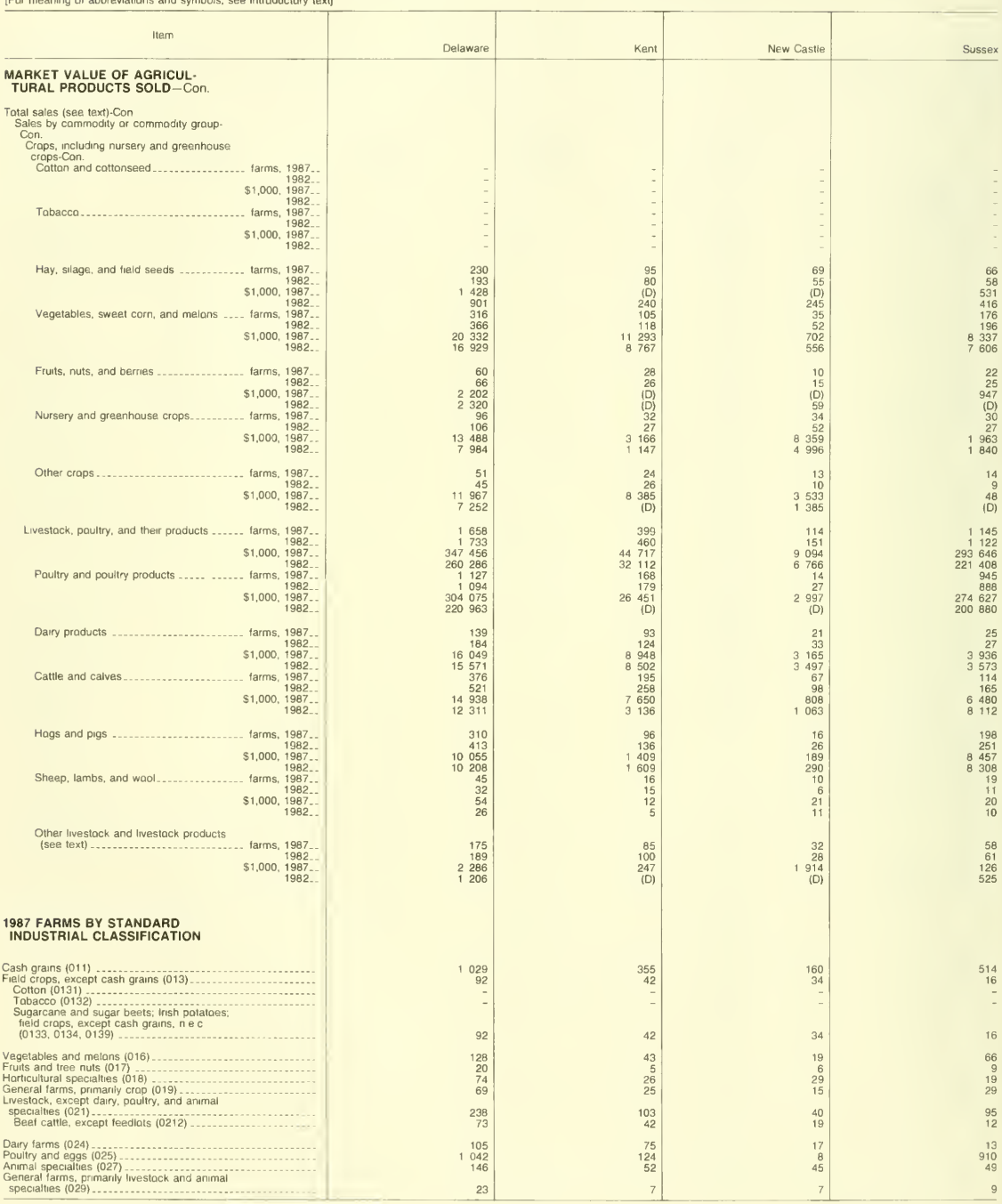

'Data tor 1982 excludes abnormal farms

2Data for 1982 includes market value of barley sold 
Table 3. Farm Production Expenses: 1987 and 1982

[Data are based on a sample of farms. For meaning of abbreviations and symbols, see introductory text]

\begin{tabular}{|c|c|c|c|c|}
\hline Item & Delaware & Kent & New Castle & Sussex \\
\hline $\begin{array}{c}\text { Total tarm production expenses ................ farms, } 1987 \ldots \\
\$ 1.000,1987 . \\
\text { Average per farm ......................... dollars, } 1987 \ldots\end{array}$ & $\begin{array}{rr}2 & 965 \\
377 & 102 \\
127 & 184\end{array}$ & $\begin{array}{r}856 \\
75839 \\
88597\end{array}$ & $\begin{array}{rr}380 \\
26 & 844 \\
70 & 642\end{array}$ & $\begin{array}{r}729 \\
274418 \\
158715\end{array}$ \\
\hline $\begin{array}{r}\text { Livestock and poultry purchased ............ tarms, } 1987 \ldots \\
1982 . \\
\$ 1,000,1987 \ldots \\
1982 \ldots\end{array}$ & $\begin{array}{rr}1 & 374 \\
1 & 320 \\
53 & 522 \\
37 & 202\end{array}$ & $\begin{array}{r}312 \\
278 \\
7887 \\
4 \quad 437\end{array}$ & $\begin{array}{r}48 \\
82 \\
828 \\
570\end{array}$ & $\begin{array}{r}1014 \\
960 \\
44 \quad 807 \\
32 \quad 196\end{array}$ \\
\hline 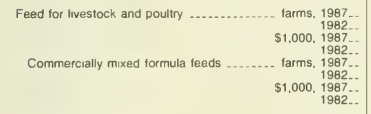 & \begin{tabular}{rr|}
1 & 637 \\
1 & 642 \\
187 & 183 \\
140 & 508 \\
1 & 341 \\
1 & 336 \\
180 & 490 \\
136 & 947
\end{tabular} & $\begin{array}{rr}432 \\
389 \\
21 & 501 \\
15 & 420 \\
294 \\
243 \\
18522 \\
14 & 514\end{array}$ & $\begin{array}{rr}110 \\
188 \\
2475 \\
2393 \\
53 \\
119 \\
1 & 903 \\
1 & 595\end{array}$ & $\begin{array}{rr}1 & 095 \\
1 & 065 \\
163 & 207 \\
122 & 696 \\
994 \\
974 \\
160 & 065 \\
120 & 838\end{array}$ \\
\hline 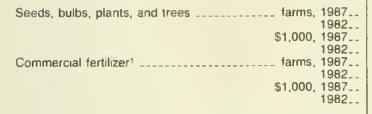 & $\begin{array}{rr}1 & 899 \\
2 & 112 \\
8 & 667 \\
6 & 964 \\
1 & 807 \\
2 & 191 \\
15 & 802 \\
19 & 993\end{array}$ & $\begin{array}{r}657 \\
675 \\
3285 \\
2730 \\
658 \\
797 \\
6750 \\
7880\end{array}$ & \begin{tabular}{rr|} 
& 264 \\
319 \\
2 & 128 \\
1 & 270 \\
295 \\
358 \\
2 & 831 \\
3 & 649
\end{tabular} & $\begin{array}{lr} & 978 \\
1 & 118 \\
3 & 254 \\
2 & 964 \\
& 854 \\
1 & 036 \\
6 & 221 \\
8 & 463\end{array}$ \\
\hline 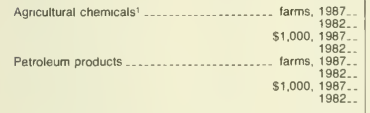 & $\begin{array}{rr}1 & 973 \\
2 & 058 \\
11 & 197 \\
10 & 868 \\
2 & 692 \\
3 & 322 \\
8 & 787 \\
13 & 435\end{array}$ & $\begin{array}{rr} & 656 \\
635 \\
4616 \\
3 & 496 \\
817 \\
1 & 006 \\
2 & 989 \\
4 & 514\end{array}$ & $\begin{array}{r}289 \\
300 \\
1 \quad 833 \\
1 \quad 750 \\
369 \\
487 \\
1187 \\
1798\end{array}$ & $\begin{array}{ll}1 & 028 \\
1 & 123 \\
4 & 748 \\
5 & 621 \\
1 & 506 \\
1 & 829 \\
4 & 612 \\
7 & 123\end{array}$ \\
\hline 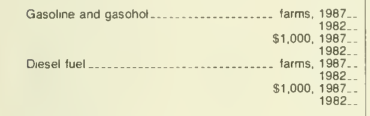 & $\begin{array}{ll}2 & 356 \\
3 & 062 \\
3 & 226 \\
5 & 192 \\
1 & 494 \\
1 & 755 \\
3 & 905 \\
5 & 019\end{array}$ & $\begin{array}{rr}739 \\
921 \\
1 & 142 \\
1 & 598 \\
522 \\
\\
588 \\
1529 \\
1 & 933\end{array}$ & $\begin{array}{l}340 \\
444 \\
384 \\
708 \\
234 \\
237 \\
477 \\
639\end{array}$ & $\begin{array}{rr}1 & 277 \\
1 & 697 \\
1 & 700 \\
2 & 887 \\
738 \\
\\
1 & 930 \\
1 & 900 \\
2 & 447\end{array}$ \\
\hline $\begin{array}{r}\text { Natural gas . ........... farms, } 1987 . . \\
\\
\$ 1,000,1987 . \\
1982 .\end{array}$ & $\begin{array}{r}93 \\
27 \\
331 \\
79\end{array}$ & $\begin{array}{r}23 \\
1 \\
29 \\
\text { (D) }\end{array}$ & $\begin{array}{r}30 \\
-\overline{-} \\
14 \overline{7} \\
-\end{array}$ & $\begin{array}{r}40 \\
26 \\
155 \\
\text { (D) }\end{array}$ \\
\hline 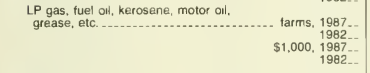 & $\begin{array}{rr}1 & 873 \\
(\text { (NA) } \\
1 & 325 \\
3 & 144\end{array}$ & $\begin{array}{r}586 \\
\text { (NA) } \\
288 \\
(D)\end{array}$ & $\begin{array}{r}264 \\
\text { (NA) } \\
180 \\
451\end{array}$ & $\begin{array}{r}1023 \\
\text { (NA) } \\
857 \\
\text { (D) }\end{array}$ \\
\hline 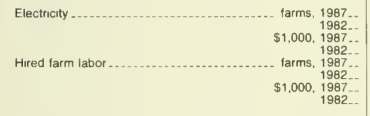 & $\begin{array}{rr}2 & 195 \\
2 & 244 \\
4 & 862 \\
4 & 554 \\
1 & 254 \\
1 & 462 \\
22 & 788 \\
17 & 893\end{array}$ & $\begin{array}{rr}583 \\
596 \\
1005 \\
925 \\
356 \\
373 \\
7641 \\
5646\end{array}$ & $\begin{array}{r}301 \\
330 \\
746 \\
608 \\
141 \\
221 \\
4223 \\
2026\end{array}$ & $\begin{array}{rr}1 & 311 \\
1 & 318 \\
3 & 112 \\
3 & 021 \\
757 \\
\\
868 \\
10 & 924 \\
10 & 220\end{array}$ \\
\hline 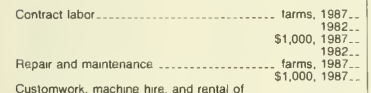 & $\begin{array}{rr}364 \\
179 \\
5 & 798 \\
1 & 296 \\
2 & 481 \\
11 & 739\end{array}$ & $\begin{array}{r}66 \\
50 \\
1348 \\
624 \\
735 \\
3765\end{array}$ & $\begin{array}{r}35 \\
32 \\
338 \\
151 \\
325 \\
1435\end{array}$ & $\begin{array}{r}263 \\
97 \\
4 \quad 112 \\
521 \\
1 \\
421 \\
6539\end{array}$ \\
\hline 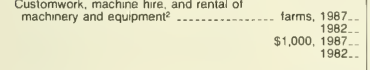 & $\begin{array}{ll}1 & 192 \\
1 & 271 \\
2 & 445 \\
2 & 606\end{array}$ & $\begin{array}{l}403 \\
382 \\
836 \\
817\end{array}$ & $\begin{array}{l}110 \\
157 \\
332 \\
487\end{array}$ & $\begin{array}{r}679 \\
732 \\
1277 \\
1303\end{array}$ \\
\hline 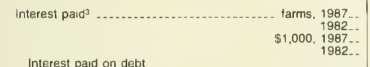 & $\begin{array}{rr}1 & 415 \\
1 & 433 \\
14 & 872 \\
16 & 948\end{array}$ & $\begin{array}{r}374 \\
469 \\
4994 \\
5331\end{array}$ & $\begin{array}{rr} & 148 \\
& 164 \\
1 & 513 \\
2 & 283\end{array}$ & $\begin{array}{r}893 \\
800 \\
8365 \\
9335\end{array}$ \\
\hline 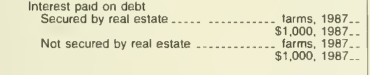 & $\begin{array}{r}1205 \\
12415 \\
500 \\
2457\end{array}$ & $\begin{array}{r}313 \\
4038 \\
146 \\
956\end{array}$ & $\begin{array}{r}97 \\
1176 \\
81 \\
337\end{array}$ & $\begin{array}{r}795 \\
7201 \\
273 \\
1163\end{array}$ \\
\hline 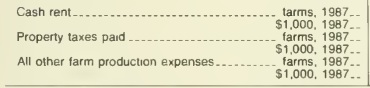 & $\begin{array}{rr}11 & 972 \\
2 & 089 \\
2 & 524 \\
2 & 591 \\
2 & 813 \\
15 & 758\end{array}$ & $\begin{array}{r}313 \\
4136 \\
741 \\
701 \\
785 \\
4383 \\
\end{array}$ & $\begin{array}{r}142 \\
2768 \\
316 \\
475 \\
361 \\
3734\end{array}$ & $\begin{array}{r}517 \\
4185 \\
1667 \\
1415 \\
1667 \\
7641\end{array}$ \\
\hline
\end{tabular}

'Data for 1987 include cost of custom applications.

2Data for 1987 exclude cost of custom applications for commercial fertilizer and agriculturai chemicals.

Jata for 1982 do not include imputation for item nonresponse 
Table 4. Net Cash Return From Agricultural Sales, Government Payments, Other FarmRelated Income, and Commodity Credit Corporation Loans: 1987 and 1982

\begin{tabular}{|c|c|c|c|c|}
\hline Item & Delaware & Kent & New Castle & Sussex \\
\hline \multicolumn{5}{|l|}{ NET CASH RETURN } \\
\hline $\begin{array}{l}\begin{array}{l}\text { Net cash return from agricultural sales for the } \\
\text { farm unit (see text) }\end{array} \\
\text { Average per farm } \\
\text { farms, } 1987 \\
\$ 1,000,1987\end{array}$ & $\begin{array}{rr}2 & 965 \\
65 & 300 \\
22 & 023\end{array}$ & $\begin{array}{rr}856 \\
10 & 076 \\
11 & 771\end{array}$ & $\begin{array}{r}380 \\
4841 \\
12739\end{array}$ & $\begin{array}{rr}1 & 729 \\
50 & 383 \\
29 & 140\end{array}$ \\
\hline 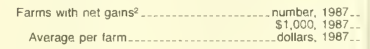 & $\begin{array}{rr}1 & 695 \\
76 & 564 \\
45 & 171\end{array}$ & $\begin{array}{rr}349 \\
14 & 378 \\
41 & 198\end{array}$ & $\begin{array}{r}158 \\
7121 \\
45072\end{array}$ & $\begin{array}{rr}1 & 188 \\
55 & 065 \\
46 & 351\end{array}$ \\
\hline $\begin{array}{c}\text { Farms with net losses.................... number, } 1987 \ldots \\
\$ 1,000,1987 \ldots \\
\text { Average per tarm....................... dollars, } 1987 \ldots\end{array}$ & $\begin{array}{rr}1 & 270 \\
11 & 265 \\
8 & 870\end{array}$ & $\begin{array}{ll}507 \\
4 & 302 \\
8 & 485\end{array}$ & $\begin{array}{r}2222 \\
10281 \\
10\end{array}$ & $\begin{array}{r}541 \\
4682 \\
8655\end{array}$ \\
\hline \multicolumn{5}{|l|}{ GOVERNMENT PAYMENTS } \\
\hline 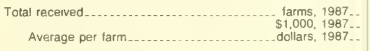 & $\begin{array}{r}549 \\
7414 \\
13505\end{array}$ & $\begin{array}{r}178 \\
2285 \\
12839\end{array}$ & $\begin{array}{rr}125 \\
2333 \\
18662\end{array}$ & $\begin{array}{r}246 \\
2796 \\
11367\end{array}$ \\
\hline $\begin{array}{r}\text { Amount recerved in cash ............... tarms, } 1987 \ldots \\
\$ 1,000,1987 \ldots\end{array}$ & $\begin{array}{r}478 \\
3513\end{array}$ & $\begin{array}{r}146 \\
1056\end{array}$ & $\begin{array}{r}116 \\
1157\end{array}$ & $\begin{array}{r}216 \\
1299\end{array}$ \\
\hline $\begin{array}{r}\text { Value of commodity certificates received..... farms, } 1987 . \\
\$ 1,000,1987 \ldots\end{array}$ & $\begin{array}{r}493 \\
3902\end{array}$ & $\begin{array}{r}167 \\
1229\end{array}$ & $\begin{array}{rr}116 \\
1175\end{array}$ & $\begin{array}{r}210 \\
1497\end{array}$ \\
\hline \multicolumn{5}{|l|}{ OTHER FARM-RELATED INCOME } \\
\hline 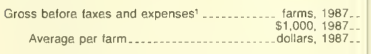 & $\begin{array}{r}757 \\
3998 \\
5282\end{array}$ & $\begin{array}{ll}1 & 260 \\
4 & 201 \\
4 & 617\end{array}$ & $\begin{array}{r}79 \\
476 \\
6024\end{array}$ & $\begin{array}{l}418 \\
2322 \\
5555\end{array}$ \\
\hline 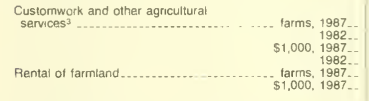 & $\begin{array}{r}272 \\
284 \\
1514 \\
1425 \\
287 \\
1151\end{array}$ & $\begin{array}{r}65 \\
80 \\
316 \\
526 \\
94 \\
355\end{array}$ & \begin{tabular}{r|r}
25 \\
39 \\
132 \\
86 \\
32 \\
160
\end{tabular} & $\begin{array}{r}182 \\
165 \\
1066 \\
814 \\
161 \\
636\end{array}$ \\
\hline 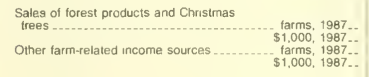 & $\begin{array}{l}113 \\
878 \\
208 \\
456\end{array}$ & $\begin{array}{r}65 \\
461 \\
83 \\
68\end{array}$ & $\begin{array}{r}16 \\
121 \\
27 \\
63\end{array}$ & $\begin{array}{r}32 \\
296 \\
98 \\
324\end{array}$ \\
\hline \multicolumn{5}{|l|}{$\begin{array}{l}\text { COMMODITY CREDIT } \\
\text { CORPORATION LOANS }\end{array}$} \\
\hline $\begin{array}{r}\text { Total ... farms. } 1987 . \\
1982 . \\
\$ 1,000.1987 \\
1982 .\end{array}$ & $\begin{array}{rr} & 143 \\
4 & 53 \\
4 & 256 \\
1 & 725\end{array}$ & $\begin{array}{r}41 \\
16 \\
1605 \\
695\end{array}$ & $\begin{array}{r}44 \\
11 \\
1205 \\
166\end{array}$ & $\begin{array}{r}58 \\
26 \\
1446 \\
864\end{array}$ \\
\hline 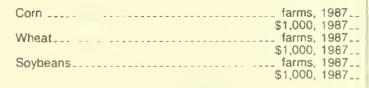 & $\begin{array}{r}133 \\
3877 \\
10 \\
20 \\
16 \\
340\end{array}$ & $\begin{array}{r}35 \\
1369 \\
3 \\
4 \\
9 \\
\text { (D) }\end{array}$ & $\begin{array}{r}44 \\
192 \\
1 \\
\text { (D) } \\
2 \\
\text { (D) }\end{array}$ & $\begin{array}{r}54 \\
1316 \\
6 \\
(D) \\
5 \\
106\end{array}$ \\
\hline 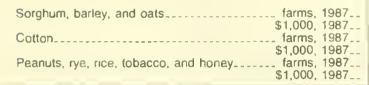 & $\begin{array}{r}6 \\
19 \\
- \\
- \\
- \\
-\end{array}$ & $\begin{array}{l}1 \\
\text { (D) } \\
- \\
- \\
-\end{array}$ & $\begin{array}{l}\bar{z} \\
\overline{-} \\
-\end{array}$ & $\begin{array}{l}5 \\
\text { (D) } \\
- \\
-\end{array}$ \\
\hline
\end{tabular}

'Data are based on a sample of tarms

2Farms with total production expenses equal to market value of agnicultural products sold are included as farms with gains

3Data for 1987 are based on a sample of farms; data for 1982 are nonsample 
Table 5. Farms, Land in Farms, and Land Use: 1987 and 1982

[Far meaning of abbreviations and symbols, see intraductory text]

\begin{tabular}{|c|c|c|c|c|}
\hline All Farms & Delaware & Kent & New Castle & Sussex \\
\hline \multicolumn{5}{|l|}{ FARMS AND LAND IN FARMS } \\
\hline 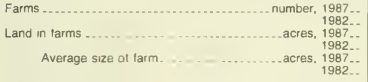 & $\begin{array}{rr}2 & 966 \\
3 & 338 \\
608 & 245 \\
655 & 465 \\
205 \\
196\end{array}$ & $\begin{array}{rr}1 & 857 \\
201 & 444 \\
210 & 109 \\
235 \\
208\end{array}$ & $\begin{array}{r}380 \\
488 \\
93 \quad 998 \\
102 \quad 023 \\
247 \\
209\end{array}$ & $\begin{array}{rr}1 & 729 \\
1 & 841 \\
312 & 803 \\
343 & 333 \\
181 \\
186\end{array}$ \\
\hline 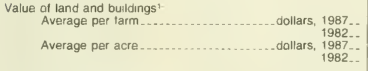 & $\begin{array}{rr}369 & 751 \\
364 & 843 \\
1 & 765 \\
1 & 829\end{array}$ & $\begin{array}{rr}402 & 674 \\
367 & 064 \\
1 & 568 \\
1 & 783\end{array}$ & $\begin{array}{rr}670 & 476 \\
528 & 150 \\
2 & 769 \\
2 & 398\end{array}$ & $\begin{array}{rr}287 & 359 \\
320 & 338 \\
1 & 607 \\
1 & 682\end{array}$ \\
\hline 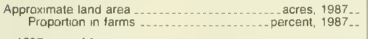 & $\begin{array}{r}1236704 \\
49.2\end{array}$ & $\begin{array}{r}380633 \\
529\end{array}$ & $\begin{array}{r}253222 \\
37.1\end{array}$ & $\begin{array}{r}602848 \\
519\end{array}$ \\
\hline $\begin{array}{c}1987 \text { size of farm } \\
1 \text { to } 9 \text { acres } \\
10 \text { to } 49 \text { acres } \\
50 \text { to } 69 \text { acres }\end{array}$ & $\begin{array}{rr} & 514 \\
2 & 018 \\
867 \\
21 & 371 \\
196 \\
\\
\\
\text { (D) }\end{array}$ & $\begin{array}{r}73 \\
347 \\
251 \\
6290 \\
61 \\
3587\end{array}$ & $\begin{array}{r}35 \\
167 \\
114 \\
2634 \\
23 \\
\text { (D) }\end{array}$ & $\begin{array}{r}406 \\
1504 \\
502 \\
12447 \\
112 \\
6451\end{array}$ \\
\hline $\begin{array}{l}70 \text { to } 99 \text { acres } \ldots 0 \text { to } 139 \text { acres } \ldots \ldots \ldots \\
140 \text { to } 179 \text { acres }\end{array}$ & $\begin{array}{rr}230 \\
18 \quad 995 \\
219 \\
25 \quad 473 \\
143 \\
22 \quad 684\end{array}$ & $\begin{array}{rr}7 & 92 \\
791 \\
71 \\
8 \quad 328 \\
44 \\
7067\end{array}$ & $\begin{array}{rr}1 & 22 \\
& 205 \\
28 \\
3 & 151 \\
& 25 \\
4 & 003\end{array}$ & $\begin{array}{r}116 \\
9499 \\
120 \\
13 \quad 994 \\
74 \\
11 \quad 614\end{array}$ \\
\hline 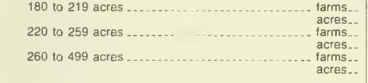 & $\begin{array}{rr}117 \\
23 & 433 \\
& 76 \\
18 & 037 \\
& 288 \\
104 & 012\end{array}$ & $\begin{array}{rr}8 & 43 \\
271 \\
29 \\
675 \\
33 \quad 035\end{array}$ & $\begin{array}{r}21 \\
4 \\
125 \\
11 \\
2 \quad 617 \\
16 \quad 369\end{array}$ & 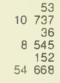 \\
\hline 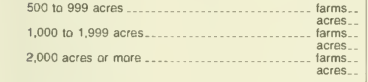 & $\begin{array}{r}188 \\
\text { (D) } \\
100 \\
\text { (D) } \\
28 \\
\text { (D) }\end{array}$ & $\begin{array}{r}57 \\
39 \\
360 \\
38 \\
50 \\
754 \\
8 \\
29 \\
539\end{array}$ & $\begin{array}{r}30 \\
\text { (D) } \\
21 \\
\text { (D) } \\
4 \\
\text { (D) }\end{array}$ & $\begin{array}{r}101 \\
70 \quad 863 \\
41 \\
54 \quad 424 \\
16 \\
58 \quad 057\end{array}$ \\
\hline 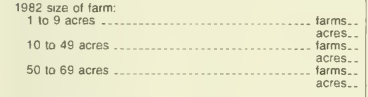 & $\begin{array}{r}503 \\
2077 \\
905 \\
22 \quad 580 \\
259 \\
14968\end{array}$ & $\begin{array}{r}87 \\
412 \\
257 \\
6586 \\
70 \\
4062\end{array}$ & $\begin{array}{r}51 \\
228 \\
132 \\
3032 \\
35 \\
2069\end{array}$ & $\begin{array}{r}365 \\
1437 \\
516 \\
12962 \\
154 \\
8837\end{array}$ \\
\hline 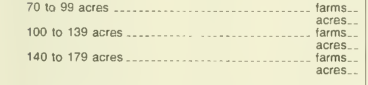 & $\begin{array}{r}313 \\
25 \quad 939 \\
264 \\
30 \quad 680 \\
176 \\
27625\end{array}$ & $\begin{array}{rr}123 \\
10 & 314 \\
103 \\
11 & 899 \\
62 \\
9851\end{array}$ & $\begin{array}{rr}38 \\
3109 \\
31 \\
3 & 594 \\
& 34 \\
5 & 250\end{array}$ & $\begin{array}{r}152 \\
12 \\
516 \\
130 \\
15 \quad 187 \\
80 \\
12 \quad 524\end{array}$ \\
\hline 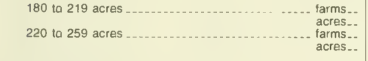 & $\begin{array}{rr} & 153 \\
30 & 159 \\
& 116 \\
27 & 734\end{array}$ & $\begin{array}{r}54 \\
10 \\
758 \\
39 \\
9331\end{array}$ & $\begin{array}{r}38 \\
7 \quad 377 \\
19 \\
4522\end{array}$ & $\begin{array}{rr}61 \\
12 & 024 \\
& 58 \\
13 & 881\end{array}$ \\
\hline 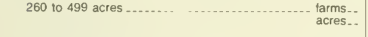 & $\begin{array}{r}322 \\
114859\end{array}$ & $\begin{array}{r}116 \\
41950\end{array}$ & $\begin{array}{r}48 \\
18 \quad 003\end{array}$ & $\begin{array}{r}158 \\
54906\end{array}$ \\
\hline 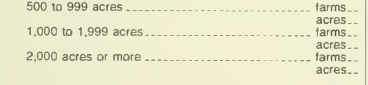 & $\begin{array}{r}211 \\
147 \quad 706 \\
90 \\
117 \quad 982 \\
26 \\
93 \quad 156\end{array}$ & $\begin{array}{r}61 \\
173 \\
31 \\
\text { (D) } \\
6 \\
\text { (D) }\end{array}$ & $\begin{array}{r}44 \\
969 \\
16 \\
\text { (D) } \\
2 \\
\text { (D) }\end{array}$ & $\begin{array}{r}106 \\
75 \quad 564 \\
43 \\
56 \quad 834 \\
18 \\
66 \quad 661\end{array}$ \\
\hline \multicolumn{5}{|l|}{$\begin{array}{l}\text { LAND IN FARMS ACCORDING TO } \\
\text { USE }\end{array}$} \\
\hline $\begin{array}{rr}\text { Total cropland............................ farms, } 1987 \ldots \\
& 1982 \ldots \\
\text { acres. } & 1987 \ldots \\
& 1982 \ldots\end{array}$ & $\begin{array}{rr}2 & 397 \\
2 & 843 \\
501 & 290 \\
521 & 104\end{array}$ & $\begin{array}{rr} & 791 \\
942 \\
170 & 377 \\
174 & 788\end{array}$ & $\begin{array}{rr}363 \\
461 \\
78 & 162 \\
84 & 120\end{array}$ & $\begin{array}{rr}1 & 243 \\
1 & 440 \\
252 & 751 \\
262 & 196\end{array}$ \\
\hline $\begin{array}{rr}\text { Harvested crapland ...................... farms, } 1987 \ldots \\
\\
1982 . . \\
\text { acres, } \begin{array}{r}1987 \ldots \\
1982 . .\end{array}\end{array}$ & $\begin{array}{rr}2 & 172 \\
2 & 700 \\
441 & 502 \\
499 & 986\end{array}$ & $\begin{array}{r}721 \\
905 \\
150674 \\
167936\end{array}$ & $\begin{array}{rr}324 \\
434 \\
64 & 358 \\
77 & 566\end{array}$ & $\begin{array}{rr}1 & 127 \\
1 & 361 \\
226 & 470 \\
254 & 484\end{array}$ \\
\hline $\begin{array}{r}\text { Cropland used only for pasture or grazing ..... farms, } 1987 . . \\
1982 . \\
\text { acres, } 1987 . . \\
1982 .\end{array}$ & $\begin{array}{r}510 \\
602 \\
10200 \\
12374\end{array}$ & $\begin{array}{r}219 \\
246 \\
3873 \\
4 \quad 312\end{array}$ & $\begin{array}{ll} & 116 \\
132 \\
2 & 643 \\
4 & 037\end{array}$ & $\begin{array}{r}175 \\
224 \\
3684 \\
4025\end{array}$ \\
\hline 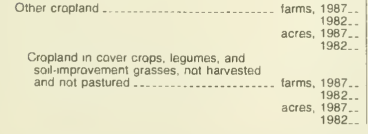 & $\begin{array}{r}874 \\
332 \\
49 \\
588 \\
8744 \\
\end{array}$ & $\begin{array}{r}272 \\
104 \\
15830 \\
2540 \\
\\
\\
32 \\
24 \\
1530 \\
479\end{array}$ & $\begin{array}{r}176 \\
104 \\
11.161 \\
2517 \\
\\
\\
\\
46 \\
\\
1115 \\
11 \\
779\end{array}$ & 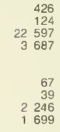 \\
\hline
\end{tabular}

See tootnotes at end of table 
Table 5. Farms, Land in Farms, and Land Use: 1987 and 1982-Con.

[For meanung of abbreviations and symbols, see introductory text]

\begin{tabular}{|c|c|c|c|c|c|}
\hline \multicolumn{2}{|l|}{ All Farms } & \multirow[t]{2}{*}{ Delaware } & \multirow[t]{2}{*}{ Kent } & \multirow[t]{2}{*}{ New Castie } & \multirow[t]{2}{*}{ Sussex } \\
\hline $\begin{array}{l}\text { LAND IN FARMS ACCORDING TO } \\
\text { USE - Con. }\end{array}$ & & & & & \\
\hline $\begin{array}{l}\text { Total cropland-Con } \\
\text { Other cropland-Con } \\
\text { Cropland on which all crops falled..... }\end{array}$ & $\begin{array}{l}\text { tarms. } 1987 .- \\
1982 . \\
\text { acres. } 1987- \\
1982 .\end{array}$ & $\begin{array}{r}188 \\
55 \\
6696 \\
1326\end{array}$ & $\begin{array}{r}76 \\
21 \\
1895 \\
290\end{array}$ & $\begin{array}{r}20 \\
15 \\
372 \\
(D)\end{array}$ & $\begin{array}{r}92 \\
19 \\
4429 \\
\text { (D) }\end{array}$ \\
\hline Cropland in cuittvated summer fallow ... & $\begin{array}{l}\text { farms. } 1987 \ldots \\
1982 . . \\
\text { acres. } 1987 . . \\
1982 .\end{array}$ & $\begin{array}{r}36 \\
18 \\
902 \\
279\end{array}$ & $\begin{array}{r}16 \\
7 \\
384 \\
176\end{array}$ & $\begin{array}{r}8 \\
6 \\
262 \\
(D)\end{array}$ & $\begin{array}{r}12 \\
5 \\
256 \\
\text { (D) }\end{array}$ \\
\hline Cropland rde........ & $\begin{array}{l}\text { farms. } 1987 . \\
1982 . . \\
\text { acres. } 1987 . . \\
1982 .\end{array}$ & $\begin{array}{r}612 \\
209 \\
37099 \\
4182\end{array}$ & $\begin{array}{r}183 \\
64 \\
12021 \\
1595\end{array}$ & $\begin{array}{r}130 \\
72 \\
9412 \\
1551\end{array}$ & $\begin{array}{r}299 \\
73 \\
15 \quad 666 \\
1036\end{array}$ \\
\hline Total woodland & $\begin{array}{l}\text { farms, } 1987 . . \\
1982 . \\
\text { acres, } 1987 . . \\
1982 .\end{array}$ & $\begin{array}{rl}1 & 239 \\
1 & 451 \\
75 & 156 \\
91 & 736\end{array}$ & $\begin{array}{r}417 \\
500 \\
20886 \\
24321\end{array}$ & $\begin{array}{r}155 \\
705 \\
7839 \\
8619\end{array}$ & $\begin{array}{r}667 \\
746 \\
46431 \\
58796\end{array}$ \\
\hline Woodland pastured ............................ & $\begin{aligned} & \text { farms, } 1987 \ldots \\
& 1982 . \\
& \text { acres. } 1987 . . \\
& 1982 . .\end{aligned}$ & $\begin{array}{rl} & 141 \\
& 197 \\
3 & 714 \\
6 & 928\end{array}$ & $\begin{array}{r}58 \\
82 \\
1910 \\
1610\end{array}$ & $\begin{array}{r}25 \\
31 \\
319 \\
875\end{array}$ & $\begin{array}{r}58 \\
84 \\
1485 \\
4443\end{array}$ \\
\hline Woodland not pastured. & $\begin{array}{l}\text { farms. } 1987 . . \\
1982 . \\
\text { acres. } 1987 \ldots \\
1982 . .\end{array}$ & $\begin{array}{rl}1 & 158 \\
1 & 338 \\
71442 \\
84808\end{array}$ & $\begin{array}{r}385 \\
463 \\
18976 \\
22711\end{array}$ & $\begin{array}{r}141 \\
189 \\
7520 \\
7744\end{array}$ & $\begin{array}{r}632 \\
686 \\
44946 \\
54353\end{array}$ \\
\hline Other land ............ & $\begin{array}{l}\text { farms, } 1987-- \\
1982- \\
\text { acres, } 1987- \\
1982-\end{array}$ & $\begin{array}{rl}2 & 042 \\
2 & 198 \\
31 & 799 \\
42 & 625\end{array}$ & $\begin{array}{r}570 \\
664 \\
10181 \\
11000\end{array}$ & $\begin{array}{r}275 \\
342 \\
7997 \\
9284\end{array}$ & $\begin{array}{rl}1 & 197 \\
1 & 192 \\
13 & 621 \\
22 & 341\end{array}$ \\
\hline $\begin{array}{l}\text { Pastureland and rangeland other than } \\
\text { cropland and woodland pastured..... }\end{array}$ & $\begin{array}{r}\text { farms. } 1987 . \\
1982 . \\
\text { acres. } 1987 \\
1982 .\end{array}$ & $\begin{array}{r}212 \\
209 \\
4824 \\
12490\end{array}$ & $\begin{array}{r} \\
88 \\
66 \\
2334 \\
2 \quad 054\end{array}$ & $\begin{array}{r}42 \\
54 \\
1072 \\
1790\end{array}$ & $\begin{array}{r} \\
82 \\
89 \\
1418 \\
8646\end{array}$ \\
\hline $\begin{array}{l}\text { Land in house lots, ponds, roads, } \\
\text { wasteland, etc .................. }\end{array}$ & $\begin{aligned} & \text { farms, } 1987 .- \\
& 1982 . \\
& \text { acres. } \\
& 1987 . \\
& 1982 .\end{aligned}$ & $\begin{array}{rr}1 & 994 \\
2 & 148 \\
26 & 975 \\
30 & 135\end{array}$ & $\begin{array}{r}553 \\
651 \\
7847 \\
8946\end{array}$ & $\begin{array}{r}267 \\
324 \\
6925 \\
7494\end{array}$ & $\begin{array}{rl}1 & 174 \\
1 & 173 \\
12 & 203 \\
13 & 695\end{array}$ \\
\hline Pastureland, all types ............................. & $\begin{array}{r}\text { larms, } 1987 . . \\
1982 . \\
\text { acres, } 1987 . . \\
1982 . .\end{array}$ & $\begin{array}{r}736 \\
858 \\
18738 \\
31792\end{array}$ & $\begin{array}{r}301 \\
333 \\
8117 \\
7976\end{array}$ & $\begin{array}{r}147 \\
171 \\
4034 \\
6702\end{array}$ & $\begin{array}{r}288 \\
354 \\
6587 \\
17 \quad 114\end{array}$ \\
\hline $\begin{array}{l}\text { Cropland diverted under annual commodity } \\
\text { acreage adjustment programs................ }\end{array}$ & $\begin{array}{l}\text { farms. } 1987 . . \\
1982 . \\
\text { acres, } 1987 . \\
1982 . .\end{array}$ & $\begin{array}{r}450 \\
39 \\
29683 \\
663\end{array}$ & $\begin{array}{r}140 \\
8 \\
8 \quad 362 \\
289\end{array}$ & $\begin{array}{r}117 \\
22 \\
(D) \\
255\end{array}$ & $\begin{array}{r}193 \\
9 \\
(\mathrm{D}) \\
119\end{array}$ \\
\hline $\begin{array}{l}\text { Cropland placed under the conservation } \\
\text { reserve program }\end{array}$ & $\begin{array}{l}\text { farms, } 1987 . \\
\text { acres, } 1987 . .\end{array}$ & $\begin{array}{r}18 \\
1141\end{array}$ & 612 & (D) $^{2}$ & (D) \\
\hline
\end{tabular}

'Data are based on a sample of farms, see text 
Table 6. Harvested Cropland by Size of Farm and Acres Harvested: 1987 and 1982

[For meaning of abbreviations and symbols, see introductory text]

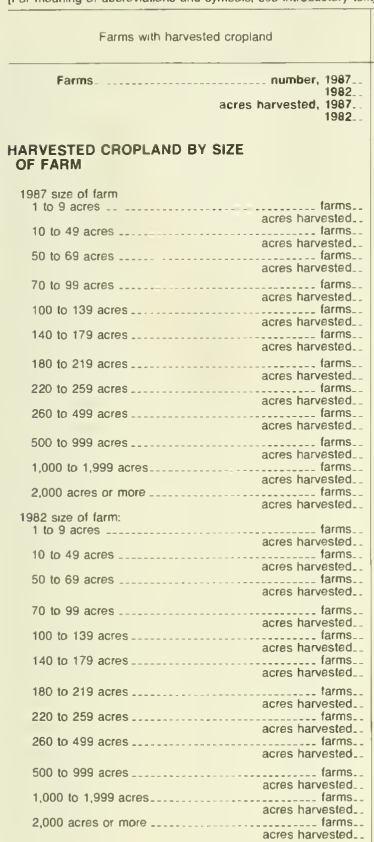

\section{HARVESTED CROPLAND BY ACRES HARVESTED}

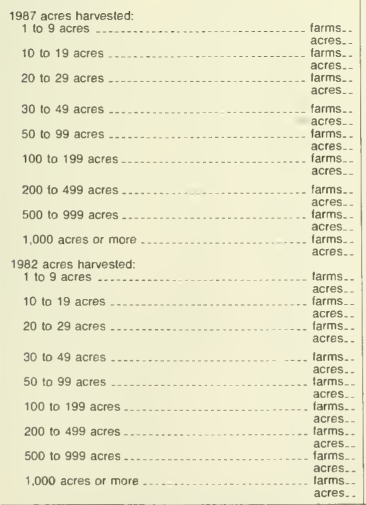

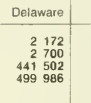

\begin{tabular}{r|r|r} 
& & \\
Kent & New Castle & Sussex \\
\hline 721 & 324 & 127 \\
905 & 634 & 1361 \\
150674 & 64558 & 226470 \\
167936 & 77566 & 254484
\end{tabular}


Table 7. Irrigation: 1987 and 1982

[For meaning of abbreviations and symbols, see introductory text]

\begin{tabular}{|c|c|c|c|c|}
\hline Farms with irrigation & Delaware & Kent & New Castle & Sussex \\
\hline $\begin{array}{ll}\text { Farms . } & \\
\text { Land in irrigated tarms ........................ acres, } & 1987 \ldots \\
& 1982 . . \\
& 1982 . .\end{array}$ & $\begin{array}{rr}384 \\
323 \\
210 & 022 \\
175 & 518\end{array}$ & 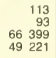 & $\begin{array}{rr} & 44 \\
11 & 47 \\
11 & 196 \\
10 & 157\end{array}$ & $\begin{array}{rr} & 227 \\
183 \\
132 & 427 \\
116 & 140\end{array}$ \\
\hline $\begin{array}{r}\text { Harvested cropland ........ farms, } 1987 . . \\
1982 . \\
\text { acres, } 1987 . \\
1982 .\end{array}$ & $\begin{array}{r}377 \\
320 \\
166 \quad 408 \\
140 \quad 247\end{array}$ & $\begin{array}{rr}111 \\
91 \\
56 & 015 \\
43 & 102\end{array}$ & $\begin{array}{rr} & 44 \\
9 & 47 \\
9 & 595 \\
9 & 176\end{array}$ & $\begin{array}{r}222 \\
182 \\
100798 \\
87969\end{array}$ \\
\hline $\begin{array}{rr}\text { Other cropland, excluding cropland } & \\
\text { pastured......... farms, } & 1987 \ldots \\
& 1982 . \\
\text { acres, } & 1987 . \\
& 1982 . .\end{array}$ & $\begin{array}{r}173 \\
41 \\
14 \\
774 \\
2123\end{array}$ & $\begin{array}{r}45 \\
8 \\
3 \quad 193 \\
206\end{array}$ & $\begin{array}{r}17 \\
9 \\
852 \\
59\end{array}$ & $\begin{array}{r}111 \\
24 \\
10729 \\
1858\end{array}$ \\
\hline $\begin{array}{r}\text { Pastureland, excluding woodland pastured .... farms, } 1987 . . \\
1982 . \\
\text { acres, } 1987 . \\
1982 . .\end{array}$ & $\begin{array}{rr}64 \\
51 \\
2 & 396 \\
3 & 074\end{array}$ & $\begin{array}{r}23 \\
17 \\
1010 \\
415\end{array}$ & $\begin{array}{r}7 \\
7 \\
28 \\
290\end{array}$ & $\begin{array}{rr} & 34 \\
& 27 \\
1 & 358 \\
2 & 369\end{array}$ \\
\hline 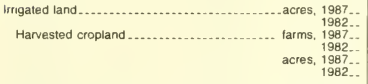 & $\begin{array}{rr}60 & 812 \\
44 & 168 \\
375 \\
318 \\
60744 \\
44014\end{array}$ & $\begin{array}{r}18521 \\
15549 \\
111 \\
91 \\
\text { (D) } \\
\text { (D) }\end{array}$ & $\begin{array}{rr}2 & 063 \\
2 & 323 \\
44 \\
\\
47 \\
\\
2 & \text { (D) } \\
2 & 323\end{array}$ & 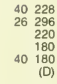 \\
\hline $\begin{array}{rr}\text { Pastureland and other land .................. tarms, } 1987 . . \\
1982 . . \\
\text { acres, } 1987 . . \\
1982 . . \\
1987 \text { irrigated acres by sıze of farm. }\end{array}$ & $\begin{array}{r}14 \\
6 \\
68 \\
154\end{array}$ & (D) & (D) & $\begin{array}{r}9 \\
4 \\
48 \\
\text { (D) }\end{array}$ \\
\hline 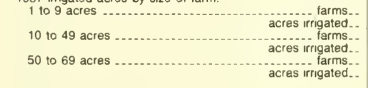 & $\begin{array}{r}32 \\
78 \\
78 \\
501 \\
7 \\
165\end{array}$ & $\begin{array}{r}11 \\
27 \\
23 \\
153 \\
2 \\
\text { (D) }\end{array}$ & $\begin{array}{r}8 \\
16 \\
15 \\
95 \\
2 \\
\text { (D) }\end{array}$ & $\begin{array}{r}13 \\
35 \\
40 \\
253 \\
3 \\
82\end{array}$ \\
\hline $\begin{array}{l}70 \text { to } 99 \text { acres } \\
100 \text { to } 139 \text { acres ... farms. } \\
140 \text { to } 179 \text { acres .... } \\
\text { acres ingated.- } \\
\text { acres irngated.. } \\
\text { acres irngated.- }\end{array}$ & $\begin{array}{r}14 \\
274 \\
24 \\
1055 \\
11 \\
853\end{array}$ & $\begin{array}{r}5 \\
(D) \\
6 \\
252 \\
3 \\
\text { (D) }\end{array}$ & \begin{tabular}{r|r}
1 \\
3 \\
3 \\
134 \\
2 \\
(D)
\end{tabular} & $\begin{array}{r}8 \\
206 \\
15 \\
669 \\
6 \\
367\end{array}$ \\
\hline 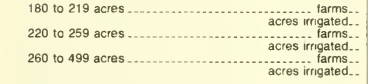 & $\begin{array}{r}19 \\
1558 \\
7 \\
196 \\
73 \\
7937\end{array}$ & $\begin{array}{r}2 \\
(\mathrm{D}) \\
3 \\
3 \\
22 \\
1857\end{array}$ & \begin{tabular}{r|}
1 \\
$(\mathrm{D})$ \\
\\
5 \\
5
\end{tabular} & $\begin{array}{r}16 \\
1289 \\
4 \\
193 \\
46 \\
5 \quad 456\end{array}$ \\
\hline $\begin{array}{l}500 \text { to } 999 \text { acres ............ } \\
1,000 \text { to } 1,999 \text { acres.......... } \\
2,000 \text { acres or more }\end{array}$ & $\begin{array}{rr}62 \\
17 & 116 \\
& 39 \\
14 & 184 \\
& 18 \\
16 & 895\end{array}$ & $\begin{array}{r}16 \\
3393 \\
14 \\
5918 \\
6 \\
\text { (D) }\end{array}$ & $\begin{array}{r}3 \\
374 \\
3 \\
264 \\
1 \\
\text { (D) }\end{array}$ & $\begin{array}{r}43 \\
13 \quad 349 \\
22 \\
8002 \\
11 \\
10 \\
1027\end{array}$ \\
\hline $\begin{array}{l}1982 \text { irrigated acres by size of farm: } \\
1 \text { to } 9 \text { acres } \\
10 \text { to } 49 \text { acres } \\
50 \text { to } 69 \text { acres }\end{array}$ & $\begin{array}{r}29 \\
57 \\
47 \\
296 \\
13 \\
321\end{array}$ & $\begin{array}{r}7 \\
13 \\
13 \\
\text { (D) } \\
1 \\
\text { (D) }\end{array}$ & $\begin{array}{r}17 \\
31 \\
11 \\
58 \\
- \\
-\end{array}$ & $\begin{array}{r}5 \\
13 \\
23 \\
\text { (D) } \\
12 \\
\text { (D) }\end{array}$ \\
\hline $\begin{array}{l}70 \text { to } 99 \text { acres } \\
100 \text { to } 139 \text { acres } \ldots \text { acres ifrigated.. } \\
140 \text { to } 179 \text { acres }\end{array}$ & $\begin{array}{r}12 \\
382 \\
21 \\
1121 \\
14 \\
742\end{array}$ & $\begin{array}{r}5 \\
(D) \\
11 \\
603 \\
3 \\
\text { (D) }\end{array}$ & $\begin{array}{r}1 \\
(D) \\
\overline{1} \\
\text { (D) }\end{array}$ & $\begin{array}{r}6 \\
260 \\
10 \\
518 \\
10 \\
488\end{array}$ \\
\hline 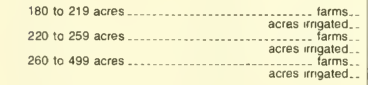 & $\begin{array}{rr}17 \\
1 & 121 \\
13 \\
1 & 029 \\
52 \\
5 \quad 506\end{array}$ & $\begin{array}{r}3 \\
136 \\
5 \\
(D) \\
16 \\
1809\end{array}$ & $\begin{array}{r}180 \\
1 \\
(D) \\
5 \\
375\end{array}$ & $\begin{array}{r}11 \\
805 \\
7 \\
507 \\
31 \\
322\end{array}$ \\
\hline 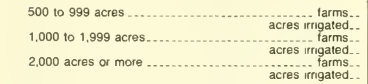 & $\begin{array}{r}60 \\
14 \quad 132 \\
31 \\
9013 \\
014 \\
10 \quad 448\end{array}$ & $\begin{array}{r}17 \\
475 \\
9 \\
\\
\text { (D) } \\
3 \\
4 \\
4 \\
046\end{array}$ & $\begin{array}{r}6 \\
887 \\
2 \\
(0) \\
-\end{array}$ & $\begin{array}{rr}8 & 37 \\
470 \\
20 \\
4 & 640 \\
11 \\
6 & 402\end{array}$ \\
\hline
\end{tabular}


Table 8. Machinery and Equipment on Place: 1987 and 1982

[Data are based on a sample of farms. For meaning of abbreviations and symbols, see introductory text]

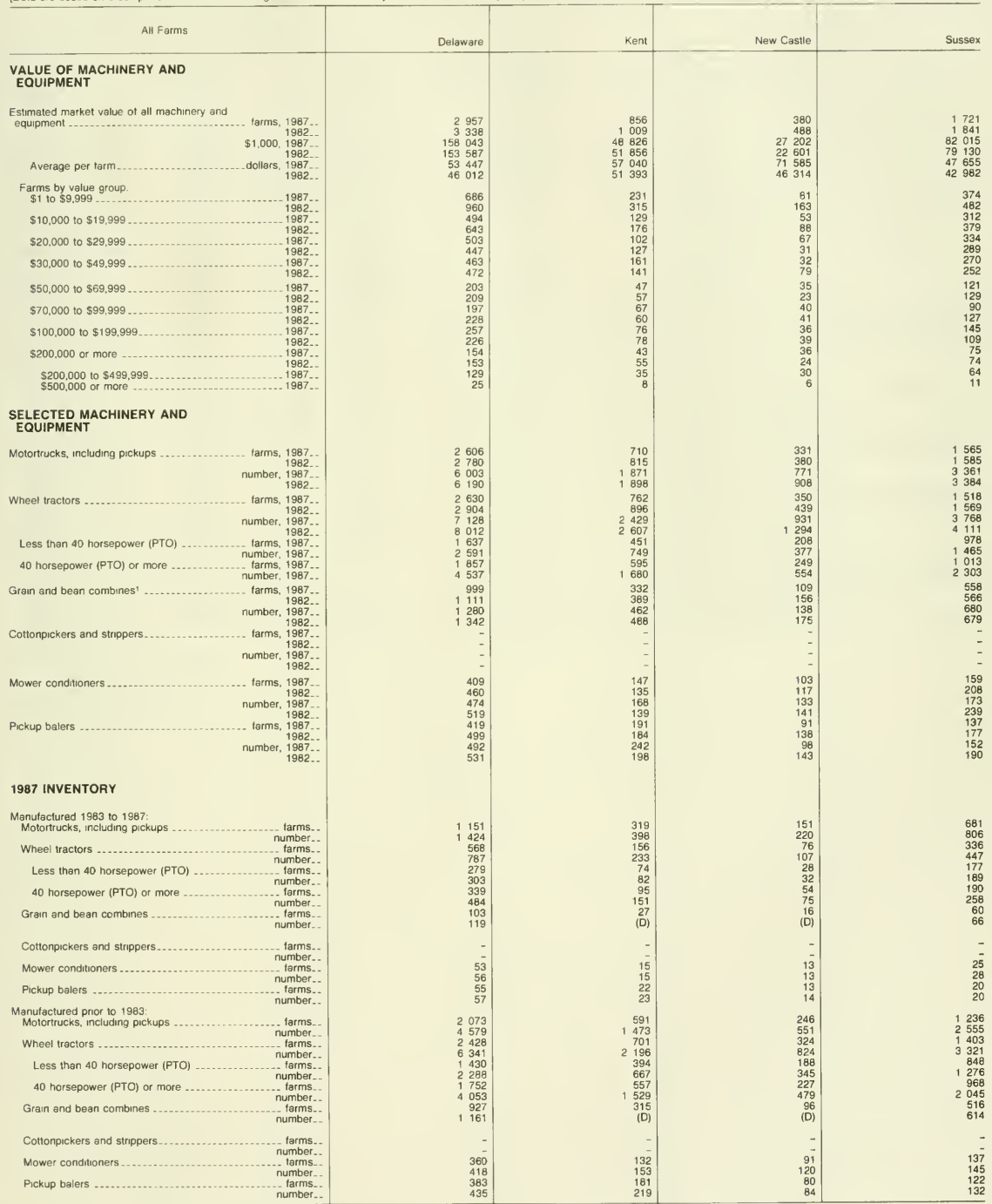

'Data for 1982 include self-propelied only. 
Table 9. Agricultural Chemicals Used, Including Fertilizer and Lime: 1987 and 1982

[Data are based on a sample of farms. For meaning of abbreviations and symbols, see introductory text]

\begin{tabular}{|c|c|c|c|c|}
\hline Chemicals used & Detaware & Kent & New Castie & Sussex \\
\hline $\begin{array}{rr}\text { Commercial fertuizer } & \\
\text { acres on which used, } & 1982 . \\
& 1982 . .\end{array}$ & $\begin{array}{rr}1 & 807 \\
2 & 191 \\
368 & 068 \\
408 & 758\end{array}$ & $\begin{array}{r}658 \\
797 \\
146 \quad 320 \\
143 \quad 521\end{array}$ & $\begin{array}{rr} & 295 \\
358 \\
59 & 743 \\
69 & 555\end{array}$ & $\begin{array}{rr}1 & 854 \\
1 & 036 \\
162 & 005 \\
195 & 682\end{array}$ \\
\hline $\begin{array}{r}\text { Cropland fertilized, except pastureland ........ farms, } 1987 . . \\
1982 . \\
\text { acres on which used, } 1987 \\
1982 .\end{array}$ & $\begin{array}{rr}1 & 725 \\
2 & 107 \\
363 & 365 \\
400 & 710\end{array}$ & $\begin{array}{r}644 \\
775 \\
144 \\
453 \\
141 \quad 861\end{array}$ & $\begin{array}{ll} & 266 \\
& 330 \\
58 & 750 \\
68 & 315\end{array}$ & $\begin{array}{rr}1 & 815 \\
1 & 002 \\
160 & 162 \\
190 & 534\end{array}$ \\
\hline $\begin{array}{r}\text { Pastureland and rangeland fertilized .......... farms, } 1987 \ldots \\
1982 \\
\qquad \text { acres on which used, } 1987 . \\
1982 .\end{array}$ & $\begin{array}{r}267 \\
212 \\
4703 \\
8048\end{array}$ & $\begin{array}{r}116 \\
91 \\
1867 \\
1660\end{array}$ & $\begin{array}{r}61 \\
43 \\
993 \\
1240\end{array}$ & $\begin{array}{rr} & 90 \\
78 \\
1 & 843 \\
5 & 148\end{array}$ \\
\hline $\begin{array}{r}\text { Lime . farms, } 1987 . . \\
1982 . \\
\qquad \begin{array}{r}\text { acres on which used. } 1987 . \\
1982 . \\
\text { tons, } 1987 . \\
1982 .\end{array}\end{array}$ & \begin{tabular}{lr|} 
& 643 \\
& 766 \\
66 & 386 \\
58 & 639 \\
67 & 144 \\
58 & 379
\end{tabular} & $\begin{array}{ll} & 267 \\
222 \\
27 & 822 \\
21 & 965 \\
29 & 046 \\
22 & 204\end{array}$ & $\begin{array}{rr} & 123 \\
11 & 189 \\
11 & 390 \\
12 & 697 \\
12 & 534 \\
12 & 189\end{array}$ & $\begin{array}{ll} & 253 \\
& 355 \\
27 & 174 \\
23 & 977 \\
25 & 564 \\
23 & 986\end{array}$ \\
\hline $\begin{array}{l}\text { Sprays, dusts, granules, fumigants, etc. to } \\
\text { control- } \\
\text { Insects on hay and other crops ........... farms, } 1987 \ldots \\
1982 . \\
\text { acres on which used, } 1987 . \\
1982 .\end{array}$ & $\begin{array}{r}837 \\
896 \\
150 \quad 686 \\
170 \quad 101\end{array}$ & $\begin{array}{r}235 \\
238 \\
46 \quad 129 \\
49303\end{array}$ & $\begin{array}{ll} & 164 \\
& 165 \\
31 & 951 \\
34 & 509\end{array}$ & $\begin{array}{rr}438 \\
793 \\
72606 \\
86 & 289\end{array}$ \\
\hline 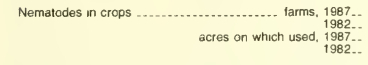 & $\begin{array}{rr}79 \\
188 \\
12 & 225 \\
23 & 010\end{array}$ & $\begin{array}{rr}6 \\
1068 \\
29 \\
2923\end{array}$ & $\begin{array}{rr} & 18 \\
71 \\
3 & 060 \\
10 & 153\end{array}$ & $\begin{array}{rr} & 55 \\
97 \\
8097 \\
9 & 934\end{array}$ \\
\hline $\begin{array}{rr}\text { Diseases in crops and orchards ............ farms, } 1987 \ldots & 1982 \\
\text { acres on which used, } & 1987 \ldots \\
& 1982 \ldots\end{array}$ & $\begin{array}{r}214 \\
214 \\
23904 \\
16975\end{array}$ & $\begin{array}{rr} & 75 \\
67 \\
9 & 990 \\
6 & 279\end{array}$ & $\begin{array}{rr}36 \\
69 \\
3 \quad 843 \\
5301\end{array}$ & $\begin{array}{r}103 \\
78 \\
10071 \\
5 \quad 395\end{array}$ \\
\hline $\begin{aligned} & \text { Weeds, grass, or brush in crops and } \text { farms, } 1987 \\
& \text { pasture } 1982 . \\
& \text { acres on which used, } 1987 \\
& 1982 .\end{aligned}$ & \begin{tabular}{rr|}
1 & 522 \\
1 & 768 \\
331 & 691 \\
381 & 813
\end{tabular} & $\begin{array}{rr} & 485 \\
& 576 \\
108 & 566 \\
128 & 819\end{array}$ & $\begin{array}{rr}214 \\
230 \\
44806 \\
52311\end{array}$ & $\begin{array}{rr} & 823 \\
962 \\
178 & 319 \\
200 & 683\end{array}$ \\
\hline $\begin{aligned} & \text { Chemicats used for defoliation or for growth } \\
& \text { control of crops or thinning of frutt ........... farms, } 1987 .- \\
& 1982 . \\
& \text { acres on which used, } 1987 \\
& 1982 .\end{aligned}$ & $\begin{array}{rr}105 \\
95 \\
11 & 351 \\
8 & 664\end{array}$ & $\begin{array}{rr} & 64 \\
7 & 26 \\
7 & 590 \\
2 & 099\end{array}$ & $\begin{array}{r}10 \\
18 \\
1005 \\
1993\end{array}$ & $\begin{array}{rr} & 31 \\
51 \\
2756 \\
4 & 572 \\
\end{array}$ \\
\hline
\end{tabular}




\section{Table 10. Tenure and Characteristics of Operator and Type of Organization: 1987 and 1982}

[For meaning of abbreviations and symbols, see introductory text]

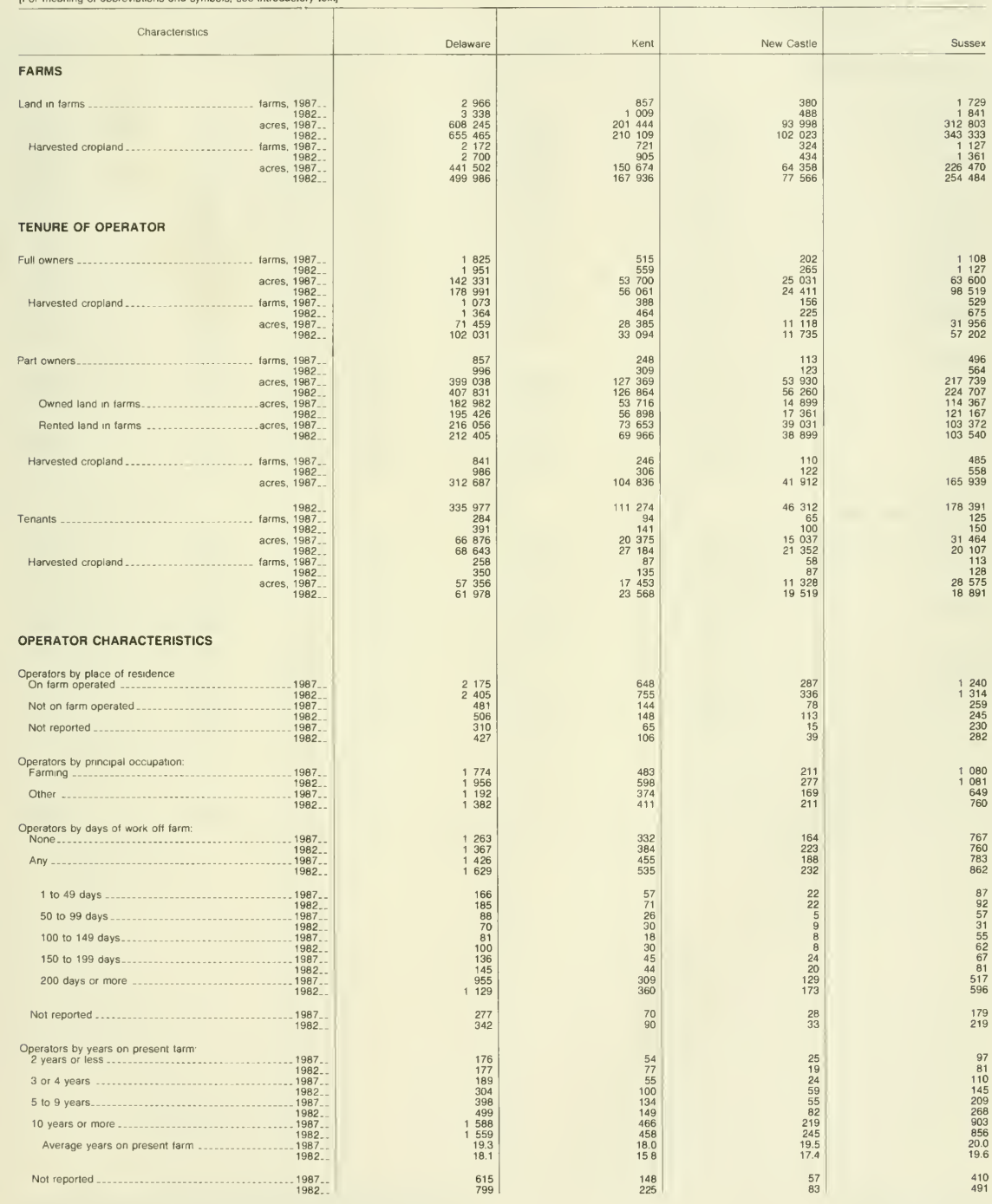


Table 10. Tenure and Characteristics of Operator and Type of Organization: 1987 and $1982-$ Con.

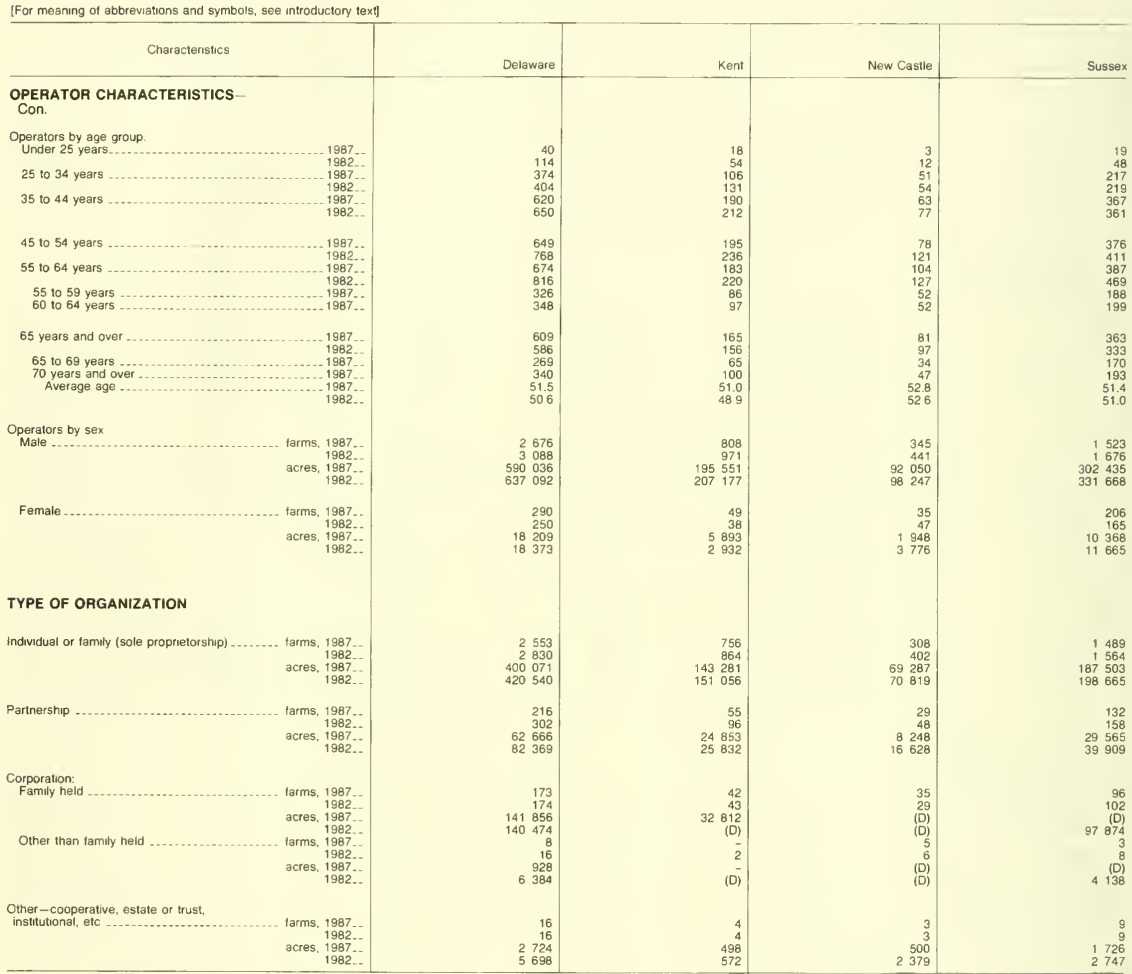


Table 11. Cattle and Calves-Inventory and Sales: 1987 and 1982

[For meaning of abbreviations and symbols, see introductory text]

\begin{tabular}{|c|c|c|c|c|}
\hline Item & Delaware & Kent & New Castie & Sussex \\
\hline \multicolumn{5}{|l|}{ INVENTORY } \\
\hline $\begin{array}{rr}\text { Cattle and calves ......................... farms, } 1987 . . \\
\\
1982 . \\
\text { number, } 1987 \ldots \\
1982 . .\end{array}$ & $\begin{array}{rr} & 461 \\
& 660 \\
31 & 191 \\
33 & 360\end{array}$ & $\begin{array}{rr}226 \\
319 \\
16 & 179 \\
15 & 398\end{array}$ & $\begin{array}{r}83 \\
113 \\
4619 \\
5471\end{array}$ & $\begin{array}{rr}152 \\
228 \\
10393 \\
12491\end{array}$ \\
\hline \multicolumn{5}{|l|}{ Farms by inventory } \\
\hline 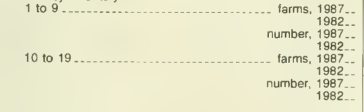 & $\begin{array}{r}188 \\
265 \\
810 \\
1016 \\
74 \\
121 \\
1007 \\
1662\end{array}$ & $\begin{array}{r}85 \\
114 \\
358 \\
464 \\
36 \\
56 \\
503 \\
778\end{array}$ & $\begin{array}{r}33 \\
42 \\
150 \\
157 \\
11 \\
16 \\
138 \\
234\end{array}$ & $\begin{array}{r}70 \\
109 \\
302 \\
395 \\
27 \\
49 \\
366 \\
650\end{array}$ \\
\hline 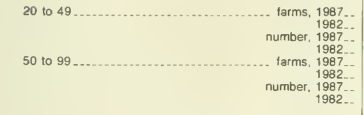 & $\begin{array}{rr}85 \\
124 \\
2616 \\
3 & 737 \\
& 46 \\
& 70 \\
3 & 246 \\
4 & 833\end{array}$ & $\begin{array}{rr} & 45 \\
77 \\
1 & 457 \\
2 & 326 \\
& 21 \\
& 29 \\
1 & 469 \\
1 & 984\end{array}$ & $\begin{array}{r}16 \\
14 \\
483 \\
409 \\
10 \\
26 \\
650 \\
1763\end{array}$ & $\begin{array}{r}24 \\
33 \\
676 \\
1002 \\
15 \\
15 \\
1127 \\
1086\end{array}$ \\
\hline 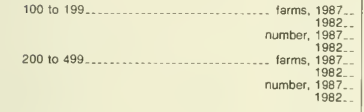 & $\begin{array}{rr} & 32 \\
4 & 48 \\
6 & 550 \\
& 29 \\
& 26 \\
7 & 780 \\
6 & 760\end{array}$ & $\begin{array}{rr} & 19 \\
25 \\
2507 \\
(D) \\
16 \\
14 \\
14 \\
4345 \\
3504\end{array}$ & $\begin{array}{r}5 \\
9 \\
755 \\
1293 \\
7 \\
6 \\
1 D \\
1615\end{array}$ & $\begin{array}{r}8 \\
14 \\
1135 \\
(D) \\
6 \\
6 \\
1041 \\
104 \\
164\end{array}$ \\
\hline 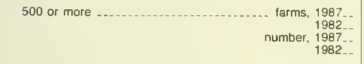 & $\begin{array}{rr}11 & 7 \\
835 \\
8 & 802\end{array}$ & $\begin{array}{r}4 \\
4 \\
540 \\
\text { (D) }\end{array}$ & (D) & $\begin{array}{r}2 \\
2 \\
\text { (D) } \\
\text { (D) }\end{array}$ \\
\hline $\begin{array}{r}\text { Cows and heifers that had calved ........... farms, } 1987 \ldots \\
1982 . \\
\text { number, } 1987 \ldots \\
1982 \ldots\end{array}$ & $\begin{array}{r}358 \\
517 \\
11541 \\
15085\end{array}$ & $\begin{array}{ll} & 183 \\
6 & 261 \\
6 & 220 \\
6 & 976\end{array}$ & $\begin{array}{rr} & 54 \\
86 \\
2 & 124 \\
3 & 066\end{array}$ & $\begin{array}{r}121 \\
170 \\
3 \\
5047\end{array}$ \\
\hline $\begin{array}{lr}\text { Beet cows ...... farms, } 1987 \ldots \\
& 1982 \ldots \\
& \text { number, } 1987 \ldots \\
1987 \text { iarms by inventory. } & 1982 \ldots \\
& \end{array}$ & $\begin{array}{rr}216 \\
311 \\
2 & 187 \\
5 & 129\end{array}$ & $\begin{array}{r}82 \\
121 \\
788 \\
1283\end{array}$ & $\begin{array}{r}37 \\
52 \\
374 \\
889\end{array}$ & $\begin{array}{rr} & 97 \\
138 \\
1 & 025 \\
2 & 957\end{array}$ \\
\hline 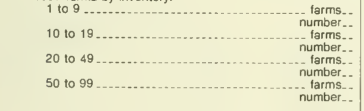 & $\begin{array}{r}155 \\
587 \\
19 \\
(D) \\
36 \\
\text { (D) } \\
6 \\
\text { (D) }\end{array}$ & $\begin{array}{r}64 \\
246 \\
4 \\
10) \\
13 \\
389 \\
1 \\
\text { (D) }\end{array}$ & $\begin{array}{r}25 \\
\text { (D) } \\
2 \\
(\mathrm{D}) \\
10 \\
\text { (D) } \\
- \\
-\end{array}$ & $\begin{array}{l}66 \\
\text { (D) } \\
13 \\
(D) \\
13 \\
\text { (D) } \\
5 \\
\text { (D) }\end{array}$ \\
\hline 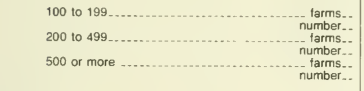 & $\begin{array}{l}- \\
- \\
- \\
- \\
- \\
-\end{array}$ & $\begin{array}{l}- \\
\overline{-} \\
\overline{-} \\
-\end{array}$ & $\begin{array}{l}- \\
\overline{-} \\
- \\
= \\
-\end{array}$ & $\begin{array}{l}\overline{-} \\
\overline{-} \\
\overline{-}\end{array}$ \\
\hline 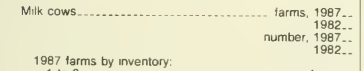 & $\begin{array}{rr}169 \\
948 \\
9354 \\
9356\end{array}$ & $\begin{array}{rr} & 113 \\
& 163 \\
5 & 432 \\
5 & 693\end{array}$ & $\begin{array}{rr} & 20 \\
1 & 39 \\
1 & 750 \\
2 & 177\end{array}$ & $\begin{array}{rr} & 36 \\
2 & 46 \\
2 & 172 \\
2 & 086\end{array}$ \\
\hline $\begin{array}{c}10 \text { to } 19 \\
20 \text { to } 49\end{array} 50$ to $99 \ldots \ldots \begin{array}{c}\text { farms } \\
\text { number }\end{array}$ & \begin{tabular}{rr|}
49 \\
99 \\
27 \\
$(D)$ \\
25 \\
\\
$(D)$ \\
31 \\
2133
\end{tabular} & $\begin{array}{r}35 \\
72 \\
26 \\
\text { (D) } \\
14 \\
412 \\
19 \\
\text { (D) }\end{array}$ & $\begin{array}{r}2 \\
\text { (D) } \\
- \\
5 \\
\text { (D) } \\
6 \\
\text { (D) }\end{array}$ & $\begin{array}{r}12 \\
\text { (D) } \\
1 \\
\text { (D) } \\
6 \\
\text { (D) } \\
6 \\
\text { (D) }\end{array}$ \\
\hline $\begin{array}{r}100 \text { to } 199 \\
200 \text { to } 499 \\
500 \text { or more }\end{array}$ & $\begin{array}{r}29 \\
720 \\
8 \\
238 \\
- \\
-\end{array}$ & $\begin{array}{r}13 \\
\text { (D) } \\
6 \\
\text { (D) } \\
- \\
-\end{array}$ & $\begin{array}{r}6 \\
\text { (D) } \\
1 \\
\text { (D) } \\
- \\
-\end{array}$ & $\begin{array}{r}10 \\
\text { (D) } \\
1 \\
\text { (D) } \\
- \\
-\end{array}$ \\
\hline $\begin{array}{rr}\text { Heifers and heifer calves ................... farms, } 1987 \ldots \\
1982 \ldots \\
\text { number, } 1987 \ldots \\
1982 \ldots \\
\end{array}$ & $\begin{array}{r}316 \\
418 \\
8130 \\
8970\end{array}$ & $\begin{array}{rr}170 \\
227 \\
4320 \\
5129\end{array}$ & $\begin{array}{r}50 \\
1763 \\
17674\end{array}$ & $\begin{array}{rr} & 96 \\
& 128 \\
2 & 047 \\
2 & 167\end{array}$ \\
\hline $\begin{array}{r}\text { Steers, steer calves, bulls, and bull calves ... farms, } 1987 . . \\
1982 . \\
\text { number, } 1987 . . \\
1982 \ldots\end{array}$ & $\begin{array}{rr}306 \\
11 & 467 \\
9 & 305\end{array}$ & $\begin{array}{ll} & 158 \\
& 227 \\
5 & 639 \\
3 & 293\end{array}$ & $\begin{array}{r}54 \\
81 \\
732 \\
731\end{array}$ & $\begin{array}{rr} & 94 \\
& 159 \\
5 & 149 \\
5 & 281\end{array}$ \\
\hline
\end{tabular}


Table 11. Cattle and Calves-Inventory and Sales: 1987 and 1982-Con.

[For meaning of abbreviations and symbols, see introductory text]

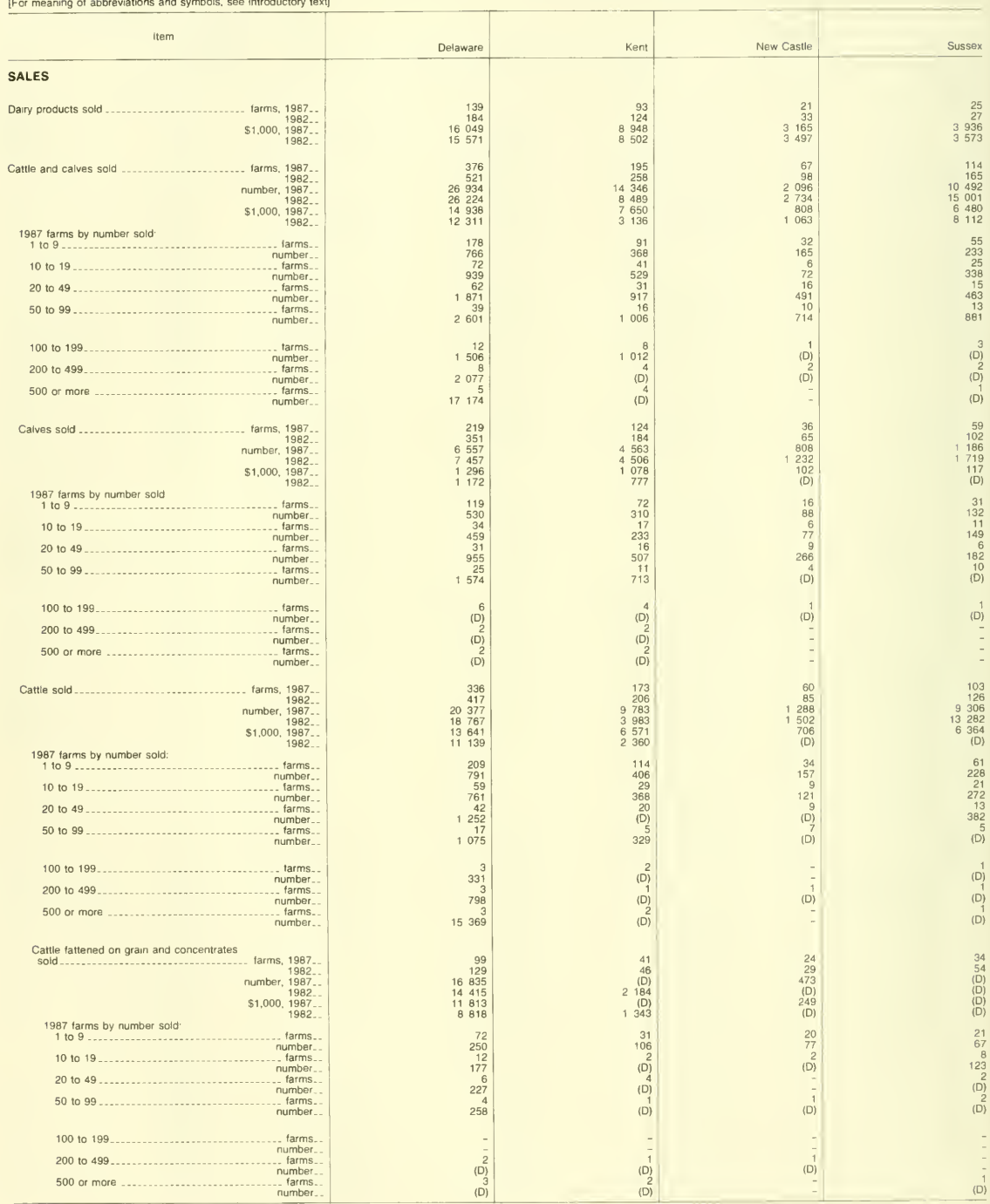


Table 12. Hogs and Pigs-Inventory, Litters, and Sales: 1987 and 1982

[For meaning of abbreviations and symbols, see introductory text]

\begin{tabular}{|c|c|c|c|c|}
\hline Item & Delaware & Kent & New Castle & Sussex \\
\hline \multicolumn{5}{|l|}{ INVENTORY } \\
\hline $\begin{array}{rr}\text { Hogs and pigs . ............................ tarms, } 1987 .- & 1982 . \\
\text { number, } & 1987 . . \\
& 1982 .\end{array}$ & $\begin{array}{rr} & 301 \\
421 \\
49 & 714 \\
54 & 411\end{array}$ & $\begin{array}{rr}97 \\
147 \\
5 & 958 \\
9 & 555\end{array}$ & $\begin{array}{rr}15 \\
30 \\
1 & 079 \\
2 & 325\end{array}$ & $\begin{array}{r}189 \\
244 \\
42 \\
677 \\
42 \quad 531\end{array}$ \\
\hline 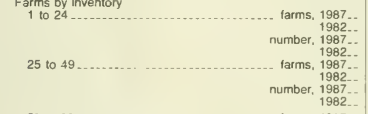 & $\begin{array}{r}123 \\
200 \\
982 \\
1576 \\
56 \\
50 \\
1874 \\
1687\end{array}$ & $\begin{array}{r}58 \\
92 \\
403 \\
712 \\
19 \\
17 \\
(D) \\
603\end{array}$ & $\begin{array}{r}7 \\
17 \\
43 \\
94 \\
3 \\
3 \\
(D) \\
76\end{array}$ & $\begin{array}{r}58 \\
91 \\
536 \\
770 \\
34 \\
30 \\
1131 \\
1008\end{array}$ \\
\hline 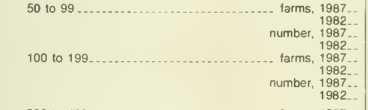 & $\begin{array}{rr} & 47 \\
3 & 66 \\
4 & 350 \\
4 & 23 \\
& 42 \\
2 & 744 \\
5 & 441\end{array}$ & $\begin{array}{r}12 \\
17 \\
815 \\
1070 \\
2 \\
12 \\
10 \\
109 \\
169\end{array}$ & $\begin{array}{r}3 \\
6 \\
169 \\
504 \\
\overline{2} \\
\overline{2} \\
\text { (D) }\end{array}$ & $\begin{array}{r}32 \\
43 \\
2166 \\
2779 \\
21 \\
28 \\
\text { (D) } \\
\text { (D) }\end{array}$ \\
\hline 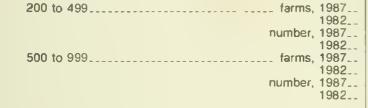 & $\begin{array}{rr} & 23 \\
7 & 412 \\
9 & 600 \\
15 \\
19 \\
10 & 136 \\
12 & 956\end{array}$ & $\begin{array}{r}3 \\
6 \\
(D) \\
1846 \\
2 \\
1 \\
\text { (D) } \\
\text { (D) }\end{array}$ & $\begin{array}{r}2 \\
\text { (D) } \\
\overline{-} \\
\overline{2} \\
\text { (D) }\end{array}$ & $\begin{array}{r}18 \\
25 \\
5887 \\
7 \quad 754 \\
13 \\
16 \\
10 \\
10956\end{array}$ \\
\hline 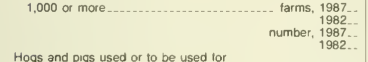 & $\begin{array}{rr} & 14 \\
23 & 416 \\
18 & 798\end{array}$ & $\begin{array}{r}1 \\
2 \\
(D) \\
\text { (D) }\end{array}$ & $\overline{-}$ & $\begin{array}{l}13 \\
10 \\
\text { (D) } \\
\text { (D) }\end{array}$ \\
\hline $\begin{array}{rr}\text { Hogs and pigs used or to be used lor } \\
\text { breeding.......... farms, } 1987 . . \\
1982 . \\
\text { number, } 1987 . . \\
1982 .\end{array}$ & $\begin{array}{r}203 \\
287 \\
10813 \\
8689\end{array}$ & $\begin{array}{r}53 \\
96 \\
1166 \\
1239\end{array}$ & $\begin{array}{r}9 \\
17 \\
230 \\
306\end{array}$ & $\begin{array}{ll} & 141 \\
& 174 \\
9 & 417 \\
7 & 144\end{array}$ \\
\hline $\begin{array}{l}\begin{array}{r}1987 \text { farms by inventory: } \\
1 \text { to } 24 \\
25 \text { to } 49 \\
50 \text { to } 99 \\
100 \text { or more }\end{array} \text { Other hogs and pigs } \\
\text { number, } \begin{array}{r}1987 \\
1982 .\end{array} \\
\text { number } \\
\text { norms }\end{array}$ & $\begin{array}{r}141 \\
22 \\
16 \\
24 \\
7766 \\
267 \\
364 \\
38 \\
901 \\
45722\end{array}$ & $\begin{array}{r}47 \\
2 \\
1 \\
3 \\
\text { (D) } \\
83 \\
123 \\
4792 \\
8316\end{array}$ & $\begin{array}{r}7 \\
1 \\
\overline{1} \\
1 \\
14 \\
14 \\
27 \\
849 \\
2019\end{array}$ & $\begin{array}{r}87 \\
19 \\
15 \\
20 \\
7037 \\
170 \\
214 \\
33 \\
260 \\
35 \\
387\end{array}$ \\
\hline \multicolumn{5}{|l|}{ LITTERS } \\
\hline $\begin{array}{r}\text { Litters of pigs farrowed between- } \\
\text { Dec. } 1 \text { of preceding year and Nov. } 30 \ldots \ldots \text { tarms, } 1987 . . \\
1982 . \\
\text { number, } 1987 \ldots \\
1982 \ldots\end{array}$ & $\begin{array}{rr}211 \\
292 \\
13 & 197 \\
10 & 887\end{array}$ & $\begin{array}{r}54 \\
98 \\
1749 \\
1610\end{array}$ & $\begin{array}{r}10 \\
17 \\
397 \\
389\end{array}$ & $\begin{array}{r}147 \\
177 \\
11051 \\
8888\end{array}$ \\
\hline 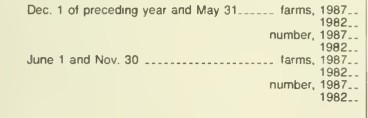 & $\begin{array}{r}179 \\
259 \\
6597 \\
5875 \\
183 \\
259 \\
6600 \\
5012\end{array}$ & $\begin{array}{r}44 \\
88 \\
877 \\
874 \\
48 \\
83 \\
872 \\
736\end{array}$ & $\begin{array}{r}8 \\
16 \\
214 \\
207 \\
7 \\
15 \\
183 \\
182\end{array}$ & $\begin{array}{r}127 \\
155 \\
5506 \\
4794 \\
128 \\
161 \\
5545 \\
4094\end{array}$ \\
\hline \multicolumn{5}{|l|}{ SALES } \\
\hline $\begin{array}{l}\text { Hogs and pigs sold .... farms, } 1987 .- \\
1982 . \\
\text { number, } 1987 . . \\
1982 \ldots \\
\$ 1,000,1987 . \\
1987 \text { farms by number sold: } \\
1982 \ldots\end{array}$ & $\begin{array}{rr}310 \\
413 \\
109600 \\
101972 \\
10055 \\
10208\end{array}$ & $\begin{array}{rr} & 96 \\
136 \\
15 & 343 \\
14 & 429 \\
1 & 409 \\
1 & 609\end{array}$ & $\begin{array}{rr} & 16 \\
26 \\
2671 \\
2492 \\
189 \\
290\end{array}$ & $\begin{array}{rr} & 198 \\
91 & 251 \\
85 & 051 \\
8 & 457 \\
8 & 308\end{array}$ \\
\hline $\begin{array}{c}1 \text { to } 24 \\
25 \text { to } 49 \\
50 \text { to } 99 \ldots 0\end{array}$ & $\begin{array}{r}77 \\
748 \\
66 \\
221 \\
53 \\
3619 \\
41 \\
5271\end{array}$ & $\begin{array}{r}29 \\
250 \\
23 \\
790 \\
15 \\
1023 \\
19 \\
2531\end{array}$ & $\begin{array}{r}8 \\
46 \\
3 \\
(0) \\
1 \\
(0) \\
- \\
-\end{array}$ & $\begin{array}{r}40 \\
452 \\
40 \\
\text { (D) } \\
37 \\
\text { (D) } \\
22 \\
2740\end{array}$ \\
\hline 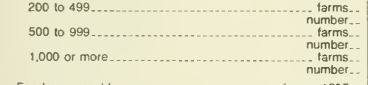 & $\begin{array}{rr}7552 \\
25 \\
20 \\
13 \quad 756 \\
28 \\
76 \quad 433\end{array}$ & $\begin{array}{r}6 \\
\text { (D) } \\
1 \\
\text { (D) } \\
3 \\
\text { (D) }\end{array}$ & $\begin{array}{r}1 \\
\text { (D) } \\
2 \\
\text { (D) } \\
1 \\
\text { (D) }\end{array}$ & $\begin{array}{r}18 \\
5241 \\
247 \\
11 \quad 776 \\
24 \\
67508\end{array}$ \\
\hline 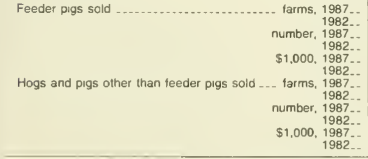 & $\begin{array}{rr} & 74 \\
30 & 594 \\
27 & 544 \\
1 & 503 \\
1 & 268 \\
& 285 \\
& 363 \\
79 & 006 \\
74 & 428 \\
8 & 552 \\
8 & 940\end{array}$ & $\begin{array}{r}31 \\
50 \\
4975 \\
2887 \\
218 \\
102 \\
87 \\
117 \\
10 \\
1168 \\
11542 \\
1191 \\
1507\end{array}$ & $\begin{array}{r}6 \\
11 \\
1859 \\
403 \\
96 \\
14 \\
13 \\
21 \\
812 \\
2089 \\
93 \\
276\end{array}$ & $\begin{array}{rr} & 37 \\
& 77 \\
23 & 760 \\
24 & 254 \\
1 & 188 \\
1 & 151 \\
185 \\
185 \\
& 225 \\
67 & 826 \\
60 & 797 \\
7 & 269 \\
7 & 157 \\
\end{array}$ \\
\hline
\end{tabular}


Table 13. Sheep and Horses - Inventory and Sales: 1987 and 1982

[For meaning of abbreviations and symbols, see introductory text]

\begin{tabular}{|c|c|c|c|c|}
\hline item & Delaware & Kent & New Castle & Sussex \\
\hline $\begin{array}{r}\text { Sheep and lambs inventory ................ farms, } 1987 . . \\
1982 . \\
\text { number. } 1987 . \\
1982 .\end{array}$ & $\begin{array}{r}50 \\
41 \\
1667 \\
681\end{array}$ & $\begin{array}{r}16 \\
16 \\
590 \\
121\end{array}$ & $\begin{array}{r}12 \\
10 \\
507 \\
186\end{array}$ & $\begin{array}{r}22 \\
15 \\
570 \\
374\end{array}$ \\
\hline $\begin{array}{l}1987 \text { tarms by inventory } \\
1 \text { to } 24 \\
25 \text { to } 99 \\
100 \text { to } 299 \\
300 \text { to } 999 \\
1.000 \text { or more. }\end{array}$ & $\begin{array}{r}33 \\
12 \\
5 \\
- \\
-\end{array}$ & $\begin{array}{r}10 \\
4 \\
2 \\
- \\
-\end{array}$ & $\begin{array}{l}6 \\
4 \\
2 \\
- \\
-\end{array}$ & $\begin{array}{r}17 \\
4 \\
1 \\
- \\
-\end{array}$ \\
\hline $\begin{array}{rr}\text { Ewes } 1 \text { year old or older .................... farms, } 1987 . \\
\\
\text { number, } 1982 . \\
1982 \\
\text { n }\end{array}$ & $\begin{array}{r}45 \\
33 \\
1006 \\
358\end{array}$ & $\begin{array}{r}16 \\
14 \\
375 \\
69\end{array}$ & $\begin{array}{r}10 \\
6 \\
221 \\
105\end{array}$ & $\begin{array}{r}19 \\
13 \\
410 \\
184\end{array}$ \\
\hline $\begin{array}{r}\text { Sheep and tambs shorn ................. farms, } 1987 . \\
1982 \\
\text { number, } 1987 . \\
1982 \\
\text { pounds of wool, } 1987 . \\
1982\end{array}$ & $\begin{array}{rr}39 \\
29 \\
1099 \\
474 \\
7081 \\
3 & 033\end{array}$ & $\begin{array}{r}13 \\
14 \\
346 \\
82 \\
2 \quad 199 \\
586\end{array}$ & $\begin{array}{r}10 \\
5 \\
281 \\
140 \\
+836 \\
955\end{array}$ & $\begin{array}{r}16 \\
10 \\
472 \\
252 \\
3046 \\
1492\end{array}$ \\
\hline $\begin{array}{r}\text { Sheep and lambs sold .................... farms, } 1987 \ldots \\
1982 . \\
\text { number, } 1987 . \\
1982 .\end{array}$ & $\begin{array}{r}38 \\
24 \\
772 \\
469\end{array}$ & $\begin{array}{r}15 \\
8 \\
161 \\
101\end{array}$ & $\begin{array}{r}8 \\
6 \\
314 \\
159\end{array}$ & $\begin{array}{r}15 \\
10 \\
297 \\
209\end{array}$ \\
\hline $\begin{array}{r}\text { Sheep, lambs, and wool sold ............... farms, } 1987 \ldots \\
1982 . \\
51,000,1987 . \\
1982 .\end{array}$ & $\begin{array}{l}45 \\
32 \\
54 \\
26\end{array}$ & $\begin{array}{r}16 \\
15 \\
12 \\
5\end{array}$ & $\begin{array}{r}10 \\
6 \\
21 \\
11\end{array}$ & $\begin{array}{l}19 \\
11 \\
20 \\
10\end{array}$ \\
\hline $\begin{array}{r}\text { Horses and ponies inventory ............ farms, } 1987 \ldots \\
1982 . \\
\text { number, } 1987 \ldots \\
1982 \ldots\end{array}$ & $\begin{array}{rr}425 \\
476 \\
3029 \\
3 & 514\end{array}$ & $\begin{array}{rl} & 198 \\
227 \\
1 & 216 \\
1 & 533\end{array}$ & $\begin{array}{r}87 \\
89 \\
842 \\
922\end{array}$ & $\begin{array}{r}140 \\
160 \\
971 \\
1059\end{array}$ \\
\hline $\begin{array}{r}\text { Horses and ponies sold ................... farms, } 1987 . . \\
1982 . \\
\text { number, } 1987 . \\
1982 \ldots \\
\$ 1,000,1987 \ldots \\
1982 \ldots\end{array}$ & 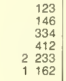 & $\begin{array}{r}66 \\
82 \\
140 \\
213 \\
219 \\
476\end{array}$ & $\begin{array}{r}26 \\
21 \\
110 \\
50 \\
1910 \\
191\end{array}$ & $\begin{array}{r}31 \\
43 \\
84 \\
149 \\
105 \\
496\end{array}$ \\
\hline
\end{tabular}


Table 14. Poultry-Inventory and Sales: 1987 and 1982

[For meaning of abbreviations and symbols, see introductory text]

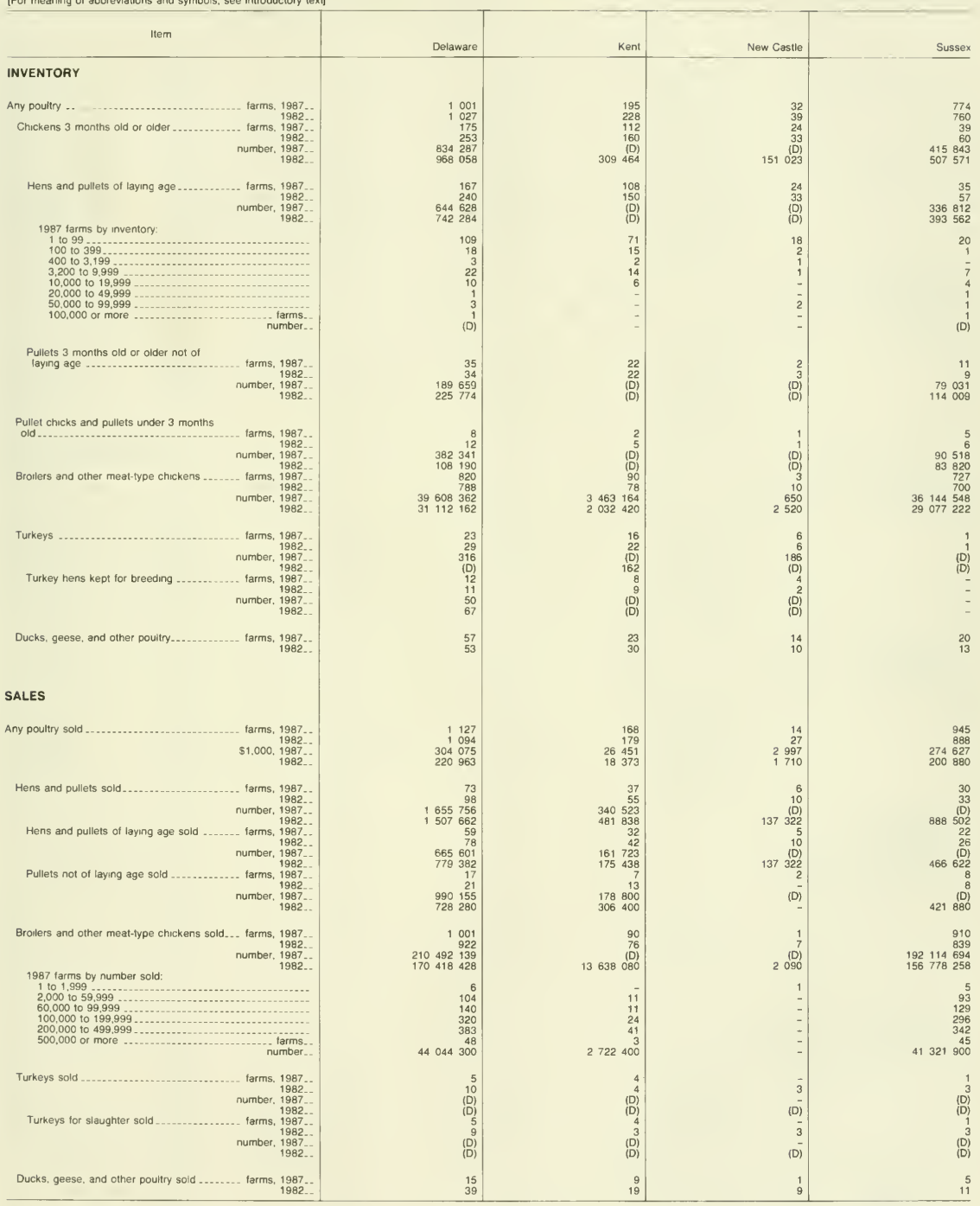


Table 15. Selected Crops: 1987 and 1982

[For meaning of abbreviations and symbols, see introductory text]

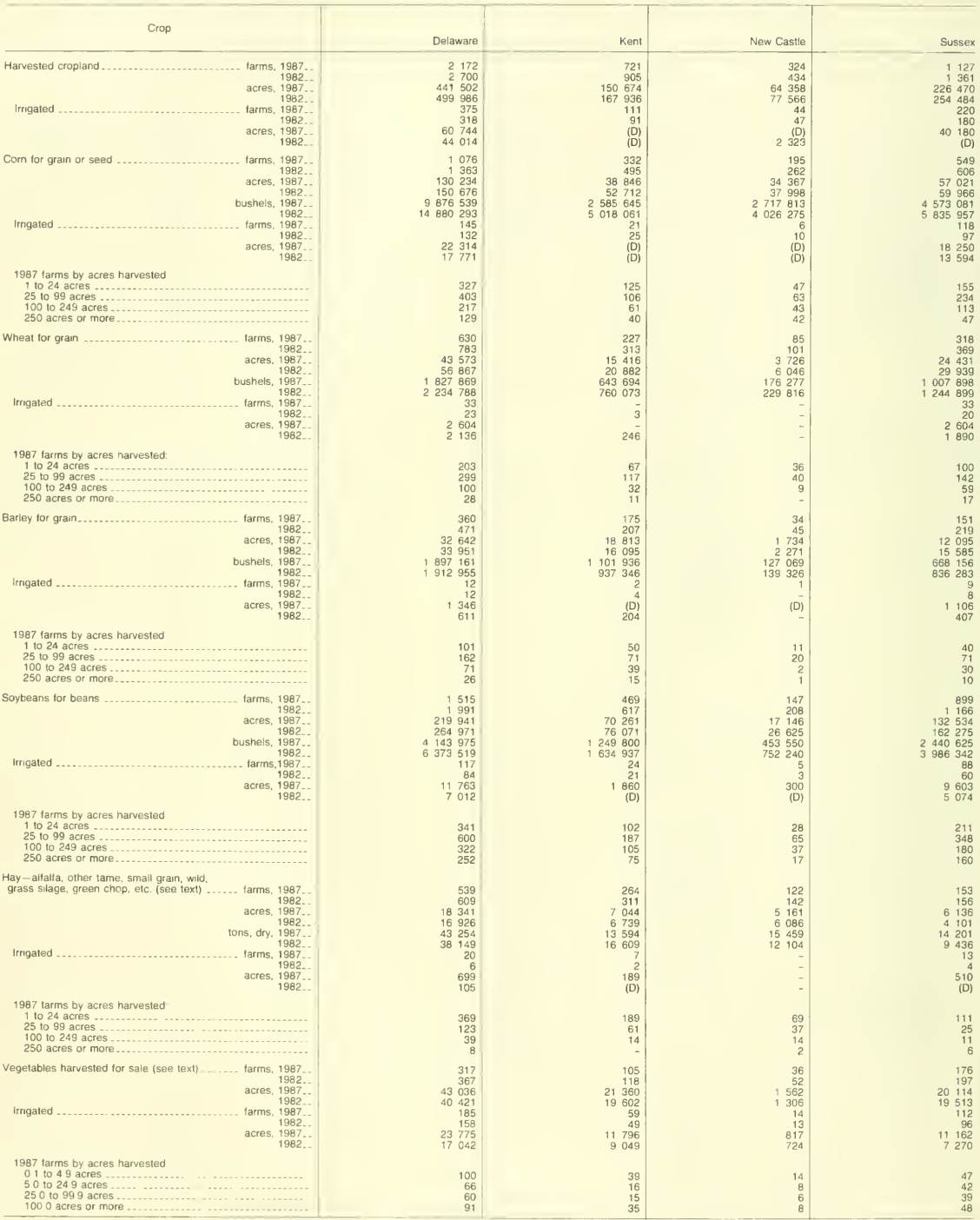


Table 16. Farms With Sales of $\$ 10,000$ or More: 1987 and 1982

[Data for 1987 include abnormal farms For meaning of abbreviations and symbals, see introductory text]

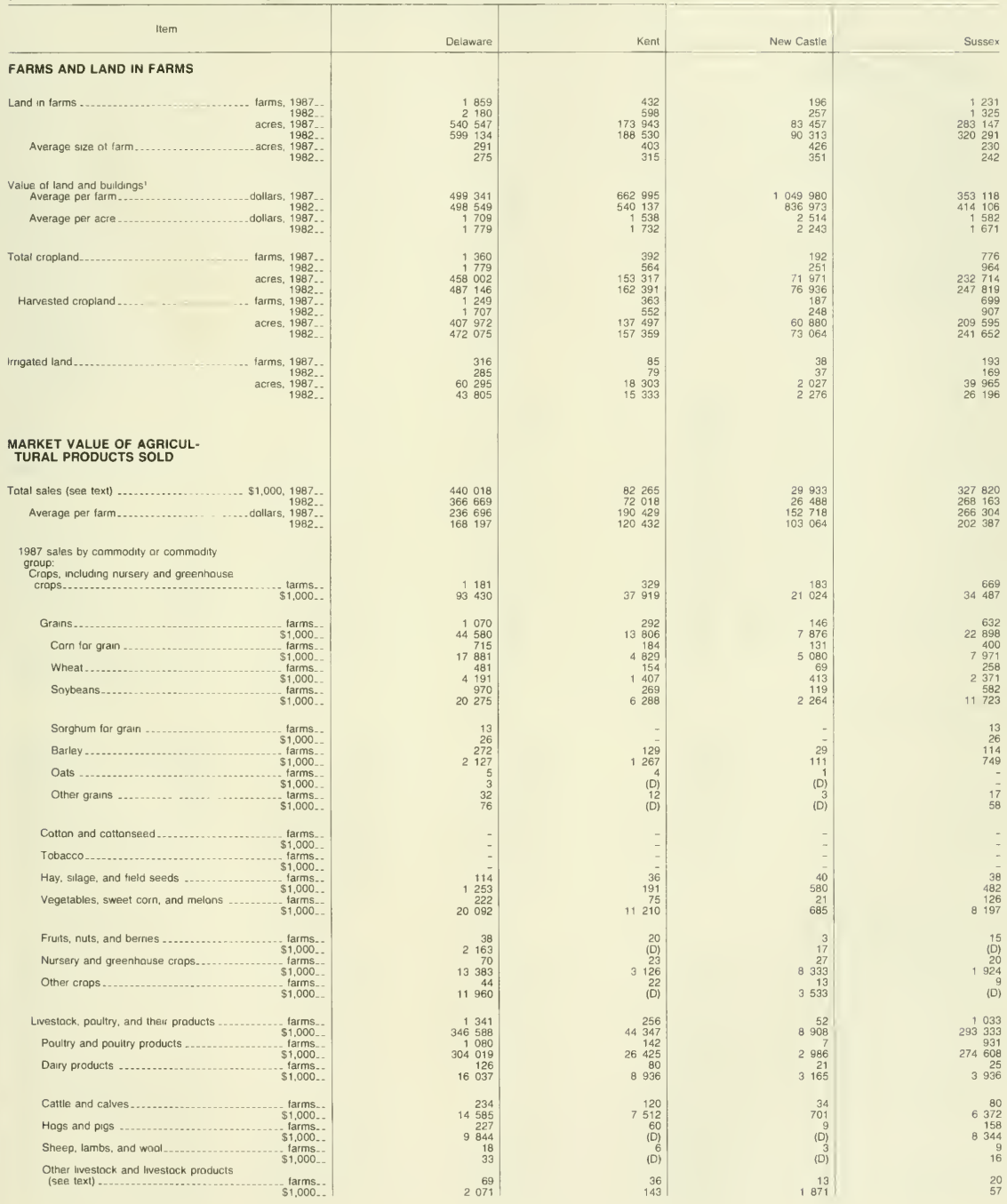


Table 16. Farms With Sales of $\$ 10,000$ or More: 1987 and $1982-$ Con.

IData for 1987 include abnormal farms For meaning of abbreviations and symbols, see introductory text

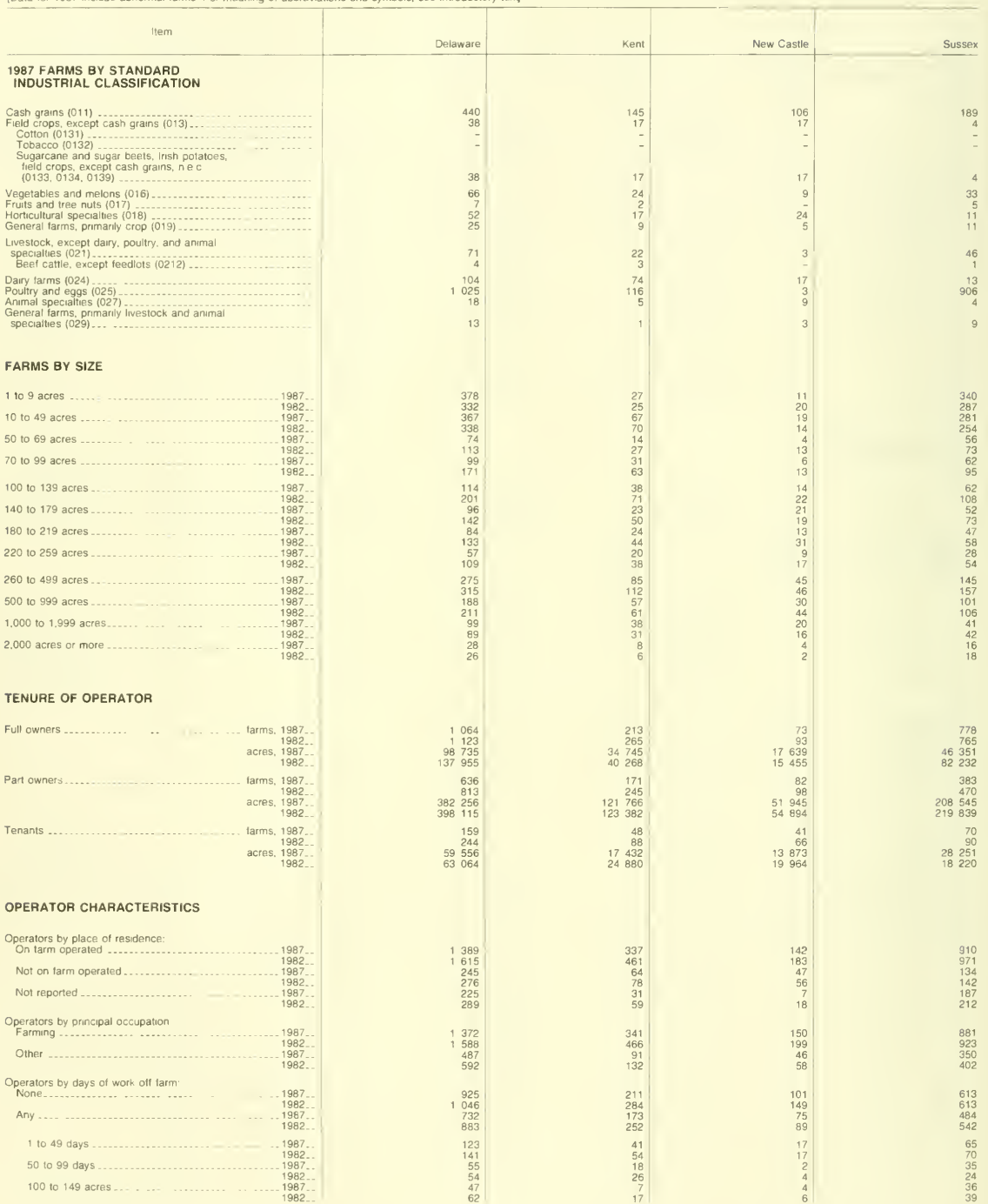

See footnotes at end of table 
Table 16. Farms With Sales of $\$ 10,000$ or More: 1987 and $1982-$ Con.

[Data for 1987 include abnormal farms For meaning of abbreviations and symbols, see introductory tex]]

\begin{tabular}{|c|c|c|c|c|}
\hline Item & Delaware & Kent & New Castle & Sussex \\
\hline \multicolumn{5}{|l|}{$\begin{array}{l}\text { OPERATOR CHARACTERISTICS - } \\
\text { CON. }\end{array}$} \\
\hline \multicolumn{5}{|l|}{ 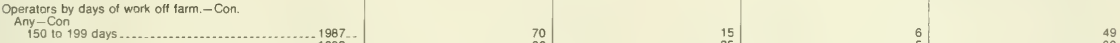 } \\
\hline 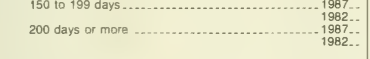 & $\begin{array}{r}70 \\
90 \\
437 \\
536\end{array}$ & $\begin{array}{r}15 \\
25 \\
92 \\
130\end{array}$ & $\begin{array}{r}6 \\
5 \\
46 \\
57\end{array}$ & $\begin{array}{r}49 \\
60 \\
299 \\
349\end{array}$ \\
\hline 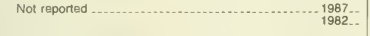 & $\begin{array}{l}202 \\
251\end{array}$ & $\begin{array}{l}48 \\
62\end{array}$ & $\begin{array}{l}20 \\
19\end{array}$ & $\begin{array}{l}134 \\
170\end{array}$ \\
\hline $\begin{array}{l}1987 \text { operators by years on present farm: } \\
2 \text { years or less ... } \\
3 \text { or } 4 \text { years } \\
5 \text { to } 9 \text { years. } \\
\text { 10 years or more } \\
\text { Average years on present farm }\end{array}$ & $\begin{array}{r}85 \\
119 \\
235 \\
+020 \\
19.5\end{array}$ & \begin{tabular}{r|}
20 \\
31 \\
64 \\
247 \\
18.6
\end{tabular} & \begin{tabular}{r|r}
7 \\
10 \\
24 \\
128 \\
20.8
\end{tabular} & $\begin{array}{r}58 \\
78 \\
147 \\
645 \\
19.7\end{array}$ \\
\hline 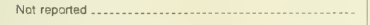 & 400 & 70 & 27 & 303 \\
\hline $\begin{array}{l}1987 \text { operators by age group: } \\
\text { Under } 25 \text { years } \\
25 \text { to } 34 \text { years } \\
35 \text { to } 44 \text { years } \\
45 \text { to } 54 \text { years } \\
55 \text { to } 64 \text { years } \\
65 \text { years and over } \\
\text { Average age }\end{array}$ & \begin{tabular}{l|}
22 \\
260 \\
425 \\
426 \\
419 \\
307 \\
50.0
\end{tabular} & $\begin{array}{r}11 \\
64 \\
101 \\
97 \\
94 \\
65 \\
49.3\end{array}$ & $\begin{array}{r}1 \\
29 \\
35 \\
30 \\
66 \\
35 \\
52.1\end{array}$ & $\begin{array}{r}10 \\
167 \\
289 \\
299 \\
259 \\
207 \\
49.9\end{array}$ \\
\hline \multicolumn{5}{|l|}{ TYPE OF ORGANIZATION } \\
\hline 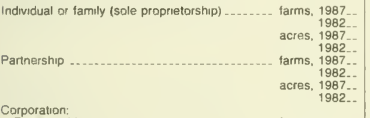 & $\begin{array}{r}1537 \\
1765 \\
338062 \\
370 \quad 933 \\
161 \\
225 \\
58948 \\
\text { (D) }\end{array}$ & $\begin{array}{r}352 \\
487 \\
117 \quad 174 \\
132 \quad 320 \\
43 \\
66 \\
\text { (D) } \\
\text { (D) }\end{array}$ & $\begin{array}{r}150 \\
188 \\
60 \quad 114 \\
59 \quad 729 \\
19 \\
19 \\
36 \\
1 D \\
16 \quad 259\end{array}$ & $\begin{array}{r}1935 \\
11090 \\
160 \quad 774 \\
178 \quad 884 \\
99 \\
123 \\
\quad(D) \\
38 \quad 366\end{array}$ \\
\hline 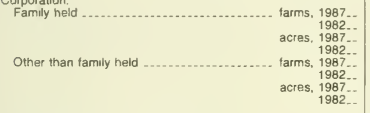 & $\begin{array}{r}146 \\
162 \\
(\mathrm{D}) \\
139700 \\
5 \\
13 \\
1 \mathrm{D} \\
4954 \\
49\end{array}$ & $\begin{array}{r}35 \\
40 \\
256 \\
\text { (D) } \\
\overline{2} \\
\text { (D) }\end{array}$ & $\begin{array}{r}24 \\
26 \\
\text { (D) } \\
\text { (D) } \\
2 \\
4 \\
\text { (D) } \\
\text { (D) }\end{array}$ & $\begin{array}{r}87 \\
96 \\
\text { (D) } \\
\text { (D) } \\
3 \\
7 \\
\text { (D) } \\
\text { (D) }\end{array}$ \\
\hline $\begin{array}{l}\text { Other-cooperative, estate or trust, } \\
\text { institutional, etc. ...................... farms, } 1987 . . \\
\\
\text { acres, } 1982 . \\
1982 .\end{array}$ & $\begin{array}{r}10 \\
15 \\
2464 \\
\text { (D) }\end{array}$ & $\begin{array}{r}2 \\
3 \\
\text { (D) } \\
\text { (D) }\end{array}$ & $2 . \begin{array}{r}1 \\
3 \\
379\end{array}$ & $2 \begin{array}{r}7 \\
9 \\
(D 47 \\
747\end{array}$ \\
\hline \multicolumn{5}{|l|}{$\begin{array}{l}1987 \text { FARM PRODUCTION } \\
\text { EXPENSES }\end{array}$} \\
\hline 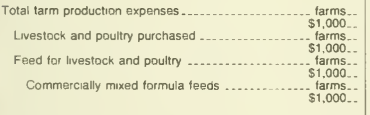 & $\begin{array}{rl}1 & 866 \\
370 & 478 \\
1 & 200 \\
53 & 253 \\
1 & 300 \\
186 & 851 \\
1 & 189 \\
180 & 414\end{array}$ & $\begin{array}{r}433 \\
7362 \\
212 \\
7746 \\
267 \\
21371 \\
205 \\
18491\end{array}$ & $\begin{array}{r}199 \\
25603 \\
15 \\
753 \\
34 \\
2375 \\
238 \\
1896\end{array}$ & $\begin{array}{r}1234 \\
271513 \\
973 \\
94754 \\
11005 \\
163104 \\
956 \\
160027\end{array}$ \\
\hline 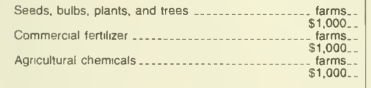 & $\begin{array}{rl}1 & 169 \\
8 & 287 \\
1 & 052 \\
14 & 974 \\
1 & 197 \\
10 & 385\end{array}$ & $\begin{array}{rl}359 \\
3 & 160 \\
351 \\
6394 \\
348 \\
4264\end{array}$ & $\begin{aligned} 167 \\
2073 \\
177 \\
2740 \\
1786 \\
1764\end{aligned}$ & $\begin{array}{rl} & 643 \\
3 & 054 \\
524 \\
5 & 839 \\
663 \\
4 & 357\end{array}$ \\
\hline 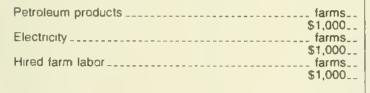 & $\begin{array}{rl}1 & 662 \\
8 & 172 \\
1 & 637 \\
4 & 690 \\
1 & 091 \\
22 & 594\end{array}$ & $\begin{array}{r}408 \\
2750 \\
363 \\
937 \\
270 \\
7539\end{array}$ & $\begin{array}{r}194 \\
1079 \\
180 \\
702 \\
120 \\
4170\end{array}$ & $\begin{array}{rr}1 & 060 \\
4 & 343 \\
1 & 094 \\
3 & 052 \\
701 \\
10884\end{array}$ \\
\hline $\begin{array}{c}\text { Contract labor...... } \\
\$ 1,000 .- \\
\text { Feparr and maintenance } \\
\$ 1,000 .-\end{array}$ & $\begin{array}{r}342 \\
5753 \\
1649 \\
11006\end{array}$ & $\begin{array}{rr}58 \\
1322 \\
390 \\
3515\end{array}$ & $\begin{array}{r}30 \\
328 \\
171 \\
1319\end{array}$ & $\begin{array}{l}254 \\
4104 \\
11088 \\
6172\end{array}$ \\
\hline $\begin{array}{l}\text { Customwark, machine hire, and rental of } \\
\text { machinery and equipment .................... tarms.. } \\
\$ 1,000 \ldots\end{array}$ & $\begin{array}{r}792 \\
2210\end{array}$ & $\begin{array}{l}217 \\
748\end{array}$ & $\begin{array}{r}71 \\
302\end{array}$ & $\begin{array}{r}504 \\
1160\end{array}$ \\
\hline 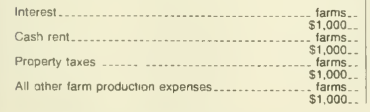 & $\begin{array}{rr}1 & 137 \\
14 & 258 \\
707 \\
10709 \\
1745 \\
2101 \\
1 \quad 865 \\
15 & 234\end{array}$ & $\begin{array}{r}284 \\
4846 \\
228 \\
4057 \\
382 \\
534 \\
433 \\
4179\end{array}$ & $\begin{array}{r}182 \\
1328 \\
98 \\
2698 \\
153 \\
345 \\
198 \\
3625\end{array}$ & $\begin{array}{rl} & 771 \\
8 & 083 \\
381 \\
3 & 954 \\
1 & 210 \\
1 & 221 \\
1 & 234 \\
7 & 431\end{array}$ \\
\hline
\end{tabular}


Table 16. Farms With Sales of $\$ 10,000$ or More: 1987 and $1982-$ Con.

[Data for 1987 include abnormal farms For meaning of abbreviations and symbols, see introductory text]

\begin{tabular}{|c|c|c|c|c|}
\hline item & Delaware & Kent & New Castle & Sussex \\
\hline \multicolumn{5}{|l|}{ MACHINERY AND EQUIPMENT' } \\
\hline 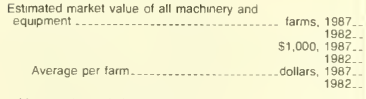 & $\begin{array}{rr}1 & 866 \\
2 & 181 \\
137 & 284 \\
139 & 412 \\
73 & 571 \\
63 & 921\end{array}$ & $\begin{array}{ll} & 433 \\
& 599 \\
40 & 183 \\
46 & 417 \\
92 & 800 \\
77 & 492\end{array}$ & $\begin{array}{rr} & 199 \\
24 & 257 \\
19 & 849 \\
121 & 103 \\
77 & 201\end{array}$ & $\begin{array}{rr}1 & 234 \\
1 & 325 \\
73 & 002 \\
73 & 154 \\
59 & 159 \\
55 & 210\end{array}$ \\
\hline 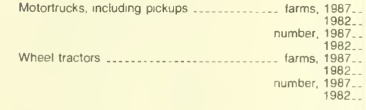 & $\begin{array}{ll}1 & 717 \\
1 & 969 \\
4 & 682 \\
5 & 122 \\
1 & 670 \\
2 & 014 \\
5 & 110 \\
6 & 381\end{array}$ & $\begin{array}{r}379 \\
537 \\
1353 \\
1508 \\
399 \\
578 \\
1540 \\
1999\end{array}$ & $\begin{array}{l}187 \\
224 \\
603 \\
706 \\
185 \\
255 \\
666 \\
975\end{array}$ & $\begin{array}{ll}1 & 151 \\
1 & 208 \\
2 & 726 \\
2 & 908 \\
1 & 086 \\
1 & 181 \\
2 & 904 \\
3 & 407\end{array}$ \\
\hline 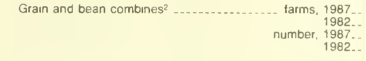 & $\begin{array}{r}702 \\
927 \\
948 \\
1 \\
132\end{array}$ & $\begin{array}{l}210 \\
303 \\
317 \\
402\end{array}$ & $\begin{array}{r}97 \\
133 \\
124 \\
144\end{array}$ & $\begin{array}{l}395 \\
491 \\
507 \\
586\end{array}$ \\
\hline \multicolumn{5}{|l|}{ LIVESTOCK AND POULTRY } \\
\hline $\begin{aligned} \text { Cattle and calves inventory ................. farms, } & 1987 \ldots \\
& 1982 \ldots \\
\text { number, } & 1987 \ldots \\
& 1982 \ldots\end{aligned}$ & $\begin{array}{ll} & 271 \\
& 436 \\
29 & 286 \\
31 & 156\end{array}$ & $\begin{array}{r}131 \\
208 \\
15 \\
257 \\
14 \\
378\end{array}$ & $\begin{array}{rr} & 38 \\
63 \\
4 & 039 \\
4 & 917\end{array}$ & $\begin{array}{r}102 \\
165 \\
9990 \\
11861\end{array}$ \\
\hline $\begin{array}{r}\text { Cows and helfers that had calved ........... tarms, } 1987 \ldots \\
1982 . . \\
\text { number, } 1987 \ldots \\
1982 .\end{array}$ & 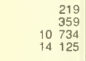 & $\begin{array}{rr}113 \\
179 \\
5852 \\
6562\end{array}$ & $\begin{array}{rr}25 \\
54 \\
1 & 872 \\
2 & 790\end{array}$ & $\begin{array}{rr}81 \\
126 \\
3010 \\
4773\end{array}$ \\
\hline 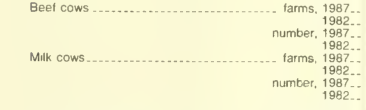 & $\begin{array}{rr} & 104 \\
188 \\
1 & 471 \\
4 & 299 \\
132 \\
198 \\
9 \\
9 & 263 \\
9 & 826\end{array}$ & $\begin{array}{r}36 \\
65 \\
496 \\
937 \\
84 \\
130 \\
5356 \\
5625\end{array}$ & $\begin{array}{r}9 \\
25 \\
\text { (D) } \\
658 \\
18 \\
32 \\
32 \\
\text { (D) } \\
2132\end{array}$ & $\begin{array}{r}59 \\
98 \\
(D) \\
2704 \\
30 \\
36 \\
\\
2069 \\
20\end{array}$ \\
\hline $\begin{array}{l}\text { Heifers and heifer calves .................... farms, } 1987 \ldots \\
\text { number, } 1987 \ldots \\
\text { Steers, steer calves, bulls, and bull calves ... farms, } 1987 \ldots \\
\text { number, } 1987 \ldots\end{array}$ & $\begin{array}{r}204 \\
7592 \\
182 \\
10 \quad 960\end{array}$ & $\begin{array}{r}108 \\
4032 \\
54 \\
5373\end{array}$ & $\begin{array}{r}26 \\
(D) \\
24 \\
\text { (D) }\end{array}$ & $\begin{array}{r}70 \\
\text { (D) } \\
64 \\
\text { (D) }\end{array}$ \\
\hline 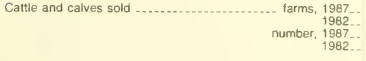 & 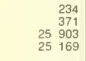 & $\begin{array}{rr} & 120 \\
187 \\
13 & 931 \\
8 & 059\end{array}$ & $\begin{array}{rr} & 34 \\
61 \\
1 & 809 \\
2 & 375\end{array}$ & $\begin{array}{rr}80 \\
123 \\
10 & 163 \\
14 & 735\end{array}$ \\
\hline 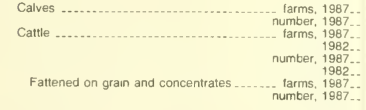 & $\begin{array}{rr}151 \\
6 \quad 184 \\
209 \\
308 \\
19 \\
1819 \\
18 \quad 236 \\
16 \\
49 \\
612\end{array}$ & $\begin{array}{r}84 \\
420 \\
407 \\
155 \\
9511 \\
3752 \\
21 \\
\text { (D) }\end{array}$ & $\begin{array}{r}20 \\
660 \\
32 \\
55 \\
1149 \\
101 \\
10 \\
421\end{array}$ & $\begin{array}{r}47 \\
1 \quad 104 \\
70 \\
98 \\
9059 \\
\text { (D) } \\
18 \\
\text { (D) }\end{array}$ \\
\hline $\begin{array}{r}\text { Hogs and pigs inventory..................... farms, } 1987 \ldots \\
1982 . . \\
\text { number, } 1987 \ldots \\
1982 .\end{array}$ & $\begin{array}{r}220 \\
328 \\
48308 \\
52 \quad 642\end{array}$ & $\begin{array}{r}59 \\
104 \\
5522 \\
8722\end{array}$ & $\begin{array}{r}9 \\
21 \\
991 \\
2101\end{array}$ & $\begin{array}{rr} & 152 \\
& 203 \\
41 & 795 \\
41 & 819\end{array}$ \\
\hline 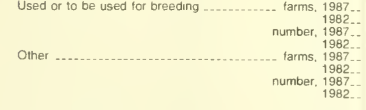 & 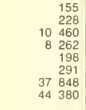 & $\begin{array}{rr} & 37 \\
& 72 \\
1 & 087 \\
1 & 017 \\
& 49 \\
& 87 \\
4 & 435 \\
7 & 705\end{array}$ & $\begin{array}{r}6 \\
13 \\
219 \\
273 \\
8 \\
19 \\
772 \\
1828\end{array}$ & 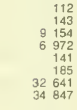 \\
\hline 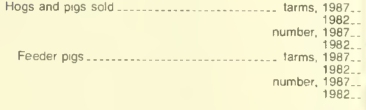 & $\begin{array}{r}227 \\
333 \\
107 \\
028 \\
98 \quad 963 \\
53 \\
104 \\
29785 \\
25 \quad 634\end{array}$ & $\begin{array}{r}60 \\
102 \\
(\mathrm{D}) \\
13017 \\
20 \\
38 \\
4638 \\
1910\end{array}$ & $\begin{array}{r}9 \\
17 \\
(0) \\
2 \quad 341 \\
3 \\
7 \\
17 \\
789 \\
329\end{array}$ & $\begin{array}{rr} & 158 \\
& 214 \\
90 & 238 \\
83 & 605 \\
& 30 \\
& 59 \\
23 & 358 \\
23 & 395\end{array}$ \\
\hline 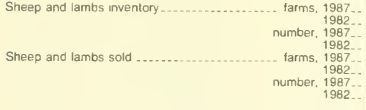 & $\begin{array}{r}19 \\
21 \\
1024 \\
297 \\
16 \\
12 \\
444 \\
225\end{array}$ & $\begin{array}{r}6 \\
9 \\
\text { (D) } \\
\text { (D) } \\
6 \\
4 \\
\text { (D) } \\
\text { (D) }\end{array}$ & $\begin{array}{r}3 \\
2 \\
\text { (D) } \\
\text { (D) } \\
2 \\
2 \\
\text { (D) } \\
\text { (D) }\end{array}$ & $\begin{array}{r}10 \\
10 \\
460 \\
124 \\
8 \\
6 \\
240 \\
109\end{array}$ \\
\hline 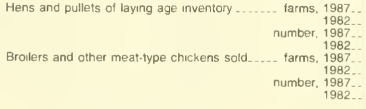 & $\begin{array}{rr} & 82 \\
146 \\
641 & 454 \\
738 & 241 \\
995 \\
\\
909 \\
210484 \\
1709 \\
1788 & 935\end{array}$ & $\begin{array}{r}56 \\
99 \\
\text { (D) } \\
174 \\
90 \\
74 \\
\text { (D) } \\
\text { (D) }\end{array}$ & $\begin{array}{r}10 \\
15 \\
\text { (D) } \\
\text { (D) } \\
\overline{3} \\
\text { (D) }\end{array}$ & $\begin{array}{r}16 \\
336 \quad 287 \\
(D) \\
905 \\
832 \\
1 \mathrm{D}) \\
156 \quad 750 \quad 295\end{array}$ \\
\hline
\end{tabular}


Table 16. Farms With Sales of $\$ 10,000$ or More: 1987 and $1982-$ Con.

[Data for 1987 include ebnormal farms. For meaning of abbreviations and symbols, see introductory text]

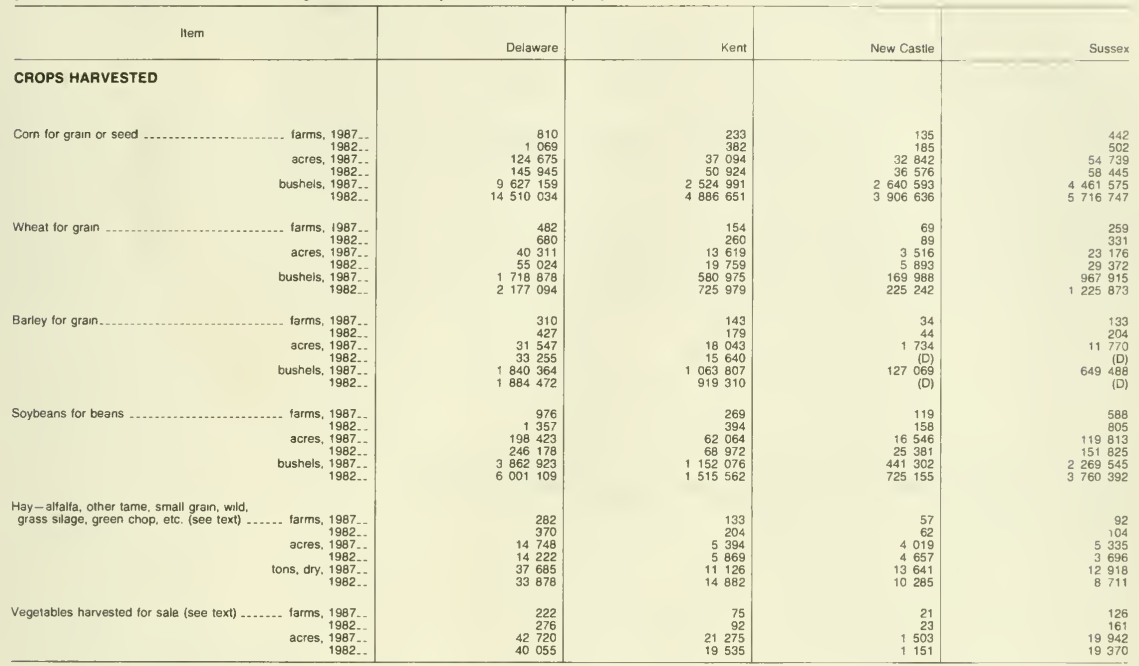

1 Data are based on a sample of farms.

2Data for 1982 include self-propelled only 
Table 17. Milk Goats-Inventory and Sales: 1987 and 1982

\begin{tabular}{|c|c|c|c|c|c|c|c|c|}
\hline \multirow{3}{*}{ Geographic area } & \multirow{2}{*}{\multicolumn{2}{|c|}{ Inventory }} & \multicolumn{6}{|c|}{ Sales } \\
\hline & & & \multicolumn{2}{|c|}{ Milk goats } & \multicolumn{2}{|c|}{ Goat milk } & \multirow[b]{2}{*}{ Farms } & \multirow{2}{*}{$\begin{array}{r}\text { Total sales } \\
(\$ 1,000)\end{array}$} \\
\hline & Farms & Number & Farms & Number & Farms & Gallons & & \\
\hline \multicolumn{9}{|l|}{ STATE TOTAL } \\
\hline $\begin{array}{r}\text { Delaware ........... } 1987 \ldots \\
1982 .\end{array}$ & $\begin{array}{l}22 \\
35\end{array}$ & $\begin{array}{l}110 \\
208\end{array}$ & $\begin{array}{l}11 \\
16\end{array}$ & $\begin{array}{l}133 \\
109\end{array}$ & $\begin{array}{l}2 \\
3\end{array}$ & $\begin{array}{l}\text { (D) } \\
\text { (D) }\end{array}$ & $\begin{array}{r}13 \\
(\mathrm{NA})\end{array}$ & $\begin{array}{l}\text { (D) } \\
\text { (D) }\end{array}$ \\
\hline \multicolumn{9}{|l|}{ COUNTIES, 1987} \\
\hline $\begin{array}{l}\text { Kent } \\
\text { Sussex }\end{array}$ & $\begin{array}{l}12 \\
10\end{array}$ & $\begin{array}{l}65 \\
45\end{array}$ & $\begin{array}{l}7 \\
4\end{array}$ & $\begin{array}{l}\text { (D) } \\
\text { (D) }\end{array}$ & $\overline{2}$ & (D) & $\begin{array}{l}7 \\
6\end{array}$ & ${ }^{3}$ \\
\hline
\end{tabular}

Table 18. Angora Goats-Inventory and Sales: 1987 and 1982

[Not pubished for this State]

Table 19. Mink and Their Pelts - Inventory and Sales: 1987 and 1982 [Not published for this State]

Table 20. Colonies of Bees and Honey-Inventory and Sales: 1987 and 1982

[For meaning of abbreviations and symbols, see introductory text]

\begin{tabular}{|c|c|c|c|c|c|c|c|c|}
\hline \multirow{3}{*}{ Geographic area } & \multirow{2}{*}{\multicolumn{2}{|c|}{ Inventory }} & \multicolumn{6}{|c|}{ Sales } \\
\hline & & & \multicolumn{2}{|c|}{ Colonies of bees } & \multicolumn{2}{|c|}{ Honey } & \multirow[b]{2}{*}{ Farms } & \multirow{2}{*}{$\begin{array}{l}\text { Total sales } \\
(\$ 1,000)\end{array}$} \\
\hline & Farms & Number & Farms & Number & Farms & Pounds & & \\
\hline \multicolumn{9}{|l|}{ STATE TOTAL } \\
\hline $\begin{array}{r}\text { Delaware ............ } 1987 . . \\
1982 \ldots\end{array}$ & $\begin{array}{l}65 \\
68\end{array}$ & $\begin{array}{l}843 \\
832\end{array}$ & $\begin{array}{l}5 \\
3\end{array}$ & $\begin{array}{c}101 \\
\text { (D) }\end{array}$ & $\begin{array}{l}20 \\
15\end{array}$ & $\begin{array}{l}17916 \\
13265\end{array}$ & $\begin{array}{r}23 \\
\text { (NA) }\end{array}$ & $\begin{array}{l}19 \\
\text { (D) }\end{array}$ \\
\hline \multicolumn{9}{|l|}{ COUNTIES, 1987} \\
\hline $\begin{array}{l}\text { Kent } \\
\text { New Castle ............... }\end{array}$ & $\begin{array}{r}27 \\
9\end{array}$ & $\begin{array}{r}364 \\
73\end{array}$ & 1 & (D) & $\begin{array}{l}6 \\
3\end{array}$ & $\begin{array}{l}6794 \\
2800\end{array}$ & $\begin{array}{l}7 \\
3 \\
3\end{array}$ & $\stackrel{9}{9}$ \\
\hline Sussex ................................ & 29 & 406 & 4 & (D) & 11 & 8322 & 13 & 7 \\
\hline
\end{tabular}


Table 21. Fish Sales: 1987 and 1982

[Not published for this State]

Table 22. Miscellaneous Poultry-Inventory and Sales: 1987 and 1982

[For meaning of abbreviations and symbols, see introductory text]

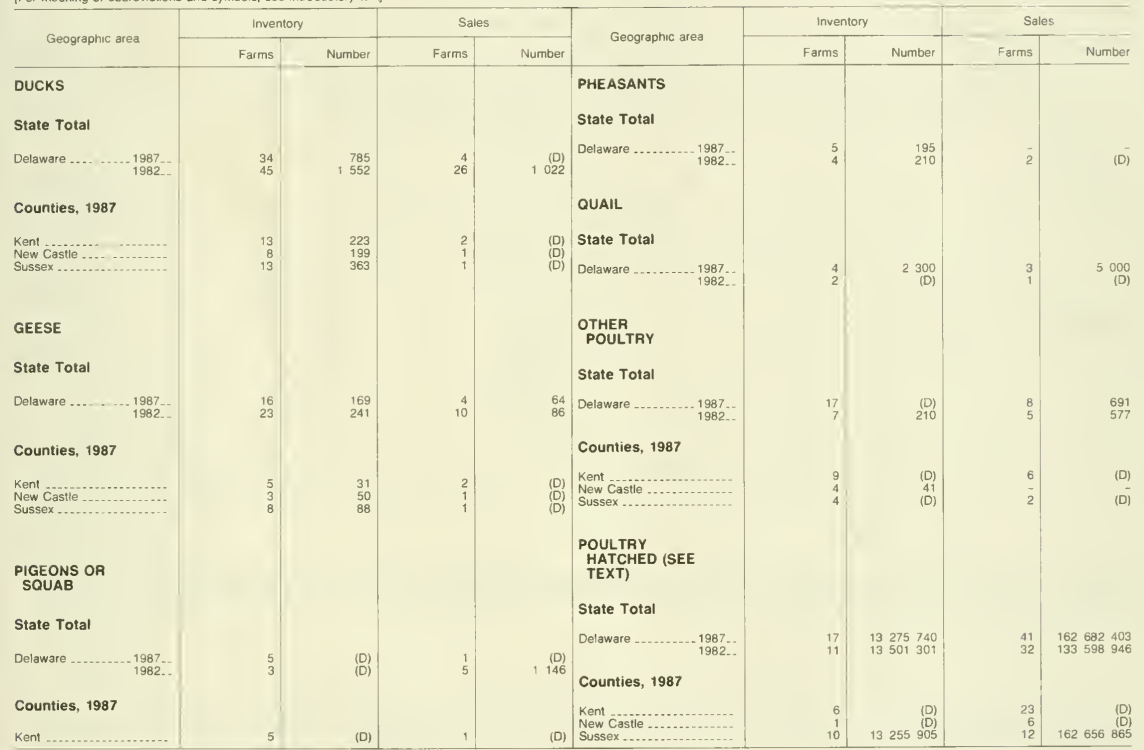

\section{Table 23. Miscellaneous Livestock and Animal Specialties-Inventory and}

\section{Sales: 1987 and 1982}

[For meaning of abbreviations and symbols, see introductory text]

\begin{tabular}{|c|c|c|c|c|c|c|c|c|c|c|c|}
\hline \multirow{2}{*}{ Geographic area } & \multicolumn{2}{|c|}{ Inventory } & \multicolumn{3}{|c|}{ Sales } & \multirow[b]{2}{*}{ Geographic area } & \multicolumn{2}{|c|}{ Inventory } & \multicolumn{3}{|c|}{ Sales } \\
\hline & Farms & Number & Farms & Number & $\begin{array}{r}\text { Sales } \\
(\$ 1,000) \\
\end{array}$ & & Farms & Number & Farms & Number & $\begin{array}{r}\text { Sales } \\
(\$ 1,000) \\
\end{array}$ \\
\hline $\begin{array}{l}\text { MULES, } \\
\text { BURAOS, AND } \\
\text { DONKEYS } \\
\text { State Total }\end{array}$ & & & & & & $\begin{array}{l}\text { MULES, } \\
\text { BURROS, AND } \\
\text { DONKEYS- } \\
\text { Con. }\end{array}$ & & & & & \\
\hline $\begin{array}{l}\text { Delaware ........... } 1987 . . \\
1982 . . \\
\text { Counties, } 1987\end{array}$ & $\begin{array}{r}22 \\
7\end{array}$ & $\begin{array}{l}43 \\
14\end{array}$ & $\begin{array}{l}2 \\
3\end{array}$ & (D) 7 & (D) & $\begin{array}{l}\text { Counties, 1987- } \\
\text { Con. }\end{array}$ & & & & & \\
\hline $\begin{array}{l}\text { Kent } \\
\text { New Castle }\end{array}$ & $\begin{array}{l}9 \\
6\end{array}$ & $\begin{array}{r}21 \\
9\end{array}$ & 1 & (D) & (D) & Sussex ................ & 7 & 13 & 1 & (D) & (D) \\
\hline
\end{tabular}


Table 23. Miscellaneous Livestock and Animal Specialties - Inventory and Sales: 1987 and $1982-$ Con.

[For meaning of abbreviations and symbols, see introductory text]

\begin{tabular}{|c|c|c|c|c|c|c|c|c|c|c|c|}
\hline \multirow[b]{2}{*}{ Geographic area } & \multicolumn{2}{|c|}{ Inventory } & \multicolumn{3}{|c|}{ Sales } & \multirow{2}{*}{ Geographic area } & \multicolumn{2}{|c|}{ Inventory } & \multicolumn{3}{|c|}{ Sales } \\
\hline & Farms & Number & Farms & Number & $\begin{array}{r}\text { Sales } \\
(\$ 1,000)\end{array}$ & & Farms & Number & Farms & Number & $\begin{array}{r}\text { Sales } \\
(\$ 1,000)\end{array}$ \\
\hline State Total & & & & & . & $\begin{array}{l}\text { GOATS, EXCEPT } \\
\text { ANGORA AND } \\
\text { MILK-COn. } \\
\text { Counties, } 1987\end{array}$ & & & & & \\
\hline Delaware ........ 1987.. & 62 & & 29 & 326 & (NA) & $\begin{array}{l}\text { Kent } \\
\text { New Castle } \\
\text { Sussex } \ldots \ldots \ldots \ldots\end{array}$ & $\begin{array}{r}15 \\
5 \\
27\end{array}$ & $\begin{array}{r}51 \\
14 \\
290\end{array}$ & $\begin{array}{r}2 \\
2 \\
15\end{array}$ & $\begin{array}{l}\text { (D) } \\
\text { (D) } \\
\text { (D) }\end{array}$ & $\begin{array}{l}\text { (D) } \\
\text { (D) } \\
7\end{array}$ \\
\hline Delaware .......... 1987.. & $\begin{array}{l}62 \\
62\end{array}$ & $\begin{array}{l}465 \\
409\end{array}$ & 29 & 153 & (NA) & $\begin{array}{l}\text { RABBITS AND } \\
\text { THEIR PELTS }\end{array}$ & 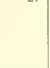 & 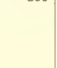 & 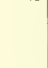 & 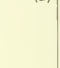 & \\
\hline Counties, 1987 & & & & & & State Total & & & & & \\
\hline $\begin{array}{l}\text { Kent } \\
\text { New Castle } \\
\text { Sussex }\end{array}$ & $\begin{array}{r}25 \\
5 \\
32\end{array}$ & $\begin{array}{r}116 \\
14 \\
335\end{array}$ & $\begin{array}{r}9 \\
2 \\
18\end{array}$ & $\begin{array}{l}(D) \\
\text { (D) } \\
213\end{array}$ & $\begin{array}{l}\text { (NA) } \\
\text { (NA) } \\
\text { (NA) }\end{array}$ & $\begin{array}{l}\text { Delaware ......... } 1987 . . \\
\text { Counties, } 1987\end{array}$ & $\begin{array}{l}19 \\
14\end{array}$ & $\begin{array}{r}974 \\
1010\end{array}$ & $\begin{array}{l}8 \\
8\end{array}$ & $\begin{array}{r}1925 \\
\text { (D) }\end{array}$ & $\begin{array}{l}12 \\
\text { (D) }\end{array}$ \\
\hline & & & & & & $\begin{array}{l}\text { Kent } \\
\text { New Castle } \\
\text { Sussex }\end{array}$ & $\begin{array}{l}7 \\
5 \\
7\end{array}$ & $\begin{array}{l}468 \\
\text { (D) } \\
\text { (D) }\end{array}$ & $\begin{array}{l}3 \\
2 \\
3\end{array}$ & $\begin{array}{r}720 \\
(D) \\
(D)\end{array}$ & $\begin{array}{l}\text { (D) } \\
\text { (D) } \\
\text { (D) }\end{array}$ \\
\hline $\begin{array}{l}\text { GOATS, EXCEPT } \\
\text { ANGORA AND } \\
\text { MILK }\end{array}$ & & & & & & $\begin{array}{l}\text { OTHER } \\
\text { LIVESTOCK } \\
\text { AND } \\
\text { LIVESTOCK } \\
\text { PRODUCTS }\end{array}$ & & & & & \\
\hline & & & & & & State Total & & & & & \\
\hline $\begin{array}{r}\text { Delaware } \ldots . . .1987 \ldots . . . \\
1982 . .\end{array}$ & $\begin{array}{l}47 \\
28\end{array}$ & $\begin{array}{r}355 \\
\text { (D) }\end{array}$ & $\begin{array}{l}19 \\
12\end{array}$ & $\begin{array}{r}193 \\
\text { (D) }\end{array}$ & (D) & $\begin{array}{r}\text { Delaware ....... } 1987 \ldots \\
1982 \ldots\end{array}$ & (NA) & $\begin{array}{l}(x) \\
(x)\end{array}$ & $\begin{array}{r}5 \\
\text { (NA) }\end{array}$ & $\begin{array}{l}(x) \\
(x)\end{array}$ & $\stackrel{9}{-}$ \\
\hline
\end{tabular}

Table 24. Grains-Corn, Sorghum, Wheat, and Other Small Grains: 1987 and 1982

[For meaning of abbreviations and symbols, see introductory text]

\begin{tabular}{|c|c|c|c|c|c|c|c|c|c|c|}
\hline \multirow{3}{*}{ Geographic area } & \multicolumn{5}{|c|}{1987} & \multicolumn{5}{|c|}{1982} \\
\hline & \multicolumn{3}{|c|}{ Harvested } & \multicolumn{2}{|c|}{ Irrigated } & \multicolumn{3}{|c|}{ Harvested } & \multicolumn{2}{|c|}{ Imigated } \\
\hline & Farms & Acres & Quantity & Farms & Acres & Farms & Acres & Quantity & Farms & Acres \\
\hline \multicolumn{11}{|l|}{$\begin{array}{l}\text { CORN FOR GRAIN OR SEED } \\
\text { (BUSHELS) }\end{array}$} \\
\hline \multicolumn{11}{|l|}{ State Total } \\
\hline $\begin{array}{l}\text { Delaware } \\
\text { Counties }\end{array}$ & 1076 & 130234 & 9876539 & 145 & 22314 & 1363 & $\cdot 150676$ & 14880293 & 132 & 17771 \\
\hline $\begin{array}{l}\text { Kent } \\
\text { New Castle } \\
\text { Sussex }\end{array}$ & $\begin{array}{l}332 \\
195 \\
549\end{array}$ & $\begin{array}{ll}38 & 846 \\
34 & 367 \\
57 & 021\end{array}$ & $\begin{array}{lll}2 & 585 & 645 \\
2 & 717 & 813 \\
4 & 573 & 081\end{array}$ & $\begin{array}{r}21 \\
6 \\
118\end{array}$ & $\begin{array}{r}\text { (D) } \\
\text { (D) } \\
18250\end{array}$ & $\begin{array}{l}495 \\
262 \\
606\end{array}$ & $\begin{array}{l}52712 \\
37996 \\
59966\end{array}$ & $\begin{array}{lll}5 & 018 & 061 \\
4 & 026 & 275 \\
5 & 835 & 957\end{array}$ & $\begin{array}{l}25 \\
10 \\
97\end{array}$ & $\begin{array}{r}\text { (D) } \\
\text { (D) } \\
13594\end{array}$ \\
\hline \multicolumn{11}{|l|}{$\begin{array}{l}\text { SORGHUM FOR GRAIN OR } \\
\text { SEED (BUSHELS) }\end{array}$} \\
\hline \multicolumn{11}{|l|}{ State Total } \\
\hline \multicolumn{11}{|l|}{$\begin{array}{l}\text { WHEAT FOR GRAIN } \\
\text { (BUSHELS) }\end{array}$} \\
\hline \multicolumn{11}{|l|}{ State Total } \\
\hline Delaware $\ldots . . . . . . . . . . . . .$. & 630 & 43573 & 1827869 & 33 & 2604 & 783 & 56867 & 2234788 & 23 & 2136 \\
\hline \multicolumn{11}{|l|}{ Counties } \\
\hline $\begin{array}{l}\text { Kent } \\
\text { New Castle } \\
\text { Sussex }\end{array}$ & $\begin{array}{r}227 \\
85 \\
318\end{array}$ & $\begin{array}{rr}15 & 416 \\
3 & 726 \\
24 & 431\end{array}$ & $\begin{array}{r}643694 \\
176277 \\
1007898\end{array}$ & $\overline{3}$ & 2604 & $\begin{array}{l}313 \\
101 \\
369\end{array}$ & $\begin{array}{r}20882 \\
6046 \\
29939\end{array}$ & $\begin{array}{r}760073 \\
229816 \\
+244899\end{array}$ & $\begin{array}{r}3 \\
20\end{array}$ & $\begin{array}{r}246 \\
1890\end{array}$ \\
\hline
\end{tabular}


Table 24. Grains-Corn, Sorghum, Wheat, and Other Small Grains: 1987 and 1982-Con.

[For meaning of abbreviations and symbols, see introductory text]

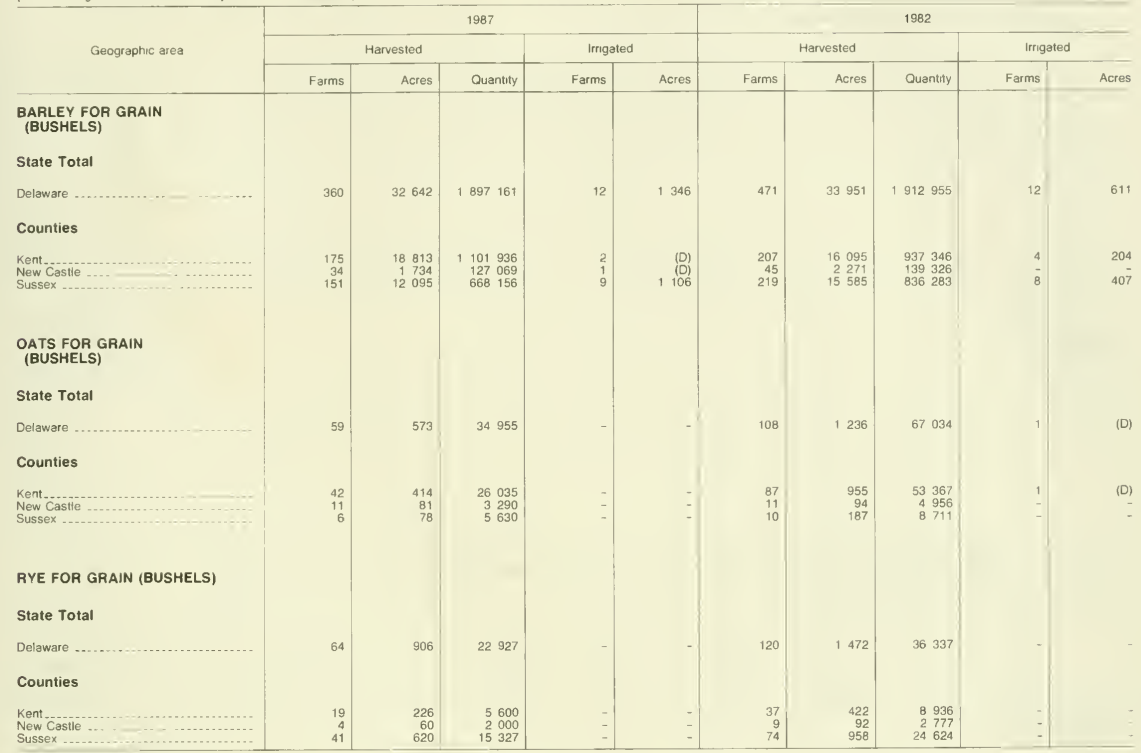

\section{Table 25. Cotton, Tobacco, Soybeans, Dry Beans and Peas, Potatoes, Sugar Crops, and Peanuts: 1987 and 1982}

[For meaning of abbreviations and symbols, see introductory text]

\begin{tabular}{|c|c|c|c|c|c|c|c|c|c|c|}
\hline \multirow{3}{*}{ Geographic area } & \multicolumn{5}{|c|}{1987} & \multicolumn{5}{|c|}{1982} \\
\hline & \multicolumn{3}{|c|}{ Harvested } & \multicolumn{2}{|c|}{ Irrigated } & \multicolumn{3}{|c|}{ Harvested } & \multicolumn{2}{|c|}{ Imigated } \\
\hline & Farms & Acres & Quantity & Farms & Acres & Farms & Acres & Quantity & Farms & Acres \\
\hline \multicolumn{11}{|l|}{$\begin{array}{l}\text { SOYBEANS FOR BEANS } \\
\text { (BUSHELS) }\end{array}$} \\
\hline \multicolumn{11}{|l|}{ State Total } \\
\hline Delaware $\ldots$............. & 1515 & 219941 & 4143975 & 117 & 11763 & 1991 & 264971 & $6 \quad 373519$ & 84 & 7012 \\
\hline $\begin{array}{l}\text { Kent } \\
\text { New Castle } \\
\text { Sussex }\end{array}$ & $\begin{array}{l}469 \\
147 \\
899\end{array}$ & $\begin{array}{rr}70 & 261 \\
17 & 146 \\
132 & 534\end{array}$ & $\begin{array}{r}1249800 \\
453550 \\
2440625\end{array}$ & $\begin{array}{r}24 \\
5 \\
88\end{array}$ & $\begin{array}{r}1860 \\
300 \\
9603\end{array}$ & $\begin{array}{r}617 \\
208 \\
1166\end{array}$ & $\begin{array}{rr}76 & 071 \\
26 & 625 \\
162 & 275\end{array}$ & $\begin{array}{r}1634937 \\
752240 \\
3986342\end{array}$ & $\begin{array}{r}21 \\
3 \\
60\end{array}$ & $\begin{array}{l}\text { (D) } \\
\text { (D) } \\
5074\end{array}$ \\
\hline \multicolumn{11}{|l|}{$\begin{array}{l}\text { DRY EDIBLE BEANS, } \\
\text { EXCLUDING DRY LIMAS } \\
\text { (CWT) }\end{array}$} \\
\hline \multicolumn{11}{|l|}{ State Total } \\
\hline Delaware .... & 3 & (D) & (D) & 1 & (D) & 4 & 172 & 1900 & -1 & \\
\hline
\end{tabular}


Table 25. Cotton, Tobacco, Soybeans, Dry Beans and Peas, Potatoes, Sugar Crops, and Peanuts: 1987 and $1982-$ Con.

[For meaning of abbreviations and symbols, see introductory text]

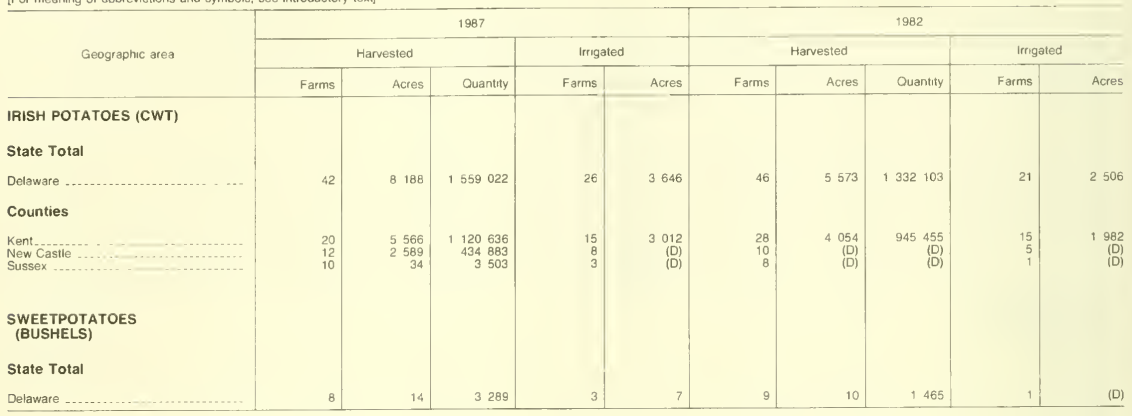

Table 26. Field Seeds, Grass Seeds, Hay, Forage, and Silage: 1987 and 1982

[For meaning of abbreviations and symbols, see introductory text]

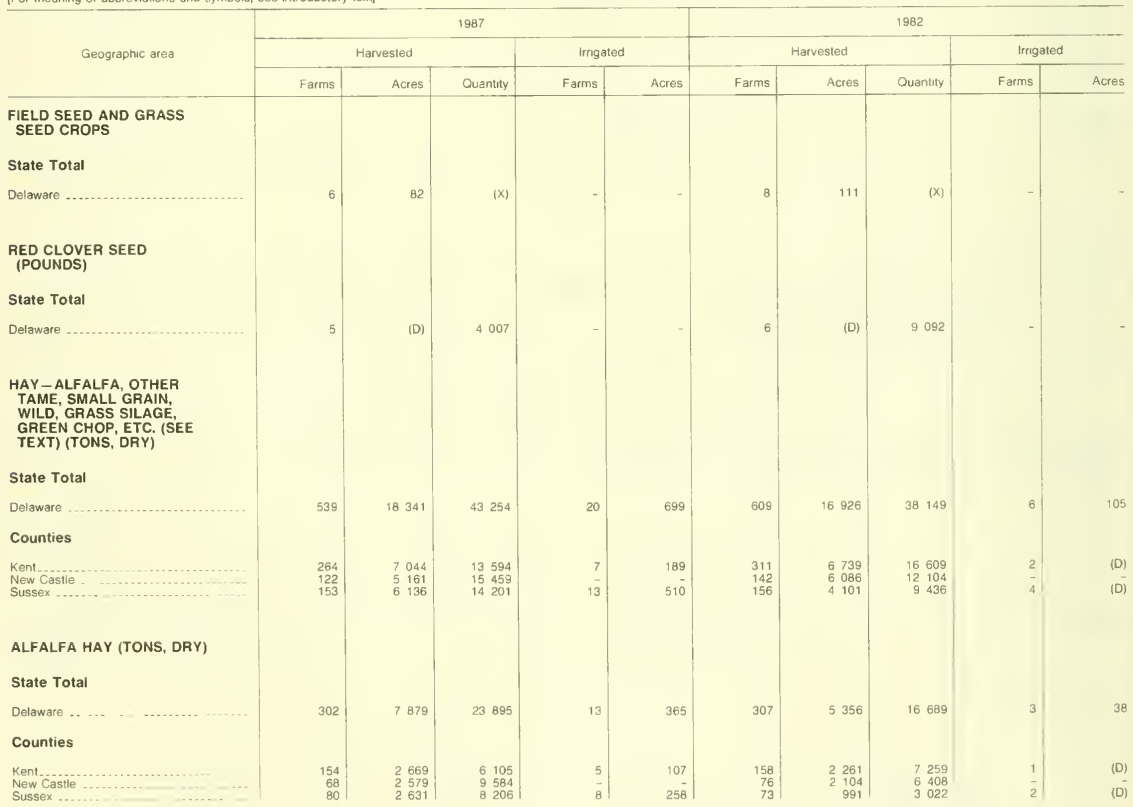

168 DELAWARE

1987 CENSUS OF AGRICULTURE -COUNTY DATA 
Table 26. Field Seeds, Grass Seeds, Hay, Forage, and Silage: 1987 and 1982-Con.

[For meaning of abbrevations and symbols, see introductory text]

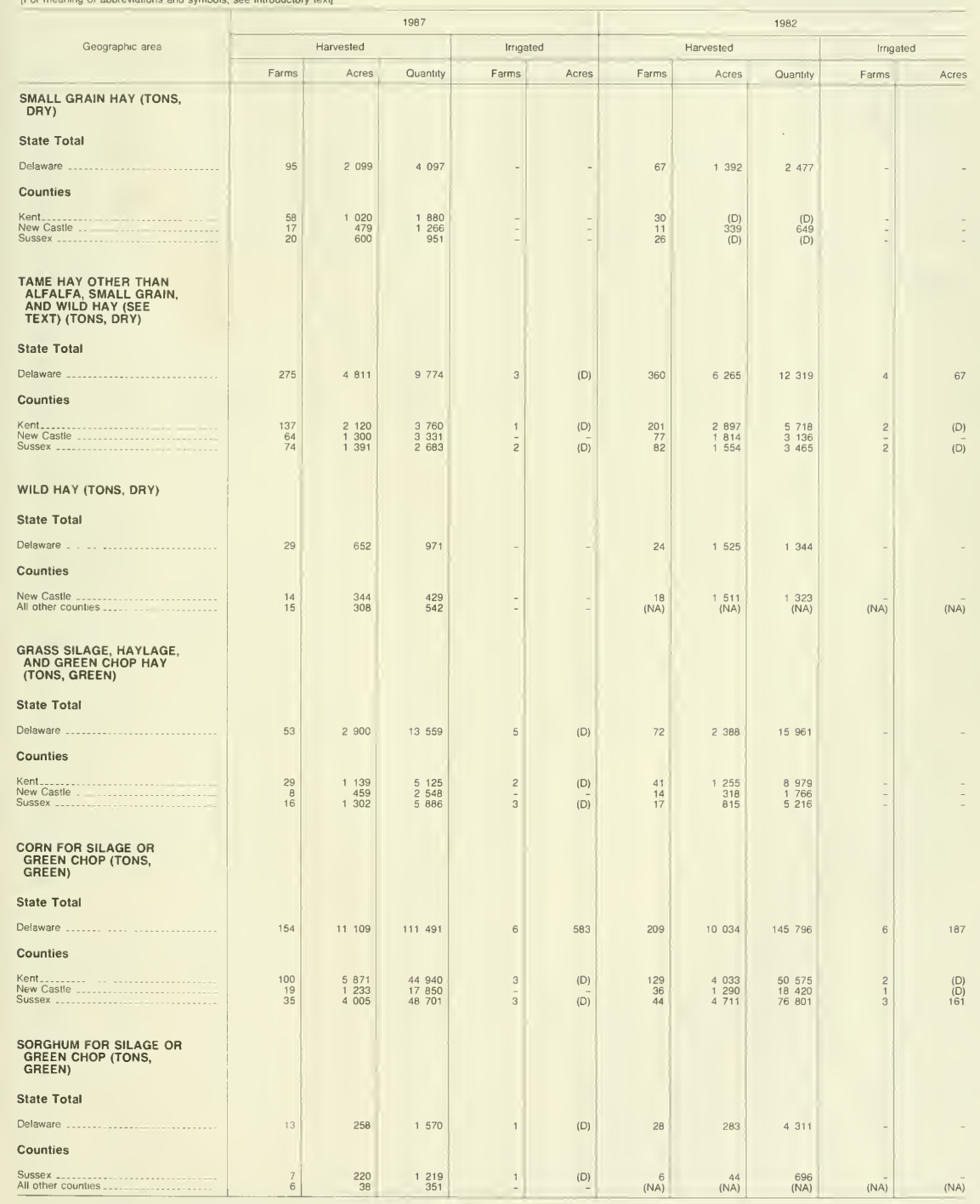


Table 27. Vegetables, Sweet Corn, and Melons Harvested for Sale: 1987 and 1982

[For meaning of abbreviations and symbols, see introductory text]

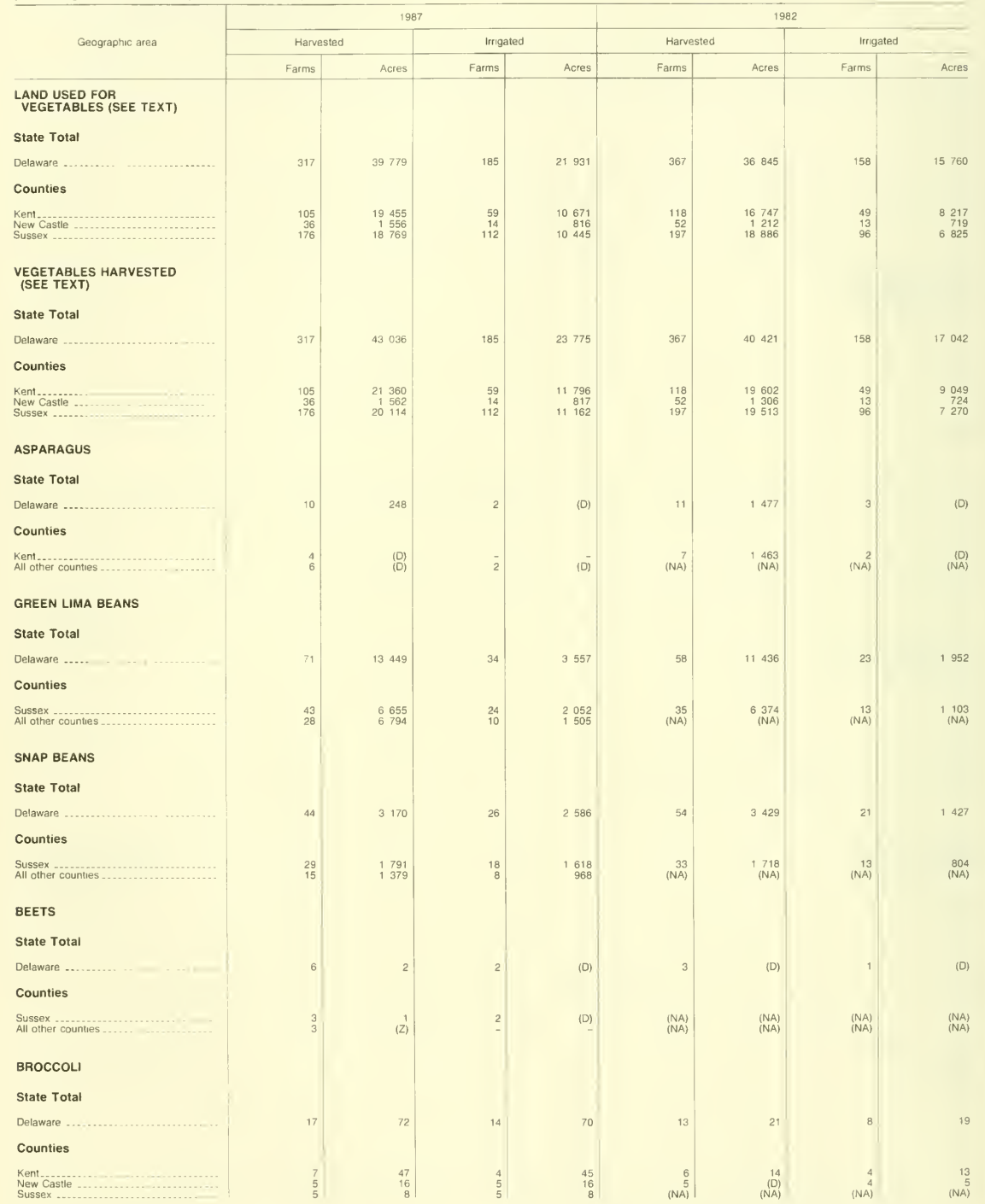


Table 27. Vegetables, Sweet Corn, and Melons Harvested for Sale: 1987 and 1982-Con.

[For meaning of abbreviations and symbols, see introductory text]

\begin{tabular}{|c|c|c|c|c|c|c|c|c|}
\hline \multirow{3}{*}{ Geographic area } & \multicolumn{4}{|c|}{1987} & \multicolumn{4}{|c|}{1982} \\
\hline & \multicolumn{2}{|c|}{ Harvested } & \multicolumn{2}{|c|}{ Irrigated } & \multicolumn{2}{|c|}{ Harvested } & \multicolumn{2}{|c|}{ Irrigated } \\
\hline & Farms & Acres & Farms & Acres & Farms & Acres & Farms & Acres \\
\hline \multicolumn{9}{|l|}{ HEAD CABBAGE } \\
\hline \multicolumn{9}{|l|}{ State Total } \\
\hline Delaware ............. & 15 & (D) & 11 & (D) & 18 & 508 & 12 & 479 \\
\hline \multicolumn{9}{|l|}{ Counties } \\
\hline $\begin{array}{l}\text { Kent } \\
\text { New Castle } \\
\text { Sussex }\end{array}$ & $\begin{array}{l}6 \\
4 \\
5\end{array}$ & $\begin{array}{l}\text { (D) } \\
(\mathrm{D}) \\
11\end{array}$ & $\begin{array}{l}4 \\
3 \\
4\end{array}$ & $\begin{array}{l}\text { (D) } \\
\text { (D) } \\
10\end{array}$ & $\begin{array}{l}6 \\
7 \\
5\end{array}$ & $\begin{array}{l}\text { (D) } \\
26 \\
\text { (D) }\end{array}$ & $\begin{array}{l}4 \\
5 \\
3\end{array}$ & $\begin{array}{l}\text { (D) } \\
26 \\
\text { (D) }\end{array}$ \\
\hline \multicolumn{9}{|l|}{ CANTALOUPS } \\
\hline \multicolumn{9}{|l|}{ State Total } \\
\hline Delaware... & 72 & 284 & 51 & 190 & 74 & 298 & 45 & 153 \\
\hline \multicolumn{9}{|l|}{ Counties } \\
\hline $\begin{array}{l}\text { Kent } \\
\text { New Castle ... }\end{array}$ & $\begin{array}{r}14 \\
7\end{array}$ & $\begin{array}{l}47 \\
40\end{array}$ & $\begin{array}{l}9 \\
4\end{array}$ & (D) & $\begin{array}{r}14 \\
6\end{array}$ & $\begin{array}{r}58 \\
7\end{array}$ & $\begin{array}{l}8 \\
4\end{array}$ & (D) \\
\hline Sussex ....... & 51 & 198 & 38 & 147 & 54 & 233 & 33 & 123 \\
\hline \multicolumn{9}{|l|}{ CARROTS } \\
\hline \multicolumn{9}{|l|}{ State Total } \\
\hline Delaware ...... & 10 & 680 & 6 & 472 & 10 & 1193 & 5 & 238 \\
\hline \multicolumn{9}{|l|}{ CAULIFLOWER } \\
\hline \multicolumn{9}{|l|}{ State Total } \\
\hline Delaware ...... & 12 & 73 & 9 & 72 & 15 & 101 & 12 & 98 \\
\hline \multicolumn{9}{|l|}{ Counties } \\
\hline $\begin{array}{l}\text { New Castle } \\
\text { All other counties...................... }\end{array}$ & $\begin{array}{l}6 \\
6\end{array}$ & $\begin{array}{r}5 \\
68\end{array}$ & $\begin{array}{l}4 \\
5\end{array}$ & $\begin{array}{r}5 \\
67\end{array}$ & (NA) & $\begin{array}{l}(\mathrm{D}) \\
\text { (NA) }\end{array}$ & $\begin{array}{r}6 \\
\text { (NA) }\end{array}$ & $\begin{array}{l}(\mathrm{D}) \\
(\mathrm{NA})\end{array}$ \\
\hline \multicolumn{9}{|l|}{ COLLARDS } \\
\hline \multicolumn{9}{|l|}{ State Total } \\
\hline Delaware ..... & 4 & 2 & 2 & (D) & 2 & (D) & 1 & (D) \\
\hline \multicolumn{9}{|l|}{$\begin{array}{l}\text { GREEN COWPEAS AND } \\
\text { GREEN SOUTHERN PEAS }\end{array}$} \\
\hline State Total & & & & & & & - & \\
\hline Delaware .... & 3 & (D) & 3 & (D) & 3 & (D) & - & - \\
\hline \multicolumn{9}{|l|}{ CUCUMBERS AND PICKLES } \\
\hline \multicolumn{9}{|l|}{ State Total } \\
\hline 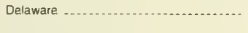 & 31 & 1985 & 19 & 1825 & 67 & 1755 & 43 & 1723 \\
\hline \multicolumn{9}{|l|}{ Counties } \\
\hline $\begin{array}{l}\text { Kent } \\
\text { All other counties }\end{array}$ & $\begin{array}{l}20 \\
11\end{array}$ & $\begin{array}{r}1560 \\
424\end{array}$ & $\begin{array}{r}13 \\
6\end{array}$ & $\begin{array}{r}1412 \\
413\end{array}$ & $\begin{array}{r}27 \\
\text { (NA) }\end{array}$ & $\begin{array}{r}1342 \\
\text { (NA) }\end{array}$ & $\begin{array}{r}15 \\
(\mathrm{NA})\end{array}$ & $\begin{array}{l}1327 \\
\text { (NA) }\end{array}$ \\
\hline \multicolumn{9}{|l|}{ OKRA } \\
\hline State Total & & & & & & & & \\
\hline Delaware... & 4 & 1 & 21 & (D) & 5 & 2 & 2 & (D) \\
\hline
\end{tabular}


Table 27. Vegetables, Sweet Corn, and Melons Harvested for Sale: 1987 and 1982-Con.

[For meaning of abbreviations and symbols, see introductory text]

\begin{tabular}{|c|c|c|c|c|c|c|c|c|}
\hline \multirow{3}{*}{ Geographic area } & \multicolumn{4}{|c|}{1987} & \multicolumn{4}{|c|}{1982} \\
\hline & \multicolumn{2}{|c|}{ Harvested } & \multicolumn{2}{|c|}{ irrigated } & \multicolumn{2}{|c|}{ Harvested } & \multicolumn{2}{|c|}{ Imigated } \\
\hline & Farms & Acres & Farms & Acres & Farms & Acres & Farms & Acres \\
\hline \multicolumn{9}{|l|}{$\begin{array}{l}\text { GREEN PEAS, EXCLUDING } \\
\text { GREEN COWPEAS }\end{array}$} \\
\hline \multicolumn{9}{|l|}{ State Total } \\
\hline $\begin{array}{l}\text { Delaware } \\
\text { Counties }\end{array}$ & \multicolumn{4}{|c|}{ Counties } & 67 & 10093 & 35 & 4426 \\
\hline $\begin{array}{l}\text { Kent } \\
\text { New Castie } \\
\text { Sussex } \ldots \ldots\end{array}$ & $\begin{array}{r}30 \\
3 \\
33\end{array}$ & $\begin{array}{r}5555 \\
235 \\
4061\end{array}$ & $\begin{array}{r}18 \\
3 \\
24\end{array}$ & $\begin{array}{l}3213 \\
235 \\
2304\end{array}$ & $\begin{array}{l}26 \\
10 \\
31\end{array}$ & $\begin{array}{r}5647 \\
394 \\
4052\end{array}$ & $\begin{array}{r}16 \\
4 \\
15\end{array}$ & $\begin{array}{l}2612 \\
290 \\
1525\end{array}$ \\
\hline \multicolumn{9}{|l|}{ HOT PEPPERS } \\
\hline \multicolumn{9}{|l|}{ State Total } \\
\hline $\begin{array}{l}\text { Delaware } \ldots . . .1 \ldots \ldots \\
\text { Counties }\end{array}$ & \multicolumn{3}{|c|}{ Counties } & 63 & 40 & 96 & 8 & 25 \\
\hline $\begin{array}{l}\text { Kent } \\
\text { New Castie } \\
\text { Sussex }\end{array}$ & $\begin{array}{r}31 \\
3 \\
13\end{array}$ & $\begin{array}{l}137 \\
\text { (D) } \\
\text { (D) }\end{array}$ & $\begin{array}{r}14 \\
1 \\
7\end{array}$ & $\begin{array}{l}50 \\
(\mathrm{D}) \\
\text { (D) }\end{array}$ & $\begin{array}{r}35 \\
3 \\
\text { (NA) }\end{array}$ & $\begin{array}{r}88 \\
\text { (D) } \\
\text { (NA) }\end{array}$ & $\begin{array}{r}7 \\
\text { (NA) }\end{array}$ & $\begin{array}{l}\text { (D) } \\
\text { (NA) }\end{array}$ \\
\hline \multicolumn{9}{|l|}{ SWEET PEPPERS } \\
\hline \multicolumn{9}{|l|}{ State Total } \\
\hline Delaware ............. & 36 & 142 & 24 & 84 & 38 & 133 & 16 & 62 \\
\hline $\begin{array}{l}\text { Kent } \\
\text { New Castie } \\
\text { Sussex }\end{array}$ & $\begin{array}{r}21 \\
3 \\
12\end{array}$ & $\begin{array}{r}123 \\
1 \\
18\end{array}$ & $\begin{array}{r}16 \\
1 \\
7\end{array}$ & $\begin{array}{r}69 \\
(D) \\
(D)\end{array}$ & $\begin{array}{r}21 \\
6 \\
11\end{array}$ & $\begin{array}{r}110 \\
3 \\
20\end{array}$ & $\begin{array}{l}7 \\
2 \\
7\end{array}$ & $\begin{array}{l}44 \\
\text { (D) } \\
\text { (D) }\end{array}$ \\
\hline \multicolumn{9}{|l|}{ PUMPKINS } \\
\hline $\begin{array}{l}\text { Delaware ...... } \\
\text { Counties }\end{array}$ & 33 & 120 & 16 & 80 & 22 & 43 & 7 & 20 \\
\hline $\begin{array}{l}\text { Kent } \\
\text { New Castle } \\
\text { Sussex }\end{array}$ & $\begin{array}{r}7 \\
5 \\
21\end{array}$ & $\begin{array}{r}39 \\
9 \\
73\end{array}$ & $\begin{array}{r}5 \\
11\end{array}$ & 32 & $\begin{array}{r}\text { (NA) } \\
5 \\
15\end{array}$ & $\begin{array}{r}(\mathrm{NA}) \\
(\mathrm{D}) \\
26\end{array}$ & $\begin{array}{r}\text { (NA) } \\
1 \\
5\end{array}$ & $\begin{array}{l}\text { (NA) } \\
\text { (D) } \\
\text { (D) }\end{array}$ \\
\hline \multicolumn{9}{|l|}{ SPINACH } \\
\hline 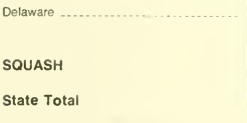 & 5 & 501 & 5 & 501 & 4 & (D) & 3 & 395 \\
\hline $\begin{array}{l}\text { Delaware .......... } \\
\text { Counties }\end{array}$ & 16 & 247 & 10 & 102 & 18 & 166 & 9 & 82 \\
\hline $\begin{array}{l}\text { Kent ........ } \\
\text { Sussex }\end{array}$ & $\begin{array}{r}5 \\
11\end{array}$ & (D) & $\begin{array}{l}3 \\
7\end{array}$ & (D) & $\begin{array}{r}3 \\
13\end{array}$ & (D) & $\begin{array}{l}2 \\
5\end{array}$ & $\begin{array}{l}\text { (D) } \\
\text { (D) }\end{array}$ \\
\hline SWEET CORN & & & & & & & & \\
\hline State Total & & & & & & & & \\
\hline $\begin{array}{l}\text { Delaware .... } \\
\text { Counties }\end{array}$ & 109 & 7643 & 68 & 5739 & 140 & 6120 & 64 & 4043 \\
\hline $\begin{array}{l}\text { Kent } \\
\text { New Castle } \\
\text { Sussex } \ldots \ldots \ldots \ldots \ldots \ldots\end{array}$ & $\begin{array}{l}30 \\
24 \\
55\end{array}$ & $\begin{array}{ll}3 & 681 \\
1 & 001 \\
2 & 962\end{array}$ & $\begin{array}{r}21 \\
8 \\
39\end{array}$ & $\begin{array}{l}3166 \\
327 \\
2246\end{array}$ & $\begin{array}{l}35 \\
39 \\
66\end{array}$ & $\begin{array}{l}2451 \\
595 \\
3075\end{array}$ & $\begin{array}{r}19 \\
7 \\
38\end{array}$ & $\begin{array}{l}1958 \\
233 \\
1853\end{array}$ \\
\hline
\end{tabular}

172 DELAWARE 
Table 27. Vegetables, Sweet Corn, and Melons Harvested for Sale: 1987 and 1982-Con.

[For meaning of abbreviations and symbols, see introductory text]

\begin{tabular}{|c|c|c|c|c|c|c|c|c|}
\hline \multirow{3}{*}{ Geographic area } & \multicolumn{4}{|c|}{1987} & \multicolumn{4}{|c|}{1982} \\
\hline & \multicolumn{2}{|c|}{ Harvested } & \multicolumn{2}{|c|}{ Irrigated } & \multicolumn{2}{|c|}{ Harvested } & \multicolumn{2}{|c|}{ Imgated } \\
\hline & Farms & Acres & Farms & Acres & Farms & Acres & Farms & Acres \\
\hline \multicolumn{9}{|l|}{ TOMATOES } \\
\hline \multicolumn{9}{|l|}{ State Total } \\
\hline Delaware ..... & 63 & 887 & 38 & 661 & 74 & 1096 & 31 & 535 \\
\hline \multicolumn{9}{|l|}{ Counties } \\
\hline $\begin{array}{l}\text { Kent } \\
\text { New Castie } \\
\text { Sussex }\end{array}$ & $\begin{array}{r}8 \\
12 \\
43\end{array}$ & $\begin{array}{r}97 \\
41 \\
749\end{array}$ & $\begin{array}{r}5 \\
4 \\
29\end{array}$ & $\begin{array}{l}\text { (D) } \\
\text { (D) } \\
579\end{array}$ & $\begin{array}{l}16 \\
14 \\
44\end{array}$ & $\begin{array}{r}162 \\
43 \\
891\end{array}$ & $\begin{array}{r}4 \\
6 \\
21\end{array}$ & $\begin{array}{l}\text { (D) } \\
\text { (D) }\end{array}$ \\
\hline \multicolumn{9}{|l|}{ TURNIPS } \\
\hline \multicolumn{9}{|l|}{ State Total } \\
\hline Delaware .... & 7 & 37 & 2 & (D) & 10 & 14 & 5 & (D) \\
\hline \multicolumn{9}{|l|}{ Counties } \\
\hline $\begin{array}{l}\text { New Castle } \\
\text { All other counties ................ }\end{array}$ & $\begin{array}{l}3 \\
4\end{array}$ & $\begin{array}{l}25 \\
12\end{array}$ & $\overline{2}$ & (D) & (NA) & $\begin{array}{l}\text { (D) } \\
(\mathrm{NA})\end{array}$ & (NA) & $\begin{array}{l}\text { (D) } \\
\text { (NA) }\end{array}$ \\
\hline \multicolumn{9}{|l|}{ MIXED VEGETABLES } \\
\hline \multicolumn{9}{|l|}{ State Total } \\
\hline Delaware .... & 4 & 14 & 3 & 9 & 12 & 24 & 1 & (D) \\
\hline \multicolumn{9}{|l|}{ WATERMELONS } \\
\hline \multicolumn{9}{|l|}{ State Total } \\
\hline Delaware .... & 103 & 1589 & 70 & 1246 & 119 & 1311 & 62 & 923 \\
\hline \multicolumn{9}{|l|}{ Counties } \\
\hline 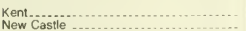 & $\begin{array}{r}13 \\
3\end{array}$ & 89 & $\begin{array}{l}8 \\
1\end{array}$ & (D) & $\begin{array}{r}13 \\
3\end{array}$ & $\begin{array}{r}112 \\
3\end{array}$ & $\begin{array}{l}6 \\
3\end{array}$ & $\begin{array}{r}69 \\
3\end{array}$ \\
\hline $\begin{array}{l}\text { New Castle } \\
\text { Sussex ........................................ }\end{array}$ & 87 & 1498 & 61 & 1227 & 103 & 1196 & 53 & $851^{3}$ \\
\hline \multicolumn{9}{|l|}{ OTHER VEGETABLES } \\
\hline \multicolumn{9}{|l|}{ State Total } \\
\hline Delaware ..... & 7 & 7 & 3 & 5 & 3 & (D) & 2 & (D) \\
\hline Counties & & & & & & & & \\
\hline $\begin{array}{l}\text { Kent } \\
\text { Sussex }\end{array}$ & $\begin{array}{l}3 \\
4\end{array}$ & $\begin{array}{l}4 \\
3\end{array}$ & $\stackrel{2}{1}$ & (D) & (NA) & $\begin{array}{l}\text { (NA) } \\
\text { (D) }\end{array}$ & $\begin{aligned}(N A) \\
2\end{aligned}$ & $\begin{array}{l}\text { (NA) } \\
\text { (D) }\end{array}$ \\
\hline
\end{tabular}

Table 28. Fruits and Nuts: 1987 and 1982

[For meaning of abbreviations and symbols, see introductory text]

\begin{tabular}{|c|c|c|c|c|c|c|c|c|c|}
\hline \multirow{2}{*}{ Geographic area } & \multicolumn{3}{|c|}{ Total } & \multicolumn{2}{|c|}{ Trees or vines not of bearing age } & \multicolumn{2}{|c|}{ Trees or vines of bearing age } & \multicolumn{2}{|c|}{ Harvested } \\
\hline & Farms & Acres & Trees or vines & Farms & Number & Farms & Number & Farms & Pounds \\
\hline \multicolumn{10}{|l|}{$\begin{array}{l}\text { LAND IN } \\
\text { ORCHARDS }\end{array}$} \\
\hline State Total & & & & & & & & & \\
\hline $\begin{array}{l}\text { Delaware ........... 1987 } \\
\text { Counties, } 1987\end{array}$ & $\begin{array}{l}36 \\
47\end{array}$ & $\begin{array}{ll}1 & 378 \\
1 & 289\end{array}$ & $\begin{array}{l}(x) \\
(x) \\
(x)\end{array}$ & $\begin{array}{l}(x) \\
(x)\end{array}$ & $\begin{array}{l}(x) \\
(x)\end{array}$ & $\begin{array}{l}(x) \\
(x) \\
(x)\end{array}$ & $\begin{array}{l}\left(\begin{array}{l}(X) \\
(x)\end{array}\right.\end{array}$ & $\begin{array}{l}(x) \\
(x)\end{array}$ & $\begin{array}{l}\left(\begin{array}{l}(x) \\
(x) \\
(x)\end{array}\right.\end{array}$ \\
\hline $\begin{array}{l}\text { Kent } \\
\text { New Castie } \\
\text { Sussex }\end{array}$ & $\begin{array}{r}13 \\
6 \\
17\end{array}$ & $\begin{array}{l}\text { (D) } \\
\text { (D) } \\
\text { (D) }\end{array}$ & $\begin{array}{l}\left(\begin{array}{l}x \\
(x) \\
(x)\end{array}\right) \\
(x)\end{array}$ & $\begin{array}{l}(x) \\
(X) \\
(X)\end{array}$ & $\begin{array}{l}(X) \\
(X) \\
(X)\end{array}$ & $\begin{array}{l}(X) \\
(X) \\
(X)\end{array}$ & $\begin{array}{l}(x) \\
(x) \\
(x)\end{array}$ & $\begin{array}{l}(X) \\
(X) \\
(X)\end{array}$ & $\begin{array}{l}(x) \\
(x) \\
(x)\end{array}$ \\
\hline
\end{tabular}


Table 28. Fruits and Nuts: 1987 and 1982-Con.

[For meaning of abbreviations and symbols, see introductory text]

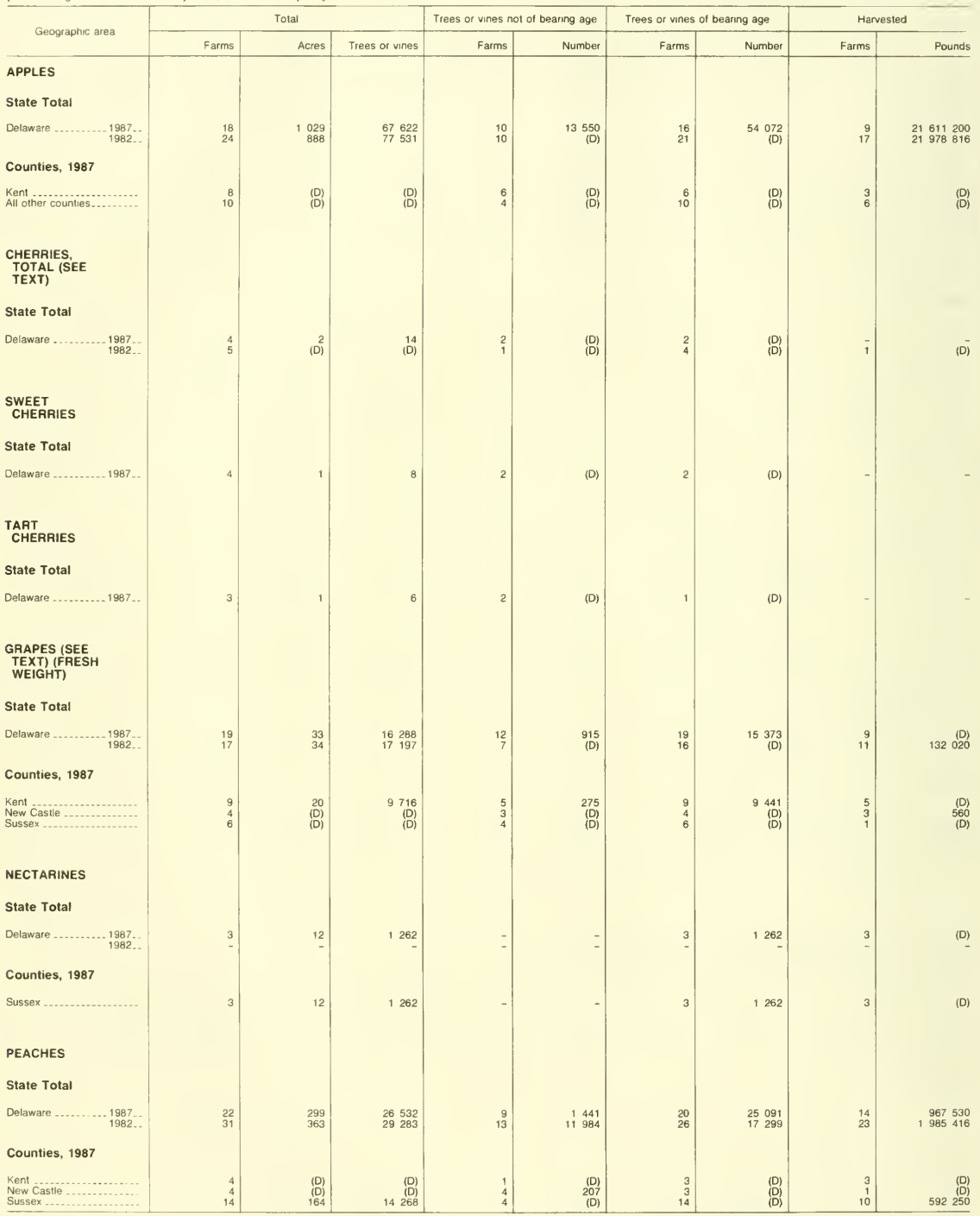


Table 29. Berries Harvested for Sale: 1987 and 1982

[For meaning of abbreviations and symbols, see introductory text]

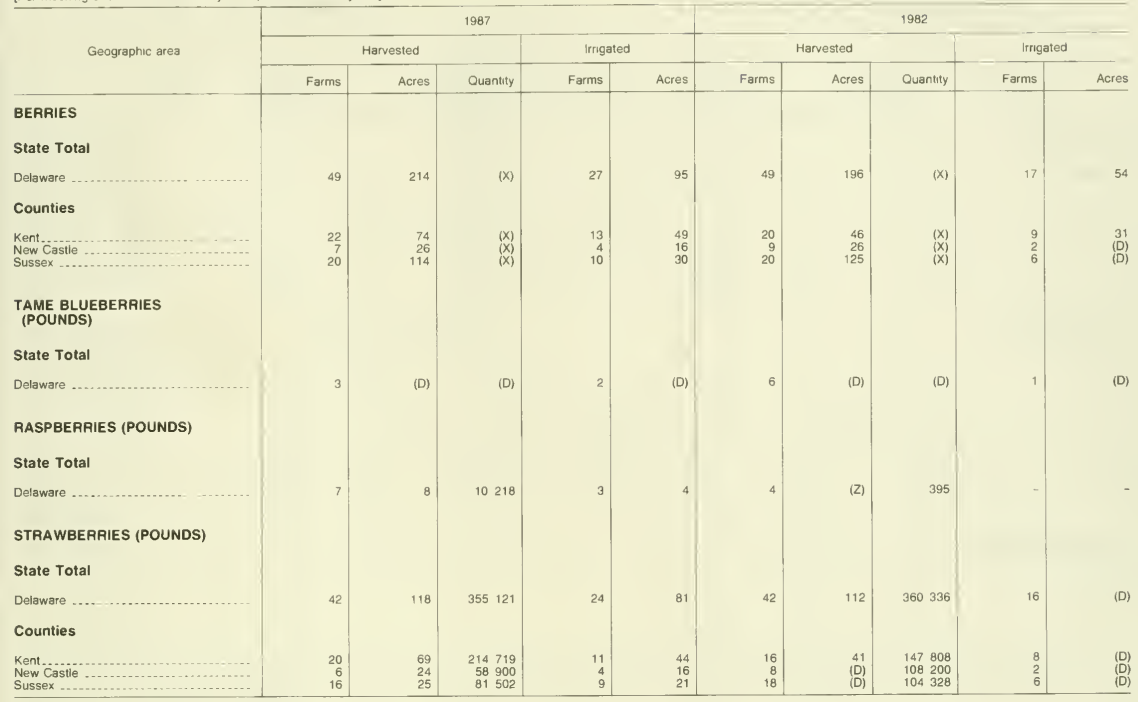

Table 30. Nursery and Greenhouse Crops, Mushrooms, and Sod Grown for Sale: 1987 and 1982

[For meaning of abbreviations and symbols, see introductory text]

\begin{tabular}{|c|c|c|c|c|c|c|c|c|}
\hline \multirow[b]{2}{*}{ Geographic area } & \multicolumn{4}{|c|}{1987} & \multicolumn{4}{|c|}{1982} \\
\hline & Farms & $\begin{array}{l}\text { Sq. } \mathrm{ft} \text {. under } \\
\text { glass or other } \\
\text { protection }\end{array}$ & $\begin{array}{r}\text { Acres in the } \\
\text { open }\end{array}$ & $\begin{array}{r}\text { Saies } \\
(\$ 1,000)\end{array}$ & Farms & $\begin{array}{r}\text { Sq ft. under } \\
\text { glass or other } \\
\text { protection }\end{array}$ & $\begin{array}{r}\text { Acres in the } \\
\text { open }\end{array}$ & $\begin{array}{r}\text { Sales } \\
(\$ 1,000) \\
\end{array}$ \\
\hline \multicolumn{9}{|l|}{$\begin{array}{l}\text { NURSERY AND } \\
\text { GREENHOUSE CROPS } \\
\text { (SEE TEXT) }\end{array}$} \\
\hline State Total & & & & & & & & \\
\hline Delaware & 96 & 1310210 & 951 & 13488 & 106 & 1340520 & 1280 & 7984 \\
\hline 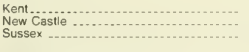 & $\begin{array}{l}32 \\
34 \\
30\end{array}$ & $\begin{array}{r}107887 \\
1001367 \\
200956\end{array}$ & $\begin{array}{r}635 \\
241 \\
75\end{array}$ & $\begin{array}{ll}3 & 166 \\
8 & 359 \\
1 & 963\end{array}$ & $\begin{array}{l}27 \\
52 \\
27\end{array}$ & $\begin{array}{rrr}66 & 920 \\
1 & 110 & 150 \\
163 & 450\end{array}$ & $\begin{array}{l}472 \\
449 \\
359\end{array}$ & $\begin{array}{ll}1 & 147 \\
4 & 996 \\
1 & 840\end{array}$ \\
\hline \multicolumn{9}{|l|}{$\begin{array}{l}\text { NURSERY, FLORICULTURE, } \\
\text { VEGETABLE AND FLOWER } \\
\text { SEED CROPS, SOD, ETC., } \\
\text { GROWN IN THE OPEN, } \\
\text { IRRIGATED (SEE TEXT) }\end{array}$} \\
\hline State Total & & & & & & & & \\
\hline $\begin{array}{l}\text { Delaware .... } \\
\text { Counties }\end{array}$ & 32 & $(x)$ & 512 & (X) & 27 & (X) & 428 & (X) \\
\hline $\begin{array}{l}\text { Kent } \\
\text { New Castie } \\
\text { Sussex }\end{array}$ & $\begin{array}{r}13 \\
4 \\
15\end{array}$ & $\begin{array}{l}(X) \\
(X) \\
(X)\end{array}$ & $\begin{array}{r}\text { (D) } \\
\text { (D) } \\
49\end{array}$ & $\begin{array}{l}(x) \\
(x) \\
(x)\end{array}$ & $\begin{array}{r}9 \\
8 \\
10\end{array}$ & $\begin{array}{l}(x) \\
(x) \\
(x)\end{array}$ & $\begin{array}{r}368 \\
22 \\
38\end{array}$ & $\begin{array}{l}(X) \\
(X) \\
(X)\end{array}$ \\
\hline
\end{tabular}


Table 30. Nursery and Greenhouse Crops, Mushrooms, and Sod Grown for Sale: 1987 and 1982-Con.

[For meaning of abbrevations and symbols, see introductory text]

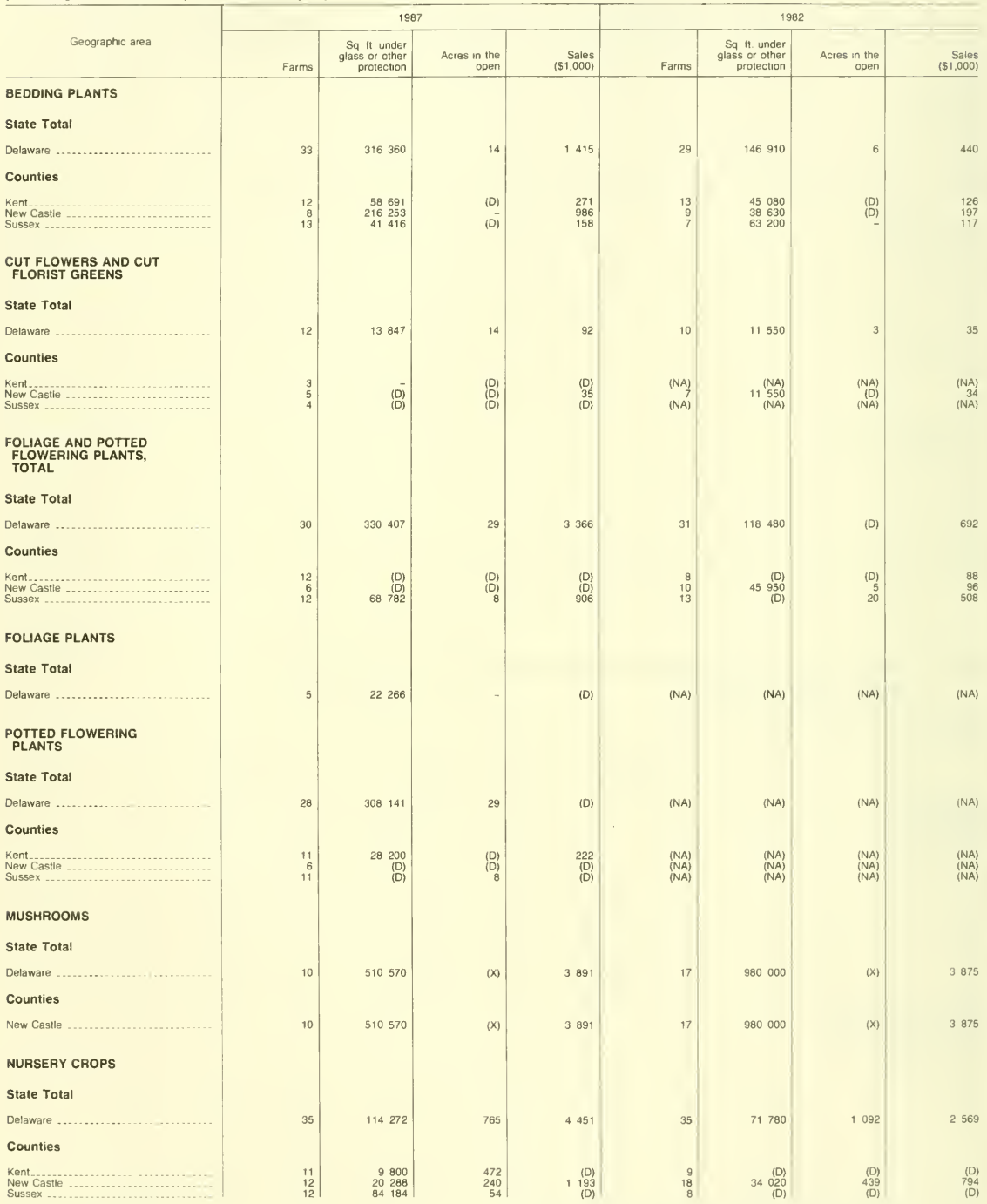


Table 30. Nursery and Greenhouse Crops, Mushrooms, and Sod Grown for Sale: 1987 and 1982-Con.

[For meaning of abbreviations and symbols, see introductory text]

\begin{tabular}{|c|c|c|c|c|c|c|c|c|}
\hline \multirow[b]{2}{*}{ Geographic area } & \multicolumn{4}{|c|}{1987} & \multicolumn{4}{|c|}{1982} \\
\hline & Farms & $\begin{array}{r}\text { Sq. ft. under } \\
\text { glass or other } \\
\text { protection }\end{array}$ & $\begin{array}{r}\text { Acres in the } \\
\text { open }\end{array}$ & $\begin{array}{r}\text { Sales } \\
(\$ 1,000)\end{array}$ & Farms & $\begin{array}{l}\text { Sq ft under } \\
\text { glass or other } \\
\text { protection }\end{array}$ & $\begin{array}{r}\text { Acres in the } \\
\text { open }\end{array}$ & $\begin{array}{r}\text { Sales } \\
(\$ 1.000)\end{array}$ \\
\hline \multicolumn{9}{|l|}{$\begin{array}{l}\text { GREENHOUSE } \\
\text { VEGETABLES }\end{array}$} \\
\hline State Total & & & & & & & & \\
\hline Delaware ... & 5 & 14254 & (X) & 32 & 2 & (D) & $(X)$ & (D) \\
\hline
\end{tabular}

Table 31. Other Crops: 1987 and 1982

[For meaning of abbreviations and symbols, see introductory text]

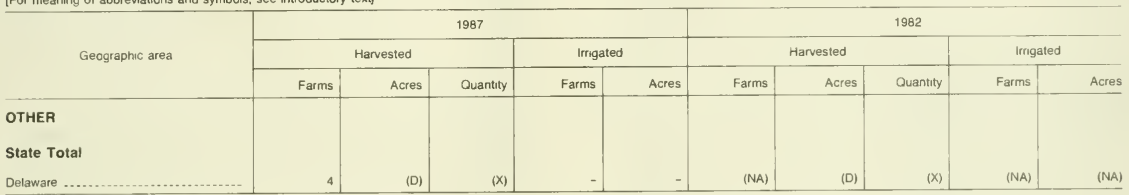

Table 32. Farms Operated by Black and Other Races by Value of Sales and Occupation: 1987 and 1982

Table 33. Farms Operated by Black and Other Races by Tenure: 1987 and 1982

[For classification of social and ethric groups, see text For meaning of abbreviations and symbols, see introductory text]

\begin{tabular}{|c|c|c|c|c|c|c|c|c|c|}
\hline \multirow[b]{2}{*}{ Geographic area } & \multicolumn{3}{|c|}{ Full owners } & \multicolumn{3}{|c|}{ Part owners } & \multicolumn{3}{|c|}{ Tenants } \\
\hline & Number & $\begin{array}{r}\text { Land in } \\
\text { farms } \\
\end{array}$ & $\begin{array}{c}\text { Harvested } \\
\text { cropland }\end{array}$ & Number & $\begin{array}{r}\text { Land in } \\
\text { farms }\end{array}$ & $\begin{array}{c}\text { Harvested } \\
\text { cropland }\end{array}$ & Number & $\begin{array}{c}\text { Land in } \\
\text { tarms }\end{array}$ & $\begin{array}{l}\text { Harvested } \\
\text { cropland }\end{array}$ \\
\hline STATE TOTAL & & & & & & & & & \\
\hline $\begin{array}{l}\text { Delaware ........... 1987... } \\
\text { COUNTIES, } 1987\end{array}$ & $\begin{array}{l}34 \\
33\end{array}$ & $\begin{array}{l}1715 \\
1213 \\
1213\end{array}$ & $\begin{array}{l}469 \\
764\end{array}$ & $\begin{array}{l}12 \\
21\end{array}$ & $\begin{array}{ll}1 & 858 \\
3 & 154 \\
3\end{array}$ & $\begin{array}{l}1605 \\
2809 \\
2\end{array} 809$ & $\begin{array}{l}6 \\
5\end{array}$ & $\begin{array}{r}81 \\
584\end{array}$ & $\begin{array}{r}69 \\
484\end{array}$ \\
\hline $\begin{array}{l}\text { Kent } \\
\text { New Castie - } \\
\text { Sussex .... }\end{array}$ & $\begin{array}{r}7 \\
3 \\
24 \\
\end{array}$ & $\begin{array}{r}\text { (D) } \\
\text { (D) } \\
1307 \\
\end{array}$ & $\begin{array}{l}166 \\
303\end{array}$ & $\begin{array}{r}1 \\
11 \\
\end{array}$ & $\begin{array}{l}\text { (D) } \\
\text { (D) }\end{array}$ & $\begin{array}{l}\text { (D) } \\
\text { (D) }\end{array}$ & $\frac{3}{3}$ & $\begin{array}{l}\text { (D) } \\
\text { (D) }\end{array}$ & $\begin{array}{l}\text { (D) } \\
\text { (D) }\end{array}$ \\
\hline
\end{tabular}




\section{Table 34. Operators by Selected Racial Groups: 1987 and 1982}

[For meaning of abbreviations and symbols, see introductory text]

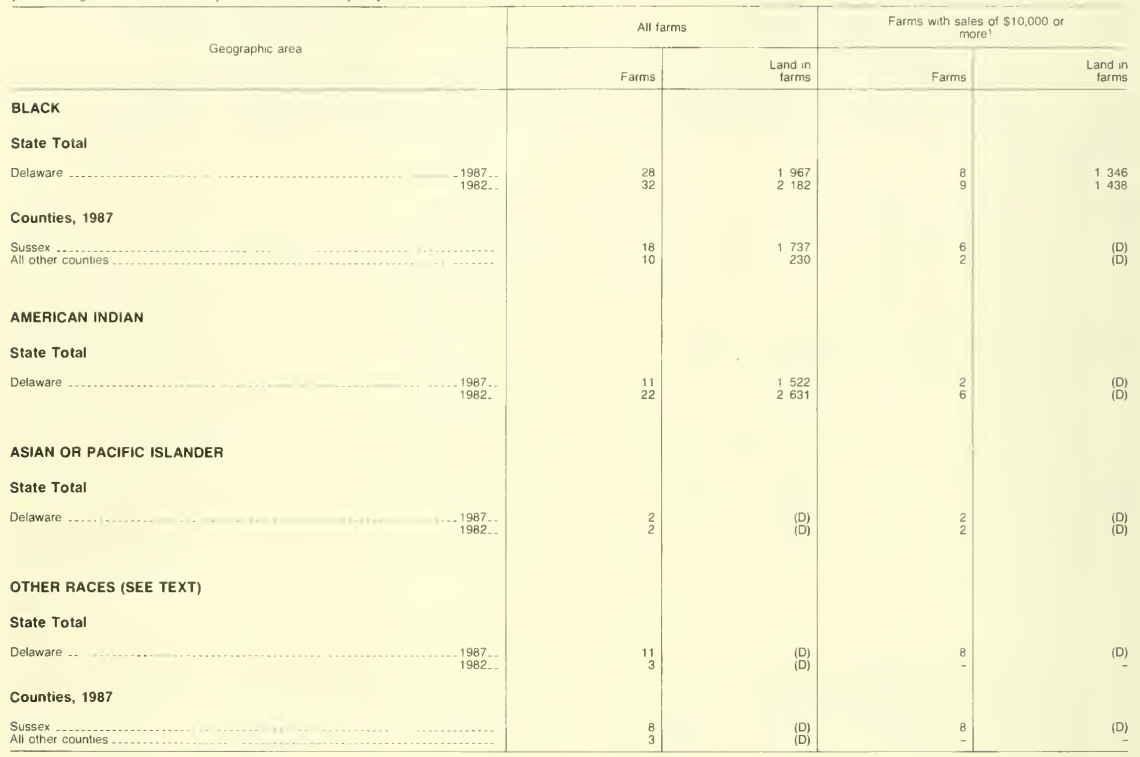

'Data for 1982 exclude abnormal farms.

Table 35. Operators of Spanish Origin: 1987 and 1982

[For classification of Spanish onigin, see text. For meaning of abbreviations and symbols, see introductory text]

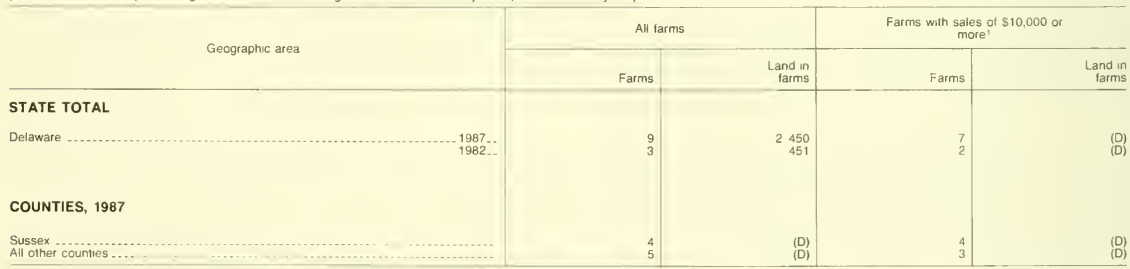

'Data for 1982 exclude abnormal tarms

Table 36. Farms With Grazing Permits: 1987

[Not published for this State] 


\section{APPENDIX A. General Explanation}

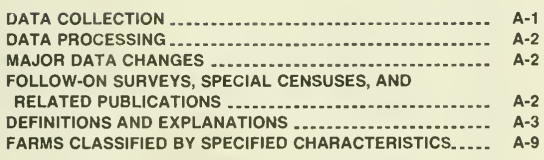

\section{DATA COLLECTION}

\section{Method of Enumeration}

All agriculture censuses beginning with the 1969 census primarily have used mailout/mailback data collection. Direct enumeration methods, however, continue to be used for the agriculture census in Puerto Rico, Guam, the U.S. Virgin Islands, American Samoa, and the Commonwealth of the Northern Mariana Islands.

\section{Mail List}

The mail list for the 1987 census was comprised of individuals, businesses, and organizations that could be readily identified as being associated with agriculture. The list was assembled from the records of the 1982 census, administrative records of the Internal Revenue Service (IRS), and the statistical records of the U.S. Department of Agriculture (USDA). In addition, lists of large or specialized operations, such as nurseries and greenhouses, specialty crop farms, poultry farms, fish farms, livestock farms, and cattle feedlot operations, were obtained from State and Federal agencies, trade associations, and similar organizations. Lists of companies having one or more establishments (or locations) producing agricultural products were obtained from the 1982 census and updated using the information from the Standard Statistical Establishment List maintained by the Census Bureau. Exhaustive record linkage, unduplication, and mathematical modeling yielded a final mail list of 4.1 million names and addresses that had a substantial probability of being a farm operation.

\section{Report Forms}

In 1987, three different report forms were used-a two-page, a four-page, and a six-page form to minimize the reporting burden, particularly for small farms and places less likely to be farms. The six-page sample form and the four-page nonsample form are the same, except sections 23 through 28 have been added to the sample form to obtain supplemental information from a sample of farms.
The information collected in these sections will give the Bureau of the Census a good basis for making estimates of these data for other farms included in the census. The two-page form does not have as many questions or as much detail as the four-page and six-page forms. The four-page form has 11 regional versions and the six-page form has 13 regional versions. Both forms have different crops prelisted. Appendix D contains copies of both the two-page and six-page forms.

The six-page form was mailed to $1,104,000$ addressees on the mail list, including all those expected to be large (based on expected sales or acreage) or unique (farms operated by multiestablishment companies or nonprofit organizations), all those in Alaska and Hawaii, and a sample of other addressees. The two-page form was mailed to 906,000 addressees. These were expected to be small farms or less likely to be farms. The four-page form was mailed to the remaining $2,079,000$ addressees. Further discussion of the criteria used to determine which form was mailed to an addressee is provided in the Census Sample Design section of appendix C.

\section{Initial Mailing}

The report forms were mailed in mid-December 1987 to the approximately 4,089,000 individuals, businesses, and organizations on the mail list. The mail packages included a report form, a cover letter with a description of the purposes and uses of the census on the reverse side, an information sheet containing instructions for completing the form, and a postage-paid return envelope. Additional special instructions were included with report forms sent to grazing associations; feedlot operations; institutional organizations; Indian reservations; firms with multiple farm or ranch operations; and producers of poultry under contract, bees and honey, fish, laboratory animals, and nursery and greenhouse crops.

To provide additional help to farmers in completing their reports, copies of an Agriculture Census Guide booklet were sent to vocational agriculture instructors, USDA county offices of the Agriculture Stabilization and Conservation Service, and the Cooperative Extension Service. The Guide contained descriptions and definitions of various items in more detail thar the instructions included with each report form. Representatives of the above agencies graciously consented to assist farmers in completing their report forms. 


\section{Followup Procedures}

A thank you/reminder card was mailed to those on the mail list in mid-January 1988 . Five followup letters, three of which were accompanied by a report form, were sent to nonrespondents at 4-week intervals starting in mid-February and continuing until early June 1988.

Telephone calls were made to all large farms who had not responded. In addition, telephone calls were made to a sample of other nonrespondents in counties that had a response rate of less than 75 percent. A nonresponse adjustment procedure was used to represent the final nonrespondent farms in the census results. A description of this procedure is included in the Census Estimation section of appendix $C$.

\section{DATA PROCESSING}

Selected report forms were reviewed prior to keying the data. These included reports with attached correspondence and reports with remarks or no positive data on the front page.

The data from each report form were subjected to a detailed item-by-item computer edit. The edit performed comprehensive checks for consistency and reasonableness, corrected erroneous or inconsistent data, supplied missing data based on similar farms within the same county, and assigned farm classification codes necessary for tabulating the data. Substantial computer-generated changes to the data were clerically reviewed and verified.

In the computer edit, farms with sales, acreage, or commodities exceeding specified levels were tested for historical comparability. Key items, such as acreage and sales, were compared for substantial changes between 1982 and 1987. Sizeable historical differences were resolved or verified by telephone, if necessary. Respondents who reported sales or acreage above specified levels on nonsample forms were sent correspondence requesting the additional sample data. Prior to publication, tabulated totals were reviewed by statisticians to identify inconsistencies and potential coverage problems. Comparisons were made with previous census data, estimates published by the USDA, and other available data.

\section{MAJOR DATA CHANGES}

Prior to each agriculture census, the Census Bureau reviews the content of the census forms to eliminate questions no longer needed and to identify new items necessary to meet user needs and to better describe the agricultural situation in our Nation. Data requests are solicited from farmers, farm organizations, land grant colleges and universities, State and federal agencies, and members of the Census Advisory Committee on Agricultural Statistics. Each agency and organization is asked to identify and justify its specific data needs. The following data inquiries were added to the 1987 report form:
Income from farm-related sources

Acres under the Conservation Reserve Program

Payments received for participation in federal farm programs

Grazing permits by source

Additional data on production expenses were added in 1987:

Repair and maintenance expenses

Cash rent

Property taxes paid

All other production expenses

The following separate data inquiries were eliminated from the 1987 form:

Storage capacity for petroleum products

Number of hired farm and ranch workers

Value of agricultural products sold directly to individuals

for human consumption

Source of irrigation water

Tons of commercially mixed feed

Expenditures for coal, wood, and coke

Selected machinery items: automobiles, corn heads for combines, and field forage harvesters

Chinchillas

Worms

Tropical and baitfish

\section{FOLLOW-ON SURVEYS, SPECIAL CENSUSES, AND RELATED PUBLICATIONS}

In addition to the 1987 Census of Agriculture for the 50 States, Puerto Rico, Guam, the U.S. Virgin Islands, American Samoa, and the Commonwealth of the Northern Mariana Islands, the census of agriculture program includes the 1988 Farm and Ranch Irrigation Survey, the 1988 Agricultural Economics and Land Ownership Survey, and the 1988 Census of Horticultural Specialties.

The 1988 Farm and Ranch Irrigation Survey provides data on water use by irrigated farms and ranches. Data include: the amount of water applied by crop, method of water distribution, source of water, and energy costs for pumping water. Data from this survey will be published as volume 3 , part 1 .

The 1988 Agricultural Economics and Land Ownership Survey provides detailed data on debts, expenses, taxes, credits, assets, land ownership, and farm and off-farm income for farm operators. Many of these items, as well as detailed data on landlord characteristics, are being collected from the landlords of the farms involved in the survey. Data from this survey will be published as volume 3, part 2.

The 1988 Census of Horticultural Specialties covers operations growing and selling $\$ 2,000$ or more of horticultural products such as greenhouse products, outdoorgrown floricultural products, nursery products, mushrooms, and sod. These data will be published as volume 4 .

Additional publications of the 1987 Census of Agriculture data include the Agricultural Atlas of the United States (previously called the Graphic Summary), Coverage Evaluation, Ranking of States and Counties, and Government Payments and Market Value of Agricultural Products Sold. 
The Agricultural Atlas of the United States presents the Nation's agriculture graphically illustrated by dot and multicolor pattern maps. The maps provide displays on size and type of farm, land use, farm tenure, market value of products sold, crops harvested, livestock inventories, and other characteristics of farms. This report will be published as volume 2, part 1.

The Coverage Evaluation report provides estimates of the completeness of the 1987 Census of Agriculture for the United States, geographic regions, and selected States and groups of States. Estimates with their associated sample reliability are provided for farms not on the mail list, farms classified as nonfarms, duplicate farms, and nonfarms classified as farms. This report will be published as volume 2, part 2.

The Ranking of States and Counties report ranks the leading States and counties for selected items in the 1987 census and provides comparative data from the 1982 census. This report will be published as volume 2, part 3 .

A new publication on Government Payments and Market Value of Agricultural Products Sold presents 1987 data for the United States and each State. The U.S. table has a format similar to volume 1, U.S. table 52 and presents summary data by size of farm. This report will be published as volume 2, part 5 .

\section{DEFINITIONS AND EXPLANATIONS}

The following definitions and explanations provide a more detailed description of the terms used in this publication than are available in the tables or on the report form. For an exact wording of the questions on the 1987 census report forms and the information sheet which accompanied these forms, see appendix D. Most definitions of terms are the same as those used in earlier censuses. The more important exceptions are also noted here.

Farms or farms reporting-The term "farms" or "farms reporting" in the presentation of data denotes the number of farms reporting the item. For example, if there are 3,710 farms in a State and 842 of them had 28,594 cattle and calves, the data for those farms reporting cattle and calves would appear as:

$$
\text { Cattle and calves--.---.--farms-- }
$$

number--

Land in farms-The acreage designated in the tables as "land in farms" consists primarily of agricultural land used for crops, pasture, or grazing. It also includes woodland and wasteland not actually under cultivation or used for pasture or grazing, provided it was part of the farm operator's total operations. Large acreages of woodland or wasteland held for nonagricultural purposes were deleted from individual reports during the processing operations. Land in farms includes acres set aside under annual commodity acreage programs as well as acres in the Conservation Reserve Program for places meeting the farm definition.
Land in farms is an operating unit concept and incluoies land owned and operated as well as land rented from others. Land used rent free was to be reported as land rented from others. All grazing land, except land used under government permits on a per-head basis, was included as "land in farms" provided it was part of a farm or ranch. Land under the exclusive use of a grazing association was to be reported by the grazing association and included as land in farms. All land in Indian reservations used for growing crops or grazing livestock was to be included as land in farms. Land in reservations not reported by individual Indians or non-Indians was to be reported in the name of the cooperative group that used the land. In some instances, an entire Indian reservation was reported as one farm.

Land area-The approximate land area of counties and States represents the total land area as determined by records and calculations as of January 1, 1988. These data are updated periodically; however, the acreages shown for 1987 are essentially the same as for 1982 . Any differences between the land area for 1987 and 1982 are due to annexations and other changes affecting county boundaries.

Land in two or more counties-With few exceptions, the land in each farm was tabulated as being in the operator's principal county. The principal county was defined as the one where the largest value of agricultural products was raised or produced. It was usually the county containing all or the largest proportion of the land in the farm or viewed by the respondent as his/her principal county. For a limited number of Midwest and Western States, this procedure has resulted in the allocation of more land in farms to a county than the total land area of the county. To minimize this distortion, separate reports were required for large farms identified from the 1982 census as having more than one farm unit. Other reports received showing land in more than one county were separated into two or more reports if the data would substantially affect the county totals.

Value of land and buildings-Respondents were asked to report their estimate of the current market value of land and buildings owned, rented or leased from others, and rented or leased to others. Market value refers to the value the land and buildings would sell for under current market conditions. If the value of land and buildings was not reported, it was estimated using the average value of land and buildings from a similar farm in the same geographic area. 
Harvested cropland-This category includes land from which crops were harvested or hay was cut, and land in orchards, citrus groves, vineyards, nurseries, and greenhouses. Land from which two or more crops were harvested was counted only once, even though there was more than one use of the land.

Cropland used only for pasture or grazing-This category includes land used only for pasture or grazing that could have been used for crops without additional improvement. Included also was all cropland used for rotation pasture and land in government diversion programs that were pastured. However, cropland that was pastured before or after crops were harvested was to be included as harvested cropland rather than cropland for pasture or grazing.

Other cropland-This category includes cropland not harvested and not grazed which was used for cover crops, soil improvement crops, land on which all crops failed, cultivated summer fallow, idle cropland, and land planted in crops that were to be harvested after the census year.

Total woodland-This category includes natural or planted woodlots or timber tracts, cutover and deforested land with young growth which has or will have value for wood products, land planted for Christmas tree production, and woodland pastured. Land covered by sagebrush or mesquite was to be reported as other pastureland and rangeland or other land.

Woodland pastured-This category includes all woodland used for pasture or grazing during the census year. Woodland or forest land pastured under a per-head grazing permit was not counted as land in farms and therefore, was not included in woodland pastured.

Cropland in annual commodity acreage adjustment programs-This category includes land diverted or set aside under the provisions of the Federal Commodity Acreage Program. These data are for the acres of cropland taken out of production by growers of wheat, cotton, rice, corn, sorghum, barley, and oats, and devoted to conservation uses. Information was not obtained as to which crops would have been grown on the acres set aside.

Cropland in the Conservation Reserve Program (CRP)-This category includes acres of "highly erodible" cropland taken out of agricultural production and planted to protective cover crops or reforested. The CRP was established through the 1985 Food Security Act and provides for annual rental payments and shared costs of conservation practices through a 10-year contract with the USDA. Appendix B presents data on places with all their cropland enrolled in the Conservation Reserve Program and which were not counted as farms in the 1987 census.
Irrigated land-This category includes all land watered by any artificial or controlled means, such as sprinklers, furrows or ditches, and spreader dikes. Included are supplemental, partial, and preplant irrigation. Each acre was to be counted only once regardless of the number of times it was irrigated or harvested.

Operator-The term "operator" designates a person who operates a farm, either doing the work or making day-to-day decisions about such things as planting, harvesting, feeding, and marketing. The operator may be the owner, a member of the owner's household, a hired manager, a tenant, a renter, or a sharecropper. If a person rents land to others or has land worked on shares by others, he/she is considered the operator only of the land which is retained for his/her own operation. For partnerships, only one partner is counted as the operator. If it is not clear which partner is in charge, then the senior or oldest active partner is considered the operator. For census purposes, the number of operators is the same as the number of farms. In some cases, the operator was not the individual named on the address label of the report form, but another family member, a partner, or a hired manager who was actually in charge of the farm operation.

Operator characteristics-All operators were asked to report place of residence, principal occupation, days of off-farm work, year in which his/her operation of the farm began, age, race, sex, and Spanish origin. If race, age, sex, and principal occupation were not reported, they were imputed based on information reported by farms with similar acreage, tenure, and value of sales. No imputations were made for nonresponse to place of residence, Spanish origin, off-farm work, or year began operation. Operators of Spanish origin were tabulated by reported race.

Farm production expenses-In 1987, additional specific expense items and a category for all other farm production expenses were added to the selected farm production expenses collected in 1982. Consequently, we are publishing total farm production expenses in 1987. The expenses are limited to those incurred in the operation of the farm business. Expenses include the share of the expenditures provided by landlords, contractors, and partners in the operation of the farm business. Property taxes paid by landlords are excluded. Expenditures for nonfarm activities; farm-related activities such as providing customwork for others, the production and harvest of forest products, and recreational services; and household expenses are excluded. In 1987, as in other recent censuses, operators producing crops, livestock, or poultry under contract often were unable or unwilling to estimate the cost of production inputs furnished by the contractors. As a consequence, extensive estimation was required for contract producers.

Commercial fertilizer-The expense for commercial fertilizer is the amount spent on fertilizer during 1987 
including the cost of custom application. The cost of custom application was excluded from the 1982 and 1978 data.

Agricultural chemicals-These expenses include the cost of all insecticides, herbicides, fungicides, and other pesticides, including the cost of custom application. Data exclude commercial fertilizer purchased. The cost of custom application was excluded from the 1982 and 1978 data. The cost of lime was excluded from the 1987 and 1982 data, but included in 1978 .

Customwork, machine hire, and rental of machinery and equipment-These expenses include costs incurred for having customwork done on the place and for renting machines to perform agricultural operations. The cost of cotton ginning is excluded. The cost of labor involved in the customwork service is included in the customwork expense. The cost of custom application of fertilizer and chemicals was included in the 1982 and 1978 customwork data, but is included in expenditures for these items in 1987. The cost of hired labor for operating rented or hired machinery is included as a hired farm and ranch labor expense.

Interest-In 1987, separate data were collected for interest paid on debts secured by real estate and interest paid on debts not secured by real estate. In 1982 , only total interest expenses were collected.

Market value of agricultural products sold-This category represents the gross market value before taxes and production expenses of all agricultural products sold or removed from the place in 1987 regardless of who received the payment. It includes sales by the operator as well as the value of any shares received by partners, landlords, contractors, or others associated with the operation. In addition, it includes receipts from placing commodities in the Commodity Credit Corporation (CCC) loan program in 1987. It does not include payments received for participation in federal farm programs nor does it include income from farm-related sources such as customwork and other agricultural services, or income from nonfarm sources.

The value of crops sold in 1987 does not necessarily represent the sales from crops harvested in 1987. Data may include sales from crops produced in earlier years and exclude some crops produced in 1987, but held in storage and not sold. For commodities, such as sugar beets and wool, sold through a co-op which made payments in several installments, respondents were requested to report the total value received in 1987 .

The value of agricultural products sold was requested of all operators. If the operator failed to report this information, estimates were made based on the amount of crops harvested, livestock or poultry inventory or number sold. Extensive estimation was required for operators growing crops or livestock under contract.
Caution should be used when comparing sales in 1987 with sales reported in earlier censuses. Sales figures are expressed in current dollars and have not been adjusted for inflation or deflation.

Government payments-This category is limited to direct cash or generic commodity certificate (PIK) payments received by the farm operator in 1987. It includes deficiency and diversion payments; wool payments; payments from the Dairy Termination Program, the Conservation Reserve Program, other conservation programs, and all other federal farm programs under which payments were made directly to farm operators.

Other farm-related income-The 1987 report form included a new inquiry on income from farm-related sources. These data consist of gross income in 1987 before taxes and expenses from the sales of farm by-products and other sales and services closely related to the principal functions of the farm business. These data are for income producing activities that are primarily a by-product or supplemental to the farm operation. They exclude income from business activities that are separate from the farm business.

Customwork and other agricultural services-This income includes gross receipts received by farm operators for providing services for others such as planting, plowing, spraying, and harvesting. Income from customwork and other agricultural services is generally included in the agriculture census if it is closely related to the farming operation. However, it is excluded if it constitutes a separate business or is conducted from another location.

Rental of farmland-This income includes gross cash rent or share payments received from renting out farmland; payments received from the lease or sale of allotments for crops such as tobacco; and payments received for livestock pastured on a per-head, permonth, or per-pound basis. It excludes rental income from nonfarm property.

Sales of forest products-This income includes gross receipts from the sales of Christmas trees, standing timber, maple products, gum for naval stores, firewood, and other forest products from the farm business. It excludes income from nonfarm timber tracts and sawmill businesses.

Other farm-related income sources-This income includes gross receipts from hunting leases, fishing fees, camping, other recreational services, patronage dividends of cooperatives, sales of farm by-products, and other sales and services closely related to the farm business. It excludes income from nonfarm businesses.

Commodity Credit Corporations loans-This category includes loans for corn, wheat, soybeans, sorghum, barley, oats, cotton, peanuts, rye, rice, tobacco, and honey. 
Agricultural chemicals used, including fertilizer and lime-For each type of agricultural chemical, the acres treated were to be reported only once even if the acres were fertilized or limed more than once. If multipurpose chemicals were used, the acres treated for each purpose were to be reported.

Fish and other aquacultural products-The raising of fish and other aquacultural products in captivity is included in the agriculture census. Production in salt water is considered not to be in captivity and is excluded from the census.

Bees and honey-Bee and honey production was enumerated and tabulated in the county in which the home farm was located even though hives are often moved from farm to farm over a wide geographic area.

Citrus enumeration - In the 1987 census, reports for selected citrus caretakers in Arizona, Florida, and Texas were obtained by direct enumeration. A citrus caretaker is an organization or person caring for or managing citrus groves for others. This special enumeration has been used in recent censuses because of the difficulty in identifying and enumerating absentee grove owners who often do not know the information that is needed to adequately complete the census report. Each citrus caretaker was enumerated as a farm operator and requested to complete one report form for all groves cared for and to furnish a list of grove owners' names, addresses, and acres of citrus. The names on the lists were matched to completed grove owners' report forms to eliminate duplication. The caretaker also was requested to inform the grove owner that he had already reported for the citrus under his care and that the grove owner was not to report the citrus again. In the 1987 census, 7 caretakers in Arizona reported 175 grove owners having 12,000 acres of citrus; the 65 caretakers in Florida reported 3,000 grove owners having 170,000 acres of citrus; and 20 caretakers in Texas reported 800 grove owners having 14,500 acres of citrus.

Crop year or season covered-Acres and quantity harvested are for the calendar year 1987 except for citrus fruits, avocados, olives; vegetables in Florida; sugarcane in Florida and Texas; and pineapples and coffee in Hawaii.

Citrus fruits - The data for Florida relate to the quantity harvested in the September 1986 through July 1987 harvest season, except limes that were harvested in the April 1987 through March 1988 harvest season. The data for Texas relate to the quantity harvested in the September 1986 through May 1987 harvest season. The data for States, other than Florida and Texas, relate to the quantity harvested in the 1986-87 harvest season.

Avocados-The data for California relate to the quantity harvested in the November 1986 through November 1987 harvest season and for Florida the April 1987 through March 1988 harvest season.
Olives-The data for California relate to the quantity harvested in the September 1986 through March 1987 harvest season.

Vegetables-The data for Florida relate to the crop harvested in the September 1986 through August 1987 harvest season.

Sugarcane for sugar-The data for Florida relate to the cuttings from November 1986 through April 1987, and for Texas the cuttings from October 1986 through April 1987.

Pineapples-The data for Hawaii relate to the quantity harvested in the year ending May 31, 1987.

Coffee-The data for Hawaii relate to the 1986-87 crop.

Acres and quantity harvested-Crops were reported in whole acres, except for the following crops which were reported in 10ths of acres: Irish potatoes, sweetpotatoes, tobacco, fruit and nut crops including land in orchards, berries, vegetables, and nursery and greenhouse crops; and in Hawaii, taro, ginger root, and lotus root. Totals for crops reported in 10ths of acres were rounded to whole acres at the aggregate level during the tabulation process.

If two or more crops were harvested from the same land during the year, the acres would be counted for each crop. Therefore, the total acres of all crops harvested generally exceeds the acres of cropland harvested. An exception to this procedure is hay crops. When more than one cutting of hay was taken from the same acres, the acres are counted only once but the quantity harvested includes all cuttings. However, hay cut for both dry hay and green chop or silage would be reported for each applicable crop. For interplanted crops or "skip-row" crops, acres were to be reported according to the portion of the field occupied by each crop.

If a crop was planted but not harvested, the acres were not to be reported as harvested. These acres were to be reported in the "land use" section under the appropriate cropland items - cropland used only for pasture or grazing, cropland used for cover crops, cropland on which all crops failed, or cropland idle.

Corn and sorghum hogged or grazed were to be reported as "cropland harvested" and not as "cropland used only for pasture or grazing." Crop residue left in fields and later hogged or grazed was not reported as cropland pasture.

Quantity harvested was not obtained for crops such as vegetables; nursery and greenhouse crops; corn cut for dry fodder, hogged or grazed; and sorghum, hogged or grazed.

Acres of land in bearing and nonbearing fruit orchards, citrus or other groves, vineyards, and nut trees were to be reported as harvested cropland regardless of whether the crop was harvested or failed. However, abandoned orchards were to be reported as cropland idle, not as harvested cropland and the individual abandoned orchard crop acres were not to be reported. 
Land in orchards-This category includes land in bearing and nonbearing fruit trees, citrus or other groves, vineyards, and nut trees of all ages, including land on which all fruit crops failed. Respondents were instructed not to report abandoned plantings and plantings of less than 20 total fruit, citrus, or nut trees, or grapevines.

Crop units of measure-The regional report forms allowed the operator to report the quantity of field crops harvested in a unit of measure commonly used in the region. When the operator reported in a unit of measure different than the unit of measure published, the quantity harvested was converted to the published unit of measure.

Grapes could be reported in dry weight or fresh weight; plums and prunes in fresh weight, or prunes in dry weight; and in Hawaii, coffee in pounds parchment or pounds cherry, and macadamia nuts in pounds husked, unshelled or pounds shelled. For other fruit and nut crops and citrus, the operator was given a choice of units of measure of pounds, tons, or boxes. The quantity harvested for these crops is published in pounds.

Write-in crops-To reduce the length of the report form, only the major crops for the region were prelisted. For other crops, the respondent was requested to look at a list of crops in each section and write in the crop name and its code. For crops that had no individual code listed on the report form, the respondent was to write in the crop name and code the crop into the appropriate "all other" category for that section. Write-in crops coded as "all other" were reviewed and assigned a specific code when possible. Crops not assigned a specific code were left in the appropriate "all other" category.

In some cases, the reviewers were unable to determine the specific crop reported by the respondent because of incomplete or generalized crop names. To ensure proper coding, most of these respondents were telephoned. Reports for those not telephoned were changed on the basis of other reports for the area.

Misreported or miscoded crops-In a few instances, tabulated data may be inaccurate because respondents misunderstood or misinterpreted questions on the report form. Data may have been reported on the wrong line or in the wrong section, or the wrong crop code may have been placed beside the name of a write-in crop. Some of these errors as well as some keying errors may not have been identified during processing and therefore, were not corrected. Reports with significant acres of unusual crops for the area were examined to minimize the possibility that they were in error.

\section{"See text" References}

Items in the tables which carry the note "See text" are explained or defined in this section.
Data are based on a sample of farms-For 1987 , 1982 , and 1978, selected data were collected from only a sample of farms. These data are subject to sampling error. For 1987 , the six-page sample form was mailed to all large and specialized farms (based on expected sales, acres, or standard industrial classification), all farms in Alaska and Hawaii, and approximately 17 percent of all other farms. Sample sections 23 through 28 of the 1987 census forms included inquiries on production expenses, commercial fertilizer and lime, chemicals, machinery and equipment, value of land and buildings, and income from farm-related sources. Estimates of the reliability of county totals for selected items are shown in table $\mathrm{F}$ of appendix $\mathrm{C}$.

Operators of Spanish origin-No imputation was made for those not responding to the question on Spanish origin.

Farms operated by Black and other races-This category includes Blacks, American Indians, Asian and Pacific Islanders, and all other racial groups other than White.

All other races-This category is primarily limited to persons native to or of ancestry from Mexico, the Caribbean, and Central and South America.

Total sales-This item represents the gross market value of all agricultural products sold before taxes and expenses in the census year including livestock, poultry, and their products; and crops, including nursery crops and hay. Respondents were asked to include landlords' and contractors' shares. The value of commodities placed in CCC loans are included as sold. In 1987, all farms including abnormal farms were tabulated by size based on reported sales. In 1982 and 1978, abnormal farms were included in the total sales figure, but excluded from the detailed size breakdowns. Abnormal farms include institutional farms, experimental and research farms, and Indian reservations.

Farms with sales of less than $\$ 1,000-$ This category includes all farms with actual sales of less than $\$ 1,000$, but having the production potential for sales of $\$ 1,000$ or more. These farms normally could be expected to sell $\$ 1,000$ or more of agricultural products.

Net cash return from agricultural sales for the farm unit-This category is derived by subtracting total operating expenditures from the gross market value of agricultural products sold. Depreciation and the change in inventory values are excluded from expenditures. Production expenditures may be understated on part owner and tenant farms because property taxes paid by landlords are excluded. Other landlord expenditures, such as insurance or rent paid, which are not readily known to renters may also be omitted or understated. Gross sales include sales by the operator as well as the share of sales received by 
partners, landlords, and contractors. Consequently, the net cash return is that of the farm unit rather than the net farm income of the operator.

Other livestock and livestock products-This category includes all livestock and livestock products not listed separately.

Value of livestock and poultry on farms-Data for the value of livestock and poultry on farms were obtained by multiplying the inventory of each major age and sex group by State average prices. The State average prices for cattle, hogs, sheep, Angora goats, hens and pullets of laying age, and turkeys were obtained primarily from data published by the National Agricultural Statistics Service, USDA. Prices applied to other livestock and poultry were census-derived averages based primarily on reported value of sales in the census.

Poultry hatched-This category includes all poultry hatched on the place during the year and placed or sold. Incubator egg capacity on December 31,1987 , is tabulated under the column heading "Inventory" and the number of poultry hatched and placed or sold is under the heading "Sales."

Hay-alfalfa, other tame, small grain, wild, grass silage, green chop, etc.-Data shown for hay represent all hay crops, including grass silage, haylage, and hay crops cut and fed green (green chop). In production data, dry tons represent dry tonnage for the various hay categories and dry weight equivalents for grass silage and hay cut and fed green. The conversion used was 3 tons of green weight to 1 ton of dry weight.

Tame hay other than alfalfa, small grain, and wild hay-Data shown represent dry tons of hay harvested from clover, lespedeza, timothy, Bermuda grass, Sudan grass, and other types of legume and tame grasses.

Grapes-Farm operators were given the option of reporting the quantity of grapes harvested in dry weight or fresh weight. For publication purposes, all quantities of grapes harvested have been converted to pounds of fresh weight. The conversion used was 4 pounds fresh weight to 1 pound dry weight.

Plums and prunes-Farm operators were given the option of reporting the quantity of plums and prunes harvested in dry weight or fresh weight. For publication purposes, all quantities of plums and prunes harvested have been converted to pounds of fresh weight. The conversion used was 3 pounds fresh weight to 1 pound dry weight.

Cherries-For 1987, cherries were reported as "sweet cherries," "tart cherries," or "cherries" depending on the regional form the respondent completed. On regional forms for States where cherries are an important fruit crop, "sweet cherries" and "tart cherries" were listed separately. On the other regional forms, either "cherries" were listed or could be written in. For publication purposes, "cherries, total" could be shown along with the individual breakdown of "sweet cherries," "tart cherries," or "cherries, not specified." "Cherries, not specified" is used to account for cherries where the "sweet" and "tart" breakdown was not asked or where respondents wrote in "cherries" but did not specify or code the kind of cherry. All the individual cherry items may not be shown. Data for "sweet cherries," "tart cherries," and "cherries, not specified" are not available for 1982 .

Other fruits and nuts-Data shown for other fruits and nuts relate to any fruits and nuts not having a specific code on the 1987 report form.

Land used for vegetables-Data are for the total land used for vegetable crops. The acres are reported only once, even though two or more harvests of a vegetable or more than one vegetable were harvested from the same acres.

Vegetables harvested for sale-The acres of vegetables harvested is the summation of the acres of individual vegetables harvested. All of the individual vegetable items may not be shown.

Nursery and greenhouse crops grown for sale-These data are a summation of the individual items reported. All of the individual items may not be shown.

Nursery, floriculture, vegetable and flower seed crops, sod, etc., grown in the open, irrigated-Data refer to farms reporting irrigated nursery, floriculture, vegetable and flower seeds, sod, bedding plants, etc., grown in the open.

Other grains-These data are for the total market value of other grains sold including dry edible beans, dry lima beans, buckwheat, dry southern peas (cowpeas), emmer and spelt, flaxseed, mixed grains, lentils, mustard seed, dry edible peas, popcorn, proso millet, rice, rye for grain, safflower, sunflower seed, triticale, and wild rice.

Value of crop production-This item represents the estimated value of all crops harvested during the 1987 crop year. Data for the value of crops harvested were obtained by multiplying the average estimated value per unit by the reported acres or quantity harvested. Generally, harvested units of production (pounds, bushels, bales, etc.) were multiplied by State estimates of prices per unit. If only acres harvested were reported, State estimates for value of production per acre were used. The State average production price and production value per acre used in these calculations were obtained usually from publications of the National Agricultural Statistics Service, USDA. When 
USDA estimates were not available, Bureau of the Census statisticians made estimates using available sources such as data from adjacent States, respondent report forms, county extension agents, and other persons knowledgeable about specific crops.

\section{FARMS CLASSIFIED BY SPECIFIED CHARACTERISTICS}

State tables 48 through 53 present detailed 1987 data for all farms classified by specified characteristics-tenure of operator, type of organization, age and principal occupation of operator, size of farm (acres), value of agricultural products sold, and standard industrial classification. Other tables include data classified by value of sales groups or other characteristics of the farm or the operator.

Farms by value of agricultural products sold or value of sales - In 1987, all farms were tabulated by size based on reported sales. In 1982 and earlier censuses, abnormal farms were not tabulated based on sales size. In the tables on market value of agricultural products sold, the sales of abnormal farms in 1982 and earlier censuses were included in the total sales figure, but excluded from the detailed size categories. Abnormal farms included institutional farms, experimental and research farms, and Indian reservations. The category "farms with sales of less than $\$ 1,000$ " included all farms with actual sales of less than $\$ 1,000$ but having the production potential for sales of $\$ 1,000$ or more. These farms normally could be expected to sell $\$ 1,000$ or more of agricultural products.

The sales size categories used in this report are consistent with the standard business size categories issued by Office of Management and Budget (OMB) in 1982. In State table 52, data are presented for four sales size categories between $\$ 10,000$ and $\$ 49,999$. This provides users with bridge data under both the OMB and the 1978 census classifications. For the 1992 census, data will be presented only for the OMB sales size categories of $\$ 10,000$ to $\$ 24,999$ and $\$ 25,000$ to $\$ 49,999$.

Abnormal farms-This category includes institutional farms, experimental and research farms, and Indian reservations. Institutional farms include those operated by hospitals, penitentiaries, churches, schools, grazing associations, and government agencies. In 1987 and 1982, nongovernmental units such as church farms and Future Farmers of America camps were classified as abnormal farms only when 50 percent or more of their products produced and intended for human consumption were utilized by the organization.

Farms by tenure of operator-The classifications of tenure used in the 1987 census were:

Full owners, who operate only land they own.
Part owners, who operate land they own and also land they rent from others.

Tenants, who operate only land they rent from others or work on shares for others.

Farms by type of organization-All farms were classified by type of organization in the 1987 census. The classifications used were:

Individual or family (sole proprietorship), excluding partnership and corporation.

Partnership, including family partnership.

Corporation, including family corporation.

Other, cooperative, estate or trust, institutional, etc.

Corporations were subclassified by two additional characteristics into:

1. Family held

Other than family held

2. More than 10 stockholders 10 or less stockholders

Farms by age and principal occupation of operator-Data on age and principal occupation were requested from all operators in 1987. The principal occupation classifications used were:

Farming-The operator spent 50 percent or more of his/her worktime in 1987 in farming or ranching.

Other-The operator spent more than 50 percent of his/her worktime in 1987 in occupations other than farming or ranching.

Farms by size-All farms were classified into selected size groups according to the total land area in the farm. The land area of a farm is an operating unit concept and includes land owned and operated as well as land rented from others. Land rented to or assigned to a tenant was considered the tenant's farm and not the owner's.

Farms by standard industrial classification- In 1987, all agricultural production establishments (farms, ranches, nurseries, greenhouses, etc.) were classified by type of activity using the standard industrial classification (SIC) system. These classifications, found in the 1987 SIC Manual ${ }^{1}$, are used to promote uniformity and comparability in the presentation of statistical data collected by various agencies.

'Standard Industrial Classification Manual: 1987. For sale by Superintendent of Documents, U.S. Government Printing Office, Washington, DC 20402. Stock No. 041-001-003-14-2. 
An establishment primarily engaged in crop production (major group 01) or production of livestock and animal specialties (major group 02) is classified in the four-digit industry and three-digit industry group which accounts for 50 percent or more of the total value of sales of its agricultural products. If the total value of sales of agricultural products of an establishment is less than 50 percent from a single four-digit industry, but 50 percent or more from the products of two or more four-digit industries within the same three-digit industry group, the establishment is classified in the miscellaneous industry of that industry group. Otherwise, it is classified as a general crop farm in industry 0191 or a general livestock farm in industry 0291. Establishments that derive 50 percent or more of the value of sales from horticultural specialties of industry group 018 are classified in industry 0181 or 0182 according to their primary activity.

Characteristics of all farms by selected SIC groupings are shown in State tables 18 and 53. The SIC groupings shown in State table 53, together with the associated products (value of sales representing 50 percent or more of the value of agricultural products sold during the year) on which the classification is based, are as follows:

Cash grains (011)-Wheat, rice, corn, soybeans, barley, buckwheat, cowpeas, dry field and seed beans and peas, flaxseed, lentils, milo, mustard seed, oats, popcorn, rye, safflower, sorghum, sunflowers, and other small grains.

Cotton (0131)-Cotton and cottonseed.

Tobacco (0132)-Tobacco.

Sugarcane, sugar beets, Irish potatoes, hay, peanuts, and other field crops (0133, 0134, 0139)-Sugarcane, sugar beets, Irish potatoes, alfalfa, broomcorn, clover, grass seed, hay, hops, mint, peanuts, sweetpotatoes, timothy, and yams.

Vegetables and melons (016)-Vegetables and melons grown in the open.
Fruits and tree nuts (017)-Berries, grapes, tree nuts, citrus fruits, deciduous tree fruits, avocados, bananas, coffee, dates, figs, olives, pineapples, and tropical fruit.

Horticultural specialties (018)-Bedding plants, bulbs, florists' greens, flower and vegetable seeds, flowers, foliage, fruit stocks, nursery stock, ornamental plants, shrubberies, sod, mushrooms, and vegetables grown under cover.

General farms, primarily crops (019)-Crops, including horticultural specialties, but less than 50 percent of sales from any single three-digit industry group.

Livestock, except dairy, poultry, and animal specialties (021)-Cattle, calves, hogs, sheep, goats, goat's milk, mohair, and wool.

Beef cattle, except feedlots (0212)-Production or feeding of beef cattle, except feedlots.

Dairy farms (024)-Production of cows' milk and other dairy products and raising of dairy heifer replacements.

Poultry and eggs (025)-Chickens, chicken eggs, turkeys, ducks, geese, pheasants, pigeons, quail, and squab.

Animal specialties (027)-Fur-bearing animals, rabbits, horses, ponies, bees, fish in captivity except fish hatcheries, worms, and laboratory animals.

General farms, primarily livestock and animal specialties (029) - Livestock and animal specialties and their products, but less than 50 percent of sales from any single three-digit industry group.

The SIC manual was revised for 1987. Animal aquaculture (0273) was established as a new industry and horticultural specialties, not elsewhere classified (0189) was deleted. 


\section{APPENDIX B. \\ Places With All Cropland in the Conservation Reserve \\ Program}

The Food Security Act of 1985 established the Conservation Reserve Program (CRP). This program provides annual payments for highly erodible cropland enrolled in the program and meeting its conservation requirements. It also requires that the land be taken out of agricultural production for 10 years.

The 1987 Census of Agriculture includes Conservation Reserve acreage as land in farms on operations that meet the census farm definition. For census purposes, a farm is any place from which agricultural products of $\$ 1,000$ or more were produced and sold or normally would have been sold during the census year. Operations which placed all of their cropland in the CRP and did not otherwise meet the farm definition based upon sales, livestock inventories, planted crops, or other criteria for potential sales were not included as farms in the census tabulations.
The following table provides CRP data for places not meeting the census farm definition ("whole farm" CRP places). It also contains separate but corresponding CRP data for farms included in the census tabulations. In addition to State data, detailed county data are presented for counties with three or more "whole farm" CRP places reported. For counties with less than three "whole farm" CRP places reported, their data are combined and reported in "all other counties."

The data for "whole farm" CRP places are not complete for all counties. The census mail list was developed from sources which indicated the farm had agricultural production activity. It was not designed to cover all "whole farm" CRP places. Therefore, the data for these places are limited to what was reported in the census and have not been adjusted to account for nonresponse, incomplete coverage, and reporting errors.

\section{Land in Conservation Reserve Program: 1987}

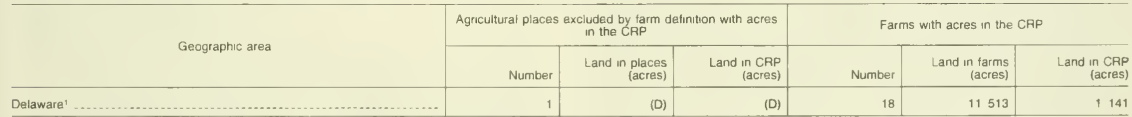

'Data are published for counties with 3 agricultural piaces or more reporting CRP acres. Counties with less than 3 places are combined under "all other counties." 



\section{APPENDIX C. Statistical Methodology} MAIL LIST MODEL ......................

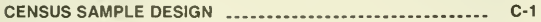
CENSUS ESTIMATION ............ C-1 CENSUS SAMPLING ERROR ................. C-3

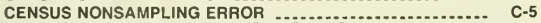
EDITING DATA AND IMPUTATION FOR ITEM NONRESPONSE

TABLES:

A. PERCENT OF STATE TOTALS CONTRIBUTED BY WHOLE FARM NONRESPONSE ESTIMATION: 1987

8. RELIABILITY ESTIMATES FOR NUMBER OF FARMS IN A COUNTY REPORTING A COMPLETE COUNT ITEM: 1987

C. RELIABILITY ESTIMATES FOR NUMBER OF FARMS IN A COUNTY REPORTING A SAMPLE ITEM: 1987

D. RELIABILITY ESTIMATES OF STATE TOTALS: 1987

E. RELIABILITY ESTIMATES OF PERCENT CHANGE IN STATE TOTALS: 1982 TO 1987

F. RELIABILITY ESTIMATES OF COUNTY TOTALS: 1987

G. STATE COVERAGE EVALUATION ESTIMATES OF FARMS NOT ON THE MAIL LIST: 1987

\section{MAIL LIST MODEL}

A statistical discriminant model was developed to predict the probability that a mail list addressee operated a farm. The model was used to identify the 4.1 million records from the preliminary census mail list of 6.0 million records that would receive a census of agriculture report form. Records from the 1982 census mail list were used to build the model. Record characteristics such as the source of the mail list record (see appendix A for a description of record sources), number of source lists on which the record appeared, expected value of agricultural sales, and geographic location were used to separate mail list records into model groups. The proportion of 1982 census farm records in each group was calculated to provide an estimate of the probability that an addressee in the group operated a farm.

Using these same group definitions, the 1987 census mail list records were separated into groups, each with an associated estimate of farm probability from the model. The 4.1 million mail list records in groups with the largest estimate of farm probability were selected to receive the census report form. A large percentage of the 1.9 million records that were dropped from the 6.0 million preliminary census mail list were nonfarm records from the previous census. This procedure was used to obtain a more complete census enumeration without excessive respondent burden and data collection cost.

\section{CENSUS SAMPLE DESIGN}

Each of the 4.1 million name and address records on the census mail list was designated to receive one of three different types of census report forms. The three forms were the nonsample census form (a four-page form), the sample form (a six-page form), and the short form (a two-page form). Sections 1 through 22 of the sample form were identical to sections on the nonsample census form. However, the sample form contained additional sections on farm production expenditures, usage of fertilizers and insecticides, value of machinery and equipment, value of land and buildings, and farm-related income. The short form contained abbreviated versions of the sections on the nonsample census form. These three different forms were used to reduce the response burden of the census, while providing quality information on a large number of data items at the county level.

The sample form was mailed to all mail list records in Alaska and Hawaii and to a sample of records in other States identified when the mail list was constructed. Addresses were selected into the sample with certainty if they were expected to have large total values of agricultural products sold or large acreage, if they were firms with two or more farms, or if they had other special characteristics. When a nonsample large farm was identified during processing, a supplemental form that contained the additional data inquiries was mailed. All farms in counties with less than 100 farms in 1982 were included in the sample with certainty; counties containing 100 to 199 farms in 1982 were systematically sampled at a rate of 1 in 2; and counties containing 200 or more farms in 1982 were systematically sampled at a rate of 1 in 6 . This differential sample scheme was used to provide reliable data for sections 23 through 28 of the report form for all counties.

To determine which mail list records would receive the short form, all mail list records not designated for the sample were sorted into model groups according to farm probability as specified by the mail list model. The 906,000 mail list records in the model groups with the lowest probability of being farms and with an expected total value of agricultural product sales less than $\$ 20,000$ were designated to receive the short form. The remaining mail list records were selected to receive the nonsample census form.

\section{CENSUS ESTIMATION}

The 1987 Census of Agriculture used two types of statistical estimation procedures. These estimation 
procedures accounted both for nonresponse to the data collection and for the sample data collection. These procedures are used because some farm operators never respond to the census despite numerous attempts to contact them, and not all farm operators are requested to provide the sample data items.

\section{Whole Farm Nonresponse Estimation}

A statistical estimation procedure was used to account for the census farms among mail list nonrespondents that were not designated for telephone followup. A stratified systematic sample of eligible census nonrespondents were mailed a simplified report form. Five sample strata were defined based on form type, expected value of sales, and previous census status. The report form was designed to provide sufficient information to determine farm status. Additional mail and telephone contacts were made to survey nonrespondents to obtain sufficient response for survey estimates.

Estimates of the proportion of census nonrespondents that operated farms were made for each stratum in the State using survey results and applied to the total number of census nonrespondents in that stratum. A synthetic estimation procedure was used to estimate the number of census nonrespondents that operated farms for each county by stratum. This estimation procedure is based on the assumption that the distribution of farms in a stratum by county is the same for census nonrespondents as for census respondents.

Within each stratum in a county, a noninteger nonresponse weight was calculated and assigned to each eligible respondent farm record. The procedure used for calculating the nonresponse weight assumed the eligible census respondents and the nonrespondent farm operations in a county had similar characteristics within each stratum. The noninteger nonresponse weight was the ratio of the sum of the estimated number of nonrespondent farms (using nonresponse survey results) and the number of eligible census respondent farms to the number of eligible census respondent farms. Stratum controls were established to ensure that this weight was never greater than 2.0. The noninteger nonresponse weight was used in the estimation of the final weight for the sample items. It was randomly rounded to an integer weight of either 1 or 2 for each record for tabulating the complete count items.

The procedure assumed that we obtain complete response from large and unique farm operations because these cases received intensive telephone followup during census processing. In situations where addressees could not be contacted by telephone or refused to cooperate, secondary sources such as Agricultural Stabilization and Conservation Service offices or county extension agents were asked to provide information as to whether or not the addressee had agricultural activities. Data from previous census reports for the specific addressee, in conjunction with other information, were used to complete the census report form.
Table A quantifies the effect of the nonresponse esti. mation procedure on selected census data items. The percentage of the census value contributed by nonresponse estimation as provided in this table indicates the potential for bias in published figures resulting from this procedure. The estimates provided in these tables do not reflect the effect of nonresponse to individual data items on respondents' census report forms. The effect of this item nonresponse is discussed further under Census Nonsampling Error.

Table A. Percent of State Totals Contributed by Whole Farm Nonresponse Estimation: 1987

\begin{tabular}{|c|c|}
\hline Item & Percent of total \\
\hline $\begin{array}{l}\text { Farms } \\
\text { Value of land and buildings } \\
\text { Market value of agricultural products sold - } \$ 1,000- \\
\text { Harvested cropland } \\
\text { Corn for grain or seed } \\
\text { Wheat for grain } \\
\text { Livestock and poultry inventory: } \\
\text { Cattle and calves } \\
\text { Hogs and pigs } \\
\text { Hens and pullets of laying age }\end{array}$ & $\begin{array}{r}12.7 \\
6.3 \\
6.7 \\
1.2 \\
5.6 \\
4.7 \\
6.0\end{array}$ \\
\hline
\end{tabular}

\section{Sample Estimation}

All respondent sample records received a sample weight. The sample data estimates the actual figures that would have resulted from a complete census of the items in sections 23 through 28 of the report form. The estimates were obtained from an iterative ratio estimation procedure that resulted in the assignment of a weight to each record containing sample items. For any given county, a sample item total was estimated by multiplying the data items for each farm in the county by the corresponding sample weight and summing overall sample records in the county.

Each sample farm was assigned one sample weight to be used to produce estimates for all sample items. For example, if the weight given to a sample farm had the value 5 , all sample data items reported by that farm would be multiplied by 5 . The weight assigned a certainty farm was 1 . The estimation procedure used to assign weights was performed for each county.

Within a county, the ratio estimation procedure for farms was performed in three steps using three variables. The first variable contained eight 1987 total value of agricultural production (TVP) groups. Both the second and third variables, Standard Industrial Classification (SIC) code and farm acreage, contained two groups. The variable groups were as follows: 
TVP

$\$ 1$ to $\$ 999$

$\$ 1,000$ to $\$ 2,499$

SIC

01 All crops

02 All live-

stock

$\$ 2,500$ to $\$ 4,999$

$\$ 5,000$ to $\$ 9,999$

$\$ 10,000$ to $\$ 24,999$

$\$ 25,000$ to $\$ 49,999$

$\$ 50,000$ to $\$ 99,999$

$\$ 100,000$ or more

The first step in the estimation procedure was to partition the sample records into 32 mutually exclusive initial post strata formed by combining the three variable groups. This produced a three dimensional array where the cells of the array corresponded to the initial post strata groups. Each sample farm record was assigned an initial weight equal to the ratio of the total farm count to the sample farm count, expanded for nonresponse estimation, for the cell containing the sample farm. This weight was approximately equal to the inverse of the probability of selecting a farm for the census sample.

The second step in the estimation procedure was to combine, if necessary, the cells of the array (prior to the repeated ratio estimation) to increase the reliability of the ratio estimation procedure. Any cell within the array that either contained less than 10 sample farms or had a ratio of total farms to sample farms that was more than 2 times the mail sample rate was collapsed with another cell (in the same variable) according to a specified collapsing pattern. New total farm counts and sample farm counts were computed for each of the collapsed cells (final post strata) and were used in the ratio estimation procedure to calculate final sample weights.

In the third step in the ratio estimation procedure, complete counts for the three variables (TVP, SIC, acreage) were used to compute the marginals of the array defined by the final post strata. Factors were then applied to expanded sample totals in each cell of the array to obtain agreement with the row marginal (TVP) complete counts. The sample totals then had factors applied to obtain agreement with the column marginal (SIC) complete counts. Lastly, the sample totals had factors applied to obtain agreement with the depth marginal (acreage) complete counts. This procedure that requires the row totals, then the column totals, and then the depth totals to agree with the complete counts for the rows, columns, and depths, respectively, is continued iteratively until the process converges (the marginal totals agree with the complete count totals).

The ratio of the adjusted total farm count to the sample farm count obtained from the second iteration of the estimation procedure was the noninteger final post stratum sample weight assigned to the sample farm records in that post stratum. The noninteger sample weight, the product of the noninteger final post stratum sample weight and the nonresponse weight, was randomly rounded to an integer weight for tabulation. If, for example, the final weight for the farms in a particular group was 7.2, then one-fifth of the sample farms in this group were randomly assigned a weight of 8 and the remaining four-fifths received a weight of 7 .

\section{CENSUS SAMPLING ERROR}

Sampling error in the census data results from the nonresponse sample and the census sample data collection. Census items were classified as either complete count or sample data items. The complete count items were asked of all farm operators. The complete count data items included land in farms, harvested cropland, livestock inventory and sales, crop acreages, quantities harvested and crop sales, land use, irrigation, government loans and payments, conservation acreage, type of organization, and operator characteristics (sections 1 through 22 of the census report form). Variability in the complete count data items is considerably smaller than in the sample items as the variation is due only to the nonresponse sample estimation procedure. The sample items were asked of approximately 25 percent of the total census farm operators. The sample data items included farm production expenditures, fertilizer and chemical usage, farm machinery and equipment, value of land and buildings, and farm-related income (sections 23 through 28 of the census report form). Variability in the estimates of sample items is due both to the census sample selection and estimation procedure and the nonresponse sample estimation procedure.

The sample for the 1987 Census of Agriculture is one of a large number of possible samples of the same size that could have been selected using the same sample design. Estimates derived from the different samples would differ from each other. The difference between a sample estimate and the average of all possible sample estimates is called the sampling deviation. The standard error or sampling error of a survey estimate is a measure of the variation among the estimates from all possible samples, and thus is a measure of the precision with which an estimate from a particular sample approximates the average result of all possible samples. The percent relative standard error of estimate is defined as the standard error of the estimate divided by the value being estimated multiplied by 100 . If all possible samples were selected, each of the samples were surveyed under essentially the same conditions, and an estimate and its standard error were calculated from each sample, then:

1. Approximately 67 percent of the intervals from one standard error below the estimate to one standard error above the estimate would include the average value of all possible samples.

2. Approximately 90 percent of the intervals from 1.65 standard errors below the estimate to 1.65 standard errors above the estimate would include the average value of all possible samples. 
The computations involved to define the above confidence statements are illustrated in the following example. Assume that the estimate of number of farms for the State is 94,382 and the relative standard error of the estimate (percent) is .1 percent $(0.001)$. Multiplying 94,382 by 0.001 yields 94, the standard error. Therefore, a 67-percent confidence interval is 94,288 to 94,476 (i.e., 94,382 plus or minus 94). If corresponding confidence intervals were constructed for all possible samples of the same size and design, approximately 2 out of 3 (67 percent) of these intervals would contain the figure obtained from a complete enumeration. Similarly, a 90 percent confidence interval is 94,227 to 94,538 (i.e., 94,382 plus or minus 1.65 $\times 94)$.

Tables $B$ and $C$ provide the reliability estimates of the estimated number of farms in a county reporting complete count and sample items, respectively. Both tables show the percent relative standard errors for selected estimated number of farms in a county reporting an item. These are derived from a regression equation. The parameters of the regression equation were estimated using the estimated number of farms in a county reporting the complete count or sample item as the independent variable and the standard error of that estimate as the dependent variable for all counties in the State.

Table B. Reliability Estimates for Number of Farms in a County Reporting a Complete Count Item: 1987

\begin{tabular}{|c|c|}
\hline Farms & $\begin{array}{r}\text { Relative standard } \\
\text { error of estimate } \\
\text { (percent) }\end{array}$ \\
\hline $\begin{array}{l}\text { Number of farms reporting: } \\
250 \\
100 \\
150 \\
2000 \\
1,000 \\
2,000\end{array}$ & $\begin{array}{r}13.8 \\
8.0 \\
5.6 \\
4.3 \\
2.9 \\
2.2 \\
1.5 \\
.9 \\
.6 \\
.5 \\
.3 \\
.2\end{array}$ \\
\hline
\end{tabular}
form.

Note: Complete count items are items in sections 1 to 22 of the report

To illustrate the use of these tables, assume that the estimate of the number of farms reporting hogs and pigs for a particular county, as given in county table 12 , is 89 . Since hogs and pigs is a complete count data item, refer to table $B$ and select the estimated relative standard error of the estimate from the row whose value is equal to or just less than the estimated number of farms, 89. For this example, the relative standard error of the estimate comes from the row for 75 farms reporting. For sample data items, follow the same procedure using table $\mathrm{C}$. In counties that had less than 100 farms in the 1982 Census of Agriculture, table $\mathrm{C}$ does not apply because the farms in these counties were sampled with certainty (1 in 1), and thus, the reliability estimates for the number of farms in these counties are smaller than for counties that were sampled at lower rates ( 1 in 2 or 1 in 6 ).

Table C. Reliability Estimates for Number of Farms in a County Reporting a Sample Item: 1987

\begin{tabular}{|c|c|}
\hline Farms & $\begin{array}{l}\text { Relative standard } \\
\text { error of estimate } \\
\text { (percent) }\end{array}$ \\
\hline Number of farms reporting: & $\begin{array}{r}26.6 \\
21.1 \\
17.5 \\
14.6 \\
10.8 \\
8.6 \\
6.0 \\
3.8 \\
2.6 \\
1.9 \\
1.3 \\
1.0\end{array}$ \\
\hline
\end{tabular}

Note: Sample items are items in sections 23 to 28 of the report form.

Table D presents the relative standard error of selected State data items for all farms and for all farms with sales of $\$ 10,000$ or more. The percent relative standard error of the estimate for complete count data measures the variation associated with the sample-based adjustment for whole farm nonresponse. The percent relative standard error of the estimate for sample items measures both the sampling error due to the nonresponse sample estimation procedure and the census sample selection and estimation procedure. The reliability of State estimates may vary substantially from State to State. Generally, State estimates for a given data item are less reliable than the corresponding U.S. estimate.

Table E presents the standard error (not relative standard error) for percent change in State totals from 1982 to 1987. The general purpose of the percent change estimate is to provide a relative measure of the difference in a characteristic between censuses. The relative change for a given characteristic is defined as the ratio of the difference of the 1987 and the 1982 estimate for that characteristic to the 1982 estimate. This ratio is multiplied by 100 to obtain the percent change. The percent standard error of a percent change estimate, then, is the standard error of the ratio multiplied by 100 .

Table F presents the relative standard error for county totals for 10 major complete count items and 7 sample items. The relative standard error of the estimate (percent) for the same item differs among counties in a State. Reasons for this are differences among counties in (1) the total number of farms, (2) the number of large farms included with certainty, (3) the size classifications of the farms sampled, (4) the amount of nonresponse, (5) the general agricultural characteristics, and (6) the specific characteristic being measured. 


\section{CENSUS NONSAMPLING ERROR}

The accuracy of the census counts are affected by the joint effects of the sampling errors described in the previous section and nonsampling errors. Extensive efforts were made to compile a complete and accurate mail list for the census, to design an understandable report form and instructions, and to minimize processing errors through the use of quality control, verification, and check measures on specific operations. Nonsampling errors arise from incompleteness of the census mail list, duplication in the mail list, incorrect data reporting, errors in editing of reported data, and errors in imputation for missing data. These specific nonsampling errors are further discussed in this section. Evaluation studies will be conducted to measure the extent of certain nonsampling errors such as coverage error, classification error, and item imputation.

\section{Census Coverage}

The main objective of the census of agriculture is to obtain a complete and accurate enumeration of U.S. farms with accurate data on all aspects of the agricultural operation. However, the cost and availability of resources for this enumeration place restrictions on operationally feasible data collection methodologies. The past five agriculture censuses have been conducted by mail enumeration with telephone contact for selected nonrespondents. The completeness of such an enumeration thus depends to a large extent on the coverage of farm operations by the census mail list.

Historically, the census of agriculture has included approximately 90 percent of the farms in the United States and over 96 percent of the agricultural production. Complete enumeration of agricultural operations satisfying the farm definition of $\$ 1,000$ or more in agricultural sales is complicated by fluctuations in agricultural operations qualifying for enumeration, the variety of arrangements under which farms are operated, the multiplicity of names used by an operation, the number of operations in which an operator participates, the accuracy of data reporting, etc. A new mail list is compiled for each census because no current single list of agricultural operations is comprehensive.

An evaluation of census coverage has been conducted for each census of agriculture since 1945. The evaluation provides estimates of the completeness of census farm count and major census data items. In addition, the evaluation helps to identify problems in the census enumeration and provide information that can form the basis for improvements. The results of the 1987 Coverage Evaluation program will be published in volume 2, part 2.

The evaluation of coverage conducted in 1987 was designed to measure errors in the census mail list and in farm classification. Mail list error includes a measurement of farms not on the census mail list (undercount), and a measurement of farms enumerated more than once in the census (overcount). Classification error includes a measurement of farms classified as nonfarms in the census (undercount) and of nonfarms classified as farms in the census (overcount). Classification error arises from reporting and processing errors. Mail list undercount dominates all coverage errors. Net coverage error is defined as the difference of undercounted and overcounted farms. Measurements of these errors, as well as a description of the complete coverage program, will be available in the Coverage Evaluation report.

\section{Mail List Coverage}

A major problem with the use of a mail list for the census of agriculture enumeration is the difficulties that are encountered in compiling a complete list. The percentage of farms on the census mail list varies considerably by State. Several reasons have contributed to farm operators' names not being included on the census mail list-the operation may have been started after the mail list was developed, the operation may be so small as not to appear in agricultural related source lists used in compiling the census list, or the operation may have been falsely classified as a nonfarm prior to mailout. A large proportion of the farms not included on the mail list were small in both acres and sales of agricultural products.

The 1987 Census of Agriculture Coverage Evaluation used the area segment sample of the 1987 June Enumerative Survey (JES) of the National Agricultural Statistical Service (NASS) to estimate farms not on the census mail list. The Census Bureau contracted with the NASS to augment the JES data collection and receive survey data under the confidentiality protection afforded by Title 13 , U.S. Code, from all residents of area sample segments with agricultural activity. These survey records were matched to the census mail list. Records that did not match were mailed a census of agriculture report form to estimate mail list coverage. Estimates of farms not on the census mail list used the capture-recapture dual frame estimator that will be described in the Coverage Evaluation report.

Table $\mathrm{G}$ provides coverage evaluation estimates of the number of farms not on the mail list and selected characteristics of those farms with their percent relative standard error. The table also provides an estimate of characteristics of farms not on the mail list as a percentage of total farms in the State. The estimate of total farms in the State is based on census farm count and the estimated number of farms not on the census mail list. This estimate of total farms in the State was not adjusted for classification and list duplication errors. Estimates of these errors will be made at the regional rather than the State level and will be available in the Coverage Evaluation report. The table provides the standard error (not relative standard error) of this percent estimate.

\section{Respondent and Enumerator Error}

Incorrect or incomplete responses to the mailed census report form or to the questions posed by a telephone 
enumerator introduce error into the census data. Such incorrect information can lead, in some cases, to incorrect enumeration of farms. This type of reporting error is measured by the Classification Error Study discussed later in this section. To reduce all types of reporting error, questions were phrased as clearly as possible based on tests of the census report form, and detailed instructions for completing the report form were provided to each addressee. In addition, each respondent's answers were checked for completeness and consistency.

\section{Item Nonresponse}

Nonresponse to particular questions on the census report that we would logically or statistically expect to be present may create a type of nonsampling error in both complete count and sample data. When information reported for another farm with similar characteristics is used to edit or impute for item nonresponse, the data may be biased because the characteristics of the nonrespondents have not been observed and may differ from those reported by respondents. Any attempt to correct the data for nonresponse may not completely reflect this difference either at the element level (individual farm operation) or on the average.

\section{Processing Error}

The many steps of processing of each census report form are sources for the introduction of nonsampling error. The processing of the census report forms includes clerical screening for farm activity, computerized check-in of report forms and followup of nonrespondents, keying and transmittal of completed report forms, computerized editing of inconsistent and missing data, review and correction of individual records referred from the computer edit, review and correction of tabulated data, and electronic data processing. These operations undergo a number of quality control checks to ensure as accurate an application as possible, yet some errors are not detected and corrected.

\section{Classification Error}

An evaluation study of classification errors was conducted in the 1987 Census of Agriculture as part of the census coverage evaluation program. A sample of mail list respondents was selected, and these addresses reenumerated to determine whether they were a farm or nonfarm. A farm status determination was made based on the evaluation questionnaire and compared with the status based on the data reported on the census form. Differences in status were reconciled.

In past censuses, the proportion of farms undercounted due to classification errors was higher for farms with small values of sales. The classification error rate was higher for (1) livestock farms than crop farms, (2) farms with a small number of acres than larger farms, or (3) tenant farms than full or part-owner farms. Results from the 1987 classification error study will be published in the Coverage Evaluation report.

\section{EDITING DATA AND IMPUTATION FOR ITEM NONRESPONSE}

For the 1987 Census of Agriculture, as in previous censuses, all reported data were keyed and then edited by computer. The edits were used to determine whether the reports met the minimum criteria to be counted as farms in the census. Computer edits also performed a series of complex, logical checks of consistency and completeness of item responses. They provided the basis for deciding to accept, impute (supply), delete, or alter the reported value for each data record item.

Whenever possible, edit imputations, deletions, and changes were based on component or related data on the respondent's report form. For some items, such as operator characteristics, data from the previous census were used when available. Values for other missing or unacceptable reported data items were calculated based on reported quantities and known price parameters.

When these and similar methods were not available and values had to be supplied, the imputation process used information reported for another farm operation in a geographically adjacent area with characteristics similar to those of the farm operation with incomplete data. For example, a farm operation that reported acres of corn harvested, but did not report quantity of corn harvested, was assigned the same bushels of corn per acre harvested as that of the last nearby farm with similar characteristics that reported acceptable yields during that particular execution of the computer edit. The imputation for missing items in each section of the report form was conducted separately; thus, assigned values for one operation could come from more than one respondent.

Prior to the imputation operation, a set of default values and relationships were assigned to the possible imputation variables. The relationships and values varied depending on the item being imputed. For example, different default values were assigned for several standard industrial classification and total value of sales categories when imputing hired farm labor expenses. These values and item relationships for the possible imputation variables were stored in the computer in a series of matrices. The computer records were sorted by reported State and county, where the county sequence was based on similar types of farms and agricultural practices.

Each execution of the computer edit consisted of records from only one State. For a given execution of the edit, the stored entries in the various matrices were retained in the computer only until a succeeding record having acceptable characteristics for some sections of the report form was processed by the computer. Then the acceptable responses 
of the succeeding operation replaced those previously stored. When a record processed through the edit had unreported or unacceptable data, the record was assigned the last acceptable ratio or response from an operation with a similar set of characteristics. Once each execution of the computer edit for a State was completed, the possible imputation variables were reset to the default values and relationships for subsequent executions.

After the initial computer edit, keyed reports not meeting the census farm definition were reviewed to ensure that the data were keyed correctly. Edit referrals were generated for about 30 percent of the reports included as farms, and they were also reviewed for keying accuracy and to ensure that the computer edit actions were correct. If the results of the computer edit were not acceptable, corrections were made and the record was reedited. More extensive discussions of the edit and item imputation methodology with measures of the extent of imputation in the census estimates will be provided in a separate research report.

Tables $D$ through $G$ follow. 
Table D. Reliability Estimates of State Totals: 1987

[For meaning of abbreviations and symbols, see introductory text]

\begin{tabular}{|c|c|c|c|c|}
\hline \multirow[b]{2}{*}{ Item } & \multicolumn{2}{|c|}{ All farms } & \multicolumn{2}{|c|}{ Farms with sales of $\$ 10.000$ or more } \\
\hline & $\begin{array}{r}\text { Total } \\
\text { (number) }\end{array}$ & $\begin{array}{r}\text { Relative standard } \\
\text { eiror of estimate } \\
\text { (percent) }\end{array}$ & $\begin{array}{r}\text { Total } \\
\text { (number) }\end{array}$ & $\begin{array}{r}\text { Relative standard } \\
\text { error of estimate } \\
\text { (percent) }\end{array}$ \\
\hline $\begin{array}{l}\text { Farms } \\
\text { Land in tarms } \\
\text { Average size of } \mathrm{farm} \ldots \ldots \ldots\end{array}$ & $\begin{array}{rr}2 & 966 \\
608 & 245 \\
& 205\end{array}$ & $\begin{array}{l}2 \\
5 \\
7\end{array}$ & $\begin{array}{rr}1 & 859 \\
540 & 547 \\
291\end{array}$ & $\begin{array}{l}2 \\
4 \\
7\end{array}$ \\
\hline $\begin{array}{l}\text { Value of land and buidings }{ }^{1} \\
\text { Average per farm } \\
\text { Average per acre }\end{array}$ & $\begin{array}{rr}1096 & 313 \\
369 & 751 \\
1 & 765\end{array}$ & $\begin{array}{l}1.9 \\
2.7 \\
3.9\end{array}$ & $\begin{array}{rr}931 & 771 \\
499 & 341 \\
1 & 709\end{array}$ & $\begin{array}{l}1.9 \\
3.1 \\
4.0\end{array}$ \\
\hline $\begin{array}{l}\text { Estimated market value of all machinery and equipment }{ }^{1} \ldots \ldots \ldots \ldots . . . \\
\text { Average per farm }\end{array}$ & $\begin{array}{rr}158 & 043 \\
53 & 447\end{array}$ & $\begin{array}{l}2.2 \\
41\end{array}$ & $\begin{array}{rr}137 & 284 \\
73 & 571\end{array}$ & $\begin{array}{l}2.3 \\
49\end{array}$ \\
\hline $\begin{array}{l}\begin{array}{c}\text { Farms by size } \\
1 \text { to } 9 \text { acres } \\
10 \text { to } 49 \text { acres } \\
50 \text { to } 179 \text { acres } \ldots \ldots\end{array} \\
\end{array}$ & $\begin{array}{r}514 \\
2018 \\
867 \\
21371 \\
788 \\
(D)\end{array}$ & $\begin{array}{l}1.5 \\
2.0 \\
12 \\
1.4 \\
1.3 \\
1.4\end{array}$ & \begin{tabular}{rr|}
1 & 378 \\
382 \\
367 \\
8 & 248 \\
383 \\
41 & 074
\end{tabular} & $\begin{array}{l}1.1 \\
1.4 \\
1.0 \\
1.0 \\
1.3 \\
1.6\end{array}$ \\
\hline 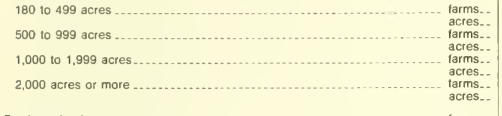 & $\begin{array}{r}481 \\
482 \\
188 \\
\text { (D) } \\
100 \\
\text { (D) } \\
28 \\
\text { (D) }\end{array}$ & $\begin{array}{r}1.6 \\
1.5 \\
17 \\
1.7 \\
- \\
- \\
- \\
-\end{array}$ & $\begin{array}{r}416 \\
1307 \\
188 \\
\text { (D) } \\
99 \\
\text { (D) } \\
28 \\
\text { (D) }\end{array}$ & $\begin{array}{l}1.4 \\
1.4 \\
1.6 \\
1.6 \\
- \\
- \\
-\end{array}$ \\
\hline 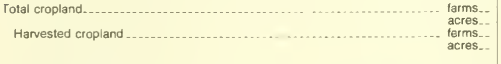 & \begin{tabular}{rr|}
2 & 397 \\
501 & 290 \\
2 & 172 \\
441 & 502
\end{tabular} & $\begin{array}{l}4 \\
5 \\
5 \\
.5\end{array}$ & $\begin{array}{rr}1 & 360 \\
458 & 002 \\
1 & 249 \\
407 & 972\end{array}$ & $\begin{array}{l}4 \\
4 \\
4 \\
4\end{array}$ \\
\hline $\begin{array}{l}\begin{array}{c}\text { Acres harvested: } \\
1 \text { to } 9 \text { acres }\end{array} \\
10 \text { to } 49 \text { acres } \ldots \ldots \ldots \\
50 \text { to } 99 \text { acres } \ldots \ldots \ldots \ldots\end{array}$ & $\begin{array}{r}313 \\
1363 \\
634 \\
15686 \\
356 \\
24 \quad 869\end{array}$ & \begin{tabular}{l|}
2.4 \\
3.0 \\
1.6 \\
1.7 \\
2.2 \\
22
\end{tabular} & $\begin{array}{r}116 \\
461 \\
164 \\
4560 \\
158 \\
11348\end{array}$ & $\begin{array}{l}2.1 \\
2.5 \\
1.8 \\
2.3 \\
2.2 \\
2.3\end{array}$ \\
\hline $\begin{array}{l}200 \text { to } 499 \text { acres } \ldots \ldots \ldots \ldots \ldots \ldots \\
500 \text { to } 999 \text { acres } \ldots \ldots \ldots \ldots \ldots \\
1,000 \text { acres or more } \ldots \ldots \ldots \ldots \\
\text { acres.. }\end{array}$ & $\begin{array}{rr}293 \\
41 & 001 \\
349 \\
110690 \\
141 \\
101 \quad 280 \\
86 \\
146 \quad 613\end{array}$ & $\begin{array}{l}2.1 \\
2.1 \\
1.7 \\
1.7 \\
1.4 \\
1.3 \\
- \\
-\end{array}$ & $\begin{array}{rr}241 \\
34 & 278 \\
343 \\
109 \\
432 \\
141 \\
101 \quad 280 \\
86 \\
146 \\
613\end{array}$ & $\begin{array}{l}2.0 \\
2.0 \\
1.6 \\
1.5 \\
1.3 \\
1.2 \\
- \\
-\end{array}$ \\
\hline $\begin{array}{l}\text { Cropland used only tor pasture or grazing .... } \\
\text { Other cropland }\end{array}$ & $\begin{array}{rr}510 \\
10 & 200 \\
874 \\
49 & 588\end{array}$ & $\begin{array}{l}1.8 \\
2.0 \\
1.1 \\
1.1\end{array}$ & $\begin{array}{rr}225 \\
679 \\
575 \\
43051\end{array}$ & $\begin{array}{r}1.7 \\
2.1 \\
1.0 \\
.9\end{array}$ \\
\hline $\begin{array}{l}\text { migated land } \\
\text { Acres Irrigated: }\end{array}$ & 60812 & $\begin{array}{r}1.4 \\
.5\end{array}$ & $\begin{array}{r}316 \\
60 \quad 295\end{array}$ & $\begin{array}{r}1.1 \\
.5\end{array}$ \\
\hline 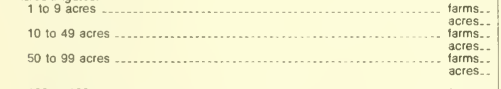 & $\begin{array}{r}103 \\
280 \\
78 \\
1664 \\
60 \\
4244\end{array}$ & $\begin{array}{l}3.3 \\
3.9 \\
4.2 \\
4.7 \\
2.7 \\
2.8\end{array}$ & $\begin{array}{r}55 \\
149 \\
59 \\
\text { (D) } \\
59 \\
\text { (D) }\end{array}$ & $\begin{array}{l}2.4 \\
2.6 \\
4.3 \\
4.8 \\
2.5 \\
2.6\end{array}$ \\
\hline $\begin{array}{l}100 \text { to } 199 \text { acres } 200 \text { to } 499 \text { acres } \\
500 \text { to } 999 \text { acres } \\
1,000 \text { acres or more }\end{array}$ & $\begin{array}{rr} & 50 \\
6850 \\
& 58 \\
15 \quad 640 \\
17 & 27 \\
17 & 847 \\
14 & 8 \\
14 & 287\end{array}$ & $\begin{array}{r}3.0 \\
2.9 \\
1.9 \\
1.5 \\
- \\
- \\
- \\
-\end{array}$ & $\begin{array}{r}50 \\
6850 \\
58 \\
15 \quad 640 \\
27 \\
17847 \\
14 \quad 8 \\
14 \quad 287\end{array}$ & $\begin{array}{l}2.5 \\
2.6 \\
1.8 \\
1.4 \\
- \\
- \\
-\end{array}$ \\
\hline 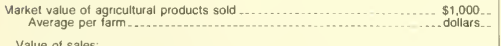 & $\begin{array}{l}443575 \\
149553\end{array}$ & .13 & $\begin{array}{l}440018 \\
236696\end{array}$ & $\begin{array}{l}1 \\
.3\end{array}$ \\
\hline 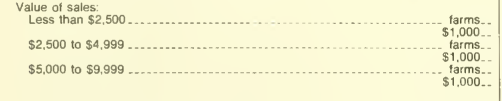 & $\begin{array}{r}540 \\
521 \\
270 \\
977 \\
297 \\
2059\end{array}$ & $\begin{array}{l}1.8 \\
2.6 \\
3.0 \\
3.0 \\
2.7 \\
2.7\end{array}$ & $\begin{array}{l}- \\
\overline{-} \\
\overline{-}\end{array}$ & $\begin{array}{l}\text { - } \\
\text { - }\end{array}$ \\
\hline 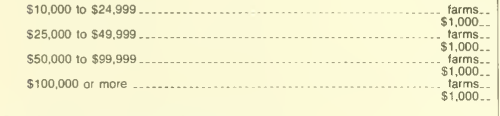 & $\begin{array}{rr}4 & 280 \\
546 \\
214 \\
7 & 632 \\
257 \\
18 & 614 \\
1 & 108 \\
409 & 227\end{array}$ & \begin{tabular}{r|}
2.6 \\
2.7 \\
27 \\
2.7 \\
23 \\
24 \\
- \\
-
\end{tabular} & $\begin{array}{r}280 \\
4546 \\
214 \\
7632 \\
257 \\
18614 \\
1108 \\
409227\end{array}$ & $\begin{array}{l}2.1 \\
2.2 \\
2.4 \\
2.4 \\
2.1 \\
2.1 \\
- \\
-\end{array}$ \\
\hline 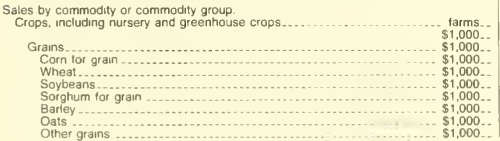 & \begin{tabular}{rr|}
1 & 992 \\
96 & 118 \\
46 & 701 \\
18 & 294 \\
4 & 438 \\
21 & 654 \\
& 36 \\
2 & 190 \\
6 \\
63
\end{tabular} & $\begin{array}{r}5 \\
3 \\
5 \\
6 \\
1.0 \\
7 \\
164 \\
12 \\
148 \\
3.3\end{array}$ & $\begin{array}{rr}1 & 181 \\
93 & 430 \\
44 & 580 \\
17 & 881 \\
4 & 191 \\
20 & 275 \\
& 26 \\
2 & 127 \\
& 3 \\
& 76\end{array}$ & $\begin{array}{r}.5 \\
2 \\
5 \\
5 \\
9 \\
7 \\
72 \\
22 \\
1.2 \\
17.0 \\
3.0\end{array}$ \\
\hline
\end{tabular}

See tootnotes at end ot table 
Table D. Reliability Estimates of State Totals: 1987-Con.

[For meaning of abbreviations and symbols, see introductory text]

\begin{tabular}{|c|c|c|c|c|}
\hline \multirow[b]{2}{*}{ Item } & \multicolumn{2}{|c|}{ All farms } & \multicolumn{2}{|c|}{ Farms with sales of $\$ 10,000$ or more } \\
\hline & $\begin{array}{r}\text { Total } \\
\text { (number) }\end{array}$ & $\begin{array}{r}\text { Relative standard } \\
\text { error of estimate } \\
\text { (percent) }\end{array}$ & $\begin{array}{r}\text { Total } \\
\text { (number) }\end{array}$ & $\begin{array}{r}\text { Relative standard } \\
\text { error of estimate } \\
\text { (percent) }\end{array}$ \\
\hline \multicolumn{5}{|l|}{$\begin{array}{l}\text { Sales by commodity or commodity group-Con } \\
\text { Crops, including nursery and greenhouse crops-Con. }\end{array}$} \\
\hline 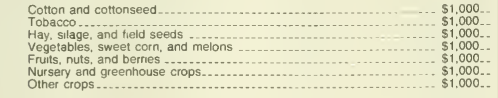 & $\begin{array}{rr}1 & 428 \overline{8} \\
20 & 332 \\
2 & 202 \\
13 & 488 \\
11 & 967\end{array}$ & $\begin{array}{r}2 . \overline{9} \\
4 \\
16 \\
\overline{3}\end{array}$ & \begin{tabular}{rr|}
1 & $25 \overline{3}$ \\
20 & 092 \\
2 & 163 \\
13 & 383 \\
11 & 960
\end{tabular} & $\begin{array}{r}30 \\
\overline{0} \\
1.5 \\
\overline{3}\end{array}$ \\
\hline 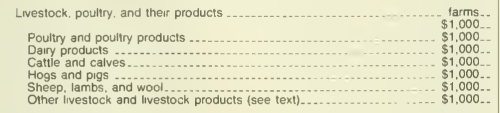 & $\begin{array}{rr}1 & 658 \\
347 & 456 \\
304 & 075 \\
16 & 049 \\
14 & 938 \\
10 & 055 \\
& 54 \\
2 & 286\end{array}$ & $\begin{array}{r}6 \\
1 \\
1 \\
6 \\
4 \\
12 \\
13.4 \\
3.7\end{array}$ & $\begin{array}{rr}1 & 341 \\
346 & 588 \\
304 & 019 \\
16 & 037 \\
14 & 585 \\
9 & 844 \\
3 & 33 \\
2 & 071\end{array}$ & $\begin{array}{r}4 \\
1 \\
1 \\
.6 \\
4 \\
1.1 \\
4.5 \\
4.0\end{array}$ \\
\hline $\begin{array}{l}\begin{array}{l}\text { Farms by standard industrial classification: } \\
\text { Cash grains }(011) \\
\text { Field crops, except cash grains }(013) \ldots \ldots \ldots\end{array} \\
\text { Vegetables and melons }(016)\end{array}$ & $\begin{array}{rr}1 & 029 \\
289 & 411 \\
& 92 \\
29 & 065 \\
128 \\
52 & 830\end{array}$ & $\begin{array}{r}1.1 \\
1.0 \\
4.9 \\
2.0 \\
3.9 \\
.9\end{array}$ & $\begin{array}{r}440 \\
242 \quad 769 \\
38 \\
26 \quad 176 \\
66 \\
51 \quad 330\end{array}$ & $\begin{array}{r}1.3 \\
1.0 \\
5.3 \\
1.8 \\
3.8 \\
.9\end{array}$ \\
\hline $\begin{array}{l}\text { Fruits and tree nuts }(017) \\
\text { Horticultural specialties }(018) \\
\text { General farms, primarity crop }(019)\end{array}$ & $\begin{array}{rr}2 & 205 \\
74 \\
2 & 913 \\
69 \\
18 & 178\end{array}$ & $\begin{array}{r}10.5 \\
4.9 \\
- \\
5.5 \\
2.8\end{array}$ & $\begin{array}{rr}1 & 7 \\
536 \\
52 \\
2465 \\
25 \\
16285\end{array}$ & $\begin{array}{r}10.1 \\
4.3 \\
-\overline{-} \\
4 . \overline{4} \\
2.7\end{array}$ \\
\hline 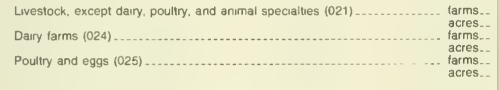 & \begin{tabular}{rr|}
34 & 238 \\
105 \\
105 \\
36 & 884 \\
1 & 042 \\
121 & 689
\end{tabular} & \begin{tabular}{r|}
3.1 \\
2.0 \\
2.9 \\
13 \\
6 \\
4
\end{tabular} & $\begin{array}{rr}71 \\
24 & 910 \\
104 \\
(D) \\
1025 \\
121 & 106\end{array}$ & $\begin{array}{r}3.8 \\
1.7 \\
2.7 \\
1.2 \\
5 \\
4\end{array}$ \\
\hline $\begin{array}{l}\text { Animal specialties (027) } \\
\text { General tarms, primanly livestock and animal speciaities (029) } \ldots . . . . . . \text { farms.. } \\
\text { acres.. }\end{array}$ & $\begin{array}{rr}5 & 146 \\
& 23 \\
15 & 262\end{array}$ & $\begin{array}{r}4.4 \\
5.4 \\
10.0 \\
1.5\end{array}$ & $\begin{array}{r}18 \\
1916 \\
13 \\
14771\end{array}$ & $\begin{array}{l}82 \\
6.1 \\
81 \\
1.2\end{array}$ \\
\hline 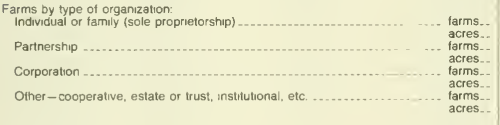 & $\begin{array}{rr}2 & 553 \\
400 & 071 \\
& 216 \\
62 & 666 \\
181 \\
142 & 784 \\
16 \\
2724\end{array}$ & $\begin{array}{r}4 \\
6 \\
2.4 \\
2.1 \\
2.0 \\
6 \\
12.0 \\
59\end{array}$ & $\begin{array}{rr}1 & 537 \\
338 & 062 \\
161 \\
58 & 948 \\
151 \\
141 & 073 \\
10 \\
2 & 464\end{array}$ & $\begin{array}{r}.3 \\
6 \\
2.2 \\
2.1 \\
1.3 \\
.5 \\
13.4 \\
6.1\end{array}$ \\
\hline $\begin{array}{l}\begin{array}{l}\text { Tenure of operator: } \\
\text { Full owners }\end{array} \\
\text { Part owners } \\
\text { Tenants }\end{array}$ & $\begin{array}{rr}1 & 825 \\
142 & 331 \\
857 \\
399 & 038 \\
& 284 \\
66 & 876\end{array}$ & $\begin{array}{r}6 \\
1.3 \\
1.1 \\
6 \\
2.5 \\
1.9\end{array}$ & $\begin{array}{rr}1 & 064 \\
98 & 735 \\
636 \\
382 & 256 \\
& 159 \\
59 & 556\end{array}$ & $\begin{array}{r}6 \\
1.5 \\
8 \\
.6 \\
2.7 \\
1.9\end{array}$ \\
\hline $\begin{array}{l}\begin{array}{l}\text { Operators by principal occupation: } \\
\text { Farming }\end{array} \\
\text { Other }\end{array}$ & \begin{tabular}{rr|}
1 & 774 \\
508 & 992 \\
1 & 192 \\
99 & 253
\end{tabular} & $\begin{array}{r}6 \\
5 \\
9 \\
1.8\end{array}$ & $\begin{array}{rr}1 & 372 \\
476 & 957 \\
487 \\
63 & 590\end{array}$ & $\begin{array}{r}4 \\
.5 \\
1.1 \\
24\end{array}$ \\
\hline $\begin{array}{l}\begin{array}{c}\text { Operators by sex } \\
\text { Male }\end{array} \\
\text { Female } \\
\text { Average age of operator } \ldots \ldots \ldots \\
\text { farms... }\end{array}$ & $\begin{array}{r}2676 \\
590 \quad 036 \\
290 \\
18 \quad 209 \\
51.5\end{array}$ & $\begin{array}{r}3 \\
5 \\
22 \\
43 \\
.6\end{array}$ & $\begin{array}{r}1656 \\
526 \quad 232 \\
203 \\
14315 \\
50.0\end{array}$ & $\begin{array}{r}3 \\
4 \\
1.9 \\
4.8 \\
6\end{array}$ \\
\hline $\begin{array}{l}\text { Cropland under federal acreage reduction programs: } \\
\text { Annual commodity acreage adjustment programs } \ldots \ldots \ldots \\
\text { Conservation reserve program }\end{array}$ & $\begin{array}{r}450 \\
29683 \\
18 \\
1 \quad 141\end{array}$ & $\begin{array}{r}1.4 \\
.9 \\
6.1 \\
5.7\end{array}$ & $\begin{array}{r}383 \\
28660 \\
15 \\
1124\end{array}$ & $\begin{array}{r}1.2 \\
8 \\
2.1 \\
3.4\end{array}$ \\
\hline 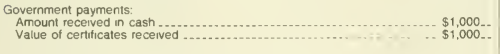 & $\begin{array}{l}3513 \\
3902\end{array}$ & $\begin{array}{l}7 \\
7\end{array}$ & $\begin{array}{ll}3 & 421 \\
3 & 749\end{array}$ & .6 \\
\hline 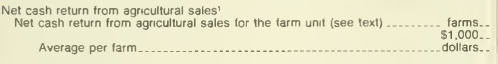 & $\begin{array}{rr}2 & 965 \\
65 & 300 \\
22 & 023\end{array}$ & $\begin{array}{r}5 \\
24 \\
44\end{array}$ & $\begin{array}{r}1866 \\
68350 \\
36 \quad 629\end{array}$ & $\begin{array}{r}.6 \\
22 \\
4.6\end{array}$ \\
\hline 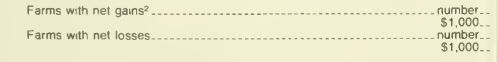 & $\begin{array}{rr}1 & 695 \\
76 & 564 \\
1 & 270 \\
-11 & 265\end{array}$ & $\begin{array}{r}169 \\
3 \\
5 \\
(Z)\end{array}$ & $\begin{array}{rr}1 & 462 \\
76 & 116 \\
404 \\
-7 & 767\end{array}$ & $\begin{array}{r}17.0 \\
.3 \\
5 \\
(Z)\end{array}$ \\
\hline 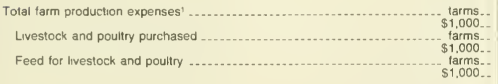 & $\begin{array}{rr}2 & 965 \\
377 & 102 \\
1 & 374 \\
53 & 522 \\
1 & 637 \\
187 & 183\end{array}$ & $\begin{array}{r}4 \\
4 \\
2.7 \\
6 \\
26 \\
6\end{array}$ & $\begin{array}{rr}1 & 866 \\
370 & 478 \\
1 & 200 \\
53 & 253 \\
1 & 300 \\
186 & 851\end{array}$ & $\begin{array}{r}.5 \\
4 \\
21 \\
.6 \\
2.0 \\
.6\end{array}$ \\
\hline
\end{tabular}

See footnotes at end of table 
Table D. Reliability Estimates of State Totals: 1987-Con.

[For meaning of abbreviations and symbols, see introductory text]

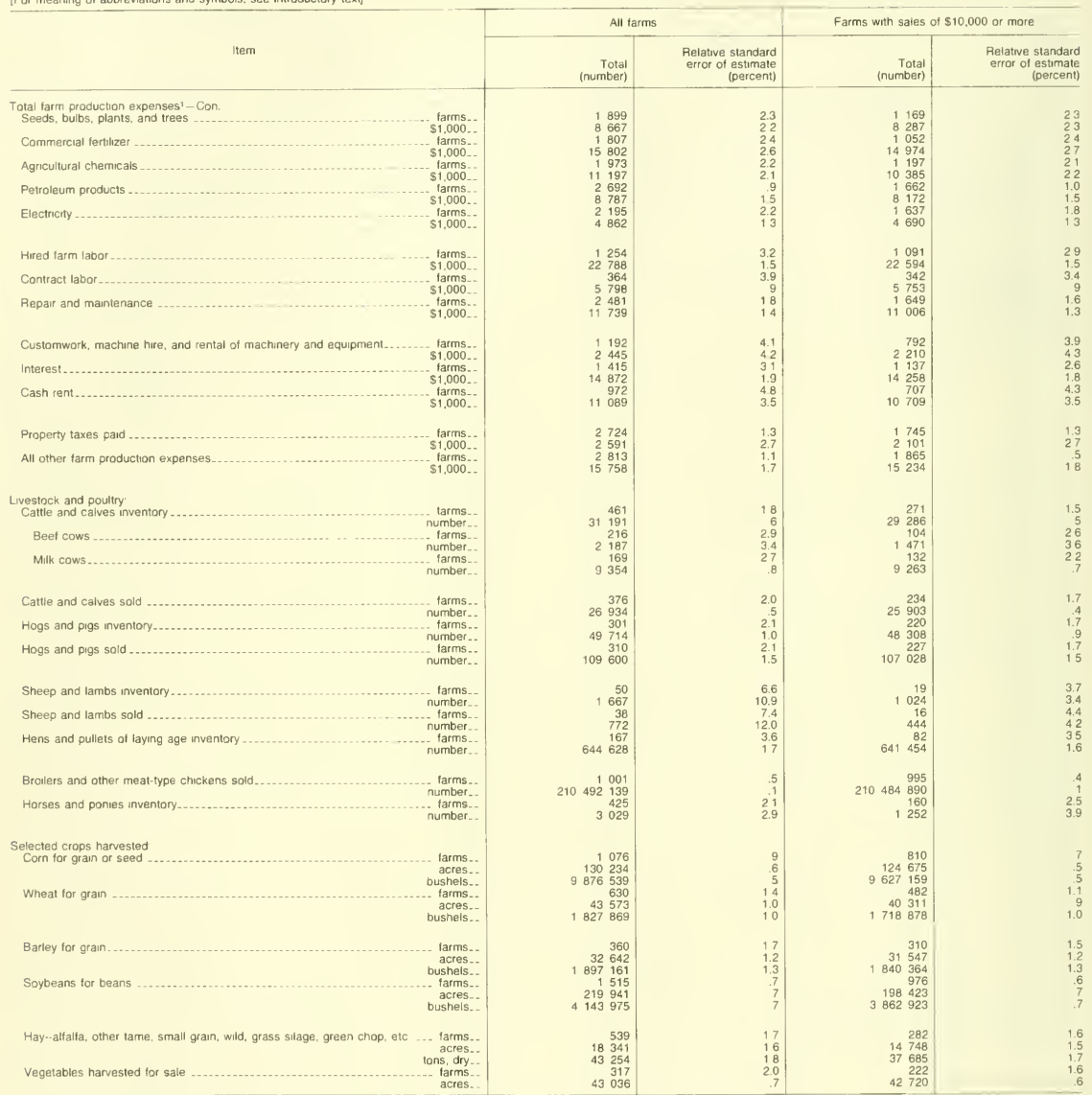

'Data are based on a sample of farms

2Farms with total production expenses equal to market value of agncultural products sold are included as farms with gains 
Table E. Reliability Estimates of Percent Change in State Totals: 1982 to 1987

[For meaning of abbreviations and symbols, see introductory text]

\begin{tabular}{|c|c|c|c|c|}
\hline \multirow[b]{2}{*}{ Item } & \multicolumn{2}{|c|}{ All farms } & \multicolumn{2}{|c|}{ Farms with sales of $\$ 10,000$ or more } \\
\hline & Percent change & $\begin{array}{r}\text { Standard error } \\
\text { of estimate } \\
\text { (percent) }\end{array}$ & Percent change & $\begin{array}{l}\text { Standard error } \\
\text { of estimate } \\
\text { (percent) }\end{array}$ \\
\hline $\begin{array}{l}\text { Farms } \\
\text { Land in farms }\end{array}$ & $\begin{array}{r}-11.1 \\
-7.2\end{array}$ & $\begin{array}{l}7 \\
6\end{array}$ & $\begin{array}{r}-147 \\
-98\end{array}$ & $\begin{array}{l}5 \\
5\end{array}$ \\
\hline . & 1.3 & 48 & 2 & 5.3 \\
\hline Total cropland........ & -15.7 & .7 & -23.6 & .5 \\
\hline $\begin{array}{l}\text { acres. } \\
\text { farms... } \\
\text { acres... }\end{array}$ & $\begin{array}{r}-38 \\
-19.6 \\
-11.7\end{array}$ & $\begin{array}{l}7 \\
7 \\
6\end{array}$ & $\begin{array}{r}-60 \\
-268 \\
-13.6\end{array}$ & $\begin{array}{l}5 \\
5 \\
5\end{array}$ \\
\hline Irrigated land...................... farms... & $\begin{array}{l}18.9 \\
37.7\end{array}$ & $\begin{array}{r}1.7 \\
.8\end{array}$ & $\begin{array}{l}10.9 \\
37.6\end{array}$ & $\begin{array}{r}13 \\
.7\end{array}$ \\
\hline 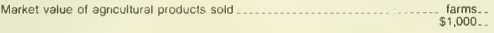 & $\begin{array}{r}-11.1 \\
19.7\end{array}$ & $\begin{array}{l}7 \\
.4\end{array}$ & $\begin{array}{r}-14.7 \\
20.0\end{array}$ & $\begin{array}{l}5 \\
3\end{array}$ \\
\hline Crops, including nursery and greenhouse crops... & -20.4 & 7 & -27.5 & 5 \\
\hline Livestock, poultry, and their products . & $\begin{array}{r}-12.8 \\
-4.3\end{array}$ & $\begin{array}{l}4 \\
7\end{array}$ & $\begin{array}{r}-12.8 \\
-5.2\end{array}$ & $\frac{3}{5}$ \\
\hline Poultry and poultry products & 33.5 & 2 & 33.6 & 2 \\
\hline Poultry and poultry products ................. & 37.6 & 2 & 37.6 & 2 \\
\hline \multicolumn{5}{|l|}{$\begin{array}{l}\text { Selected farm production expenses'. } \\
\text { Livestock and poultry purchased }\end{array}$} \\
\hline Livestock and poultry purchased ... & $\begin{array}{r}4.1 \\
43.9\end{array}$ & $\begin{array}{l}45 \\
1.9\end{array}$ & $\begin{array}{r}4 \\
43.8\end{array}$ & $\begin{array}{l}3.7 \\
1.9\end{array}$ \\
\hline Feed for livestock and poultry ..... & -3 & 42 & -45 & 3.5 \\
\hline Seeds, bulbs, plants, and trees .... & $\begin{array}{r}33.2 \\
-10.1\end{array}$ & $\begin{array}{l}1.9 \\
3.3\end{array}$ & $\begin{array}{r}33.8 \\
-312\end{array}$ & 1.9 \\
\hline Seeds, bulbs, plants, and trees ...... & $\begin{array}{r}-10.1 \\
24.5\end{array}$ & 47 & $\begin{array}{l}-21.2 \\
24.3\end{array}$ & $\begin{array}{l}2.9 \\
4.8\end{array}$ \\
\hline Commercial fertilizer ${ }^{2} \ldots$ & -17.5 & 29 & -261 & 2.8 \\
\hline$\$ 1,000$. & $\begin{array}{r}-21.0 \\
-41\end{array}$ & $\begin{array}{l}3.2 \\
3.3\end{array}$ & $\begin{array}{l}-217 \\
-188\end{array}$ & $\begin{array}{l}3.3 \\
2.9\end{array}$ \\
\hline 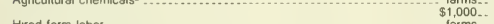 & $\begin{array}{r}-4.1 \\
3.0\end{array}$ & 36 & -14 & 3.6 \\
\hline Hired farm labor ............. & $\begin{array}{r}-14.2 \\
27.4\end{array}$ & $\begin{array}{l}4.6 \\
73\end{array}$ & -9.5 & 46 \\
\hline $\begin{array}{l}\$ 1,000 .- \\
\text { farms.. } \\
\text { sime }\end{array}$ & $\begin{array}{c}27.4 \\
-1.3\end{array}$ & 49 & $\begin{array}{l}28.0 \\
-9.4\end{array}$ & $\begin{array}{l}7.4 \\
42\end{array}$ \\
\hline$\$ 1,000$.. & -12.2 & 3.2 & -146 & 31 \\
\hline \multicolumn{5}{|l|}{$\begin{array}{l}\text { Livestock and poultry inventory } \\
\text { Cattle and caives........ }\end{array}$} \\
\hline Cattle and caives........... & $\begin{array}{l}-30.2 \\
-6.5\end{array}$ & 1.3 & $\begin{array}{r}-37.8 \\
-6.0\end{array}$ & $\begin{array}{r}1.0 \\
5\end{array}$ \\
\hline Hogs and pigs .............. & -285 & 1.5 & -32.9 & 1.2 \\
\hline Hens and pullets of laying age.... & $\begin{array}{r}-8.6 \\
-30.4\end{array}$ & 2.5 & $\begin{array}{r}-82 \\
-43.8\end{array}$ & 2.9 \\
\hline 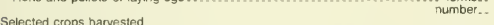 & -13.2 & 1.5 & -13.1 & 1.4 \\
\hline $\begin{array}{l}\text { Selected crops harvested } \\
\text { Corn for grain or seed }\end{array}$ & -21.1 & 9 & -242 & 7 \\
\hline Sorghum for gran or seed... & 250.0 & 21.5 & 220.0 & 20.4 \\
\hline Wheat for grain ................. & -19.5 & 1.2 & -291 & (1) 8 \\
\hline acres.. & -23.4 & 8 & -267 & .7 \\
\hline \multirow{2}{*}{ 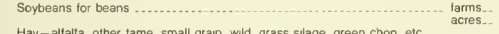 } & -23.9 & .7 & -28.1 & .6 \\
\hline & -17.0 & & -19.4 & \\
\hline (see text) & $\begin{array}{r}-11.5 \\
84\end{array}$ & $\begin{array}{l}1.6 \\
1.9\end{array}$ & $\begin{array}{r}-23.8 \\
3.7\end{array}$ & $\begin{array}{l}1.2 \\
1.6\end{array}$ \\
\hline Vegetables harvested for sale (see text) ..... & $\begin{array}{r}-136 \\
65\end{array}$ & 17 & $\begin{array}{r}-196 \\
67\end{array}$ & 1.3 \\
\hline 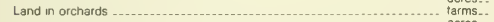 & -234 & 6.0 & $\begin{array}{r}-105 \\
94\end{array}$ & 7.2 \\
\hline acres.. & 6.9 & 12 & 9.4 & 1.0 \\
\hline
\end{tabular}

'Data are based on a sample of farms.

2Data tor 1987 include cost of custom applications

Data for 1982 do not include imputation for item nonresponse. 
Table F. Reliability Estimates of County Totals: 1987

[For meaning of abbreviations and symbols, see introductory text]

\begin{tabular}{|c|c|c|c|c|c|c|c|c|c|c|c|c|c|c|c|c|}
\hline \multirow[b]{2}{*}{ Geographic area } & \multicolumn{2}{|l|}{ Farms } & \multicolumn{4}{|c|}{ Land in farms } & \multicolumn{2}{|c|}{$\begin{array}{l}\text { Average value of land } \\
\text { and buildings per tarm' }\end{array}$} & \multicolumn{2}{|c|}{$\begin{array}{c}\text { Estumated market value } \\
\text { of all machinery and } \\
\text { equipment }\end{array}$} & \multicolumn{4}{|c|}{ Harvested cropland } & \multicolumn{2}{|c|}{ Irrigated land } \\
\hline & $\begin{array}{r}\text { Total } \\
\text { (number) }\end{array}$ & $\begin{array}{r}\text { Relative } \\
\text { standard } \\
\text { error of } \\
\text { estimate } \\
\text { (percent) }\end{array}$ & & $\begin{array}{r}\text { Total } \\
\text { (acres) }\end{array}$ & \multicolumn{2}{|c|}{$\begin{array}{r}\text { Relative } \\
\text { standard } \\
\text { error of } \\
\text { estimate } \\
\text { (percent) }\end{array}$} & $\begin{array}{r}\text { Value } \\
\text { (dollars) }\end{array}$ & $\begin{array}{c}\text { Relative } \\
\text { standard } \\
\text { error of } \\
\text { estimate } \\
\text { (percent) }\end{array}$ & \multicolumn{2}{|r|}{$\begin{array}{r}\text { Relative } \\
\text { standard } \\
\text { error of } \\
\text { estimate } \\
\text { (percent) }\end{array}$} & \multicolumn{4}{|c|}{$\begin{array}{r}\text { Relative } \\
\text { standard } \\
\text { error of } \\
\text { estumate } \\
\text { (percent) }\end{array}$} & $\begin{array}{r}\text { Total } \\
\text { (acres) }\end{array}$ & $\begin{array}{r}\text { Relative } \\
\text { standard } \\
\text { error of } \\
\text { estumate } \\
\text { (percent) }\end{array}$ \\
\hline $\begin{array}{l}\text { Kent } \\
\text { New Castle } \\
\text { Sussex }\end{array}$ & $\begin{array}{r}857 \\
380 \\
1729 \\
\end{array}$ & $\begin{array}{l}5 \\
8 \\
3 \\
\end{array}$ & & $\begin{aligned} 201444 \\
93998 \\
312803\end{aligned}$ & & $\begin{array}{r}9 \\
11 \\
6\end{array}$ & $\begin{array}{ll}402 & 674 \\
670 & 476 \\
287 & 359\end{array}$ & $\begin{array}{l}6.6 \\
7.6 \\
6.3\end{array}$ & $\begin{array}{l}48826 \\
27202 \\
82015\end{array}$ & $\begin{array}{l}3.4 \\
9.4 \\
2.2\end{array}$ & \multicolumn{2}{|c|}{$\begin{array}{r}150674 \\
64358 \\
226470\end{array}$} & & $\begin{array}{r}.9 \\
1.1 \\
.7\end{array}$ & $\begin{array}{rr}18 & 521 \\
2 & 063 \\
40 & 228 \\
\end{array}$ & $\begin{array}{r}7 \\
73 \\
.7\end{array}$ \\
\hline \multirow[b]{2}{*}{ Geographic area } & \multicolumn{2}{|c|}{$\begin{array}{l}\text { Cattie and catves } \\
\text { inventory }\end{array}$} & \multicolumn{4}{|c|}{ Hogs and pigs inventory } & \multicolumn{2}{|c|}{ Corn for grain or seed } & \multicolumn{2}{|c|}{ Wheat for grain } & \multicolumn{4}{|c|}{ Soybeans for beans } & \multicolumn{2}{|c|}{$\begin{array}{l}\text { Market value of agricultural } \\
\text { products sold }\end{array}$} \\
\hline & $\begin{array}{r}\text { Total } \\
\text { (number) }\end{array}$ & $\begin{array}{r}\text { Relative } \\
\text { standard } \\
\text { error of } \\
\text { estimate } \\
\text { (percent) }\end{array}$ & & $\begin{array}{r}\text { Total } \\
\text { (number) }\end{array}$ & $\begin{array}{l}\text { Retat } \\
\text { stand } \\
\text { error } \\
\text { estim } \\
\text { (perce }\end{array}$ & $\begin{array}{l}\text { ative } \\
\text { dard } \\
\text { r of } \\
\text { nate } \\
\text { cent) }\end{array}$ & $\begin{array}{r}\text { Tota } \\
\text { (acres }\end{array}$ & $\begin{array}{r}\text { Relative } \\
\text { standard } \\
\text { error of } \\
\text { estimate } \\
\text { (percent) }\end{array}$ & $\begin{array}{r}\text { Total } \\
\text { (acres) }\end{array}$ & $\begin{array}{r}\text { Relative } \\
\text { standard } \\
\text { error of } \\
\text { estimate } \\
\text { (percent) }\end{array}$ & & $\begin{array}{r}\text { Total } \\
\text { (acres) }\end{array}$ & $\begin{array}{l}\text { Rela } \\
\text { stan } \\
\text { erro } \\
\text { estir } \\
\text { (perc }\end{array}$ & $\begin{array}{l}\text { ative } \\
\text { dard } \\
\text { ar of } \\
\text { nate } \\
\text { cent) }\end{array}$ & $\begin{array}{l}\text { Tot: } \\
\text { (acre }\end{array}$ & $\begin{array}{r}\text { Relative } \\
\text { standard } \\
\text { error of } \\
\text { estumate } \\
\text { (percent) }\end{array}$ \\
\hline $\begin{array}{l}\text { Kent } \\
\text { New Castle } \\
\text { Sussex }\end{array}$ & $\begin{array}{rl}16 & 179 \\
4 & 619 \\
10 & 393\end{array}$ & $\begin{array}{r}1.0 \\
19 \\
.8\end{array}$ & & $\begin{array}{rr}5 & 958 \\
1 & 079 \\
42 & 677\end{array}$ & & $\begin{array}{l}2.9 \\
4.2 \\
1.0\end{array}$ & $\begin{array}{ll}38 & 846 \\
34 & 367 \\
57 & 021\end{array}$ & $\begin{array}{r}1.3 \\
13 \\
.7\end{array}$ & $\begin{array}{rr}15 & 416 \\
3 & 726 \\
24 & 431\end{array}$ & $\begin{array}{l}20 \\
24 \\
13\end{array}$ & & $\begin{array}{rr}70 & 261 \\
17 & 146 \\
132 & 534\end{array}$ & & $\begin{array}{r}1.3 \\
2.2 \\
9\end{array}$ & $\begin{array}{rr}83 & 60 \\
30 & 41 \\
329 & 55\end{array}$ & $\begin{array}{l}3 \\
5 \\
1 \\
\end{array}$ \\
\hline \multirow{3}{*}{ Geographic area } & \multicolumn{16}{|c|}{ Selected farm production expenses? } \\
\hline & \multicolumn{3}{|c|}{$\begin{array}{l}\text { Livestock and poultry } \\
\text { purchased }\end{array}$} & \multicolumn{4}{|c|}{ Commercial fertilizer } & \multicolumn{2}{|c|}{ Hired farm labor } & \multicolumn{4}{|c|}{ Petroleum products } & \multicolumn{3}{|c|}{ Electricity for the farm business } \\
\hline & $\begin{array}{r}\text { Total } \\
(\$ 1,000)\end{array}$ & $\begin{array}{l}\text { Rela } \\
\text { standard } \\
\text { of estur } \\
\text { (perc }\end{array}$ & $\begin{array}{l}\text { ative } \\
\text { error } \\
\text { nate } \\
\text { cent) }\end{array}$ & & $\begin{array}{r}\text { Total } \\
\$ 1,000)\end{array}$ & & $\begin{array}{r}\text { Relative } \\
\text { indard error } \\
\text { of estimate } \\
\text { (percent) }\end{array}$ & $\begin{array}{r}\text { Total } \\
(\$ 1,000)\end{array}$ & $\begin{array}{r}\text { Relative } \\
\text { standard error } \\
\text { of estumate } \\
\text { (percent) }\end{array}$ & & & $\begin{array}{r}R \\
\text { standar } \\
\text { of es } \\
\text { (pe }\end{array}$ & $\begin{array}{l}\text { lative } \\
\text { error } \\
\text { imate } \\
\text { rcent) }\end{array}$ & & $\begin{array}{r}\text { Total } \\
(\$ 1,000)\end{array}$ & $\begin{array}{r}\text { Relative } \\
\text { standard error } \\
\text { of estimate } \\
\text { (percent) }\end{array}$ \\
\hline $\begin{array}{l}\text { Kent } \\
\text { New Castle } \\
\text { Sussex.................... }\end{array}$ & $\begin{array}{r}7887 \\
828 \\
44807\end{array}$ & & $\begin{array}{r}2.9 \\
40 \\
.5\end{array}$ & & $\begin{array}{ll}6 & 750 \\
2 & 831 \\
6 & 221\end{array}$ & & $\begin{array}{l}4.7 \\
45 \\
35\end{array}$ & $\begin{array}{rr}7641 \\
44223 \\
10 \quad 924\end{array}$ & $\begin{array}{l}13 \\
72 \\
1.1\end{array}$ & & $\begin{array}{l}989 \\
187 \\
612\end{array}$ & & $\begin{array}{l}3.2 \\
4.3 \\
1.6\end{array}$ & & $\begin{array}{rr}1 & 005 \\
746 \\
3 \quad 112 \\
\end{array}$ & $\begin{array}{l}3.2 \\
4.9 \\
1.3\end{array}$ \\
\hline
\end{tabular}

'Data are based on a sample of farms 
Table G. State Coverage Evaluation Estimates of Farms Not on the Mail List: 1987

[Data are based on a sample of farms; see text For meaning of abbreviations and symbols, see introductory text]

\begin{tabular}{|c|c|c|c|c|}
\hline \multirow[b]{2}{*}{ Item } & \multicolumn{2}{|c|}{ Not on mal list } & \multicolumn{2}{|c|}{ Percent not on mast list } \\
\hline & Total number & $\begin{array}{r}\text { Relative standard error } \\
\text { of estimate } \\
\text { (percent) }\end{array}$ & Total percent & Standard error of percent \\
\hline $\begin{array}{l}\text { Farms } \\
\text { Land in farms }\end{array}$ & 5630 & $\begin{array}{l}10.7 \\
493\end{array}$ & $\begin{array}{r}7.7 \\
9\end{array}$ & $\begin{array}{r}5 \\
(2)\end{array}$ \\
\hline $\begin{array}{l}\text { Farms by size } \\
\text { Less than } 50 \text { acres } \ldots \ldots \ldots \ldots \\
50 \text { acres or more } \ldots \ldots\end{array}$ & (S) & (S) & $\begin{array}{l}\text { (S) } \\
\text { (S) }\end{array}$ & $\begin{array}{l}\text { (S) } \\
\text { (S) }\end{array}$ \\
\hline Harvested cropland ... .................... larms... & 2770 & $\begin{array}{l}16.3 \\
95.1\end{array}$ & $\begin{array}{r}68 \\
6\end{array}$ & ( 5 ( ) \\
\hline $\begin{array}{l}\begin{array}{l}\text { Farms by value of sales: } \\
\text { Less than } \$ 10,000 \\
\text { Less than } \$ 2,500 \\
\$ 2,500 \text { to } \$ 9,999\end{array} \\
\$ 10,000 \text { or more }\end{array}$ & $\begin{array}{r}247 \\
\text { (S) } \\
\text { (S) }\end{array}$ & $\begin{array}{r}10.7 \\
\text { (S) } \\
\text { (S) }\end{array}$ & $\begin{array}{r}182 \\
\text { (S) } \\
\text { (S) }\end{array}$ & $\begin{array}{l}1.0 \\
\text { (S) } \\
\text { (S) }\end{array}$ \\
\hline Market value of agricultural products sold ......................... $\$ 1,000 \ldots$ & 501 & 23.8 & .1 & (Z) \\
\hline $\begin{array}{l}\text { Farms by standard industral classification: } \\
\text { Crops (01) } \\
\text { Livestock (02) }\end{array}$ & (S) & (S) & (S) & (S) \\
\hline 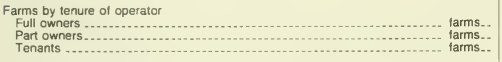 & $\begin{array}{r}247 \\
-\end{array}$ & $\begin{array}{r}10.7 \\
-\end{array}$ & \begin{tabular}{r|r}
11.9 \\
- \\
-
\end{tabular} & $\begin{array}{l}7 \\
- \\
-\end{array}$ \\
\hline $\begin{array}{l}\text { Operators by principal occupation. } \\
\text { Farming } \\
\text { Other }\end{array}$ & $24 \overline{7}$ & 10.7 & 17.2 & $1 . \overline{0}$ \\
\hline Average age of operator ........................... years.. & 50.2 & 21.5 & (x) & (x) \\
\hline
\end{tabular}

Note 1: Farms classified as nonfarms, nonfarms classified as farms, and farms appearing more than once in the census are not accounted for in these estimates, but will be provided in Note 1: Farms classified as nonfarms, nonfarms classified as farms, and
the 1987 Coverage Evaluation publication. See appendix $\mathrm{C}$ for futher explanation.

Note 2: Detail may not add to total due to rounding 



\section{APPENDIX D. Report Form and Information Sheet}

OUE BYFEBRUARY 1,1988

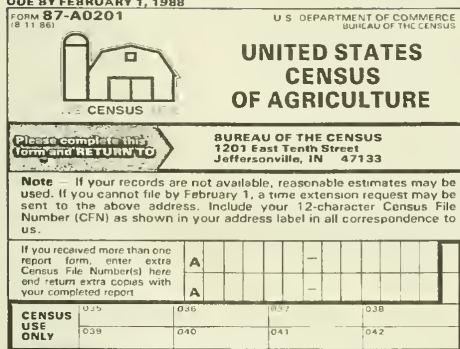

SECTOWFII ACREAGE IN 1987 Report land owned, rented, or used st by you, your spouse, or by the partnership, corporation, or organization for which you are reporting. Include ALL LAND. organization for which you are reporting. Include
REGARDLESS OF LOCATION OR USE cropland. pastureland, rangeland, woodland, idle land. house lots, etc. It the acres you gaerated in 1987 changed during the vear.
vefer to the INFORMATION SMEET, section ing

1. All land owned

2. All land rented or leased FROM OTHERS, including land worked by you on shares, used rent free, in exchange for services. payment of taxes, etc. Include leased Federal, State, and railroad land. (DO NOT include land used on a per head basis under a grazing permit.) Also complete item 5 below.

3. All land rented or leased TO OTHERS, including land worked on shares by others and land subleased. Also complete item 6 below

4. Acres in "THIS PLACE" - ADD acres owned litem 11 and acres rented (item 2), then SUBTRACT acres rented TO OTHERS (item 3 ), and enter the resuit in this space.

For this census report these are the acres in "THIS PLACE.

None Number of acres

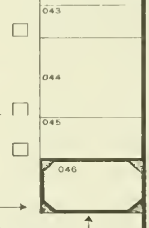
If the entry Is zero Drt these are the aCNOS hM

S. If you rented land FROM OTHERS (item 2), enter the following information for each landlord.

\begin{tabular}{|c|c|c|}
\hline Name of lanciord & Malling address (include ZIP Code) & Number of acres \\
\hline & & \\
\hline & & \\
\hline
\end{tabular}

6. If you rented land TO OTHERS (item 3), enter the following information for each renter.

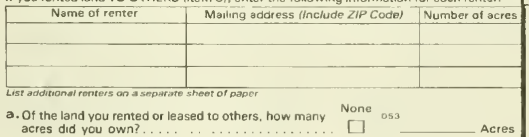

7. Did you have any grazing permits on a per-head basis?
oss
, $\square$ Yes - Mark $(x)$ all boxes which apply
(3) Forest Service
${ }_{2} \square$ No-Gotoitem \&
$\{4$ Taytor Grazing Sec. 3 (BLM) ${ }_{5} \square$ Indian Land
${ }_{6} \square$ Other Specify,

8. LOCATION OF AGRICULTURAL ACTIVITY FOR "THIS PLACE"
. In what county was the largest value of your agricultural products
rased ot produced?
b. If you also had agricultural operations in any other countylies), enter the
county name(s), etc.

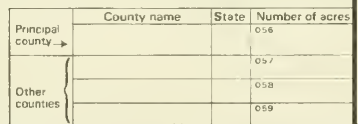

INSTRUCTIONS - Please report your crops in the appropriate section. Use section 7 to report ONLY thoss CROPS NOT listed in sections 2 through 6 and section 8. DO NOT INCLUDE crops grown on land rented to others.

SECTIOKLe ] Were any of the following CROPS harvested from "THIS PLACE" in 1987 ?

1. Corn (field) for grain or seed iRepon quantity ans
dir shelted-weight bassis.)

2. Com (field) for silage or green chap

3. Soybeans for beans

4. Beans, diy edible

5. Wheat for gran

6. Oats for grain

7. Barley for grain

8. Rye for grain

9. Sorghum for grain or seed

10. Sorghum for silage or green chop iDo nar include sorghum suden crosses.).

11. Tobacco - all types

12. Potatoes, Irish

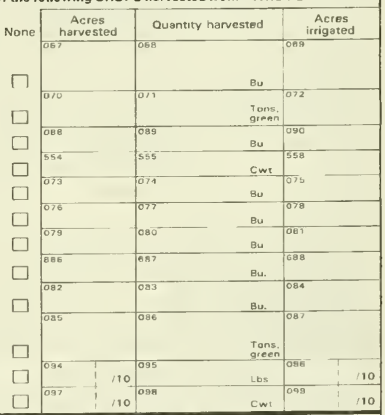

SECTION or harvested from "THIS PLACE" in 19877

Inctude sorghum-iudan crosses end hay cut trom pastures.

S3 , $\square$ YES - Complete this section $2 \square$ NO - Go to section 4

11 curtings were made for borh dry hay and grass silage. haylage, or green choo from the same fields, report the ocreage in the appropriate rems un
HAY and aIso Under GRASS SILAGE, HAYLAGE, and GRFEN CHOO.

1. ORY HAY iff rwo or more cuttings of dry hav were made from the same acres, report
acres only once, but report fotal tons from all acres onty
cutrings.

a. Alfalfa and alfalfa mixtures for hay or dehydrating

b. Small grain hay oats, wheat, barley, rye, etc.

c. Other tame dry hay - clover, lespedeza, timothy, Sudangrass, meadow and pasture grasses, etc.

d. Wild hay

2. GRASS SILAGE, MAYLAGE, AND GREEN CHDP "it two or more cuttings werc made from the same acres, report all cuttings. I.

3. HAY SOLD - Did you sell any hay or grass silage in 1987 ? IAeporr velu or grass silage in 1987 ? 1 if
of hay sold in section 9 , trem 3 )

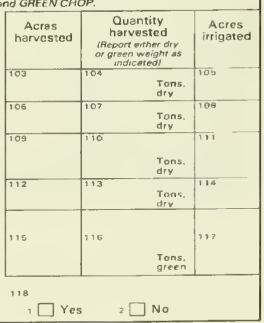

PENALTY FOA FAILURE TOREPOAT 


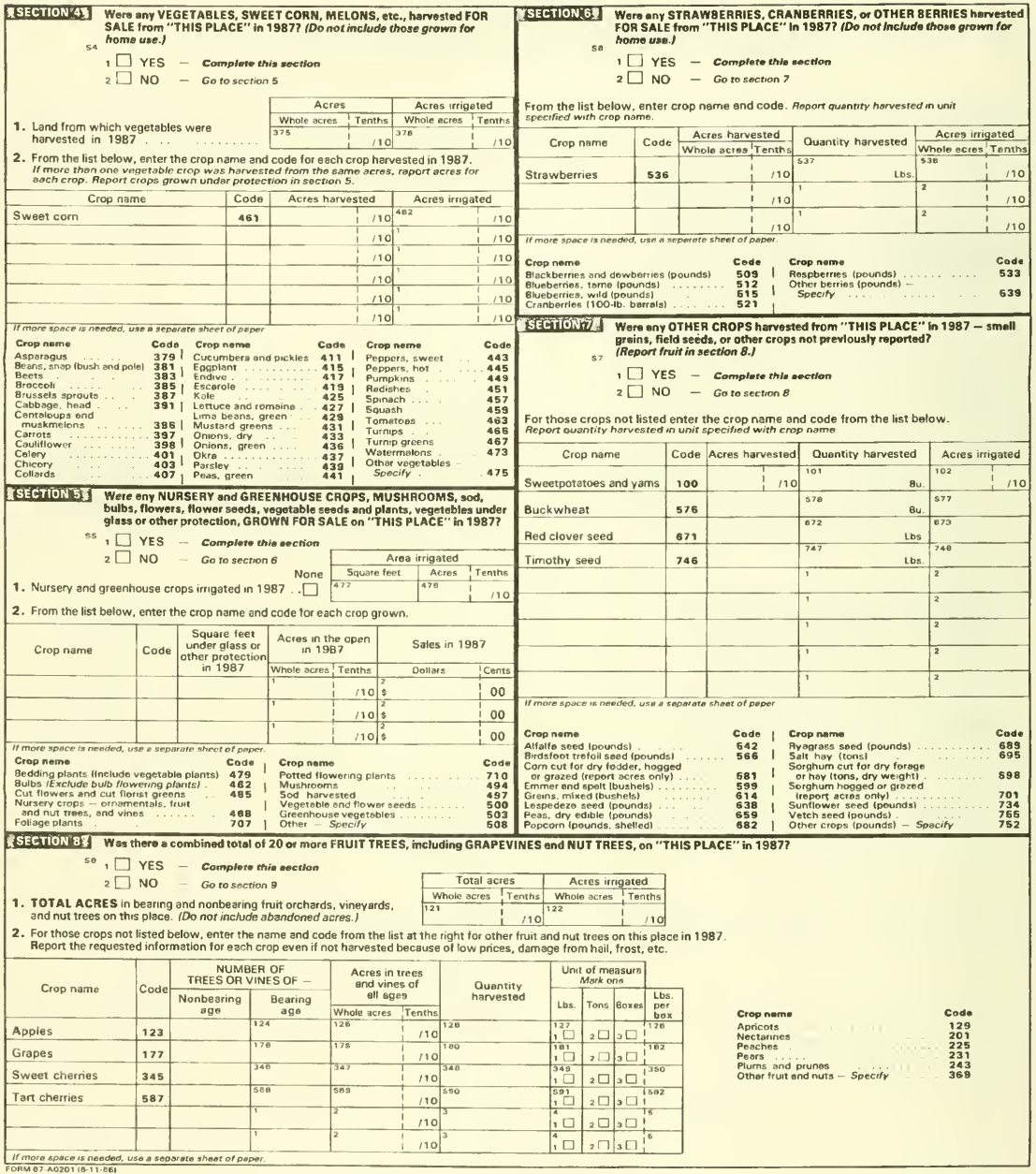

Page 2 
SSECTION'97 GROSS VALUE of CROPS SOLD trom "THIS PLACE" in 1987, BEFORE taxes and expenses (Refer to the INFORMATION SHEET, section 9 .)

Report your best estimate of the value for each of the following groups of crops sold from this place in 1987. Include the value of the landlord's and/or contractor's share, estimating if necessary. Include value of Govarnment CCC loans.

1. Grains, soybeans and other beans sold in 1987

a. Com for grain

b. Wheat

c. Soybeans

d. Sorghum for grain

๑. Barley

1. Oats

g. Other - rye, dry beans, dry peas, popcom sunflower seed, buckwheat, etc.

2. Tobacco

3. Hay, silage, field seeds, and grass seeds

4. Vegetables, sweet corn, and melons - IDo not include Insh potatoes and sweatpotatoes, report them in item 6 below

5. Fruits, nuts, and berries - apples, peaches, grapes, cherries, crenbernes, strawberries, blueberries, etc.

6. Other crops - Irish pota1oes, sweetpotatoes, etc. (Do not includo nursery and graenhouse croos.)

Soecify

\begin{tabular}{|c|c|c|}
\hline None & Dollars & Cants \\
\hline$\square$ & $\begin{array}{l}773 \\
5\end{array}$ & 00 \\
\hline$\square$ & $\begin{array}{l}74 \\
5\end{array}$ & 00 \\
\hline & $\begin{array}{l}773 \\
5\end{array}$ & 00 \\
\hline & $\begin{array}{l}778 \\
\$\end{array}$ & 00 \\
\hline ¿ & $\begin{array}{l}777 \\
\$\end{array}$ & 00 \\
\hline & $\begin{array}{l}778 \\
5\end{array}$ & 00 \\
\hline & 779 & i \\
\hline & $\$$ & ao \\
\hline & $\begin{array}{l}781 \\
\$\end{array}$ & 00 \\
\hline & $\begin{array}{l}782 \\
\$\end{array}$ & 00 \\
\hline & 783 & 1 \\
\hline & $s$ & 00 \\
\hline & 784 & i \\
\hline & $\$$ & 00 \\
\hline$\square$ & 788 & 00 \\
\hline
\end{tabular}

SECTIONB 1 How were the ACRES in this place USED in 1987 ?

1. Copy acres in "THIS PLACE" from section 1, item 4, page 1 NOTE: For items 2 to 5 below, if land was used for mora than one purpose in 1987 repart it in the FIRST land use histed below that apolies. For sxample, report croplan

\section{CROPLAND}

a. Cropland harvested - Include all iand from which crops were harvested or hay was cut, and all land in orchards,

b. Cropland used only for pasture or grazing - Include rotation oasture and grszing land that could have been used for crops without doitional imorovements.

c. Cropland used for cover crops, legumes, and soil improvement grasses, but NOT harvested and NOT pastured.

d. Cropland on which all crops fasled - IExceotron: Do not report hera land in orchards and vinevards on which tha croo
favied Such acreage is to ba reported in rtem $2 a$ )

•. Cropland in cultivated summer fallow

f. Cropland idte

3. Woodland-inchude all
woodlots end limber tracts and a. Woodland pastured woodlots and limber tracts and
cutovar and daforasted land $\left\{\begin{array}{l}\text { a. Woodland not pasture } \\ \text { b. Wh voung }\end{array}\right.$

Other pastureland and rangaland - Include any pasturalan Other pastureland and rangaland
other than crooland and woodland pasture.

5. All other land - Land in house lots, ponds, roads, wasteland, etc. - Include sny land not roported in items 2 through 4 above. 6. TOTAL ACRES - Add the ecrea reported in items 2 through 5

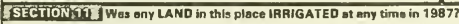

Irrigated tand is all land watared by any artificial or controlled means - sorinklers, furrows or dirches, soreader dikes, etc. Include suopiemental, partiat, and oreplant irngetion.

$$
\begin{aligned}
& 1 \square \text { YES - Complete this soction } \\
& 2 \square \text { NO }- \text { Go to section } 12
\end{aligned}
$$

1. How many acres of harvested land were irrigated? include land from which hay was cut and land in bearing and

2. How many acres of pastureland, rangeland, and any other lands not included in item 1 above were irrigated?

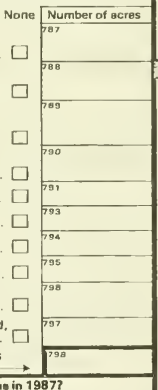

SECTIOTh 2: Were any ACRES in this place SET ASIDE, DIVERTED, OR IDLED

$\sin$ under FEDERAL acreage reduction programs in 1987 ?

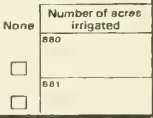
$\square$ YES
Comploto this section
$2 \square$ NO
Go to section 13

1. How many acres were set aside (or divarted) undar ANNUAL commodity acreage adjustment programs?

2. How many acres were under the CONSERVATION RESERVE PROGRAM (10 year, CRP)?

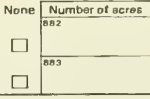

SECrIONh13 Did you or snyone else have any CATTLE or CALVES on this place in 1987 ?

513

$1 \square$ YES - Complete this soction

$2 \square$ NO - Go to section 14

- DECEMBER 31, 1987 INVENTORY

1. CATTLE AND CALVES of all agas

TTotal of $s, b, c$, and $d$ balow

a.
had calved.

b. MILK COWS kept for production of milk or crearn for sale or home use-include dry
milk cows and milk herfers that had caived. c. HEIFERS ANO HEIFER CALVES - iDo not include
herfars that had calved.) $\ldots \ldots \ldots \ldots \ldots \ldots \ldots$

d. STEERS, STEER CALVES, BULLS. AND BULL CALVES

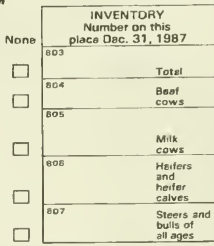

\section{- CATtLE AND CALVES SOld}

FROM THIS PLACE IN 1987

include thosa fed on this place on a

contract or custom bisis. Also reoon
as sold cattle moved from this olace

to o foedilot for further feeding.

2. Calves weighing less than 500 pounds

3. Cattle, including calves weighing

500 pounds or mora

\$. Of the total cattle sold, how many were FATTENED on this place on GRAIN or CONCENTRATES for 30 days or more and SOLD for SLAUGHTER?

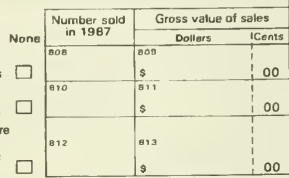

\section{- DAIRY PRODUCTS SOLD FROM} THIS PLACE IN 1987

4. Gross value of selas of DAIRY PRODUCTS

from this place in 1987 - Include mink, cream

buttar, etc.

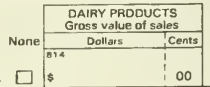

s14, $\square$ YES - Complete this section

$$
2 \square \text { NO - Go to section } 15
$$

- DECEMBER 31, 1987 INVENTORY

1. HOGS and PIGS of all ages (Total of $a$ and $b$ below)

a. HOGS and PIGS used or to be used for 8REEDING

b. OTHER HOGS and PIGS

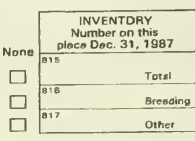

- LITTERS FARROWED

2. LITTERS FARROWED on this place between -

a. December 1, 1986 and May 31, 1987

b. June 1, 1987 and November 30,1987

None Number of littars

$81 a$

819

- hOGS AND PIGS SOLD

3. HOGS and PIGS SOLD from this place in 1987

4. Of the hogs and pigs sold, how many were

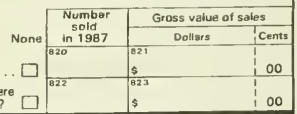

GECT WR

515 ,

1 $\square$ YES - Complote this soction

$2 \square$ NO - Go to section 16

1. SHEEP and LAMBS of all ages

a. EWES 1 year old or older

\begin{tabular}{|c|c|c|}
\hline \multirow[b]{2}{*}{ None } & \multicolumn{2}{|c|}{ Gross value of eales } \\
\hline & Dollars & Cents \\
\hline & $\begin{array}{l}823 \\
\$\end{array}$ & 00 \\
\hline
\end{tabular}

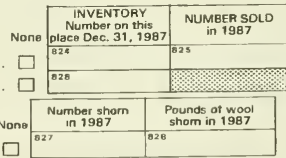

2. SHEEP and LAMBS SHORN

What was the gross value of sales of SHEEP, 


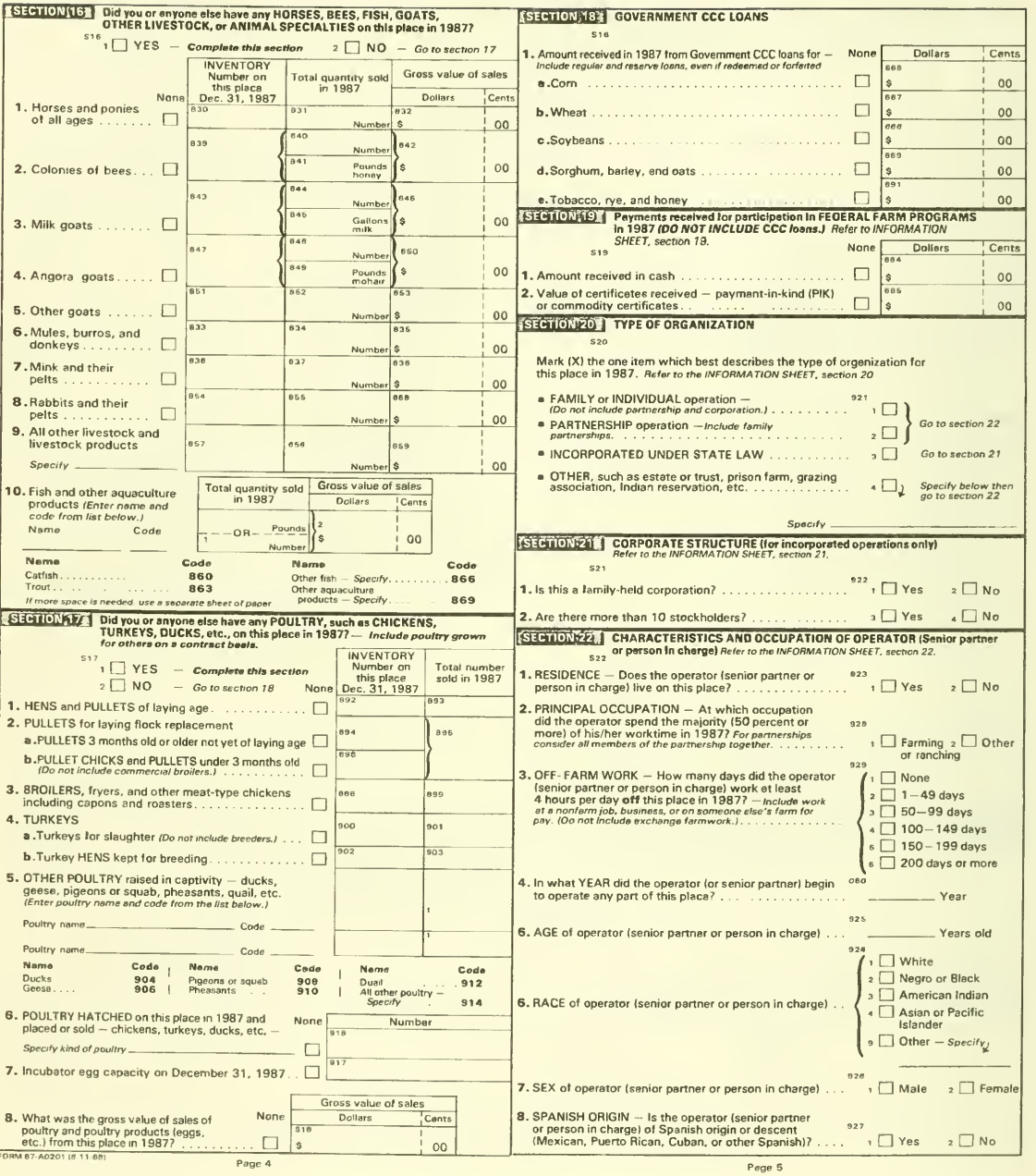



[SECTIONE23] PRODUCTION EXPENSES pald by you and othars for this
place in 1987

Include your best estimates of expenses paid by you, your landlord, contractors, buyers, and others for production of crops, livestock, and other agricultural products in 1987 . 100 NOT INCLUOE expenses connected with performing customwork for others: operatian of nonfarm activities, businesses, or services; or household expenses not related to the farm business.)

1. Livestock and poultry purchesed - cattle, calves, hogs, pigs, sheep, lambs, goats, horses, chicks, poults, started pullets, etc.

2. Feed purchased for livestock and poultry - grain. hay, silage, mixed feeds, concentrates, etc.

a. Commercially mixed formula feeds purchased complete, supplement, concentrates, premixes. (Do not include ingredients. purchesed separetely, such as soybean meet, cottonseed

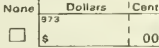

3. Seed cost - for com, other grains, soybeans, tobacco, cotton, etc. - include plants and trees purchesed. .....

4. Commercial fertilizer purchased - all forms, including rock phosphate and grpsum. Include cost of custom epplicertions.

5. Agricultural chemicals purchasad - Insecticides, herbicides, fungicides, other pesticides, etc, - Include cost of custom applications. (Do not include hime.)

6. Gasoline and other petroleum fuel and oil purchased lor the larm business -

a. Gasoline and gasohol.

b. Diesel tuel

c. Natural gas

d. LP gas, fuel oil, kerosene, motor oil, grease, etc.

7. Electricity for the farm business - 1Do nor include household expenses.)

8. Hired farm and ranch labor - also include employer's cost for social security, workman's compensation, insurance prerniums, pension plans, etc. (SEe INFORMATION SHEET)

9. Contract labor - Include expenditures for lsbor, such as harvesting of fruit, vegetables, bernes. etc. performed on a contrect basis by e contractor, crew leader, a cooperative, etc.

10. Repair and maintenance expenses for the upkeap of buildings, motor vehicles, and farm oquipment

11. Customwork, machine hire and rental of machinery and equipmemt - include expenditures for use of equipment and for customwork such as grinding and mixing feed, plowing, combuning, com picking. drying, silo filling, spraying, dusting, fertilizing, etc. 10 fertilizer and chemicals.)

12. Interest paid on debts - ISES INFORMATION SHEET) a. Secured by real estate.

b. Not secured by real estate

13. Cash rent peid for land and bulldings in 1987 1Do not include grazing tees $t$.

14. Proparty taxes paid - inchude farm real estate. machunery, fivestock, etc. for the ferm business. 10 o not include

15. All other production expenses - inchude insurence. water, anumal heolth costs. grazing fees. markating charges, miscellianeous farm suppliss, otc. Do not include deprectetion. household expenses
farm business.l

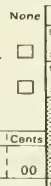

00

$\square \leqslant$

0

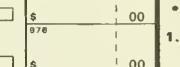

Were anY INSECTICIOES, HERBICIDES, FUNGICIDES, NEMATICIDES, OTHER PESTICIDES, or OTHER CHEMICALS used on this place in 1987 ?

525

$1 \square$ YES - Complete this section $\quad 2 \square$ NO - Go to section 26

Include any materials provided by you, your lendlords, or contractors. For each item listed, report acres only once. II multipurpose chemicals were used, report acreage treated for each purpose.

1. Sprays, dusts, granules, fumıgants, etc.. (fungicide, herbicide, insecticide. nematicidel to control -

a. Insects on crops, including hay

b. Nematodee in crops

c. Dlseeses in crops and orchards (blights. smuts, rusts, etc.l

d. Weeds, grass, or brush in crops and pasture Include both pre-emergance and post emergence.

2. Chemicals for dafoliation or for growth control of crops or thinning of fruit

SECTIOWI26 I MACHINERY AND EQUIPMENT on this place on December 31, 1987 -
Include onfy equipment used for agricultural operations in 1986 of 7987.

- Value of ALL machinery and equipment on this place, December 31, 1987

1. What is the estimated market value of ALL machinery. equipment, and implements usually kept on this place and used lor the farm or ranch business? - include cars, trucks, tractors, combines, plows, disks, harrows, dryers. pumps, motors, irngerion equipment, doiry equipment including milkers and bulk tanks, ivestock feeders. grinding and mixing

SELECTED machinery and equipment on this place, December 31, 1987. (Report only if used in 1986 or 1987.)

2. Motortrucks - Include pickups

3. Wheel tractors other than garden tractors and motor tillers

a. Less than 40 horsepower (PTO).

b. 40 hor sepower (PTO) or more

00

4. Grain and bean combines, all types

5. Cotton pickers and strippers

6. Mower conditioners.

7. Pickup balers - Include rectsngle

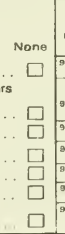

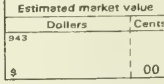
f SECTIONIZS JESTIMATED CURRENT MARKET VALUE OF LAND and 527 BUILDINGS

Please give your best ESTIMATE of the CURRENT MARKET VALUE of land and buildings for all acres reported in section 1 , items 1,2 , and 3 , page 1 .

1. All land owned

2. All land rented or leased FROM OTHERS

3. All land rented or leased TO OTHERS

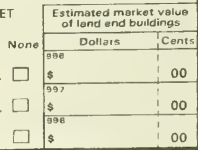

BECTIOHES JINCOME FROM FARM - RELATED SOURCES IN 1987

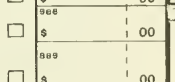

1. Customwork and other agricultural services provided for farmers and others - plowing, planting. spraying. harvesting. preparation of products lor market, etc. (If customwork is e separste business, refer to INFORMATION
SHEET, sectnon 28).

2. Gross cash rent or share payments received from renting out lamland or payments received from lease or sale of allotments - Include perments for hvestock pestured an

3. Sales of forest products and Christmas trees - Include meple products. naval stores, firowood, etc.

Was any COMMERCIAL FERTILIZER,
LIME used on this place during 1987?

, $\square$ YES - Complete this section ${ }_{2} \square$ NO - Go to sectron 25

None Acres fertilized

1. Acres of cropland fertilized in 1987- Tho not include cropland for pestures reported in section to, item $2 b$.

. Acres of pastureland and rangeland fertilized in 1987 reported in section 10 , iterns $2 \mathrm{~b}$ and 4

$\square$

. Recreationel services, patronage dividends of cooperatives, and ather income which is CLOSELY RELATEO to the agricultural operation on this place -

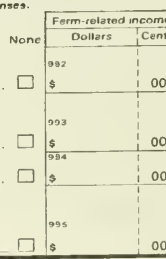

SECTIONZT) PERSON COMPLETING THIS REPORT - Please print

3. LIME - tons of lime used and acres on which applied - tDo nor include tend plester of gyosum or hime for sanitstion.)

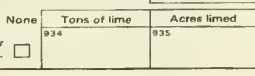




\section{UNITED STATES CENSUS OF AGRICULTURE}

\section{Special Reporting Instructions}

\section{Who Should Report}

WE NEED A REPLY FROM EVERYONE RECEIVING A REPORT FORM, INCLUDING individuals, landlords, tenants, partnerships. corporations, institutions, and THOSE NOT CONDUCTIN AGRICULTURAL OPERATIONS. Each case included in the census has a unique Census File Number (CFN). In order to make the census results as complete and accurate as possible, we need to obtain information about every CFN.

2. If You Raceived More Than One Report Form for an Operation Complete only ONE report form for an operation. Write "Duplicate" near the address label of each extra report form. Also, write the 11-digit census tile number(s) of the DUPLICATE report(s) ON THE COMPLETEO REPORT in the space provided to the left of the address label. Return the extra reportis) in the same envelope with your completed report form so that we can correct our records.

\section{If You No Longer Farm}

If you had agricultural operations at any time during 1987, please report all agricultural activity during the year. Bepor all land on your report all agricultural activity during the year. Report all land on your
census form that you owned or rented. Also, report your 1987 crop and livestock production and 1987 sales.

Explain on the first page of the report form for on a separate sheet of paper) that you quit farming or ranching and give the approximate date and the name and address of the present operator, if known.

4. If You Never Farmed or Have No Assoclation With Agriculture Please write a note on the report form near the address label explaining this and return the form so that we can correct our records. In our efforts to make the census as complete as possible, we obtained lists from various sources. We tried to eliminate duplicate and nonfarm addresses, however, it was not always possible to do so.

5. If You Heve More Than One Agricultural Operetion Complete a report form for EACH SEPARATE and OISTINCT production unit, i.e., each individual farm, ranch, feedlot, greenhouse, etc., or combination of farms, etc., for which you maintain SEPARATE records of operating expenses and sales, livestock and other inventories, crop acreages, and production.

\section{If You Have a Partnership Operation}

Complete only ONE report for the entire partnership's agricultural operation and include all partners' shares on the one report. If members of the partnership also operate separate farms or ranches in addition to the partnership farming operation, separate report forms should be completed for each individual operation.

If two or more report forms were received for the same operation, mark eech additional form as a "Duplicate." Return the duplicate report's) in the same envelope with the completed partnership report, where the same envelope with the completed partnership report, Where partier) has completed a report for the partnership (provide name and partner) has completed of partnership.'

\section{Landlord's or Contractor's Share}

If you rented or leased land trom others or had a contract for the production of agricultural products, include both your share and the landlord's or contractor's share of the production, sales, and expenses so your census report form will be complete for "THIS PLACE."

If you do not know the landlord's or contractor's share, include your BEST ESTIMATE, If you do not have records available for all data items. use your best estimate.

\section{How to Enter Your Response}

Enter your replies in the proper spaces, on the correct lines, and in the units requested, i.e., dollars, bushels, tons, etc. Write any explanation outside the answer spaces or on a separate sheet of paper.

All dollar figures may be entered in whole dollars. CENTS ARE NOT REQUIRED.

Enter whole numbers except where tenths are requested, such as acres of potatoes harvested. If you have $1 / 2,1 / 3$, or $1 / 4$ of an acre, convert to tenths. For example, convert $1 / 2$ to $5 / 10,1 / 3$ to $3 / 10,1 / 4$ to $2 / 10$.

The census report form will contain sections and questions which do not apply to you. When this occurs, mark the "None" or "No" box and go on to the next item or section.

\section{Instructions For Specified Sections}

- Section 1 - ACREAGE IN 1987

Your answers to this section will determine the land (Acres in "THIS PLACE") referred to in the rest of the report form. When answering the acreage questions, include the land associated with your agricultural operations in 1987 whether in production or not. Include all land that you owned or rented during 1987 even if only for part of the year. Do not include any unrelated residential or commercial land.

IF YOU OUIT FARMING DURING 1987 - Complete the report form for the portion of the year that you did farm. Explain on the report form in the space to the left of the address label for on another sheet of paper) when you stopped farming and include the name and address of the person now using the land. Report all land in section 1 in whole acres.

Item 1 - All Lend Owned - Report all land owned in 1987 whether held under title, purchase contract or mortgage, homestead law, or as heir or trustee of an undivided estate. Include all land owned by you and/or your spouse, or by the partnership, corporation, or organization for which you are reporting.

Item 2 - All Land Rented or Leased FROM OTHERS Report all land rented by you or your operation even though the landlord may have supplied materials or supervision.

INCLUOE in item 2:

a. Land for agricultural use that you rented from others for cash

b. Land you worked on a share basis (crop or livestock)

c. Land owned by someone else that you used rent-free

d. Federal, State, Indian reservation, or railroad land rented or leased by the acre

DO NOT INCLUDE in item 2 :

Land used on a per-head or animal unit license or permit basis, such as section 3 of the Taylor Grazing Act, National Forest, or Indian reservation permit land. If you had any of these permits, mark "yes" to item 7

Item 3 - All Land Rented or Leased TO OTHERS - Include all land rented out for any purpose if it was part of the acreage reported in items 1 and 2 . A report form will be obtained from

INCLUDE in item 3

a. Owned land rented to others for cash or a share of crops or livestock

b. Land you rented from someone and then subleased to someone else

c. Land worked for you by someone for a share of crops or livestock

d. Land which you allowed others to use rent-free

Item 4 - Acres in "THIS PLACE" - This figure will show the total of all land you operated at any time in 1987.

If item 4, Acres in "THIS PLACE" is " 0 " and:

a. You raised any crops or had any livestock or poultry on THIS PLACE" in 1987, complete the report.

b. All your land was operated by a renter or sharecropper. complete item 6 (name and address of rentersl, skip to and complete section 29, and explain brielly, "all land rented out," etc. Mail form in return envelope.

c. You did not have any agricultural activity on owned or rented land in 1987 , complete section 29 and explain briefly, such as "retired," "sold farm," and date. Give name and address of current operator if known and return form.

Sections 2 through 8 - CROPS

Sections 2 through 8 provide spece for reporting crops harvested during the 1987 crop year from the land shown in section 1, item 4 (Acres in "THIS PLACE") of your report. Please report your crops in the appropriate sections. Do NOT include any crops grown on land rented or leased TO OTHERS, or worked by others on shares during 1987

Acres harvested - Enter the acres harvested in 1987. Round fractions to whole acres except where tenths are requested by "110" in the reporting box, such as for potatoes. 
Quantity harvested - If your unit of measure is different than the unit on the report form, please convert your figure for the quantity harvested to the unit requested. If the harvest was incomplete by December 31, 1987, please report the quantity harvested and estimated quantity to be harvested.

Acres irrigeted - For each crop irrigated, report number of acres irrigated. Irrigation is defined as land watered by artificial or controlled means - sprinklers, furrows or ditches, spreader dikes, purposeful flooding, etc. Include acres that received supplemental, partial, and/or preplant irrigation. Do not report water applied in transplanting tobacco plants, trees, or vegetables as irrigation. Leave "Acres irrigated" blank for crops that are not irrigated.

How to Report Crops Harvested

Sections 2 and 3 - Report only for the listed crops.

Sections 4 through 8 - To report: (1) find the crop name and the code number from the list in the section: (2) enter crop name and code in the first two columns of the first available answer line in the section: in the first two columns of the first available answer line in the section; you harvested a crop not listed in sections 4 through 8 , use the "Other" you harvested a crop not listed in sections 4 through 8 , use

Double Cropping - If two or more crops were harvested from the same land (double cropping) report the total acres and production of each harvested crop in the appropriate section(s) of the report form Example: In 1987 you harvested 1,230 bushels of wheat from 40 acres, then on the same 40 acres planted soybeans, from which you
harvested 1,550 bushels. You irrigated the soybeans but not the wheat.

\begin{tabular}{|c|c|c|c|}
\hline $\begin{array}{l}\text { Were ony o } \\
\text { "THIS PLA }\end{array}$ & \multicolumn{3}{|c|}{$\begin{array}{l}\text { Were ony of the following cROPS harvested from } \\
\text { "THis PLACE" in } 1987 \text { ? }\end{array}$} \\
\hline & \begin{tabular}{|c|} 
Acres \\
harvested
\end{tabular} & $\begin{array}{c}\text { Quantity } \\
\text { harvested }\end{array}$ & $\begin{array}{c}\text { Acres } \\
\text { irrigated }\end{array}$ \\
\hline 1. Cottan ......... & 091 & Bales. & 093 \\
\hline 2. Soybeans for beans $\square$ & ${ }^{088} 40$ & $1,550 \mathrm{Bu}$. & ${ }^{090}<0$ \\
\hline 3. Wheat for grain.... & ${ }^{073} 40$ & $1,230 \mathrm{Bu}$. & 075 \\
\hline 4. Oats tor grain. & 076 & $0>7$ Bu. & 078 \\
\hline
\end{tabular}

Interplanted Crops - If two crops were grown at the same time in alternating strips in the same field, report the portion of the field used for each crop.

Example: A 60 acre field was planted in cotton and soybeans, with two rows of cotton followed by an area of the same width planted in soybeans. No irrigation was used. Thirty acres of soybeans and 30 acres of cotton would be reported in the appropriate section(s).

Sklp Row Planting - If a crop is planted in an alternating pattern of planted and non-planted rows, such as two rows planted and two rows skipped $(2 \times 2)$, report the portion of the field occupied by the crop in the appropriate section for that crop, and report the skipped portion as "Cropland idle" in section 10 , item $2 t$

- Section 4 - VEGETABLES - Report acres of vegetables harvested FOR SALE or commercial processing. Do not include vegetables grown FOR SALE or commercial processing. Do not include vegetables grown
for home use. Report the total acreage of each vegetable crop harvested. Example: In 1987 you harvested 10 acres of lettuce from a field, then replanted the field in lettuce and harvested the 10 acres again. Both crops of lettuce were irrigated. Enter only 10 acres of land from which vegetables were harvested and 10 acres irrigated in item 1 of section 4 . but write in 20 acres of lettuce harvested and 20 acres of lettuce irrigated in item 2 of section 4 .

- Section 8 - FRUITS and NUTS - In counting the combined tota of 20 or more trees and vines, include those for home use as well as of 20 or more trees and vines, include those for home use as well as those maintained for sale of the production. Acres in trees or vines that have been abandoned should not be included;

If crops other than fruit and nut trees and vines were interplanted with trees or vines, report the total acres for the orchard crop in section 8 and the total acres of the interplanted crop in the appropriate section.

\section{- Section 9 - GROSS VALUE OF CROPS SOLD}

Report the value of all crops sold from "THIS PLACE" in 1987. regardless of the year they were harvested or who owned the land. Be sure to report gross values before deducting expenses and taxes. Include Government CCC loans received for "THIS PLACE"' in 1987 Include payments received in 1987 from cooperatives or marketing organizations for crops produced on "THIS PLACE" regardless of the organizations for crops produced on "TH
year in which the crops were harvested.

Also include as sales, your estimate of the value of any crop removed from "THIS PLACE" in trade for services, such as hay cut in exchange for fence repair, clearing, or other services. It the sale price or market value is not known, give your best estimate of the crop's market value when removed from "THIS PLACE."

DO NOT INCLUDE crops or crop products purchased from others and later sold.

\section{- Section 10 - USE OF ACRES IN "THIS PLACE"}

This section is used to classify the acres in 'THIS PLACE' reported in section 1 , item 4 . (Do not include any acres you rented to others reported in section 1 , item 3 ). The sum of the acres entered in various categories should equal total acres in "THIS PLACE."

Land Used for More Than One Purpose - Do not report the Land Used for More Than One Purpose- Oo not report the or all of your land was used for more than one listed purpose
1987 , report that land only in the first category listed. For example, if you plowed under a cover crop, and planted and example, if you plowed under a cover crop, and planted and harvested a grain crop, report the land in item 20 . "Cropland crops, legumes, etc." (item 2c).

Double Cropping - When more than one crop was harvested from the same land in 1987, report that land only ONCE as "Cropland harvested," in item 2 a.

Interplanted Crops - If you interplanted crops, such as cotton in an orchard, report the total land used for both crops only ONCE, as "Cropland harvested," in item 2 a. Sklp Row Planted Crops - Report the acres that represent the
total nonplanted or skipped rows as "Cropland idle, "item $2 f$. The acres that represent the planted rows should be reported as "Cropland harvested," in item 2a.

\section{Section 12 - ACRES SET ASIDE, DIVERTED, OR IDLED
UNDER FEDERAL ACREAGE REDUCTION UNDER FEDERAL ÁC
PROGRAMS IN 1987}

Include in item 2 all ecres in "THIS PLACE" retired from production and placed, by long-term contract, into the Conservation Reserve should be included.

\section{- Sections 13 through 17 -LIVESTOCK, POULTRY, OTHER} LIVESTOCK, O
SPECIALTIES

Animals and Poultry to Inciude in the Report - Report all animals, poultry, and animal specialties on "this place" (section 1 , item 4) on December 31, 1987. Include all owned by you and 1. item 4) on December 31 , Include animals on unfenced lands. any kept by you for others. Include animals on unfenced lands, National Forest land, district land, Cooperative grazing land, or rangeland administered by the Bure 34 of Land Management on a per-head or lease basis. Animals in transit on December 31,1987 . or animals on a short-term pasture (such as be reported by the person who had control of the animals.

Anlmals and Poultry to Exclude from the Report - Do not report animals or poultry kept on land rented to others or kept under a share arrangement on tand rented to others. Do not include animals quartered in feedlots which are not a part of "this place." Animals kept on a place not operated by you are to be included on the report for that place.

Animals Bought and Sold - DO NOT REPORT ANY ANIMALS BOUGHT AND THEN RESOLD WITHIN 30 DAYS. Such purchases and sales are considered "dealer" transactions, and are not included in this census.

Number Sold - Report all animals and poultry sold or removed from "this place" in 1987, without regard to ownership or who shared in the receipts. Include animals sold for a landlord or given to a landlord or others in trade or in payment for goods or services. to a NOT report number sold for any livestock or poultry kept on another place. Dairy Termination Program or "'Whole-Herd Dairy Buy-Out Program" - The amount received in 1987 from the Government under the dary termination program should be included in section 19, item 1987 should be reported in section 13 .

Animals Moved to Another Plece - For animals moved from Animais moved to Another Place - For animals moved from "this place" to another place, such as for further feeding, report animals as "sold" and give your best

Fat Cattle Sold - Cattle fattened on grain or concentrates for 30 days or more and sold for slaughter are reported in section 13 item 3 a.

DO NOT INCLUDE WITH FATTENED CATTLE SOLD:

a. Cattle and calves sold for further feeding

b. Veal ca'ves, or any calves weighing less than 500 pounds

c. Dairy cows fed only the usual dairy ration before being sold 
Value of Sales - Report the total gross value of animals and poultry sold or removed from "this place" in 1987 without deducting production or marketing expenses (cost of feed, cost of livestock purchased, cost of hauling and selling, etc.). If the sale price or market value is not known, give your best estimate of their market value when they left "this place." Do NOT report the value of sales of any livestock and poultry owned by you but kept and sold from a place you did not operate.

Contract and Custom Feeding Operations - Livestock or poultry kept by you on "this place" on a contract or custom basis should be included on this report REGARDLESS OF OWNERSHIP. Report as "INVENTORY" numbers of animals or poultry on the place on December 31, 1987. Report as "SOLD" animals and poultry kept on a contract or custom basis and removed or sold from the place in 1987 . If the sale price or market value is not known, give your best estimat of the market value of the animals or poultry when they left the place.

\section{- Section 16 - HORSES, BEES, FISH, GOATS, OTHER LIVESTOCK,}

Item 2 - If you owned BEES - Report all colonies or hives of bees and honey operations conducted by you, regardless of where the hives were kept most of the year. Report hives or colonies, pounds of honey sold. and value of sates.

Items 7 and 8 - Mink pelts and rabbit pelts should be included in number sold and value of sales, but not in inventory.

Item 9 - Other Livestock and Livestock Products - Include in all other livestock and livestock products manure, beeswax, and any other animal products sold from "this place" in 1987. Pleese indicate units used in reporting.

Item 10 - Fish and Other Aquaculture Products - Report number of pounds sold and gross value of sales for each. Enter name and code from list.

\section{- Section 17 - POUltar}

The person who furnished the housing and labor should report the poultry operation on his/her report form regardless of who owns the birds. Report as sold poultry that were taken or moved trom the place
in 1987 .

\section{- Section 18 - AMOUNT RECEIVED FROM GOVERNMENT}

Item 1 - Report the amount received under the regular or reserve program for commodities placed under CCC loan during 1987 . Include amount received even if commodity was redeemed or forfeited prior to

Do not include CCC Ioans received to build crop storage facilities or amount received for storage payments in the reserve program.

\section{- Section 19 - FEDERAL PAYMENTS RECEIVED}

Report all payments received from Federal Farm Programs in 1987 regardless of whether payment was made in cash or commodity certificates. Include cash payments in item 1 . In item 2 . include the value of any certificates held or the value received from sale or redemption of any certificates in 1987.

Federal payments include receipts from Federal programs such as deficiency payments, "Whole-herd dairy buy-out." support price payments, indemnity programs, disaster payments, paid land diversion, inventory reduction payments, payments recaived for

\section{- Section 20 - TYPE OF ORgANIZATION}

Use the following defintions to determine the type of organization for your operation:

Family or Individual Operation - Defined as farm or business organization controlled and operated by an individual (sole proprietor) Include family operations that are not incorporeted and not operated under a partnership agreement.

Partnership Operation - Defined as two or more persons who have agreed on the amount of their contribution (capital and effort) and the distribution of profits. Co-ownership of land by husband and wife or joint filing of income tax forms by husband and wife DOES NOT foint filing of income tax forms by husband and wife DOES NOT contributions, decisionmakıng. profits, and liabilities exists. Production under contract or under a share rental agreement DOES NOT constitute under contract
a partnership.

Incorporated Under State Law - A corporation is defined as a legal entity or artificial person created under the laws of a State to carry on a business. This definition does not include cows of a State to carry on a type of corporation should be reported in section 21.

Other - Such as cooperatives Idefined as an incorporated or unincorporated enterprise or an association created and formed jointly by the members), estate or trust (defined as a fund of money or property administered for the benefit of another individual or organization). prison farm, grazing association, Indian reservation, institution run by a government or religious entity, etc.
- Section 21 - CORPORATE STRUCTURE

This section is to be answered by corporations only. Answer both items. A family held corporation has more than 50 percent of its stock owned by persons related by blood or marriage.

\section{- Section 22 - CHARACTERISTICS AND OCCUPATION OF}

This section collects informatıon about the operator of "this place" delined as the individual owner, the operator, the senior partner, or person in charge for the type of organization reported in section 20.

For Family or Individual Operation - Complete this section for the operator.

For Partnership Operations - Answer all items, except item 2 for the "Senior Pertner "The "Senior Partner" is the individual who is mainly responsible for the agricultural operations on "this place." not necessarily the person senior in age. If each partner shares equally in the day-to-day management decisions, consider the oldest as the "Senior Partner." For item 2 (Principal

Occupation) consider all members of the partnership together. Please include as "farming" worktime at all types of agricultural enterprises, including work at greenhouses, nurseries, mushroom production, ranching. feedlots, broiler feeding, etc.

For Corporations and Other Operations (Cooperatives, Estates, etc.) - Complete section 22 for the person in charge, such as a hured manager, business manager, or other person primarily responsible for the on-site, day-to-day operation of the farm or ranch business.

Item 4 - Yeer Began Operation - Report the first year the operator or senior partner began to operate any pan of "this place" on a continuous basis. If the operator returned to a place previously operated, report the year operations were resumed.

\section{- Section 23 - PRODUCTION EXPENSES paid by you and} others for "this place" in 1987

Include farm production expenses paid by you, your landlord. contractors, or anyone else for crops, livestock, or poultry produced on "this place." Include expenses incurred in 1987 even if they were not paid for in 1987. Please estimate if exact figures are not known. Refer to the individual expenditure items below for further explanations.

Livestock and Poultry Purchesed - Report the cost of cattle. calves, hogs, pigs, sheep, lambs, horses, goats, chicks, pullets. poults, etc., including breeding stock and dairy cows. Contract poults, etc., including breeding stock and dairy cows. Contract growers or custom feeders who did not own or purchase the livestock or poultry themselves should estimate the value of the
cattle, catves, pigs, baby chicks, pullets, etc, at the time they came onto the place.

Feed Purchased for Livestock and Poultry - Report the purchase cost of corn, sorghum, oats, barley, other grains silage, hay, mixed feed, concentrates, etc., fed to livestock and poultry on "'this place." Contract livestock and poultry growers should estimate the value of feed provided by the contracting company. Custom feedyards should include feed costs for all cattle fed even if the owners of the cattle were billed for the feed. Feed raised on "this place" should not be reported as purchased.

Cost of Hired Farm and Ranch Labor - Include gross salaries and wages, commissions, dismissal pay, vacation pay, and paid bonuses paid to hired workers, family members, hired managers. administrative and clerical employees, and salaried corporate officers. Also, include supplemental cost for benefits such as employer's social security contributions, unemployment employer's social security contributions, unemployment medical insurance, pension plans, etc.

Contract Labor - Includes the labor costs of workers lurnished on a crew leader, or cooperative for harvesting vegetables or fruit, shearing sheep, or similiar farm activities. Do not include costs for building or repair work done by a construction contractor. Include the cost of customwork or machine hire in item 11 .

Repair and Meintenance Expenses for the Upkeep of Buildings, Motor Vehicles, and Farm Equipment - Include the Buildings, Motor Vehicles, and Farm Equipment- include the cost of repairs and upkeep of farm machinery, vehicles, building include repairs to vehicles not used in the farm business or for include repairs to vehicles not used in the farm business or for equipment used only for performing customwork for others. Oo the cost of additions to existing buildings. 
Interest Expense Pald on Debts - Report all interest expenses paid in 1987 for the farm business. Include interest on loans secured by land and buildings (real estate) in item $12 \mathrm{a}$. Include all loans not secured by real estate such as for fertilizer, feed, and seed in item $12 \mathrm{~b}$. Include interest paid on CCC loans. Do not include interest associated with activities not related to production of crops or livestock on "this place" such as land or buildings rented to others, packing sheds, or feed mills that provide services to others. Do not include interest on owner/operator dwelling where amount is separated from interest on other land and buildings on "this place."

Cash Rent Paid for Lond and Buildings In 1987 - Report rent paid in cash during 1987 for land and buildings in "this place." Do not include rent paid for operator dwelling or other nonfarm property. Do not include the value of shares of crops or livestock paid to lendlords.

Property Taxes Paid - Include real estate property taxes you peid on the acres and buildings you operated and used in the farm business.

Do not include:

a. Property taxes on land or buildings rented to someone else

b. Taxes paid by landlords

c. Property taxes paid on other property not associated with the farm business

d. Income and excise taxes

All Other Production Expenses - Farm production costs not previously listed should be reported here. In addition to items listed on the report form, include bookkeeping charges, tax preparation fees. postage, advertising. commission for sale of cattle, and fees paid for farm-related advice or for farm consultants. Do not include depreciation or expenditures for the purchase of land and buildings or new or used machinery.

- Section 24 - COMMERCIAL FERTILIZER AND LIME

Report acres on which commercial fertilizer (items 1 and 2) or lime (item 31 was applied during 1987 . If any acreage was fertilized or limed more than once, report acres ONLY ONCE in each item. Repor expense for commercial fertilizer purchased, excluding lime, in section 23, item 4.
- Section 26 - MACHINERY AND EQUIPMENT

The estimated market value in item 1 refers to ALL mechinery and equipment kept primarily on "this place" and used for the farm business. Report the value in its present condition, not the replacenent or depreciated value. Specialized equipment, which replacement or depreciated value. Specialized eauipment, which is an integrel part of a building

- Section 27 - ESTIMATED CURRENT MARKET VALUE OF LAND AND BUILDINGS

The value for each of the three listed categories should be your estimate of the value of the land and buildings if they were sold in estimate of the value of the land and buildings if they were sold in the current market. The real estate tax assessment value shou not be used unless that value represents a full market value assessment and the land and buildings could reasonably be assumed to be sold at that price. Do not deduct real estate marketing charges from your estim
not the value on a per acre basis.

- Section 28 - INCOME FROM FARM - RELATED SOURCES IN 1987 Item 1 through 4 refer only to those income producing activities for which you use part of the land, machinery, equipment, labor or capital normally used on "this place, and which you do not gross amounts received before taxes and expenses.

Item 1 - Customwork - Do not report income for customwork or agricultural services provided to others if operated as an entirely separate business from your agricultural operations.

Item 2 - Rental Income - Do not include rental income from nonfarm property.

Item 3 - Forest Products - Include only those forest products r Christmas trees cut from "this place." not items cut from other or Christmas trees cut from "this place, " not items cut from o nonfarm timess.

Item 4 - Other Farm-Related Income - Include income from hunting leases, fishing fees, and other recreational services, sales hunting leases, fishing fees, and other recreational services, sales of farm by -products, end other business or income closely related to the agricultural operation on "this place." Include dividends business done with farmer-owned cooperatives. Do not enter previously reported farm sales or income from investments not associated with the farm. Do not include retirement pensions or social security benefits received. 


\section{UNITED STATES CENSUS OF AGRICULTURE}

NOTICE - Response to this Inquiry is requir ad by law (ritle 13, U.S. Code). 8y the same law YOUR REPORT TO THE CENSUS BUREAU IS CONFIDENTIAL. It may be seen only by swom Census employees and may be ueed The law also provides that coptes retained in your files are immune from legol process.

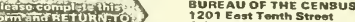

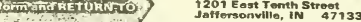

Noto - If your racords ara not avalable, reasonable estimates may be to the above address, toclude your 12 - tharacter Census fust may be sant

as shown in your address lebel in all correspondence to ve.

It you received more than ona

Cengus file Numberis) hare

and return extra copies with

vour completed report

CENSUS 035

USE

039

038

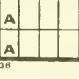

SECTOKT:

1. At any time during 1987, did you plent, grow, or heve any:

- Hay or tobacco?

- Other crops?

- Greenhouse or nursery crops?

$\square$ Yes

$\square$ No

2. At any time during 1987, did you raise, sell, or keep any:

- Cattle, hogs, sheep, or goatg? Horses or ponies?

- Chickens or other poultry? Fish in captivity?

- Other enimel specialties?

$$
\square \text { Yes } \square \text { No }
$$

If You answered YES to EITHEA of these questions, go to SECTION 2

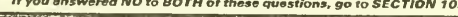

[SECTIOTI2 ACREAGE IN 1987 Peport land owned, rented, or used by you, your spouse, or by the partnership, corporation, or organization for which you are reporting. Include ALL LAND. REGARDLESS OF LOCATION OR USE - cropland, pastureland, rangeland, woodland, idle land, house lots, etc.

1. All land owned

2. All land rented or leased FROM OTHERS, including land worked by you on shares, used rent free, in exchange for services, payment of taxes, etc. Include leased Federal, State, and railroad land. (DO NOT include land used on a per-head basis under a grazing permit.)

3. All land rented or leased TO OTHERS, including land worked on shares by others and land subleased. Also complete item 5 below.

4. Acres in "THIS PLACE" - ADD acres owned (item 1) and acres rented (item 2), then SUBTRACT acres rented TO OTHERS (item 3), and enter the result in this space. None Number of acres

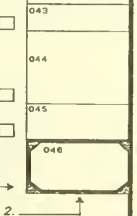
5. Of the land you rented or leased to others, how many None os acres did you own?

6. In what county was the largest value of your agricultural products raised or produced?

\section{BSETIOT3 LAND USE and IRRIGATION}

- PART A - How were the ACRES in this plece used in 1987 ?

1. Cropland harvested - Include all tond from which crops were harvested or hay was cut, and all land in orchands. citrus

groves. vineyords, and nursery and greenhouse crops.

2. Cropland on which all crops failed - Exception: Do not

report here land in orchards and vineyards on which the crop failed.

3. Cropland idle, cropland used for cover crops, or cropland in cultivated summer fallow

4. Cropland used only for pasture, woodland pastured and other pastureland and rangeland

5. All other woodland, wasteland, houselots, etc. not reported in items 1 through 4 above

\section{- PART B - IRRIgation}

1. How many acres of harvested land were irrigated? Inchude land from which hay was cut and lend in bearing and nonbearing

2. How many acres of pastureland, rangelend, and any other lands not included in item 1 above were irrigated?
Please correct amors in name, address, and ZIP Code. ENTER street and number if not shown.

\section{SECTION 4}

PART A - CROPS HARVESTED from "THIS PLACE" In 1987.

To not include crops grown on land rented to others.

1. Hay crops -

a. Alfalfa and alfalfa mixtures

b. Small grain hay

c. Wild hay

d. Other hay

Specify kind

2. Corn for grain or seed

3. Soybeans for beans

4. Wheat for grain

5. Tobacco - all types

6. Potatoes, Irish $-1 D 0$ not include those grown for home use.

\begin{tabular}{|c|c|c|}
\hline \multirow{2}{*}{ None } & $\begin{array}{c}\text { Acres } \\
\text { harvested }\end{array}$ & Quantity harvest \\
\hline & 103 & 104 \\
\hline & 100 & 107 \\
\hline & 112 & $\because 3$ \\
\hline & 102 & 110 \\
\hline & 067 & Oes \\
\hline
\end{tabular}

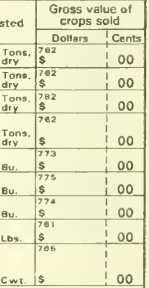

7. All vegetables for sale Do not include those grown Specify kindis)

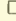

$\square$

$\square$<smiles>c1ccc(-c2cnccn2)cc1</smiles>

87-A0400

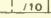

cwr.

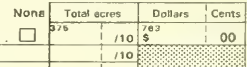

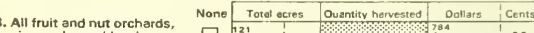
vineyards, and berries specify $\square$

$$
k \text { lnd (s) }\{
$$

9. Other crops - For addrtional crops, enter the crop name end code from the list below.

Report quantity barvested in the unit specified with crop neme

\begin{tabular}{|l|l|l|l|}
\hline Crop name & Code & $\begin{array}{c}\text { Acres } \\
\text { harvested }\end{array}$ & Quantity harvested \\
\hline & & & 1 \\
\hline & & & 1 \\
\hline
\end{tabular}

Gross value of Doliors Cents

Crop name

Barley for grain (bushels)

Code I Cropname

Corn for gilege or green chap (tons, green)

079 Oots for grain thushelsi

\begin{tabular}{l|l}
$\$$ & 00 \\
$\$$ & 00
\end{tabular}

Cotton (bales)

- PART B - NURSERY and GREENHOUSE CROPS GROWN FOR SALE on "THIS PLACE" in 1987

\begin{tabular}{|c|c|c|c|c|c|c|c|}
\hline \multirow[t]{2}{*}{ Crop name } & \multirow{2}{*}{ Code } & \multirow{2}{*}{\multicolumn{2}{|c|}{$\begin{array}{c}\text { Square faut } \\
\text { under glass or } \\
\text { other protection }\end{array}$}} & \multicolumn{2}{|c|}{$\begin{array}{c}\text { Acres in the open } \\
\text { in } 1987\end{array}$} & \multicolumn{2}{|c|}{ Sales in 1987} \\
\hline & & & & Whole acres & Tenths & Dollars & Cents \\
\hline & & & & & 110 & & 100 \\
\hline \multicolumn{8}{|c|}{ se separare sheot of paper. } \\
\hline $\begin{array}{l}\text { Crop name } \\
\text { Badding plants linclud } \\
\text { Curt fiowers and cut fl } \\
\text { Nursery crops - orna } \\
\text { and nut trees, and }\end{array}$ & $\begin{array}{l}\text { eens } \\
\text { s, fruit }\end{array}$ & 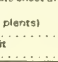 & $\begin{array}{l}\text { Code I } \\
478 \\
4861 \\
4881\end{array}$ & $\begin{array}{l}\text { Crop name } \\
\text { Potted flowe } \\
\text { Foliage plant } \\
\text { Greenhouge } \\
\text { Other - Spe }\end{array}$ & $\begin{array}{l}\text { eting pl } \\
\text { its } \\
\text { vegeta } \\
\text { ocify. }\end{array}$ & & $\begin{array}{r}\text { Code } \\
710 \\
707 \\
603 \\
606 \\
\end{array}$ \\
\hline
\end{tabular}

From the list below, enter the crop name and code for each crop grown. 
SEETUONES LIVESTOCK and POULTAY

- PART A - CATtLE and CALVES

1. CATTLE and CALVES of all eges ........

a. BEEF COWS - includa boof hoifors that

b. MILK COWS kept for production of milk of cream for sale or home use - Include dry
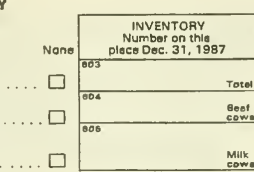

None

2. Velue of DAIRY PROOUCTS sold in 1987 Include milk, cream, butter, efe. . . . . . . .

- cattle and calyes sold FROM THIS PLACE IN 1987 include os sold cottio moved from fooding.

3. Calves less than 500 pounds

4. Cattia - Include celves 500 pounds or more

a. Of ALL cettle sold, how many were FATTENED on this place on GRAIN or CONCENTRATES for 30 days or more and SOLD for SLAUGHTER?

- PART 8 - HOGS and PIGS

1. HOGS and PIGS of all eges

c. HOGS and PIGS used or to be used for breeding
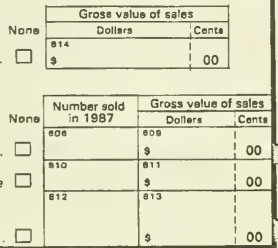

2. HOGS end PIGS SOLD from this place in $1987 . .$. .

3. Ol the hogs and pigs sold, how many were sold es FEEDER PIGS for further feeding?

- PART C - SHEEP and LAMBS

1. SHEEP and LAMBS of all ages

•. EWES 1 yeer old or older. .

2. SHEEP and LAMBS SHORN in 1987 .................

\section{None 027}

\begin{tabular}{|c|c|c|c|}
\hline \multirow[b]{2}{*}{ None } & \multirow{2}{*}{$\begin{array}{c}\text { Number sold } \\
\text { In } 1987\end{array}$} & \multicolumn{2}{|c|}{ Gross valus of seles } \\
\hline & & Dollers & Conts \\
\hline & 620 & 821 & \\
\hline & & $\$$ & 00 \\
\hline & 622 & 823 & \\
\hline & & 9 & 00 \\
\hline
\end{tabular}

None

$\square$

Number on this NUM8ER SOLD \begin{tabular}{|l|l|}
$\begin{array}{l}\text { Number on this } \\
\text { plece Dec. 31. } 1987\end{array}$ & In 1987 \\
\hline 824 & 025
\end{tabular}

820

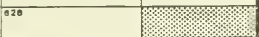

3hat was the gross velue of sales of SHEEP, LAMBS, and WOOL from this place in 1987 ?

- PART O - POULTRY

1. HENS and PULLETS

c. HENS end PULLETS of laying ege

b. FULLETS 3 montha old or older not yet of laying ege for layer replecement

c. PULLETS under 3 months old for layer replacement

2. BROILERS, fryers, other meat-type chickens

3. TURKEYS for slaughter (Do not include breaders.)

4. OTHER POULTRY (Entor name/code from bolow.)

Poutry name

Nams/codb

do Mamalcodo

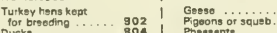

5. Value of POULTRY and POULTRY PRODUCTS leggs, etc.l sold from this place in $1987 ?$.

in $19877 \ldots . . . .4$. Codo

\begin{tabular}{|c|c|c|}
\hline & Number] & ool \\
\hline \multirow[b]{2}{*}{ None } & \multicolumn{2}{|c|}{ Gross velue of sales } \\
\hline & Dollers & Ceme \\
\hline & 828 & 00 \\
\hline
\end{tabular}

- PART E - MORSES, OTHER LIVESTOCK, ARIMIAL SPECIALTIES, and FISH

1. Horses and ponies \begin{tabular}{|l|l|}
\hline None \begin{tabular}{|c|} 
INVENTORY \\
Number on this \\
placo Doc.31, 1987
\end{tabular} & $\begin{array}{c}\text { Total quantity } \\
\text { sold in } 1987\end{array}$ \\
\hline 030 & 031 \\
\hline
\end{tabular} of all gges......

2. Colonies of bees. Grnss value of sales 1.39

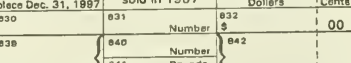

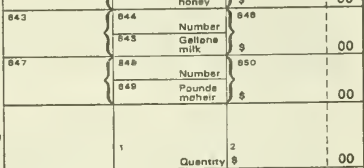

Milk gosts

4. Angora goats

5. Other livestock, fish. animal products. (Enter ngme/code from bolow.)

Name _Code C_

Nams/codo Nome/codo

and their poits 85 Other llvestock, fieh,

goots ... pits...851, 8nd their products... 857

SECTUULG] GOVERNMENT CCC LOANS Amount recelved in 1987 from Government CCC loans. Includa regular

Specity cropls) \begin{tabular}{ll:l}
\hline None & Dollars & Cents \\
\hline$\square$ & 100 \\
\hline
\end{tabular}

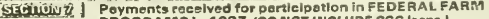
ROGRAMS In 1987 (OO NOT INCLUDE CCClOens.)

1. Amount received in cesh ......................

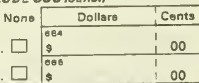
(PIK) or commodity certificates............ $\square$

SEciluAs I Acros in thla plece SET ASIDE, DIVEATED, or IDLEO under FEDEAAL scrasge raductlon programs in 1987

1. How meny ecres were set aside (or diverted) under ANNUAL commodity acreage edjustment programs?

None Number of acres

. How many acres were under the CONSERVATION RESERVE PROGRAM (10 ygar, CRP)?

SEGUUUUS \ CMARACTERISTICS ONd OCCUPATION OF OPERATOR (Senlor partnst or parson in charge)

1. RESIDENCE - Does the operator live on this ${ }^{323}$ plece?

2. PAINCIPAL OCCUPATION - At which occupation did the operator spend the majority (50 percent or more) of his/her worktime in 1987 ? For pertnerships consider

920

3. OFF-FARM WORK - How many days did the ${ }^{329}(\square$ None operator work at least 4 hours per day off this $\quad{ }^{2} \square 1-49$ days place in 1987 ? - Includo work at o nanferm job,
businoss. or on someone else's form for pay. (Do not ${ }_{3} \square$ 50-99 days

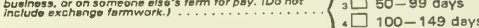
${ }^{4} \square 100-149$ days
${ }_{6} \square \quad 150-199$ days ( $\square 200$ days or more

4. In whet YEAR did the operator begin to operate ${ }^{\text {DeO }}$ In whet YEAR did the operator begin to operate
any part of this pleca? $\ldots \ldots \ldots \ldots \ldots \ldots \ldots$

5. AGE of operato

8. RACE of operator

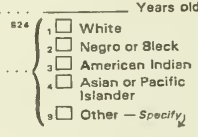

7. SEX of operato

8. SPANISH ORIGIN - Is the Operetor of Spenish ${ }^{927}$ origin or descent (Mexicen, Puerto Pican, Cuben, or other Spenish)?

$\square$ Yes $\quad{ }_{2} \square$ No SECIIÓJUUU I PERSON COMPLETING THIS REPORT - Plosso print Nomo $\left.\right|^{990}$ Doto

Telephone number 990 Deto 


\section{UNITED STATES CEMSUS OF AGRICULTURE}

\section{Special Feporâing Insâtuctions}

\section{Who Should Raport}

WE NEED A REPLY FRDM EVERYONE RECEIVING A REPORT FORM. INCLUDING individuals, landlords, tenants, partnerships, AGRICULTURAL OPEFATIONS. Each case included in the census hes a unique Census File Number (CFN). In ordar to make the censu: results as complete and accurate as possible, we need to obtain

2. If You Recelved Moro Than Ono Report Form for an Oparation Complete only ONE report form for an operation. Write "Duplicatc" near the address label of each extra report form. Also. Write the 11 digit census tile number(s) of the DUPLICATE report(s) ON THE label. Return the extra report(s) in the same envelope with your completed report form so that we can correct our records.

3. If You No Longer Farm

If you had agricultural operations at any time during 1987. please report all agricultural ectivity during the year. Report all land on your census rorm that you owned or rented. Also

Explain on the first nage of the report form for on a separate sheet of poper) thet vou quit farming or ranching and give the appraximate
date and the nome and address of the present operator, if known.

4. If You Never Farmed or Have No Association With Agricuiture Please write a note on the report torm near.the address label cxplaining this and return the form so that we can correct our records. In out efforts to make the census as complete as possible. we obtained list addresses, however, it was not always possible to do so.

5. Ir You Have More Thon One Agricuttural Operation

Complete a report form for EACH SEPARATE and DISTINCT production unit, $1 c$.. each individual farm, ranch, fecdlot. greenhouse, etc., or combination of farms, etc., for which you maintain SEPAFATE records of operating cxpenses and sales.

\section{If You Have a Portnarship Operation}

Complete only ONE report for the entire partnership's agricultural operation and include ell partners' sharcs on the one report. If members of the partnershup also operate separate farms or ranches in should be completed for each individual operation.

If two or more report forms were recelved for the same operation. mark each additional form as a "Duplicate." Return the duplicate reporits in the same envelope with the comploted partnership report. Whare possible. Or write a note on the duplicate report, such as. (provide name and CFN of partnerstup."

\section{Londlord's or Contractor's Share}

If you rented or leased land from others or had a contract for the production of agricultural products, include bott your share and the landlord's or contractor's share of the production, sales, and expenses
so your census report form will be complete for "THIS PLACE. If you do not know the landlord's or contractor's share, include vour items. use your best estumate.

8. How to Entar Your Response

Enter your replies in the propar spaces, on the correct lines, and in the units requested. ..... dollars. bushels, tons, etc. Write any explanation Alt dollar tigures may be entered in whole dollars. CENTS ARE NOT

Enter whole numbers except where tenths are requested, such as acre

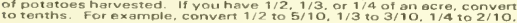
The census report form will contain sections and questions which do not apply to you. When this occurs, mark the "None" or "No" box and

\section{Instructions For Specified Sections}

\section{- Saction 2 - ACREAGE IN 1987}

Your answers to this section will determine the land (Acres in

When answering the acreage questions, include the land associated with your agricultural operations in 1987 whather in production of with your agricultural operations in 1987 whather in production or only for part of the year. Do not include any unrelated residential or commercial land

Report all land in section 2 in whole acres. ftem 1-All Land Owned - Report all land owned in 1987 whether held under title, purchased contract or mortgage, homesteed tew, or as heir or trustee of an undivided estate. Include all land owned by you and/or your spouse, or by the

Item 2 - All Land Rented or Losed FROM OTHERS - Peport all land rented by you or your operation even though the landiord may have supplied materials or supervision.

INCLUDE in rem 2 :

a. Land for agricultural use that you rented from others for cash

b. Land you worked on a share basis (crop or livestock)

c. Land owned by someone else that you used rent-free

d. Federal, State, Indian reservetion, or railroad land ranted or

DO NOT INCLUDE in item 2

Land used on a per head or animal unit license or permit besis, such as section 3 of the Taylor Grazing Act. National Forest, or Indian permit land.

Item 3 - All Land Ranted or Leased TO OTHERS - Include all land rented out for any purpose if it was part of the ocreage report in tenants to cover the operations on that land.

INCLUDE in item 3:

a. Owned land rented to others for cash or a share of crops or livestock

b. Land you rented from someone and then subleesed to someone else

c. Land worked for you by someone for a share of crops or

d. Land which you allowed others to use rent-free Item $4-$ Acres in "THIS PLACE" T This figure will show the total
of all land you operated at any time in 1987 .

If item 4, Acres in "THIS PLACE" is " $O$ " and:

a. You raised any crops or had any livestock or poultry on "THIS

b. All your land was operated by a renter or sharecropper, skip to and complete section 10, and explain briefly. "All land rented out." etc. Mail form in return envelope.

c. You did not have any agricultural activity on owned or rented land in 1987. complete section 10 and explain briefly. such os "retired." "sold farm." and date. Give nam.

\section{n Section 3 - LAND USE AND IRRIGATION}

This section is used to classify the acres in "THIS PLACE" raported in section 2, item 4. Do not include any acres you rented to others reportcd in scction 2 , item 3 . The sum of the acres entered in various categories should equal total acres in "THIS PLACE."

Land $U$ sed for More Then One Purpose - Do not report the same acreage for more than one of the listed purposes. If part or all of your land was used for more than one listed purpose in 1987, report that crop and later used the samo land for pesture, report the land in part A. item 1. "Cropland harvested."

Double Cropping - When more than one crop was harvested from , report that lend only ONCE as "Cropland harvested, " in part A, item 1 of this scction.

Interplented Crops - If you interplented crops, such as cotton in an orchard, report the total land used for both crops only ONCE. as Skip Row Planted Crops - Report the acres that represent the total ecres that represent the planted rows should bo reported as "Cropland harvested." part A, item 1 .

Irrigation is defined as land watored by artificial or controlled maans - sprinklers, furrows or ditches, spreader dikes, nurposetul łlooding, etc Include acres that receive supplemental, partial, a

\section{Section 4 - CROPS}

This section provides space for raporting crops harvasted during the 1987 crop year from the land shown in section 2, item 4 listed on the form. For these crops iust report acres harvested. quantity harvested, and value of sales. If you produced crops not and report the acres harvested, quantity harvested. and the value 
DONOT INCLUDE

- Any crops grown on land rented or leased TO OTHERS, or

b. Crops or crop products purchased from others and later sold.

Acres Harvested - Enter the acres harvested in 1987. Round Acres Harvested - Enter the acres harvasted in 1987 . Round
in the reporting box. such as potatoes.

Quantity Harvested - If your unit of measure is different then the on the report form. please convert your figure for the quantity December 31.1987 , please repor? the quantity harvestcd and the estimated quantity to be harvested.

Gross Value of Crops Sold - Report the value of all crops sold from who owned the land. Be sure to report gross value befnre deducting expenses and taxes. Include Government CCC loans received for COOPEretives or markating organizations for crops produced on "THIS

Item 7 - Vegetebles - Report acres of vegetables hervested FOR SALE or commercial processing. Do not include vegetables grown for

Itern 8 - Frult Orchards, Cierus, Vineyards, and Nut Trees home use as well as those maintained for sale of their production. Acres in trees and vines that heve been abandoned should not be included, these acres should be included in section 3, part A, item 3 "Cropland idle.

If crops other than fruit and nut trees and vines were interplanted with trees or vines. report the total acres for the orchard crop in item 8 and

Item 9 - Other Crops - To report: (1) find the crop name and the in the first two columns of the first evalable answer line under item 9 . (3) enter the information that is requested in thic remaining columns. If you harvested a crop not listed, use the "OTHER" code and specify the crop name. If you need additional space, use a separate sheet of gross value of crop(e) sold

\section{- SECtion 5 - LIVESTOCK, POULTRY, OTHER LIVESTOCK, OR}

Pats A, B, C, and D - LIVESTOCK AND POULTFY

Animals and Poultry to Include in the Report - Report al animals, poultry, and anmal specialties on "this place" (section 2 , item 4) on December 31, 1987. Include all owned by you and any kept by you for others. Include animals on unfenced lands. National Forest land, district lond. cooperative grazing association land, or rangeland administered by the Bureau of Land Management on a
per-head or lease basis. Animals in transit on December 31 . 1987 . or
anmals on a short-term pasture isuch as wheat pasture or crop residual on a per head or lease basis should be reported by the person who had control of the animals.

Animals and Poultry to Exclude from the Report - Do not report animals or poultry kept on land rented to others or kept under a share arrangement on land rented to others. Do not include enimals quertered inteedis for that place.

Animals Bought and Sold - DO NOT REPORT ANY ANIMALS and sales are considered ". W WITHIN 30 OAYS, Such purchases in this census.

Numbor Sold - Peport all enimals and poultry sold or removed from the receipts Include animals sold for to ownership or who shared in or others in trade or in payment for goods or services. Do NOT report number sold for any livestock or poultry kept on another place.

Dairy Terminetion Program or "Whole-Herd Deiry Buy-Out Program" - The amount received in 1987 from the Government under the dairy termination program should be included in section 7 .
item 1 . Dairy cattle and catves sold should be reported in section 5 .
part A.

Animals Moved to Anokher Place - For animals moved from "'thrs place : to another place, such as for further feeding. report animals a "sold" and give your best estimate of their market value when they

Fat Catzle Sold - Cattle fattened on grain or concentrates for 30 days or more and sold for slaughter are reportod in section 5 . part A.

OO NOT INCLUDE WITH FATTENED CATTLE SOLD:

a. Cattle and celves sold for further fceding

b. Dairy cows fed only the usual dairy ration betore being sold

c. Veal calves, or any calves weighing less than 500 pounds
Velue of Seles - Report the rota! gross value of animats and poultry sold or removed from "this place" in 1987 without deducting production or marketing expenses (cost of feed, cast of ivestock . when they left " this place." DO NOT report the value of sales of any livestock and poutty owned by you but kept and sold from a place you did not operate.

Contrect and Custom Feeding Operations - Livestock or poultry kept by you on "this place" On a contract or custom basis should be included on this report REGARDLESS OF OWNER the place on December 31,1987 . Report as .'SOLD. animals and poultry kept on a contract or custom basis and removed or sold from the place in 1987 . If the sale price or market value is not known, give your best estimate of

The person who furnished the housing and labor should report the pout'ryoperation on his/her report form regardless of who owned the from the place in 1987 .

\section{PaRE - HORSES, OTHER LIVESTOCK, ANIMAL}

II you owned BEES - Report ell colonies or hives of bees and honey operations conducted by you, regardless of where the hives were and value of seles.

Othar Livestock and Livestock Products - Include in all other livestock and livestock products manure, beeswax, and any other animal products sold from "this place" in 1987 . Mink pelts and rabbi inventory.

Fish and Other Aqueculture Products - Report quantity sold and gross value of salos for each.

\section{- Secion 8 - AMOUNT RECEIVEO FROM GOVERNMENT CCC}

Item 1 - Report the amount received under the regular or roserve program for commodities placed under CCC loan during 1987 Include amount received even if comr
forfeited prior to December 31,1987

Do not include CCC loans received to build crop storage facilities or amount received for storage payments in the reserve program.

- Section 7 - FEDERAL PAYMENTS RECEIVED

Report all payments received from Federal Farm Programs in 1987 regardless of whether payment was made in cash or commodity certificatcs. Includo cash payments in item 1 , in itern 2 , include th value of any certificates held or the value receivod from sale or redemption of any certificates in 1987

Federal payments include receipts from Federel programs such as deficiency payments. "Whole-Herd Dairy Buy Out," support price payments, indemnity programs, disaster payments, paid land
diversion, inventory reduction payments, payments received for approved soll and water conservation projects. etc.

- SECIION 8 - ACRES SET ASIDE, OIVERTED. OR IDLED UNDER

Include in item 2 all acres in "this place" retired from production and pleced, by long-term contract, into the Conservation Reserve Program. Acres plece

\section{SECtion 9-CHARACTERISTICS AND DCCUPATION OF}

This section collects information about the operator of "this place" defined as the individual owner, the operator, the senior partner, or person in charge.

For Family or Individual Operation - Complete this section for the operator.

For Partnership Operations - Answer all items, except item 2 , for the "Senior Partner." The "Senior Pertner" is the individual who is mainly responsible for the agricultural operations on "this plece. " not necessarily the person senior in age. If each partncr shares equally the day-to day management decisions, consider the oldest as the members of the partnership together. Please include as " farming or ranching. " worktime at all types of eqricultural enterprises, includin work et greenhouses, nurseries, mushroom production, ranching. feedlotis, broiler feeding. etc. For Corporatlon and Other Operations (Cooperatives, Estates, the on-site, day to day operation of the farm or ranch business.

Item 4 - Year Began Operation - Report the first year the operator or senior partner began to operate any part of "this place" operated, report the year operazions were resumed. 



\section{INDEX}

(Index items not reported for the State will not appear in designated tables)

\begin{tabular}{|c|c|c|c|c|c|}
\hline Item & $\begin{array}{r}\text { State } \\
\text { tables }\end{array}$ & $\begin{array}{r}\text { County } \\
\text { tables }\end{array}$ & Item & $\begin{array}{r}\text { State } \\
\text { tables }\end{array}$ & $\begin{array}{r}\text { County } \\
\text { tables }\end{array}$ \\
\hline A & & & B-Con. & & \\
\hline Abnormal farms.... & 19 & - & Bluegrass seed, & & \\
\hline Acreage reduction & & & $\begin{array}{l}\text { Kentucky................... } \\
\text { Boysenberries ............. }\end{array}$ & $\begin{array}{r}44 \\
-\end{array}$ & $\begin{array}{l}26 \\
29\end{array}$ \\
\hline 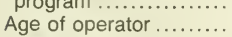 & $\begin{array}{l}7,10,48-53 \\
1,16,48-53\end{array}$ & $1,10,16$ & Breeding hogs and & & \\
\hline Agricultural products sold, & $1,10,70-30$ & , & pigs .................. & $32,48-53$ & $\begin{array}{r}12,16 \\
27\end{array}$ \\
\hline $\begin{array}{l}\text { market value........... } \\
\text { Agricultural services }\end{array}$ & $1,2,10,18,4$ & $1,2,16$ & 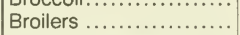 & $1,20-22,48-53$ & $1,14,16$ \\
\hline income ................. & $5,48-53$ & 4 & Bromegrass seed.. & - & 26 \\
\hline Alfalfa hay.............. & $43,44,48-53$ & 26 & Brussels sprouts... & - & 27 \\
\hline Alfalfa seed ............ & 43,44 & 26 & Buckwheat............... & - & 24 \\
\hline Almonds .................. & $45,48-53$ & 28 & 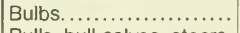 & 46 & 30 \\
\hline $\begin{array}{l}\text { American Indian } \\
\text { operator ................. }\end{array}$ & (2) & 34 & $\begin{array}{l}\text { Bulls, bull calves, steers, } \\
\text { and steer calves...... }\end{array}$ & $20,25,48-53$ & 11,16 \\
\hline Angora goats............. & 41 & 18 & Burros, donkeys, and & & \\
\hline Apples .................. & $45,48-53$ & 28 & mules $\ldots \ldots \ldots \ldots \ldots$ & 41 & 23 \\
\hline Apricots.......................... & 42 & 28 & 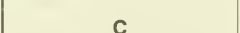 & & \\
\hline Aquacultural products .. & 41 & 21 & $C$ & & \\
\hline Artichokes .............. & - & 27 & Cabbage....... & - & 27 \\
\hline Asian or Pacific Islander & & & Cantaloups ... & 44 & 27 \\
\hline operator ........ & 17 & 34 & Carrots................... & - & 27 \\
\hline Asparagus ............... & - & 27 & Cash, government farm & & \\
\hline Assets, value ............ & $\begin{array}{r}1,10-12,18 \\
47,48-53\end{array}$ & $1,5,8,16$ & $\begin{array}{l}\text { programs payments ... } \\
\text { Cash rent, expenses.... }\end{array}$ & $\begin{array}{r}5 \\
3,10,48-53\end{array}$ & $\begin{array}{r}4 \\
3,16\end{array}$ \\
\hline Austrian winter peas.... & - & 26 & Cash rent or share & & \\
\hline Avocados......... & 45 & 28 & $\begin{array}{l}\text { payments received, } \\
\text { farm-related income... }\end{array}$ & $5,48-53$ & 4 \\
\hline B & & & Cattish sales ............. & $25.277^{-}$ & 21 \\
\hline & & & Cattle and calv & $\begin{array}{r}1,10,20,23,27,41 \\
48-53\end{array}$ & $1,11,16$ \\
\hline $\begin{array}{l}\text { Bahia grass seed ......... } \\
\text { Balers, pickup ........... }\end{array}$ & $13,48-53$ & $\begin{array}{r}26 \\
8\end{array}$ & $\begin{array}{c}\text { Cattle and calves sales, } \\
\text { value. }\end{array}$ & $\begin{array}{r}2,20,26,31,47 \\
48-53\end{array}$ & $2,11,16$ \\
\hline Bananas ................ & 45 & 28 & 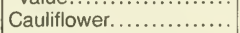 & $\begin{array}{r}20 \\
-40-80\end{array}$ & 27 \\
\hline $\begin{array}{l}\text { Barley for grain............ } \\
\text { Barley for grain sales. }\end{array}$ & $1,42-44,48-53$ & $1,15,16,24$ & Celery................... & - & 27 \\
\hline value.................... & $2,48-53$ & 2,16 & $\begin{array}{l}\text { Certificates, govern- } \\
\text { ment farm programs }\end{array}$ & & \\
\hline Beans, dry edible ....... & $42-44,48-53$ & $15,16,25$ & payments................ & & 4 \\
\hline Beans, dry lima .... & - & 25 & Chemicals, expenses... & $1,3,10,15,47,48-53$ & 3,16 \\
\hline Beans, green lima ....... & - & 27 & Chemicals used ......... & $15,48-53$ & 9 \\
\hline 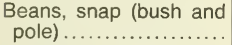 & $44,48-53$ & 27 & $\begin{array}{l}\text { Cherries. .................... } \\
\text { Chickens } 3 \text { months old }\end{array}$ & $45,48-53$ & 28 \\
\hline Beans, soybeans........ & $1,42-44,48-53$ & $1,15,16,25$ & or older......... & $1,20,21,23,48-53$ & 1,14 \\
\hline Bedding plants .... & 46 & 30 & Chicory ............ & & 27 \\
\hline Beef cows...... & $1,20,25,29,48-53$ & $1,11,16$ & Chinese cabbage... & - & 27 \\
\hline Bees, colonies .. & 41 & 20 & Chinese or ming peas.. & - & 27 \\
\hline Beets, sugar........ & $42-44,48-53$ & $1,15,16,25$ & Christmas trees and & & \\
\hline Beets, table ..... & - & 27 & forest products sales, & & \\
\hline Bentgrass seed. & - & 26 & farm-related income. & $5,48-53$ & 4 \\
\hline Bermuda grass $\mathrm{s}$ & - & 26 & Citrus fruit ... & $45,48-53$ & 28 \\
\hline Berries................. & $42,44,48-53$ & 29 & Clover seed .............. & - & 26 \\
\hline Birdsfoot trefoil seed ... & - & 26 & Coffee $\ldots \ldots \ldots \ldots \ldots \ldots$ & 45 & 28 \\
\hline Blackberries .............. & - & 29 & Collards................... & - & 27 \\
\hline $\begin{array}{l}\text { Black operators and other } \\
\text { races } \ldots \ldots \ldots \ldots \ldots \ldots \ldots . . . . . . .\end{array}$ & $16,17,48-53$ & $32-34$ & $\begin{array}{l}\text { Colonies of bees.......... } \\
\text { Combines, grain and }\end{array}$ & 41 & 20 \\
\hline Blueberries .............. & 44 & 29 & bean, all types .......... & $13,48-53$ & 16 \\
\hline
\end{tabular}




\section{INDEX-Con.}

(Index items not reported for the State will not appear in designated tables)

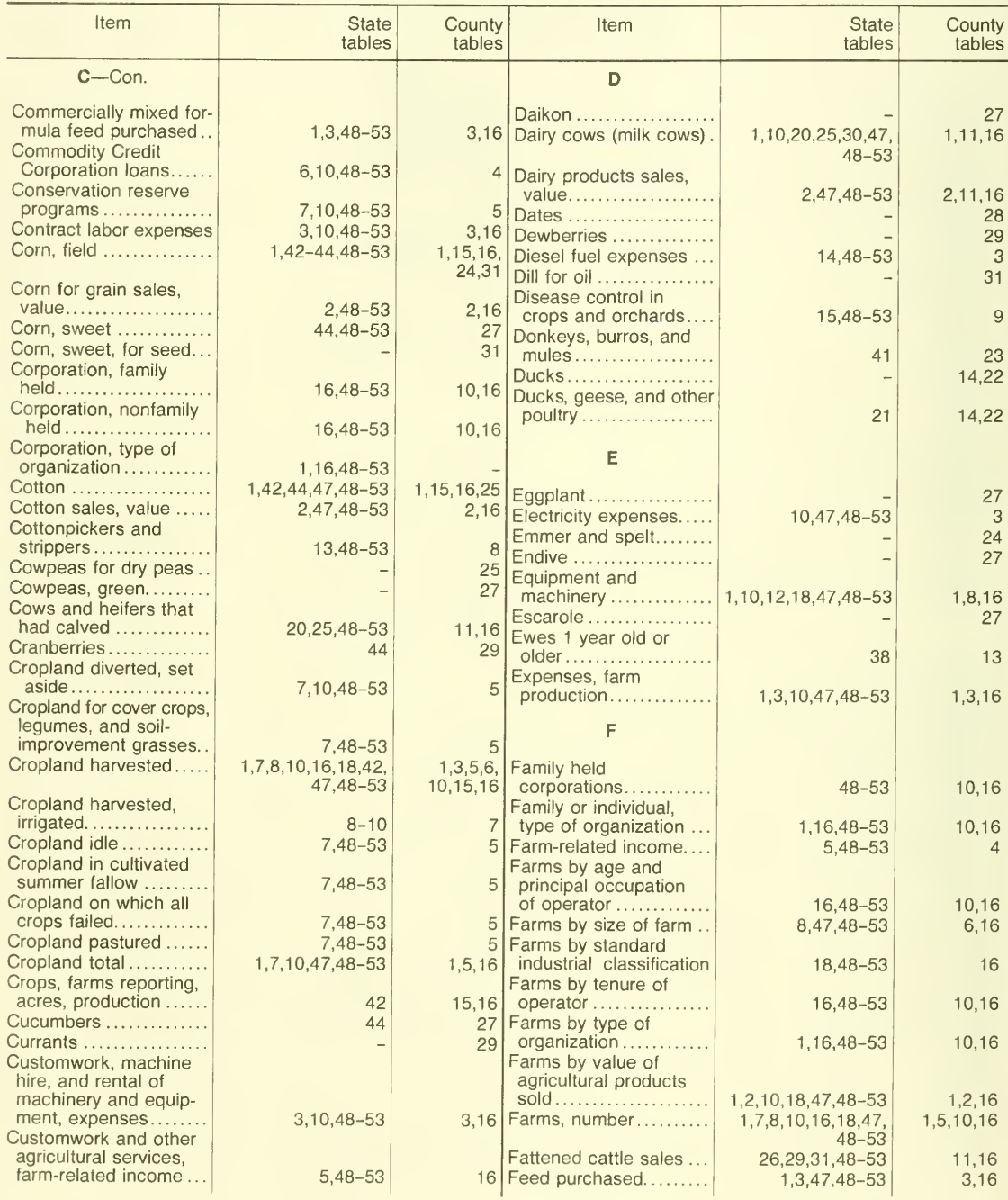


INDEX-Con.

(Index items not reported for the State will not appear in designated tables)

\begin{tabular}{|c|c|c|c|c|c|}
\hline Item & $\begin{array}{r}\text { State } \\
\text { tables }\end{array}$ & $\begin{array}{r}\text { County } \\
\text { tables }\end{array}$ & Item & $\begin{array}{r}\text { State } \\
\text { tables }\end{array}$ & $\begin{array}{r}\text { County } \\
\text { tables }\end{array}$ \\
\hline F-Con. & & & G-Con. & & \\
\hline Feeder pigs sales....... & $20,33,35-37,48-53$ & 12 & Grapes................. & $45,48-53$ & 28 \\
\hline Female operators.. & $16,17,48-53$ & 10 & Grass silage, haylage, & & \\
\hline Fertilizer applied ..... & $15,48-53$ & 9 & and green chop hay... & 43,44 & 26 \\
\hline Fertilizer expenses...... & $3,10,15,47,48-53$ & 3,16 & Grazing permits ......... & - & 36 \\
\hline Fescue seed ............. & 44 & 26 & Grease, LP gas, fuel & & \\
\hline Field seed crops ......... & $44,48-53$ & 26 & oil, kerosene, motor oil, & & \\
\hline Figs $\ldots \ldots \ldots \ldots \ldots \ldots \ldots$ & - & 28 & etc., expenses .......... & $14,48-53$ & 3 \\
\hline Filberts................. & 45 & 28 & Greenhouse crops ....... & 42,46 & 30 \\
\hline Fish sales ............... & 41 & 21 & Greenhouse vegetables & 46 & 30 \\
\hline Flaxseed $\ldots \ldots \ldots \ldots \ldots$ & $42,44,48-53$ & 24 & Guar ...................... & $\overline{-}$ & 31 \\
\hline $\begin{array}{l}\text { Florist greens and } \\
\text { flowers, cut........... }\end{array}$ & - & 30 & Guavas .................. & 45 & 28 \\
\hline $\begin{array}{l}\text { Flower and vegetable } \\
\text { seeds .................... }\end{array}$ & 46 & 30 & H & & \\
\hline Flowering plants, & & & Hatcheries... & (1) & \\
\hline $\begin{array}{l}\text { potted } \ldots \ldots \ldots \ldots \ldots \ldots \\
\text { Flowers and florist }\end{array}$ & 46 & 30 & $\begin{array}{l}\text { Hay crops } \ldots \ldots \ldots \ldots \ldots \\
\text { Hay, silage, and field }\end{array}$ & $1,42-44,48-53$ & $1,15,16,26$ \\
\hline greens, cut .............. & 46 & 30 & seeds sales, value..... & $2,47,48-53$ & 2,16 \\
\hline Foliage plants ........... & 46 & 30 & Haylage, grass silage, and & & \\
\hline $\begin{array}{l}\text { Forest products and } \\
\text { Christmas trees sales }\end{array}$ & & & green chop hay.......... & 43,44 & $\begin{array}{l}26 \\
28\end{array}$ \\
\hline farm-related income... & $5,48-53$ & 4 & Heifers and heifer & & 20 \\
\hline Foxtail millet seed ...... & & 26 & calves $\ldots \ldots \ldots \ldots$. & $20,25,48-53$ & 11,16 \\
\hline Fruit crops............... & 45 & $15,16,28$ & Hens and pullets of & & \\
\hline Fruits, nuts, and berries & & & laying age $\ldots \ldots \ldots \ldots$ & $20,21,48-53$ & 14,16 \\
\hline $\begin{array}{l}\text { sales, value } \ldots \ldots \ldots \ldots . . . \\
\text { Fuel oil, kerosene, motor }\end{array}$ & $2,47,48-53$ & 2,16 & Herbs ......................... & & 31 \\
\hline oil, grease, LP gas, etc., & & & expenses.............. & $3,10,47,48-53$ & 3,16 \\
\hline expenses................ & $14,48-53$ & 3 & Hogs and pigs.......... & $1,10,20,32,35,47$ & $1,12,16$ \\
\hline Full owners .............. & $16,48-53$ & 10,16 & & $48-53$ & \\
\hline G & & & $\begin{array}{l}\text { Hogs and pigs sales, } \\
\text { value............................. }\end{array}$ & $2,20,33,36,47,48-53$ & $2,12,16$ \\
\hline & & & Hogs, litters farrowed.. & $34,37,48-53$ & \\
\hline Garlic . & - & 27 & Honey sales............. & 41 & 20 \\
\hline Gas, natural, expenses. & $14,48-53$ & 3 & Honey tangerines ....... & - & 28 \\
\hline $\begin{array}{l}\text { Gasoline and other } \\
\text { petroleum fuel and oil }\end{array}$ & & & $\begin{array}{l}\text { Honeydew melons } \ldots \ldots \\
\text { Hops .................... }\end{array}$ & & $\begin{array}{l}27 \\
31\end{array}$ \\
\hline expenses...$\ldots \ldots \ldots \ldots$ & $14,48-53$ & 3,16 & Horses and ponies..... & $20,41,48-53$ & 13 \\
\hline Gasoline expenses & $14,48-53$ & 3 & & & \\
\hline Geese ................ & - & 22 & I & & \\
\hline $\begin{array}{l}\text { Geese, ducks, and } \\
\text { other poultry........ }\end{array}$ & 41 & $4,22, \quad ; \quad$, & & & \\
\hline Ginger root ......... & - & 31 & $\begin{array}{l}\text { Income from farm-related } \\
\text { sources }\end{array}$ & & \\
\hline Goat milk sales .... & 41 & 17 & Income, see net cash & $5,48-53$ & 10 \\
\hline Goats .............. & $41,48-53$ & 23 & return ................. & $4,48-53$ & 4 \\
\hline Goats, Angora..... & 41 & 23 & Individual or family, type & & \\
\hline Goats, milk ........ & 41 & 17 & of organization ........... & $1,16,48-53$ & 10,16 \\
\hline Goats, other ............ & 41 & 23 & Insects, chemical & & \\
\hline $\begin{array}{l}\text { Government farm } \\
\text { programs payments ... }\end{array}$ & $5,10,47,48-53$ & 4 & $\begin{array}{l}\text { control ..................... } \\
\text { Interest debt not secured }\end{array}$ & $15,48-53$ & 9 \\
\hline Grain hay ............... & 43,44 & 26 & by real estate......... & $3,48-53$ & 3 \\
\hline Grain sales, value....... & $2,47,48-53$ & 2,16 & Interest, debt secured & & \\
\hline & $44,48-53$ & 16 & by real estate ........... & $3,48-53$ & \\
\hline Grapefruit.. & 45 & 28 & Interest expenses...... & $1,3,10,47,48-53$ & 3,16 \\
\hline
\end{tabular}




\section{INDEX-Con.}

(Index items not reported for the State will not appear in designated tables)

\begin{tabular}{|c|c|c|c|c|c|}
\hline Item & $\begin{array}{r}\text { State } \\
\text { tables }\end{array}$ & $\begin{array}{r}\text { County } \\
\text { tables }\end{array}$ & Item & $\begin{array}{r}\text { State } \\
\text { tables }\end{array}$ & $\begin{array}{l}\text { County } \\
\text { tables }\end{array}$ \\
\hline I-Con. & & & M & & \\
\hline Irish potatoes....... & $1,42-44,48-53$ & $1,15,16,25$ & Macadamia nuts ........ & 45 & 28 \\
\hline $\begin{array}{l}\text { Irrigated farms and } \\
\text { acres } \ldots \ldots \ldots \ldots \ldots \ldots\end{array}$ & $1,8-10$ & 1,7 & $\begin{array}{l}\text { Machine hire, rental } \\
\text { of machinery and equip- } \\
\text { ment, and customwork }\end{array}$ & & \\
\hline$J$ & & & $\begin{array}{l}\text { expenses................ } \\
\text { Machinery and }\end{array}$ & $3,10,48-53$ & 3,16 \\
\hline Jojoba ....... & - & 31 & $\begin{array}{r}\text { equipment } \ldots \ldots \ldots \ldots \ldots \\
\text { Male operators ........... }\end{array}$ & $\begin{array}{r}1,10,12,18,47,48-53 \\
16,17,48-53\end{array}$ & $\begin{array}{r}1,8,16 \\
10\end{array}$ \\
\hline $\mathbf{K}$ & & & Mangoes.................. & - & 28 \\
\hline Kale ................. & - & 27 & Melons .................... & $2507^{-}$ & \\
\hline $\begin{array}{l}\text { Kentucky bluegrass } \\
\text { seed .................. }\end{array}$ & 44 & 26 & Milk cows (dairy cows) & $\begin{array}{r}1,10,20,25,30,47 \\
48-53\end{array}$ & $1,11,16$ \\
\hline $\begin{array}{l}\text { Kerosene, motor oil, } \\
\text { grease, LP gas, fuel oil, }\end{array}$ & & & $\begin{array}{l}\text { Milk goats } \ldots \ldots \ldots \ldots \\
\text { Millet, proso .......... }\end{array}$ & $\begin{array}{l}41 \\
44\end{array}$ & $\begin{array}{l}17 \\
24\end{array}$ \\
\hline etc., expenses ......... & $14,48-53$ & 3 & Millet seed, foxtail... & - & 26 \\
\hline Kiwifruit ......... & & 28 & Mink and their pelts..... & 41 & 19 \\
\hline Kumquats..... & - & 28 & 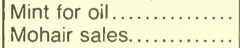 & $\begin{array}{l}44 \\
41\end{array}$ & $\begin{array}{l}31 \\
18\end{array}$ \\
\hline 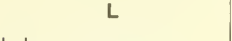 & & & $\begin{array}{l}\text { Motor oil, grease, LP } \\
\text { gas, fuel oil, kerosene, }\end{array}$ & & \\
\hline $\begin{array}{l}\text { Labor expenses.......... } \\
\text { Land and buildings, }\end{array}$ & & 3,16 & etc., expenses .......... & $14,48-53$ & 3 \\
\hline $\begin{array}{l}\text { value................ } \\
\text { Land in farms ....... }\end{array}$ & $\begin{array}{r}1,10,11,47,48-53 \\
1,7,8,10,16,18,47\end{array}$ & $\begin{array}{r}5,16 \\
1,5,10,16\end{array}$ & pickups ................. & $13,48-53$ & 8,16 \\
\hline Land owned. & $\begin{array}{r}48-53 \\
10,48-53\end{array}$ & & $\begin{array}{l}\text { Mower conditioners..... } \\
\text { Mules, burros, and }\end{array}$ & $13,48-53$ & 8 \\
\hline Land rented from others & $\begin{array}{r}10,48-53 \\
48-53\end{array}$ & - & donkeys ................ & 41 & 23 \\
\hline Land rented to others .. & $48-53$ & $\begin{array}{l}- \\
-\end{array}$ & Mungbeans for beans.. & - & 31 \\
\hline $\begin{array}{l}\text { Land set aside in federal } \\
\text { farm programs ......... }\end{array}$ & & & 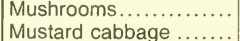 & $\begin{array}{r}46 \\
-\end{array}$ & $\begin{array}{l}30 \\
27\end{array}$ \\
\hline $\begin{array}{l}\text { farm program } \\
\text { Land use ...... }\end{array}$ & $\begin{array}{r}7,10,48-53 \\
7,87-53\end{array}$ & 1 & Mustard greens ............ & $\overline{-}$ & 27 \\
\hline Lemons........ & $\begin{array}{r}7,8,10,47,48-53 \\
45\end{array}$ & $\begin{array}{r}5 \\
28\end{array}$ & Mustard seed............ & - & 24 \\
\hline Lentils............ & 44 & $\begin{array}{l}28 \\
25\end{array}$ & 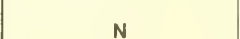 & & \\
\hline Lespedeza seed & - & 26 & & & \\
\hline Lettuce and romaine.... & 44 & 27 & Natural gas expenses. & $14,48-53$ & 3 \\
\hline Lima beans, dry.... & - & 25 & Nectarines ............... & - & 28 \\
\hline $\begin{array}{l}\text { Lima beans, green ....... } \\
\text { Lime applied............. }\end{array}$ & $1548-53$ & 27 & $\begin{array}{l}\text { Nematode control in } \\
\text { crops } \ldots \ldots \ldots \ldots \ldots \ldots \ldots . \ldots . \ldots\end{array}$ & $15,48-53$ & 9 \\
\hline Limes ............. & $\begin{array}{r}15,48-53 \\
-\end{array}$ & $\begin{array}{r}9 \\
28\end{array}$ & Net cash return from & & Y \\
\hline Litters farrowed .......... & $34,37,48-53$ & 12 & agricultural sales....... & $4,48-53$ & 4 \\
\hline $\begin{array}{l}\text { Livestock and livestock } \\
\text { products sold ........... }\end{array}$ & 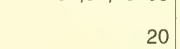 & 12 & 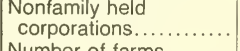 & $16,48-53$ & 10,16 \\
\hline Livestock and poultry... & 20 & 1,16 & Number of farms......... & $1,7,8,10,16,18,47$ & $1,5,10,16$ \\
\hline $\begin{array}{l}\text { Livestock and poultry } \\
\text { purchased................ }\end{array}$ & $1,3,10,47,48-53$ & 3,16 & Nursery and greenhouse & & \\
\hline $\begin{array}{l}\text { Livestock, poultry, and } \\
\text { their products sales, }\end{array}$ & & & $\begin{array}{l}\text { crops } \ldots \ldots \ldots \ldots \ldots \ldots . . . \ldots \\
\text { Nursery and greenhouse }\end{array}$ & 42,46 & 30 \\
\hline value.................. & $1,2,10,18,20,47$ & $1,2,16$ & crops sales, value.... & $2,42,46,47,48-53$ & $2,16,30$ \\
\hline $\begin{array}{l}\text { Loans, Commodity Credit } \\
\text { Corporation.............. }\end{array}$ & $6,10,48-53$ & 4 & trees, etc. ............... & 46 & 30 \\
\hline Loganberries ............ & - & 29 & 0 & & \\
\hline $\begin{array}{l}\text { LP gas, fuel oil, kerosene, } \\
\text { motor oil, grease, etc., } \\
\text { expenses ............... }\end{array}$ & $14,48-53$ & 3 & $\begin{array}{l}\text { Oat sales, value } \ldots \ldots \ldots \ldots \\
\text { Oats for grain ............ } \\
\text { Occupation of operator. }\end{array}$ & $\begin{array}{r}2,48-53 \\
1,42-44,48-53 \\
1,16,48-53\end{array}$ & $\begin{array}{r}2,16 \\
1,15,16,24 \\
1,10,16\end{array}$ \\
\hline
\end{tabular}


INDEX-Con.

(index items not reported for the State will not appear in designated tables)

\begin{tabular}{|c|c|c|c|c|c|}
\hline Item & $\begin{array}{r}\text { State } \\
\text { tables }\end{array}$ & $\begin{array}{r}\text { County } \\
\text { tables }\end{array}$ & Item & $\begin{array}{r}\text { State } \\
\text { tables }\end{array}$ & $\begin{array}{r}\text { County } \\
\text { tables }\end{array}$ \\
\hline O-Con. & & & P-Con. & & \\
\hline Off-farm work by & & & Pimientos .... & & \\
\hline operator ................. & $1,16,48-53$ & $1,10,16$ & Pineapples... & $1,42,44,48-53$ & $1,15,16,31$ \\
\hline Okra $\ldots \ldots \ldots \ldots \ldots \ldots \ldots$ & & 27 & Pistachios ... & & 28 \\
\hline & $\overline{-}$ & 28 & Plums ............ &, 45 & 28 \\
\hline $\begin{array}{l}\text { Onions, dry and green.. } \\
\text { Operator characteristics- }\end{array}$ & 44 & 27 & Pomegranates........... & - & 28 \\
\hline residence, age, race, & & & $\begin{array}{l}\text { Ponies and horses....... } \\
\text { Popcorn. }\end{array}$ & $\begin{array}{r}20,41,48-53 \\
44\end{array}$ & 13 \\
\hline $\begin{array}{l}\text { occupation, off-farm } \\
\text { work sex } \text { Spanish }\end{array}$ & & & 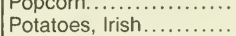 & $\begin{array}{r}1,42-44,48-53 \\
44\end{array}$ & $\begin{array}{r}24 \\
1.15,16.25\end{array}$ \\
\hline $\begin{array}{l}\text { work, sex, Spanish } \\
\text { origin, years on }\end{array}$ & & & Potatoes, sweet............ & $42,44,48-53$ & $\begin{array}{r}1,10,10,25 \\
25\end{array}$ \\
\hline present farm ........... & $16,17,48-53$ & 10,16 & Poultry and poultry & & \\
\hline Oranges. & 45 & 28 & products sales, v & $2,20,48-53$ & 2,16 \\
\hline Orchardgrass seed ..... & - & 26 & Poultry hatched ...... & - & 22 \\
\hline Orchards................ & $1,42-44,48-53$ & $1,15,16,28$ & Principal occupation & & \\
\hline Organization of farm.... & $1,16,48-53$ & 10,16 & of operator $\ldots \ldots \ldots \ldots$. & $1,16,48-53$ & $1,10,16$ \\
\hline $\begin{array}{l}\text { Other farm production } \\
\text { expenses. }\end{array}$ & $5,48-53$ & 3,16 & $\begin{array}{l}\text { Production expenses ... } \\
\text { Property taxes, }\end{array}$ & $1,3,10,47,48-53$ & $1,3,16$ \\
\hline Other field crops sales, & $40-53$ & 3,10 & expenses.............. & $3,10,48-53$ & 3,16 \\
\hline value.................. & $2,48-53$ & 2,16 & Proso millet.............. & 44 & 24 \\
\hline Other grains sales, value & $2,48-53$ & 2,16 & Prunes .................... & 45 & 28 \\
\hline Other livestock and live- & & & Pullets .............. & 22 & 14 \\
\hline $\begin{array}{l}\text { stock products sales, } \\
\text { value...................... }\end{array}$ & $2,47,48-53$ & 2,16 & Pumpkins.... & - & 27 \\
\hline Other poultry ............. & & 22 & $\mathbf{Q}$ & & \\
\hline Owned land ............... & $10,48-53$ & - & & & \\
\hline $\mathbf{P}$ & & & Quail .......... & - & 22 \\
\hline & & & $\mathbf{R}$ & & \\
\hline $\begin{array}{l}\text { Papayas ................... } \\
\text { Parsley }\end{array}$ & 45 & $\begin{array}{l}28 \\
27\end{array}$ & & & \\
\hline Part owners .. & 20 & 27 & Rabbits and their pelts . & 41 & 23 \\
\hline $\begin{array}{l}\text { Part owners .. } \\
\text { Partnership, ty }\end{array}$ & $16,48-53$ & 10,16 & Race of operator......... & $16,48-53$ & 34 \\
\hline organization & $1,16,48-53$ & 10,16 & $\begin{array}{l}\text { Radishes } \ldots \ldots \ldots \ldots \ldots \\
\text { Rangeland } \ldots \ldots \ldots \ldots \ldots \ldots\end{array}$ & $7,48-53^{-}$ & $\begin{array}{r}27 \\
5\end{array}$ \\
\hline Passion fruit.. & - & 28 & Rapeseed .................. & - & 31 \\
\hline $\begin{array}{l}\text { Pastureland and grazing } \\
\text { land. }\end{array}$ & & & Raspberries ............. & 44 & 29 \\
\hline Pastureland and other & $7,48-53$ & 5 & Redtop seed ............. & - & 26 \\
\hline land irrigated ........... & & 7 & $\begin{array}{l}\text { Rent paid in cash, } \\
\text { expenses ............. }\end{array}$ & $3,10,48-53$ & 3,16 \\
\hline Payroll expenses........ & $1,3,10,47,48-53$ & 3,16 & Rent received, farm- & & \\
\hline Peaches .......... & $45,48-53$ & 28 & related income........ & $5,48-53$ & 4 \\
\hline Peanuts for nuts & $42-44,48-53$ & $1,15,16,25$ & Repair and maintenance & & \\
\hline Pears .................. & 45 & 28 & expenses................ & $3,10,48-53$ & 3,16 \\
\hline Peas, Austrian winter ... & - & 26 & Residence of operator.. & $16,48-53$ & 10,16 \\
\hline Peas, Chinese or ming . & - & 27 & Rhubarb ................ & - & 27 \\
\hline Peas, dry edible & 44 & 25 & Rice............. & $1,42-44,48-53$ & $1,15,16,24$ \\
\hline Peas, green . & $44,48-53$ & 27 & Romaine and lettuce ... & 44 & 27 \\
\hline Pecans....... & $45,48-53$ & 28 & Rye for grain ........... & 42,44 & 24 \\
\hline Peppers....... & - & 27 & Ryegrass seed. & 44 & 26 \\
\hline Persimmons ........... & - & 28 & - & & \\
\hline Petroleum products & $01014=0$ & & $\mathbf{S}$ & & \\
\hline $\begin{array}{l}\text { expenses....... } \\
\text { Pheasants ...... }\end{array}$ & $3,10,14.48-53$ & $\begin{array}{r}3,16 \\
22\end{array}$ & & & \\
\hline $\begin{array}{l}\text { Pheasants ....... } \\
\text { Pickup balers... }\end{array}$ & $13,48-5 \overline{3}$ & $\begin{array}{r}22 \\
8\end{array}$ & Safflower ................. & - & 24 \\
\hline 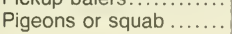 & - & $\begin{array}{r}8 \\
22\end{array}$ & $\begin{array}{l}\text { Sales of agricultural } \\
\text { products } \ldots \ldots \ldots \ldots \ldots \ldots\end{array}$ & $1,2,10,18,47,48-53$ & $1,2,16$ \\
\hline
\end{tabular}




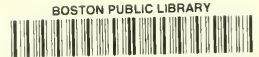 \\ 39999063135485 INDEX-Con.}

(Index items not reported for the State will not appear in designated tables)

\begin{tabular}{|c|c|c|c|c|c|}
\hline Item & $\begin{array}{r}\text { State } \\
\text { tables }\end{array}$ & $\begin{array}{r}\text { County } \\
\text { tables }\end{array}$ & Item & $\begin{array}{r}\text { State } \\
\text { tables }\end{array}$ & $\begin{array}{r}\text { County } \\
\text { tables }\end{array}$ \\
\hline S-Con. & & & $\mathbf{T}$-Con. & & \\
\hline Salt hay....... & - & 31 & Tenure of operator....... & $16,48-53$ & 10,16 \\
\hline Seeds, bulbs, plants, and & & & Timothy seed.............. & & 26 \\
\hline trees purchase & $3,10,48-53$ & 3 & Tobacco ......... & $1,42-44,48-53$ & $1,15,16,25$ \\
\hline Set aside programs, & & & Tobacco sales, value. & $2,47,48-53$ & 2,16 \\
\hline acreage................. & $7,10,48-53$ & 5 & Tomatoes.............. & $44,48-53$ & 27 \\
\hline Sex of operator .......... & $16,17,48-53$ & 10 & Tractors, wheel. . & $13,48-53$ & 8,16 \\
\hline Shallots................ & & 27 & Triticale .......... & - & 24 \\
\hline Sheep and lambs ....... & $10,20,38,39,48-53$ & $1,13,16$ & Trout sales ...... & - & 21 \\
\hline Sheep and lambs shorn & $38,48-53$ & 13 & Trucks, including & & \\
\hline $\begin{array}{l}\text { Sheep, lambs, and wool } \\
\text { sales, value ............ }\end{array}$ & $8,47,48-53$ & 13,16 & pickups ........... & $\begin{array}{l}13,48-53 \\
24,48-53\end{array}$ & $\begin{array}{r}8,16 \\
14\end{array}$ \\
\hline Size of farm, average... & $1,48-53$ & 1,16 & Turnip greens & $\begin{array}{r}1,24,48-53 \\
-\end{array}$ & $\begin{array}{l}14 \\
27\end{array}$ \\
\hline Small grain hay .......... & 43,44 & 26 & Turnips............... & - & 27 \\
\hline $\begin{array}{l}\text { Snap beans, bush and } \\
\text { pole....................... }\end{array}$ & -53 & & Type of farm ............ & $18,48-53$ & 16 \\
\hline Sod ..................... & $\begin{array}{r}44,48-53 \\
46\end{array}$ & 30 & Type of organization.... & $1,16,48-53$ & $1,10,16$ \\
\hline Sorghum.. & $1,42-44,48-53$ & $\begin{array}{r}1,15,16,24 \\
26,31\end{array}$ & V & & \\
\hline $\begin{array}{l}\text { Sorghum for grain sales, } \\
\text { value........................ }\end{array}$ & $2,48-53$ & 20,01 & $\begin{array}{l}\text { Value of agricultural } \\
\text { products sold ....... }\end{array}$ & $1,2,10,18,47,48-53$ & $1,2,16$ \\
\hline $\begin{array}{l}\text { Southern peas } \\
\text { (cowpeas), dry ................ }\end{array}$ & $2,70-30$ & 25 & $\begin{array}{l}\text { Value of land and } \\
\text { buildings } \ldots \ldots \ldots \ldots \ldots . . . . .\end{array}$ & $1,10,18,48-53$ & 5,16 \\
\hline $\begin{array}{l}\text { Southern peas } \\
\text { (cowpeas), green... }\end{array}$ & - & 27 & $\begin{array}{l}\text { Value of machinery } \\
\text { and equipment......... }\end{array}$ & $1,10,12,18,47,48-53$ & $1,8,16$ \\
\hline Soybeans............... & $1,42-44,48-53$ & $1,15,16,25$ & $\begin{array}{l}\text { Vegetable and flower } \\
\text { seeds. }\end{array}$ & 46 & 30 \\
\hline $\begin{array}{l}\text { Soybeans sales, value.. } \\
\text { Spanish origin, }\end{array}$ & $2,48-53$ & 2,16 & Vegetables, greenhouse & $\begin{array}{l}40 \\
46\end{array}$ & 30 \\
\hline operators of ............ & $16,17,48-53$ & 35 & $\begin{array}{l}\text { Vegetables harvested for } \\
\text { sale }\end{array}$ & $142-44,48-53$ & 1151627 \\
\hline $\begin{array}{l}\text { Spelt and emmer ........ } \\
\text { Spinach }\end{array}$ & - & 24 & Vegetables, sweet corn, & & $1,15,10,21$ \\
\hline $\begin{array}{l}\text { Spinach } \ldots \ldots \ldots \ldots \ldots \ldots \\
\text { Squash } \ldots \ldots \ldots \ldots \ldots \ldots \ldots\end{array}$ & - & 27 & and melons sales, value & $2,47,48-53$ & 2,16 \\
\hline Standard industrial & & & Vetch seed $. . . \ldots \ldots \ldots . .$. & & 26 \\
\hline $\begin{array}{l}\text { classification of } \\
\text { Steers, steer calve }\end{array}$ & $18,48-53$ & 2,16 & w & & \\
\hline & 20,$25 ; 48-53$ & 11,16 & Walnuts, Engl & $45,48-53$ & 28 \\
\hline Strawberries... & 43,44 & 29 & Wate & - & 27 \\
\hline Sudangrass seed & & 26 & Watermelons & 44 & 27 \\
\hline Sugar beets ....... & $42-44,48-53$ & $1,15,16,25$ & Weeds, chemical & & \\
\hline Sugarcane....... & $1,42-44,48-53$ & $1,15,16,25$ & control........ & $15,48-53$ & 9 \\
\hline Sunflower seed & $1,42,44,48-53$ & $1,15,16,24$ & Wheat for grain & $1,42-44,48-53$ & $1,15,16,24$ \\
\hline Sweet corn.... & $44 ; 48-53$ & 27 & Wheat sales, value & $2,48-53$ & 2,16 \\
\hline Sweet corn for & & 31 & Wheatgrass se & - & 26 \\
\hline Sweet potatoes & $42,44,48-53$ & 25 & Wheel tractors ........... & $13,48-53$ & 8,16 \\
\hline $\mathbf{T}$ & & & $\begin{array}{l}\text { Wild hay } \ldots \ldots \ldots \ldots \ldots \\
\text { Wild rice } \ldots \ldots \ldots \ldots\end{array}$ & & $\begin{array}{l}26 \\
24\end{array}$ \\
\hline & & & Woodland......................... & $1,7,48-53$ & 5 \\
\hline Tame dry hay & 43,44 & 16,26 & Wool, pounds shorn .... & $38,48-53$ & 13 \\
\hline Tangelos....... & & 28 & Work off-farm by & & \\
\hline $\begin{array}{l}\text { Tangerines .... } \\
\text { Taro........... }\end{array}$ & - & $\begin{array}{l}28 \\
31\end{array}$ & operator ................ & $1,16,48-53$ & $1,10,16$ \\
\hline $\begin{array}{l}\text { Taxes, property, } \\
\text { expenses........... }\end{array}$ & & 3 & $\mathbf{Y}$ & & \\
\hline Tenant operated farms . & $16,48-53$ & 10,16 & Years on present farm. . & $47,48-53$ & 16 \\
\hline
\end{tabular}




\section{CENSUS OF AGRICULTURE}

Results of the 1987 Census of Agriculture are being published in a series of reports which provide data for each county (or equivalent), each State, the United States, Puerto Rico, Guam, the Virgin Islands of the United States, American Samoa, and the Northern Mariana Islands. The publications include statistics on the number of farms; land in farms; farm and operator characteristics; livestock, poultry, and their products; crop production and value; operating expenditures; irrigation; and other characteristics of farms.

Publication order forms may be obtained from Data User Services Division, Customer Services, Bureau of the Census, Washington, DC 20233, any U.S. Department of Commerce district office, or by calling (301) 763-1113.

\begin{abstract}
ADVANCE REPORTS (AC87-A-01-000(A) TO 56-000(A)
Advance Reports are published separately for each county (or equivalent) in the United States with 10 farms or more, for each State, and the United States. The reports contain data for all agricultural operations with $\$ 1,000$ or more in actual or potential sales of agricultural products in the census year. The Advance Reports contain final data for major data items together with comparable data from the 1982 census. Included in the reports are data on number of farms, land in farms, size of farms, land use practices, farm operator characteristics, sales expenditures, machinery and equipment, livestock, poultry, dairy products sold, and major crops harvested (which vary by State). No advance reports are available for Puerto Rico, Guam, or the U.S. Virgin Islands.
\end{abstract}

\section{VOLUME 1. GEOGRAPHIC AREA SERIES (AC87-A-1 TO 56)}

State and County Data (A-1 to 50 ) are published showing detailed data in national and State tables for the United States, and in county and State tables separately for each State. These reports include data on number and size of farms; crop production; livestock, poultry, and their products; tenure, age, and occupation of operators; types of organization; value of products sold; and standard industrial classification of farms.

\section{Summary and State Data (A-51)}

- Chapter 1. National level data

- Chapter 2. State level data

Outlying Areas (A-52 to 56) provide detailed data for the regions and municipios of Puerto Rico; the election districts of Guam; the U.S. Virgin Islands; American Samoa; and Northern Mariana Islands.

\section{VOLUME 2. SUBJECT SERIES (AC87-S-1 TO 6)}

Agricultural Atlas of the United States (AC87-S-1), formerly the Graphic Summary, presents a profile of the Nation's agriculture in a series of dot and multicolor pattern maps. The maps provide displays on size and type of farm, land use, farm tenure, market value of products sold, crops harvested, livestock inventories, and other characteristics of farms.

Coverage Evaluation (AC87-S-2) provides national and regional level estimates on the completeness of the census, in terms of both the number of farms missed and selected characteristics of those farms.

Ranking of States and Counties (AC87-S-3) presents the ranking of the top 20 States and the top 100 counties of importance of selected items from the 1987 census. Comparative data from the 1982 census are included in most tables. Tables also show cumulative totals for States and counties.
History (AC87-S-4) is a concise description of the major census operations together with facsimiles of selected data tables. It explains the history of the agriculture census, farm definition, data collection and processing, and dissemination of census data.

Government Payments and Market Value of Agricultural Products Sold (AC87-S-5) shows detailed data for farms crosstabulated by combined market value of agricultural products sold and Government payments received, including detailed national data and selected data for each State.

ZIP Code Tabulations of Selected Items From the 1987 Census of Agriculture (AC87-S-6) provides tabulations by fivedigit ZIP Code for selected items from the 1987 census. Data items include number of farms, land in farms, farms by size, market value of agricultural products sold by size of sale. livestock inventory, cropland harvested, and selected crops.

\section{VOLUME 3. RELATED SURVEYS (AC87-RS-1 AND 2)}

The Farm and Ranch Irrigation Survey (AC87-RS-1) provides statistical data collected from a sample of farm operations from the 1987 Census of Agriculture. The publication offers information on acres irrigated, land use, yields of specified crops, methods of water distribution, quantity of water used by its source, and other irrigation practices.

Agricultural Economics and Land Ownership Survey (AC87. RS-2) provides data on indebtedness, expenditures, income and assets for both farm operators and landlords. This report also includes measures of credit used for purchases and expenditures, debt by type of lender, assets, off-farm income, and other land ownership data.

\section{VOLUME 4. CENSUS OF HORTICULTURAL SPECIALTIES (AC87-HOR-1)}

This report includes detailed information on the horticultural establishments with production and sales of $\$ 2,000$ or more. It provides data on number of establishments, value of sales of horticultural products, type of horticultural products, and kinds of horticultural businesses, for the United States, States, and counties.

\section{ELECTRONIC MEDIA}

Flexible Diskette-The Advance Reports of the 1987 Census of Agriculture are available on flexible diskettes. The files can be used with any compatible microcomputer employing the PC-DOS 2.0 or higher operating system. Diskettes can be obtained by calling (301) 763-4100.

Computer Tapes-Public-use computer tapes contain the same summary statistics that are found in the published reports. Two files are available for each State: data for counties and the aggregated State-level data. Order forms may be obtained from the Data User Services Division, Customer Services, Bureau of the Census, Washington, DC 20233 (or call (301) 763-4100).

Compact Disc-Read Only Memory (CD-ROM) - Data for the conterminous United States and Puerto Rico are available on CD-ROM. The CD-ROM can be obtained from the Data User Services Division, Customer Services, Bureau of the Census, Washington, DC 20233 (or call (301) 763-4100).

Online Access - National and State level data from the 1987 Census of Agriculture are available on CENDATA through two information vendors-CompuServe and DIALOG. In addition, the advance reports, highlights of the Subject Series, and Related Surveys reports, are available online from AGRIDATA. For information on these services call (301) 763-4100. 
$\lambda$ 